\title{
Cohomology with Polynomial Growth and Completion Theory
}

\author{
By \\ Nobuo SASAKURA*
}

\begin{abstract}
Contents
Introduction

Chapter I. Cohomology with polynomial growth and completion theory ..... 375

§1. Cohomology with polynomial growth ...................... 378

§2. Cohomology with algebraic division and polynomial growth.. 406

\$3. Application to analytic de Rham theory ..................... 425

Chapter II. Uniform estimations on homomorphisms of coherent sheaves..... 432

§4. Uniform estimations with bound and algebraic divisions ... 433

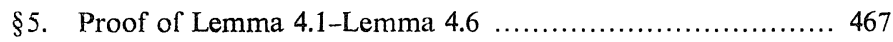

Appendix I. Elementary computations - 1

Chapter III. Polynomial growth uniform estimations for the structure sheaves of complex euclidean spaces …..................................... 500

$\S 6$. Cousin integrals and spectral sequences ...................... 501

$\S 7$. A sharpening of degeneracy theorem and polynomial growth uniform estimations ..................................... 521

Appendix 11. Elementary comp ttations - 2
\end{abstract}

\section{Introduction}

1. As the title indicates, the main purpose of the present paper is to give a unification of the following two basic theories for coherent sheaves on analytic varieties: (1) a type of cohomology theory, in which what we call polynomial growth $(=p . g$.) conditions on cochains and coverings are involved and (2) completion theory along subvarieties of a given analytic variety. Our theory is given to (algebraic) affine varieties and their analytic analogues (n.1, $\S 1.2$ ), which are more general than the affine varieties. The main body of this paper is devoted to certain explicit uniform estimations on p.g. and what we call

Communicated by S. Nakano, Novenber 15, 1979.

* Department of Mathematics, Tokyo Metropolitan University, Fukazawa, Setagaya, Tokyo 158, Japan. 
algebraic division (=a.d.) properties of coherent sheaves on such varieties (n.2). Our main application of such uniform estimations is to give:

(1) an analogue of Theorems A, B of H. Cartan in our unified cohomology theory for the two theories mentioned just above, which we simply call 'cohomology with p.g. in completion theory' (cf. Theorems 2.5, 2.6 and Theorems $1.5,1.6)$.

We will apply such a result to generalize, to varicties with singularities, the well known theorems of A. Grothendieck on algebraic and analytic de Rham theory (cf. [5]). This paper is originally developed*) to provide an analytic base of the generalization as above, in such a manner that the Stein and algebraic properties, which may be the most important properties of the varieties as above, reflect closely in getting the generalization. Our explicit formulations in (1) and in our uniform estimations are so chosen, to certain degrees, that they are convenient for the applications to the de Rham theory.

2. Concerning the two quantitative properties mentioned in $n .1$, the first one 'p.g.' is a synonym for 'rational' or 'meromorphic', when the degree $q$ of the cohomology groups is zero (Theorem 1.5), and such a property concerns most basic properties of algebraic and analytic varieties. When $q \geqq 1$, our treatments of the p.g. cohomology (=cohomology with p.g.) theory may be $a$ sharpening of purely' algebraic treatments of coherent sheaves $(\S 1)$. By the second one, algebraic division (=a.d.) property, we mean such a property that concerns the degrees of zeros of cochains etc. along (imbedded) subvarieties of an analytic variety. As we learn from the classical Hilbert zero point theorem, such a property concerns basic properties of the imbedded varieties, and is important for investigations of analytic varieties. Now our studies of the a.d. properties will be focussed on what we call open map properties (Definition 2.1) of geometric filtered complexes, such as Cech and de Rham complexes of global nature $(\S 2, \S 3)$ as well as certain local complexes formed from homomorphisms of coherent sheaves $(\S 4)$, where the filtered structures are defined by the powers of the ideals of the subvarieties. The open map property concerns that property of the degree one map in question (\$2.1), and implies standard comparison theorems in completion theories (cf. [13]). In particular, it insures:

*) Cf. $[15]_{1}$. 
(2) exactness of complexes $\rightarrow$ that of the completion of the complexes.

The open map property for the Čech complexes will insure the analogue of Theorems A, B mentioned in (1), n.1, while that for the other complexes will concern interesting a.d. properties of analytic varieties $(\S 3, \S 4$ and $\S 5$ ). Our main task in the uniform estimations is to combine those on open map properties with those on the p.g. properties of the above complexes.

Now letting the type of our cohomology theories be as above, we summarizc briefly the content of this paper.

3. Chapter I contains the basic notions and the main results of this paper. First, in Section 1.1, we summarize basic notions which are used in our p.g. cohomology theory. In Section 1.2 we give our main results in the p.g. uniform estimations (Theorems 1.1-1.4), and we derive from them an analogue of Theorems A, B in our p.g. cohomology theory (Theorem 1.5, Theorem 1.6). The cohomology theory in Section 1 concerns the p.g. properties of the complexes but not with the a.d. properties, and Theorem 1.5, Theorem 1.6 may be regarded as a prototype of the result mentioned in (1), n.1. Our proof of Theorems 1.1-1.6 will be given by using a p.g. version of standard tools for treatments of coherent sheaves, syzygies, imbedding of analytic varieties as well as extensions of cochains, and a p.g. uniform estimation on Cousin integrals (cf. Lemma 1.2-Lemma 1.4 and Theorem 1.7 in § 1.3).

Cohomology theories with p.g. conditions were studied by P. DeligneG. Maliotionist [11] and by M. Cornalba-P. A. Griffiths [2] for locally free sheaves over smooth algebraic varieties, by using the $\bar{\partial}$-estimations. The situation in our p.g. cohomology theory, where we work with what we call 'p.g. coherent sheaves (Definition 1.5)' over the analytic varieties as in 1.1 , is more general than theirs. Our method depending on Cousin integrals differ from theirs. Next, in Section 2, we generalize the p.g. uniform estimations by combining them with uniform estimations on the a.d. properties of the complexes in question. The main results in this generalization, which we call $d . p$. $(=a . d$. + p.g.) uniform estimation, as well as in the uniform estimation of this paper are given in Theorems 2.1-2.4. Such results insure the open map properties of the complexes, and our analogue of Theorems A, B in the p.g. cohomology in the completions (cf. (1), n.1) is a formal consequence of them.

4. In the first part of Chapter II, we summarize our non cohomological uniform estimations on homomorphisms of coherent sheaves (cf. §4.1). Wc 
then give a cohomological version of those estimations, and we derive, from such cohomological results, all the lemmas in Chapters I, II which concern the uniform estimations on the sheaf homomorphisms (§4.2). The uniform estimations in Section 4.1 contain results on the open map property of certain Koszul complexes, which provide a cohomological generalization of Hilber zero point theorem and are a non cohomological version of the main lemma, Lemma 2.5, in the d.p. uniform estimations in Section 2 (cf. Lemma 4.2-Lemma 4.4). Such a fact, together with an open map property of the de Rham complex (Lemma 4.7), is our main result on the open map property of the sheaf homomorphisms, and may be worthwhile pointing out in connection with our treatments of completion theories.

5. Finally, in Chapter III, we prove the key theorem, Theorem 1.7, for the geometric arguments in Section 1, Section 2 (as was indicated in Section 3), which concerns a p.g. uniform estimation of the structure sheaves of the complex euclidean spaces. We prove Theorem 1.7, by reducing it to rather elementary p.g. estimations on Cousin integrals (on the euclidean line). Our reduction depends on certain filtrations defined for the sets of the cochains (in question) and some algebraic machinaries for the filtrations, which imply a strong sharpening of the standard degerency theorem in the spectral sequence theory. The algebraic arguments and the p.g. estimations on Cousin integrals in Chapter III may owe their own interests, aparting from the applications to Chapters I, II. (For the content of Chapter III indicated soon above, see the beginning of Chapter III. We add a brief outline of Chapters I-III in the beginning of each chapter. Such an introduction may be useful for understanding of the content of each chapter and of the whole line taken in this paper.)

6. In giving the application of the cohomology theories in Section 1, Section 2 to the analytic de Rham theory ( 3 ), we should quote our results on the $C^{\infty}$-de Rham theory for certain stratified spaces, whose outline was given in $[15]_{2-4}$ and in [17]. The details of [15] ${ }_{2-4}$ and [17] will be published elsewhere in a near future. Except the part of the application to the de Rham theory in Section 3, this paper is completely self contained.

7. The author began the study of the contents of this paper and of the analytic de Rham theory in 1971, and the very early versions of the content of this paper were given in $[15]_{1}$ and [16]. Considerable parts of the explicit computations in the uniform estimation of the present paper depend on [16]. 
However, the present paper is written entirely newly from [16]. Finally, the contents of the present paper seem to deserve to be generalized in more general situations: our p.g. cohomology theory is given in a more or less categorical form. Generalizations of the content of Section 1 seems to be very desirable in that line (cf. n.6, § 1.2). Assuming the p.g. cohomology theory in Section 1, the most important facts in giving the d.p. cohomology theory are the open map properties of the geometric complexes mentioned hitherto. From the scope of the arguments in Section 2, the validity of the open map property as well as the clarifications of their geometric meanings seems to be desirable for more general classes of geometric complexes. Finally, our explicit p.g. uniform estimations and the algebraic machinaries in Chapter III seem to deserve to be tried their appiicabilities for more general types of 'cohomology with growth conditions'. The author hopes that he will try possible generalizations about what are mentioned above. We also hope that the contents of the present paper provide a basis for possible generalizations.

\section{Chapter $\mathbb{1}$. Cohomology with Polymomial Growth and Completion Theory}

Here, for convenience of reading of Chapter 1, we illustrate the basic notions and the styles of the formulations in our p.g. uniform estimations. For this we first let $\mathbb{C}^{n}$ be a complex euclidean space and $z$ coordinates of it. We set $g:=|z|+1, \mathfrak{D}:=$ structure sheaf of $\mathbb{C}^{n}$, and, for an element $\alpha=\left(\alpha_{1}, \alpha_{2}\right) \in \mathbb{R}^{+2}$, we define:

$$
\left\{\begin{array}{l}
H^{0}\left(\mathbb{C}^{n}, \mathfrak{D} ; g\right)_{\alpha}:=\left\{\varphi \in \Gamma\left(\mathbb{C}^{n}, \mathfrak{D}\right) ;|\varphi(P)|<\alpha_{1} g(P)^{\alpha_{2}} \text { in } \mathbb{C}^{n}\right\}, \\
H^{0}\left(\mathbb{C}^{n}, \mathfrak{D} ; g\right)_{\mathrm{p} . \mathrm{g}}:=\cup_{\alpha \in \mathbb{R}^{+2}} H^{0}\left(\mathbb{C}^{n}, \mathfrak{D} ; g\right)_{\alpha} .
\end{array}\right.
$$

We then recall that a classical consequence of Cauchy integral formula implies:

$$
H^{0}\left(\mathbb{C}^{n}, \mathfrak{D} ; g\right)_{\text {p.g }} \cong\{\text { polynomials in } z\} \text {. }
$$

Next letting $X$ be a complex space, we may say that

$$
\left\{\begin{array}{l}
\operatorname{Coh}(X) \\
\operatorname{Cov}(X)
\end{array}\right\}:=\text { collection of all }\left\{\begin{array}{l}
\text { coherent sheaves over } X \\
\text { open coverings of } X
\end{array}\right\}
$$

and the cochain map

$$
C^{q}: \operatorname{Coh}(X) \times \operatorname{Cov}(X) \ni(\widetilde{e s}, \mathscr{A}) \longrightarrow C^{q}(\mathscr{A}, \mathbb{E})
$$

constitute the underlying data for the cohomology theories of coherent sheaves 
over $X$. Now our first task in Section 1 is to give p.g. versions of $(0)_{2},(0)_{3}$, which yield a generalization of the sets in $(0)_{1}$, with respect to the underlying varieties, coherent sheaves and cohomology degrees. Our main results in Section 1 for the case of the degree $=0$ are a generalization of $(0)_{1}^{\prime}$, while those for degree $\geqq 1$ insures the vanishing property of the cohomology groups*): in Section 1.1 we introduce some abstract notions, p.g. filtration, $q$-structure $e^{* *)}$ of abelian sheaves and p.g. functions; we see that such notions suffice to generalize the sets as in $(0)_{1}$ to general abelian sheaves (Definition 1.45) and to give a p.g. version of $\operatorname{Coh}(X)$, denoted by $\operatorname{Coh}(X)_{\mathrm{p} . \mathrm{g}}$, to reduced complex spaces. Also, using the p.g. and a 'distance function' of a topological space $X$, we define for each subset $Y$ of $X$ what we call ' $p . g$. covering of $Y$ in $X$ ', in a concrete p.g. fashion (Definition 1.7).

In the first part of Section 1.2, we attach, to our analytic varieties in the main body of Chapter I (cf. n.1, §1.2), a p.g. version of $\operatorname{Cov}(X)$, denoted by $\operatorname{Cov}(X)_{\text {p.g }}$, by using the arguments in Section 1.1. Next take a p.g. sheaf $\mathfrak{H}$ $\in \operatorname{Coh}(X)_{\text {p.g. }}$ Then, using a p.g. version of the cochain map as in $(0)_{3}$, we attach to $\mathfrak{H}$ what we call 'p.g. cochain collection', $\mathbb{C}^{*}(X, \mathfrak{H})_{\mathrm{p} . \mathrm{g}}$ in symbol. Such a p.g. collection contains all necessary sets of p.g. cochains in our p.g. uniform estimations, and may be the most basic underlying data for our p.g. cohomology theory. We note that the above p.g. collections, $\operatorname{Cov}(X)_{\mathrm{p} . \mathrm{g}}$ and $C^{*}(X, \mathfrak{H})_{\mathrm{p} . \mathrm{g}}$, are parametrized in a certain concrete fashion, where the main part of the parameter space is a product $\boldsymbol{R}^{+s}(s>0)$; we define what we call p.g. estimation maps, which are a concrete transformation of $\boldsymbol{R}^{+s}(s>0)$. The main results in Section 1, Theorems 1.1-1.6, are given to the p.g. cochain collections. We use the p.g. estimation maps for the explicit estimations in those results.

In Section 2 we generalize the p.g. cohomology theory in Section 1 to what we called the d.p. cohomology theory (cf. Introduction). We give a generalization of the p.g. cochain collection, which we call d.p. cochain collection (\$2.2). The main uniform estimations, Theorems 2.1-2.4, of Section 2 as well as of this paper are formulated in terms of the latter cochain collections (\$2.2). The content of Section 2 is more general than the one of Section 1, by the introduction of the new factor of what we called the a.d. estimations (cf. Introduction). However, the algebraic style of the formulations in Section 2 will be given

*) Cf. Theorem 1.5 and Theorem 1.6. Also see Introduction.

**) This is an obvious abstraction of the 'absolute value' as in $(0)_{1}$ to general abelian sheaves (cf. Definition 1.41). 
parallelly to the one in Section 1.

\section{Terminologies and Notations}

Here we summarize some terminologies and notations, which are used throughout the present paper.

1. First letting $X$ be a topological space, we set

$$
\left\{\begin{array}{l}
\operatorname{Ouv}(X) \\
\operatorname{Ab}(X)
\end{array}\right\}:=\text { collection of all }\left\{\begin{array}{l}
\text { open sets of } X \\
\text { abelian sheaves over } X
\end{array}\right\}
$$

For an element $\mathscr{A}=\left\{A_{\mu}\right\} \in \operatorname{Cov}_{0}(X)$ we set $|\mathscr{A}|=\cap_{\mu} A_{\mu}$. We use the symbol $\mathscr{N}^{q} \mathscr{A}(q \geqq 1)$ for the $q$-nerve of $\mathscr{A}$ :

$$
\mathscr{N}^{q} \mathscr{A}:=\left\{\mathscr{A}_{\mu}=\left(A_{1} \prec \cdots \prec A_{q}\right) \subset \mathscr{A} ;\left|\mathscr{A}_{\mu}\right| \neq \phi\right\} .
$$

2. For a positive number $a$, we set:

$$
\mathbb{R}_{a}^{+}:=\left\{r \in \mathbb{R}^{+} ; r \geqq a\right\} .
$$

We use the set $\boldsymbol{R}_{1}^{+}$frequently in the uniform estimations in this paper. Also we use the symbol $\boldsymbol{R}^{+}$, as usual, for the set $\{r \in \mathbb{R} ; r>0\}$. Moreover, for a subset $\boldsymbol{T}$ of $\boldsymbol{R}$, we use the set:

$$
\mathbb{T}_{a}^{+}:=\{t \in \mathbf{T} ; t \geqq a\} .
$$

We use such a set for the case $\boldsymbol{T}=\mathbb{Z}=$ set of all integers (cf. Chapter II). Next, for an element $\sigma=\left(\sigma_{1}, \sigma_{2}\right) \in \boldsymbol{R}_{1}^{+2}$, we define:

$$
\boldsymbol{R}_{\sigma}^{+2}:=\left\{\left(r_{1}, r_{2}\right) \in \mathbb{R}^{+} \times \mathbb{R}^{+} ; r_{i} \geqq \sigma_{i}(i=1,2)\right\} .
$$

When $\sigma=(1,1)$, we use the symbol $\boldsymbol{R}_{1}^{+2}$ also for $\mathbb{R}_{\sigma}^{+2}$. (This symbol is concordant to $\boldsymbol{R}_{1}^{+2}=\boldsymbol{R}_{1}^{+} \times \boldsymbol{R}_{1}^{+}$(cf. (2) $\left.)_{1}\right)$ ). Thirdly, for elements $\alpha=\left(\alpha_{1}, \alpha_{2}\right) \in \mathbb{R}^{+2}$ and $a \in \boldsymbol{R}^{+}$, we set:

$$
\alpha a:=\alpha_{1} a^{\alpha_{2}} .
$$

Moreover, for elements $\sigma=\left(\sigma_{1}, \sigma_{2}\right), \sigma^{\prime}=\left(\sigma_{1}^{\prime}, \sigma_{2}^{\prime}\right) \in \mathbb{R}^{+2}$, we write $\sigma \geqq \sigma^{\prime}$, if $\sigma_{1} \geqq \sigma_{1}^{\prime}$ and $\sigma_{2} \geqq \sigma_{2}^{\prime}$. When $\sigma_{1}>\sigma_{1}^{\prime}$ or $\sigma_{2}>\sigma_{2}^{\prime}$, we write $\sigma>\sigma^{\prime}$. 


\section{$\S 1$. Cohomology with Polynomial Growth}

\section{§1.1. Polynomial Growth Conditions}

1. Cochain collection. First letting $X$ be a topological space and $(\mathscr{A}, \mathfrak{F})$ an element of $\operatorname{Cov}_{0}(X) \times \mathrm{Ab}(X)$, we make

Definition 1.1. By $q$-th cochain (resp. cocycle) collection for $(\mathscr{A}, \mathbb{J})$, we mean the collection of all subsets of $C^{q}(\mathscr{A}, \mathfrak{F})$ (resp. $Z^{q}(\mathscr{A}, \mathfrak{F})$ ). We use the symbols $\mathbb{C}^{q}(\mathscr{A}, \mathfrak{F}), \mathbb{Z}^{q}(\mathscr{A}, \mathfrak{F})$ for such collections.

Here we check

Proposition 1.1. The following two facts are equivalent:

$$
Z^{q}(\mathscr{A}, \mathfrak{F}) \subset \delta C^{q-1}(\mathscr{A}, \mathfrak{F}) .
$$

$(1.1)_{2}$ For each $\mathscr{D} \in \mathbb{Z}^{q}(\mathscr{A}, \mathfrak{F})$, there is an element $\mathscr{D}^{\prime} \in \mathbb{C}^{q-1}(\mathscr{A}, \mathfrak{F})$ so that

$$
\mathscr{D} \subset \delta \mathscr{D}^{\prime} \text {. }
$$

Proof. Taking $\mathscr{D}$ to be $Z^{q}(A, \mathfrak{F}) \in Z^{q}(\mathscr{A}, \mathfrak{F}),(1.1)_{2}^{\prime}$ insures $(1.1)_{2}$. Conversely setting $\mathscr{D}^{\prime}=C^{q-1}(\mathscr{A}, \mathfrak{F}) \in \mathbb{C}^{q-1}(\mathscr{A}, \mathfrak{F})$, we have $(1.1)_{2}$ from $(1.1)_{1}$.

In our later arguments, we do not work with the 'whole spaces' $C^{q}(\mathscr{A}, \mathfrak{F}), \ldots$ but with what we call p.g. subgroups, $C^{q}(\mathscr{A}, \mathfrak{F})_{\text {p.g }}$ in symbol, ... of $C^{q}(\mathscr{A}, \mathfrak{F}), \ldots$, which are characterized by concrete p.g. conditions (cf. $\S 1.2$. See also n.2 soon below). We will derive corresponding facts to $(1.1)_{1}$ ('vanishing property' in p.g. cohomology theory) from correspondences of $(1.1)_{2}$. In the later arguments, the former is also a formal consequence of the latter, but the converse is not true. Our main subject in Section 1 will be to get similar inclusions to $(1.1)_{2}$ in our p.g. cohomology, by making explicit similar correspondences to the one: $\mathscr{D} \rightarrow \mathscr{D}^{\prime}$ in $(1.1)_{2}$. Our main results in such a direction are given in Theorems 1.1-1.6 in Section 1.2. The remainder of Section 1.1 will be devoted to define what we call p.g. cochain collection, which is a family of elements of $\mathbb{C}^{q}(\mathscr{A}, \mathfrak{F})$ (characterized by concrete p.g. conditions), by making clear basic notions in the definition of such collections. The arguments will be given in a somewhat abstract fashion.

2. P.g. filtration. We begin $n .2$ by making a definition, which will play a basic role to define the collection mentioned at the end of $n .1$. 


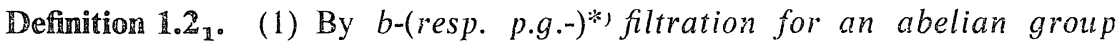
$\mathscr{B}$, we mean a map $0: \mathbb{R}^{+} \rightarrow 2^{\mathscr{H}}$ (resp. $\Psi^{P}: \mathbb{R}^{+2} \rightarrow 2^{\mathscr{O}}$ ) satisfying:

$(1.2)_{1} \quad \theta(a) \ni 0$ for each $a \in \mathbb{R}^{+}$(resp. $\Psi(\alpha) \ni 0$ for each $\alpha \in \mathbb{R}^{+2}$ ).

$(1.2)_{2} \quad \theta\left(a^{\prime}\right) \supset \theta(a)$ if $a^{\prime} \geqq a$ (resp. $\Psi\left(\alpha^{\prime}\right) \supset \Psi(\alpha)$ if $\left.\alpha^{\prime} \geqq \alpha\right)$.

$(1.2)_{3} \quad$ (Archimedian property) For any $a, a^{\prime} \in \mathbb{R}^{+}$(resp. $\alpha, \alpha^{\prime} \in \mathbb{R}^{+2}$ ), there is an element $a^{\prime \prime} \in \mathbb{R}^{+}\left(\right.$resp. $\left.\alpha^{\prime \prime} \in \mathbb{R}^{+2}\right)$ so that

$(1.2)_{3}^{\prime} \quad \theta\left(a^{\prime \prime}\right) \supset\left\{b \pm b^{\prime}:\left(b, b^{\prime}\right) \in \partial(a) \times \theta\left(a^{\prime}\right)\right\}$ (resp. $\Psi\left(\alpha^{\prime \prime}\right) \supset\left\{b \pm b^{\prime}\right.$

$$
\left.\left(b, b^{\prime}\right) \in \Psi(\alpha) \times \Psi\left(\alpha^{\prime}\right)\right\} .
$$

(2) We call $\cup_{a \in \mathbb{R}^{+}} \theta(a), \cup_{\alpha \in \mathbb{R}^{+2}} \Psi(\alpha)$ respectively $\left.\theta-b d d^{*}\right)$ and $\Psi \cdot p . g$. subgroups of $\mathscr{B}$. (When no confusions occur, we drop the terms 'bdd' and 'p.g.' from the terminology just above.)

Definition 1.22 . We say that $b$-filtrations $\theta_{1}, \theta_{2}: R^{+} \rightarrow 2^{\text {B }}$ are equivalent, if $\theta_{i}\left(\mathbb{R}^{+}\right)(i=1,2)$ are cofinal with respect to the increasing inclusion as in $(1.2)_{2}$.

Defination $1.22_{3}$. Letting $\mathscr{B}$ ' be a subgrcup of $\mathscr{B}$ (resp. $\omega: \mathscr{B} \rightarrow \mathscr{B}^{\prime \prime}$ a homomorphism of abelian group), we call the b-filtration $0^{\prime}: \mathbb{R}^{+} \ni a \rightarrow 2^{\mathscr{B}^{\prime}} \ni \theta(a) \cap \mathscr{B}^{\prime}$ (resp. $\omega_{*} \theta: \mathbb{R}^{+} \ni a \rightarrow 2^{\mathscr{B}} \exists \omega O(a)$ ) the one induced from $\mathscr{B}$ to $\mathscr{B}^{\prime}$ (resp. to $\mathscr{B}^{\prime \prime} b y$ $\omega)$. Moreover, letting $\tilde{\theta}, \tilde{0}^{\prime \prime}$ te b-filtrations of $\mathscr{B}, \mathscr{B}^{\prime \prime}$ we say that $\tilde{\theta}, \hat{\theta}^{\prime \prime}$ are compatible with $\omega$, if, for each $b \in \mathbb{R}^{+}$, we have:

$$
\omega \hat{\theta}(b) \subset \hat{\theta}^{\prime \prime}\left(b^{\prime}\right) \text {, with a suitable } b^{\prime} \in \mathbb{R}^{+} .
$$

The 'induced filtutions', 'equivalence" and 'compatibility" as above are defined for p.g. filtrations in the similar manner to Definition $1.2_{2}$. Wow taking $\mathscr{B}$ to be $C^{q}(\mathscr{A}, \mathfrak{F})$ in $n .1$, let $\psi$ be the p.g. flltration in Definition $1.2_{1}$.

Definition 1.3. By q-th $\Psi$-p.g. cochain and cocycle collections for $(\mathscr{A}, \mathbb{F})$, we mean

$$
\begin{aligned}
& \mathbb{C}^{q}(\mathscr{A}, \widetilde{\mho} ; \Psi)_{\mathrm{p} . \mathrm{g}}:=\Psi\left(\mathbb{R}^{+2}\right)\left(\subset 2^{C^{q}(\mathscr{A}, \widetilde{)})}\right), \\
& \mathbb{Z}^{q}(\mathscr{A}, \tilde{\mho} ; \Psi)_{\mathrm{p} . \mathrm{g}}:=\widetilde{\Psi}\left(\mathbb{R}^{+2}\right)\left(\subset 2^{Z^{q}(\mathscr{N}, \Im)}\right),
\end{aligned}
$$

where $\tilde{\Psi}$ is the induced filtration of $\Psi$ to $Z^{q}(\mathscr{A}, \mathscr{F})$ (Definition $\left.1.2_{3}\right)$. Also we set

$(1.3)_{1}^{\prime} \quad C^{q}(\mathscr{A}, \mathscr{W} ; \Psi)_{\mathrm{p} . \mathrm{g}}:=\Psi^{\prime}$-p.g. subgroup of $C^{q}(\mathscr{A}, \mathfrak{F})\left(\right.$ Definition $\left.1.2_{1}\right)$.

$(1.3)_{1}^{\prime \prime} \quad Z^{q}(\mathscr{A}, \mathscr{F} ; \Psi)_{\mathrm{p} . \mathrm{g}}:=C^{q}(\mathscr{A}, \mathbb{W} ; \Psi)_{\mathrm{p} . \mathrm{g}} \cap Z^{q}(\mathscr{A}, \mathbb{W})$.

Moreover, when $\mathscr{A}$ consists of the single element $X$ (i.e. $\mathscr{A}=\{X\}$ ), we set

*) 'b'=initial of 'bounded' and 'bdd'=abbreviation of 'bounded'. 


$$
\Gamma(X, \mathfrak{F} ; \Psi)_{\mathrm{p} \cdot \mathrm{g}}:=Z^{0}(\mathscr{A}, \mathfrak{F} ; \Psi)_{\mathrm{p} \cdot \mathrm{g}}
$$

Now we will construct p.g. filtration in a geometric manner. For this we first make a definition, which is a slice abstraction of 'absolute value' for analytic functions etc. in the standard meaning.

Definition 1.4 . (1) By q-structure of $₹ \in \mathrm{Ab}(X)$, we mean a family $\theta=\left\{\theta_{U} ; U \in \operatorname{Ouv}(X)\right\}$ of b-filtrations $\theta_{U}: \boldsymbol{R}^{+} \rightarrow 2^{\mathscr{B}}, \mathscr{B}=\Gamma(U, \mathfrak{F})$, satisfying:

$(1.3)_{2} \rho \theta_{U^{\prime}}(a) \supset \theta_{U}(a)$ for any $U^{\prime} \supset U$ and $a \in \mathbb{R}^{+}$, where $\rho=$ restriction: $\Gamma\left(U^{\prime} ; \mathfrak{f}\right)$ $\rightarrow \Gamma(U, \mathfrak{F})$.

(1.3) 3 The stalk $\theta_{P}$ of $\theta$ at $P \in X$ (i.e. $\theta_{P}: \mathbb{R}^{+} \ni a \rightarrow \theta_{P}(a):=\lim _{U \rightarrow P} \theta_{U}(a)$ ) satisfies $(1.3)_{3}^{\prime} \quad \theta_{P}$ is a b-filtration for $\mathfrak{F}_{P}$, and $\mathfrak{F}_{P}=\cup_{a \in \boldsymbol{R}^{+}} \theta_{P}(a)$.

(2) For an element $\varphi_{P} \in \mathfrak{F}_{P}$ we call $\inf \left(a \in \boldsymbol{R}^{+} ; \theta_{P}(a) \ni \varphi_{P}\right)\left(\in \mathbb{R}^{+} \cup 0\right)$ the $\theta$-absolute value of $\varphi_{P}$.

We call the pair $(\mathfrak{F}, \theta)$ simply $q$-sheaf. When there is no fear of confusions, we write the symbol ' $(\mathscr{F}, \theta)$ ' also as ' $F$ '. Letting $\left(X, \mathfrak{D}_{X}\right)$ be a reduced complex space, we define a q-structure $\theta_{X}=\left\{\theta_{U} ; U \in \operatorname{Ouv}(X)\right\}$ by

$$
\theta_{U}: \boldsymbol{R}^{+} \ni a \rightarrow \Gamma\left(U, \mathfrak{D}_{X}\right)_{a}:=\left\{\varphi \in \Gamma\left(U, \mathfrak{D}_{X}\right) ;|\varphi(P)|<a \text { on } U\right\},
$$

where || denotes the absolute value (in the standard sense).

Definition 1.4 $4_{2}$. We call $\theta_{X}$ the standard q-structure of $D_{X}$. One check easily that the absolute value defined by the standard q-structure coincides with that in $(1.3)_{4}$. For $\mathfrak{D}_{X}^{k}$ we define the standard q-structure by $\theta_{X}^{k}=\left\{\theta_{U}^{k}\right\}_{U}$, where $\theta_{U}^{k}$ assigns to each $a \in \boldsymbol{R}^{+}$the $k$-times direct sum of the subset in (1.3) ${ }_{4}$ (of $\left.\Gamma\left(U, \mathfrak{D}_{\mathbf{x}}\right)\right)$. We define q-structures for general coherent sheaves in n.3. Now returning to the pair $(\mathscr{A}, \mathfrak{F})$ in Definition $1.4_{1}$, take a subsheaf $\mathfrak{F}^{\prime}$ of $\mathfrak{F}$ and a homomorphism $\omega: \mathfrak{F} \rightarrow \widetilde{\mho}^{\prime \prime}$.

Definition 1.4. . By q-structure for $\mathfrak{F}^{\prime}, \mathfrak{F}^{\prime \prime}$ induced from $\mathfrak{F},(\mathfrak{F}, \omega)$, we mean

$$
\begin{aligned}
& \theta^{\prime}:=\left\{\theta_{U}^{\prime}: \boldsymbol{R}^{+} \ni a \rightarrow \theta_{U}^{\prime}(a):=\theta_{U}(a) \cap \Gamma\left(U, \mathfrak{F}^{\prime}\right)\right\}_{U}, \\
& \omega_{*} \theta:=\left\{\omega^{*} \theta_{U} ; \boldsymbol{R}^{+} \ni a \rightarrow \omega \theta_{U}(a)\right\}_{U},
\end{aligned}
$$

where $\theta$ is the q-structure of $\mathfrak{F}$ as in Definition $1.4_{1}$.

Next the following simple definition plays basic roles not only in our geometric construction of p.g. filtrations but also in many aspects of later arguments:

Definition 1.44. By a defining function of p.g. structure of $X$ (or, simply, a 
p.g. function), we simply mean a map $g: X \rightarrow \mathbb{R}_{1}^{+}$. We call $(X, g)$ simply a p.g. pair.

Now, using the p.g. pair $(X, g)$ and the q-sheaf $(\mathfrak{F}, \theta)$ as in Definition $1.4_{1}$, we define p.g. filtration for $(\mathscr{A}, \mathfrak{F})$ in the following fashion.

Definition 1.45. (1) We say that an element $\varphi \in C^{q}(\mathscr{A}, \mathfrak{F})$ is $(g, \theta)-\alpha-$ growth $\left(\alpha \in \mathbb{R}^{+2}\right)$, if for each $\mathscr{A}^{\prime} \in \mathscr{N}^{q+1} \mathscr{A}$, we have

$(1.3)_{6}\left|\varphi_{\mathscr{A}^{\prime}}(Q)\right|_{\theta}<\alpha \cdot g(Q)$ on $\left|\mathscr{A}^{\prime}\right|$, where ||$_{\theta}=\theta$-absolute value (Definition $\left.1.4_{1}\right)$.

(2) By $(g, \theta)-p . g$. filtration for $C^{q}(\mathscr{A}, \mathfrak{F})$, we mean

$(1.3)_{7} \quad \Psi_{g . \theta}: \mathbb{R}^{+2} \ni \alpha \rightarrow$ the subset of $C^{q}(\mathscr{A}, \mathfrak{F})$ consisting of all $(g, \theta)$ - $\alpha$-growth cochains.

The following notation will be used in the later arguments (cf. n.3, §1.1 and $\S 1.2)$ :

$(1.3)_{6}^{\prime}\left\{\begin{array}{l}C^{q}\left(\mathscr{A}, \mathfrak{F} ; \Psi_{g, \theta}\right)_{\alpha}:=\Psi_{g, \theta}(\alpha) \\ C^{q}\left(\mathscr{A}, \mathfrak{F} ; \Psi_{g, \theta}\right)_{\mathrm{p} . \mathrm{g}}:=\cup_{\alpha} \Psi_{g, \theta}(\alpha), \text { where } \alpha \text { runs through } \mathbb{R}^{+2} .\end{array}\right.$

Letting the subsheaf $\mathfrak{F}^{\prime}$ of $\mathfrak{F}$ and the homomorphism $\omega: \mathfrak{F} \rightarrow \mathbb{F}^{\prime \prime}$ be as in Definition $1.4_{1}$, we use the symbols $\theta^{\prime}, \omega_{*} \theta$ for the q-structures for $\widetilde{F}^{\prime}, \mathbb{F}^{\prime \prime}$, which are induced from $\theta$ to $\mathfrak{F}^{\prime}$ (resp. $\theta$ to $\mathfrak{F}^{\prime \prime}$ by $\omega$ ). Then we easily have

Proposition 1.2. $\Psi_{g, \theta^{\prime}}=\left(\Psi_{g, \theta}\right)^{\prime}$ and $\Psi_{g, \omega^{*} \theta}=\omega_{*} \Psi_{g, \theta}$, where the right sides are induced from $\Psi_{g, \theta}$ to $C^{q}\left(\mathscr{A}, \widetilde{\mho}^{\prime}\right)$ and to $C^{q}\left(\mathscr{A}, \mathfrak{F}^{\prime \prime}\right)$ by $\omega_{*}$.

In $\mathrm{n} .3$ soon below we define a p.g. filtration for certain coherent sheaves in a more explicit manner.

3. P.g. coherent sheaf. Letting $\left(X, \mathfrak{D}_{X}\right)$ be a reduced complex space, take a p.g. function $g$ of $X$ (Definition $1.4_{4}$ ). We begin $n .3$ by giving a p.g. condition on coherent sheaves over $X$, which is used in the remainder of this paper.

Definition 1.5. By a $(g)$-p.g. resolution of an $\mathfrak{D}_{X}$-coherent sheaf $\mathfrak{R}$, we mean a pair $\overline{\mathscr{K}}=\left(\omega,\left\{K_{j}\right\}_{j=1}^{p-1}\right)$ consisting of an $\mathfrak{D}_{X}$-homomorphism $\omega$ and matrices $K_{j}(1 \leqq j \leqq p-1)$ with entries in $\Gamma\left(X, D_{X} ; \Psi_{g, \theta_{X}}\right)_{\mathrm{p} . \mathrm{g}}$. The pair $\overline{\mathscr{K}}$ must satisfy a resolution as follows:

$$
0 \longrightarrow D_{X}^{k_{p}} \stackrel{K_{p-1}}{\longrightarrow \cdots \cdots} \stackrel{K_{1}}{\longrightarrow} \mathfrak{D}_{X}^{k_{1}} \stackrel{\omega}{\longrightarrow} \mathfrak{\Omega} \longrightarrow 0 .
$$

(For later convenience we call $\mathfrak{D}_{X}^{k_{1}}$ in $(1.4)_{1}$ the 'first resolution part' of $\mathfrak{\Omega}$.) 
Convention 1.1. (1) By a p.g. coherent sheaf over $X$, we mean a pair $\mathfrak{I}=(\Re, \mathscr{K})$ as above; starting with the sheaf $\mathfrak{K}$, our explicit uniform estimations depend not only on $\mathfrak{K}$ but also on a resolution like $(1.4)_{1}$. The terminology ' $p . g$. sheaf' as above is convenient for later purposes.

(2) When there is no fear of confusions we use the symbol ' $\mathfrak{5}$ ' for also ' $\Omega$ '.

We arrange here some data which are useful in later arguments: first, writing $(X, g)$ as $X$, we set

$$
\operatorname{Coh}(\mathbb{X})_{\text {p.g }}:=\text { collection of all p.g. coherent sheaves over } X \text {. }
$$

We define a map (length map)

$$
\lg : \operatorname{Coh}(\mathbb{X})_{\text {p.g }} \ni \mathfrak{H} \rightarrow \mathbb{Z}^{+} \ni p_{\text {. }}(=\text { length of the resolution of } \mathfrak{H})
$$

(cf. $\left.(1.4)_{1}\right)$, and we also define an increasing filtration of Coh $(\mathbb{X})_{\mathrm{p} . \mathrm{g}}$ :

$$
\operatorname{Coh}^{p}(\mathbf{X})_{\mathrm{p} . \mathrm{g}}:=\left\{\mathfrak{S} \in \operatorname{Coh}(\mathbb{X})_{\mathrm{p} . \mathrm{g}} ; p_{\mathfrak{g}} \leqq p\right\}
$$

Our coherent sheaves in later arguments are in $\operatorname{Coh}(\mathbb{X})_{\text {p.g. }}$. Next letting the p.g. sheaf $\mathfrak{H}$ be as in $(1.4)_{1}$, we mean by standard $q$-structure of $\mathfrak{S}$ the one induced from $\omega: \mathfrak{D}^{k_{1}} \rightarrow \mathfrak{S}$ (cf. Definition $1.4_{2}$ and $(1.4)_{1}$ ). This q-structure $\theta_{\mathfrak{5}}$ is determined by $\omega$, while that of $D^{k_{1}}$ is uniquely determined by the analytic structure of $X$ (cf. n.2); we may say that $\theta_{\mathfrak{5}}$ is determined 'uniquely' by the analytic structure of $(\mathfrak{S}, X)$. Now letting $\Psi_{g, \theta_{\mathfrak{F}}}$ denote the $\left(g, \theta_{\mathfrak{g}}\right)$-p.g. filtration for $C^{q}(\mathscr{A}, \mathfrak{S})$ (cf. Definition $1.4_{5}$ ), we get $\Psi_{g, \theta_{\mathfrak{5}}}$-p.g. cochain collection etc., which are obtained by applying Definition 1.3 to $\Psi_{g, \theta_{5}}$. For later notational convenience we arrange here some notation for such collections. (The key point in the arrangement is: (1) to drop the term $\theta_{5}$ from $\Psi_{g, \theta_{55}}, \ldots$ and (2) to write $\Psi_{g}:=\Psi_{g, 0_{\mathfrak{j}}}$ simply as ' $g$ ', when no notational ambiguities take place.) Thus we have:

$(1.4)_{6}\left\{\begin{array}{l}\mathbb{C}^{q}(\mathscr{A}, \mathfrak{H} ; g)_{\text {p.g }} \\ \mathbb{Z}^{q}(\mathscr{A}, \mathfrak{H} ; g)_{\text {p. g }}\end{array}\right\}:=\Psi_{g}-$ p.g. $\left\{\begin{array}{l}\text { cochain } \\ \text { cocycle }\end{array}\right\}$ collection for $(\mathscr{A}, \mathfrak{H})$.

$(1.4)_{7} \quad C^{q}(\mathscr{A}, \mathfrak{H} ; g)_{\text {p.g }}:=\Psi_{g}-$ p.g. subgroup of $C^{q}(\mathscr{A}, \mathfrak{H})$.

$(1.4)_{8}\left\{\begin{array}{l}C^{q}(\mathscr{A}, \mathfrak{S} ; g)_{\alpha} \\ Z^{q}(\mathscr{A}, \mathfrak{S} ; g)_{\alpha}\end{array}\right\}:=\left\{\begin{array}{l}\text { set of }\left(g, \theta_{\mathfrak{L}}\right)-\alpha-\text { growth cochains with value in } \mathfrak{S} \\ C^{q}(\mathscr{A}, \mathfrak{S} ; g)_{\alpha} \cap Z^{q}(\mathscr{A}, \mathfrak{S}) .\end{array}\right\}$.

(For the above sets, collections and subgroup, see (1.3) ${ }_{6}^{\prime}$ and (1.3) $)_{1}$.) Also we will abbreviate ' $\Psi_{q}$-p.g.' and ' $\left(g, \theta_{52}\right)$-' in the above terminologies simply as 
' $g$ '. The data as in $(1.4)_{6-8}$ will be frequently used in the remainder of Section 1 (cf. $§ 1.2, \S 1.3$ ). Using the above notation we have the following easily from Proposition 1.2.

Proposition 1.3. $C^{q}(\mathscr{A}, \mathfrak{H} ; g)_{\alpha}=\omega C^{q}\left(\mathscr{A}, \mathfrak{D}_{X}^{k_{1}} ; g\right)_{\alpha} \quad$ (cf. Definition $1.4_{3}$ ).

(The similar relation to the above holds for $C^{q}(A, \mathfrak{s} ; g)_{\mathrm{p} . \mathrm{g}}$ and $Z^{q}(A, \mathfrak{S}$; $g)_{\text {p.g. }}$ ) We finish n.3 by the following remark for convenience of later arguments (cf. §1.3).

Remark 1.1. (1) For $\mathfrak{D}_{X}^{k}(k>0)$ one can attach the p.g. coherent sheaf in the following manner: $0 \rightarrow \mathfrak{D}_{X}^{k} \stackrel{i}{\longrightarrow} \mathfrak{D}_{X}^{h} \rightarrow 0$, with the identity $i$. This trivial realization of $\mathfrak{D}_{X}^{k}$ as the p.g. coherent sheaf is useful in later arguments. Unless we say otherwise, we mean by the 'p.g. coherent sheaf $\mathfrak{D}_{X}$ ' the above (trivial) one.

(2) Next we define a subcollection of $\operatorname{Coh}(X)_{\mathrm{p} \cdot \mathrm{g}}$ :

$$
\operatorname{Coh}^{\prime}(X)_{\mathrm{p} . \mathrm{g}}:=\left\{\tilde{S} \in \operatorname{Coh}(X)_{\mathrm{p} . \mathrm{g}},\right.
$$

where the first resolution $\omega: D_{X}^{k} \rightarrow 5$ (cf. Definition 1.5) is defined by a matrix $K$ (i.e. $\omega=K)$, with entries in $\left.\Gamma\left(X, \mathfrak{D}_{Y} ; g\right)_{\mathrm{p} . \mathrm{g}}\right\}$. Note that $\mathfrak{S}$ is a subsheaf of $D_{X}^{k^{\prime}}$, with $k^{\prime}=$ length of columns of $K$, and we have a p.g. filtration for $\mathfrak{S}$ by means of the inclusion: $\mathfrak{S} \hookrightarrow \mathfrak{D}_{X}^{k^{\prime}}$ (Definition $1.4_{2}$ and Proposition 1.3). Writing this filtration as $\Psi_{g}^{\prime}$, the set of $\Psi_{g}^{\prime}$-ce-growth cochains with value in $\mathfrak{S}$ (cf. (1.4) $)_{8}$ ) is explicitly as follows:

$(1.4)_{10} \quad C^{q}\left(\mathscr{A}, \mathfrak{j} ; \Psi_{g}^{\prime}\right)_{\alpha}=C^{q}\left(\mathscr{A}, \mathfrak{D}_{X}^{h} ; g\right)_{\alpha} \cap C^{q}(\mathscr{A}, \mathfrak{g}) \quad\left(\mathrm{cf} .(1.4)_{5}\right)$.

In Section 1.3 we give a comparison of $\Psi_{g}^{\prime}$ and the standard p.g. filtration $\Psi_{g}$, which is a key fact in our p.g. uniform estimations (Lemma 1.2).

4. $\mathbb{P}$.g. covering. Here we assume that the p.g. pair $(X, g)$ is as in n.2, and we fix a map $d: X \times X \rightarrow[0, \infty)$, to which we impose the single condition: $d=0$ on the diagonal $\Delta_{X}$ of $X$. We define a type of p.g. covering, which is used in the main body of this paper. For this letting $P$ be a point of $X$ we use the symbol $\tilde{U}_{r}(P):=\{Q \in X ; d(P, Q)<r\}$. Then taking a subset $Y$ of $X$ and an element $\sigma \in \mathbb{R}_{1}^{+2}$ we make

Deimition $1.6_{1}$. By $g-p . g$. covering of $Y$ of size $\sigma$ in $X$, we mean the following collection of elements of $2^{x}$

$$
\mathscr{A}_{\sigma}(Y ; g):=\left\{\tilde{U}_{\sigma}(P ; g) ; P \in Y\right\},
$$

where $\tilde{U}_{\sigma}(P ; g):=\tilde{U}_{r}(P)$, with $r=\{\sigma g(P)\}^{-1}$. Next take subsets $X^{\prime}, Y^{\prime}$ of $X$ 
satisfying $Y^{\prime} \subset Y \cap X^{\prime}$ and an element $\sigma^{\prime} \in \boldsymbol{R}_{1}^{+2}$ satisfying $\sigma^{\prime} \geqq \sigma$.

Definition $1.6_{2}$. We call the map

$$
s: \mathscr{B}_{\sigma^{\prime}}\left(Y^{\prime} ; g\right) \ni \tilde{U}_{\sigma}^{\prime}(P ; g) \longrightarrow \mathscr{A}_{\sigma}(Y ; g) \ni \tilde{U}_{\sigma}(P ; g)
$$

p.g. refining map (from the left side to right side). Here the left side denotes the $g$-p.g. covering of $Y^{\prime}$ of size $\sigma$ in $X^{\prime}$, and $\tilde{U}_{\sigma}^{\prime}(P ; g):=\tilde{U}_{\sigma}(P ; g) \cap X^{\prime}$. Fixing $Y$ (resp. $X^{\prime}, Y^{\prime}$ ), the p.g. covering in $(1.5)_{1}$ (resp. the p.g. map in $(1.5)_{2}$ ) is determined uniquely by $\sigma$ (resp. $\sigma, \sigma^{\prime}$ ). This fact will be useful to fix our ideas and to simplify arguments in later explicit uniform estimations (cf. $\$ 1.3$ and \$4). Also such coverings and maps are suitable for our geometric applications of the uniform estimations to geometric situations (cf. §1.2, §2.2 and §3). The coverings and the refining maps in the main body of this paper will be the ones in $(1.5)_{1,2}$.

Now, by Definitions $1.6_{1,2}$ we have introduced all necessary basic notions to define what we call 'p.g. cohomology theory for analytic varieties'; the first basic datum is the p.g. function $g: X \rightarrow \boldsymbol{R}_{1}^{+}$which is used to measure the p.g. properties of cochains and coverings. The q-structure for abelian sheaves is used to define the p.g. condition on cochains (cf. Definition 1.41). Finally 'distance function $d$ ' is used to define the p.g. condition of the coverings. As was checked in n.3, the q-structure for coherent sheaves may be regard as determined by the underlying analytic structure of the varieties; we may regard the p.g. function $g$ and the 'distance function' $d$ are most basic 'additional data' to the analytic varieties, which are used to define what we call p.g. cohomology for those varieties. In order to emphasize this, we will sometimes call $(X, g)$ and $(X, g, d)$ as just above 'p.g. pair' and 'p.g. triple'.

5. Finally we arrange here certain concrete maps, which are used in later explicit estimations: first, by a positive monomial, we mean $M(t)=a t^{b}(a, b>0)$, where $t$ is a variable. We call a map $\mathscr{L}: \mathbb{R}^{+2} \ni\left(\sigma_{1}, \sigma_{2}\right) \rightarrow \mathbb{R}^{+2} \ni\left(\tilde{\sigma}_{1}, \tilde{\sigma}_{2}\right)$ to be of 'exponential linear type' (or, simply, 'el-map'), if $\tilde{\sigma}_{1}=M\left(\sigma_{1}\right) \exp M^{\prime}\left(\sigma_{2}\right)$, $\tilde{\sigma}_{2}=L\left(\sigma_{2}\right)$, with a positive monomial $M$, a finite sum $M^{\prime}$ of positive monomials and a linear function $L(t)=c t ; c>0$. It is easily checked that a composition of el-maps is also an el-map. Next making a notational convention:

$$
a \cdot\left(\alpha_{1}, \alpha_{2}\right)=\left(a \alpha_{1}, \alpha_{2}\right) \text { for any } a, \alpha_{1} \text { and } \alpha_{2} \in \mathbb{R}^{+} \text {, }
$$

we set 
$(1.6)_{1}^{\prime}$

$$
\mathbb{E}_{\mathrm{p} . \mathrm{g}}^{\prime}:=\left\{E^{\prime}: \mathbb{R}^{+} \times \mathbb{R}^{+2} \ni(r, \sigma) \longrightarrow \mathbb{R}^{+} \times \mathbb{R}^{2} \ni\left(r^{\prime}, \sigma^{\prime}\right)\right\},
$$

where $r^{\prime}=M_{1}(r), \sigma^{\prime}=M_{2}\left(r^{-1}\right) \cdot \mathscr{L}_{1}(\sigma)$,

$$
\begin{aligned}
\mathbb{E}_{\mathrm{p} . \mathrm{g}}:=\left\{E:\left(\mathbb{R}^{+} \times \mathbb{R}^{+2}\right)\right. & \times \mathbb{R}^{+} \ni(r, \sigma: \alpha) \longrightarrow \\
& \left.\left(\mathbb{R}^{+} \times \mathbb{R}^{+2}\right) \times \mathbb{R}^{+2} \ni\left(r^{\prime}, \sigma^{\prime} ; \alpha^{\prime}\right)\right\},
\end{aligned}
$$

where $\left(r^{\prime}, \sigma^{\prime}\right)=E^{\prime}(r, \sigma)$, with an element $\mathbb{E}^{\prime} \in \mathbb{E}_{\mathrm{p} . \mathrm{g}}^{\prime}, \quad$ and $\quad \alpha^{\prime}=M_{3}\left(r^{-1}\right) \cdot \mathscr{L}_{2}(\alpha+\sigma)$. (Here $M_{i}, \mathscr{L}_{j}$ are positive monomials and el-maps.) We write the correspondence: $\mathbb{E}_{\mathrm{p} . \mathrm{g}} \ni E \rightarrow \mathbb{E}_{\mathrm{p} . \mathrm{g}}^{\prime} \ni E^{\prime}$ as $\pi$, where $E, E^{\prime}$ are as

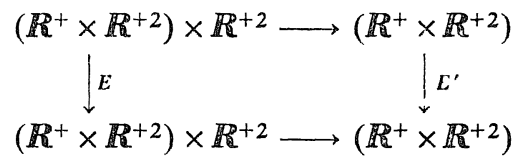

Figure $\mathrm{I}$. in $(1.6)_{2}$ (cf. Figure I).

Definition 1.7. We call an element $E \in \mathbb{E}_{\mathrm{p.g}}$ an estimation map for p.g. cohomology (or, simply, p.g.c. map) and $E^{\prime}=\pi(E) \in \mathbb{E}_{\text {p.g }}^{\prime}$ its first part.

In the later estimations, the map $E^{\prime}$ concerns those on coverings, while the map $E$ concerns those on both coverings and cochains (\$1.2): letting the element $(r ; \sigma ; \alpha)$ be as in $(1.6)_{2}$, the estimation: $(r ; \sigma) \rightarrow\left(r^{\prime} ; \sigma^{\prime}\right)=E^{\prime}(r ; \sigma)$ concerns that of coverings, while the element $\alpha$ concerns that of cochains. Note that the factorization in Figure $I$ insures that the term ' $\alpha$ ' has no influences on the correspondence: $(r ; \sigma) \rightarrow\left(r^{\prime} ; \sigma^{\prime}\right)$. We use this fact in Section 1.2. Next take p.g.c. maps $E_{1}, E_{2} \in \mathbb{E}_{\mathrm{p} . \mathrm{g}}$. Then the composition $E_{2} \circ E_{1}$ is not, in general, in $\mathbb{E}_{\text {p.g. }}$. However, define an order in $\mathbb{R}^{+} \times \mathbb{R}^{+2} \times \mathbb{R}^{+2}$ by

$$
(r ; \sigma ; \alpha)>\left(r^{\prime} ; \sigma^{\prime} ; \alpha^{\prime}\right) \Longleftrightarrow r<r^{\prime}, \sigma>\sigma^{\prime} \text { and } \alpha>\alpha^{\prime} .
$$

Then the set $\mathbb{E}_{\text {p.g }}$ is closed under the composition in the sense that there is a p.g.c. map $E_{3} \in \mathbb{E}_{\mathrm{p} . \mathrm{g}}$ satisfying

$$
(1.6)_{3} \quad E_{3}(r ; \sigma ; \alpha) \succ E_{2} \circ E_{1}(r ; \sigma ; \alpha) \text { for each }(r ; \sigma ; \alpha) \in(0,1] \times \mathbb{R}_{1}^{+2} \times \mathbb{R}_{1}^{+2} \text {. }
$$

We use this fact in later p.g. estimations frequently, without mentioning it explicitly (cf. § 1.3). Also we use the symbol: $E_{3} \succ E_{2} \circ E_{1}$ to indicate the inequality in $(1.6)_{3}$.

Finally, the p.g.c. maps as above will be used in our main results in the p.g. uniform estimations (cf. Theorem 1.1 and Theorem 1.2). Our explicit form of the p.g.c. maps are chosen in such a manner that (1) the p.g. estimations obtained by such maps insure our p.g. analogues of Theorems A, B of H. Cartan and (2) the p.g.c. maps are concordant to more elementary p.g. estimations on sheaf homomorphisms and on Cousin integrals (cf. §4 and §6). Fixing the 
explicit forms of the p.g.c. maps as above, considerable parts of the arguments will be reduced to those of p.g.c. maps, which are essentially algebraic and elementary (cf. §1.3. Also see, in particular, §4.2.).

\section{§1.2. Main Results}

Here we summarize our main results on the p.g. cohomology theory in Section 1: in n.1-n.4 we introduce some basic data, which will underlic the arguments in the cohomology theory in the remainder of Chapter I. Using such data, we give our main results on the uniform estimations in the p.g. cohomology in Theorems 1.1-1.4 (cf. n.4, n.5). Also dropping the explicit estimations in these results, we give analogues of Theorems $A_{A}, B$ of $H$. Cartan in our cohomology theory in Section 1 (cf. Theorems 1.5, 1.6 in n.6).

1. Geometric data. As was mentioned, our analytic varieties in Chapter I will be Stein varieties with suitable algebraicity (and will have similarities to affine varieties). Here we introduce such varieties.

(i) First, by a coordinated complex euclidean space, we mean a pair $\left(\mathbb{C}^{n}, z\right)$ of a complex euclidean space $\mathbb{C}^{n}$ and its coordinates $z$. When there is no fear of confusions, we use the terminology 'complex euclidean space $\mathbb{C}^{n}(z)$ ' (or, simply, 'euclidean space $\mathbb{C}^{n}(z)$ ') as a synonym for 'coordinated euclidean space $\left(\mathbb{C}^{n}, z\right)^{\prime}$. We then introduce a geometric datum:

$$
\tilde{\mathbb{X}}:=\left(\mathbb{C}^{n}(z) \times \mathbb{C}^{n^{\prime}}\left(z^{\prime}\right), \tilde{X}=\mathbb{C}^{n}(z) \times U_{0}^{\prime}, P_{0}^{\prime}\right),
$$

where $\mathbb{C}^{n}(z), \mathbb{C}^{n^{\prime}}\left(z^{\prime}\right)$ are euclidean spaces and $U_{0}^{\prime}\left(\ni P_{0}^{\prime}\right)$ is an open set of $\mathbb{C}^{n^{\prime}}$, and we set

$$
\mathrm{Am}_{0}:=\text { collection of all geometric data as in }(1.7)_{0}^{\prime} \text {. }
$$

The underlying variety of $\tilde{X}$ will be $\tilde{X}=\mathbb{C}^{n} \times U_{0}^{\prime}$. We regard $\mathbb{C}^{n} \times \mathbb{C}^{n^{\prime}}$ as the ambient space of $\tilde{X}=\mathbb{C}^{n} \times U_{0}^{\prime}$ and the point $P_{0}^{\prime}$ as the center of $\tilde{X}$. As we will see in later arguments, the uniform estimations, which are given to varieties in $\mathbb{A r}_{0}$, are most basic among the ones in Section 1.2. (In Section 1.2 we introduce two another types of varieties (cf. $(1.8)_{0}$ and $\left.(1.11)_{0}\right)$. The p.g. estimations for such varieties will be derived from the ones for varieties in $\mathrm{An}_{0}$, by using explicit relations of the former varieties to the latter; see Corollary 1.4 and Lemma 1.3 in Section 1.3.)

(ii) Next, by a (smooth) local analytic variety of affine type, we mean a 
geometric datum

$$
X=\left(\mathbb{C}^{n}(z), U_{0}, X_{0}, h, P_{0}, \mathfrak{S}_{\mathbb{X}}\right),
$$

where $X_{0}\left(\ni P_{0}\right)$ is an analytic variety in an open set $U_{0}$ of $\mathbb{C}^{n}$, and $h$ is an element of $\Gamma\left(U_{0}, \mathfrak{O}_{U_{0}}\right), \mathfrak{D}_{U_{0}}$ being the structure sheaf of $U_{0}$. Moreover, setting

$(1.8)_{0}^{\prime \prime} \quad D_{0}=$ divisor of $h\left(\right.$ in $\left.U_{0}\right), D=X_{0} \cap D_{0}$ and $X=X_{0}-D$,

the final datum $5_{X}$ in $(1.8)_{0}^{\prime \prime}$ is a $\left|h^{-1}\right|$-p.g. resolution of $\Omega_{X}$ over $U_{0}-D_{0}$ (cf. $(1.4)_{1}$ ), where $\mathfrak{D}_{X}$ is the structure sheaf of $X$ and the first term of $\mathfrak{H}_{X}$ is of the form: $\mathfrak{D}_{U_{0}-D_{0}} \stackrel{\omega}{\longrightarrow} D_{X}$ (cf. $(1.4)_{1}$ ), with the natural homomorphism $\omega$. (Here $\mathfrak{D}_{U_{0}-D_{0}}$ is the structure sheaf of $U_{0}-D_{0}$.) The datum $\mathbb{X}$ must satisfy

$(1.8)_{1} \quad D \ni P_{0}$, and $X$ is smooth,

$(1.8)_{2}$ the germs of $X_{0}, D$ at $P_{0}$ have no common irreducible components.

We set

$(1.8)_{0} \quad \operatorname{Am}_{1 a}:=$ collection of all smooth analytic varieties of affinc type (cf. $\left.(1.8)_{0}^{\prime}\right)$.

The underlying variety of $\mathbb{X}$ will be $X=X_{0}-D$. We regard $U_{0}-D_{0}$ as the ambient space of $X$ and the point $P_{0} \in X_{0}$ as the center of $X_{0}, \ldots$. The p.g. uniform estimation for $\mathbb{X} \in \mathbb{A m}_{1 a}$ will play basic roles in semi-global estimations in later arguments (cf. $\S 2$ ). (Note that we include the p.g. resolution $\mathfrak{H}_{X}$ of the structure sheaf $\mathfrak{D}_{X}$ in $(1.8)_{0}$. The resolution $\mathfrak{S}_{X}$ is used to give an explicit uniform estimations for the sheaf $\mathfrak{D}_{X}$; see $\S 1.3$.)

In the remainder of Section 1.2 we will fix geometric data $\tilde{X} \in \mathbb{A n}_{0}$ and $\mathbb{X} \in \mathbb{A n}_{1 a}$ of the form in (1.7) $)_{0}^{\prime}$ and $(1.8)_{0}^{\prime}$. In (iii) soon below we fix some additional data and notations for such varieties.

(iii) First, to $\tilde{\mathbb{X}}, \mathbb{X}$, we attach the following p.g. and distance functions:

$$
\left\{\begin{array}{l}
g_{\tilde{\mathbf{x}}} \\
g_{\mathbf{X}}
\end{array}\right\}:=\left\{\begin{array}{l}
|\tilde{z}|+1 \\
|h|^{-1}
\end{array}\right\},\left\{\begin{array}{l}
d_{\tilde{\mathbf{X}}} \\
d_{\mathbf{X}}
\end{array}\right\}:=\text { natural distance*) of }\left\{\begin{array}{l}
\mathbb{C}^{n}(z) \times \mathbb{C}^{n^{\prime}}\left(z^{\prime}\right) \\
\mathbb{C}^{n}(z)
\end{array}\right\},
$$

where $\tilde{z}=\left(z, z^{\prime}\right)$. When there is no fear of confusions, we write $d_{\tilde{X}}, d_{X}$ also as $d_{z}, d_{z}$. In our framework, p.g. and distance functions have basic meanings to define what we call p.g. cohomology theory for analytic varieties (cf. n.4, §1.1). The p.g. and distance functions for $\tilde{\mathbb{X}}, \mathbb{X}$ as above may reflect closely the analytic structures of $\tilde{X}, \mathbb{X}$ and may be natural ones for studies of p.g. properties of co-

4.) Natural distance of $C_{1}^{\prime \prime}(z)$ is defined to be $d_{z}:=-z=z^{\prime} \mid$ for $z, z^{\prime} \subset \mathbb{C}^{\prime \prime}$. 
herent sheaves over $\tilde{\boldsymbol{X}}, \boldsymbol{X}$. Our p.g. and distance functions for $\tilde{\mathbf{X}} \in \mathbf{A n}_{0}$, $\boldsymbol{X} \in \mathbf{A} \mathbf{n}_{1 a}$ in the remainder of Chapter I will be the ones in (1.9) (cf. also n.4, $\S 1.1)$.

Next, $g_{\mathbf{X}}, d_{\mathbf{X}}$ as above are determined by $\boldsymbol{X}$. We will use the symbol $\boldsymbol{X}$ also for the p.g. pair and triple: $\left(X, g_{\mathbf{X}}\right)$ and $\left(X, g_{\mathbf{X}}, d_{\mathbf{X}}\right)$ (cf. n.4, §1.1). Also when there is no fear of confusion, we use $\boldsymbol{X}$ for its underlying variety $X$. For the variety $\boldsymbol{X} \in \mathbf{A n}_{0}$ we use the similar notational convenience to the above.

2. P.g. parametrization. Letting the analytic varieties $\tilde{X} \in \mathbf{A n}_{0}$ and $\boldsymbol{X} \in \mathbf{A} \mathbf{n}_{1 a}$ be as hitherto, we attach to them what we call 'p.g. cochain collections'. Such collections will contain all necessary sets of cochains in our p.g. uniform estimations. The arguments will be given parallelly to $\tilde{\boldsymbol{X}}, \boldsymbol{X}$. For this we set $(1.9)_{0}^{\prime} \quad\left(\boldsymbol{X}^{*}, X^{*}, P_{0}^{*}\right):=\left(\tilde{\boldsymbol{X}}, X, P_{0}^{\prime}\right)$ or $\left(\boldsymbol{X}, X, P_{0}\right) \quad\left(\mathrm{cf} .(1.7)_{0}^{\prime},(1.8)_{0}^{\prime}\right)$, and $D_{0, \boldsymbol{X}^{*}}:=U_{0}^{\prime}$ or $D$. We construct the p.g. collection in the following three steps.

First we define a parametrization of open submanifolds of $\boldsymbol{X}^{*}$ by

$(1.9)_{1} \quad v_{\mathbf{X}^{*}}: \nu_{\mathbf{X}^{*}}:=D_{0, \mathbf{X}^{*}} \times \boldsymbol{R}^{+} \ni v=\left\{\begin{array}{l}\left(P^{\prime} ; r\right) \\ (P ; r)\end{array}\right\} \longrightarrow\left\{\begin{array}{l}\tilde{\boldsymbol{X}}_{r}\left(P^{\prime}\right):=\mathbb{C}^{n} \times U_{r}\left(P^{\prime}\right) \\ \boldsymbol{X}_{r}(P):=U_{r}(P) \cap \boldsymbol{X}\end{array}\right\}$,

where $U_{r}\left(P^{\prime}\right), U_{r}(P)$ are the discs in $\boldsymbol{C}^{n^{\prime}}, \boldsymbol{C}^{n}$ of center $P^{\prime}, P$ and radius $r$, and we set

$$
\operatorname{Ouv}^{\prime}\left(\boldsymbol{X}^{*}\right)_{\mathrm{p} . \mathrm{g}}:=v_{\boldsymbol{X}^{*}}\left(\boldsymbol{\nu}_{X^{*}}\right) .
$$

(The manifolds in $\operatorname{Ouv}^{\prime}\left(\boldsymbol{X}^{*}\right)_{\text {p.g }}$ do not share particular p.g. properties. But the use of the suffix 'p.g.' will be concordant to the arguments henceforth.)

Next forming a parameter space $\boldsymbol{\mu}_{\boldsymbol{X}^{*}}:=\boldsymbol{\nu}_{\mathbf{X}^{*}} \times \boldsymbol{R}_{1}^{+2}$, we define the following parametrization of elements of $\operatorname{Cov}_{0}\left(\boldsymbol{X}^{*}\right)$ :

$$
u_{\mathbf{X}^{*}}: \boldsymbol{\mu}_{\mathbf{X}^{*}}=\boldsymbol{\nu}_{\mathbf{X}^{*}} \times \boldsymbol{R}_{1}^{+2} \ni \mu=(v ; \sigma) \longrightarrow \mathscr{A}_{\sigma}\left(\boldsymbol{X}_{r}^{*}\left(P^{*}\right)\right),
$$

where $\boldsymbol{X}_{r}^{*}\left(P^{*}\right)=\tilde{\boldsymbol{X}}_{r}\left(P^{\prime}\right)$ or $\boldsymbol{X}_{r}(P)$ is as in $(1.9)_{1}$, and

$(1.9)_{2}^{\prime \prime} \mathscr{A}_{\sigma}\left(\boldsymbol{X}_{\boldsymbol{r}}^{*}\left(P^{*}\right)\right):=g_{\boldsymbol{X}^{+}}$-p.g. covering of $\boldsymbol{X}_{r}^{*}\left(P^{*}\right)$ of size $\sigma$ in $\boldsymbol{X}^{*}$ (cf. $\left.(1.6)_{1}\right)$.

We then set

$$
\operatorname{Cov}_{0}\left(X^{*}\right)_{p \cdot g}:=u_{X^{*}}\left(\mu_{X^{*}}\right)
$$

Thirdly taking an element $\mathfrak{S} \in \operatorname{Coh}\left(\boldsymbol{X}^{*}\right)_{\text {p.g }}$ (Definition 1.5$)$, we define a parametrization of sets of p.g. cochains with values in $\mathfrak{H}$ by 
(1.9) ${ }_{3} \quad C_{, \mathfrak{j}}^{q}: \lambda_{\mathbf{X}^{*}}:=\boldsymbol{\mu}_{\mathbf{X}^{+}} \times \boldsymbol{R}_{1}^{+2} \ni \lambda=(\mu ; \alpha) \longrightarrow C^{q}\left(\mathscr{A}_{\sigma}\left(\boldsymbol{X}_{r}^{*}\left(P^{*}\right), \mathfrak{S}\right)_{\alpha}\right):=$ set of all $\alpha$-growth cochains with value in $\mathfrak{S}$ (cf. $\left.(1.4)_{8}\right)$,

and we set

$$
\boldsymbol{C}^{q}\left(\mathbb{X}^{*}, \mathfrak{S}\right)_{\mathrm{p} . \mathrm{g}}:=C_{\mathfrak{g}}^{q}\left(\lambda_{\mathbf{X}^{*}}\right)
$$

We define a parametrization $Z^{q}$ by changing ' $C^{q}$ ' to ' $Z$ ' .

\section{P.g. parametrization table}

$$
\begin{aligned}
& \lambda_{\mathbf{X}^{*}}=\mu_{\mathbf{X}^{*}} \times \boldsymbol{R}_{1}^{+2} \ni \lambda=(\mu ; \alpha) \stackrel{c_{\mathfrak{b}}^{q}}{\longrightarrow} \mathbb{C}^{q}\left(\mathbb{X}^{*}, \mathfrak{S}\right)_{\mathrm{p}, \mathrm{g}} \ni C^{q}\left(A_{\sigma}\left(\mathbb{X}_{r}^{*}\left(P^{*}\right)\right), \mathfrak{S}\right)_{\alpha}, \\
& \boldsymbol{\mu}_{\mathbf{X}^{*}}=\nu_{\mathbf{X}^{*}} \times \boldsymbol{R}_{1}^{+2} \ni \mu=(v ; \sigma) \stackrel{u_{X^{*}}}{\longrightarrow} \operatorname{Cov}_{0}\left(\mathbb{X}^{*}\right)_{\mathrm{p} . \mathrm{g}} \ni \mathscr{A}_{\sigma}\left(\mathbb{X}_{r}^{*}\left(P^{*}\right)\right)_{\alpha}, \\
& \boldsymbol{\nu}_{\mathbf{X}^{*}}=D_{0, \mathbf{X}^{*}} \times \mathbb{R}^{+} \ni v=\left(P^{*} ; r\right) \stackrel{v_{\mathbf{X}^{*}}}{\longrightarrow} \operatorname{Ouv}^{\prime}\left(\mathbb{X}^{*}\right)_{\mathrm{p} . \mathrm{g}} \ni \mathbb{X}_{r}^{*}\left(P^{*}\right) \text {. }
\end{aligned}
$$

The manifolds, their coverings and the sets of the cochains in our p.g. uniform estimations for $X^{*}$ will be taken from $\operatorname{Ouv}^{\prime}\left(\mathbb{X}^{*}\right)_{\mathrm{p} . \mathrm{g}}, \operatorname{Cov}_{0}\left(\mathbb{X}^{*}\right)_{\mathrm{p} . \mathrm{g}}$ and $C^{q}\left(X^{*}, \mathfrak{H}\right)_{\text {p.g. }}$. The last collection contains all necessary data in our p.g. uniform estimations. In order to emphasize the role of such a collection in our uniform estimation, we make

Definition 1.8. We call $\mathbb{C}^{q}\left(\boldsymbol{X}^{*}, \mathfrak{H}\right)_{\mathrm{p} . \mathrm{g}} q-$ th p.g. cochain collection for $\mathfrak{h}$.

3. Estimation data. In the uniform estimations in n.4, we use the p.g.c. estimation map $E \in \mathbb{E}_{\mathrm{p} . \mathrm{g}}$ and its first part $E^{\prime} \in \boldsymbol{E}_{\mathrm{p} . \mathbf{g}}^{\prime}$ (Definition 1.7). Next note that our uniform estimation does not work for all elements of $\lambda_{\mathbf{X}^{*}}$ (cf. (1.9) $)_{3}$ ) but for elements of a suitable subset

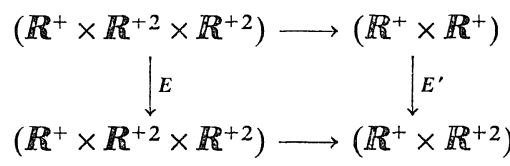

Figure I. of $\lambda_{X^{*}}$. More precisely, take subsets $U_{1}^{\prime}\left(\ni P_{0}^{\prime}\right)$ of $U_{0}^{\prime}$ and $U_{1}\left(\ni P_{0}\right)$ of $U_{0}$ (cf. $\left.(1.7)_{0},(1.8)_{0}^{\prime}\right)$, and we set $D_{1, \mathbf{X}^{k}}=U_{1}^{\prime}$ or $\left(D \cap U_{1}\right)\left(\subset D_{0, X^{*}}\right)$. Also taking elements $\tilde{r}=\tilde{r}_{\boldsymbol{X}^{*}} \in \mathbb{R}^{+}$and $\tilde{\sigma}=\tilde{\sigma}_{\mathbf{X}^{*}} \in \mathbb{R}_{1}^{+2}$, we set*)

$$
\nu_{\mathbf{X}^{*}}^{\prime}:=D_{1 ; \mathbf{X}^{*}} \times(0, \tilde{r}), \mu_{\mathbf{X}^{*}}^{\prime}:=\nu_{\mathbf{X}^{*}}^{\prime} \times \mathbb{R}_{\tilde{\sigma}}^{+2} \text { and } \lambda_{\mathbf{X}^{*}}^{\prime}=\mu_{\mathbf{X}^{*}} \times R_{1}^{+2} .
$$

We fix this restricted parameter space in the remainder of Section 1. Our p.g. uniform estimations for $\boldsymbol{X}^{*}$ in Section 1 will work for all elements of $\boldsymbol{X}_{\mathbf{X}^{*}}{ }^{*}$

4. Main results. Now, using the sets of the coverings and the cochains in $(1.9)_{1-3}$ and the estimation maps as in $n .3$, we will give our main results on the

*) $\boldsymbol{R}_{\tilde{\sigma}}^{+2}:=\left\{\sigma \in \boldsymbol{R}^{+2} ; \sigma \geqq \tilde{\sigma}\right\}$ (cf. the end of Introduction of Chapter I). 
p.g. uniform estimations for $\mathbf{X}^{*}=\tilde{X} \in \mathbf{A n}_{0}$ or $\boldsymbol{X} \in \mathbf{A n}_{1 a}$. First we will be concerned with Cech coboundary operator.

Theorem 1.1 (P.g. uniform estimation for Cech operator $\left.\delta=\delta_{\mathbf{X}^{\star}}\right)$. There is a map*) $\varepsilon_{\delta}: \operatorname{Coh}\left(\boldsymbol{X}^{*}\right)_{\mathrm{p} . \mathrm{g}} \ni \mathfrak{S} \rightarrow \mathbb{E}_{\mathrm{p} . \mathrm{g}} \ni E_{\mathfrak{g}}(q>0)$, with which we have:

$$
\begin{gathered}
s^{*} Z^{q}\left(\mathscr{A}_{\sigma}\left(\boldsymbol{X}_{r}^{*}\left(P^{*}\right)\right), \mathfrak{S}\right)_{\alpha} \subset \delta C^{q-1}\left(\mathscr{A}_{\sigma^{\prime}}\left(\mathbf{X}_{r^{\prime}}^{*}\left(P^{*}\right)\right), \mathfrak{S}\right)_{\alpha^{\prime}}, \\
\text { with }\left(r^{\prime} ; \sigma^{\prime} ; \alpha^{\prime}\right)=E_{\mathfrak{S}}(r ; \sigma ; \alpha) .
\end{gathered}
$$

Here the parameter $\left(P^{*} ; r ; \sigma ; \alpha\right)$ is in $\lambda_{X^{+}}^{\prime}\left(\subset D_{1: X^{*}} \times \mathbb{R}^{+} \times \mathbb{R}_{1}^{+2} \times \mathbb{R}_{1}^{+2}\right)$. Moreover, $s=$ p.g. refining map $p^{* *)}: \mathscr{A}_{\sigma^{\prime}}\left(\mathbb{X}_{r^{\prime}}^{*}\left(P^{*}\right)\right) \hookrightarrow \mathscr{A}_{\sigma}\left(\mathbb{X}_{r}^{*}\left(P^{*}\right)\right)$ (cf. Definition 1.6 $\left.{ }_{2}\right)$.

Next we will be concerned with the resolution of $\mathfrak{g} \in \operatorname{Coh}\left(\mathbb{X}^{*}\right)_{\text {p.g }}$ (cf. also $\left.(1.4)_{2}\right)$.

Theorem 1.2 (P.g. uniform estimation for resolution). There is a map $\varepsilon_{\boldsymbol{X}}: \operatorname{Coh}\left(\boldsymbol{X}^{*}\right)_{\mathrm{p} . \mathrm{g}} \ni \mathfrak{S} \rightarrow \mathbb{E}_{\mathrm{p.g}} \ni E_{\mathfrak{g},}(q \geqq 0)$, with which we have

$$
\begin{gathered}
s^{*} Z^{q}\left(\mathscr{A}_{\sigma}\left(\mathbb{X}_{r}^{*}\left(P^{*}\right)\right), \mathfrak{s}\right)_{\alpha} \subset \omega_{\mathfrak{r}} Z^{q}\left(\mathscr{A}_{\alpha^{\prime}}\left(\mathbb{X}_{r^{\prime}}^{*}\left(P^{*}\right)\right), \mathfrak{D}_{\mathbb{X}^{*}}^{k}\right)_{\alpha^{\prime}}, \\
\text { with }\left(r^{\prime} ; \sigma^{\prime} ; \alpha\right)=E_{\mathfrak{g}}(r ; \sigma ; \alpha),
\end{gathered}
$$

where the parameter $\left(P^{*} ; r ; \sigma ; \alpha\right)$ is as in Theorem 1.1. Moreover, $\omega_{55}: D_{\mathbf{X}^{k}}^{k}$ $\rightarrow \mathfrak{S}$ is the first resolution part of $\mathfrak{S}$ (Definition 1.5).

For the proof of Theorem 1.1, Theorem 1.2, see Section 1.3. Also we give applications of Theorem 1.1, Theorem 1.2 in n.6, Section 1.2 and in Section 3. Here we add the following

Corollary 1.1. There is a map $\varepsilon_{\delta}^{\prime}: \mathbb{Z}^{+} \rightarrow \mathbb{E}_{\mathrm{p} . \mathrm{g}}^{\prime}$, which satisfies the factorization in Figure II. (In Figure II 'Ig' denotes the length map $\left(c f .(1.4)_{2}\right)$, and the projection $\pi$ is as in Figure I, n.6, §1.1).

The similar factorization to Figure II holds also

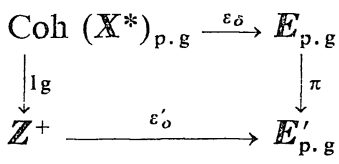

Figure II. for the map $\varepsilon_{\mathbf{X}^{*}}$ in Theorem 1.2. (Corollary 1.1 is not a consequence of Theorem 1.1. But the proof of the latter will also insure the former; see $\S 1.3$.)

Now in accordance to the parametrization table in $n .2$, we rewrite Theorem 1.1 in the following diagram:

*) Strictly, the map $s_{\delta}$ in Theorem 1.1 depends also on $q=$ cohomology degree. But the influence of $q$ on the estimation is small; we do not mention the cohomology degree in question in Theorem 1.1 and in the other estimations in Section 1.

***) For the p.g. refining map $s$, see n.4, Section 1.1. We use the symbol $s$ for the p.g. refining map in question, without mentioning it in the later arguments. 


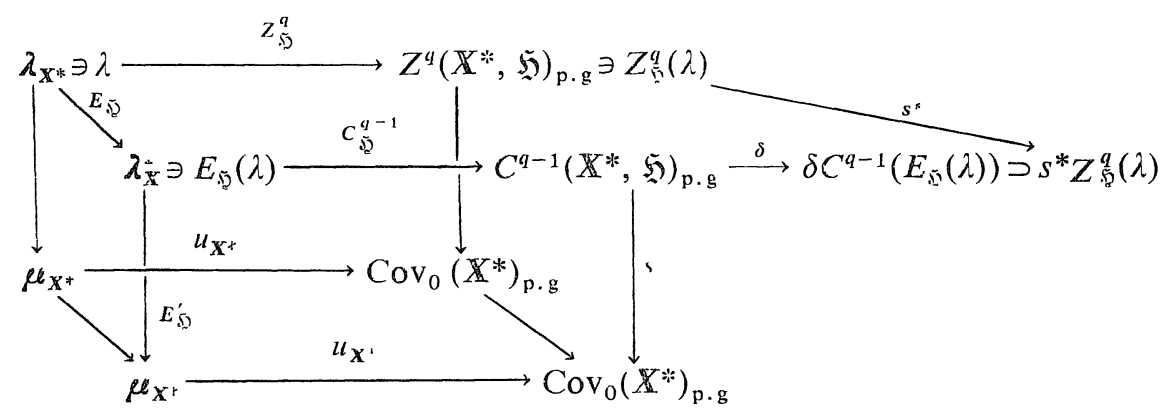

Figure III. P.g. uniform estimation for Cech operator.

(The similar diagram also holds for Theorem 1.2.) Next we assume that the variety $\mathbb{X}^{*}$ in Theorem 1.1, Theorem 1.2 is in $\mathbb{A n}_{1 a}: \mathbb{X}^{*}=\mathbb{X} \in \mathbb{A m}_{1 a}$ (cf. (1.8)o), and take a point $P \in D_{1 ; X}=\left(U_{1} \cap D\right)$. We then set $\tilde{\mu}_{X}:=(0, \tilde{r}) \times \mathbb{R}_{1}^{+2}$ (cf. $\left.(1.9)_{4}\right)$. For an element $\mu=(r ; \sigma) \in \tilde{\ell}_{X}\left(=(0, \tilde{r}) \times \mathbb{R}_{1}^{+2}\right)$, we write the p.g. covering $\mathscr{A}_{\sigma}\left(\mathbb{X}_{r}(P)\right)\left(\mathrm{c} f .(1.9)_{2}\right)$ as $\mathscr{A}_{\mu}(P)$. Moreover, we set

$(1.10)_{3} \quad C^{q}\left(\mathscr{A}_{\mu}(P), \mathfrak{\mathfrak { S }}\right)_{\mathrm{p}, \mathrm{g}}:=\cup_{\alpha \in \mathbb{R}_{1}^{+2}} C^{q}\left(\mathscr{A}_{\mu}(P), \mathfrak{S}\right)_{\alpha} \quad\left(=g_{X}\right.$-p.g. subgroup*) of $\left.C^{q}\left(\mathscr{A}_{\mu}, \mathfrak{5}\right)\right)$.

Then, from the explicit formulations of Theorem 1.1, Theorem 1.2 and from the factorization in Figure I, n.3, Section 1.2 (of the p.g.c. maps $E_{\text {. }} \in \mathbb{E}_{\text {p.g }}$ ), we easily have

Corollary 1.2.**) We have the inclusions:

$$
\left\{\begin{array}{l}
s^{*} Z^{q}\left(\mathscr{A}_{\mu}(P), \mathfrak{H}\right)_{\mathrm{p} . \mathrm{g}} \subset \delta C^{q-1}\left(\mathscr{A}_{\mu^{\prime}}(P), \mathfrak{H}\right)_{\mathrm{p} . \mathrm{g}}(q \geqq 1), \\
s^{*} Z^{q}\left(\mathscr{A}_{\mu}(P), \mathfrak{S}\right)_{\mathrm{p} . \mathrm{g}} \subset \omega_{\tilde{\mathfrak{H}}} Z^{q}\left(\mathscr{A}_{\mu^{\prime}}(P), \mathfrak{S}\right)_{\mathrm{p} . \mathrm{g}}(q \geqq 0),
\end{array}\right.
$$

where $\mu^{\prime}$ is a suitable element of $\tilde{\mu}_{\mathbf{x}}$.

Corollary 1.2 is given in terms of the p.g. subgroups as in $(1.10)_{4}$, and may be more suitable for geometric applications than Theorem 1.1, Theorem 1.2, where we used the sets of the cochains, $C^{q}\left(\mathscr{A}_{\mu}(P), \mathfrak{H}\right)_{\alpha}, \ldots$ We use Corollary 1.2 in n.6.

Here we make a remark on the explicit estimation in Theorem $1.1^{* * *)}$.

Remark $1.2_{1}$. As may be clear from its formulation, the estimation map

*) Cf. $(1.4)_{7}$.

**) The similar fact to Corollary 1.2 holds also for $\tilde{\mathbf{X}} \in \mathrm{An}_{0}$. But we do not use such a fact (cf. n.6, §1.2).

****) The similar remark also holds for Theorem 1.1. 
$E_{\mathfrak{j}} \in \boldsymbol{E}_{\mathrm{p} . \mathrm{g}}$ is taken independently from the point $P^{*}$, which is the origin of the manifold $\boldsymbol{X}_{r}^{*}(P)$ in question. When the variety $\boldsymbol{X}^{*}=\mathbf{X} \in \mathbf{A n}_{1 a}$, the divisor $D_{1 ; \mathbf{X}^{*}}\left(\ni P^{*}\right)$ has, in general, singularities, and the above independence is never of obvious nature. As we will see in Section 1.3, Section 4.2 and in Section 5.1, this independence is insured by certain uniform estimations on Weierstrass polynomials and the coherency theorem of K. Oka (or, more precisely, the structure of the proof of his theorem). From its formulation, we may regard that the coherency theorem insures a uniform structure of the coherent sheaves with respect to the points on the analytic varieties. The independence mentioned just above plays a very basic role in our treatments of the cohomology theories in this paper (cf. $\S 2, \S 3$ ). As in the case of theories of coherent sheaves, where no explicit estimations are involved, the coherency theorem of $\mathrm{K}$. Oka will play the basic role in our cohomology theory in this paper. Next Corollary 1.1 and Corollary 1.3 concern a type of uniform estimations with respect to the p.g. sheaves on the analytic varieties in question. Though we do not use those results in this paper, the factorizations in Corollary 1.1 and Corollary 1.3 may be useful, when one concerns a family of p.g. coherent sheaves.

Remark 1.2. . The remark here is of technical nature for the proof of Theorem 1.1. Letting $\boldsymbol{X}^{*}$ be a variety in $\mathbf{A n}_{1 a}$ or $\mathbf{A n}_{0}$, we use the phrase 'Theorem 1.1 holds for $\mathfrak{D}_{\mathbf{X}^{*}}$ ' as a synonym for that $(1.10)_{1}$ holds for $\mathfrak{D}_{\mathbf{X}^{*}}$, with a suitable p.g.c. map $E_{\mathbf{X}^{*}}$. Next, letting $\mathscr{C}$ be a collection of p.g. coherent sheaves over $\boldsymbol{X}^{*}$, we use the phrase 'Theorem 1.1 holds for $\mathscr{C}$ ' as a synonym for ' $(1.10)_{1}$ holds for each $\mathfrak{S} \in \mathscr{C}^{\prime}$ (by changing $\operatorname{Coh}\left(\boldsymbol{X}^{*}\right)$ in Theorem 1.1 by $\mathscr{C}$ ). When we use this terminology, we assume that the factorization in Corollary 1.1 holds for $\mathscr{C}$. We use the similar terminology for Theorem 1.2, Theorem 1.3 and Theorem 1.4.

5. An affine analogue. Here we give an analogue of the results in $n .4$ to affine varieties. The content here is chiefly given for purpose of geometric application (cf. n.6 soon below and §3). We do not give corresponding explicit estimations to Theorem 1.1, Theorem 1.2 to affine varieties*). Our results here will be given in a similar form to Corollary 1.2. In order to formulate such results, we first mean by smooth imbedded affine variety a datum $\boldsymbol{X}^{\prime}$ as follows

*) For the explicit estimations for Theorem 1.3, Theorem 1.4, see Section 1.3 and Section 4.2 , where the proof of these theorems is given. 


$$
\boldsymbol{X}^{\prime}=\left(\mathbb{C}^{n}(z), X^{\prime}, \mathfrak{H}_{\mathbf{X}^{\prime}}\right),
$$

where $X^{\prime}$ is a smooth affine variety in a euclidean space $\mathbb{C}^{n}(z)$ (cf. n.1, §1.2) and $\mathfrak{S}_{\boldsymbol{X}^{\prime}}$ is a $(|z|+1)$-p.g. resolution of the structure sheaf*) $\mathfrak{D}_{\boldsymbol{X}^{\prime}}$ of $\boldsymbol{X}^{\prime}$ over $\mathbb{C}^{n}$. We then set

$(1.11)_{0} \quad$ Aff: = collection of all smooth imbedded affine varieties.

Letting an element $\boldsymbol{X}^{\prime} \in \mathrm{Aff}$ be of the form in $(1.11)_{0}$, the underlying analytic variety is the affine variety $X^{\prime}$. In this paper, unless we say otherwise, we regard*) $\left(\boldsymbol{X}^{\prime}, \mathfrak{D}_{\mathbf{x}^{\prime}}\right)$ as the analytic variety. When we regard it as the algebraic variety, we write it as $\left(\mathbb{X}_{\text {alg }}^{\prime}, \mathfrak{D}_{\mathbf{X}^{\prime}, \text { al }}\right)$, where the underlying topology is that of Zariski. The p.g. and distance functions for $\mathbb{X}^{\prime}$ will be $g_{\mathbf{X}^{\prime}}=|z|+1, d_{\mathbf{X}^{\prime}}:=$ induced distance from the natural one $d_{z}$ of $\mathbb{C}^{n}(z)$. We write the p.g. pair $\left(\mathbb{X}^{\prime}, g_{\mathbf{X}^{\prime}}\right)$ and triple $\left(\mathbb{X}^{\prime}, g_{\mathbf{X}^{\prime}}, d_{\mathbf{X}^{\prime}}\right)$ also as $\mathbf{X}^{\prime}$ (cf. also n.1, §1.2).

Next setting $\boldsymbol{\mu}_{X^{\prime}}:=\mathbb{R}_{1}^{+2}$, our coverings will be taken from the family

$$
\operatorname{Cov}_{0}\left(\mathbb{X}^{\prime}\right)_{\text {p.g }}:=\left\{\mathscr{A}_{\sigma}\left(\mathbb{X}^{\prime}\right) ; \sigma \in \mu_{X^{\prime}}\right\},
$$

where $\mathscr{A}_{\sigma}\left(\mathbb{X}^{\prime}\right)$ : $=g_{X^{\prime}}$-p.g. covering of $\mathbb{X}^{\prime}$ of size $\sigma$ in $X^{\prime}$ (Definition $1.6_{1}$ ). Taking an element $\mathfrak{S}^{\prime} \in \operatorname{Coh}\left(\mathbb{X}^{\prime}\right)_{\text {p.g }}$, our underlying datum for the p.g. cohomology (given to $\mathfrak{S}^{\prime}$ ) will be the following p.g. group:

$$
C^{q}\left(\mathscr{A}_{\sigma}\left(\boldsymbol{X}^{\prime}\right), \mathfrak{H}^{\prime}\right)_{\mathrm{p} . \mathrm{g}}:=g_{\mathbf{X}^{\prime}} \text {-subgroup of } C^{q}\left(\mathscr{A}_{\sigma}\left(\mathbb{X}^{\prime}\right), \mathfrak{H}^{\prime}\right)
$$

(Definition 1.3 and $(1.4)_{8}$ ).

Thirdly we will use el-maps $\mathscr{L}: \mathbb{R}^{+2} \rightarrow \mathbb{R}^{+2}$ (cf. n.5, § 1.1) in our estimations soon below. We set

$$
\mathbb{L}=\text { collection of all el-maps . }
$$

For each $q \in \mathbb{Z}^{+} \cup 0$ we fix a restricted parameter space $\boldsymbol{\beta}_{\mathbf{X}^{\prime}}^{\prime}:=\mathbb{R}_{\tilde{\sigma}}^{+2}$, with an element $\tilde{\sigma}=\tilde{\sigma}_{\boldsymbol{X}^{\prime}} \in \mathbb{R}_{1}^{+2}$; our uniform estimations will work for $\boldsymbol{\mu}_{\mathbf{X}^{\prime}}^{\prime}$ (cf. also n.3, $\S 1.2$. Now we give an analogue of Theorem 1.1, Theorem 1.2 to $\mathbb{X}^{\prime}$ in the following fashion.

Theorem 1.3 (P.g. uniform estimation for Cech operator $\delta=\delta_{\mathbf{X}}$ ). There is a map $\varepsilon_{\delta}: \operatorname{Coh}\left(\mathbb{X}^{\prime}\right)_{\text {p.g }} \ni \mathfrak{H}^{\prime} \rightarrow \mathbb{L} \ni \mathscr{L}_{\mathfrak{S}^{\prime}}(q>0)$, with which we have

$$
s^{*} Z^{q}\left(\mathscr{A}_{\sigma}\left(\boldsymbol{X}^{\prime}\right), \mathfrak{S}^{\prime}\right)_{\mathrm{p} . \mathrm{g}} \subset \delta C^{q-1}\left(\mathscr{A}_{\sigma^{\prime}}\left(\boldsymbol{X}^{\prime}\right), \mathfrak{H}^{\prime}\right)_{\mathrm{p} . \mathrm{g}}, \text { with } \sigma^{\prime}=\mathscr{L}_{\mathfrak{S}^{\prime}}(\sigma) .
$$

Theorem 1.4 (P.g. uniform estimation for resolution). There is a map

*) As in n. 3 we use the symbol $\boldsymbol{X}^{\prime}$ also for its underlying variety $X^{\prime}$. 
$\varepsilon_{\mathbf{X}^{\prime}}: \operatorname{Coh}\left(\boldsymbol{X}^{\prime}\right)_{\text {p.g }} \ni \mathfrak{S}^{\prime} \rightarrow \mathbb{L} \ni \mathscr{L}_{\mathfrak{S}^{\prime}}(q \geqq 0)$, with which we have

$(1.12)_{2} \quad s^{*} Z^{q}\left(\mathscr{A}_{\sigma}\left(\boldsymbol{X}^{\prime}\right), \mathfrak{S}^{\prime}\right)_{\mathrm{p} . \mathrm{g}} \subset \omega_{\mathfrak{g}^{\prime}} Z^{q}\left(\mathscr{A}_{\sigma^{\prime}}\left(\boldsymbol{X}^{\prime}\right), \mathfrak{S}^{\prime}\right)_{\mathrm{p} . \mathrm{g}}$, with $\sigma^{\prime}=\mathscr{L}_{\mathfrak{5}^{\prime}}(\sigma)$,

where $\omega_{\mathfrak{S}^{\prime}}: \mathfrak{D}_{\mathbf{X}^{\prime}}^{k} \rightarrow \mathfrak{H}^{\prime}$ is the first resolution of $\mathfrak{S}^{\prime}$ (cf. Definition 1.5).

In the above $\sigma$ is in the restricted parameter space $\mu_{X^{\prime}}^{\prime}$. Also, corresponding to Corollary 1.2 , we have

Corollary 1.3. We have the following factorization

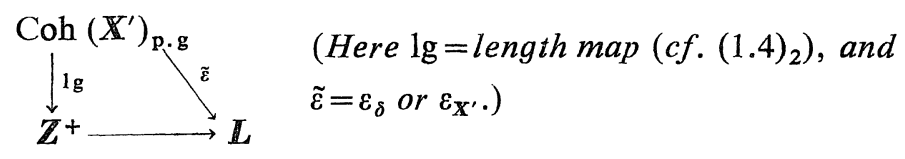

The proof of the above results is given in Section 1.3 (cf. also $§ 4$ ).

6. P.g. complexes. Here we give our analogue of Theorems A, B of $\mathbf{H}$. Cartan to the p.g. cohomology theory. For this we fix the following data as in Theorems 1.1-1.4 (cf. also Corollary 1.2):

$$
\left\{\begin{array}{l}
\text { the local analytic variety } \mathbf{X} \in \mathbf{A n}_{1 a} \text {, the point } P \in D, \\
\text { and the parameter space } \tilde{\mu}_{X}=(0, \tilde{r}) \times \mathbb{R}_{\tilde{\sigma}}^{+2}, \\
\text { the affine variety } \boldsymbol{X}^{\prime} \text { and the parameter space } \boldsymbol{\mu}_{X^{\prime}}^{\prime},
\end{array}\right.
$$

and we set

$(1.13)_{1} \quad\left(\mathbb{X}^{*}, \mu_{\mathbf{X}}^{*}\right)=\left(\mathbb{X}, \tilde{\mu}_{\mathbf{X}}\right)$ or $\left(\mathbb{X}^{\prime}, \mu_{\mathbf{X}^{\prime}}^{\prime}\right)$, and $\mathscr{A}_{\mu}=\mathscr{A}_{\sigma}\left(\mathbb{X}_{r}(P)\right)$ or $\mathscr{A}_{\sigma}\left(\mathbb{X}^{\prime}\right)$, for each $\mu=(r ; \sigma) \in{\tilde{\boldsymbol{\varepsilon}_{X}}}_{\mathbf{X}}=(0, \tilde{r}) \times \mathbb{R}_{\tilde{\sigma}}^{+2}$ or $=\sigma \in \boldsymbol{\mu}_{\mathbf{X}^{\prime}}^{\prime}=\mathbb{R}_{\tilde{\sigma}}^{+2}$, where the point $P \in D$ is as in Corollary 1.2.

Letting the p.g. sheaf $\mathfrak{S}^{*}=\mathfrak{5}$ or $\mathfrak{H}^{\prime}$ be as in Theorem 1.1, or Theorem 1.3, we make

Definition $1.9_{1}$. By p.g. Čech complex for $\left(\mathscr{A}_{\mu}, \mathfrak{S}^{*}\right)$, we mean

$$
0 \longrightarrow C^{0}\left(\mathscr{A}_{\mu}, \mathfrak{S}^{*}\right)_{\mathrm{p} . \mathrm{g}} \longrightarrow \cdots \longrightarrow C^{q}\left(\mathscr{A}_{\mu}, \mathfrak{S}^{*}\right)_{\mathrm{p} . \mathrm{g}} \longrightarrow
$$

We write this complex as $C^{*}\left(\mathscr{A}_{\mu}, \mathfrak{S}^{*}\right)_{\mathrm{p} . \mathrm{g}}$.

Next, assuming that $\mathfrak{S}^{*}$ is of the form in $(1.4)_{1}$, we call the following complex q-th p.g. resolution complex for $\mathfrak{H}^{*}$ :

$$
\begin{aligned}
0 \longrightarrow Z^{q}\left(\mathscr{A}_{\mu}, \mathfrak{D}_{X^{*}}^{k}\right)_{\mathrm{p} . \mathrm{g}} & \stackrel{K_{p-1}}{\longrightarrow} \cdots \stackrel{K_{1}}{\longrightarrow} Z^{q}\left(\mathscr{A}_{\mu}, \mathfrak{D}_{\mathbf{X}^{*}}^{k_{1}}\right)_{\mathrm{p} . \mathrm{g}} \\
\stackrel{\omega_{\mathfrak{g}^{*}}}{\longrightarrow} Z^{q}\left(\mathscr{A}_{\mu}, \mathfrak{S}^{*}\right)_{\mathrm{p} . \mathrm{g}} & \longrightarrow 0 .
\end{aligned}
$$

Thirdly, we regard $\tilde{\boldsymbol{\mu}}_{\boldsymbol{X}^{*}}=\tilde{\boldsymbol{\mu}}_{\boldsymbol{X}}$ or $\boldsymbol{\mu}_{\boldsymbol{X}^{\prime}}^{\prime}$ as the ordered set in the following 
manner (cf. $\left.(1.13)_{0}\right)$ :

$$
\mu=(r ; \sigma) \succ \mu^{\prime}=\left(r^{\prime} ; \sigma^{\prime}\right) \Longleftrightarrow r<r^{\prime}, \sigma>\sigma^{\prime}, \text { and } \mu=\sigma \succ \mu^{\prime}=\sigma^{\prime} .
$$

Then letting $X_{P}$ denote the germ of $X$ at $P$ we make

Definition $1.9_{2}$. By p.g. Cech complex for $\left(\mathbb{X}_{P}, \mathfrak{5}\right)$ or $\left(\mathbb{X}^{\prime}, \mathfrak{S}^{\prime}\right)$, we mean $(1.13)_{5} C^{*}\left(\mathbb{X}_{P}, \mathfrak{S}\right)_{\mathrm{p} . \mathrm{g}}:=\lim _{\mu \rightarrow} C^{*}\left(\mathscr{A}_{\mu}, \mathfrak{S}\right)_{\mathrm{p} . \mathrm{g}}, C^{*}\left(\mathbb{X}^{\prime}, \mathfrak{S}^{\prime}\right)_{\mathrm{p} . \mathrm{g}}:=\lim _{\mu \rightarrow} C^{*}\left(\mathscr{A}_{\mu}, \mathfrak{S}^{\prime}\right)_{\mathrm{p} . \mathrm{g}}$

We define 'p.g. resolution complex for $\left(\mathbb{X}_{P}, \mathfrak{S}\right)$ or $\left(\mathbb{X}^{\prime}, \mathfrak{S}^{\prime}\right)$ ' by operating the similar limit procedure to $(1.13)_{5}$ to the complex in $(1.13)_{3}$.

Writing the $q$-th cohomology groups of the p.g. Cech complexes in Definition $1.9_{3}$ as $H^{q}\left(\mathbb{X}_{P}, \mathfrak{S}\right)_{\text {p.g }}, H^{q}\left(\mathbb{X}^{\prime}, \mathfrak{S}^{\prime}\right)_{\text {p.g }}$, we have the following theorem directly from Corollary 1.2 and Theorem 1.3.

Theorem 1.5. $H^{q}\left(\mathbb{X}_{P}, \mathfrak{S}\right)_{\mathrm{p} . \mathrm{g}} \cong 0$ and $H^{q}\left(\mathbb{X}^{\prime}, \mathfrak{S}^{\prime}\right)_{\mathrm{p} . \mathrm{g}} \cong 0(q>0)$.

Next applying the standard syzygy arguments to Corollary 1.2 and Theorem 1.4, we easily have

Lemma 1.1. The $q$-th p.g. resolution complexes $(q \geqq 0)$ for $\left(\mathbb{X}_{P}, \mathfrak{S}\right)$ and $\left(\mathbf{X}^{\prime}, \mathfrak{5}^{\prime}\right)$ are exact (Definition $\left.1.9_{2}\right)$ :

$$
\begin{gathered}
(1.13)_{6} \quad 0 \longrightarrow \lim _{\mu \rightarrow} Z^{q}\left(\mathscr{N}_{\mu}(P), \mathfrak{D}_{X}^{k_{p}}\right)_{\mathrm{p} . \mathrm{g}} \stackrel{K_{p-1}}{\longrightarrow} \cdots \stackrel{K_{1}}{\longrightarrow} \lim _{\mu \rightarrow} Z^{q}\left(\mathscr{A}_{\mu}(P), \mathfrak{D}_{X}^{k_{1}}\right)_{\mathrm{p} . \mathrm{g}} \\
\stackrel{\omega_{\mathfrak{S}}}{\longrightarrow} \lim _{\mu \rightarrow} Z^{q}\left(\mathscr{A}_{\mu}(P), \mathfrak{S}\right)_{\mathrm{p} . \mathrm{g}} \longrightarrow 0,
\end{gathered}
$$

where we set $\mathscr{A}_{\mu}(P):=\mathscr{A}_{\sigma}\left(\mathbb{X}_{r}(P)\right)$.

(and the similar exact sequence for $\left(\mathbb{X}^{\prime}, \mathbf{s}^{\prime}\right)$ ).

Now, in order to determine $H^{0}\left(\mathbb{X}_{P}, \mathfrak{S}\right)_{\mathrm{p} . \mathrm{g}}, H^{0}\left(\mathbb{X}^{\prime}, \mathfrak{S i}^{\prime}\right)_{\mathrm{p} . \mathrm{g}}$, we let $\theta_{P}, \theta_{\mathbf{X}^{\prime}}$ denote the natural homomorphisms from the algebraic objects to the analytic ones

$(1.13)_{7} \quad \theta_{P}: \mathfrak{D}_{X_{0}}(* D)_{P} \rightarrow H^{0}\left(\mathbb{X}_{P}, \mathfrak{D}_{\mathbf{X}}\right)_{\mathrm{p} . \mathrm{g}}, \theta_{\mathbf{X}^{\prime}}: \Gamma\left(\mathbb{X}_{\mathrm{alg}}^{\prime}, \mathfrak{D}_{\mathbb{X}^{\prime} ; \mathrm{alg}}\right) \rightarrow H^{0}\left(\mathbb{X}^{\prime}, \mathfrak{D}_{\mathbf{X}^{\prime}}\right)_{\mathrm{p} . \mathrm{g}}$, where

$(1.13)_{7}^{\prime} \mathfrak{D}_{X_{0}}(* D):=$ sheaf (over $X_{0}$ ) of meromorphic functions with the pole $D$.

Then we have

Theorem 1.6. $6_{1}$. The homomorphisms $\theta_{P}$ and $\theta_{\mathbf{X}^{\prime}}$ are isomorphic. 
If $\boldsymbol{X}^{\prime}=\boldsymbol{C}^{n}$ then Theorem $1.6_{1}$ is a classically well known consequence of Cauchy integral formula.*) If $X_{0}$ is smooth, then we get Theorem 1.6 also easily from Hartogus theorem on removable singularities (in the codimension one case). For general $\boldsymbol{X}, \boldsymbol{X}^{\prime}$ we derive Theorem 1.6 from what are mentioned just above (cf. n.4, §1.3). Finally, applying the standard syzygy arguments to Theorem $1.6_{1}$ and Lemma 1.1, we easily have**)

Theorem 1.6. $\mathbf{2}_{2}$. The following complexes are exact:

$$
\left\{\begin{array}{l}
\mathfrak{D}_{\mathbf{X}}^{k_{2}} \stackrel{K_{1}}{\longrightarrow} \mathfrak{D}_{\mathbf{X}}^{k_{1}} \stackrel{\omega_{\mathfrak{S}}}{\longrightarrow} H^{0}\left(\boldsymbol{X}_{P}, \mathfrak{H}\right)_{\mathrm{p} . \mathrm{g}} \longrightarrow 0, \\
\Gamma\left(\boldsymbol{X}_{\mathrm{a} 1 \mathrm{~g}}^{\prime}, \mathfrak{D}_{\mathbf{X}^{\prime}, \mathrm{alg}}^{k_{2}}\right) \stackrel{K_{1}^{\prime}}{\longrightarrow} \Gamma\left(\boldsymbol{X}_{\mathrm{alg}}^{\prime}, \mathfrak{D}_{\mathbf{X}^{\prime}, \mathrm{alg}}^{k_{1}}\right) \stackrel{\omega_{\mathfrak{g}}}{\longrightarrow} H^{0}\left(\boldsymbol{X}^{\prime}, \mathfrak{H}^{\prime}\right)_{\mathrm{p} . \mathrm{g}} \longrightarrow 0 .
\end{array}\right.
$$

Theorem 1.5 and Theorem 1.6 are our p.g. analogues of Theorems A and B of H. Cartan ([1]). Applications of Theorem 1.5 and Theorem 1.6 will be given in Section 3.

Remark 1.3. . In [21], H. Yamaguchi showed an analogue of Theorem 1.5 and Theorem 1.6 to algebraic locally free coherent sheaves over affine varieties, by using Theorem 1.5 and Theorem 1.6. Next, note that, in Theorems 1.1-1.6, we gave a more or less categorical treatments of the p.g. uniform estimations. At present, we lack the notion of 'p.g. maps'. In this direction, S. Kamiya ([6]) gave some functorial treatments of our p.g. cohomology theory. It seems to be quite desirable to give a suitable functorial generalization of our p.g. cohomology theory in Section 1 .

Remark $1.3_{2}$. As was mentioned, ${ }^{* * *}$ ) cohomology theories with p.g. conditions were studied by P. Deligne-G. Maliotionist ([11]) and by M. CorbalnaP. A. Griffiths ([2]) for locally free algebraic coherent sheaves over smooth algebraic varieties. Our results for p.g. coherent sheaves over the analytic varieties as in Section 1.2, together with the result of H. Yamaguchi ([21]), are more general than theirs. In particular, the independence assertions mentioned in Remark 1.2 are not found in [2], [11]. Also, our proof of Theorems 1.1-1.6 depends on p.g. estimations on the uniform estimations on homomorphisms on coherent sheaves in Section 1.3 and on Cousin integrals (cf. Chapter III), and is entirely different from ones in [2], [11], which use the $\bar{\partial}$-estimations.

*) Cf. $(0)_{1}$ in the introduction of Chapter I.

**) Cf. also Lemma 1.2 in Section 1.2.

***) Cf. Introduction. 


\section{§1.3. A Key Theorem and Key Lemmas}

Here we will reduce Theorems 1.1-1.4 in Section 1.2 to a key theorem, Theorem 1.7, and key lemmas, Lemmas 1.2-1.4. The latter is proven in Chapter III and Section 4.

1. A key theorem. First setting

$(1.14)_{0} \quad$ Euc $^{\prime}:=$ collection of all products $\mathbb{C}^{n}(z) \times \mathbb{C}^{n^{\prime}}\left(z^{\prime}\right)$ of coordinated euclidean spaces (n.1, § 1.2),

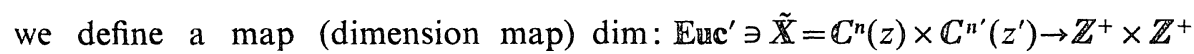
$\ni\left(n, n^{\prime}\right)$. The p.g. and distance functions for $\tilde{X}$ are $g_{\tilde{\mathbf{X}}}=|\tilde{z}|+1$, with $\tilde{z}=\left(z, z^{\prime}\right)$, and $d_{\tilde{\mathbf{X}}}:=d_{\tilde{z}}$ (=natural distance of $\tilde{\mathbf{X}}$ ) (cf. n.1, § 1.2). Taking an element $\left(P^{\prime} ; r, \sigma ; \alpha\right) \in \lambda_{\tilde{\mathbf{x}}}:=\boldsymbol{C}^{n^{\prime}} \times(0,1] \times \boldsymbol{R}_{1}^{+2} \times \mathbb{R}_{1}^{+2}$, the set of the cochains in Theorem 1.7 soon below is as follows:

$(1.14)_{1}^{\prime} C^{q}\left(\mathscr{A}_{\sigma}\left(\tilde{\mathbf{X}}_{r}\left(P^{\prime}\right)\right), \mathfrak{D}_{\tilde{\mathbf{X}}}\right)_{\alpha}:=$ set of all $g_{\tilde{\mathbf{X}}^{-} \alpha \text {-growth cochains with value in }}$ the structure sheaf $\mathfrak{D}_{\tilde{\mathbf{X}}}$ of $\tilde{\mathbb{X}}$ (cf. $(1.4)_{8}$ ),

where

$(1.14)_{2}^{\prime} \mathscr{A}_{\sigma}\left(\tilde{\mathbf{X}}_{r}\left(P^{\prime}\right)\right):=g_{\tilde{\mathbf{X}}}$-p.g. covering of $\tilde{\mathbb{X}}_{r}\left(P^{\prime}\right):=\mathbb{C}^{n} \times U_{r}\left(P^{\prime}\right)$ of size $\sigma$ in $\mathbb{C}^{n} \times \mathbb{C}^{n^{\prime}}$, with $U_{r}\left(P^{\prime}\right):=\left\{Q^{\prime} \in \mathbb{C}^{n^{\prime}}\left(z^{\prime}\right) ; d_{z^{\prime}}\left(Q^{\prime}, P^{\prime}\right)<r\right\}$ (Definition 1.6.6.* $\left.{ }^{*}\right)$

Then the following theorem is most basic among the results in Section 1.3.

Theorem 1.7 (P.g. uniform estimation for $\mathfrak{D}_{\tilde{\mathbf{x}}}$; $\left.\tilde{\mathbf{X}}=\mathbb{C}^{n} \times \mathbb{C}^{n^{\prime}}\right)$. There is a map $\varepsilon_{\delta}: \mathbb{E u c}^{\prime} \ni \tilde{\mathbb{X}} \rightarrow \mathbb{E}_{\mathrm{p} . \mathrm{g}} \ni$ $E_{\tilde{\mathbf{X}}}(q>0)$, which is factored as in Figure $\mathbf{I}$, and with which we have the following for each $\tilde{\mathbf{X}} \in \mathbf{E u c}^{\prime}$ :

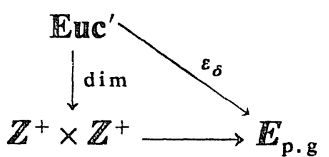

Figure I.

$$
s^{*} Z^{q}\left(\mathscr{A}_{\sigma}\left(\tilde{X}_{r}\left(P^{\prime}\right)\right), \mathfrak{D}_{\tilde{\mathbf{x}}}\right)_{\alpha} \subset \delta C^{q-1}\left(\mathscr{A}_{\sigma^{\prime}}\left(\tilde{X}_{r^{\prime}}(P)\right), \mathfrak{D}_{\tilde{\mathbf{X}}}\right)_{\alpha^{\prime}},
$$

with $\left(r^{\prime}, \sigma^{\prime} ; \alpha^{\prime}\right)=E_{\tilde{\mathbf{X}}}(r, \sigma ; \alpha)$, where $\left(P^{\prime} ; r, \sigma ; \alpha\right)$ is in $\lambda_{\tilde{\mathbf{X}}}=\mathbb{C}^{n^{\prime}} \times \cdots \times \mathbb{R}_{1}^{+2}$.

As may be clear from its formulation, Theorem 1.7 will be most basic for the proof of Theorems 1.1-1.6 (among the p.g. uniform estimations given in Section 1.3). The proof of Theorem 1.7 will be given in Chapter III in an independent manner from the contents**) of Chapters I, II. Here we derive a

*) $d_{z}$, = natural distance in $\mathbb{C}^{n}\left(z^{\prime}\right)$.

**) Cf. Introduction 
consequence of Theorem 1.7. For this we first set

$(1.14)_{3} \quad$ Euc: $=$ collection of all euclidean spaces $\mathbb{X}^{\prime}=\mathbb{C}^{n}(z)$.

We denote by ' $\operatorname{dim}_{0}$ ' the map: $\mathbb{E u c} \ni \mathbb{X}^{\prime} \rightarrow \mathbb{Z}^{+} \ni n$, and we also define a map

$(1.14)_{4} \quad I^{\prime}: \mathbb{E u c} \ni X^{\prime}=\mathbb{C}^{n}(z) \longrightarrow \mathbb{A} f \ni\left(\mathbb{C}^{n}(z), \mathbb{X}^{\prime}=\mathbb{C}^{n}, \mathfrak{S}_{\mathbf{X}^{\prime}}\right)$,

where $\mathfrak{S}_{\mathbf{X}^{\prime}}$ denotes the trivial resolution of $\mathfrak{D}_{\mathbf{X}^{\prime}}: 0 \rightarrow \mathfrak{D}_{\mathbf{X}^{\prime}} \rightarrow \mathfrak{D}_{\mathbf{X}^{\prime}} \rightarrow 0$ (cf. Remark 1.1). By means of $I^{\prime}$ we regard an element $X^{\prime}=\mathbb{C}^{n}(z) \in$ Euc as the element of Aff; we use the terminology for $\mathbf{A f f}\left(\right.$ n.5, § 1.2) for $\mathbb{X}^{\prime}$. In particular, the p.g. and distance functions for $\mathbb{X}^{\prime}$ are $g_{X^{\prime}}=|z|+1$ and $d_{\mathbf{X}^{\prime}}=$ natural distance of $\mathbb{C}^{n}(z)(\mathrm{n} .4, \S 1.2)$. Next taking an element $(\sigma, \alpha) \in \boldsymbol{\lambda}_{X^{\prime}}=\mathbb{R}_{1}^{+2} \times \mathbb{R}_{1}^{+2}$ we set $(1.14)_{4}^{\prime} C^{q}\left(\mathscr{A}_{\sigma}\left(\boldsymbol{X}^{\prime}\right), \mathfrak{D}_{\mathbf{X}^{\prime}}\right)_{\alpha}:=$ set of $g_{\mathbf{X}^{\prime}}-\alpha$-growth cochains with value in $\mathfrak{H}$, where $\mathscr{A}_{\sigma}\left(\mathbb{X}^{\prime}\right):=g_{\mathbf{X}^{\prime}}$-p.g. covering of $\mathbb{X}^{\prime}$ (in $\mathbb{X}^{\prime}$ ) of size $\sigma$ (Definition $1.6_{1}$ ).

We use el-maps for the estimations in Corollary 1.4 soon below. We set $(1.14)_{4}^{\prime \prime} \quad \tilde{\mathbb{L}}=\mathbb{L} \times \mathbb{L}$, with $\mathbb{L}=$ collection of all el-maps (cf. $(1.11)_{3}$ ).

To an element $\tilde{\mathscr{L}}=\left(\mathscr{L}_{1}, \mathscr{L}_{2}\right) \in \tilde{\mathbb{H}}=\mathbb{L} \times \mathbb{L}$ we attach a map

$$
(1.14)_{4}^{\prime \prime \prime} \quad \tilde{\mathscr{L}}: \mathbb{R}^{+2} \times \mathbb{R}^{+2} \ni(\sigma, \alpha) \longrightarrow \mathbb{R}^{+2} \times \mathbb{R}^{+2} \ni\left(\mathscr{L}_{1}(\sigma), \mathscr{L}_{2}(\alpha+\sigma)\right) .
$$

We then have

Corollary 1.4. There is a map $\varepsilon: \mathbb{E a c} \ni \mathbb{X}^{\prime}=\mathbb{C}^{n}(z) \rightarrow$ $\tilde{\mathbb{L}} \ni \tilde{\mathscr{L}}_{\mathbf{X}^{\prime}}(q>0)$, whichs atisfies Figure II, and with which we have the following for each $\mathbb{X}^{\prime} \in \mathbb{E u c}$ :

$(1.14)_{5} s^{*} Z^{q}\left(\mathscr{A}_{\sigma}\left(\mathbb{X}^{\prime}\right), \mathcal{D}_{\mathbf{X}^{\prime}}\right)_{\alpha} \subset \delta C^{q-1}\left(\mathscr{A}_{\sigma^{\prime}}\left(\mathbb{X}^{\prime}\right), \mathfrak{D}_{\mathbf{X}^{\prime}}\right)_{\alpha^{\prime}}$,

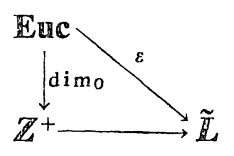

Figure II. with $\left(\sigma^{\prime} ; \alpha^{\prime}\right)=\tilde{\mathscr{L}}_{\mathbf{X}^{\prime}}(\sigma ; \alpha)$, where $(\sigma ; \alpha)$ is in $\lambda_{\mathbf{X}^{\prime}}\left(=\mathbb{R}_{1}^{+2} \times \mathbb{R}_{1}^{+2}\right)$.

Proof. Letting $U_{1}^{\prime}$ denote the disc in the euclid line $\mathbb{C}(w)$ with the center 0 (=origin of $\mathbb{C}$ ) and radus $r=1$, we identify $\mathbb{C}^{n}$ with $\mathbb{C}^{n} \times 0 \subset \mathbb{C}^{n} \times U_{1}^{\prime}$. We write the projection: $\mathbb{C}^{n} \times U_{1}^{\prime} \rightarrow \mathbb{C}^{n}$ as $\pi_{X^{\prime}}$. Then, writing the left side of $(1.14)_{5}$ as $Z^{q}$, we have $\pi_{X^{\prime}}^{*} Z^{q} \subset Z^{q}\left(\mathscr{A}_{\sigma}\left(\mathbb{C}^{n} \times U_{1}^{\prime}\right) \text {, D) }\right)_{\alpha}$, where $\mathfrak{D}:=$ structure sheaf of $\mathbb{C}^{n} \times U_{1}^{\prime}$. Apply Theorem 1.7 to the right-hand side of the inclusion just above, and we restrict the resulting inclusions of the form $(1.14)_{1}$ to $\mathbb{C}^{n}\left(\cong \mathbb{C}^{n} \times 0\right)$. Comparing the explicit estimations in Theorem 1.7 and Corollary 1.4 (cf. also the explicit form of the p.g.c. maps and el-maps in $n .5, \S 1.1$, we get easily $(1.14)_{5}$.

q.e.d.

From $(1.14)_{5}$ and $(1.12)_{1}$, Theorem 1.3 , we easily have 
Corollary 1.4'. Theorem $1.7 \rightarrow$ Theorem 1.3 for each $\left.{ }^{*}\right) \mathfrak{D}_{\mathbf{X}^{\prime}} ; \mathbb{X}^{\prime} \in \mathbb{E}$ Euc.

The right side will be our starting point of the proof of Theorem 1.3 and Theorem 1.4 (cf. n.2-n.5 soon below).

2. Sheaf homomorphisms. Letting $\mathbb{X}^{*}$ be one of $\tilde{X} \in \mathbb{A}_{0}, \mathbb{X} \in \mathbb{A m}_{1 a}$ or $\mathbb{X}^{\prime} \in \mathbb{A}$ f title of n.2, which will be most basic in deriving Thecrems 1.1-1.4 from Theorem 1.7. For this taking an element $\mathfrak{S} \in \operatorname{Coh}^{\prime}\left(\mathbb{X}^{*}\right)_{\text {p.g }}$ (cf. $\left.(1.4)_{8}\right)$, we recall that such a sheaf $\mathfrak{S}$ is endowed with two natural p.g. filtrations: The first one, $\Psi$ in symbol, is induced from the first resolution $K: \mathfrak{D}_{\mathbb{X}^{n}}^{k} \rightarrow \mathfrak{S}$ of $\mathfrak{S}$, where $K$ is a matrix with entries ${ }^{* * *}$ in $\Gamma\left(\mathbb{X}^{*}, \mathfrak{D}_{\mathbf{X}^{*}} ; g_{\mathbb{X}^{+}}\right)_{\mathrm{p} . \mathrm{g}}$ (cf. $\left.(1.4)_{8}\right)$, and has been used hitherto in Section 1. The second one, $\Psi^{\prime}$, is induced from the inclusion: $\mathfrak{S} \hookrightarrow \mathfrak{D}_{\mathbb{X}^{\prime}}^{\tilde{k}}, \tilde{k}$ $=$ length of columns of $K$, and letting the parameter***) $(P ; r, \sigma, \alpha)$ or $(\sigma ; \alpha)$ $\in \mathcal{h}_{X^{*}}^{\prime}:=\boldsymbol{\beta}_{X}^{\prime} \times \mathbb{R}_{1}^{+2}$ have the similar meaning to Theorem 1.2 or Theorem 1.4, the set of $\alpha$-cochains is defined by $\Psi^{\prime}$ as follows (cf. (1.4) $)_{9}$ ):

$$
C^{q}\left(\mathscr{A}_{\sigma}\left(\mathbb{Y}^{*}\right), \mathfrak{H} ; \Psi^{\prime}\right)_{\alpha}:=C^{q}\left(\mathscr{A}_{\sigma}\left(\mathbb{L}^{* *}\right), \mathfrak{S}\right) \cap C^{q}\left(\mathscr{A}_{\sigma}\left(\mathbb{H}^{*}\right), \mathbb{D}_{X^{*}}^{\tilde{L}}\right)_{\alpha} .
$$

(Here $\mathbb{Y}^{*}$ denotes the manifold $\mathbb{X}_{r}^{*}(P)$ or $\mathbb{X}^{\prime}$ as in Theorem 1.2 and Theorem 1.4.) We write the corresponding set to $\Psi$ explicitly as follows (cf. (1.4) $)_{8}$ ):

$$
C^{q}\left(\mathscr{U}_{\sigma}\left(Y^{*}\right), \mathfrak{5} ; \Psi\right)_{\alpha}:=K C^{q}\left(\mathscr{A}_{\sigma}\left(Y^{*}\right), \mathfrak{D}_{X^{*}}^{k}\right)_{\alpha} \text {. }
$$

Now we give the key lemma, mentioned soon above, in terms of a comparison of the sets of the cochains in $(1.15)_{1,2}^{\prime}$.

Lenma 1.2 (P.g. uniform estimations for $\mathfrak{D}_{X^{-}}$ hoinomorphisnis).****) There are maps $\varepsilon_{\mathbb{X}^{*}}$ :

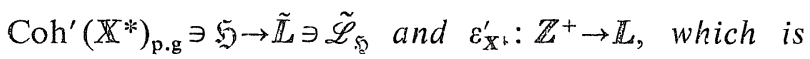
factored as in Figure III, and with which we have

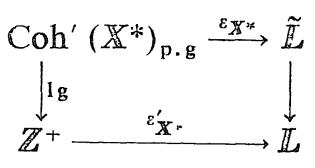

Figure III. $(1.15)_{1} s^{*} C^{q}\left(\mathscr{A}_{\sigma}\left(\mathbb{Y}^{*}\right), \mathfrak{S} ; \Psi^{\prime}\right)_{\alpha} \subset C^{q}\left(\mathscr{A}_{\sigma^{\prime}}\left(\mathbb{X}^{*}\right), \mathfrak{S} ; \Psi\right)_{\alpha^{\prime}}$ with $\left(\sigma^{\prime} ; \alpha^{\prime}\right)=\tilde{\mathscr{L}}_{\mathfrak{S}}(\sigma ; \alpha)$, where the parameter $(\sigma ; \alpha)$ is as in $(1.15)_{1}^{\prime}$.

For the proof of Lemma 1.2, see Section 4. (See also Remark 1.4 at the cnd of Section 1.3.)

*) Cf. Remark 1.2.

**) $g_{X^{2}}=$ p.g. function for $X^{*}=|\tilde{z}|+1,\left|h_{X}^{-1}\right|$ or $|z|+1$ (cf. Theorem 1.2 and Theorem 1.4).

***) For the sets of the parameter $\lambda_{\delta}^{\prime}{ }^{*}$, see $\mathrm{n} .2$ and n.5, Section 1.2.

****) For the scts $\tilde{\mathbb{L}}, \mathbb{L}$ of estimation maps, see (1.14) . Also the set $\mathbb{L}$ in Figure III is the first component of $\tilde{L}=\mathbb{L}, \mathbb{L}$, and $\lg =$ length map (cf. (1.4) $)$. 
3. Consequences of Lemma 1.2. First we prove the implication:

Corollary 1.5. Theorem $1.7+$ Lemma $1.2 \rightarrow$ Theorem 1.2 and Theorem 1.1 for $\mathbf{A n}_{0}$.

Proof. Take an element $\tilde{\boldsymbol{X}} \in \mathbf{A} \mathbf{n}_{0}$. Then, applying Theorem 1.7 to the right-hand side of $(1.11)_{2}$, Theorem 1.2 , we easily have

$(1.15)_{2} \quad$ Theorem 1.2 for $\mathbf{A n}_{0} \longrightarrow$ Theorem 1.1 for $\mathbf{A n}_{0} \quad$ (cf. n.4, §1.2).

We prove the left side inductively on $\operatorname{Coh}^{p}(\tilde{\mathbf{X}})_{\text {p.g }}(p=1,2, \ldots)$ (cf. $\left.(1.4)_{2}\right)$, using the standard syzygy arguments: if $p=1$ then $\mathfrak{H} \cong \mathfrak{D}_{X}^{k}(k>0)$, and we have directly Theorem 1.2 from Theorem 1.7 (cf. $\S 1.2$ ). Assume that (1) $p \geqq 2$, (2) Theorem 1.2 holds $^{*)}$ for $\operatorname{Coh}^{p-1}(\tilde{\boldsymbol{X}})_{\mathrm{p} . \mathrm{g}}$ and $(3) \mathfrak{H} \in \operatorname{Coh}^{p}(\tilde{\boldsymbol{X}})_{\mathrm{p} . \mathrm{g}}$. Writing $\mathfrak{H}$ as: $\cdots \rightarrow \mathfrak{D}_{\tilde{X}}^{k^{\prime}}$ $\stackrel{K_{1}}{\longrightarrow} \bigcup_{\tilde{X}}^{k} \rightarrow \mathfrak{H} \rightarrow 0$, we define an element $\mathfrak{S}_{1} \in \operatorname{Coh}^{p-1}(\tilde{\boldsymbol{X}})_{\text {p.g }} \cap \operatorname{Coh}^{\prime}(\tilde{\boldsymbol{X}})_{\text {p.g }}$ to be: $\cdots \rightarrow \mathfrak{D}_{\mathbb{X}}^{k^{\prime}} \stackrel{K_{1}}{\longrightarrow} \mathfrak{H}_{1}\left(\subset \mathfrak{D}_{\tilde{X}}^{k}\right) \rightarrow 0$. Now taking a parameter $\left(P^{\prime} ; r, \sigma: \alpha\right) \subset \lambda^{\prime} \tilde{\boldsymbol{X}}\left(\subset U_{0}^{\prime}\right.$ $\times \boldsymbol{R}^{+} \times \boldsymbol{R}^{+2} \times \boldsymbol{R}^{+2}$ ) (cf. Theorem 1.2), we set

$$
Z^{\prime q}:=\left\{\varphi \in C^{q}\left(\mathscr{A}_{\sigma}(P ; r), D_{\mathbb{X}}^{k}\right)_{\alpha} ; \omega \delta \varphi=0\right\},
$$

where we set $\mathscr{A}_{\sigma}(P ; r)=\mathscr{A}_{\sigma}\left(\tilde{\boldsymbol{X}}_{r}(P)\right)$. Then letting the p.g. filtrations $\Psi_{1}^{\prime}, \Psi_{1}$ for $\mathfrak{H}_{1}$ have the similar meanings to $\Psi, \Psi^{\prime}$ for $\mathfrak{H}$ (as in Lemma 1.2), we have $\delta Z^{\prime q} \subset C^{q+1}\left(\mathscr{A}_{\sigma}(P ; r), \mathfrak{H}_{1} ; \Psi_{1}^{\prime}\right)_{\alpha}$. Applying Lemma 1.2 and Theorem $1.1^{* *)}$ to the right side, we get

$$
s^{*} \delta Z^{q} \subset Z^{q+1}\left(\mathscr{A}_{\alpha}(P ; r), \mathfrak{H}_{1} ; \Psi_{1}\right)_{\alpha^{\prime}} \subset \delta C^{q}\left(\mathscr{A}_{\sigma^{\prime \prime}}\left(P ; r^{\prime \prime}\right), \mathfrak{H}_{1} ; \Psi_{1}\right)_{\alpha^{\prime \prime}},
$$

where $\left(\sigma^{\prime} ; \alpha^{\prime}\right)=\mathscr{L}_{\mathfrak{g}_{1}}(\sigma ; \alpha)$ and $\left(r^{\prime \prime}, \sigma^{\prime \prime} ; \alpha^{\prime \prime}\right)=E_{\mathfrak{p}_{1}}\left(r, \sigma^{\prime} ; \alpha^{\prime}\right)$ are defined as in Lemma 1.2 and Theorem 1.2.

It is clear that (b) insures

$$
s^{*} Z^{q} \subset Z^{q}\left(\mathscr{A}_{\sigma^{\prime \prime}}, \mathfrak{D}_{\tilde{X}}^{k}\right)_{\alpha^{\prime \prime}}+C^{q}\left(\mathscr{A}_{\sigma^{\prime \prime}}, \mathfrak{S}_{1}\right)_{\alpha^{\prime \prime}},
$$

where $\mathscr{A}_{\sigma^{\prime \prime}}=\mathscr{A}_{\sigma^{\prime \prime}}\left(P ; r^{\prime \prime}\right)$. Finally operating the homomorphism $\omega$ to the both sides of (c), we get the desired inclusion $(1.11)_{1}$, Theorem 1.2.

q.e.d.

For later quotations, we rewrite Corollary 1.5 in the form

$(1.15)_{3}$ Theorem $1.7 \stackrel{\text { syzygy(Lemma 1.2) }}{\longrightarrow}$ Theorem 1.2 for $\mathbf{A n}_{0}$

$$
\longrightarrow \text { Theorem } 1.1 \text { for } \mathbf{A n}_{0} \text {. }
$$

Next take subsets of $\boldsymbol{A}, \boldsymbol{A}^{\prime}$ of $\mathbf{A n}_{1 a}, \mathbf{A f f}(\mathrm{n} .1, \mathrm{n} .5, \S 1.2)$. Then the similar syzygy

*) For this terminology, see Remark $1.2_{2}$.

**) By the induction hypothesis we have Theoren 1.2 for $\operatorname{Coh}^{p-1}(\boldsymbol{X})_{\mathrm{p} . \mathrm{g}}$; by $(1.15)_{2}$ we have Theorem 1.1 for $\operatorname{Coh}^{p-1}(X)_{\text {p.g }}$. 
arguments to Corollary 1.5 insure

Proposition 1.4. If Theorem 1.1 holds for each $\mathfrak{D}_{\boldsymbol{X}} ; \mathbb{X} \in \mathbb{A}$ (resp. Theorem 1.3 holds for each $\left.\mathfrak{D}_{\mathbf{X}^{\prime}} ; \mathbb{X}^{\prime} \in \mathcal{A}^{\prime}\right)$, then we have Theorem 1.1 and Theorem 1.2 for $\boldsymbol{A}$ (resp. Theorem 1.3 and Theorem 1.4 for $\mathcal{A}^{\prime}$ ).

Taking $\boldsymbol{A}^{\prime}$ to be Euc (n.1, § 1.3), Proposition 1.4 and Corollary 1.4 insure

Corollary 1.6. Theorem $1.7 \rightarrow$ Theorem 1.3 for each $\mathfrak{D}_{\mathbf{X}^{\prime}} ; \mathbb{X}^{\prime} \in$ Euc $\stackrel{\text { syzygy(Lemma 1.2) }}{\longrightarrow}$ Theorem 1.3 and Theorem 1.4 for $\mathbb{E}$ Euc.

We give here an analogue of Corollary 1.6 to $\operatorname{An}_{1 a}$. For this we define a subcollection $\mathrm{An}_{1 a}^{0}$ of $\mathbf{A n}_{1 a}$ as follows:

$(1.15)_{4} \quad \mathbf{A n}_{1 a}^{0}:=\left\{\mathbb{X} \in \mathbf{A n}_{1 a} ; \mathbf{X}\right.$ is of the form: $\left.\left(\mathbb{C}^{n}(z), U_{0}, X_{0}, h_{\mathbf{X}}, \mathfrak{S}_{\mathbf{X}}, P_{0}\right)\right\}$

(cf. $\left.(1.8)_{0}\right)$, where $X_{0}$ coincides with the ambient space $U_{0}$. Moreover, $\mathfrak{H}_{\mathbf{X}}$ is the trivial resolution of $\mathfrak{D}_{\mathbf{X}}: 0 \rightarrow \mathfrak{D}_{\mathbf{X}} \rightarrow \mathfrak{D}_{\mathbf{X}} \rightarrow 0$ (Remark 1.1), where $\mathbb{X}:=X_{0}-D$, with the divisor $D$ of $h_{\boldsymbol{X}}$ (in $X_{0}=U_{0}$ ). Thus $\mathbb{X}$ coincides with the ambient space $U_{0}-D$. This property is similar to the one of $\mathbb{X}^{\prime}=\mathbb{C}^{n^{\prime}}(z) \in \mathbb{E u c}(\subset \mathbb{A f f}$ ) (cf. $\left.(1.14)_{3}\right)$, and $\mathbf{A n}_{1 a}^{0} \subset \mathbf{A n}_{1 a}$ has a similar role to $\mathbb{E u c} \subset \mathbf{A f f}$.

Corollary 1.7. Theorem 1.1 and Theorem 1.2 for $\mathbf{A n}_{0} \rightarrow$ Theorem 1.1 for $\mathfrak{D}_{\boldsymbol{X}} ; \boldsymbol{X} \in \mathbf{A n}_{1 a}^{0} \rightarrow$ Theorem 1.1 and Theorem 1.2 for $\mathrm{An}_{1 a}^{0}$.

The second implication follows from Proposition 1.4. The first is proven in n.4, by imbedding $\boldsymbol{X} \in \mathbf{A n}_{1 a}^{0}$ to higher dimensional euclidean spaces (cf. Lemma 1.3 in $n .4$ soon below).

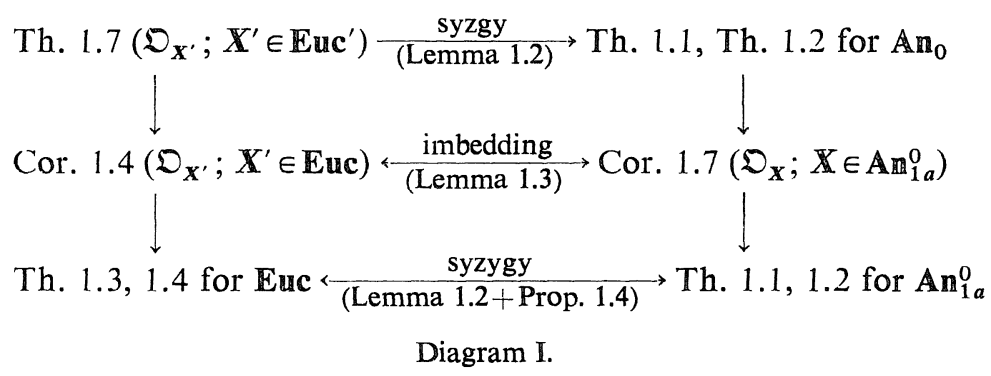

(The theorems at the bottom will be the starting point of the final part of the proof of Theorems 1.1-1.4 (cf. (n.5).)

4. Imbedding. First take a euclidean line $\mathbb{C}(w)$. Using the similar notation to $(1.15)_{4}$, we define a map 


$$
I: \mathbb{A n}_{1 a}^{0} \ni \mathbb{X} \longrightarrow \mathbb{A n}_{0} \ni \tilde{\mathbb{X}}=\left(\mathbb{C}(w) \times \mathbb{C}^{n}(z), \tilde{\mathbb{X}}=\mathbb{C} \times U_{0}, P_{0}\right)
$$

where $\mathbb{X}=X_{0}-D$, with $X_{0}=U_{0} . \quad$ Setting*) $h_{\tilde{X}}:=1-h_{X^{\prime}}$ (cf. $\left.(1.15)_{4}\right), S=$ locus of $h_{\tilde{\boldsymbol{X}}}$ in $\tilde{\mathbb{X}}$ and $\pi_{\mathbb{X}}=$ biregular map: $S \rightarrow \mathbb{X}=U_{0}-D$, we use the imbedding $\pi_{\mathbf{X}}^{-1}: \mathbb{X}=U_{0}-D \rightarrow S$ and the p.g. sheaf $\mathfrak{H}_{S}: 0 \rightarrow \mathfrak{D}_{\tilde{\mathbf{X}}} \stackrel{h_{\tilde{X}}}{\longrightarrow} \mathfrak{D}_{\tilde{\mathbf{X}}} \rightarrow \mathfrak{D}_{S} \rightarrow 0$ over $\tilde{\mathbf{X}}$ for the proof of Lemma 1.3 soon below. $\left(D_{\tilde{X}}, S_{S}\right.$ are the structure sheaves of $\tilde{X}, S$.) Letting the parameter $(P ; r ; \sigma ; \alpha) \in \lambda_{X}^{\prime}\left(\subset U_{0} \times(0, \tilde{r}) \times \mathbb{R}_{1}^{+2} \times \mathbb{R}_{1}^{+2}\right)$ be as in Theorem 1.2, we set

$(1.16)_{1}\left\{\begin{array}{l}C^{q}\left(\mathscr{A}_{\sigma}\left(\mathbb{X}_{r}(P)\right), \mathfrak{D}_{X}\right)_{\alpha} \\ C^{q}\left(\mathscr{A}_{\sigma}\left(\mathbb{X}_{r}(P)\right), \mathfrak{S}_{S}\right)_{\alpha}\end{array}\right\}:=$ set of $g:=\left\{\begin{array}{l}\left|h^{-1}\right| \\ |z|+|w|+1\end{array}\right\}-\alpha$-growth cochains with value in $\left\{\begin{array}{l}\mathfrak{D}_{\boldsymbol{X}} \\ \mathfrak{H}_{S}\end{array}\right\}$ (cf. $(1.4)_{7}$ and Definition $1.4_{5}$ ),

where

$(1.16)_{2}\left\{\begin{array}{l}\mathscr{A}_{\sigma}\left(\mathbb{X}_{r}(P)\right) \\ \mathscr{A}_{\sigma}\left(\tilde{\mathbf{X}}_{r}(P)\right)\end{array}\right\}:=g$ - p.g. covering of $\left\{\begin{array}{l}\mathbb{X}_{r}(P) \\ \tilde{\mathbf{X}}_{r}(P)\end{array}\right\}$ of size $\sigma$ in $\left\{\begin{array}{l}\mathbb{X}=U_{0}-D \\ \tilde{\mathbb{X}}=\mathbb{C} \times U_{0}\end{array}\right\}$.

$\left(\right.$ Here $\mathbb{X}_{r}(P)=\mathbb{X} \cap U_{r}(P)$ and $\tilde{\mathbb{X}}_{r}(P):=\mathbb{C} \times U_{r}(P)$, with the disc in $U_{r}(P) \subset \mathbb{C}^{n}$, are as in $(1.9)_{2}, \S 1.2$.) Then the following lemma compares the sets in $(1.16)_{1}$.

Lemma 1.3 (P.g. uniform estimation for imbedding). There is an element $E_{\boldsymbol{X}} \in \mathbb{E}_{\mathrm{p} . \mathrm{g}}(q \geqq 0)$, with which we have $(1.16)_{3}\left\{\begin{array}{l}C^{q}\left(\mathscr{A}_{\sigma^{\prime}}\left(\mathbb{X}_{r}(P)\right), \mathfrak{D}_{\mathbf{X}}\right)_{\alpha^{\prime}} \supset s_{\mathbf{X}}^{*}\left(\pi_{\mathbf{X}}^{-1}\right)^{*} \omega_{S}^{*} C^{q}\left(\mathscr{A}_{\sigma}\left(\tilde{\mathbf{X}}_{r}(P)\right), \mathfrak{S}_{S}\right)_{\alpha} \\ s_{S}^{*} \pi_{\mathbb{X}}^{*} Z^{q}\left(\mathscr{A}_{\sigma}\left(\mathbb{X}_{r}(P)\right), \mathfrak{D}_{\mathbf{X}}\right)_{\alpha} \subset \omega_{S}^{*} Z^{q}\left(\mathscr{A}_{\sigma^{\prime}}\left(\tilde{\mathbb{X}}_{r^{\prime}}(P)\right), \mathfrak{S}_{S}\right)_{\sigma^{\prime}},\end{array}\right.$ with $\left(r^{\prime} ; \sigma^{\prime} ; \alpha^{\prime}\right)=E_{\mathbf{X}}(r ; \sigma ; \alpha)$, where $\omega_{S}^{*}=$ natural homomorphism. ${ }^{* *)} \quad \mathfrak{S}_{S} \rightarrow D_{S}$ and $s_{\boldsymbol{X}}$ and $s_{S}$ are the 'p.g. refining maps'***) in $\mathbb{X}, S$.

We check Lemma 1.3 in Section 4.2. Corollary 1.7 follows from Lemma 1.3 as follows: apply Theorem 1.1 for $\mathbb{A m}_{0}$ to $\mathfrak{J}_{S}$, which is a p.g. coherent sheaf over $\mathbb{C} \times U_{0}$. Then we have the inclusion of the form $(1.10)_{1}$ for the right side of the second inclusion in $(1.16)_{3}$. Using the first inclusion in $(1.16)_{3}$, we pull back this inclusion to $\mathbb{X}$ (by means of $\pi_{\mathbf{X}}$ ). Then we have the desired inclusion in $(1.10)_{1}$ for $D_{\mathbf{X}}$.

*) Here we understand that $C^{n}(z) \times C^{n^{\prime}}\left(z^{\prime}\right)$ in Theorem 1.2 is $\mathbb{C}(w) \times \mathbb{C}^{n}(z)$ and that $U_{0}^{\prime}\left(\subset \mathbb{C}^{n^{\prime}}\left(z^{\prime}\right)\right)$ is $U_{0}\left(\subset \mathbb{C}^{n}(z)\right)$.

**) Note that $\mathfrak{S}_{S}$ and $\mathfrak{D}_{S}$ are obtained by regarding the structure sheaf of $S$ as the sheaves over $\tilde{\mathbb{X}}, S$.

***) For the precise form of the refining maps $s_{5}, s_{1}$, sce $(1.13)_{2}$ in Proposition 4.7. 
Finally, we will complete Diagram I in the following fashion.

Th. 1.3, Th. 1.4 for $\mathbb{E}$ ald<smiles>C1C[C@H]2CCC[C@@H]2C1</smiles>

Th. $1.3\left(\bigcirc_{\mathbf{X}^{\prime}} ; \mathbb{X}^{\prime} \in \mathbb{A f f}\right)$

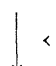

7 b. 1.3, Th. $1 .+$ for $\mathbb{A}$ ff
Th. 1.1, Th. 1.2 for $\mathbb{A}_{1 a}^{0}$

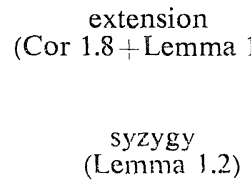

(Lemma 1.2)

Th. $1.1(2) ; \mathbb{X} \in \mathbb{A} \mathbb{n}$,

Th. 1.1, Th. 1.2 for $\mathrm{An}_{1 a}$

Diagram II.

(The second implication is insured by Proposition 1.4; Corollary 1.8 and Lemma 1.4 will be given soon below.) By Diagrams I and II, the remaining task for the proof of Theorems $1.1-1.4$ is to prove

Corollary 1.8. Wc have the following implications:

$(1.17)_{1}\left\{\begin{array}{l}\text { Theorem 1.1, Theorem 1.2 for } \mathbb{A m}_{1 a}^{0} \\ \text { Theorem 1.3, Theorem 1.4 for } \mathbb{E} \text { Euc }\end{array}\right\} \Longrightarrow$

$\left\{\begin{array}{l}\text { Theorem 1.1, for each } D_{\mathbf{X}} ; \mathbb{X} \in \mathbb{A}_{1 a} \\ \text { Theorem 1.3, for each } D_{\mathbf{X}^{\prime}} ; X^{\prime} \in \mathbb{A} \text { if }\end{array}\right.$

For the proof of Corollary 1.8, we attach to elements of $\mathbb{A}_{1 a}$, Afif their ambient spaces:

$(1.17)_{2} \quad J:\left\{\begin{aligned} \mathbb{A} n_{1 a} \ni \mathbb{X}=\left(\mathbb{C}^{n}(z), U_{0}, X_{0}, h, \mathfrak{S}_{X}, P_{0}\right) \longrightarrow \\ \mathbb{A n}_{1 a}^{0} \ni Y=\left(\mathbb{C}^{n}(z), U_{0}, U_{0}, h, \mathfrak{S}_{Y}, P_{0}\right), \\ \mathbb{A} \ni \mathbb{X}^{\prime}=\left(\mathbb{C}^{n}(z), \mathbb{X}^{\prime}, \mathfrak{S}_{X^{\prime}}\right) \longrightarrow \mathbb{E u c} \ni Y^{\prime}=\left(\mathbb{C}^{n}(z), Y^{\prime}=\mathbb{C}^{n}, \mathfrak{S}_{X^{\prime}}\right) .\end{aligned}\right.$

(For the above notation, see $(1.8)_{0}$ and $(1.11)_{0}$. In particular, $5_{X}$ is the p.g. resolution of the structure sheaf $D_{X}$ of $\mathbb{X}=X_{0}-D$, with $D=$ locus of $h$ on $\mathbb{X}_{0}$, and $\mathfrak{S}_{\boldsymbol{Y}}$ is the trivial resolution of ${ }^{*} \mathbf{Y}=U_{0}-D_{0}: 0 \rightarrow \mathfrak{D}_{\mathbb{Y}} \rightarrow \mathfrak{D}_{\mathbf{Y}} \rightarrow 0$, with the pole $D_{0}$ of $h$ on $U_{0}$.) We will prove Corollary 1.8 by extending cochains on $X$ its ambient space $\mathbb{Y}$.

5. Extensions of cochains. Letting the variety $\mathbb{X} \in \mathbb{A n}_{1 a}$ and $\mathbb{X}=J(\mathbb{X})$ be as in $(1.17)_{2}$ and letting the paraneter $(P ; r ; \sigma ; \alpha) \subset \boldsymbol{\lambda}_{X}^{\prime}$ have the similar meaning to Lemma 1.3, we compare the following sets of the cochains*):

$(1.17)_{1}^{\prime}\left\{\begin{array}{l}C^{q}\left(\mathscr{A}_{\sigma}\left(\mathbb{X}_{r}(P)\right), \mathscr{D}_{x}\right)_{\alpha} \\ C^{q}\left(\mathscr{N}_{\sigma}\left(\mathbb{Y}_{r}(P)\right), \mathfrak{S}_{\mathbb{X}}\right)_{\sigma}\end{array}\right\}:=$ sel of all $g\left(=\left|h^{-1}\right|\right)-\alpha$-growth cochains with value in $\left\{\begin{array}{l}\mathfrak{D}_{X} \\ \mathfrak{S}_{X}\end{array}\right\}$

t) Cf. (1.4) and (1.3)". 
where

$$
\begin{gathered}
(1.17)_{1}^{\prime \prime}\left\{\begin{array}{l}
\mathscr{A}_{\sigma}\left(\boldsymbol{X}_{r}(P)\right) \\
\mathscr{A}_{\sigma}\left(\boldsymbol{Y}_{r}(P)\right)
\end{array}\right\}:=g \text {-p.g.-covering of }\left\{\begin{array}{l}
\boldsymbol{X}_{r}(P):=\boldsymbol{X} \cap U_{r}(P) \\
\boldsymbol{Y}_{r}(P):=\boldsymbol{Y} \cap U_{r}(P)
\end{array}\right\} \\
\text { of }{ }^{*)} \text { size } \sigma \text { in }\left\{\begin{array}{l}
\boldsymbol{X}=X_{0}-D \\
\boldsymbol{Y}=U_{0}-D_{0}
\end{array}\right\} .
\end{gathered}
$$

Also letting the affine variety $\boldsymbol{X}^{\prime} \in \mathrm{Aff}$ and $\boldsymbol{Y}^{\prime}=\boldsymbol{C}^{n}(z)$ be as in $(1.17)_{2}$, we will compare the following sets

$$
\begin{gathered}
(1.17)_{2}^{\prime}\left\{\begin{array}{l}
C^{q}\left(\mathscr{A}_{\sigma}(\boldsymbol{X}), \mathfrak{D}_{\mathbf{X}}\right)_{\alpha} \\
C^{q}\left(\mathscr{A}_{\sigma}(\boldsymbol{Y}), \mathfrak{D}_{\mathbf{X}}\right)_{\alpha}
\end{array}\right\}:=\text { set of all } g(=|z|+1) \text { - } \alpha \text {-growth cochains with } \\
\text { value in }\left\{\begin{array}{l}
\mathfrak{D}_{\mathbf{X}} \\
\mathfrak{H}_{\mathbf{X}}
\end{array}\right\},
\end{gathered}
$$

where

$$
(1.17)_{2}^{\prime \prime} \quad\left\{\begin{array}{l}
\mathscr{A}_{\sigma}\left(\boldsymbol{X}^{\prime}\right) \\
\mathscr{A}_{\sigma}\left(\boldsymbol{Y}^{\prime}\right)
\end{array}\right\}:=g \text {-p.g. coverings of }\left\{\begin{array}{l}
\boldsymbol{X}^{\prime} \\
\boldsymbol{Y}^{\prime}
\end{array}\right\} \text { of size } \sigma \text { in }\left\{\begin{array}{l}
\boldsymbol{X}^{\prime} \\
\boldsymbol{Y}^{\prime}
\end{array}\right\} .
$$

(In the above, the parameter $(\sigma ; \alpha)$ is in $\lambda_{X^{\prime}}^{\prime}\left(\subset \boldsymbol{R}_{1}^{+2} \times \boldsymbol{R}_{1}^{+2}\right)$ (cf. Lemma 1.2). Then we have

Lemma 1.4 (P.g. uniform estimation for extension). Take suitable $E_{\boldsymbol{X}}$ $\in \boldsymbol{E}_{\mathrm{p} . \mathrm{g}}$ and $\tilde{\mathscr{L}}_{\mathbf{x}} \in \tilde{\boldsymbol{L}}(q \geqq 0)$. Then we have

$$
\begin{aligned}
&(1.17)_{3}\left\{\begin{array}{l}
s^{*} Z^{q}\left(\mathscr{A}_{\sigma}\left(\boldsymbol{X}_{r}(P)\right), \mathfrak{D}_{\mathbf{X}}\right)_{\alpha} \subset \omega_{\mathbf{X}} Z^{q}\left(\mathscr{A}_{\sigma^{\prime}}\left(\boldsymbol{Y}_{r^{\prime}}(P)\right), \mathfrak{H}_{\mathbf{X}}\right)_{\alpha^{\prime}} \\
s^{*} Z^{q}\left(\mathscr{A}_{\sigma}\left(\boldsymbol{X}^{\prime}\right), \mathfrak{D}_{\mathbf{X}^{\prime}}\right)_{\alpha} \subset \omega_{\mathbf{X}^{\prime}} Z^{q}\left(\mathscr{A}_{\alpha^{\prime}}\left(\boldsymbol{Y}^{\prime}\right), \mathfrak{H}_{\mathbf{X}^{\prime}}\right)_{\alpha^{\prime}},
\end{array}\right\} \text { where } \\
&\left\{\begin{array}{l}
\left(r^{\prime}, \sigma^{\prime} ; \alpha^{\prime}\right)=E_{\boldsymbol{X}}(r, \sigma ; \alpha) \\
\left(\sigma^{\prime} ; \alpha^{\prime}\right)=\tilde{\mathscr{L}}_{\boldsymbol{X}^{\prime}}(\sigma ; \alpha)
\end{array}\right\} .
\end{aligned}
$$

Here $\omega_{\mathbf{X}}$ and $\omega_{\boldsymbol{X}^{\prime}}$ are the natural homomorphisms: $\mathfrak{S}_{\mathbf{X}} \rightarrow \mathfrak{D}_{\mathbf{X}}$ and $: \mathfrak{H}_{\boldsymbol{X}^{\prime}} \rightarrow \mathfrak{D}_{\boldsymbol{X}^{\prime}}$.

(We prove Lemma 1.4 , by extending cochains on $\boldsymbol{X}$ to $U_{0}-D_{0}, \ldots$ (cf. §4.2).)

Now Corollary 1.8 is derived from Lemma 1.4 as follows. First applying the Theorem 1.1 (for $\mathfrak{H}_{\mathbf{X}}$ ) to the first inclusion in $(1.17)_{3}$, we get the inclusion of the form $(1.10)_{1}$ for the right side of the former inclusion. Then, operating $\omega_{\mathbf{X}}$ to that inclusion (of the form $(1.10)_{1}$ ), we have the desired inclusion of the form $(1.10)_{1}$ for $\mathfrak{D}_{\boldsymbol{x}}$. This insures the check of first implication in $(1.17)_{1}$. The second implication is checked in the similar manner to the above. Thus we have Corollary 1.8, and we also finish the proof of Theorems 1.1-1.4 (cf. also the

*) $U_{r}(P):=\operatorname{disc}$ in $C^{n}$ as in $(1.9)_{1}$, Section 1.2 . 
remark soon below Corollary 1.8).*

6. Proof of Theorem 1.6. First recall that we checked the comparison of 'meromorphic' and 'p.g.' in Theorem 1.6 for elements of $\mathbf{A n}_{1 a}^{0}$ and $\mathbb{E u c}$ (cf. n.6, § 1.2). Then, using the extension of the cochains in Lemma 1.4 for $\mathbf{A n}_{1 a}, \mathbf{A f f}$, we get Theorem 1.6 for $\mathbf{A n}_{1 a}$, Aff from the corresponding facts**) for $\mathbf{A n}_{1 a}^{0}$, Euc. Thus we have shown that, for the proof of Theorems 1.1-1.6, it suffices to prove the key theorem, Theorem 1.7 and the key lemmas, Lemma 1.2-Lemma 1.4.

For convenience of understanding of the logical structure of Section 1.3, we summarize Diagrams I, II and the content of n.6, Section 1.3 as follows:

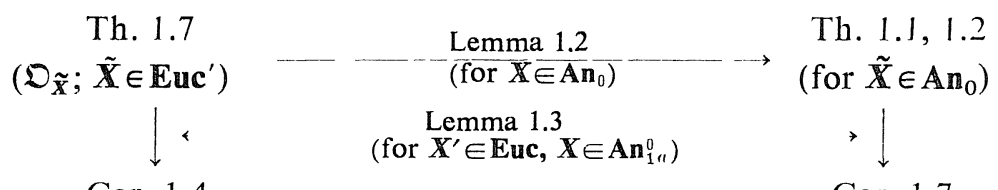

Cor. 1.4

$\left(\mathfrak{D}_{\boldsymbol{X}^{\prime}} ; \boldsymbol{X}^{\prime} \in \mathbf{E u c}\right)$

Th. $1.6_{1}$ for $\boldsymbol{X}^{\prime} \in \mathbb{E u c}$

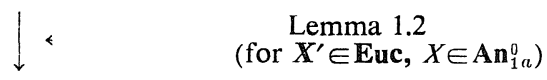

Th. 13, 1.4

(for $\left.\boldsymbol{X}^{\prime} \in \mathbf{E u c}\right)$

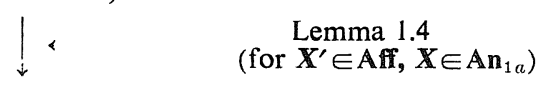

Th. 1.3

$\left(\mathfrak{D}_{\mathbf{X}^{\prime}} ; \boldsymbol{X}^{\prime} \in \mathbf{A f f}\right)$

Th. $1.6_{1}$ for $\boldsymbol{X}^{\prime} \in \mathbf{A f f}$

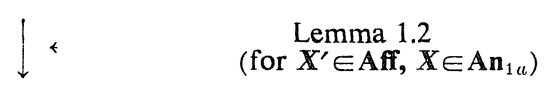

Th. $1.3,1.4$

(for $\mathbf{X}^{\prime} \in \mathbf{A f f}$ )

Cor. 1.7

$\left(\mathfrak{D}_{\mathbf{X}} ; \mathbb{X} \in \mathrm{An}_{1 a}^{0}\right)$

Th. $1.6_{1}$ for $\mathbb{X} \in \mathbf{A n}_{1 a}^{0}$

Th. 1.1, 1.2

(for $\mathbb{X} \in \mathbb{A n}_{1 a}^{0}$ )

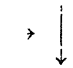

Th. 1.1

$\left(\mathfrak{D}_{\mathbf{X}} ; \mathbb{X} \in \mathrm{An}_{\mathbf{n}_{a}}\right)$

Th. 1.6 for $\mathbf{X} \in \mathbb{A n}_{1 a}$

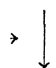

Th. 1.1, Th. 1.2

(for $\mathbb{X} \in \mathbb{A n}_{1 a}$ ).

Diagram III.

We will finish Section 1.3 by a technical remark for the proof of Lemma 1.2.

Remark 1.4. Letting the varieties $\mathbb{X} \in \mathbb{A} \mathbf{n}_{1 a}, \mathbb{X}^{\prime} \in \mathbf{A f f}$ be as in Lemma 1.2, we define the following subcollections of $\operatorname{Coh}^{\prime}(\mathbb{X})_{\mathrm{p} . \mathrm{g}}$, $\operatorname{Coh}^{\prime}\left(\mathbb{X}^{\prime}\right)_{\mathrm{p} . \mathrm{g}}$ (cf. n. $\mathrm{n} .2, \S 1.3$ and $\left.(1.4)_{9}^{\prime}\right)$ :

*) See also Diagrams I, II.

**) Also, in this step, we use Theorem 1.2 and, Theorem 1.4 for $\mathrm{An}_{1 a}^{0}$, Euc. This follows from Lemma 1.2 (cf. n.3, §1.3), and our use of those theorems is legitimate (cf. also Diagram III at the end of $\S 1.3$ ). 


$$
\operatorname{Coh}^{\prime \prime}\left(\mathbb{X}^{*}\right)_{\mathrm{p} . \mathrm{g}}:=\left\{5 \in \operatorname{Coh}^{\prime}\left(\mathbb{X}^{*}\right)_{\mathrm{p} . \mathrm{g}}\left(\mathrm{cf} .(1.4)_{9}^{\prime}\right)\right\},
$$

where $\mathbb{X}^{*}=\mathbb{X} \in \mathbb{A n}_{1 a}$ or $=\mathbb{X}^{\prime} \in \mathbb{A}$ f , and writing sictly in the form of $(1.4)_{1}$,

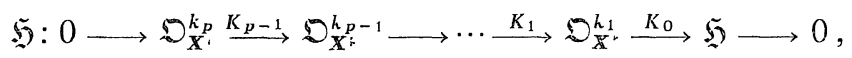

the element 5 must satisfy

$(1.18)_{1}^{\prime \prime}$ the entries of $K_{j}(0 \leqq j<p)$ are in $\Gamma\left(X_{0}, \mathfrak{D}_{X_{0}}(* D)\right)$ or $\Gamma\left(\mathbb{X}_{\mathrm{alg}}^{\prime}, \mathfrak{D}_{X^{\prime},: 1 \mathrm{~g}}\right)$, according as $\mathbb{X}^{*}=\mathbb{X} \in \mathbb{A m}_{1 a}$ or $=\mathbb{X}^{\prime} \in \mathbf{A} \mathbb{f}$.

(Recall that, for an element $\mathfrak{S} \in \mathrm{Coh}^{\prime}\left(\mathbb{K}^{* *}\right)_{p, g}$, the corresponding condition to $(1.18)_{1}^{\prime \prime}$ is 'the entries of $K_{j}$ are p.g. with respect to the p.g. function $g_{\mathbf{X}^{\prime}}=|\tilde{z}|+1$ or $\left.|z|+1^{\prime}\left(\mathrm{cf} .(1.4)_{9}\right)\right)$.

Now recall that Lemma 1.2 was given to $\operatorname{Coh}^{\prime}\left(\mathbb{X}^{*}\right)_{\text {p.g }}$. Here we $\left.\operatorname{check}^{*}\right)$ $(1.18)_{2}$ one can replace ' $\mathrm{Coh}^{\prime}\left(\mathbb{X}^{*}\right)_{\mathrm{pg}}$ ' by ' $\mathrm{Coh}^{\prime \prime}\left(\mathbb{X}^{*}\right)_{\mathrm{p} . \mathrm{g}}$ ' in Lemma 1.2.

First, if $\mathbb{X} \in \mathbb{A n}_{1 a}^{0}$ or $\mathbb{X}^{\prime} \in \mathbb{E} u \mathfrak{c}$, then the comparison of 'p.g.' and 'meromorphic (or, rational) ' in Theorem $1.6_{1}$ is a well known fact (cf. n.6, §1.2), and (1.18) is legitimate. On the other hand, Diagram III insures that 'Lemma 1.2 for $\mathrm{An}_{1 a}^{0}, \mathbb{E u c}$ as well as the extension of cochains in Lemma 1.4' imply Theorem $1.6_{1}$ for general $\mathbb{X} \in \mathbb{A n}_{1 a}$ and $\mathbb{X}^{\prime} \in \mathbb{A}$ ff. Thus we have (1.18) 2 .

\section{§2. Cohomology with Algebraic Division and Polynomial Growth}

This section contains the main results of this paper: First, in Section 2.1, we summarize some algebraic notions used in Section 2. Using them we give our main results of Section 2 as well as of this paper in Section 2.2. In Section 2.3 we reduce the results of Section 2.2 to those in Section 1, by using some uniform estimations on the a.d. and p.g. properties of coherent sheaves (cf. also Introduction).

\section{§2.1. Algebraic Division Conditions}

1. Open map property. We begin Section 2.1 by arranging some terminologies, which will be used in later arguments. First, a filtered group is, as usual, a decreasing sequence $\mathscr{B}=\{B(m)\}_{m=0}^{\infty}$ of abelian groups $B(m)$. When

*) Note that Lemma 1.4 follows from an estimation on local parametrization of analytic varieties, and is independent from I.cmma 1.2 (n.5, \$4.2). Also we use Lemma 1.3 in Diagram III. This lemuna is also proven independently from Lemma 1.2 (n.6, §4.2). 
there is no fear of confusions we write $B(0)$ also as $B$. By a filtered complex we mean such a one

$$
0 \longrightarrow E \stackrel{e}{\longrightarrow} \mathscr{C}^{0} \longrightarrow \cdots \longrightarrow \mathscr{C}^{q} \stackrel{d q}{\longrightarrow} \cdots,
$$

where $d_{q}$ is a homomorphism of filtered groups and $e$ is that of abelian groups. Letting $\mathscr{C}^{\prime *}: 0 \rightarrow E \stackrel{e}{\longrightarrow} \mathscr{C}^{\prime \jmath} \rightarrow \cdots \rightarrow \mathscr{C}^{\prime} q \rightarrow$ be an another filtered complex, a homomorphism $\omega: \mathscr{C}^{*} \rightarrow \mathscr{C}^{\prime *}$ is a collection $\omega=\left\{\omega^{\prime},\left\{\omega_{q}\right\}_{q=0}\right\}$ of homemorphisms $\omega_{q}: \mathscr{C}^{q} \rightarrow \mathscr{C}^{\prime q}$ (of filtered groups) and that of abelian groups $\omega^{\prime}: E \rightarrow E^{\prime}$ satisfying the standard commutativity condition. Next let $\mathscr{C}=\left\{\mathscr{C}_{\mu}^{*} ; \mu \in \mathscr{\ell}\right\}$ be a direct system of filtered complexes. Writing $\mathscr{C}_{\mu}^{*}$ as: $0 \rightarrow E_{\mu} \stackrel{e}{\longrightarrow} \mathscr{C}_{\mu}^{0} \rightarrow \cdots \rightarrow \mathscr{C}_{\mu}^{\mu} \stackrel{d}{\longrightarrow}$ (cf. $\left.(2.1)_{1}\right)$ and $\mathscr{C}_{\mu}^{q}$ as $\left\{C_{\mu}^{q}(m)\right\}_{m=0}$, we make

Defimition $2.1_{1}$. We say that $\mathscr{C}$ has open mup property (resp. is e-exact) if, for each $q \in \mathbb{Z}^{+} \cup 0$ and $\mu \in \mu$, there is an element $\mu^{\prime}>\mu$, with which $(2.1)_{2}$ (resp. $(2.1)_{3}$ ) below holds

$(2.1)_{2}$ there is a map $a: \mathbb{Z}^{+} \rightarrow \mathbb{Z}^{+}$so that $d_{\mu^{\prime}} C_{\mu^{\prime}}^{q}(m) \supset \rho_{\mu^{\prime} \mu} Z^{q+1}\left(C_{\mu}^{*}(a(m))\right)$, $(2.1)_{3} \quad d_{\mu^{\prime}} C_{\mu^{\prime}}^{q-1} \supset \rho_{\mu^{\prime} \mu} Z^{q}\left(C_{\mu}^{*}\right)(q \geqq 1), e_{\mu^{\prime}} E_{\mu^{\prime}} \supset \rho_{\mu^{\prime} \mu} Z^{0}\left(C_{\mu}^{*}\right)(q=0)$ where $C_{\mu}^{q-1}=C_{\mu}^{q-1}(0)$ and $C_{\mu}^{*}=\sum_{q \geqq 0} C_{\mu}^{q}$. (If ${ }^{*} \mu=1$ then $(2.1)_{2}$ is equivalent to say that $d_{\mu}: C_{\mu}^{q} \rightarrow \mathbb{Z}^{q+1}\left(C_{\mu}^{*}\right)$ is an open map, with respect to the topology determined by $C_{k}^{q}, \ldots$ ) The following equivalent condition to $(2.1)_{2}$ is useful in later arguments:

$(2.1)_{2}^{\prime}$ there is a map $b: \mathbb{Z}^{+} \rightarrow \mathbb{Z}^{+}$satisfying $\lim _{m \rightarrow} b(m)=\infty$ and $d_{\mu^{\prime}} C_{\mu^{\prime}}^{q}(b(m))$ $\supset \rho_{\mu^{\prime} \mu} Z^{q+1}\left(C_{\mu}^{*}(m)\right)$ for $m \gg 0$.

The open map property is important because we have*)

Proposition 2.1 $\mathbb{1}_{1}$. If $\mathscr{C}$ satisfies the open map property and is ge-exact, then we have

$(2.1)_{4}\left\{\begin{array}{l}\lim _{\mu \rightarrow} H^{q}\left(\lim _{\leftarrow m} C_{\mu}^{*} / C_{\mu}^{*}(m)\right) \cong \lim _{\mu \rightarrow}\left(\lim _{\leftarrow m} H^{q}\left(C_{\mu}^{*} / C_{\mu}^{*}(m)\right)\right) \cong 0(q \geqq 1), \\ \lim _{\mu \rightarrow} Z^{0}\left(\lim _{\leftarrow m} C_{\mu}^{*} / C_{\mu}^{*}(m)\right) \cong \lim _{\mu \rightarrow}\left(\lim _{\leftarrow m} Z^{0}\left(C_{\mu}^{*} / C_{\mu}^{*}(m)\right)\right) \cong \lim _{\mu \rightarrow}\left(\lim _{\leftarrow m} \theta_{\mu m} e_{\mu} E_{\mu}\right)\end{array}\right.$ where $\theta_{\mu m}$ is the natural homomorphism: $C_{\mu}^{0} \rightarrow C_{\mu}^{0} / C_{\mu}^{0}(m)$.

Remark 2.1. Take a Noetherian ring $\mathfrak{D}$, an ideal $\mathfrak{I}$ of $\Phi$ and a complex $C^{*}$ of $\mathfrak{D}$-modules:**) $0 \rightarrow C^{0} \rightarrow \cdots \rightarrow C^{q} \rightarrow \cdots$. We set $\mathscr{C}^{q}:=\left\{\mathfrak{J}^{m} C^{q}\right\}_{m=0}^{\infty}$. Then

*) For the proof of Proposition $2.1_{1}$ and for roles of the open map property in other standard comparison theorems in the completion theory, see M. Noumi [13].

**) We understand that the augumentation map e: $\mathrm{E} \rightarrow C^{0}$ is of the form: $E=0$ and $e=$ zero map. We use the similar notations in later arguments (cf. Definition $2.5_{2}$ ). 
Artin-Rees theorem insures that if $\mathscr{C}^{*}$ is exact then $\mathscr{C}^{*}$ satisfies the open map property. Also it is well known that the above theorem insures the exactness of the completion of $\mathscr{C}^{*}$ (cf. [12]). In spite of this basic character, the open map property seems to have been not taken up in general situations. In our context, the open map property was first conjectured for de Rham complexes by S. Lubkin in 1971 (in our conversations). The open map property for the de Rham complex is our starting point of our studies in Section 2. The open map properties will be given for some geometric complexes of local and global nature (cf. § 2.3 and B, §4.2). The most substantial part of Section 2 will concern that property for certain Cech complexes of global nature.

Finally, letting $\mathscr{C}^{*}=\left\{\mathscr{C}_{\mu}^{*} ; \mu \in \boldsymbol{\mu}\right\}$ be as in Definition $2.1_{1}$, take an another direct system $\mathscr{C}^{\prime}=\left\{\mathscr{C}_{\mu}^{\prime *} ; \mu \in \boldsymbol{\mu}\right\}$ of filtered complexes. Writing $\mathscr{C}_{\mu}^{*}, \mathscr{C}_{\mu}^{\prime *}$ as $0 \rightarrow E_{\mu} \rightarrow \mathscr{C}_{\mu}^{0} \rightarrow \cdots \rightarrow \mathscr{C}_{\mu}^{q} \rightarrow$ and $0 \rightarrow E_{\mu}^{\prime} \rightarrow \mathscr{C}_{\mu}^{\prime 0} \rightarrow \cdots \rightarrow \mathscr{C}_{\mu}^{\prime q} \rightarrow \cdots$, we jassume that the complexes (of abelian groups) $0 \rightarrow E_{\mu} \rightarrow C_{\mu}^{0} \rightarrow \cdots \rightarrow C_{\mu}^{q}$ and $0 \rightarrow E_{\mu}^{\prime} \rightarrow C_{\mu}^{\prime 0} \rightarrow \cdots \rightarrow C_{\mu}^{\prime q}$ $\rightarrow$ coincide. $\left(\right.$ Here $\left.C_{\mu}^{q}=C_{\mu}^{q}(0), \ldots\right)$

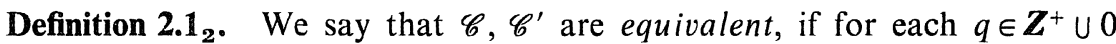
and $\mu \in \mu$, there is an element $\mu^{\prime}>\mu$, with which we have the following for each $m \gg 0$ :

$$
\rho_{\mu^{\prime} \mu} C_{\mu}^{q}(m) \subset C_{\mu^{\prime}}^{\prime q}\left(m^{\prime}\right), \text { with an element } m^{\prime} \in \boldsymbol{Z}^{+} \text {satisfying } \lim _{m \rightarrow \infty} m^{\prime}=\infty,
$$
and if the converse relation to this holds.

Proposition 2.12. Assume that $\mathscr{C}, \mathscr{C}^{\prime}$ are equivalent. If $\mathscr{C}$ satisfies the open map property, then $\mathscr{C}^{\prime}$ satisfies that condition.

2. A.d. filtration. Let $X$ be a topological space, $\mathfrak{D}$ a sheaf of ring over $X, \mathfrak{R}$ an $\mathfrak{D}$-module and $f=\left(f_{j}\right)_{j=1}^{s}$ a subset of $\Gamma(X, \mathfrak{D})$. We write $\left\{f_{j}^{m}\right\}_{j=1}^{s}$ as $\mathscr{F}^{m}$. By $m$-th standard homomorphism for $\mathcal{f}$, we mean the homomorphism $F^{m}: \mathfrak{D}^{s} \ni \varphi=\left(\varphi_{j}\right) \rightarrow \mathfrak{D} \ni \sum_{j} f_{j}^{m} \varphi_{j}(1 \leqq j \leqq s)$, and we write the image $F^{m} \mathfrak{D}^{s} \subset \mathfrak{D}$ also as $f^{m} \mathfrak{D}$. We use the symbol $f^{m} \mathfrak{r}$ for the $\mathfrak{D}$-submodule of $\mathfrak{h}$, which is spanned by elements $\varphi_{m} \cdot \varphi$, with $\varphi_{m} \in f^{m} \mathfrak{O}$ and $\varphi \in \mathfrak{K}$.

Next take an element $\left.\mathscr{A} \in \operatorname{Cov}_{0}(X){ }^{*}\right)$ We then make the following definition for later terminological convenience.

Definition 2.2. By $\mathscr{F}$-a.d. filtered group of $C^{q}(\mathscr{A}, \mathfrak{R})$ (or, $q$-th $\not$-a.d.filtered cochain group for $(\mathscr{A}, \mathfrak{\Re})$ ), we mean the following:

*) $\operatorname{Cov}_{0}(X)=2^{\operatorname{Ouv}(X)}$ (cf. the end of the introduction of Chapter I). 


$$
\left\{C^{q}\left(\mathscr{A}, \mathcal{f}^{m} \Omega\right)\right\}_{m=0}^{\infty} \text {, with } \mathscr{f}^{0} \Omega=\Omega .
$$

In $\mathrm{n} .3$ soon below we will combine Definition 2.2 with the p.g. filtration in Definition $1.2_{1}$.

3. D.p. filtration.*) First taking an abelian group $\mathscr{B}$ and a map $\Phi$ : $\left(\mathbb{Z}^{+} \cup 0\right) \times \boldsymbol{R}^{+2} \ni(m, \alpha) \rightarrow 2^{\mathscr{Q}}$, we denote by $\Phi_{m}$ the restriction of $\Phi$ to $m \times \mathbb{R}^{+2}$ $\left(\cong \boldsymbol{R}^{+2}\right)$. Setting $\mathscr{B}(m ; \Phi)_{\mathrm{p} . \mathrm{g}}=\cup_{\alpha \in \boldsymbol{R}^{+2}} \Phi_{m}(\alpha)(\subset \mathscr{B})$, we make

Definition 2.3. . We say that $\Phi$ is a $d . p$. filtration of $\mathscr{B}$, if we have (2.3) $\Phi_{1} \Phi_{m}: \boldsymbol{R}^{+2} \rightarrow 2^{\mathscr{B}}$ is a p.g. filtration for each $m \in \mathbb{Z}^{+} \cup 0$ (Definition $1.2_{1}$ ). $(2.3)_{2} \quad \mathscr{B}(m ; \Phi)_{\mathrm{p} . \mathrm{g}} \subset \mathscr{B}\left(m^{\prime} ; \Phi\right)_{\mathrm{p} . \mathrm{g}}$ for any $m^{\prime} \leqq m$, and $\Phi_{m}, \Phi_{m^{\prime}}$ are compatible with the inclusion: $\mathscr{B}(m ; \Phi)_{\text {p.g }} \hookrightarrow \mathscr{B}\left(m^{\prime} ; \Phi\right)_{\text {p.g }}$ (Definition $\left.1.2_{3}\right)$.

Next letting the datum $(X, \mathfrak{D}, \mathfrak{\Omega}, f)$ be as in n.2, we take a p.g. function $g: X$ $\rightarrow \boldsymbol{R}_{1}^{+}$(Definition $\left.1.4_{4}\right)$. We will define a d.p. filtration for $C^{q}(\mathscr{A}, \mathfrak{R})$ by means of $(f, g)$. For this we assume that $\Omega$ is a homomorphic image of $\mathfrak{D}^{k}: \mathfrak{D}^{k}$ $\stackrel{\omega}{\longrightarrow} \mathfrak{K} \rightarrow 0$ and that $\mathfrak{D}$ is endowed with a q-structure $\theta$ (Definition $1.4_{1}$ ). We endow $\Omega$ with the induced q-structure, $\theta_{\Omega}$ in symbol, from $(\theta, \omega)$ (Definition $\left.1.4_{3}\right)$. Recall that $(g, \theta),\left(g, \theta_{\mathscr{R}}\right)$ define p.g. filtrations for $C^{q}\left(\mathscr{A}, \mathfrak{D}^{k}\right), C^{q}(\mathscr{A}, \Re)$ (Definition $1.4_{5}$ ). For an element $\alpha \in \mathbb{R}_{1}^{+2}$ we set

$(2.3)_{3}\left\{\begin{array}{l}C^{q}\left(\mathscr{A}, \mathfrak{D}^{k} ; g\right)_{\alpha} \\ C^{q}(\mathscr{A}, \Omega ; g)_{\alpha}\end{array}\right\}:=$ set of $\left\{\begin{array}{l}(g, \theta) \\ \left(g, \theta_{\Omega}\right)\end{array}\right\} \alpha$-growth cochains with valve

$$
\text { in }\left\{\begin{array}{l}
\mathfrak{D}^{k} \\
\mathfrak{S}
\end{array}\right\}\left(\mathrm{cf} .(1.6)_{3}\right) \text {, where }
$$

$$
C^{q}(\mathscr{A}, \mathfrak{R} ; g)_{\alpha}=\omega C^{q}\left(\mathscr{A}, \mathfrak{O}^{k} ; g\right)_{\alpha} \quad \text { (cf. Proposition 1.3). }
$$

We use the symbol $F^{m}$ (=m-th stan-

dard homomorphism for $f): \mathfrak{D}^{s} \rightarrow \mathfrak{D}$ (cf. n.2) for its $k$-times direct sum $\mathfrak{D}^{s k}:=\mathfrak{D}^{s}+\cdots+\mathfrak{D}^{s} \stackrel{F^{m}+\cdots+F^{m}}{\longrightarrow} \mathfrak{D}^{k}=$ $\mathfrak{D}+\cdots+\mathfrak{D}$. Assuming that $\ell^{\mathfrak{C}} \Gamma(X$,

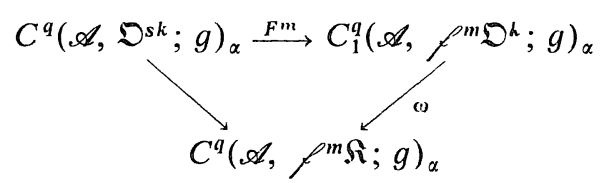

Figure I. $\mathfrak{D}, g)_{\text {p.g }}$, we make

Definition 2.3. . By left and right $(\mathscr{F}, g)$-d.p. filtration of $B=C^{q}(\mathscr{A}, \mathfrak{R})$, we mean the following maps:

$(2.3)_{3}\left\{\begin{array}{l}l_{1, g} \\ { }_{r} \Psi_{t, g}\end{array}\right\}:\left(\mathbb{Z}^{+} \cup 0\right) \times \mathbb{R}^{+2} \ni(m, \alpha) \longrightarrow 2^{\mathscr{R}} \ni\left\{\begin{array}{l}F^{m} C^{q}\left(\mathscr{A}, D^{s k} ; g\right)_{\alpha} \\ C^{q}(\mathscr{A}, \Re ; g)_{\alpha} \cap C^{q}\left(\mathscr{A}, \mathcal{f}^{m} \mathfrak{S}\right) .\end{array}\right.$

*) 'd.p'='a.d.'+'p.g.' (cf. n. 2., §2.1 and §1.1). 
We use the following notation for the first set in the right side of $(2.3)_{3}$ :

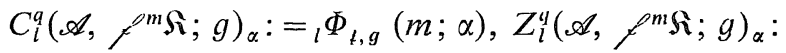

$$
\begin{aligned}
& ={ }_{l} \Phi_{l, g}(m ; \alpha) \cap \delta^{-1}(0) \text {. }
\end{aligned}
$$

Note that $(2.3)_{3}$ implies

$$
C_{l}^{q}\left(\mathscr{A}, f^{m} \mathfrak{S} ; g\right)_{\alpha}:=\omega C_{l}^{q}\left(\mathscr{A}, f^{m m \mathfrak{D}^{k}} ; g\right)_{\alpha} \quad \text { (cf. also Figure I). }
$$

(Here and in Figure I, we regard $\mathfrak{D}^{k}$ as the trivial p.g. sheaf: $0 \rightarrow \mathfrak{D}^{k} \stackrel{i}{\longrightarrow} \mathfrak{D}^{k} \rightarrow 0$, with the identity $i(\operatorname{Remark} 1.1)$.) We use the notation $C_{r}^{q}\left(\mathscr{A}, \mathcal{f}^{m} \mathfrak{i} ; g\right)_{\alpha}, \ldots$ for the second set of $(2.3)_{3}$. Moreover, we set

$$
C_{\tilde{c}}^{q}\left(\mathscr{A}, f^{m \Re} ; g\right)_{\mathrm{p} . \mathrm{g}}:=\cup_{\alpha \in \mathbb{R}_{1}^{+2}} C_{\tilde{c}}^{q}\left(\mathscr{A}, f^{m_{\mathfrak{i}}} ; g\right)_{\alpha} \quad\left(\mathrm{cf} .(2.3)_{4}\right),
$$

where the symbol $\tilde{c}$ indicates the symbol ' $l$ ' or ' $r$ '.

The left filtration ${ }_{l} \Phi_{l ; g}$ makes use of informations of the left side of the homomorphism $\omega \cdot F^{m}: \mathfrak{D}^{s k} \rightarrow \mathfrak{S}$ and the definition of the sets in $(2.3)_{4}$ is concordant to the similar sets in the p.g. cohomology theory in Section 1 (cf. (1.4) $)_{8}$ ). The left filtration is suitable for later explicit uniform estimation (cf. §2.2). The right filtration ${ }_{r} \Phi_{l ; g}$ is, as we will see soon later, suitable for applications to the completion theory.

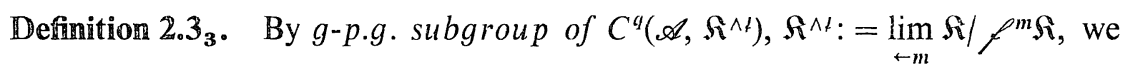
mean

$$
C^{q}\left(\mathscr{A}, \mathfrak{S}^{\wedge \ell} ; g\right)_{\mathrm{p} . \mathrm{g}}:=\lim _{\leftarrow m} C^{q}(\mathscr{A}, \mathfrak{\Re}, g)_{\mathrm{p} . \mathrm{g}} / C_{r}^{q}\left(\mathscr{A}, f^{m} \mathfrak{s} ; g\right)_{\mathrm{p} . \mathrm{g}} .
$$

The word 'subgroup' is justified by the following

Proposition 2.2. . There is a natural injection

$$
i: C^{q}\left(\mathscr{A}, \mathfrak{R}^{\wedge t} ; g\right)_{\mathrm{p} . g} \hookrightarrow C^{q}\left(\mathscr{A}, \mathfrak{R}^{\wedge \ell}\right) \text {. }
$$

Proof. First, from $(2.3)_{3}$, we have the exact sequence:

(a) $0 \longrightarrow C_{r}^{q}\left(\mathscr{A}, f^{m} \mathfrak{S}_{\mathrm{i}} ; g\right)_{\mathrm{p} . \mathrm{g}} \longrightarrow C^{q}(\mathscr{A}, \mathfrak{i} ; g)_{\mathrm{p} . \mathrm{g}} \stackrel{\mu_{m} \cdot i}{\longrightarrow} C^{q}(\mathscr{A}, \mathfrak{K}) / C^{q}\left(\mathscr{A}, f^{m} \mathfrak{r}_{\mathrm{i}}\right)$, where the homomorphism $\mu_{m}$ is induced from the natural one: $\mathfrak{A} \rightarrow \mathfrak{S} / f^{m} \mathfrak{R}$ and $i=$ inclusion: $C^{q}(\mathscr{A}, \Re ; g)_{\mathrm{p} . \mathrm{g}} \hookrightarrow C^{q}(\mathscr{A}, \mathfrak{R})$. (Thus we use the information of the right side of (a) in the definition of the right filtration ${ }_{r} \Phi_{k ; g}$.) It is easy to get $(2.3)_{8}$ from (a).

q.e.d.

Concerning the right and left filtrations, we remark:*)

*) If we replace the symbol ' $C_{r}^{q}$ ' in (2.3) ${ }_{7}$ by ' $C_{1}^{q}$, then the corresponding fact to (2.3) ${ }_{8}$ fails in general; the right filtration is more suitable than the left one for application to the completion theory. 
Proposition 2.2. $\quad C_{r}^{q}\left(\mathscr{A}, f^{m} \Re ; g\right)_{\mathrm{p} . \mathrm{g}} \supset C_{l}^{q}\left(\mathscr{A}, f^{m} \Re ; g\right)_{\mathrm{p} . \mathrm{g}}$.

This follows directly from the definition of the both sides; see $(2.3)_{\sigma}^{\prime}$. In Lemma $2.2_{1}$, $\$ 2.2$, we show that the above two filtrations are 'equivalent' for the varieties of the type in Section 1.

4. D.p.c. estimation map. Finally we introduce an estimation map, which will be used in the main estimations in Section 2 (cf. Theorems 2.1-2.2,$\S 2.2$ ).

Defimition 2.4. By a d.p.c. estimation map, we mean a collection $E=(\widetilde{E}$, $\exp M, L$ ), where $\tilde{E}$ is a p.g.c. estimation map $\in \mathbb{E}_{\text {p.g }}$ (Definition 1.5 ), $M$ is a positive monomial (n.5, $\S 1.1$ ) and $L$ is a linear map: $\mathbb{R}^{+} \ni m \rightarrow \mathbb{R}^{+} \ni \mathrm{cm} ; c>0$. Recall that $E$ is a map : $\mathbb{D}:=\left(\mathbb{R}^{+} \times \mathbb{R}^{+2} \times \mathbb{R}^{+2}\right) \ni(r ; \sigma ; \alpha) \rightarrow \mathbb{D}=\left(\mathbb{R}^{+} \times \mathbb{R}^{+2} \times \mathbb{R}^{+2}\right)$ $\ni\left(r^{\prime} ; \sigma^{\prime} ; \alpha^{\prime}\right)$. We regard $E$ as the map :*)

$(2.4)_{1} \quad E: \mathbb{D} \times\left(\mathbb{Z}^{+} \cup 0\right) \ni(r ; \sigma ; \alpha) \times m \rightarrow \mathbb{D} \times\left(\mathbb{Z}^{+} \cup 0\right) \ni\left(r^{\prime} ; \sigma^{\prime} ; a^{\prime} \cdot \alpha^{\prime}\right) \times[L(m)]$, where $a^{\prime}=\exp M(m)$.

(In the later estimations, we write the parameter space $\mathbb{D} \times\left(\mathbb{Z}^{+} \cup 0\right)$ as $\left(\mathbb{R}^{+} \times \mathbb{R}^{+2}\right)$ $\times\left(\mathbb{Z}^{+} \cup 0\right) \times \mathbb{R}^{+2}$; see $\S 2.2$.) Note that the correspondence: $\left({ }^{*} ; \sigma\right) \rightarrow\left(r^{\prime} ; \sigma^{\prime}\right)$ is given by the first part $E^{\prime}$ of $\tilde{E}\left(\in \mathbb{E}_{\mathrm{p} . \mathrm{g}}^{\prime}\right.$ ) (cf. Definition 1.5). We call $E^{\prime}$ also the first part of $E$. The correspondence: $\left(\mathbb{Z}^{+} \cup 0\right) \ni m \rightarrow\left(\mathbb{Z}^{+} \cup 0\right) \ni[L(m)]$ will concern the 'a.d. part' of the cochains (cf. §2.2). We call this correspondence 'the a.d. part of $E$ '. The map $E$ is factored as follows:

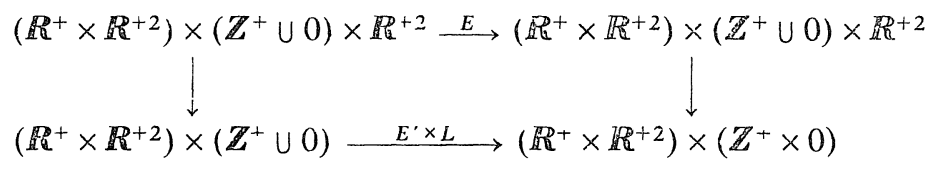

(Here ' $L$ ' denotes the a.d. part of $E$.)

We set:

$$
\mathbb{E}_{\mathrm{d} . \mathrm{p}}=\text { collection of all d.p.c. estimation maps. }
$$

Concerning the decomposition $E_{1} \circ E_{2}$ of the elements $E_{i} \in \mathbb{E}_{\text {d.p }}(i=1,2)$ the similar 'associativity law' to (1.6) $)_{3}$ (given for the p.g.c. maps) holds. We use this fact frequently in the sequel of this paper, without mentioning it.

*) Writing $\alpha^{\prime} \in \mathbb{R}_{1}^{+2}$ as $\left(\alpha_{1}^{\prime}, \alpha_{2}^{\prime}\right)$, we set $a \alpha^{\prime}:=\left(a \alpha_{1}^{\prime}, \alpha_{2}^{\prime}\right)$ (cf. $\left.(1.6)_{0}^{\prime}\right)$. 


\section{§2.2. Main Results}

1. Case of local variety. Letting the local variety $\boldsymbol{X}=\left(\mathbb{C}^{n}(z), U_{0}, X_{0}\right.$, $h, \ldots$ ) be as in Theorem 1.1 (cf. also $\left.(1.8)_{0}\right)$, we fix a finite subset $f=\left(f_{j}\right)_{j=1}^{s}$ $\subset \Gamma\left(X_{0}, O_{X_{0}}\right)$ satisfying (1) $f_{j}\left(P_{0}\right)=0(1 \leqq j \leqq S)$ and (2) $f_{j} \not \equiv 0\left(X_{0 ; P_{0} ; v}\right)(1 \leqq j \leqq s)$ for each irreducible component $X_{0 ; P_{0} ; v}$ of the germ of $X_{0}$ at $P_{0}$. Here we generalize Theorem 1.1, Theorem 1.2 to the present d.p. cohomology theory, which is given to the pair $(\mathbb{X}, f)$. As in Section 1 , the underlying variety for the arguments here is $X=X_{0}-D$, where $D=$ locus of $h$. The p.g. properties of cochains etc. are measured, as in Section 1, by the p.g. function $g_{X}:=\left|h^{-1}\right|$, while the a.d. properties of the cochains will be measured by $f$. As in Section 1 we use the symbol $\boldsymbol{X}$ also for $X$ and $\left(\boldsymbol{X}, g_{\mathbf{X}}\right)$. When there is no fear of confusion, we use ' $\mathbf{X}$ ' also for $\left(\mathbb{X}, g_{\mathbf{X}}, f\right)$ and $(\mathbb{X}, f)$.

(i) D.p. parametrization. Here we generalize the p.g. parametrization in n.2, $\S 1.2$ to the present d.p. cohomology theory. First the parametrization of the coverings here is same as that in $n .3, \S 1.2$ (cf. $\left.(1.8)_{2}\right)$ :

$$
\begin{aligned}
u_{\mathbf{X}}: \mu_{\mathbf{X}}:=D_{\mathbf{X}} \times \boldsymbol{R}^{+} \times \mathbb{R}^{+2} \ni \mu & =(P ; r ; \sigma) \\
& \longrightarrow \operatorname{Cov}_{0}(\mathbf{X})_{\mathrm{p} . \mathrm{g}} \ni \mathscr{A}_{\mu}:=\mathscr{A}_{\sigma}\left(\boldsymbol{X}_{r}(P)\right),
\end{aligned}
$$

where we write $D$ as $D_{\mathbf{X}}$. Also the manifold $\boldsymbol{X}_{r}(P)$ and its p.g. covering $\mathscr{A}_{\mu}$ are as in $(1.9)_{2}$.

Next, we form a product $\tau_{\mathbf{X}}:=\boldsymbol{\mu}_{\mathbf{X}} \times\left(\boldsymbol{Z}^{+} \cup 0\right) \times \boldsymbol{R}_{1}^{+2}$, and, for an element $\mathfrak{H} \in$ $\operatorname{Coh}(\boldsymbol{X})_{\text {p.g }}$ (Definition 1.5), we define the following parametrization of sets of cochains :

$$
\begin{aligned}
\tau_{\boldsymbol{X}}:=\mu_{\boldsymbol{X}} \times\left(\mathbb{Z}^{+} \cup 0\right) \times \boldsymbol{R}^{+2} \ni \tau & =(\mu ; m ; \alpha) \\
\stackrel{c_{\mathfrak{g}}^{q}}{\longrightarrow} & \left.C_{1}^{q}\left(\mathscr{A}_{\mu}, \boldsymbol{f}^{m} \mathfrak{S} ; g\right)_{\alpha} \quad \text { (cf. (2.3) }\right) .
\end{aligned}
$$

We define a parametrization $Z_{5}^{q}$ by changing ' $C_{1}^{q}$ ' to ' $Z_{1}^{q}$ ' (cf. (2.3) $)_{4}$ ). Then, we generalize the p.g. cochain collection in $(1.9)_{3}^{\prime}$ as follows:

$$
\begin{aligned}
\boldsymbol{C}_{1}^{q}(\boldsymbol{X}, \mathfrak{H})_{\mathrm{p} \cdot \mathbf{g}}^{d}:=C_{\mathfrak{J}}^{q}\left(\tau_{\mathbf{X}}\right) & =\left\{C_{1}^{q}\left(\mathscr{A}_{\mu}, \mathscr{f}^{m} \mathfrak{H} ; g\right)_{\alpha} ;(\mu ; m ; \alpha) \in \tau_{\mathbf{X}}\right. \\
& \left.=\boldsymbol{\mu}_{\mathbf{X}} \times\left(\boldsymbol{Z}^{+} \cup 0\right) \times \boldsymbol{R}_{1}^{+2}\right\} .
\end{aligned}
$$

We call $\boldsymbol{C}_{1}^{q}(\boldsymbol{X}, \mathfrak{S})_{\text {p.g }}^{d}$ the $q$-th $(f, g)$-d.p. cochain collection for $\mathfrak{H}$. We define $(f, g)$-d.p. cocycle collection $\mathbb{Z}_{1}^{q}(\boldsymbol{X}, \mathfrak{S})_{\mathrm{p} . \mathrm{g}}^{d}$ by changing the symbol $C_{\mathfrak{S}}^{q}$ in $(2.5)_{3}$ to $Z_{5}^{q}$. Such collections contain all necessary sets of cochains in the d.p. uniform 
estimations in n.1. We will fix the p.g. sheaf $\mathfrak{H}$ as above in the remainder of Section 2.

$$
\begin{aligned}
\boldsymbol{\tau}_{\mathbf{X}}=\boldsymbol{\mu}_{\mathbf{X}} \times\left(\mathbb{Z}^{+} \cup 0\right) \times \boldsymbol{R}_{1}^{+2} \ni \tau=(\mu ; m ; \alpha) \stackrel{C_{\mathfrak{g}}^{q}}{\longrightarrow} \mathbb{C}_{1}^{q}(\mathbb{X}, \mathfrak{H})_{\mathrm{p} . \mathrm{g}}^{d} \\
\\
C_{1}^{q}\left(\mathscr{A}_{\sigma}\left(\mathbb{X}_{r}(P)\right), \mathscr{f}^{m} \mathfrak{S} ; g\right)_{\alpha} \\
\boldsymbol{\mu}_{\mathbf{X}}=D_{\mathbf{X}} \times \boldsymbol{R}^{+} \times \boldsymbol{R}_{1}^{+2} \ni \mu=(P ; \sigma, \gamma) \stackrel{u_{\mathbf{X}}}{\longrightarrow} \operatorname{Cov}_{0}(\mathbb{X})_{\mathrm{p} . \mathrm{g}} \ni \mathscr{A}_{\sigma}\left(\mathbb{X}_{r}(P)\right) .
\end{aligned}
$$

D.p. parametrization table

(ii) Estimation data. We will use the d.p.c. estimation maps $E \in \mathbb{E}_{\text {d.p }}$ (Definition 2.4) for the uniform estimations in n.1. As in Section 1.2 our uniform estimations will work for a subset of the parameter space $\tau_{\mathbb{X}}$ : letting $D_{1 ; \mathbf{X}}$ be an open subset of $D_{\mathbf{X}}$, which contains $P_{0}$ (= origin of $D_{\mathbf{X}}, \mathbb{X}$...(cf. n.1, $\S 1.2)$ ), we take an element $(\tilde{r}, \tilde{\sigma}, \tilde{m}) \in \mathbb{R}^{+} \times \mathbb{R}_{1}^{+2} \times \mathbb{Z}^{+}$. We the form a subset $\boldsymbol{\mu}_{\mathfrak{g}}=D_{1 ; \mathbf{X}} \times(0, \tilde{r}) \times \boldsymbol{R}_{\tilde{\sigma}}^{+2}$ of $\boldsymbol{\mu}_{\mathbf{X}}=D_{\mathbf{X}} \times \mathbb{R}^{+} \times \mathbb{R}_{1}^{+2}$ and $\left.{ }^{*}\right) \quad \tau_{\mathfrak{\xi}}^{\prime}=\boldsymbol{\mu}_{\mathfrak{5}} \times \mathbb{Z}_{\tilde{m}}^{+} \times \mathbb{R}_{1}^{+2}$ of $\boldsymbol{\tau}_{\mathbf{X}}=\boldsymbol{\mu}_{\mathbf{X}} \times\left(\mathbb{Z}^{+} \cup 0\right) \times \boldsymbol{R}_{1}^{+2}$. As in Section 1.2 we call $\boldsymbol{\tau}_{\mathfrak{g}}^{\prime}$ restricted parameter space for $H$. We fix $\tau_{\mathfrak{\xi}}^{\prime}$ in the remainder of Section 2 .

(iii) Now, using the sets of the cochains as in the table soon above (cf. also $(2.5)_{2}$ ), we generalize Theorem 1.1, Theorem 1.2 to the d.p. cohomology theory:

Theorem 2.1 (D.p. uniform estimation for Čech operator $\delta=\delta_{X}$ ). There is a d.p.c. estimation map $E_{\mathfrak{n}} \in \mathbb{E}_{\mathrm{d} . \mathrm{p}}(q>0)$, with which we have:

$$
\begin{aligned}
& s^{*} Z^{q}\left(\mathscr{A}_{\sigma}\left(\mathbb{X}_{r}(P)\right), \rho^{m} \mathfrak{H}\right)_{\alpha} \subset \delta C^{q-1}\left(\mathscr{A}_{\sigma^{\prime}}\left(\mathbb{X}_{r^{\prime}}(P)\right), f^{m^{\prime}} \mathfrak{H}\right)_{\alpha^{\prime}} \text {, where } \\
& \left(r^{\prime}, \sigma^{\prime}, m^{\prime}, \alpha^{\prime}\right)=E_{\mathfrak{S}}(r, \sigma, m, \alpha) \quad(c f . \text { Definition } 2.4) .
\end{aligned}
$$

Theorem 2.2 (D.p. uniform estimation for resolution of $\mathfrak{5})$. There is a d.p.c. map $E_{\mathfrak{5}} \in \mathbb{E}_{\mathrm{d} . \mathrm{p}}(q \geqq 0)$, with which we have:

$$
\begin{array}{ll}
(2.6)_{2} & s^{*} Z^{q}\left(A_{\sigma}\left(\mathbf{X}_{r}(P)\right), f^{m} \mathfrak{H}\right)_{\alpha} \subset \omega_{\mathfrak{g}} Z^{q}\left(\mathscr{A}_{\sigma^{\prime}}\left(\mathbb{X}_{r^{\prime}}(P)\right), f^{\left.m^{\prime} D_{\mathbb{X}}^{k}\right)_{\alpha^{\prime}}, \text { where }}\right. \\
(2.6)_{2}^{\prime} & \left(r^{\prime}, \sigma^{\prime}, m^{\prime}, \alpha^{\prime}\right)=E_{\mathfrak{g}}(r, \sigma, m, \alpha),
\end{array}
$$

and $\omega_{\mathfrak{n}}: \mathfrak{D}_{\mathbf{X}}^{k} \rightarrow \mathfrak{H}$ is the first resolution of $\mathfrak{H}$ (Definition 1.5)**)

Note that the sheaves $f^{m} \mathfrak{D}_{X}^{k}$ in $(2.6)_{2}$ are, in general, not free sheaves. In order to complete the resolution in Theorem $2.2_{1}$ we give:

*) For the sets $\boldsymbol{R}_{\tilde{\sigma}}^{+2}, \boldsymbol{Z}_{\tilde{m}}^{+}$, see the end of introduction of Chapter I. In Theorem $2.2_{2}$, we are concerned with the structure sheaf $D_{X}$, and we should understand that $\tau_{\mathfrak{g}}=\tau_{0_{X}}, \ldots$.

**) Also, in Theorems 2.1-2.2 $\left.(2.5)_{2}\right)$. 
Theorem 2.2. (D.p. uniform estimation for $\left\{f^{m} \mathfrak{D}_{\mathbf{X}}\right\}_{m=1}^{\infty}$ ). There is an element $E_{\boldsymbol{X}} \in \mathbb{E}_{\text {d.p }}(q \geqq 0)$, with which we have:

$$
\begin{array}{ll}
(2.6)_{3} & s^{*} Z^{q}\left(\mathscr{A}_{\sigma}\left(\mathbb{X}_{r}(P)\right), \mathcal{f}^{m} \mathfrak{D}_{\mathbf{X}}\right)_{\alpha} \subset F^{m^{\prime}} Z^{q}\left(\mathscr{A}_{\sigma^{\prime}}\left(\mathbb{X}_{r^{\prime}}(P)\right), \mathfrak{D}_{\mathbf{X}}^{s}\right)_{\alpha^{\prime}}, \text { where } \\
(2.6)_{3}^{\prime} & \left(r^{\prime}, \sigma^{\prime} ; m^{\prime} ; \alpha^{\prime}\right)=E_{\boldsymbol{X}}(r, \sigma ; m ; \alpha),
\end{array}
$$

and $F^{m}: \mathfrak{D}_{\mathbf{X}}^{s} \rightarrow \mathfrak{D}_{\mathbf{X}}$ is the m-th standard homomorphism for $f(n .2, \S 2.1)$.

In Theorem $2.1-2.2_{2}$, the parameter $\tau=(P ; r, \sigma ; m ; \alpha)$ is in the restricted parameter space $\tau_{\tilde{r}}^{\prime}\left(=D_{1, X} \times(0, \tilde{r}) \times \mathbb{R}_{\tilde{\sigma}}^{+2} \times \mathbb{Z}_{\tilde{m}}^{+} \times \mathbb{R}_{1}^{+2}\right)$. We will rewrite Theorem 2.1 in the following diagram (cf. also Figure III, n.4, § 1.2).

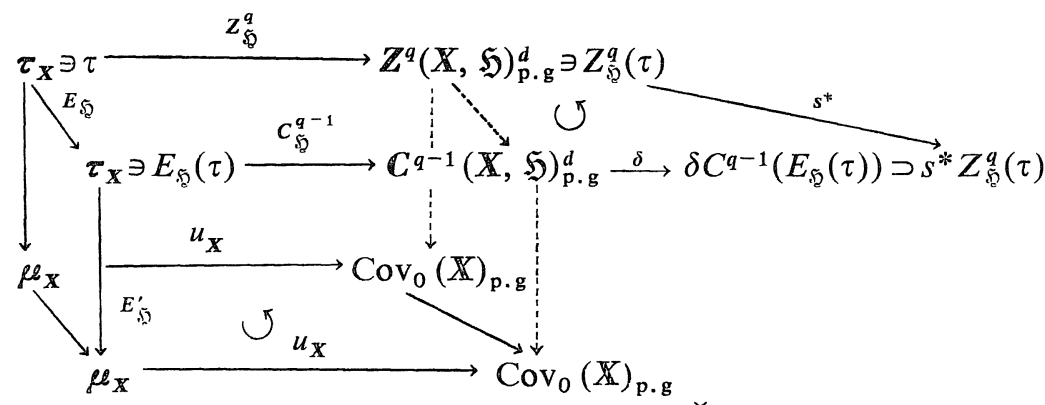

Figure I. (D.p. vanishing properties for Čech operator)*)

For the proof of Theorem 2.1-2.2 , see Section 2.3. Also applications of these results will be given in n.3, n.4, Section 2.2 and in Section 3. As we will see in Section 2.3, Theorem $2.2_{2}$ concerns an open map property of Koszul complexes, which relates to a cohomological generalization of Hilbert zero point theorem (cf Lemma 2.5; see also Introduction). Theorem $2.2_{2}$ will also fill the gap between the d.p. and p.g. estimation in Section 2 and Section 1, and may be most basic among Theorems 2.1-2.2 2 .

2. An afrime analogue. Letting the affine variety $\mathbb{X}^{\prime} \subset \mathbb{C}^{n}(z)$ be as in Theorem 1.3, we take a finite set $f^{\prime}=\left(f_{j}^{\prime}\right)_{j=1}^{s} \subset \Gamma\left(\boldsymbol{X}^{\prime}, \mathfrak{D}_{\mathbf{X}^{\prime}} ; g_{\mathbf{X}^{\prime}}\right)_{\text {p.g }}$, where the p.g. function $g_{X^{\prime}}=|z|+1$ is as in n.5, Section 1.2. Similarly to n.1, we use the symbol $\mathbb{X}^{\prime}$ also for $\left(\mathbb{X}^{\prime}, g_{\boldsymbol{X}^{\prime}}\right),\left(\boldsymbol{X}^{\prime}, f^{\prime}\right)$ and $\left(\mathbb{X}^{\prime}, g_{\mathbf{X}^{\prime}}, f^{\prime}\right)$. We generalize here Theorem 1.3 and Theorem 1.4. The set of the p.g. coverings: $\operatorname{Cov}_{0}\left(\mathbb{X}^{\prime}\right)_{\mathrm{p} . \mathrm{g}}:=\left\{\mathscr{A}_{\sigma}\left(\mathbb{X}^{\prime}\right) ; \sigma \in \boldsymbol{R}_{1}^{+2}\right\}$, where $\mathscr{A}_{\sigma}\left(\mathbb{X}^{\prime}\right)$ is the p.g. covering of $\mathbb{X}^{\prime}$ of size $\sigma$, is as in Theorem 1.3, Theorem 1.4. Next we set $\tau_{X^{\prime}}:=\mu_{X^{\prime}} \times\left(\mathbb{Z}^{+} \cup 0\right)$, with $\boldsymbol{\mu}_{\boldsymbol{X}^{\prime}}:=\mathbb{R}_{1}^{+2}$. Then taking a p.g. sheaf $\mathfrak{S}^{\prime} \in \operatorname{Coh}\left(\mathbb{X}^{\prime}\right)_{\mathrm{p} . \mathrm{g}}$ (cf. Definition $\left.1.4_{1}\right)$,

*) The map $E_{\mathfrak{g}}^{\prime}$ in Figure $I$ is the first part of $E_{\mathfrak{5}}$ (cf. n.4, §2.1). 
our d.p. cochains in $n .2$ will be parametrized as follows:

$$
\begin{aligned}
C_{5}^{q}: \tau_{X^{\prime}}=\mathbb{R}_{1}^{+2} & \times\left(\mathbb{Z}^{+} \cup 0\right) \ni(\sigma ; m) \\
& \left.\longrightarrow C_{1}^{q}\left(\mathscr{A}_{\sigma}\left(\mathbb{X}^{\prime}\right), f^{m \mathfrak{S g}^{\prime}} ; g_{X^{\prime}}\right)_{\text {p.g }} \quad \text { (cf. }(2.3)_{3}\right) .
\end{aligned}
$$

Taking an element $(\tilde{\sigma} ; \tilde{m}) \in \mathbb{R}_{1}^{+2} \times \mathbb{Z}^{+}$, we set ${ }^{*} \tau^{\prime}:=\mathbb{R}_{\tilde{\sigma}}^{+2} \times \mathbb{R}_{\tilde{m}}^{+}$. Our d.p. estimation soon below will work for elements in $\tau^{\prime}$. Thirdly, our estimation maps herc will be of the following form:

$(2.7)_{1}^{\prime} \quad E^{\prime}: \mathbb{R}_{1}^{+2} \times\left(\mathscr{L}^{+} \cup 0\right) \ni(\sigma ; m) \rightarrow \mathbb{R}_{1}^{+2} \times\left(\mathscr{Z}^{+} \cup 0\right) \ni(\mathscr{L}(\sigma),[L(m)])$, with an el-map $\mathscr{L}$ and a linear map $L=c t ; c>0$.

We write the collection of all such maps as $\mathbb{E}_{\mathrm{d} . \mathrm{p}}^{\prime}$.

Theorem 2.3 (D.p. uniform estimation for Cech operator $\delta=\delta_{\mathbf{X}^{\prime}}$ ). For $a$ suitable $E_{\text {ईg }} \in \mathbb{E}_{\text {d. p }}^{\prime}$ we have $(q \geqq 1)$ :

$(2.7)_{2} \quad s^{*} Z^{q}\left(\mathscr{A}_{\sigma}\left(\mathbb{X}^{\prime}\right)\right.$

$$
\begin{aligned}
\left.\mathscr{f}^{\prime m} \mathfrak{S}^{\prime}\right)_{\mathrm{p} . \mathrm{g}} \subset \delta C^{q-1}\left(\mathscr{A}_{\sigma^{\prime}}\left(\mathbb{X}^{\prime}\right),\right. & \left.f^{\prime m^{\prime} \mathfrak{S}^{\prime}}\right)_{\mathrm{p} . \mathrm{g}} \\
& \text { with }\left(\sigma^{\prime} ; m^{\prime}\right)=E_{\mathfrak{g}}(\sigma ; m) .
\end{aligned}
$$

Theorem 2.4. (D.p. uniform estimation for resolution). For a suitable $E_{5,} \in \mathbb{E}_{\text {d.p }}^{\prime}$ we have:

$$
\begin{aligned}
s^{*} Z^{q}\left(\mathscr{A}_{\sigma}\left(\mathbb{X}^{\prime}\right), f^{\prime m} \mathfrak{S}^{\prime}\right)_{\mathrm{p} . \mathrm{g}} \subset \omega_{\mathfrak{\mathfrak { g } ^ { \prime }}} Z^{q}\left(\mathscr{A}_{\sigma^{\prime}}\left(\mathbb{X}^{\prime}\right), \operatorname{f}^{\prime m^{\prime}} \mathfrak{D}_{\mathbb{X}^{\prime}}^{k}\right)_{\mathrm{p} . \mathrm{g}}, \\
\text { with }\left(\sigma^{\prime} ; m^{\prime}\right)=E_{\mathfrak{g}}(\sigma ; m),
\end{aligned}
$$

where $\omega_{\tilde{r}}$ is the first resolution of $\mathfrak{S}^{\prime}$ (Definition 1.5).

Theorem 2.4. (D.p. uniform estimation for $\left.\left\{f^{\prime m} \mathfrak{D}_{\boldsymbol{X}^{\prime}}\right\}_{m=0}^{\infty}\right)$. For a suitable $E_{\boldsymbol{X}^{\prime}} \in \mathbb{E}_{\text {d.p }}^{\prime}$ we have**):

$(2.7)_{4} \quad s^{*} Z^{q}\left(\mathscr{A}_{\sigma}\left(X^{\prime}\right)\right.$,

$$
\begin{aligned}
\left.\ell^{\prime m} \mathfrak{D}_{\mathbf{X}^{\prime}}\right)_{\mathrm{p} . \mathrm{g}} \subset F^{\prime m^{\prime}} Z^{q}\left(\mathscr{A}_{\sigma^{\prime}}\left(\mathbb{X}^{\prime}\right), \mathfrak{D}_{\mathbf{X}^{\prime}}^{s}\right)_{\mathrm{p} . \mathrm{g}}, \\
\\
\text { with }\left(\sigma^{\prime} ; m^{\prime}\right)=E_{\mathbb{X}^{\prime}}^{\prime}(\sigma ; m) .
\end{aligned}
$$

In the above the parameter $(\sigma ; m)$ is in $\tau^{\prime}=\mathbb{R}_{\tilde{\sigma}}^{+2} \times \mathbb{Z}_{\tilde{m}}^{+}$.

For the proof of Theorem 2.3, Theorems 2.4 1,2 , see Section 2.3.

3. Open map properties. Here we show the properties in the title for some p.g. filtered complexes. For this we first set:

*) According as we are concerned with Theorem 2.3, Theorem $2.4_{1}$ or Theorem $2.4_{2},(\tilde{\sigma} ; \tilde{m})$ depends on $\left(\mathbb{X}^{\prime}, \mathfrak{S}^{\prime}\right)$ or $\mathbb{X}^{\prime}$. Thus we should understand that $\tau^{\prime}=\tau_{\mathfrak{F}^{\prime}}^{\prime}$ or $=\tau_{X^{\prime}}^{\prime}$, according to the theorems just above.

**) $F^{\prime} m$ is the $m^{\prime}$-th standard homomorphism attached to $f^{\prime}$ (cf. n.2, $\$ 2.1$ ). 


$$
\left\{\begin{array}{l}
\left(\boldsymbol{X}^{*}, \mathfrak{H}^{*}, \rho^{*}, g^{*}\right):=\left(\boldsymbol{X}, \mathfrak{H}, f, g_{\mathbf{X}}\right) \text { or }\left(\boldsymbol{X}^{\prime}, \mathfrak{H}^{\prime}, f^{\prime}, g_{\mathbf{X}^{\prime}}\right) \\
\tilde{\boldsymbol{\mu}}_{\mathbf{X}^{*}}:=\tilde{\mu}_{\mathbf{X}}:=(0, \tilde{r}) \times \boldsymbol{R}_{\sigma}^{+2} \text { or }=\boldsymbol{\mu}_{\mathbf{X}^{\prime}}^{\prime}:=\boldsymbol{R}_{\tilde{\sigma}}^{+2}
\end{array}\right.
$$

(cf. n.1, n.2, §2.2).

We regard $\tilde{\mu}_{X^{*}}$ as the direct set in the manner as in n.6, §1.2. For an element $\mu=(r ; \sigma) \in \tilde{\mu}_{\boldsymbol{X}}=(0, \tilde{r}) \times \boldsymbol{R}_{\tilde{\sigma}}^{+2}$ or $=\sigma \in \mu_{X^{\prime}}^{\prime}$, we denote by $\mathscr{A}_{\mu}$ the p.g. covering $\mathscr{A}_{\sigma}\left(\boldsymbol{X}_{r}(P)\right.$ ) or $\mathscr{A}_{\sigma}\left(\boldsymbol{X}^{\prime}\right)$ (cf. Theorem 2.1 and Theorem 2.3). We generalize Definition 1.9 to the present d.p. cohomology theory:

Definition 2.5. . By left $\left(g^{*}, f^{*}\right)$-p.g. filtered Cech complex for $\left(\mathscr{A}_{\mu}, \mathfrak{H}^{*}\right)$, we mean the following filtered complex (cf. n.1, § 2.1):

$$
0 \longrightarrow Z^{0}\left(\mathscr{A}_{\mu}, \mathfrak{H}^{*}\right)_{\mathrm{p} . \mathrm{g}} \stackrel{i}{\longrightarrow} \mathscr{C}_{l}^{0}\left(\mathscr{A}_{\mu}, \mathfrak{H}^{*}\right)_{\mathrm{p} . \mathrm{g}}^{d} \stackrel{\delta}{\longrightarrow} \cdots \mathscr{C}_{1}^{q}\left(\mathscr{A}_{\mu}, \mathfrak{H}^{*}\right)_{\mathrm{p}, \mathrm{g}}^{d} \longrightarrow,
$$

where we set:

$$
\mathscr{C}_{1}^{q}\left(\mathscr{A}_{\mu}, \mathfrak{H}^{*}\right)_{\mathrm{p} . \mathrm{g}}^{d}:=\left\{C_{1}^{q}\left(\mathscr{A}_{\mu}, \rho^{* m} \mathfrak{H}^{*}\right)_{\mathrm{p} . \mathrm{g}}\right\}_{m=0}^{\infty} \quad\left(\mathrm{cf} .(2.3)_{6}\right),
$$

and $i$ : =inclusion: $Z^{0}\left(\mathscr{A}_{\mu}, \mathfrak{H}^{*}\right)_{\mathrm{p} . \mathrm{g}} \hookrightarrow C^{0}\left(\mathscr{A}_{\mu}, \mathfrak{H}^{*}\right)_{\mathrm{p} . \mathrm{g}}$.

Next we write $\mathfrak{H}^{*}$ in the form of $(1.4)_{1}: 0 \rightarrow \mathfrak{D}_{\mathbf{X}^{*}}^{k_{p}} \stackrel{K_{p-1}}{\longrightarrow} \cdots \stackrel{K_{1}}{\longrightarrow} \mathfrak{D}_{\mathbf{X}^{*}}^{k_{1}} \stackrel{\omega_{\mathfrak{Q}^{*}}}{\longrightarrow} \mathfrak{H}^{*}$ $\rightarrow 0$, and we set:

$$
\mathscr{Z}_{1}^{q}\left(\mathscr{A}_{\mu}, \mathfrak{S}^{*}\right)_{\mathrm{p} . \mathrm{g}}^{d}:=\left\{Z_{1}^{q}\left(\mathscr{A}_{\mu}, f^{* m} \mathfrak{H}^{*}\right)_{\mathrm{p} . \mathrm{g}}\right\}_{m=0}^{\infty} .
$$

(We define $\mathscr{Z}_{1}^{q}\left(\mathscr{A}_{\mu}, \mathfrak{D}_{\mathbf{X}^{*}}^{k_{j}}\right)_{\text {p.g }}^{d}$ in the similar manner to the above.)

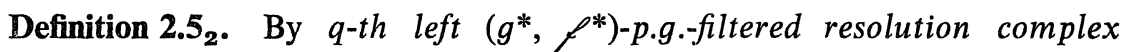
for $\left(\mathscr{A}_{\mu}, \mathfrak{H}^{*}\right)(q \geqq 0)$, we mean :

$$
\begin{aligned}
0 \longrightarrow \mathscr{Z}_{1}^{q}\left(\mathscr{A}_{\mu},\right. & \left.\mathfrak{D}_{\mathbf{X}^{*}}^{k_{p}}\right)_{\mathrm{p} . \mathrm{g}}^{d} \stackrel{K_{p-1}}{\longrightarrow} \cdots \\
& \stackrel{K_{1}}{\longrightarrow} \mathscr{Z}_{1}^{q}\left(\mathscr{A}_{\mu}, \mathfrak{D}_{\mathbf{X}^{*}}^{k_{1}}\right)_{\mathrm{p}, \mathrm{g}}^{d} \stackrel{\omega_{\mathfrak{W}}^{*}}{\longrightarrow} \mathscr{Z}_{1}^{q}\left(\mathscr{A}_{\mu}, \mathfrak{H}^{*}\right)_{\mathrm{p}, \mathrm{g}}^{d} \longrightarrow 0,
\end{aligned}
$$

where the augumentation map is understood to be the zero map (n.1, § 2.1).

We write the filtered complexes in Definitions $2.5_{1,2}$ as $\mathscr{C}_{1}^{*}\left(\mathscr{A}_{\mu}, \mathfrak{H}^{*}\right)_{\text {p.g }}^{d}$ and $\mathscr{Z}_{1}^{q}\left(\mathscr{A}_{\mu}, \mathfrak{S}^{*}\right)_{\text {p.g }}^{d}$. The right $\left(f^{*}, g^{*}\right)$-p.g.-filtered complexes $\mathscr{C}_{r}^{*}\left(\mathscr{A}_{\mu}, \mathfrak{H}\right)_{\text {p.g }}^{d}$ and $\mathscr{Z}_{r}^{q}\left(\mathscr{A}_{\mu}, \mathfrak{S}\right)_{\text {p.g }}^{d}$ will be defined similarly. Then we have:

Lemma 2.1 (Open map properties of the left p.g. filtered Cech and resolution complexes). The direct systems of the left p.g. filtered complexes $\left\{\mathscr{C}_{1}^{*}\left(\mathscr{A}_{\mu}, \mathfrak{H}^{*}\right)_{\mathrm{p.g}}\right\}_{\mu},\left\{\mathscr{Z}_{l}^{q}\left(\mathscr{A}_{\mu}, \mathfrak{H}^{*}\right)_{\mathrm{p} . \mathrm{g}}^{d}\right\}_{\mu}$ satisfy the open map property and are $\tilde{\mu}_{X^{*}}$-exact (Definition 2.1), where $\mu$ runs through $\tilde{\boldsymbol{\mu}}_{\mathbf{X}^{*}}$ (cf. $(2.8)_{0}$ ).

Proof*). Let $E_{\mathfrak{\xi}}^{\prime}:\left(\boldsymbol{R}^{+} \times \boldsymbol{R}^{+2}\right) \rightarrow\left(\boldsymbol{R}^{+} \times \boldsymbol{R}^{+2}\right)$ and $L_{\mathfrak{\xi}}: \boldsymbol{Z}^{+} \rightarrow \boldsymbol{Z}^{+} \cup 0$ be the first

*) Cf. also (2.14), n.4, §2.3, which is used in the proof of Theorem $2.2_{1}$ and Theorem $2.4_{1}$. 
and a.d. parts of the d.p.c map $E_{\mathfrak{⿷}}$ in Theorem 2.1 (cf. also n.4, §2.1). Then, letting the element $\mu=(r ; \sigma) \in{\tilde{\mu_{X}}}_{X}\left(\subset \mathbb{R}^{+} \times \mathbb{R}_{1}^{+2}\right)$ be as in Definition $2.5_{1}$, we have directly the following from $(2.6)_{1}$ and $(2.4)_{1}^{\prime}$ :

$(2.8)_{4} \quad s^{*} Z_{1}^{q}\left(\mathscr{A}_{\mu}, f^{m} \mathfrak{H}\right)_{\mathrm{p} . \mathrm{g}} \subset \delta C_{1}^{q-1}\left(\mathscr{A}_{\mu^{\prime}}, f^{m^{\prime}} \mathfrak{H}\right)_{\mathrm{p} . \mathrm{g}}$, with $\mu^{\prime}:=\left(r^{\prime} ; \sigma^{\prime}\right)=E_{52}^{\prime}(r ; \sigma)$ and $m^{\prime}=\left[L_{\mathfrak{n}}(m)\right](m \gg 0)$.

Comparing this with the numerical criterion $(2.1)_{2}^{\prime}$ for the open map property, we have that condition for the Cech complex defined for $\mathbb{X} \in \mathbb{A m}_{1 a}$. The open map property for the Čech complex for $\mathbb{X}^{\prime} \in \mathbb{A} f$ follows from Theorem 2.3 similarly to the above. Also the open map properties for the resolution complexes defined for $\mathbf{X} \in \mathbf{A n}_{1 a}$ and $\mathbf{X}^{\prime} \in \mathbb{A} f$ follow*) from Theorem $2.2_{1}$, Theorem $2.4_{1}$. Finally, the $\ell$-exactness condition for the Čech and the resolution complexes follow from the 'p.g. exactnesses', Corollary 1.2, Theorem 1.3, Theorem 1.4, and we finish the proof of Lemma 2.1 .

q.e.d.

For the right d.p. filtration we have the similar fact to Lemma 2.1:

Lemma 2.2 (Open map properties of the right d.p. filtrations). The direct systems of the right p.g. filtered complexes $\left\{\mathscr{C}_{r}^{*}\left(\mathscr{A}_{\mu}, \mathfrak{H}^{*}\right)_{\mathrm{p} . \mathrm{g}}^{d}\right\}_{\mu}$ and $\left\{\mathscr{Z}_{r}^{q}\left(\mathscr{A}_{\mu}\right.\right.$, $\left.\left.\mathfrak{S}^{*}\right)_{\mathrm{p} . \mathrm{g}}^{d}\right\}_{\mu}$ satisfy the open map property and are $\mu$-exact.

By Lemma 2.1 and Proposition 2.1 2 , the following lemma insures Lemma 2.2 .

Lemma 2.3 (Equivalence of the left and right d.p. filtrations). The direct systems $\left\{\mathscr{C}_{1}^{*}\left(\mathscr{A}_{\mu}, \mathfrak{H}^{*}\right)_{\mathrm{p} . \mathrm{g}}^{d}\right\}_{\mu}$ and $\left\{\mathscr{C}_{r}^{*}\left(\mathscr{A}_{\mu}, \mathfrak{H}^{*}\right)_{\mathrm{p} . \mathrm{g}}^{d}\right\}_{\mu}$ as well as $\left\{\mathscr{Z}_{1}^{*}\left(\mathscr{A}_{\mu}, \mathfrak{S}^{*}\right)_{\mathrm{p}, \mathrm{g}}^{d}\right\}_{\mu}$ and $\left\{\mathscr{Z}_{r}^{q}\left(\mathscr{A}_{\mu}, \mathfrak{S}^{*}\right)_{\mathrm{p} . \mathrm{g}}^{d}\right\}_{\mu}$ are equivalent (Definition $2.1_{2}$ ).

Recalling the definitions of 'equivalence' and Proposition $2.2_{2}$, we see easily that the proof of the following leads to Lemma 2.3:

Lemma 2.3'. We have the inclusion:

$(2.9)_{1} \quad s^{*} C_{r}^{q}\left(\mathscr{A}_{\mu}, f^{m} \mathfrak{S}^{*}\right)_{\mathrm{p} . \mathrm{g}} \subset C_{1}^{q}\left(\mathscr{A}_{\mu,}, f^{m^{\prime}} \mathfrak{S}^{*}\right)_{\mathrm{p} . \mathrm{g}}$, with $m^{\prime}=\left[L_{\mathbb{X}^{*}}(m)\right]$, and a suitable parameter $\mu^{\prime} \in \mu_{\mathbf{X}^{*}}$, where $m \gg 0$ and $L_{\mathbb{X}^{*}}$ is chosen**) in an independent manner from $(\mu ; m)$.

The proof of Lemma $2.3^{\prime}$ is given in Section 4.2 .

*) See the footnote *), p. 416 .

**) $\mathrm{L}_{\mathbb{Z}^{*}}$ is, as in Lemma 2.1, a linear map. Also the pair $\left(\mathscr{A}_{\mu}, \mathscr{S}^{*}\right)$ has the similar meaning to Lemma 2.1 . 
4. P.g. complexes in the completion. Now, from the results in n.1-n.3, we derive a generalization of the p.g. cohomology theory in $n .6, \S 1.2$ to the completion thecry. The content here will be our main applications of the uniform estimations in Section 2 as well as in this paper. First letting the pair $\left(\mathscr{A}_{\mu}, \mathfrak{S}^{*}\right)$ and $\mathscr{f}^{*} \in \Gamma\left(\mathbb{X}^{*}, \mathfrak{D}_{\mathbf{X}^{*}}\right)$ be as in $n .3$, we define

$(2.9)_{1} C^{*}\left(\mathscr{A}_{\mu}, \hat{\mathfrak{S}}^{*}\right)_{\mathrm{p} . \mathrm{g}}:=\lim _{\leftarrow m} C^{*}\left(\mathscr{A}_{\mu}, \mathfrak{S}^{*}\right)_{\mathrm{p} \cdot \mathrm{g}} / C_{r}^{*}\left(\mathscr{A}_{\mu}, f^{* m \mathfrak{S}^{*}}\right)_{\mathrm{p} . \mathrm{g}}$, where

$$
\hat{\mathfrak{S}}^{*}:=\lim _{\leftarrow m} \mathfrak{S}^{*} / f^{* m} \mathfrak{S}^{*} \quad \text { (cf. Definition 2.3). }
$$

By Proposition $2.2_{1}$, this complex $C^{*}\left(\mathscr{A}_{\mu}, \hat{\mathfrak{S}}^{*}\right)_{\text {p.g }}$ is a subcomplex of $C^{*}\left(\mathscr{A}_{\mu}, \hat{\mathfrak{S}}^{*}\right)$. Now, denoting by $X_{P}$ the germ of $\bar{X}$ at $P$, we generalize Definition 1.9 to the completion theory as follows

Definition 2.6. (1) By p.g. Čech complex for $\left(\mathbf{X}_{P}, \hat{\mathfrak{5}}\right)$ and $\left(\mathbb{X}^{\prime}, \hat{\mathfrak{S}}^{\prime}\right)$ we mean:

$$
C^{*}\left(\mathbb{X}_{P}, \hat{\mathfrak{S}}\right)_{\mathrm{p} . \mathrm{g}}:=\lim _{\tilde{\mu} \rightarrow} C^{*}\left(\mathscr{A}_{\tilde{\mu}}, \hat{\mathfrak{S}}\right)_{\mathrm{p} . \mathrm{g}}, \quad C^{*}\left(X^{\prime}, \hat{\mathfrak{H}}^{\prime}\right)_{\mathrm{p} . \mathrm{g}}:=\lim _{\tilde{\mu}^{\prime} \rightarrow} C^{*}\left(\mathscr{A}_{\tilde{\mu}}, \hat{\mathfrak{S}}^{\prime}\right)_{\mathrm{p} . \mathrm{g}},
$$

where $\tilde{\mu}, \tilde{\mu}^{\prime}$ run through $\tilde{\mu}_{\mathbf{X}^{*}}$.

(2) By q-th p.g. resolution complex for $\left(\mathbb{X}_{P}, \hat{\mathfrak{H}}\right)$ and $\left(\mathbb{X}^{\prime}, \hat{\mathfrak{S}}^{\prime}\right)(q \geqq 0)$, we mean

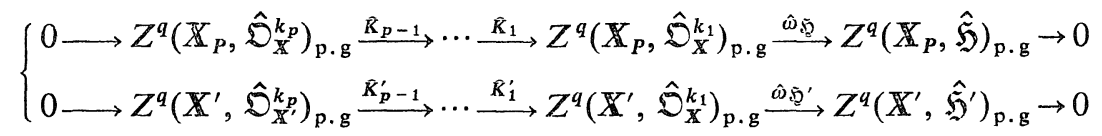

where $Z^{q}\left(\mathbb{X}_{P}, \hat{\mathfrak{H}}\right)_{\mathrm{p} . \mathrm{g}}:=C^{q}\left(\mathbb{X}_{P}, \hat{\mathfrak{H}}\right)_{\mathrm{p} . \mathrm{g}} \cap \delta^{-1}(0), \ldots$, and $\hat{K}_{p-1}, \ldots, \hat{\omega}_{\tilde{g},}, \ldots$ are the completions of $K_{p-1}, \ldots, \omega_{\mathfrak{s}}$.

Now we generalize Theorems $1.5,1.6$ to the completion theory as follows: first denoting by $H^{q}\left(\mathbb{X}_{P}, \hat{\mathfrak{H}}\right)_{\mathrm{p}_{\mathrm{g}}}$ the $q$-th cohomology group of $C^{q}\left(\mathbb{X}_{P}, \hat{\mathfrak{H}}\right)_{\mathrm{p} . \mathrm{g}}, \ldots$ we have

Theorem 2.5. $H^{q}\left(\mathbb{X}_{P}, \hat{\mathfrak{Y}}\right)_{\mathrm{p} . \mathrm{g}} \cong 0$ and $H^{q}\left(\mathbb{X}^{\prime}, \hat{\mathfrak{S}}^{\prime}\right)_{\mathrm{p} . \mathrm{g}} \cong 0(q \geqq 1)$.

This follows from Lemma 2.3 and Proposition 2.2. Next we have

Lemma 2.4. The p.g. complex in $(2.9)_{4}$ is exact.

Proof. From the open map property for the p.g. complexes (cf. Lemma 2.2), we have

$(2.9)_{4}^{\prime} \quad Z^{q}\left(\mathbb{X}_{P}, \hat{\mathfrak{H}}\right)_{\mathrm{p} . \mathrm{g}} \cong \lim _{\tilde{\mu} \rightarrow}\left(\lim _{\leftarrow m} Z^{q}\left(\mathscr{A}_{\tilde{\mu}}, \mathfrak{S}\right)_{\mathrm{p} . \mathrm{g}} / Z_{r}^{q}\left(\mathscr{A}_{\tilde{\mu}}, f^{m} \mathfrak{S}\right)_{\mathrm{p} . \mathrm{g}}\right)$ and the similar fact to $Z^{q}\left(\mathbb{X}^{\prime}, \hat{\mathfrak{S}}^{\prime}\right)_{\text {p.g. }}$.

Similarly, applying the open map property for the p.g. resolution complex 
to the right side of $(2.9)_{4}^{\prime}$, we get the desired exactiness.

q.e.d.

Finally we determine the structure of $H^{0}\left(\mathbb{X}_{p}, \hat{\mathfrak{5}}\right)_{\mathrm{p} . \mathrm{g}}$ and $H^{0}\left(\mathbb{X}^{\prime}, \hat{\mathfrak{S}}^{\prime}\right)_{\mathrm{p} . \mathrm{g}}$. For this, letting the sheaves $\mathfrak{D}_{X_{0}}(* D)$ of meromorplic functions over $X_{0}$ and $\mathfrak{D}_{\mathbf{X}^{\prime}, \mathrm{ll}}$ of (algebraic) regular functions over $\mathbb{X}_{\mathrm{alg}}^{\prime}$ be as in Theorem 1.6. (cf. also (1.13) Then we have:

Theorem 2.6. and algebraic completions to the p.g. complctions:

$$
\begin{aligned}
& (2.9)_{5} \quad \hat{O}_{P}: \hat{\mathfrak{D}}_{X_{0}}(* D)_{P} \simeq H^{C}\left(\mathbb{X}_{p}, \hat{\mathfrak{D}}_{\lambda}\right)_{\mathrm{p} . \mathrm{g}}, O_{\mathrm{X}^{\prime}}: \Gamma\left(\mathbb{X}_{\mathrm{a} 1 \mathrm{~g}}^{\prime}, \hat{\mathfrak{D}}_{X^{\prime} . \mathrm{alg}}\right) \\
& \longrightarrow H^{0}\left(\mathbb{X}^{\prime}, \hat{\mathfrak{D}}_{\mathbf{X}^{\prime}}\right)_{\mathrm{p} . \mathrm{g}},
\end{aligned}
$$

where the left sides are as follows:

$$
\begin{aligned}
& \hat{\mathfrak{O}}_{X_{0}}(* D):=\lim _{\leftarrow m} \mathfrak{D}_{X_{0}}(* D) / F^{m} \mathfrak{D}_{X_{0}}^{s}(* D), \\
& \hat{\mathfrak{D}}_{X^{\prime}, \mathrm{a} 1 \mathrm{~g}}:=\lim _{\leftarrow m} \mathfrak{D}_{X^{\prime}, \mathrm{a} 1 \mathrm{~g}} / F^{\prime m} \underbrace{}_{X^{\prime}, \mathrm{alg}}
\end{aligned}
$$

Proof. We prove the first isomorphism in $(2.9)_{5}$. The second is proven similarly. First, from the isomorphism $0_{P}: \mathfrak{D}_{\mathbf{X}_{0}}(* D)_{P} \stackrel{\sim}{\rightarrow} H^{0}\left(\mathbb{X}_{P}, \mathfrak{D}_{\mathbf{X}}\right)_{\mathrm{p} . \mathrm{g}}$, we sec that the following natural homomorphism is an jsomorphism:

(a) $\hat{0}_{P}: \mathfrak{D}_{X_{0}}(* D)_{P} \longrightarrow \lim _{\mu \rightarrow}\left(\lim _{+m} Z^{0}\left(\mathscr{A}_{\mu}(P), \mathfrak{D}_{\mathbf{X}}\right)_{\mathrm{p} . \mathrm{g}} / F^{m} Z^{0}\left(\mathscr{A}_{\mu}(P), \mathfrak{D}_{\mathbf{X}}^{\mathrm{s}}\right)_{\mathrm{p} . \mathrm{g}}\right)$, where we write $\mathscr{A}_{\mu}:=\mathscr{A}_{\sigma}\left(\mathbb{X}_{r}(P)\right)$ as $\mathscr{A}_{\mu}(P)$ (cf. also $\left.(2.8)_{0}\right)$.

On the other hand, $(2.9)_{4}^{\prime}$, Theorem $2.2_{2}$ and Lemma 2.3 imply that

$$
H^{0}\left(\mathbb{X}_{P}, \hat{D}_{\mathbf{X}}\right)_{\mathrm{p} . \mathrm{g}} \cong \text { right side of }(\mathrm{a}),
$$

and we have $(2.9)_{5}$.

q.e.d.

Finally, applying Lemma 2.4 to Thecrem 2.6 , we generalize Theorem $1.6_{2}$ to the completions $\hat{\mathfrak{S}}, \hat{\mathfrak{S}}^{\prime}$ as follows:

Theorem 2.6. $\mathbf{2}_{2}$. The following complexes are exact:

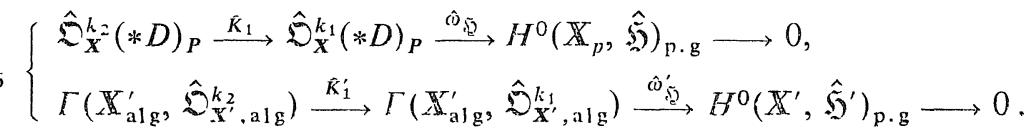

Theorem 2.5 and Theorem 2.6 are a generalization of Theorem 1.5 and Theorem 1.6 as well as our analogue of Theorems A, B of $\mathrm{H}$. Cartan in the p.g. cohomology theory in the completion. We give an application of Theorem 1.5 , Theorem 2.6 to the analytic de Rham theory in Section 3. 


\section{§2.3. Key Lemmas}

Here we give key lemmas for Theorems 2.1-2.4, which concern the open map properties of $\mathfrak{D}_{\boldsymbol{X}^{*}}$, where $\boldsymbol{X}^{*}=\boldsymbol{X}$ or $\boldsymbol{X}^{\prime}$ (cf. Lemma 2.5-Lemma 2.7). Also, using those lemmas, we prove Theorems 2.1-2.4.

1. Koszul complexes. In our proof of Theorems 2.1-2.4, we will take Theorem $2.2_{2}$ and Theorem $2.4_{2}$, which concern the a.d. properties of $\mathfrak{D}_{\mathbf{X}^{*}}$, as the starting point (cf. n.3, n.4). Recalling that the a.d. properties in Theorem 2.2, Theorem $2.4_{2}$ are measured by the powers of $f^{*}:=f$ or $f^{\prime}$, we first attach to $f^{* m}$ what we call $m$-th Koszul complex for $f^{*}$ :

$(2.10)_{0} \quad 0 \longrightarrow \mathfrak{D}_{\mathbf{X}^{*}} \stackrel{F_{0}^{* m}}{\longrightarrow} \mathfrak{D}_{\mathbf{X}^{*}}^{s}$ $\mathfrak{D}_{X^{*}}^{(s)} \stackrel{F_{P}^{* m}}{\longrightarrow} \cdots \mathfrak{D}_{X^{*}}^{s} \stackrel{F_{s}^{k_{m}} \boldsymbol{m}_{1}}{\longrightarrow} \mathfrak{D}_{\mathbf{X}^{*}} \longrightarrow 0$

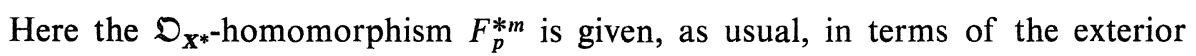
product as follows*): for a point $Q \in \boldsymbol{X}^{*}$, let $\Omega_{Q}^{p}$ denote $\mathfrak{D}_{Q}\left(:=\mathfrak{D}_{\mathbf{X}^{*}, Q}\right)$-module consisting of differential forms of degree $q$ with coefficients in $\mathfrak{D}_{Q}$. Letting $x$ be a (formal) indeterminate, we denote by $i_{Q}$ the identification: $\mathfrak{D}_{Q}^{\left(\begin{array}{c}s \\ p\end{array}\right)} \ni \varphi=$ $\left(\varphi_{J}\right)_{J} \rightarrow \Omega_{Q}^{p} \ni \sum_{J} \varphi_{J} d x_{J}$, where $J$ exhausts all indices $J=\left(j_{1}<\cdots<j_{p}\right)$. Then $F_{p}^{* m}$ is defined by: $i_{Q} F_{p}^{* m}=\wedge \omega_{m} \cdot i_{Q}$, where we set $\omega_{m}:=\sum_{j=1}^{s} f_{j}^{* m} d x_{j}$ and ' $\wedge$ ' denotes the symbols of the exterior product. Noting that $F_{s-1}^{* m}=F^{* m}$ (cf. n.2, $\S 2.1)^{* *)}$, we use the Koszul complexes in $(2.10)_{0}, \mathscr{F} * m$ in symbol, for analysis of the sheaves $f^{* m} \bigvee_{X^{*}}\left(=F^{* m} \bigvee_{\mathbf{X}^{*}}^{s}\right)$ (cf. n.2 soon below). The lemma in n.2, Lemma 2.5 , will be our key facts for the proof of Theorem $2.2_{2}$, Theorem $2.4_{2}$, which

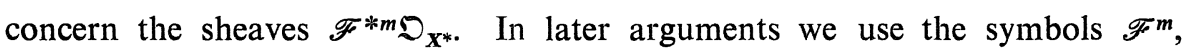
$F_{p}^{m}, \ldots$ or $\mathscr{F}^{\prime m}, F_{p}^{\prime m}, \ldots$ for $\mathscr{F}^{* m}, F_{p}^{* m}$, according as we are concerned with $\mathbf{X}^{*}$ $=\boldsymbol{X}$ or $\boldsymbol{X}^{\prime}$.) We set $\mathscr{F}^{*}=\left\{\mathscr{F}^{* m}\right\}_{m=1}^{\infty}$.

2. Open map property for $\mathscr{F}^{*}$. Letting the parameter spaces $\boldsymbol{\mu}_{\boldsymbol{X}}\left(\subset D_{\mathbf{X}}\right.$ $\times \boldsymbol{R}^{+} \times \boldsymbol{R}_{1}^{+2}$ ) and $\mu_{\boldsymbol{X}^{\prime}}:=\boldsymbol{R}_{\tilde{\sigma}}^{+2}$ be as in Theorem $2.2_{2}$, Theorem $2.4_{2}$ (cf. also $\left.(2.5)_{1},(2.7)_{1}\right)$, we form a product $\lambda_{X}:=\mu_{X} \times \boldsymbol{R}_{1}^{+2}\left(\subset D_{X} \times \boldsymbol{R}^{+} \times \boldsymbol{R}_{1}^{+2} \times \boldsymbol{R}_{1}^{+2}\right)$. Also we take a linear function $L_{0, \mathbf{X}^{*}}=c_{0, \mathbf{X}^{*}} t ; c_{0, \mathbf{X}^{*}}>0$. Then we have:

Lemma 2.5 (Open map property for $\left.\mathscr{F}^{*}\right)$. Choose suitable d.p. estimation

*) Cf. J. P. Serre [19].

**) Precisely the homomorphisms $F_{s-1}^{* m}$ and $F^{* m}$ are $: \bigcirc_{X^{*}}^{s} \ni\left(\varphi_{j}\right)_{j=1}^{s} \longrightarrow \bigcirc_{X^{*}}^{s} \ni \sum_{j=1}^{s}(-1)^{j}$ $\times f_{j}^{m} \varphi_{j}$ and $\sum_{j=1}^{s} \varphi_{j} \cdot f_{j}^{m}$. This difference of the signatures does not cause differences for the applications of the results for $F_{s-1}^{* m}$ for $F^{* m}$. 
maps*) $E_{\mathbf{X}} \in \mathbb{E}_{\mathrm{d} . \mathrm{p}}$ and $\boldsymbol{E}_{\mathbf{X}^{\prime}} \in \mathbb{E}_{\mathrm{d} . \mathrm{p}}^{\prime}$. Then, for each $(\tilde{m}, m) \in \mathbb{Z}^{+} \times \mathbb{Z}^{+}$satisfying $\tilde{m}>L_{0, x^{*}}(m)$, we have the following inclusion $(1 \leqq s<p)$ :

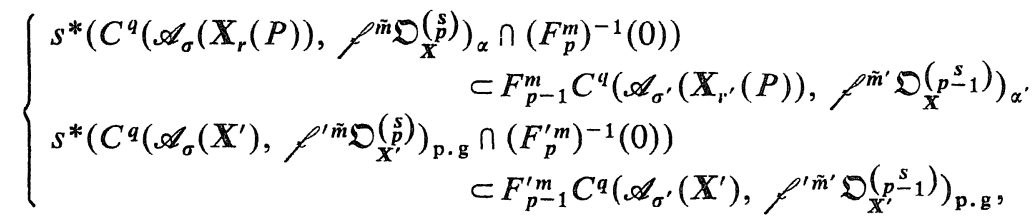

with $\left(r^{\prime} ; \sigma^{\prime} ; \tilde{m} \tilde{m}^{\prime} ; \alpha^{\prime}\right)=E_{\mathbf{X}}(r ; \sigma ; \tilde{m} ; \alpha)$ and $\left(\sigma^{\prime} ; \tilde{m^{\prime}}\right)=E_{\mathbf{X}^{\prime}}(\sigma ; \tilde{m})$. Here the parameters $(P ; r ; \sigma ; \alpha)$ are in $\lambda_{\mathbf{X}}\left(\subset D_{1, \mathbf{X}} \times \boldsymbol{R}^{+} \times \mathbb{R}_{1}^{+2} \times \mathbb{R}_{1}^{+2}\right)$ and $\sigma$ is in $\boldsymbol{\mu}_{\mathbf{X}^{\prime}}\left(\subset \boldsymbol{R}_{1}^{+2}\right)$.

If we fix an element $m \in \mathbb{Z}^{+}$, which defines the homomorphisms $F_{p}^{m}$, then Lemma 2.5 insures the open map property for $F_{p}^{m}$. As we will see soon below, Lemma 2.5 plays the most basic role in getting the d.p. uniform estimations in Section 2 from the p.g. estimations in Section 1. Also Lemma 2.5 will concern a cohomological generalization of Hilbert zero point theorem (cf. Part B, §4.1). Lemma 2.5 will play the most important roles in the lemmas given in Section 2.3.

3. Here we will prove the following implication:

Lemma 2.6 (Reduction of d.p. uniform estimations to p.g. uniform estimations).

$$
\left\{\begin{array}{l}
\text { Th. } 1.1 \\
\text { Th. } 1.3
\end{array}\right\}+\text { Lemma } 2.5 \text { for }\left\{\begin{array}{l}
\mathscr{F} \\
\mathscr{F}^{\prime}
\end{array}\right\} \longrightarrow\left\{\begin{array}{l}
\text { Th. } 2.2_{2} \\
\text { Th. } 2.4_{2}
\end{array}\right\}
$$

Precisely, in the above implication, we use Theorem 1.1 and Theorem 1.3, applied to the structure sheaves $\mathfrak{D}_{\boldsymbol{X}}$ and $\mathfrak{D}_{\boldsymbol{X}^{\prime}}$. Note that Theorem 1.1 and Theorem 1.3 do not concern the a.d. properties of $\mathfrak{D}_{\mathbf{X}}, \ldots$, while those properties are the basic factor in Theorem $2.2_{2}$ and Theorem 2.4 ; we rewrite Lemma 2.6 in the following symbolical form:

$(2.10)_{2}^{\prime}$ p.g. uniform estimation for $\mathfrak{D}_{\mathbf{X}^{*}}$, open map property for $F^{*} \rightarrow$ d.p. uniform estimation for $\mathfrak{D}_{\mathbf{X}^{*}}$.

We prove Lemma 2.6 in three steps. First we introduce a type of auxiliary estimation maps, which is used to fill the gap between the estimations in Theorem $2.2_{2}$, Theorem $2.4_{2}$ and in Theorem 1.1, Theorem 1.3.

(i) Pre d.p.c. map. Denote by $\boldsymbol{D}, \mathbb{D}^{\prime}$ the parameter space $\left(\mathbb{R}^{+} \times \mathbb{R}^{+2}\right)$ $\times \mathbb{Z}^{+} \times \boldsymbol{R}^{+2}$ and $\left(\boldsymbol{R}^{+} \times \boldsymbol{R}^{+2}\right) \times \boldsymbol{R}^{+2}$, on which the d.p.c. and p.g.c. maps operate.

*) Cf. Definition 2.4 and $(2.7)_{1}^{\prime}$ 
Then we make:

Definition 2.5. By a pre d.p.c. map we mean such a map: $(2.11)_{0} E: \mathbb{D} \rightarrow \mathbb{D}^{\prime}$, where $E$ is written as $E=\mathrm{pr} \cdot \widetilde{E}$, with a d.p.c. map $\tilde{E}$ (Definition 2.4) and the projection pr: $\mathbb{D} \rightarrow \mathbb{D}^{\prime}$.

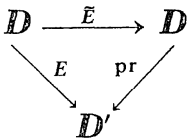

Figure I.

Take p.g.c. and d.p.c. maps $E_{1}, E_{2}$. Then, for each $\tau=(r ; \sigma ; m ; \alpha) \in(0,1)$ $\times \mathbb{R}^{+2} \times \mathbb{E}^{+} \times \mathbb{R}_{1}^{+2}$ and the pre d.p.c. map $E$ as in $(2.11)_{0}$, we have: (2.11) $)_{0}^{\prime} \quad \bar{E}_{1}(\tau) \succ E_{1} \cdot E(\tau)$, and $\bar{E}_{2}(\tau) \succ E \cdot E_{2}(\tau)$, with suitable pre d.p.c. maps $\bar{E}_{1}, \bar{E}_{2}$. (For the order $\succ$, see $(1.6)_{3}^{\prime}$.)

(ii) Letting the parameter space $\lambda_{X^{*}}^{\prime}$ and the linear function $L_{0, X^{*}}$ be as in Lemma 2.5, we check that the symbol ' $C$ ' ' in Lemma 2.5 is changed by ' $Z$ ', (by using the pre d.p.c. map instead of the d.p.c. map). (In Lemma 2.6' soon below, the parameter $(P ; r ; \sigma ; \alpha)$ or $\sigma \in \lambda_{X^{*}}^{\prime}$ is as in Lemma 2.5. Also the elements $(\tilde{m}, m) \in \mathbb{Z}^{+} \times \mathbb{Z}^{+}$satisfies: $\tilde{m}>L_{0, X^{+}}(m)$ )

Lemma $2 \mathbf{6}^{\prime}$. (1) For a suitable pre d.p.c. map $E_{\mathbf{X}}^{\prime}$ we have $(1 \leqq p<s)$ :

$(2.11)_{1} s^{* *}\left(Z^{q}\left(\mathscr{A}_{\sigma}\left(\tilde{\mathbb{X}}_{r}(P)\right), f^{\tilde{m} \mathfrak{D}_{X}^{(s)}}\right)_{\alpha} \cap\left(F_{p}^{m}\right)^{-1}(0)\right)$

$$
\subset F_{p-1}^{m} Z^{q}\left(\mathscr{A}_{\alpha^{\prime}}\left(\tilde{\mathbb{X}}_{r^{\prime}}(P)\right), \mathfrak{D}_{\boldsymbol{X}}^{(p-1}\right)_{\alpha^{\prime}}
$$

with $\left(r^{\prime} ; \sigma^{\prime} ; \alpha^{\prime}\right)=E_{\boldsymbol{X}}^{\prime}(r ; \sigma ; \tilde{m} ; \alpha)$.

(2) For a suitable el-map $\mathscr{L}_{\mathbf{X}^{\prime}}$ we have:

$(2.11)_{2} s^{*}\left(Z^{q}\left(\mathscr{A}_{\sigma}\left(\boldsymbol{X}^{\prime}\right), \mathcal{f}^{\prime \tilde{m}} \mathfrak{O}\left(\begin{array}{l}s \\ p\end{array}\right)_{X^{\prime}}\right)_{\mathrm{p} . \mathrm{g}} \cap\left(F_{p}^{\prime m}\right)^{-1}(0)\right)$

$$
\subset F_{p-1}^{\prime m} Z^{q}\left(\mathscr{A}_{\sigma^{\prime}}\left(\mathbb{X}^{\prime}\right), \mathfrak{D}_{\mathbf{X}^{\prime}}^{\left(p^{s}-1\right.}\right)_{\mathrm{p} . \mathrm{g}}
$$

with $\sigma^{\prime}=\mathscr{L}_{\mathbf{X}^{\prime}}(\sigma)$.

Proof. The proof of (1), (2) is parallel. We prove only (1). For this we first remark that. by applying Theorem 1.1 to the right side of $(2.11)_{1}$, we have: $(2.11)_{1}^{\prime} \quad s^{*}$ (left side of $\left.(2.11)_{1}\right) \subset \delta F_{p-1}^{m} C^{q-1}\left(\mathscr{A}_{\sigma^{\prime}}\left(\mathbb{X}_{r^{\prime}}(P)\right), \mathfrak{O}_{\mathbf{X}}\left(p^{s}\right)\right)_{\alpha^{\prime}}(q \geqq 1)$, where $\left(r^{\prime} ; \sigma^{\prime} ; \alpha^{\prime}\right)=E_{X}^{\prime \prime}(r ; \sigma ; \tilde{m} ; \alpha)$, with a pre d.p.c. map $E_{\tilde{X}}^{\prime \prime}$, which is determined by $E_{\tilde{X}}^{\prime}$.

(Remark that the estimation in Theorem 1.1 is given by a p.g.c. map, and from $(2.11)_{0}$, we have $\left.(2.11)_{1}^{\prime}\right) \quad$ Now, using $(2.11)_{1}^{\prime}$, the proof of Lemma $2.6^{\prime}$ is given inductively on $p:$ if $p=1$, then, remarking that $F_{0}^{m}: \mathfrak{D}_{\mathbf{X}} \rightarrow \mathfrak{D}_{\mathbf{X}}^{s}$ is injective, we have $(2.11)_{1}$ directly from Lemma 2.5. Assume that $p \geqq 2$ and that $(2.11)_{1}$ (and so $\left.(2.11)_{1}^{\prime}\right)$ holds for $\tilde{p}<p$. Take an element $\varphi$ from the left side of $(2.11)_{1}$, and we writc $\varphi$ as $\varphi=F_{p-1}^{m}\left(\rho^{\prime} \text {, with } \varphi^{\prime} \in C^{q}\left(\mathscr{A}_{\sigma^{\prime}}\left(\tilde{\mathbb{X}}_{r^{\prime}}(P)\right), f^{\prime m^{\prime}} \mathscr{D}_{\mathbf{X}}\left({ }^{s}\right)^{s}\right)\right)_{\alpha^{\prime}}$. Here 
$\left(r^{\prime} ; \sigma^{\prime} ; \tilde{m}^{\prime} ; \alpha^{\prime}\right)=\widetilde{E}_{\boldsymbol{X}}(r ; \sigma ; \tilde{m} ; \alpha)$, with a d.p.c. map $\widetilde{E}_{\boldsymbol{X}}$ as in Lemma 2.5. Applying $(2.11)_{1}^{\prime}$ for $\tilde{p}=p-1$ to $\delta \varphi^{\prime}$, we have:

$$
s^{*} \varphi^{\prime} \in F_{p-2}^{m} C^{q}\left(\mathscr{A}_{\sigma^{\prime \prime}}\left(\mathbb{X}_{r^{\prime \prime}}(P)\right), \mathscr{D}_{\mathbf{X}}^{(p-2}\right)_{\alpha^{\prime \prime}}+Z^{q}\left(\mathscr{A}_{\sigma^{\prime \prime}}\left(\mathbb{X}_{r^{\prime \prime}}(P)\right), D_{X}^{(p-1}\right)_{\alpha^{\prime \prime}},
$$

with $\left(r^{\prime \prime} ; \sigma^{\prime \prime} ; \alpha^{\prime \prime}\right)=E_{\mathbf{X}}\left(r^{\prime} ; \sigma^{\prime} ; \tilde{n}^{\prime} ; \alpha^{\prime}\right)$, where the pre d.p.c. map $\bar{E}_{\mathbf{X}}$ is determined by the maps $\widetilde{E}_{\boldsymbol{X}}$ just above and $E_{\boldsymbol{X}}^{\prime}$ in $(2.11)_{1}$ (cf. also $(2.11)_{0}$ ).

Finally, operating $F_{p-1}^{m}$ to the both sides of (a), we have $(2.11)_{1}$.

q.e.d.

(iii) Proof of Lemma 2.6. We derive Theorem 2.2 2 from (2.11), Lemma 2.6'. The proof of Theorem $2.4_{2}$ is given by using Lemma $2.6^{\prime}$ in the similar manner. Letting the linear function $L_{0}(t)=L_{0, x}(t)$ be as in Lemma 2.5, Lemma 2.6, we take a suitable linear map $L(t)$. Then we have the following for each integer $m \gg 0$ :

$$
m-m^{\prime}>L_{0}\left(m^{\prime}\right), \text { with } m^{\prime}=[L(m)] .
$$

Now take an element $\varphi \in Z^{q}\left(\mathscr{A}_{\mu}(P) \text {, } \boldsymbol{f}^{m \mathfrak{D}_{X}}\right)_{\alpha}$, where $\mathscr{A}_{\mu}(P):=\mathscr{A}_{\sigma}\left(\mathbb{X}_{r}(P)\right)$ (cf. Theorem $\left.2.2_{2}\right)$. Then setting $m^{\prime}:=[L(m)]$ and $\tilde{m}:=m-m^{\prime}$, one can write $\varphi=F_{s^{-1}}^{m} \varphi^{\prime}$, with $\varphi^{\prime} \in C^{q}\left(\mathscr{A}_{\mu}(P), f^{\tilde{m} D_{X}^{s}}\right)_{\alpha}$. By (a) one can apply Lemma $2.6^{\prime}$ to $\delta \varphi^{\prime}$, and we have:

$$
s^{*} \varphi^{\prime} \in F_{s-2}^{m} C^{q}\left(\mathscr{A}_{\sigma^{\prime}}\left(\tilde{\mathbb{X}}_{r^{\prime}}(P)\right), \mathfrak{D}_{\mathbf{X}}^{(p-2)}\right)_{\alpha^{\prime}}+Z^{q}\left(\mathscr{A}_{\sigma^{\prime}}\left(\tilde{\mathbb{X}}_{r^{\prime}}(P)\right), \mathfrak{D}_{\mathbf{X}}^{s}\right)_{\alpha^{\prime}},
$$

with $\left(r^{\prime} ; \sigma^{\prime} ; \alpha^{\prime}\right)=E_{\boldsymbol{X}}(r ; \sigma ; \tilde{m} ; \alpha)$. (Here $E_{\boldsymbol{X}}$ is the pre d.p.c. map as in Lemma 2.6.')

Operating $F_{s-1}^{m^{\prime}}$ to the both sides, we have:

$$
s^{*} \varphi \in F_{s^{-1}}^{m^{\prime}} Z^{q}\left(\mathscr{A}_{\sigma^{\prime}}\left(\tilde{\mathbb{X}}_{r^{\prime}}(P)\right), \mathfrak{D}_{\mathbf{X}}^{s}\right)_{\sigma^{\prime}} .
$$

On the other hand we see easily that the correspondence:

$$
(r ; \sigma ; m ; \alpha) \longrightarrow\left(r^{\prime} ; \sigma^{\prime} ; m^{\prime} ; \alpha^{\prime}\right)
$$

defines a d.p.c. map, which is determined by $E_{X}$ and $L(t)$. It is clear that (c) and (d) insure Theorem $2.2_{2}$.

q.e.d.

By Lemma 2.6' we see that the open map property for $\mathscr{F}^{*}$ in Lemma 2.5 suffices to get Theorem $2.2_{2}$ and Theorem $2.4_{2}$ from the p.g. uniform estimations in Section 1. In n.4 we give a lemma, which is used to get Theorem $2.2_{1}$, Theorem 2.1 from Theorem 2.2 (resp. Theorem 2.3, Theorem 2.4 from Theorem $2.4_{2}$ ).

4. Letting the p.g. sheaves $\mathfrak{H}, \mathfrak{H}^{\prime}$ be as in Theorem 2.1, Theorem 2.3, we 
assume that $\mathfrak{S}, \mathfrak{S}^{\prime}$ are in $\operatorname{Coh}^{\prime}(\boldsymbol{X})_{\text {p.g }}, \operatorname{Coh}^{\prime}\left(\boldsymbol{X}^{\prime}\right)_{\text {p.g }}$ (cf. (1.4) $)_{9}$ ). Thus $\mathfrak{S} \subset \mathfrak{D}_{\mathbf{X}}^{k}, \mathfrak{S}^{\prime}$

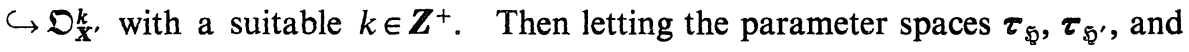
the estimation maps $E_{\mathfrak{5}} \in \boldsymbol{E}_{\mathrm{d} . \mathrm{p}}$ and $E_{\mathfrak{g}^{\prime}}^{\prime} \in \boldsymbol{E}_{\mathrm{d} \text {. p }}^{\prime}$ have the similar meanings to Theorem 2.1, Theorem 2.3, we have:

Lemma 2.7. We have the following inclusions:

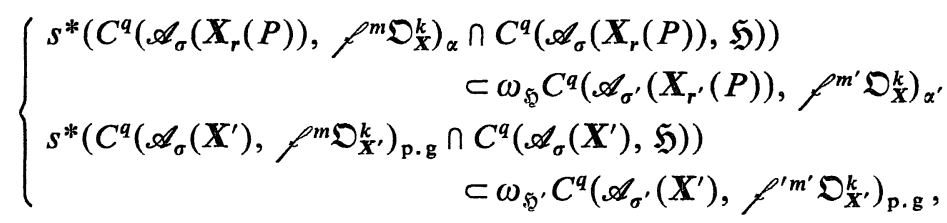

where $\left(r^{\prime} ; \sigma^{\prime} ; m^{\prime} ; \alpha^{\prime}\right)=E_{\mathfrak{5}}(r ; \sigma ; m ; \alpha)$ and $\left(\sigma^{\prime} ; m^{\prime}\right)=E_{\mathfrak{g}^{\prime}}^{\prime}(\sigma ; m)$, and the parameters $(P ; r ; \sigma ; m ; \alpha)$ and $(\sigma ; m)$ are in $\tau_{\mathfrak{g}}\left(\subset \boldsymbol{D} \times \boldsymbol{R}^{+} \times \boldsymbol{R}_{1}^{+2} \times \boldsymbol{Z}^{+} \times \boldsymbol{R}_{1}^{+2}\right)$ and in $\boldsymbol{\tau}_{\mathfrak{S}^{\prime}}\left(\subset \boldsymbol{R}_{1}^{+2} \times \boldsymbol{Z}^{+}\right)$. Moreover, $\omega_{\mathfrak{n}}: \mathfrak{D}_{\mathbf{X}}^{k^{\prime}} \rightarrow \mathfrak{D}_{X}^{k}, \ldots$ are the first resolution of $\mathfrak{S}, \ldots($ Remark 1.1).

We prove Lemma 2.7 in Section 4.2. Note that Lemma 2.7 concerns the exact complexes, and is of Artin-Rees theorem type. The role of Lemma 2.7 in our d.p. estimations in Section 2 is similar to that of the above theorem in the completions of rings (cf. [12]). Here we check the implication:

(2.13) Th. $2.2_{2}+$ Lemma $2.7 \rightarrow$ Th. $2.2_{1} \rightarrow$ Th. 2.1 (and Th. $2.4_{2}+$ Lemma 2.7 $\rightarrow$ Th. $2.4_{1} \rightarrow$ Th. 2.3).

(From a simple observation, ${ }^{*}$ ) we see that Theorem $2.2_{1,2}$, together with Theorem 1.1, imply Theorem 2.1. Here we check the first implication in (2.13).) The key fact for (2.13) is the following inclusion, which is similar to $(2.11)_{1}^{\prime}$ :

$$
\begin{aligned}
s^{*}\left(Z^{q}\left(\mathscr{A}_{\sigma}\left(\tilde{\boldsymbol{X}}_{r}(P)\right), f^{m} \mathfrak{Q}_{\mathbf{X}}^{k}\right)_{\alpha} \cap\right. & \left.Z^{q}\left(\mathscr{A}_{\sigma}\left(\tilde{\mathbf{X}}_{r}(P)\right), \mathfrak{I}\right)\right) \\
& \subset \omega_{\mathfrak{F}} Z^{q}\left(\mathscr{A}_{\sigma^{\prime}}\left(\tilde{\mathbf{X}}_{r^{\prime}}(P)\right), f^{m} \mathfrak{D}_{\boldsymbol{X}^{k^{\prime}}}\right)_{\alpha^{\prime}} .
\end{aligned}
$$

(This follows using the similar inductive arguments to n.3 (on the length of $\mathfrak{S}$ ).) Actually, remarking that $Z^{q}\left(\mathscr{A}_{\sigma}\left(\boldsymbol{X}_{r}(P)\right), f^{m} \mathfrak{G}\right)_{\alpha} \subset($ left side of $(2.14))$, we easily have Theorem 2.21 from (2.14), and we also have the first implication in (2.13).

We will conclude Section 2.3 by the following proposition.

Proposition 2.3. For the proof of Theorems 2.1-2.4, it suffices to prove Lemma 2.5, Lemma 2.7 and Lemma 2.3.

For the proof of these lemmas, see Section 4.2.

*) As in n.3, we consider only the case of the local variety $X \in \mathbf{A n}_{1 a}$. 
Remark 2.2. Here we make some remarks, which are used in the later arguments. First, we recall that we saw the following implication in $(2.10)_{2}$ :

$$
\text { Lemma } 2.5 \longrightarrow \text { Th. } 2.2_{2} \text {. }
$$

Next we remark that the open map property in Lemma 2.5 is given in terms of the symbol ' $C$ '. Using the similar (syzygy) arguments to Lemma 2.6', we see easily that Lemma 2.5 and Theorem $2.2_{2}$ enable us to change the symbol ' $C^{q}$ ' in (2.12) in Lemma 2.7 to the one ' $Z$ ' '. (Namely we have the following inclusion):

$$
\begin{aligned}
s^{*}\left(Z^{q}\left(\mathscr{A}_{\sigma}\left(\tilde{\mathbf{X}}_{r}(P)\right), f^{m} \mathfrak{D}_{X}^{(s)}\right)_{\mathrm{p} . \mathrm{g}}\right. & \left.\cap\left(F_{p}^{m}\right)^{-1}(0)\right) \\
& \subset F_{p-1}^{m} Z^{q}\left(\mathscr{A}_{\alpha^{\prime}}\left(\tilde{\mathbf{X}}_{r^{\prime}}(P)\right), f^{m^{\prime}} \mathfrak{D}_{X}(p-1)\right)_{\mathrm{p} . \mathrm{g}} .
\end{aligned}
$$

By $(2.15)_{1}$, we have the following implication:

$$
\text { Lemma } 2.5 \longrightarrow(2.15)_{2} \text {. }
$$

Thirdly, as we checked in (2.14), the symbol ' $C^{q}$ ' in Lemma 2.7 is changed to ' $Z$ ' ' (by using Lemma 2.7 and Theorem 2.22). This fact, together with $(2.15)_{1}$, insures the implication:

$$
\text { Lemma } 2.5+\text { Lemma } 2.7 \longrightarrow \text { (2.14) }
$$

We use $(2.15)_{1-3}$ in the proof of Proposition 4.2 (in n.4, §4.2).

\section{§3. Application to Analytic de Rham Theory}

Here we summarize our applications of the cohomology theories in Section 1, Section 2 to the analytic de Rham theory. As was mentioned, we use here our results on $C^{\infty}$-de Rham theory for certain stratified spaces and real analytic varieties, which were announced in $[15]_{2-4}$ and [16] (cf. Lemma 3.2 and Lemma 3.3). The details of those results will be published elsewhere in a near future (cf. [18]).

1. Letting the smooth local variety $\mathbb{X}=X_{0}-D$ and the smooth affine variety $\boldsymbol{X}^{\prime}$ be as in Section 1 , Section 2, we set*).

$$
\left\{\begin{array}{l}
\Omega_{\mathbf{X}^{\prime}, \text { alg }} \\
\Omega_{\mathbf{X}}(* D)
\end{array}\right\}:=\text { sheaf over }\left\{\begin{array}{l}
\boldsymbol{X}_{\mathrm{alg}}^{\prime} \\
\mathbf{X}_{0}
\end{array}\right\} \text { of }\left\{\begin{array}{l}
\text { rational differential forms, } \\
\text { meromorphic differential forms } \\
\text { with the pole } D \text {. }
\end{array}\right.
$$

Moreover, let the subvarieties $\boldsymbol{V}, \boldsymbol{V}^{\prime}$ of $\mathbf{X}_{0}, \mathbb{X}^{\prime}$ in Section 2, we set:

*) Similarly to Section 1, Section 2, $\boldsymbol{X}_{\mathrm{a} 1 \mathrm{~g}}^{\prime}$ and $\boldsymbol{V}_{\mathrm{a} 1 \mathrm{~g}}^{\prime}$ denote the algebraic varieties whose underlying analytic varieties are $\boldsymbol{X}^{\prime}, \boldsymbol{V}^{\prime}$. 
$(3.1)_{0}^{\prime}$

$$
\begin{aligned}
& \widehat{\Omega}_{\boldsymbol{X}}(* D):=\lim _{\leftarrow m} \Omega_{\boldsymbol{X}}(* D) / \widetilde{\boldsymbol{I}} \boldsymbol{m}_{V} \Omega_{\boldsymbol{X}}(* D), \\
& \widehat{\Omega}_{\boldsymbol{X}^{\prime}, \mathrm{alg}}:=\lim _{\leftarrow m} \Omega_{\mathbf{X}^{\prime}, \mathrm{alg}} / \Im_{V^{\prime}, \text { alg }}^{m} \Omega_{\mathbf{X}^{\prime}, \mathrm{alg}},
\end{aligned}
$$

where $\Im_{\boldsymbol{V}}$ and $\boldsymbol{I}_{\boldsymbol{V}^{\prime} \text {,alg }}$ denote the ideal sheaves of $V$ and $V_{\text {alg }}^{\prime}$. We write $\mathscr{V}-D$ as $\tilde{V}$, and, taking a point $P \in D \cap \mathbb{V}$, we denote by $\boldsymbol{X}_{P}, \tilde{V}_{P}$ the germs of $\boldsymbol{X}, \tilde{\boldsymbol{V}}$ at $P$. We then set:

(3.1) $)_{0}^{\prime \prime} H^{*}\left(\mathbb{X}_{P}, \mathbb{C}\right):=\lim _{U \rightarrow P} H^{*}(X \cap U, \mathbb{C})$, where $U$ exhaust all neighborhoods of $P$ in $X_{0}$ (and we define $H^{*}\left(\tilde{V}_{P}, \mathscr{C}\right)$ similarly).

Then we have*):

Theorem 3.1. $H^{*}\left(\mathbb{X}_{P}, \mathbb{C}\right) \cong H^{*}\left(\Omega_{\mathbf{X}}(* D)_{P}\right)$, and $H^{*}\left(\mathbb{X}^{\prime}, \mathbb{C}\right) \cong H^{*}\left(\Gamma\left(\mathbb{X}_{\mathrm{alg}}^{\prime}\right.\right.$, $\left.\left.\widehat{\Omega}_{\mathbf{X}^{\prime}, \mathrm{alg}}\right)\right)$.

Theorem 3.2. $H^{*}\left(\tilde{V}_{P}, \mathbb{C}\right) \cong H^{*}\left(\widehat{\Omega}_{X}(* D)_{P}\right), \quad$ and $\quad H^{*}\left(\mathscr{V}^{\prime}, \mathbb{C}\right) \cong H^{*}\left(\Gamma\left(\mathbb{V}_{\mathrm{alg}}^{\prime}\right.\right.$, $\left.\widehat{\Omega}_{X^{\prime}, \text { alg }}\right)$.

(Theorem 3.1 is given to the smooth varieties $\mathbb{X}, \mathbb{X}_{P}$, while Theorem 3.2 is a generalization of Theorem 3.1 to the varieties $V, \tilde{V}_{P}$, which have in general singularities.) Except the concrete style of the formulation, Theorem 3.1 is the well known theorems of A. Grothendieck in [5]. The second algebraic isomorphism in Theorem 3.2 is due to P. Deligne (cf. [7]). The first analytic isomorphism in Theorem 3.2 seems to have been not known. In the both theorems, the analytic isomorphisms are stronger than the algebraic ones. (The analytic isomorphisms, together with standard Gaga arguments, lead to the algebraic ones. The proof of the former is harder than the latter (cf. [5]).) We note that the arguments in [5], [7] use the resolution theorem of H. Hironaka. We also note that, in the proof of the first isomorphism in Theorem 3.1, [5] uses a comparison theorem of $\mathrm{H}$. Grauert-R. Remmert (on the behaviors of coherent sheaves under proper maps). As we will see soon later, our proof of Theorem 3.1 and Theorem 3.2 is largely different from the arguments in [5], [7]. Our proof is more close to that of the holomorphic de Rham theorem for Stein manifolds (cf. H. Cartan [1]):

(3.1) $H_{1} H^{*}(Y, \mathbb{C}) \cong H^{*}\left(\Gamma\left(Y, \Omega_{Y}\right)\right)$, where $Y$ is a Stein manifold and $\Omega_{Y}$ is the sheaf of holomorphic differential forms over $Y$.

As is well known, $(3.1)_{1}$ is a formal consequence of the following facts:

*) See the footnote *), p. 425 . 
$(3.1)_{2} H^{*}\left(Y, \Omega_{Y}\right) \cong 0(q \geqq 1), H^{0}\left(Y, \Omega_{Y}\right) \cong \Gamma\left(Y, \Omega_{Y}\right)$ (Theorems A, B of H. Cartan for $\Omega_{Y}$ ) .

(3.1) 3 Exactness of $\Omega_{Y}: 0 \longrightarrow C \longrightarrow \Omega_{Y}^{0} \cdots \longrightarrow \Omega_{Y}^{p} \stackrel{d}{\longrightarrow} \cdots \quad$ (Poincaré lemma).

Theorems A, B are a main result in the theory of Stein varieties, while $(3.1)_{3}$ is based on the analytic contractibility of the analytic manifolds. Our proof of Theorem 3.1 and Theorem 3.2 is patterned on the proof of $(3.1)_{3}$ indicated above. We also use a topological fact (Lemma 3.5), whose correspondence does not appear in the proof of $(3.1)_{1}$.

2. First letting $\Omega_{\mathbb{X}}, \Omega_{X^{\prime}}$ be the sheaves of holomorphic differential forms over $\mathbb{X}, \boldsymbol{X}^{\prime}$, we set: $\widehat{\Omega}_{\mathbf{X}}:=\lim _{\leftarrow m} \Omega_{\mathbf{X}} / \mathfrak{I}_{V}^{m} \Omega_{\mathbf{X}}$ and $\widehat{\Omega}_{\mathbf{X}^{\prime}}:=\lim _{\leftarrow m} \Omega_{\mathbf{X}^{\prime}} / \mathfrak{I}_{V^{\prime}}^{m} \Omega_{\mathbf{X}^{\prime}}$, where $\mathfrak{I}_{\boldsymbol{V}}$ and $\mathfrak{I}_{V^{\prime}}$ denote the ideals of $V, V^{\prime}$. Then, from our main results, Theorem 1.5, Theorem 1.6 and Theorem 2.5, Theorem 2.6, in Section 1, Section 2, we have:

Lemma 3.1. (1) $H^{q}\left(\mathbb{X}_{P}, \Omega_{\mathbf{X}}\right)_{\mathrm{p} . \mathrm{g}} \cong 0, H^{q}\left(\mathbb{X}^{\prime}, \Omega_{\mathbf{X}^{\prime}}\right)_{\mathrm{p} . \mathrm{g}} \cong 0$, and $H^{0}\left(\mathbb{X}_{P}, \Omega_{\mathbf{X}}\right)_{\mathrm{p} . \mathrm{g}}$ $\cong \Omega_{\mathbf{X}}(* D)_{P}, H^{0}\left(\boldsymbol{X}^{\prime}, \Omega_{\mathbf{X}^{\prime}}\right)_{\mathrm{p} . \mathrm{g}} \cong \Gamma\left(\mathbf{X}_{\mathrm{alg}}^{\prime}, \Omega_{\mathbf{X}^{\prime}, \mathrm{alg}}\right)$.

(2) $H^{q}\left(\mathbb{X}_{P}, \widehat{\Omega}_{\mathbf{X}}\right)_{\mathrm{p} . \mathrm{g}} \cong 0, H^{q}\left(\mathbb{X}^{\prime}, \widehat{\Omega}_{\mathbb{X}^{\prime}}\right)_{\mathrm{p} . \mathrm{g}} \cong 0(q \geqq 1)$, and $H^{0}\left(\mathbb{X}_{P}, \hat{\Omega}_{\mathbb{X}}\right)_{\mathrm{p} . \mathrm{g}}$ $=\widehat{\Omega}_{\mathbf{X}}(* D)_{P}, H^{0}\left(\boldsymbol{X}^{\prime}, \widehat{\Omega}_{\mathbf{X}^{\prime}}\right)_{\mathrm{p} \cdot \mathrm{g}} \cong \Gamma\left(\mathbb{X}_{\mathrm{alg}}^{\prime}, \hat{\Omega}_{\mathrm{X}^{\prime}, \mathrm{alg}}\right)$.

Lemma 3.1 corresponds to $(3.1)_{2}$ in our p.g. cohomology theory and in p.g. cohomology theory in the completions. As in the proof of $(3.1)_{1}$, Lemma 3.1 will play the most basic roles in our proof of Theorem 3.1 and Theorem 3.2. Also we note that Lemma 3.1 concerns the Stein and the algebraic properties of $\boldsymbol{X}, \mathbf{X}^{\prime}$, which may be the most important properties of these varieties (cf. also Introduction).

3. Next let $j_{\boldsymbol{X}^{\prime}}$ be the injection: $\mathbb{C} \hookrightarrow \Omega_{\boldsymbol{X}^{\prime}}^{0}$, and we define:

$$
C^{q}\left(\boldsymbol{X}^{\prime}, \boldsymbol{C}\right)_{\mathrm{p} . \mathrm{g}}:=\lim _{\sigma \rightarrow} C^{q}\left(\mathscr{A}_{\sigma}\left(\boldsymbol{X}^{\prime}\right), \mathbb{C}\right)_{\mathrm{p} . \mathrm{g}},
$$

where $C^{q}\left(\mathscr{A}_{\sigma}\left(\mathbb{X}^{\prime}\right), \mathbb{C}\right)_{\mathrm{p} . \mathrm{g}}:=j^{-1} C^{q}\left(\mathscr{A}_{\sigma}\left(\mathbb{X}^{\prime}\right), \Omega_{X^{\prime}}^{0}\right)_{\mathrm{p} . \mathrm{g}}$, and the p.g. covering $\mathscr{A}_{\sigma}\left(\mathbb{X}^{\prime}\right)$ is as in Theorem 1.3.

We define $C^{q}\left(\mathbb{X}_{P}, \mathbb{C}\right)_{\text {p.g }}$ similarly to the above. Then we have:

Proposition 3.1 (P.g. Poincaré lemma). The following complexes are exact:

$$
\left\{\begin{array}{l}
0 \longrightarrow C^{q}\left(\mathbb{X}_{P}, \mathbb{C}\right)_{\mathrm{p} . \mathrm{g}} \longrightarrow C^{q}\left(\mathbb{X}_{P}, \Omega_{\mathbb{X}}^{0}\right)_{\mathrm{p} . \mathrm{g}} \stackrel{d}{\longrightarrow} C^{q}\left(\mathbb{X}_{P}, \Omega_{X}^{p}\right)_{\mathrm{p} . \mathrm{g}} \longrightarrow \\
0 \longrightarrow C^{q}\left(\mathbb{X}^{\prime}, \mathbb{C}\right)_{\mathrm{p} . \mathrm{g}} \longrightarrow C^{q}\left(\mathbb{X}^{\prime}, \Omega_{X^{\prime}}^{0}\right)_{\mathrm{p} . \mathrm{g}} \stackrel{d}{\longrightarrow} C^{q}\left(\mathbb{X}^{\prime}, \Omega_{X^{\prime}}^{p}\right)_{\mathrm{p} . \mathrm{g}} \longrightarrow
\end{array}\right.
$$

where $d$ denotes the exterior differential operator. 
Proposition 3.1 will correspond to $(3.1)_{3}$ in our proof of Theorem 3.1. The proof of Proposition 3.1 is essentially very elementary (cf. the end of Section 5.2). Next letting the finite sets $f=\left(f_{j}\right)_{j=1}^{s} \subset \Gamma\left(X_{0}, D_{X_{0}}\right)$ and $f^{\prime}=\left(f_{j}^{\prime}\right)_{j=1}^{s}$ $\subset \Gamma\left(\boldsymbol{X}^{\prime}, \mathfrak{D}_{\mathbf{x}^{\prime}}\right)_{\text {p.g }}$ be as in Section 2, we set:

$$
\Omega_{\mathbf{X}, m}^{*}:=f^{m} \Omega_{\mathbf{X}^{+}}^{*} d f^{m+1} \wedge \Omega_{\mathbf{X}}^{*-1}, \Omega_{\mathbf{X}^{\prime} m}^{*}:=f^{\prime m} \Omega_{\mathbf{X}^{\prime}}^{*}+d f^{\prime m+1} \wedge \Omega_{\mathbf{X}^{\prime}}^{*-1},
$$

and

$$
\begin{aligned}
C^{q}\left(\mathscr{A}_{\sigma}\left(\boldsymbol{X}^{\prime}\right), \Omega_{\mathbf{X}^{\prime}, m}\right)_{\mathrm{p} . \mathrm{g}}:=F^{\prime m}\left(C^{q}\left(\mathscr{A}_{\sigma}\left(\boldsymbol{X}^{\prime}\right), \Omega_{\boldsymbol{X}^{\prime}}\right)^{s}\right)_{\mathrm{p} \cdot \mathrm{g}} \\
+d \rho^{\prime m+1} \wedge C^{q}\left(\mathscr{A}_{\sigma}\left(\boldsymbol{X}^{\prime}\right), \Omega_{\mathbf{X}^{\prime}}^{*-1}\right)_{\mathrm{p} \cdot \mathbf{g}} .
\end{aligned}
$$

Also letting the p.g. covering $\mathscr{A}_{\mu}(P)$, attached to $\boldsymbol{X}_{P}$, be as in Corollary 1.2, we define the p.g. complex $C^{q}\left(\mathscr{A}_{\mu}(P), \Omega_{\boldsymbol{X}, m}\right)_{\mathrm{p} . \mathrm{g}}$ similarly to the above.

Lemma 3.2 (P.g. open map property for de Rham complex). We have the inclusions:

$$
\begin{aligned}
& \left\{\begin{array}{l}
\left(C^{q}\left(\mathscr{A}_{\mu}(P), \Omega_{X, m}^{p}\right)_{\mathrm{p} . \mathrm{g}} \cap d^{-1}(0)\right) \subset d C^{q}\left(\mathscr{A}_{\mu^{\prime}}(P), \Omega_{X, m^{\prime}}^{p-1}\right)_{\mathrm{p} \cdot \mathbf{g}} \\
\left(C^{q}\left(\mathscr{A}_{\sigma}\left(\boldsymbol{X}^{\prime}\right), \Omega_{\boldsymbol{X}^{\prime}, m}^{p}\right)_{\mathrm{p} . \mathrm{g}} \cap d^{-1}(0)\right) \subset d C^{q}\left(\mathscr{A}_{\sigma^{\prime}}\left(\boldsymbol{X}^{\prime}\right), \Omega_{\mathbf{X}^{\prime}, \tilde{m}^{\prime}}^{p-1}\right)_{\mathrm{p} . \mathbf{g}}
\end{array}\right\} \\
& (p \geqq 1, q \geqq 0) \text {, }
\end{aligned}
$$

where the parameters $\mu^{\prime}, \sigma^{\prime}$ are chosen suitably in the manner as in Lemma 2.3 . Moreover, $m^{\prime}=\left[L_{\boldsymbol{X}}(m)\right]$ and $\tilde{m}^{\prime}=\left[L_{\boldsymbol{X}^{\prime}}(m)\right]$, with linear maps $L_{\boldsymbol{X}}(t)=c_{\boldsymbol{X}} t$ and $L_{\mathbf{X}^{\prime}}(t)=c_{\mathbf{X}^{\prime}} \cdot t ; c_{\mathbf{X}}, c_{\mathbf{X}^{\prime}}>0$.

For the proof of Lemma 3.2, see Lemma 4.7 (cf. part B, §4.1) and the end of Section 5.2. Our proof of Lemma 3.2 uses certain open map properties for Koszul complexes and a.d. properties of (topological) contractibility of analytic varieties (cf. §5.2). Lemma 3.2 is no longer, of obvious nature. Now applying Proposition $2.1_{1}$ to the open map property in Lemma 3.2, we have:

Lemma 3.3 (P.g. Poincaré lemma in the completion theory). The following complexes are exact:

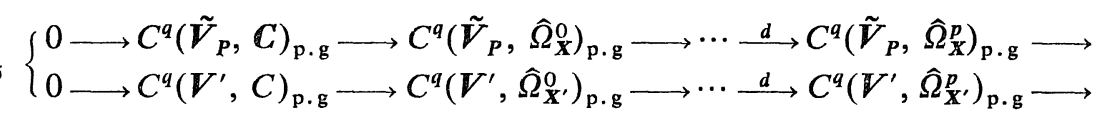

(In Lemma 3.3, the varieties in question are $\tilde{\boldsymbol{V}}, \boldsymbol{V}^{\prime}$ (instead of $\boldsymbol{X}, \boldsymbol{X}^{\prime}$ in Lemma 3.2 : by a simple observation of p.g. properties of the imbedded varieties $\tilde{\boldsymbol{V}}, \boldsymbol{V}^{\prime}$, we have: $C^{q}\left(\tilde{\boldsymbol{V}}_{\boldsymbol{P}}, \hat{\Omega}_{\mathbf{X}}^{*}\right)_{\mathrm{p} . \mathrm{g}} \cong C^{q}\left(\boldsymbol{X}_{P}, \widehat{\Omega}_{\mathbf{X}}^{*}\right)_{\mathrm{p} . \mathrm{g}}, \ldots$ (cf. [18])). Then, also from a simple observation, we easily check that $d^{-1}(0)$ at the first steps in $(3.2)_{2}$ coincide with $C^{q}\left(\boldsymbol{X}_{P}, \boldsymbol{C}\right)_{\text {p.g. }}, \ldots$ (cf. [18])).

Now, from Lemma 3.1-Lemma 3.3, we easily have: 
Lemma 3.4. We have the following isomorphisms:

$(3.2)_{6}$

$$
\left\{\begin{array}{l}
H^{*}\left(\boldsymbol{X}_{P}, \mathbb{C}\right)_{\mathrm{p} . \mathrm{g}} \cong H^{*}\left(\Omega_{\mathbf{X}}(* D)_{P}\right), \text { and } H^{*}\left(\mathbb{X}^{\prime}, \mathbb{C}\right)_{\mathrm{p} . \mathrm{g}} \cong H^{*}\left(\Gamma\left(\mathbb{X}_{\mathrm{alg}}^{\prime}, \Omega_{\boldsymbol{X}^{\prime}, \mathrm{alg}}\right)\right) \\
H^{*}\left(\tilde{\boldsymbol{V}}_{\mathbf{P}}, \mathbb{C}\right)_{\mathrm{p} . \mathrm{g}} \cong H^{*}\left(\hat{\Omega}_{\mathbf{X}}(* D)_{P}\right) \text {, and } H^{*}\left(\boldsymbol{V}^{\prime}, \mathbb{C}\right)_{\mathrm{p} . \mathrm{g}} \cong H^{*}\left(\Gamma\left(\mathbb{V}_{\mathrm{alg}}^{\prime}, \hat{\Omega}_{\boldsymbol{X}^{\prime}, \mathrm{alg}}\right)\right)
\end{array}\right\} .
$$

These isomorphisms summarize our applications of the p.g. cohomology theories as in Lemma 3.1-Lemma 3.3. In order to get Theorem 3.1 and Theorem 3.2, we should drop the term 'p.g' from the cohomology groups $H^{*}\left(\boldsymbol{X}_{\boldsymbol{P}}, \boldsymbol{C}\right)_{\mathrm{p} . \mathrm{g}}, \ldots$ in the left sides in $(3.2)_{6}$. In this step we will use our main results on p.g. $C^{\infty}$-de Rham theory for certain stratified spaces in $[15]_{2-4},[17]$.

4. Let $\boldsymbol{C}^{n}$ be the ambient (euclid) space of the local variety*) $\mathbb{X}$ and the affine variety $\boldsymbol{X}^{\prime}$. We identify $\mathbb{C}^{n}$ with the real euclid space $\mathbb{R}^{2 n}$ in a natural manner, and we fix coordinates $x=\left(x_{j}\right)_{j=1}^{2 n}$ of $\mathbb{R}^{2 n}$. The symbol $\varepsilon$ will denote the sheaf of $C^{\infty}$-(differentiable) differential forms over $\mathbb{R}^{2 n}$. Taking an open set $Y$ of $\boldsymbol{R}^{2 n}$ and a p.g. function $g: Y \rightarrow \mathbb{R}_{1}^{+}$, we set:

$(3.2)_{0} \quad \varepsilon(Y ; g)_{\mathrm{p} . \mathrm{g}}:=\left\{\varphi \in \varepsilon(Y) ; \varphi=\sum_{K} \varphi_{K} d x^{K}\right.$ satisfies the following for each suffix $K$ and each element $J \in\left(\mathbb{Z}^{+} \cup 0\right)^{2 n}$ :

(3.2) $)_{0}^{\prime}\left|D_{J} \varphi_{K}(P)\right|<\alpha_{J} g(P)$ in $Y$, with a suitable $\alpha_{J} \in \mathbb{R}_{1}^{+2}$, where $D_{J}=\partial^{J} / \partial x^{J}$. $\}$

Next taking subsets $\boldsymbol{Z}, \mathbb{Z}^{\prime}$ of $\boldsymbol{X}, \boldsymbol{X}^{\prime}$, we define:

$$
\left\{\begin{array}{c}
\mathscr{B}_{\sigma}(\mathbb{Z}) \\
\mathscr{B}_{\sigma}\left(\mathbb{Z}^{\prime}\right)
\end{array}\right\}:=g \text {-p.g. covering of }\left\{\begin{array}{l}
\mathbb{Z} \\
\mathbb{Z}^{\prime}
\end{array}\right\} \text { of size } \sigma \text { in } C^{n}\left(\cong \mathbb{R}^{2 n}\right)
$$

where $g$ is the p.g. function $\left|h_{\boldsymbol{X}}^{-1}\right|$ or $|z|+1$ of $\boldsymbol{X}$ or $\boldsymbol{X}^{\prime}$ (cf. Theorems 1.1-1.4). We use the symbols $N_{\sigma}(\mathbb{Z}), N_{\sigma}\left(\mathbb{Z}^{\prime}\right)$ for $\operatorname{supp} \mathscr{B}_{\sigma}(\mathbb{Z})$ and $\operatorname{supp} \mathscr{B}_{\sigma}\left(\mathbb{Z}^{\prime}\right)$. We may call $N_{\sigma}(\mathbb{Z}), N_{\sigma}\left(\mathbb{Z}^{\prime}\right)$ the p.g. neighborhoods of $\mathbb{Z}, \mathbb{Z}^{\prime}$ in $\mathbb{C}^{n}$ of size $\sigma$. Such p.g. neighborhoods are suitable for investigations of the p.g. properties of imbedded varieties (cf. [19]). See also Proposition 4.6, Section 4.2 of the present paper, where we discuss p.g. properties in connection with extensions of cochains from imbedded varieties to their ambient spaces.) Now we set:

$$
\left\{\begin{array}{l}
\boldsymbol{\varepsilon}\left(\boldsymbol{X}_{P}\right)_{\mathrm{p} . \mathrm{g}}:=\lim _{\sigma, r \rightarrow} \boldsymbol{\varepsilon}\left(N_{\sigma}\left(\boldsymbol{X}_{r}(P)\right), g\right)_{\mathrm{p} . \mathrm{g}}, \boldsymbol{\varepsilon}\left(\tilde{V}_{P}\right)_{\mathrm{p} . \mathrm{g}}:=\lim _{\sigma, r \rightarrow} \boldsymbol{\varepsilon}\left(N_{\sigma}\left(\tilde{V}_{\boldsymbol{r}}(P)\right), g\right)_{\mathrm{p} . \mathrm{g}} \\
\boldsymbol{\varepsilon}\left(\boldsymbol{X}^{\prime}\right)_{\mathrm{p} \cdot \mathrm{g}}:=\lim _{\sigma \rightarrow} \boldsymbol{\varepsilon}\left(N_{\sigma}\left(\boldsymbol{X}^{\prime}\right), g\right)_{\mathrm{p} . \mathrm{g}}, \boldsymbol{\varepsilon}\left(\mathbb{V}^{\prime}\right)_{\mathrm{p} . \mathrm{g}}:=\lim _{\sigma \rightarrow} \boldsymbol{\varepsilon}\left(N_{\sigma}\left(\mathbb{V}^{\prime}\right), g\right)_{\mathrm{p} . \mathrm{g}},
\end{array}\right\},
$$

*) Recall that $\boldsymbol{X}$ is of the from $\boldsymbol{X}=X_{0}-D$, with a variety $X_{0}$ in $U_{0}$. We are assuming that $U_{0} \subset \mathbb{C}^{n}$. 
where $g=\left|h_{\mathbf{X}}^{-1}\right|$ or $|z|+1$. Also the manifold $\mathbb{X}_{r}(P):=\mathbb{X} \cap U_{r}(P)$, where $U_{r}(P)$ : $=\operatorname{disc}$ in $\mathbb{C}^{n}$ of center $P$ and radius $r$, is as in Theorem 1.1. Moreover, we set $\tilde{V}_{r}(P):=\tilde{V} \cap U_{r}(P)$. Then our main result in [17] insures*):

Lemma 3.5. We have the following isomorphisms:

$$
\left\{\begin{array}{l}
H^{*}\left(\mathbb{X}_{P}, \mathbb{C}\right) \cong H^{*}\left(\varepsilon\left(\mathbb{X}_{P}\right)_{\mathrm{p} . \mathrm{g}}\right), H^{*}\left(\tilde{V}_{P}, \mathbb{C}\right) \cong H^{*}\left(\varepsilon\left(\tilde{V}_{P}\right)_{\mathrm{p} . \mathrm{g}}\right) \\
H^{*}\left(\mathbb{X}^{\prime}, \mathbb{C}\right) \cong H^{*}\left(\boldsymbol{\varepsilon}\left(\mathbb{X}^{\prime}\right)_{\mathrm{p} . \mathrm{g}}\right), H^{*}\left(\mathbb{V}^{\prime}, \mathbb{C}\right) \cong H^{*}\left(\boldsymbol{\varepsilon}\left(\mathscr{V}^{\prime}\right)_{\mathrm{p} . \mathrm{g}}\right)
\end{array}\right\} .
$$

Note that the right sides in $(3.2)_{1}$ may be regarded as $C^{\infty}$-analogues of the analytic de Rham cohomology groups as in (3.2) 6 , Lemma 3.3. Also note that the left sides in $(3.2)_{1}$ are the topological cohomology groups $H^{*}\left(\mathbb{X}_{P}, \mathbb{C}\right), \ldots$, while the left sides in $(3.1)_{6}, H^{*}\left(\mathbb{X}_{P}, \mathbb{C}\right)_{\text {p.g }}, \ldots$ contain the suffix 'p.g.' (This difference occurs from the following situation: first, in the definition of $H^{*}\left(\mathbb{X}_{P}, \mathbb{C}\right)_{\text {p.g }}$, we used the p.g. coverings $N_{\sigma}\left(\mathbb{X}_{r}(P)\right)$, which are attached to $\mathbb{X}_{r}(P)$ (cf. Definition $1.6_{2}$ ), and our use of such p.g. coverings is a main source for the suffix 'p.g.' mentioned just above. On the other hand, our proof of Lemma 3.5 is based on a type of stratified spaces attached to real analytic varieties, which we call normalized series of stratified spaces (cf. [17]). Such stratified spaces admit what we call p.g. simple coverings, where the word 'simple' is used in the similar sense to the 'simple covering' in the $C^{\text {r }}$-de Rham theorem in [21]. The simpleness as above insures that the above coverings satisfy the standard Larey condition for the constant sheaf $\mathbb{Z}$ (and so for $\mathbb{R}$ and $\mathbb{C}$ ), and they are used to determine the topological cohomology groups $H^{*}\left(\mathbb{X}_{P}, \mathbb{C}\right), \ldots$ Such coverings are also suitable for treatments of the p.g. properties of $C^{\infty}$-differential forms over analytic varieties. Using the above stratified spaces and the p.g. simple coverings of them, the proof of Lemma 3.5 is formal (cf. [17] and [18]. See also Remark 3.1 at the end of Section 3.)

5. Finally we will see that Theorem 3.1 and Theorem 3.2 are derived from Lemma 3.3 and Lemma 3.5 in a formal fashion. For this we first let $\boldsymbol{\varepsilon}_{\mathbf{X}}, \boldsymbol{\varepsilon}_{\boldsymbol{X}^{\prime}}$ denote the sheaves of $C^{\infty}$-differential forms over $\mathbb{X}, \mathbb{X}^{\prime}$. Letting the subsets $\mathbb{Z}, \mathbb{Z}^{\prime}$ of $\mathbb{X}, \boldsymbol{X}^{\prime}$ be as in (3.2) , we define 'p.g. complexes of $C^{\infty}$-differential forms': $(3.2)_{2}^{\prime} \quad \boldsymbol{\varepsilon}_{\boldsymbol{X}}(\mathbb{Z})_{\text {p.g }}$ and $\boldsymbol{\varepsilon}_{\boldsymbol{X}^{\prime}}\left(\mathbb{Z}^{\prime}\right)_{\text {p.g }}$ (in the similar manner to $(3.2)_{0}$, by using the coordinates of $\mathbb{X}, \mathbb{X}^{\prime}$ instead of those of $\mathbb{C}^{n}$ as in $\left.(3.2)_{0}\right)$.

*) Lemma 3.5 is given in [17] for local analytic varieties, and is applied to the variety $X_{P}$. On the other hand, Remarking that, the affine variety $\mathbb{X}^{\prime}$ is compactified (in $\mathbb{P}^{n}(\boldsymbol{C}) \supset \boldsymbol{C}^{n}$ ) and, applying the local results just above to each point of the completion of $\mathbf{X}^{\prime}$, we get Lemma 3.5 for $\boldsymbol{X}^{\prime}$. 
Moreover, we use the symbols $\mathscr{B}_{\sigma}^{\prime}(\mathbb{Z}), \mathscr{B}_{\sigma}^{\prime}\left(\mathbb{Z}^{\prime}\right)$ for the p.g. coverings of $\mathbb{Z}, \mathbb{Z}^{\prime}$ in $\mathbb{X}, \mathbb{X}^{\prime}$ of size $\sigma$ (cf. also (3.2) $)_{0}^{\prime \prime}$ ). We also use the symbols $N_{\sigma}^{\prime}(\mathbb{Z}), N_{\sigma}^{\prime}\left(\mathbb{Z}^{\prime}\right)$ for supp $\mathscr{B}_{\sigma}^{\prime}(\mathbb{Z})$, supp $\mathscr{B}_{\sigma}^{\prime}\left(\mathbb{Z}^{\prime}\right)$. (Thus $N_{\sigma}^{\prime}(\mathbb{Z}), N_{\sigma}^{\prime}\left(\mathbb{Z}^{\prime}\right)$ are the p.g. neighborhoods of $\mathbb{Z}, \mathbb{Z}^{\prime}$ in $\mathbb{X}, \mathbb{X}^{\prime}$.) Then, corresponding to (3.2) $)_{1}^{\prime}$, we define:

$$
\left\{\begin{array}{l}
\varepsilon_{X}\left(\mathbb{X}_{P}\right)_{\mathrm{p} \cdot \mathrm{g}}:=\lim _{\sigma, r \rightarrow} \varepsilon_{\mathbb{X}}\left(N_{\sigma}^{\prime}\left(\mathbb{X}_{r}(P)\right)\right)_{\mathrm{p} \cdot \mathrm{g}}, \boldsymbol{\varepsilon}_{\mathbf{X}}\left(\tilde{V}_{P}\right)_{\mathrm{p} . \mathrm{g}}:=\lim _{\sigma, r \rightarrow} \varepsilon_{\mathbb{X}}\left(N_{\sigma}^{\prime}\left(\tilde{V}_{r}\right)\right)_{\mathrm{p} \cdot \mathrm{g}}, \\
\varepsilon_{X^{\prime}}\left(\mathbb{X}^{\prime}\right)_{\mathrm{r} \cdot \mathrm{g}}:=\lim _{\sigma \rightarrow} \varepsilon_{X^{\prime}}\left(N_{\sigma}^{\prime}\left(\mathbb{X}^{\prime}\right)\right)_{\mathrm{p} . \mathrm{g}}, \varepsilon_{\mathbb{X}^{\prime}}\left(\mathbb{V}^{\prime}\right)_{\mathrm{p} \cdot \mathrm{g}}:=\lim _{\sigma \rightarrow} \varepsilon_{\mathbb{X}^{\prime}}\left(N_{\sigma}^{\prime}\left(\mathbb{V}^{\prime}\right)\right)_{\mathrm{p} . \mathrm{g}} .
\end{array}\right.
$$

Then it is not difficult*) to check: $H^{*}\left(\varepsilon_{\mathrm{X}}\left(\mathbb{X}_{P}\right)_{\mathrm{p} . \mathrm{g}}\right) \cong H^{*}\left(\varepsilon\left(\mathbb{X}_{P}\right)_{\mathrm{p} . \mathrm{g}}\right), \ldots$, and Lemma 3.5 is rewritten in the following manner:

$$
\left\{\begin{array}{l}
H^{*}\left(\mathbb{X}_{P}, \mathbb{C}\right) \cong H^{*}\left(\varepsilon_{X}\left(\mathbb{X}_{P}\right)_{\mathrm{p} . \mathrm{g}}\right), H^{*}\left(\tilde{V}_{P}, \mathbb{C}\right) \cong H^{*}\left(\varepsilon_{\mathbb{X}}\left(\tilde{V}_{P}\right)_{\mathrm{p} . \mathrm{g}}\right) \\
H^{*}\left(\mathbb{X}^{\prime}, \mathbb{C}\right) \cong H^{*}\left(\varepsilon_{\boldsymbol{X}^{\prime}}\left(\mathbb{X}^{\prime}\right)_{\mathrm{p} . \mathrm{g}}\right), H^{*}\left(\mathbb{V}^{\prime}, \mathbb{C}\right) \cong H^{*}\left(\varepsilon_{\mathbb{X}^{\prime}}\left(\mathbb{V}^{\prime}\right)_{\mathrm{p} . \mathrm{g}}\right)
\end{array}\right\}
$$

Now we denote by $\tau, \hat{\tau}$ the natural homomorphisms from the analytic de Rham groups to the p.g. $C^{\infty}$-de Rham groups:

$$
\tau: \Omega_{X}(* D)_{P} \longrightarrow \varepsilon_{X}(* D)_{\mathrm{p} . \mathrm{g}}, \hat{\tau}: \hat{\Omega}_{\mathbf{X}}(* D)_{P} \longrightarrow \varepsilon_{X}\left(\tilde{V}_{\mathrm{p}}\right)_{\mathrm{p} . \mathrm{g}} .
$$

Moreover, from a simple observation, we have natural homomorphisms**):

$$
\begin{aligned}
\mu: H^{*}\left(\varepsilon_{\mathbf{X}}\left(\mathbb{X}_{P}\right)_{\mathrm{p} . \mathrm{g}}\right) \longrightarrow H^{*}\left(\mathbb{X}_{P}, \mathbb{C}\right)_{\mathrm{p} . \mathrm{g}}, \hat{\mu}: H^{*}\left(\varepsilon_{X^{\prime}}\left(\tilde{V}_{P}\right)_{\mathrm{p} . \mathrm{g}}\right) \\
\longrightarrow H^{*}\left(\tilde{V}_{P}, \mathbb{C}\right)_{\mathrm{p} . \mathrm{g}} .
\end{aligned}
$$

Then we easily have the following diagrams

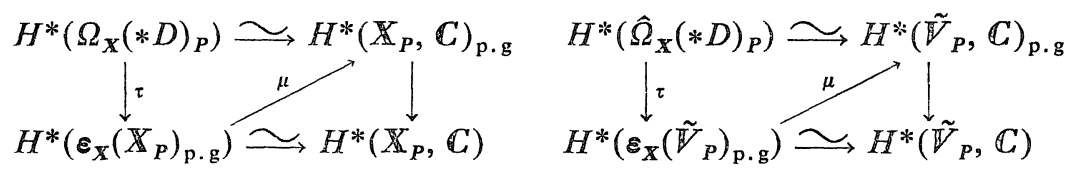

Figure I.

(and the similar diagrams for $\mathbb{X}^{\prime}$ and $\mathbb{V}^{\prime}$.) It is clear that the above diagram insures the implication:

$$
\text { Lemma 3.3+Lemma } 3.5 \longrightarrow \text { Th.3.1 and Th.3.2. }
$$

Remark 3.1. As may be clear from the content of Section 3, the most

*) From that $\mathbb{X}, \mathbb{X}^{\prime}$ are smooth, this isomorphism is rather easily checked (cf. [18]. See also Proposition 4.6 for treatments of the p.g. neighborfoods.)

**) Such homomorphisms are constructed, by using similar arguments to the one in [21], which attaches, to the closed differential forms, their cohomology classes. See also the arguments in Section 5.1 and n.5, n.6 in Section 5.2, where we give some cohomological arguments. (Such arguments have similar algebraic structures with the arguments in [211.) 
important topological fact in getting Theorem 3.1 and Theorem 3.2 is the p.g. $C^{\infty}$-de Rham isomorphism as in Lemma 3.5. Also we use some a.d. properties of (topological) contractibility of analytic varieties in getting the open map property of the de Rham complex as in Lemma 3.4 (cf. $[15]_{2}$. Also see the end of Section 5.2.) The underlying basic fact for the above topological facts is the existence of stratified spaces for analytic varieties (=normalized series of stratified spaces), which were mentioned previously. The details of the above topological facts are in $[15]_{2-4}$ and the author's forthcoming paper [18]. (The first three are outlines of what are mentioned soon above, while [18] will contain the details.)

As may be from the context of Section 3, the above topological facts are indispensable in getting Theorem 3.1 and Theorem 3.2. However, as in the case of the holomorphic de Rham theorem (3.1) $)_{1}$, and in the proof of Theorem 3.1 in [5], the most important facts in getting the analytic de Rham theorem (as in Theorem 3.1, Theorem 3.2) are the results on the coherent sheaves in Lemma 3.1.

Remark 3.2. At present, our results on the analytic de Rham theory are given separatedly, according as we are concerned with the analytic or topological aspects. The present paper covers the necessary analytic facts for the proof of Theorem 3.1, Theorem 3.2, while the necessary topological facts are summarized in $[15]_{2-4},[17]$ and in [18]. The author plans to write a survey paper on the analytic de Rham theory, which will include (1) even treatments of the analytic and topological parts as above and (2) comparisons of our methods indicated as in Section 3 and the methods taken in [5], [7].

\section{Chapter II. Uniform Estimations on Homomorphisms of Coherent Sheaves}

In Section 4.1 we summarize our non cohomological uniform estimations on homomorphisms of coherent sheaves (Lemma 4.1-Lemma 4.7). Using cohomological versions of them, we prove all the lemmas in Chapter I, Lemma 1.2-Lemma 1.4, Lemmas 2.3, 2.7 and Lemma 2.5, which concern the p.g. and a.d. uniform estimations in Chapter I (cf. §4.2. Also see Introduction). Our cohomological version of Hilbert zero point theorem*) and our non cohomological version of the main lemma, Lemma 2.5 , in the d.p. cohomology

\footnotetext{
*) Cf. Introduction.
} 
theory in Section 2 are given in n.2-n.4, Part B, Section 4.1 (cf. Lemma 4.2Lemma 4.4. Also see Introduction). The proof of the estimations in Section 4.1 is given in Section 5. In proving the open map properties*) in Section 4.1, we see that these are checked easily for smooth points of the varieties in question. We then show that those properties for the smooth points lead to the properties for 'all points' of the varieties (\$5.2). Such a method may be interesting in connection with treatments of the a.d. properties of varieties with singularities. Finally, the procedure in Section 4.2, which rewrites the non cohomological estimations in Section 4.1 in cohomological forms, is essentially algebraic; large parts of Section 4.2 is given in an abstract fashion in terms of the q-sheaves (Definition $1.4_{1}$ ). The content of Section 4.2 may be useful for general treatments of the p.g. and a.d. properties of q-sheaves.

\section{§4. Uniform Estimations with Bound and Algebraic Divisions}

\section{§4.1. Non Cohomological Estimations}

In Parts A, B we give non cohomological estimations of local forms, which concern the first and second properties in the title. In $C$ we give a global version of the results in $\mathrm{A}, \mathrm{B}$.

\section{A. Uniform Estimations with Bound}

1. Geometric underlying data. In a similar manner to Section 1.2 , we start with giving the following geometric datum:

$(4.1)_{0} \quad X:=\left(\mathbb{C}^{n}(z), U_{0}, X_{0}, X_{0}^{\prime}, P_{0}\right)$ consisting of an analytic variety $X_{0}\left(\ni P_{0}\right)$ in an open set $U_{0}$ of a euclidean space $\mathbb{C}^{n}(z)$ and a subvariety $X_{0}^{\prime}$ of $X_{0}$.

The variety $X_{0}^{\prime}$ may be empty, but should satisfy:

(4.1) $\quad X:=X_{0}-X_{0}^{\prime}$ is smooth, and $X_{0}^{\prime}$ contains $P_{0}$, if $X_{0}^{\prime} \neq \phi$.

(When $X_{0}^{\prime}$ is the divisor of an element $h \in \Gamma\left(X_{0}, \mathfrak{D}_{X_{0}}\right)$, the datum $\mathbf{X}$ is of the form which was used in Section 1.2: $\boldsymbol{X} \in \mathbb{A n}_{1 a}$ (cf. (1.8) $)_{0}$ ). Note that, in this case, $X=X_{0}-X_{\mathrm{C}}^{\prime}$ is a Stein variety. In Chapter II we do not require this condition. The datum $\mathbf{X}$ is more general than geometric data in $\mathbf{A} \mathbf{m}_{1 a}$ in Section 1.2.) We fix the geometric datum $\mathbb{X}$ in the remainder of Chapter II. The underlying

*) Sce the footnote *), p. 432. 
variety of $\boldsymbol{X}$ will be $X=X_{0}-X_{0}^{\prime}$. Moreover, for convenience of the formulations of the estimations in Section 4 , we fix subvarieties $X_{1}, X_{2}$ of $X_{0}$ satisfying

$$
X_{0} \supset X_{1} \supsetneqq X_{2} \supset X_{0}^{\prime} \text {. }
$$

2. Parametrizations. Next we will define certain sets of cross sections to coherent sheaves, which are parametrized in an explicit manner (cf. $(4.1)_{2}$ soon below). The parametrization here is of non cohomological form and is simpler than the one in Section 1, Section 2. However, the formulation of the former has some similarities to the one in the latter: first setting $\tilde{X}_{1}:=X_{1}-X_{2}$ we define a parametrization of open manifolds in $X\left(=X_{0}-X_{0}^{\prime}\right)$ :

$$
\begin{aligned}
u_{\tilde{X}_{1}}: \mu_{\tilde{X}_{1}}:=\tilde{X}_{1} \times \mathbb{R}^{+} \ni \mu=(P ; r) & \\
& \longrightarrow \operatorname{Ouv}(X) \ni \tilde{U}_{r}(P):=\{Q \in X ; d(P, Q)<r\},
\end{aligned}
$$

where $d$ is the natural distance in $\boldsymbol{C}^{n}(z)$ (cf. n.1, § 1.2).

Next taking a matrix $K: \mathfrak{D}_{X}^{v} \rightarrow D_{X}^{u}(u, v>0)$, whose entries are in $\Gamma\left(X_{0}, \mathfrak{D}_{X_{0}}\right)$, we write the image $K D_{X}^{v}\left(\subset D_{X}^{u}\right.$ ) as $\Omega$. (Here $\mathfrak{D}_{X}, D_{X_{0}}$ are the structure sheaves of $X, X_{0}$.) We use the symbols $\theta_{\Re}, \theta_{\Omega}^{\prime}$ for the q-structures of $\Omega$, which are induced from $K: D_{X}^{v} \rightarrow \Omega$ and the injection: $\Re \hookrightarrow D_{X}^{u}$ (Definition $1.4_{2}$ ). Setting $\lambda_{\tilde{X}_{1}}:=\boldsymbol{\mu}_{\tilde{X}_{1}} \times \boldsymbol{R}_{1}^{+}$, we take an element $(P ; r ; a) \in \lambda_{\tilde{X}_{1}}\left(\subset \tilde{X}_{1} \times \mathbb{R}^{+} \times \mathbb{R}_{1}^{+}\right)$. Then the sets of the cross sections, which are used in Section 4 , Section 5 , will be of the form:

$$
\left\{\begin{array}{l}
\Gamma\left(\tilde{U}_{r}(P), \Re ; \theta_{\Re}\right)_{a} \\
\Gamma\left(\tilde{U}_{r}(P), \Re ; \theta_{\Re}^{\prime}\right)_{a}
\end{array}\right\}:=\left\{\varphi \in \Gamma\left(\tilde{U}_{r}(P), \Re\right) ;\left\{\begin{array}{l}
|\varphi(Q)|_{\Re}<a \\
|\varphi(Q)|_{\Re}^{\prime}<a
\end{array}\right\} \text { in } \tilde{U}_{r}(P)\right\},
$$

where ||$_{\Re},||_{\Re}^{\prime}$ are the $\theta_{\Re}$ - and $\theta_{\Re}^{\prime}$-absolute values (Definition $1.4_{1}$ ).

Note that, by the definition of $\theta_{\Re}, \ldots$, the above sets are explicitly as follows:

$$
\begin{aligned}
& \Gamma\left(\tilde{U}_{r}(P), \Re ; \theta_{\Re}\right)_{a}=K \Gamma\left(\tilde{U}_{r}(P), \mathfrak{D}_{X}^{v} ; \theta\right)_{a}, \\
& \Gamma\left(\tilde{U}_{r}(P), \mathfrak{\Re} ; \theta_{\Re}^{\prime}\right)_{a}=\Gamma\left(\tilde{U}_{r}(P), D_{X}^{u} ; \theta^{\prime}\right)_{a} \cap \Gamma\left(\tilde{U}_{r}(P), \Re\right),
\end{aligned}
$$

where $\theta, \theta^{\prime}$ are the standard q-structures of $\mathfrak{D}_{X}^{v}, \mathfrak{D}_{X}^{\prime \prime}$ (Definition $1.4_{3}$ ).

3. Estimation maps. Letting $M$ denote the collection of all positive monomials $(\mathrm{n} .5, \S 1.1)$, we set $\tilde{\mathbf{M}}:=\mathbf{M} \times \mathbf{M}$, and we regard an element $\tilde{M}=\left(M_{1}, M_{2}\right) \in \tilde{M}=\tilde{M} \times \tilde{M}$ as a map (cf. also Figure I):

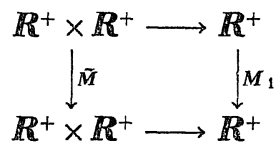

Figure I. $(4.1)_{3} \quad \tilde{M}: \boldsymbol{R}^{+} \times \boldsymbol{R}^{+} \ni(r ; a) \longrightarrow \mathbb{R}^{+} \times \boldsymbol{R}^{+} \ni\left(M_{1}(r), M_{2}(a / r)\right)$.

We use such a map in the remainder of Section 4.1. 
4. Bdd*) uniform estimation - 1 . Letting the matrix $K$ be as in n.2, we take an open subsel $U_{1}=U_{1, K}\left(\ni P_{0}\right)$ of $U_{0}$ and an element $\sigma=\sigma_{K} \in \mathbb{R}_{1}^{+2}$. We then form the following parameter spaces:

$$
\mu_{K}:=\left\{(P ; r) \in\left(U_{1} \cap \tilde{X}_{1}\right) \times \mathbb{R}^{+} ; r<\{\sigma g(P)\}^{-1}\right\},
$$

with $g(P)=d\left(P, X_{2}\right)^{-1 * *)}$, and $\lambda_{K}:=\mu_{K} \times \mathbb{R}_{1}^{+}$.

Lemma A.1' (Uniform estimation with bound-1). Take a suitable $\tilde{M}_{K} \in \tilde{M}$. Then we have:

$$
i^{*} \Gamma\left(\tilde{U}_{r}(P), \Re ; \theta_{\Re}^{\prime}\right)_{a} \subset \Gamma\left(\widetilde{U}_{r^{\prime}}(P), \Re ; \theta_{\Re}\right)_{a^{\prime}},
$$

with $\left(r^{\prime} ; a^{\prime}\right)=\tilde{M}_{K}(r ; a)$, where the parameter $(P ; r ; a)$ is in $\lambda_{K}\left(\subset \tilde{X}_{1} \times \mathbb{R}^{+} \times \mathbb{R}_{1}^{+}\right)$ and $i=$ inclusion: $\tilde{U}_{r^{\prime}}(P) \hookrightarrow \tilde{U}_{r}(P)$.

We prove Lemma $4.1^{\prime}$ in Section 5.1.

5. Bdd uniform estimation -2. Here we give an another uniform estimation, which is derived from Lemma 4.1' (cf. §5.1) and is sharper than Lemma $4.1^{\prime}$ in some aspects (cf. Remark 4.1): first take a set $h=\left\{h_{u}\right\}_{u=1}^{u_{0}} \subset \Gamma\left(X_{0}, D_{X_{0}}\right)$ satisfying $\cap_{u} D_{u}=X_{0}^{\prime}$, where $D_{u}=$ divisor of $h_{u}$ on $X_{0}$, and we set:

$(4.1)_{6} \operatorname{Coh}\left(X_{0} ; \not\right):=$ collection of all coherent sheaves $\mathfrak{5}$ over $X$, which admits a resolution of the form:

$$
0 \longrightarrow \mathfrak{D}_{X}^{k_{p}} \stackrel{K_{p-1}}{\longrightarrow} \cdots \stackrel{K_{1}}{\longrightarrow} \mathfrak{D}_{X}^{k_{1}} \stackrel{K_{0}}{\longrightarrow} \mathfrak{H}\left(\subset \mathfrak{D}_{X}^{k}\right) \longrightarrow 0,
$$

where $K_{j}(0 \leqq j \leqq p)$ are matrices, with entries in $\Gamma\left(X, \mathfrak{O}_{X}\right)$ and satisfy:

$(4.1)_{6}^{\prime \prime} \quad$ the entries of $K_{j}$ are in $\Gamma\left(X_{0}, \mathfrak{D}_{X_{0}}\left(* D_{u}\right)\right)$ (for each $\left.j, u\right)$.

Here $\mathfrak{D}_{X_{0}}\left(* D_{u}\right)$ denotes the sheaf over $X_{0}$ of meromorphic functions with pole $D_{u}$.

Now taking an open subset $U_{1, h}\left(\ni P_{0}\right)$ of $U_{0}$ and an element $\sigma_{h} \in \mathbb{R}_{1}^{+2}$, we form parameter spaces $\boldsymbol{\mu}_{h}\left(\subset X_{1} \times \mathbb{R}^{+}\right)$and $\lambda_{h}:=\boldsymbol{\mu}_{h} \times \mathbb{R}_{1}^{+}$in the similar manner to $(4.1)_{4}$ (by using $\left(U_{1, h}, \sigma_{h}\right)$ ). Then we have****)

Lemma 4.1 (Uniform estimation with bound -

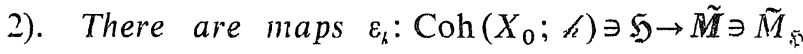
and $\varepsilon_{h}^{\prime}: \mathbb{Z}^{+} \rightarrow$ which are factored as in Figure II, and with which we have the following for each $\mathfrak{S} \in$

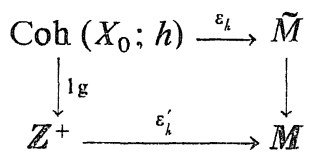

Figure II. ${ }^{* * * *}$

*) 'Bdd.' = bounded.

**) When $X_{2}=\phi$, we understand that $d\left(P, X_{2}\right)=1$.

$* * *)$ ' $l g$ ' in Figure II is the length map (cf. $(1.4)_{3}$ ). 
$\operatorname{Coh}\left(X_{0} ; h\right)$ :

$$
i^{*} \Gamma\left(\tilde{U}_{\boldsymbol{r}}(P), \mathfrak{H} ; \theta_{\mathfrak{g}}^{\prime}\right)_{a} \subset \Gamma\left(\tilde{U}_{r^{\prime}}(P), \mathfrak{H}, \theta_{\mathfrak{g}}\right)_{a^{\prime}},
$$

with $\left(r^{\prime} ; a^{\prime}\right)=\tilde{\boldsymbol{M}}_{\mathfrak{5}}(r ; a)$, where the parameter $(P ; r ; a)$ is in $\lambda_{h}\left(\subset X_{1} \times \boldsymbol{R}^{+}\right.$ $\left.\times \boldsymbol{R}_{1}^{+}\right)$.

We derive Lemma 4.1 from Lemma 4.1' (cf. § 5.1).

Here we give a remark on the formulation in Lemma 4.1' and Lemma 4.1, which we use in getting Lemma 4.1 from Lemma $4.1^{\prime}$ (cf. §5.1).

Remark $4.1_{1}$. (1) Take an element $h \in \Gamma\left(X_{0}, \mathfrak{D}_{X_{0}}\right)$, and let $\mathfrak{D}_{X_{0}}(* D)$ denote the sheaf over $X_{0}$ of meromorphic functions with pole $D$ (=divisor of $h$ ). Then, replacing the condition: 'the entries of the matrix $K$ are in $\Gamma\left(X_{0}, \mathfrak{D}_{X_{0}}\right)$ ' in Lemma 4.1 ' by 'those are in $\Gamma\left(X_{0}, D_{X_{0}}(* D)\right)$ ', we get also the similar inclusion to $(4.1)_{5}$ (in Lemma $4.1^{\prime}$ ) for $K$ (after the above change). Actually remark that $\tilde{K}=h^{d} K$ are in $\Gamma\left(X_{0}, \mathfrak{D}_{X_{0}}\right)$, with a suitable $d \in \boldsymbol{Z}^{+}$, and we apply Lemma $4.1^{\prime}$ to $\tilde{K}$. Then, recalling the explicit form of the estimation: $(r ; a) \rightarrow\left(r^{\prime} ; a^{\prime}\right)$ as in Lemma 4.1', we get easily the inclusion mentioned soon above for $K$ from the application of Lemma $4.1^{\prime}$ to $\tilde{K}$.

(2) Lemma 4.1 is sharper than Lemma $4.1^{\prime}$ in the point that (a) the parameter space $\mu_{h}$ is independent from the individual sheaf $\mathfrak{H} \in \operatorname{Coh}\left(X_{0} ; h\right)$ and (b) Lemma 4.1 satisfies Figure II (as in that lemma). The latter is used to get the similar diagrams in Corollary 1.1, Corollary 1.3 and in Lemma 1.2. Concerning the first, we remark that the open set $U_{1}=U_{1, K}$ in the parameter space $\mu_{K}$ (cf. $(4.1)_{4}$ ) is taken independently from the individual matrix $K$ as in Lemma 4.1. Actually, take an open set $U_{1}=U_{1, X}\left(\ni P_{0}\right)$ of $U_{0}$, and finite points $P_{0, v}$ $\in\left(U_{1, X} \cap X_{0}\right)$, and we attach to each $P_{0, v}$ a parameter space $\mu_{K, v}\left(\subset X \times \boldsymbol{R}_{1}^{+}\right)$ of the form (4.1) $)_{4}$, by means of a suitable element $\sigma_{K, v} \in \boldsymbol{R}_{1}^{+2}$ and an open subset $U_{K, v}\left(\ni P_{0, v}\right)\left(\subset U_{0}\right)$. Then, by Lemma 4.1' (applied to $\left.\mu_{K, v}\right)$, we have the similar inclusion to each $\left(P_{v} ; r\right) \in \mu_{K, v}$. Also assuming that $\bar{U}_{1 ; X} \subset \tilde{U}_{0} \subset U_{0}$ with a suitable open subset $\tilde{U}_{0}$ of $U_{0}$, one can assume that $U_{v} U_{k, v} \supset U_{1, X}$. From this we see that, by taking a suitable element $\sigma_{K} \in \boldsymbol{R}_{1}^{+2}$ and forming a parameter space $\mu_{K}$ by means of $\left(U_{1 ; X}, \sigma_{K}\right)$, we have the similar inclusion to $(4.1)_{5}$ for each $(P ; r) \in \boldsymbol{\mu}_{K}$, with a suitable estimation map $\tilde{M}_{K} \in \tilde{\boldsymbol{M}}$. This insures the independence of the first factor, $U_{1, X}$, of the parameter space $\mu_{K}$ mentioned just above.

(3) The similar independence from the first part of the parameter space 
$\mu_{K}$ also holds, by assuming that the entries of $K$ are in $\Gamma\left(X_{0}, \mathfrak{D}_{X_{0}}(* D)\right)$ (cf. (1)).

Remark 4.1 . In Lemma 4.1, Lemma 4.1', we used the subvarieties $X_{1}$, $X_{2}$ of $X_{0}$. In later applications, we take $\left(X_{1}, X_{2}\right)$ to be $\left(X_{0}, X_{0, \text { ing }}\right)$ or $\left(X_{0}, D\right)$, where $D$ is a divisor of $X_{0}$. The use of the varieties $X_{1}, X_{2}$ as above is convenient for the proof of Lemma 4.1. In B, Section 4.1 soon below, we use the pair $\left(X_{1}, X_{2}\right)$ as in $(4.1)_{0}$. Similar remarks to the pair $\left(X_{1}, X_{2}\right)$ just above also hold for the estimations in $B$.

\section{B. Algebraic Division Uniform Estimations}

This part concerns mainly uniform estimations on open map properties of $\mathfrak{D}_{X}$-homomorphisms (Lemma 4.2-4.5). Such results are our main results on non cohomological uniform estimations in this paper*). The proof of the results of Section 4.2 will be given in Section 5.2.

1. A.d. estimation maps.**) We begin Section 4.2 by the following

Definition 4.1. By an a.d. estimation map we mean the one: $E: \mathbb{R}^{+} \times\left(\boldsymbol{Z}^{+} \times \boldsymbol{R}^{+}\right) \ni(r ; m ; a) \longrightarrow \mathbb{R}^{+} \times\left(\mathbb{Z}^{+} \times \mathbb{R}^{+}\right) \ni\left(r^{\prime} ; m^{\prime} ; a^{\prime}\right)$, where $r^{\prime}=M_{1}(r), m^{\prime}=[L(m)]$ and $a^{\prime}=M_{2}(a / r) \cdot \exp M_{3}(m)$. Here $M_{i}(1 \leqq i \leqq 3)$ are positive monomials and $L$ is a linear function : $L=c t ; c>0$.

We then set:

$(4.2)_{1}^{\prime} \quad \mathbf{E}_{\mathrm{a} . \mathrm{d}}:=$ collection of all a.d. maps.

Letting $E \in \mathbf{E}_{\text {a.d }}$ be as in $(4.2)_{1}$ we call $M_{1}$ and $L$ the first and a.d. parts of $E$. The map

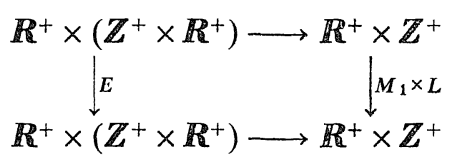

Figure I.

$E$ is factored as in Figure I. (In Figure I, the factor ' $\mathbb{R}^{+'}$ in the right side is the first factor of $\boldsymbol{R}^{+} \times\left(\mathbb{Z}^{+} \times \boldsymbol{R}^{+}\right)$.)

2. Algebraic and analytic a.d. properties. Take a finite set $\rho=\left(f_{j}\right)_{j=1}^{s}$ $\subset \Gamma\left(X_{0}, \mathfrak{D}_{X_{0}}\right)$, which vanishes at $P_{0} \in X_{0}$ (cf. $\left.(4,1)_{0}\right)$ and satisfies:

$(4.2)_{2}^{\prime} f_{j} \equiv 0\left(X_{0, i}\right)$ for each $f_{j}(1 \leqq j \leqq s)$ and each irreducible component $X_{0, i}$ of the germ of $X_{0}$ at $P_{0}$.

Then the set $f^{m}:=\left(f_{j}^{m}\right)_{j=1}^{s}$, the $m$-th homomorphism $F^{m}: \mathfrak{D}_{X}^{s} \rightarrow \mathfrak{D}_{X}$ and the sheaf $f^{m} \mathfrak{D}_{X}:=F^{m} \mathfrak{D}_{X}^{s}\left(\subset \mathfrak{D}_{X}\right)$ will have the similar meaning to n.2, Section 2.1. As in Section 2 we use $f^{m}$ to measure the a.d. properties of $\mathfrak{D}_{X}$-coherent sheaves.

*) Cf. also the beginning of Chapter II.

**) A.d. = algebraic division (cf. §2). 
On the other hand, letting $V$ be the locus of $f$, we also use*) the 'distance $|f(P)|:=\sum_{j=1}^{s}\left|f_{j}(P)\right|$ to $V$ ' to measure such properties. We will compare the above two means for the measure of the a.d. properties. For this taking an open subset $U_{1, \tilde{X}_{1}}\left(\ni P_{0}\right)$ of $U_{0}$ and an element $\sigma_{\bar{X}_{1}} \in \mathbb{R}_{1}^{+2}$, we form a subset $\boldsymbol{\mu}_{\bar{X}_{1}}$ of $\tilde{X}_{1} \times \mathbb{R}^{+}$in the manner in $(4.1)_{4}$, by using $\left(U_{1, \bar{x}_{1}}, \sigma_{\tilde{X}_{1}}\right)$. Also taking an element $\bar{m} \in \mathbb{Z}^{+}$we $\operatorname{set}^{* *)}: \tau_{\tilde{X}_{1}}:=\mu_{\tilde{X}_{1}} \times \mathbb{Z}_{\bar{m}}^{+} \times \mathbb{R}_{1}^{+}$. For an element $(P ; r ; m ; a)$ $\in \tau_{\tilde{X}_{1}}\left(\subset \tilde{X}_{1} \times \mathbb{R}^{+} \times \mathbb{Z}^{+} \times \mathbb{R}_{1}^{+}\right)$we definc:

$$
\left\{\begin{array}{l}
\Gamma\left(\tilde{U}_{r}(P), \rho^{m} \mathfrak{D}_{X}\right)_{a} \\
\Gamma\left(\tilde{U}_{r}(P), \mathfrak{D}_{X}\right)_{a}^{m}
\end{array}\right\}:=\left\{\begin{array}{l}
F^{m} \Gamma\left(\tilde{U}_{r}(P), \mathfrak{D}_{X}^{s} ; \theta\right)_{a}\left(\mathrm{cf} .(4.1)_{2}^{\prime}\right) \\
\left\{\rho \in \Gamma\left(\tilde{U}_{r}(P), \mathfrak{D}_{X}\right) ;|\varphi(Q)| \leqq a|f(Q)|^{m} \text { in } \tilde{U}_{r}(P)\right\}
\end{array}\right\} .
$$

In the above $\theta$ is the standard q-structure of $D_{Y}^{s}$ (cf. (4.1) $)_{2}$ and Definition $1.4_{1}$ ). We then have:

Lemma 4.2 (Algebraic and analytic comparison of a.d. properties). For a suitable a.d. map $E_{\bar{X}_{1}} \in \mathbf{E}_{\mathrm{a} . \mathrm{d}}$ w'e have***)

$$
i^{*} \Gamma\left(\tilde{U}_{r}(P), \mathfrak{D}_{X}\right)_{a}^{m} \subset \Gamma\left(\tilde{U}_{r^{\prime}}(P), \rho^{m^{\prime}} \mathfrak{D}_{X}\right)_{a^{\prime}},
$$

with $\left(r^{\prime} ; m^{\prime} ; a^{\prime}\right)=E_{\tilde{X}_{1}}(r ; m ; a)\left(c f .(4.2)_{1}\right)$. Here $(P ; r ; m ; a)$ is in $\tau_{\tilde{X}_{1}}\left(\subset \tilde{X}_{1}\right.$ $\left.\times \boldsymbol{R}^{+} \times \mathbb{Z}^{+} \times \mathbb{R}^{+}\right)$.

Treatments of the left side of $(4.2)_{3}$ are sometimes easier than the right side; Lemma 4.2 is useful in treatments of the a.d. properties of $\mathfrak{D}_{X}$. Next we may regard Lemma 4.2 as an analogue of the comparison of 'p.g. and meromorphic' (as in Theorem 1.6) in our treatments of the a.d. properties. Moreover, as we will see in n.3, Lemma 4.2 implies Hilbert zero point theorem for (Lemma 4.3'). Lemma 4.2 may be a basic fact in the a.d. properties of $\mathfrak{D}_{X}$.

3. Koszul complex - 1. Taking a finite set $g=\left(g_{j}\right)_{j=1}^{t} \subset \Gamma\left(X_{0}, \mathfrak{D}_{X_{0}}\right)$ satisfying the similar condition to $(4.2)_{2}^{\prime}$, we denote by $\mathscr{G}$ the Koszul complex

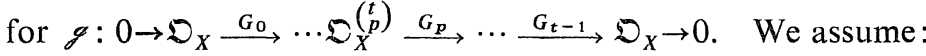
the locus $W$ of $g \subset V(=$ locus of $f)$.

Now taking an open subset $U_{1, \mathscr{s}}\left(\ni P_{0}\right)$ of $U_{0}$ and an element $\sigma_{\mathscr{G}} \in \boldsymbol{R}_{1}^{+2}$, we form a subset $\mu_{\mathscr{G}}\left(\subset \widetilde{X}_{1} \times \mathbb{R}^{+}\right)$in the manner in $(4.1)_{4}$. Also taking an element

*) By Lojasiewicz inequality, we may regard $|f(P)|$ also as the distance to $\mathrm{V}$.

**:) $\boldsymbol{Z}_{m}^{+}=\{m \in \mathbb{Z} ; m \geqq \bar{m}\}$ (cf. the introduction of Chapter. 1).

***) Precisely, the map $E_{X_{1}}$ depends on also $f$. The set $\zeta$ is a basic underlying datum in Chapter II, and we use the symbols $E_{\lambda_{1}}$ instead of $E_{X_{1}, k}, \ldots$. 
$\bar{m}=\bar{m}_{\mathscr{G}} \in \mathbb{Z}^{+}$we set: $\tau_{\mathscr{G}}:=\mu_{\mathscr{G}} \times \mathbb{Z}_{m}^{+} \times \mathbb{R}_{1}^{+}$.

Lemma 4.3 (Open map property for Koszul complex). For a suitable a.d. map $E_{\varsigma} \in \mathbb{E}_{\mathrm{a} . \mathrm{d}}$ we have $(1 \leqq p \leqq t-1)$ :

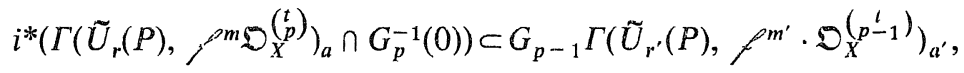

with $\left(r^{\prime} ; m^{\prime} ; a^{\prime}\right)=E_{\mathscr{g}}(r ; m ; a)$, where $(P ; r ; m ; a)$ is in $\tau_{\mathscr{g}}\left(\subset \tilde{X}_{1} \times \mathbb{R}^{+} \times \mathbb{Z}^{+}\right.$ $\left.\times \mathbf{R}_{1}^{+}\right)$.

We check that Lemma 4.2, 4.3 give a cohomological generalization of Hilbert zero point theoren. For this, taking a point $P \in \tilde{X}_{1} \cap W$, we form a filtered complex $\mathscr{C}_{P}^{*}: 0 \rightarrow \mathscr{C}_{P}^{0} \rightarrow \cdots \rightarrow \mathscr{C}_{P}^{p} \stackrel{G_{p}}{\longrightarrow} \cdots \mathscr{C}_{P}^{t-1} \rightarrow \mathscr{C}_{P}^{t} \rightarrow 0$, where $\mathscr{C}_{P}^{p}:=$ $\left\{f^{m} \bigcirc_{X, P}^{(t)}\right\}_{m=1}^{\infty}$ and the degree one map is $G_{p}(0 \leqq p \leqq t-1)$ (cf. n.1, §2.1).

Lemma $43^{\prime}$. (1) The complex $\mathscr{C}_{P}^{*}$ saitsfies the open map property (Definition 2.1 $)$. (2) The open map property for $\mathscr{C}_{P}^{*}$ at the final step*): $\mathfrak{D}_{X}^{t}$ $\stackrel{G_{t-1}}{\longrightarrow} \mathfrak{D}_{X} \rightarrow 0$ is equivalent to Hilbert zero point theorem for $(f, g): \mathfrak{D}_{X . P}$ $\supset f^{\tilde{m}} \mathfrak{D}_{X, P}$, with a suitable $\tilde{m} \in \mathbb{Z}^{+}$.

Proof. The check of (2) is easy. To see (1) take an element $\alpha \in \mathbb{R}_{1}^{+2}$. Then $f_{j} \equiv 0$ on $W$ implies: $\left|f_{j}(Q)\right| \leqq \alpha \cdot d(Q, W)$ in a small neighborhood $U_{P}$ of $P$ in $X$. By Lojasiewicz inequality we have: $\left|f_{j}(Q)^{m}\right| \leqq a \cdot|g(Q)|^{m^{\prime}}$, with suitable $m, m^{\prime} \in \mathbb{Z}^{+}$and $a \in \mathbb{R}^{+}$. Applying Lemma 4.2 to $g$, we have: $f_{j}^{m}$. $\mathfrak{D}_{X, P} \subset \mathcal{g} \mathfrak{D}_{X, P} . \quad$ By (2) this implies the open map property for $G_{t-1}: \mathfrak{D}_{X}^{t} \rightarrow \mathfrak{D}_{X}$. Finally, Lemma 4.3 insures the open map property for $G_{p}(0 \leqq p<t-1)$, and we have (1).

q.e.d.

Hilbert zero point theorem may be the most basic fact on the a.d. properties of analytic varieties. Its cohomological generalization, Lemma 4.2 and Lemma 4.3, may be also basic in treatments of the a.d. properties. (Lemma 4.3' is given for the germ $D_{X, P}$. Formulations of semi-global and global versions of Lemma $4.3^{\prime}$ will be left to interested readers.) We use Lemma 4.2 and Lemma 4.3 for computations of the Cech complexes, which are defined from Koszul complexes in the standard manner (\$5.2). Though our use of those lemmas are in an elementary level, the open map properties as in Lemma 4.2, Lemma 4.3 may be worthwhile pointing out in connection with the very basic roles of Hilbert zero point theorem and of Koszul complexes for algebraic and analytic varieties.

*) This means that $G_{t-1}\left(\nvdash^{\prime} D_{X}^{\prime}, P\right) \supset f^{m} \bigotimes_{X}$ for $m \gg 0$. Here $m^{\prime}=c(m)$, with a map $c: \mathbb{Z}^{+}$ $\rightarrow \mathbb{Z}^{+}$satisfying $\lim _{m \rightarrow} c(m)=\infty$ (cf. Definition 2.1). 
4. Koszul complex - 2. Lemma 4.3 concerns the single Koszul complex G. Here we will be concerned with the family $\mathscr{F}=\left\{\mathscr{F}^{m}\right\}_{m=1}^{\infty}$ of the $m$-th Koszul

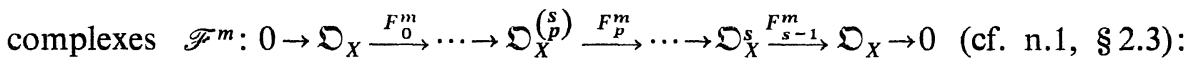
letting the parameter space $\boldsymbol{\mu}_{\tilde{X}_{1}}\left(\subset \tilde{X}_{1} \times \boldsymbol{R}^{+}\right)$be as in Lemma 4.2, we take a suitable linear function $L_{0}(t)=c_{0} t ; c_{0}>0$. Then we have:

Lemma 4.4 (Open map property for the family $\mathscr{F}=\left\{\mathscr{F}^{m}\right\}_{m=1}^{\infty}$ ). Choose a suitable a.d. map $E_{\tilde{X}_{1}} \in \mathbf{E}_{\mathrm{a} . \mathrm{d}}$. Then we have the following for each $m \in \mathbb{Z}^{+}(1$ $\leqq s \leqq p-1)$ :

$$
\begin{aligned}
i^{*}\left\{\Gamma\left(\tilde{U}_{r}(P), f^{\tilde{m}} \mathfrak{D}_{X}^{\left(\begin{array}{l}
s \\
p
\end{array}\right)}\right)_{a}\right. & \left.\cap\left(\text { kernel of } F_{p}^{m}\right)\right\} \\
& \left.\subset F_{p-1}^{m} \Gamma\left(U_{r^{\prime}}(P), f^{\tilde{m}^{\prime}} \mathfrak{D}_{X}^{(s-1}\right)^{s}\right)_{a^{\prime}},
\end{aligned}
$$

where $\left(r^{\prime} ; \tilde{m}^{\prime} ; a^{\prime}\right)=E_{\bar{X}_{1}}(r ; \tilde{m} ; a)$. Moreover, $(P ; r)$ is in $\boldsymbol{\mu}_{\bar{X}_{1}}^{\prime}\left(\subset \tilde{X}_{1} \times \boldsymbol{R}^{+}\right)$, and the a.d. exponent $\tilde{m} \in Z^{+}$of $f^{\tilde{m}}$ satisfies: $\tilde{m}>L_{0}(m)$ (cf. also Lemma 2.5).

If we fix an element $m \in Z^{+}$, which characterize the complex $F^{m}$, then (4.2) follows from Lemma 4.3. The independence of the map $E_{\bar{X}_{1}}$ from such $m \in Z^{+}$ is the key fact in Lemma 4.4. We use Lemma 4.4 to get the corresponding cohomological version, Lemma 2.5 (cf. $\$ 4.4$ ), which is the main lemma in the d.p. uniform estimations in Section 2.

5. Exact complex. Letting the set $h=\left\{h_{u}\right\}_{u=1}^{u_{0}} \subset \Gamma\left(X_{0}, \mathfrak{D}_{X_{0}}\right)$ be as in Lemma 4.1, take a coherent sheaf $\mathfrak{H} \in \operatorname{Coh}\left(X_{0} ; \not / 2\right)$ of the form in $(4.1)_{6}^{\prime}$ : $0 \rightarrow \mathfrak{D}_{X}^{k_{p}} \stackrel{K_{p-1}}{\longrightarrow} \cdots \stackrel{K_{1}}{\longrightarrow} \mathfrak{D}_{X}^{k_{1}} \stackrel{K_{0}}{\longrightarrow} \mathfrak{Y}\left(\subset \mathfrak{D}_{X}^{k}\right) \rightarrow 0$. Moreover, taking a suitable open subset $U_{1}=U_{1, \mathfrak{g}}\left(\ni P_{0}\right)$ of $U_{0}$ and elements $\sigma=\sigma_{\mathfrak{5}} \in \boldsymbol{R}_{1}^{+2}, \bar{m}=\bar{m}_{\mathfrak{5}} \in \boldsymbol{Z}^{+}$, we form parameter spaces $\mu_{\mathfrak{5}}$ and $\tau_{\mathfrak{j}}$ by*)

$$
\boldsymbol{\mu}_{\mathfrak{5}}:=\left\{(P ; r) \in\left(\tilde{X}_{1} \cap U_{1}\right) \times \boldsymbol{R}^{+} ; r<\{\sigma g(P)\}^{-1}\right\}, \boldsymbol{\tau}_{\mathfrak{5}}=\boldsymbol{\mu}_{\mathfrak{5}} \times \boldsymbol{Z}_{\bar{m}}^{+} \times \boldsymbol{R}_{1}^{+} .
$$

Lemma 4.5 (Open map property for exact complex). For a suitable a.d. map $E_{\mathfrak{g}} \in \mathbf{E}_{\mathrm{a} . \mathrm{d}}$ we have:

$(4.2)_{6} \quad i^{*}\left(\Gamma\left(\tilde{U}_{r}(P), f^{\tilde{m}} \mathfrak{D}_{X}^{k}\right)_{a} \cap \Gamma\left(\tilde{U}_{r}(P), \mathfrak{H}\right)\right) \subset K_{0} \Gamma\left(\tilde{U}_{r^{\prime}}(P), f^{\tilde{m}^{\prime}} \mathfrak{D}_{X}^{k_{1}}\right)_{a^{\prime}}$, with $\left(r^{\prime} ; \tilde{m}^{\prime} ; a^{\prime}\right)=E_{\mathfrak{5}}(r ; \tilde{m} ; a)$. Here $(P ; r ; \tilde{m} ; a)$ is in $\boldsymbol{\tau}_{\mathfrak{g}}\left(\subset \tilde{X}_{1} \times \boldsymbol{R}^{+} \times \boldsymbol{Z}_{\tilde{m}}^{+}\right.$ $\left.\times \boldsymbol{R}_{1}^{+}\right)$.

Note that Lemma 4.5 concerns an inclusion of Artin-Rees theorem type (cf. §2.1), and is used in the proof of the corresponding cohomological fact (cf. Lemma 2.7, §2.3. Also see §4.4). As we will see in Section 5.2, the proof

*) Cf. also (4.1) 4 . 
of Lemma 4.5 is easier than that of Lemma 4.3, Lemma 4.4, which concern the open map property of Koszul complexes. (Note that the Koszul complexes are not, in general, exact.)

6. Comparison of filtrations. Here we add a lemma, which is used in the proof of the comparison of the filtrations in Lemma 2.3. For this letting the sheaf $\mathfrak{S} \in \operatorname{Coh}\left(X_{0} ; \not h\right)$ be as in Lemma 4.5 , we define $\mathfrak{D}_{X}$-homomorphisms:

$$
K_{0, m}:=K_{0}+F^{m}: \mathfrak{D}_{X}^{h_{1}}+\mathfrak{D}_{X}^{s k} \ni \varphi_{1}+\varphi_{2} \longrightarrow \mathfrak{D}_{X}^{k} \ni K_{0} \varphi_{1}+F^{m} \varphi_{2},
$$

where we use the symbol $F^{m}$ of the homomorphism: $\mathfrak{D}_{X}^{s} \rightarrow \mathfrak{D}_{X}$ also for its $k$-times direct sum: $\mathfrak{D}_{X}^{s k}:=\mathfrak{D}_{X}^{s}+\cdots+\mathfrak{D}_{X}^{s} \rightarrow \mathfrak{D}_{X}^{k}:=\mathfrak{D}_{X}+\cdots+\mathfrak{D}_{X}$ (cf. n.2, §2.1).

Also we use the symbol $\mathfrak{H}_{m}$ for the image of $K_{0, m}: \mathfrak{H}_{m}:=K_{0, m}\left(\mathfrak{D}_{X}^{k_{1}+s k}\right) \subset \mathfrak{D}_{X}^{k}$.

Next, in Lemma 4.6 soon below, we use an estimation map, which is slicely different from the a.d. maps in Lemma 4.2-Lemma 4.5. For this we set:

(4.2) $)_{7}^{\prime \prime} \quad \mathbf{E}_{\mathrm{add}}^{\prime}:=$ collection of all maps $E^{\prime}: \mathbb{R}^{+} \times \mathbb{Z}^{+} \times \mathbb{R}^{+} \ni(r ; \tilde{m} ; a) \rightarrow \mathbb{R}^{+} \times \mathbb{Z}^{+} \times$ $\boldsymbol{R}^{+} \ni\left(r^{\prime} ; \tilde{m}^{\prime} ; a^{\prime}\right)$, where $\left(r^{\prime} ; a^{\prime}\right)=\tilde{M}(r ; a), m^{\prime}=[L(m)]$, with a map $\tilde{M} \in \tilde{M}$ (cf. n.1, §4.1) and a linear map $L=\mathrm{ct} ; c>0$.

Similarly to $n .1$, Section 4.2 , we call the maps $M: \mathbb{R}^{+} \ni r \rightarrow \mathbb{R}^{+} \ni r^{\prime}$ and $L: \mathbb{Z}^{+} \ni m \rightarrow \mathbb{Z}^{+} \ni m^{\prime}$ the first and a.d. parts of $E^{\prime}$.

Lemma 4.6 (Comparison of filtrations).

For a suitable map $\varepsilon_{\tilde{\tilde{n}}}: \mathbb{Z}^{+} \ni \tilde{m} \rightarrow \mathbb{E}_{\mathrm{a} . \mathrm{d}}^{\prime} \ni E_{\tilde{\tilde{g}}, \tilde{m}}$ satisfying Figure III, we have:

$$
\begin{aligned}
i^{*}\left(\Gamma\left(\tilde{U}_{r}(P), \mathfrak{D}_{X}^{k}\right)_{a}\right. & \left.\cap \Gamma\left(\tilde{U}_{r}(P), \mathfrak{S}_{\tilde{m}}\right)\right) \\
& \subset K_{0, \tilde{m}^{\prime}}\left(\tilde{U}_{r^{\prime}}(P), \mathfrak{D}_{X}^{k_{1}+s k}\right)_{a^{\prime}}
\end{aligned}
$$

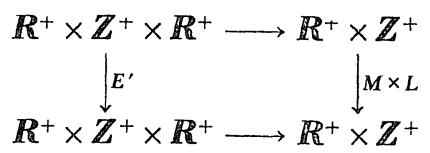

Figure II.

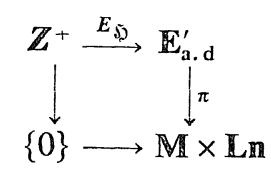

Figure III.*)

with $\left(r^{\prime} ; \tilde{n^{\prime}} ; a^{\prime}\right)=E_{\tilde{\tilde{g}}, \tilde{m}}(r ; \tilde{m} ; a)$, where $(P ; r ; \tilde{m} ; a)$ is as in Lemma 4.5 .

(In Figure III, we set $\mathbf{L n}:=\{L(t)=\mathrm{ct} ; c>0\}$. Also $\mathbf{M}:=$ collection of all positive monomials. The map $\pi: \mathbb{E}_{\text {a.d }}^{\prime} \ni E^{\prime} \rightarrow \mathbf{M} \times \operatorname{Ln} \ni(M, L)$ attaches to $E^{\prime}$ its first and a.d. parts.)

Remark 4.2. We make a remark on the estimation maps in Lemma 4.24.5 and in Lemma 4.6. For this, for an a.d. map $E \in \mathbb{E}_{\text {a.d }}$ as in (4.2) , we define a series $\left\{E_{E, \tilde{m}}^{\prime}\right\}_{m=1}^{\infty}$ of maps $E_{E, m}^{\prime} \in \mathbb{E}_{\mathrm{a} . \mathrm{d}}^{\prime}$, which satisfies Figure III, as follows:

*) The set $\{0\}$ consists of the single element $0 \in \mathbb{Z}^{+} \cup 0$. Thus Figure III claims that the first and a.d. parts of $E_{\mathfrak{5}, m}\left(m \in \mathbb{Z}^{+}\right)$are independent from $m \in \mathbb{Z}^{+}$. 
$(4.2)_{8} \quad E_{E, \tilde{m}}^{\prime}: \boldsymbol{R}^{+} \times \boldsymbol{Z}^{+} \times \boldsymbol{R}^{+} \ni(r ; \tilde{m} ; a) \longrightarrow \boldsymbol{R}^{+} \times \mathbb{Z}^{+} \times \boldsymbol{R}^{+} \ni\left(r^{\prime} ; \tilde{m}^{\prime} ; a^{\prime}\right)$, with $\left(r^{\prime} ; a^{\prime}\right)=\left(M_{1}(r), M_{2}(a / r) \exp M_{3}(m)\right)$ and $\tilde{m}^{\prime}=[L(\tilde{m})]$. (The first and a.d. parts $M_{1}, L$ of $E \in \mathbb{E}_{\text {a.d }}$ and the positive monomials $M_{2}, M_{3}$ are as in (4.2) $)_{1}$ )

The estimations in Lemma 4.2-Lemma 4.5, for example in Lemma 4.5 are given as follows:

$$
\left(r^{\prime} ; \tilde{m}^{\prime} ; a^{\prime}\right)=E_{E, \tilde{m}}^{\prime}(r ; \tilde{m} ; a) \text {, where } E \in \mathbb{E}_{\mathrm{a} . \mathrm{d}} \text { is as in Lemma 4.5. }
$$

The dependence of $E_{E, m}^{\prime}$ on $m \in \mathbb{Z}^{+}$is quite clear, and the estimation (4.2) is sharper than $(4.2)_{7}$ in Lemma 4.6 .

7. De Rham complex. Here we assume that $X_{0}$ is irreducible at the origin $P_{0}$ of $X_{0}$. We also assume that the pair $\left(X_{1}, X_{2}\right)$ is of the form: $\left(X_{0}, X_{0}^{\prime}\right)$, with a subvariety $X_{0}^{\prime}$ of $X_{0}$, and that the coordinates $z^{\prime}=\left(z_{1}, \ldots, z_{k}\right)$ provide a local parameter at each $P \in\left(X_{0}-X_{0}^{\prime}\right)$. We identify the sheaf $\Omega_{X}^{p}$ (cf. $\S 3$ ) with $\Sigma_{X}^{\left(\begin{array}{c}k \\ p\end{array}\right)}$ in the standard manner: $\Omega_{X}^{p} \ni \sum_{I} \varphi_{I} d x^{I} \stackrel{\mu}{\longrightarrow} \mathcal{D}_{X}^{\left(\begin{array}{c}k \\ p\end{array}\right)} \ni\left(\varphi_{I}\right)_{I}$, where $I$ exhausts all indices of the form: $\left(i_{1}<\cdot<\cdots<i_{p}\right)$, with $1 \leqq i_{1}<\cdots<i_{p} \leqq k$. Letting the parameter space $\tau_{X}$ be as in $n .2$, part $B$, Section 4.1 , we take a parameter $(P ; r ; a ; m) \in \tau_{X}\left(\subset X \times \mathbb{R}^{+} \times \mathbb{R}_{1}^{+} \times \mathbb{R}_{1}^{+} \times \boldsymbol{Z}^{+}\right)$, and we set:

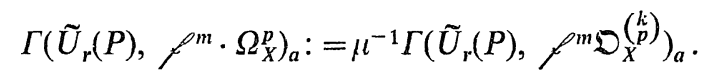

Then, letting $d_{X}$ be the exterior differential operator on $X$, we have:

Lemma 4.7 (Open map property for de Rham complex). For a suitable a.d. map $E_{X} \in \mathbf{E}_{\mathrm{a} . \mathrm{d}}$ we have $(p \geqq 1)$ :

$$
i^{*}\left(\Gamma\left(\tilde{U}_{r}(P), f^{m} \Omega_{X}^{p}\right)_{a} \cap d_{X}^{-1}(0)\right) \subset d_{X} \Gamma\left(\tilde{U}_{r^{\prime}}(P), f^{m^{\prime}} \Omega_{X}^{p-1}\right)_{a^{\prime}},
$$

with $\left(r^{\prime} ; m^{\prime} ; a^{\prime}\right)=E_{X}(r ; m ; a)$, where $\left(P ; r^{\prime} ; m^{\prime} ; a^{\prime}\right)$ is in $\boldsymbol{\tau}_{X}\left(\subset X \times \boldsymbol{R}^{+} \times \boldsymbol{R}^{+}\right.$ $\left.\times \boldsymbol{R}_{1}^{+} \times \boldsymbol{Z}^{+}\right)$.

Lemma 4.7 is derived from Theorem $1.2_{2}$, Lemma 4.3 and from our uniform estimation on the a.d. properties of (local) contractible properties of analytic varieties $\left(\mathrm{cf} .[15]_{2}\right)$. The latter concerns some topological properties of the varieties, and the details of it will be given elsewhere in a near future. We summarize the key points of the proof of Lemma 4.7 at the end of Section 5.2. Lemma 4.7 is used in the proof of our 'p.g. open map property for the de Rham complex' as in Lemma 3.2. The relation of Lemma 4.7 to Lemma 3.2 is also summarized at the end of Section 5.2.

Now, letting the parameter $(P ; r)$ be as in Lemma 4.7 , we form a filtered 
complex $\mathscr{C}_{r}^{*}(P)$ by

$$
\begin{aligned}
0 \longrightarrow \Gamma\left(\tilde{U}_{r}(P), \mathbb{C}\right) \longrightarrow & \left\{\Gamma\left(\tilde{U}_{r}(P), \Omega_{X, m}^{p}\right)\right\}_{m=0}^{\infty} \\
& \longrightarrow \cdots \stackrel{d_{X}}{\longrightarrow}\left\{\Gamma\left(U_{r}(P), \Omega_{X, m}^{p}\right)\right\}_{m=0}^{\infty} \longrightarrow
\end{aligned}
$$

where we set:

$$
\Omega_{X, m}^{p}:=f^{m} \cdot \Omega_{X}^{p}+\left(\sum_{j=1}^{s} f_{j}^{m+1} \cdot d x_{j}\right) \wedge \Omega_{X}^{p-1} .
$$

Corollary 4.1. (1) The direct system $\left\{\mathscr{C}_{r}^{*}(P) ; r \in(0 ; 1)\right\}$ satisfies the open map property.

(2) The following complex is exact (formal Poincaré lemma):

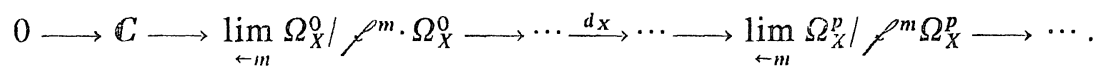

The first follows easily from Lemma 4.7 (by dropping the explicit estimation in it), and the second follows from the first by Proposition 2.1 $1_{1}$. It is in the form of (1), Corollary 4.1 that S. Lubkin conjectured the open map property for the de Rham complex (cf. § 2.1). The formal Poincare lemma (4.9)" was proved by R. Hartshorne*) and by A. Fujiki**) independently, by using the resolution theorem of $\mathrm{H}$. Hironaka. (Their methods are also independent.) The open map property in (1), Corollary 4.1 is also proven by A. Fujiki by using the resolution theorem. (His proof also uses some local contractible properties of analytic varicties.)

Remark. The content of Part B, Section 4.1 contains all examples of complexes, which we know, where the open map property hold. From the basic property of Artin-Rees theorem in the completion theory as well as from the scope of our examples of the open map properties as above, it looks like that the open map properties deserve to be studied for more general types of (geometric complexes). The author hopes that the content of Part B call attention of analytic geometeres, who are working with complexes of geometric nature (on analytic varieties).

8. Some remarks. Here we summarize some remarks for Lemmas 4.14.6, which will be used in the proof of those lemmas (cf. $\$ 5$ ).

(i) Terminologies. We begin $n .8$, by arranging some terminologies for later convenience. First recall that the estimation in Lemma 4.1' was given to 
points of $\tilde{X}_{1}=X_{1}-X_{2}$ and that the underlying homomorphism was: $K: \mathfrak{D}_{X}^{v}$ $\rightarrow \mathfrak{D}_{X}^{u}$; we will use the terminology:

$$
\text { Lemma } 4.1^{\prime} \text { holds for }\left(X_{1}, X_{2} ; K\right)
$$

as a synonym for 'the estimation $(4.1)_{5}$ (in Lemma $4.1^{\prime}$ ') holds' for the parameter $(P ; r ; a)$ as in $(4.1)_{5}$. Here $(P ; r ; a)$ should be in the parameter space of the form $\lambda_{K}$ as in $(4.1)_{5}$, and the estimation map should be of the form $M_{K} \in \boldsymbol{M}$ as in $(4.1)_{5}$. Similarly to the above we use the terminology:

$(4.3)_{2}$ Lemma 4.2 holds for $\left(X_{1}, X_{2} ; \rho\right)$ (resp. Lemma 4.3 holds for $\left(X_{1}\right.$, $\left.X_{2} ; f, \mathscr{G}\right)$, Lemma 4.4 holds for $\left(X_{1}, X_{2} ; f\right)$, Lemma 4.5 holds for $\left(X_{1}\right.$, $\left.X_{2} ; f, \mathfrak{H}\right)$ or Lemma 4.6 holds for $\left.\left(X_{1}, X_{2} ; f, \mathfrak{H}\right)\right)$

as a synonym for the following:

$(4.3)_{2}^{\prime}$ the estimation $(4.2)_{3}$ (resp. $(4.2)_{4},(4.2)_{5},(4.2)_{6}$ or $\left.(4.2)_{7}\right)$ holds for the parameter $(P ; r ; m ; a)$ as in $(4.2)_{3}$ (resp. $\left.(4.2)_{3}, \ldots\right)$.

(Here note that $(4.2)_{3}-(4.2)_{6}$ are the explicit estimations in Lemma 4.2-Lemma 4.6. Also remark that $f,(f, \mathscr{G}), \ldots$ are the underlying geometric data in Lemma 4.2-Lemma 4.6.) Moreover, for the first terminology in (4.3) ${ }_{2}$, the parameter $(P ; r ; m ; a)$ should be in the parameter space $\tau_{\tilde{X}_{1}}$ as in Lemma 4.2 and the estimation map should be of the form $E_{\tilde{\boldsymbol{X}}_{1}} \in \mathbf{E}_{\text {a.d }}$ as in Lemma 4.2. For the other terminologies in $(4.3)_{2}$, the parameter spaces and the estimation maps should be understood in the similar manner to the above.

(ii) Next taking subvarieties $X_{1}^{\prime}, X_{2}^{\prime}$ of $X_{0}$ satisfying: $X_{1}^{\prime} \supset X_{1}, X_{2}^{\prime} \subset X_{2}$, we have the following implication:

(4.3) $)_{3}^{\prime}$ Lemma 4.1' for $\left(X_{1}^{\prime}, X_{2}^{\prime} ; K\right) \longrightarrow$ Lemma $4.1^{\prime}$ for $\left(X_{1}, X_{2} ; K\right)$.

This is checked easily, by remarking that the estimations in the left and right sides are given to points in $\left(X_{1}^{\prime}-X_{2}^{\prime}\right)$ and $\left(X_{1}-X_{2}\right)$ and that the estimation in the left side is applied to the right side. (See also the explicit estimation in Lemma 4.1'.) By (4.3)' ${ }_{3}^{\prime}$ we have:

$$
\begin{aligned}
\text { Lemma } 4.1^{\prime} \text { for }\left(X_{0}, X_{0, \text { sing }}\right) \longrightarrow \text { Lemma } 4.1^{\prime} \\
\left(=\text { Lemma } 4.1^{\prime} \text { for }\left(X_{1}, X_{2} ; K\right)\right) .
\end{aligned}
$$

We prove Lemma $4.1^{\prime}$ in the form of the left side. The similar implications to the above hold for Lemma 4.2-Lemma 4.6.

(iii) Chain property. Thirdly take a subvariety $X_{3}$ of $X_{0}$ satisfying 


$$
X_{0} \supset X_{1} \supsetneqq X_{2} \supsetneqq X_{3} \supset X_{0}^{\prime} .
$$

Then we have the following implication, which will play a role in the proof of Lemma $4.1^{\prime}$ (cf. § 5.1):

Proposition 4.1. Lemma 4.1' for $\left(X_{i}, X_{i+1} ; K\right)(i=1,2) \rightarrow$ that for $\left(X_{1}\right.$, $\left.X_{3} ; K\right)$. The similar implication to the above also holds for Lemma 4.2Lemma 4.6.

Note that 'Lemma $4.1^{\prime}$ for $\left(X_{1}, X_{2} ; K\right)$ ' and 'that for $\left(X_{2}, X_{3} ; K\right)$ ' concern the same homomorphism $K$, and the inclusions in them are similar (cf. (4.1) $)_{5}$ ), except that

(4.3) 5 the point $P$ in question (cf. (4.1) $)$ ) is in $X_{1}-X_{2}$ or in $X_{2}-X_{3}$, and that the size ' $r$ ' of the manifold $\tilde{U}_{r}(P)\left(\mathrm{cf} .(4.1)_{5}\right)$ should satisfy:

$$
r<\left\{\sigma d\left(P, X_{2}\right)^{-1}\right\}^{-1} \text { or } r<\left\{\sigma d\left(P, X_{3}\right)^{-1}\right\}^{-1} .
$$

It is easy to get 'Lemma $4.1^{\prime}$ for $\left(X_{1}, X_{3} ; K\right)$ ' from the above two estimations. (The former is given to points $P \in\left(X_{1}-X_{3}\right)$, and the size $r$ of $\tilde{U}_{r}(P)$ should satisfy the inequality of the form: $r<\left\{\sigma d\left(P, X_{3}\right)^{-1}\right\}^{-1}$. It is easy to fill the gap between what is mentioned just above and $(4.3)_{5,6}$, by using elementary distance properties of analytic varieties; see also the author's forthcoming paper [18].)

(iv) Here we add a technical remark for the proof of Lemma 4.1 and Lemma 4.5, Lemma 4.6: recall that the sheaf $\mathfrak{H}$ in these lemmas is in $\operatorname{Coh}\left(X_{0}\right.$; $\not)_{\mathrm{p} . \mathrm{g}}$, where $h=\left(h_{u}\right)_{u=1}^{u_{0}}$ is a subset of $\Gamma\left(X_{0}, \mathfrak{D}_{X_{0}}\right)$. We then have:

(4.3) $)_{7}$ Lemma 4.1 for $\left(X_{0}, X_{0}^{\prime} ; K\right)$ for the case: $\# h=1 \Rightarrow$ that for the general case: $\# h \geqq 2$, where $X_{0}^{\prime}=$ locus of $h$ (and the similar fact for Lemma 4.5 and Lemma 4.6).

Actually, let $h=\left\{h_{u}\right\}_{u=1}^{u_{0}}$ be as in Lemma 4.1, we apply the left side of (4.3) to each $h_{u}\left(u=1, \ldots, u_{0}\right)$. Then the inclusion of the form in $(4.1)_{7}$, Lemma 4.1 holds for each $P \in X_{0}-D_{u}$, and the size of the manifold $\tilde{U}_{r}(P)$ (cf. (4.1) $)_{7}$ ) should satisfy: $r<\left\{\sigma d\left(P, D_{u}\right)^{-1}\right\}^{-1}$. (Here $D_{u}$ is the locus of $h_{u}$.) But $X_{0}^{\prime}=\cap_{u} D_{u}$. Also, by the Lojasiewicz inequality, we have:

(4.3) $\sigma_{8} \quad c^{\prime} d\left(P, X_{0}^{\prime}\right)<\sum_{u} d\left(P, D_{u}\right)<c \cdot d\left(P, X_{0}^{\prime}\right)$, with suitable $c, c^{\prime} \in \mathbb{R}^{+2}$. This implies:

(4.3) $\quad \tilde{c} d\left(P, X_{0}^{\prime}\right)<d\left(P, X_{u}\right)$, with a suitable index $u$, where the element $\tilde{c} \in \mathbb{R}^{+2}$ 
is determined by the element $c$ in $(4.3)_{8}$.

From this the inequality mentioned just above is replaced by $r<\left\{\sigma d\left(P, X_{0}^{\prime}\right)^{-1}\right\}^{-1}$, and we get $(4.3)_{7}$ (cf. also similar arguments in (iii)).

(v) key proposition. Here we give a key proposition for Lemmas 4.2-

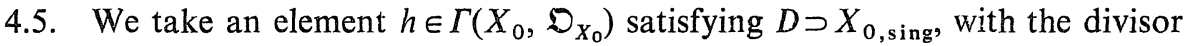
$D$ of $h$. We assume the following:

(4.4) Lemma 4.4 for $\left(X_{0}, D ; f\right)$ (resp. Lemma 4.3 for $\left(X_{0}, D ; f, \mathscr{G}\right)$ or Lemma 4.5 for $\left.\left(X_{0}, D ; f, \mathfrak{S}\right)\right)$ (cf. (4.3) $)_{1}$ ), where the Koszul complex $G$ and the sheaf $\mathfrak{H} \in \operatorname{Coh}\left(X_{0} ; h\right)$ are as in Lemma $4.3,4.5$.

Now take a suitable open set $U_{1}=U_{1, h}\left(\ni P_{0}\right)$ of $U_{0}$ and an element $\sigma=\sigma_{h} \in \boldsymbol{R}_{1}^{+2}$, we define the following parameter space (cf. $\left.(4.1)_{5}\right)$ :

$$
\mu_{h}:=\left\{(P ; r) \in\left(\left(D-X_{0, \text { sing }}\right) \cap U_{1}\right) \times \mathbb{R}^{+} ; r<\left\{\sigma d\left(P, X_{0, \text { sing }}\right)^{-1}\right\}^{-1}\right\} .
$$

Also we take a suitable $d=d_{h} \in \mathbb{Z}^{+}$, an a.d. map $E_{h} \in \mathbb{E}_{\text {a.d }}$, a linear function $L_{0, h}=c_{0, h} t ; c_{0, h}>0$ and an element $\bar{m}=\bar{m}_{h} \in \mathbb{Z}^{+}$. Then from the estimations in (4.4), we get the following weaker version of Lemma 4.2 for $\left(D, X_{0 \text {, sing }} ; \mathcal{f}\right), \ldots$, Lemma 4.5 for $\left(D, X_{0, \text { sing }} ; f, \mathfrak{H}\right)$.

Proposition 4.2. We have the following inclusions:

$(4.5)_{1} \quad i^{*} h^{d} \Gamma\left(U_{r}(P), \mathfrak{D}_{X}\right)_{a}^{\tilde{m}} \subset \Gamma\left(U_{r^{\prime}}(P), f^{\tilde{m}^{\prime}} \mathfrak{D}_{X}\right)_{a^{\prime}}$,

$(4.5)_{2} \quad i^{*} h^{d}\left(\Gamma\left(U_{r}(P), \not^{\tilde{m}} \Phi_{X}^{\left(\begin{array}{l}t \\ p\end{array}\right)}\right)_{a} \cap G_{p}^{-1}(0)\right)$

$$
\subset G_{p-1} \Gamma\left(U_{r^{\prime}}(P), f^{\tilde{m}^{\prime}} \mathfrak{D}_{X}^{\left(p_{-1}^{t}\right)}\right)_{a^{\prime}} \quad(1 \leqq p<t),
$$

$(4.5)_{3} \quad i^{*} h^{d}\left(\Gamma\left(U_{r}(P), f^{i n} \mathfrak{D}_{X}^{(s)} p\right)_{a} \cap\left(F_{p}^{m}\right)^{-1}(0)\right)$

$$
\subset F_{p-1}^{m} \Gamma\left(U_{r^{\prime}}(P), f^{\tilde{m}^{\prime}} \mathfrak{D}_{X}^{(p-1)}\right)_{a^{\prime}} \quad(p \leqq s),
$$

$(4.5)_{4} \quad i^{*} h^{d}\left(\Gamma\left(U_{r}(P), f^{m} D_{X}^{k}\right)_{a} \cap \Gamma\left(U_{r}(P), \mathfrak{H}\right)\right) \subset K_{0} \Gamma\left(U_{r^{\prime}}(P), f^{\tilde{m}^{\prime}} \bigvee_{X}^{k}\right)_{a^{\prime}}$.

The estimations is given by $\left(r^{\prime} ; \tilde{n}^{\prime} ; a^{\prime}\right)=E_{h}(r ; \tilde{m} ; a)$. Moreover, $(P ; r)$ is in $\mu_{h}\left(\subset\left(D-X_{0, \text { sing }}\right) \times \mathbb{R}^{+}\right)$. The element $\tilde{n} \in \mathbb{Z}^{+}$in $(4.5)_{1,2,4}$ satisfy $\tilde{m} \geqq \bar{m}$, and $(\tilde{m}, m) \in \mathbb{Z}^{+} \times \mathbb{Z}^{+}$in $(4.5)_{3}$ satisfies: $\tilde{m}>L_{0}(m)$. (The homomorphism $K_{0}$ in $(4.5)_{4}$ is as in Lemma 4.5.)

We prove Proposition 4.2 in Section 4.2. Note that if, we drop the term

*) Precisely, the data $\left(U_{1, h, h}\right)$ and $\left(d_{h}, E_{h}\right), \ldots$ depend also on $G$ or $H$, according as we are concerned with $(4.5)_{2}$ or (4.5) $)_{4}$. (The above data also depend on $/$. As in Lemma 4.2-4.6, we write $E_{h l}, \ldots$ instead of $E_{h, l}, \ldots$ ) 
$h^{d}$ from the left sides of $(4.5)_{1-4}$, we have Lemma 4.2 for $\left(D, X_{0, \operatorname{sing}} ; \rho\right), \ldots$ For the role of Proposition 4.2 in the proof of Lemmas 4.2-4.5, see Section 5.2.

\section{Global Version of the Results int $\mathbb{A}, \mathbb{B}$}

Taking an analytic variety*) $\tilde{\mathbb{X}}=\left(\mathbb{C}^{n}(z) \times \mathbb{C}^{n}\left(z^{\prime}\right), \tilde{X}=\mathbb{C}^{n} \times U_{0}^{\prime}, P_{1}^{\prime}\right) \in \mathbb{A m}_{0}$ (cf. $\left.(1.7)_{0}\right)$ and an affine variety*) $\mathbb{X}^{\prime} \in \mathbb{C}^{n}(z)$ (cf. $\left.(1.11)_{0}\right)$, we give here a global version $^{* *}$ ) of Lemma 4.1 to $\mathbb{X}^{*}:=\tilde{\mathbb{X}}$ or $=\mathbb{X}^{\prime}$ and that of Lemma 4.4-Lemma 4.6 to $\mathbb{X}^{\prime}$. (In the above the euclidean space $\mathbb{C}^{n}(z), \ldots$ and the open set $U_{0}^{\prime}\left(\ni P_{0}^{\prime}\right)$ in $\mathbb{C}^{n}$ are as in $(1.7)_{0}$.)

1. Global version of Lemma $4_{1}$. First taking an open set $U_{i}^{\prime}\left(\ni P_{0}^{\prime}\right)$ of $U_{0}^{\prime}$ and an element $\sigma=\sigma_{\mathbf{X}^{*}} \in \mathbb{R}_{1}^{+2}$, we attach to $\mathbb{X}^{*}$ the following parameter space (cf. also $\left.(4.1)_{1}\right)$ :

$$
\mathscr{H}_{\mathbf{X}^{*}}:=\left\{(Q ; r) \in \mathbb{X}_{1}^{*} \times \mathbb{R}^{+} ; r<\left\{\sigma g_{\mathbf{X}^{*}}(Q)\right\}^{-1}\right\},
$$

where $\mathbb{X}_{1}^{*}:=\left\{\begin{array}{c}\mathbb{C}^{n} \times U_{1}^{\prime} \\ \mathbb{X}^{\prime}\end{array}\right\}$ and $g_{\mathbf{X}^{*}}:=\left\{\begin{array}{l}|\tilde{z}|+1 \\ |z|+1\end{array}\right\}$, according as $\mathbb{X}^{*}=\left\{\begin{array}{l}\tilde{\mathbb{X}} \\ \mathbb{X}^{\prime}\end{array}\right\}$. (Recall that $g_{X^{*}}$ is the p.g. function of $\mathbb{X}^{*}$ and $\tilde{z}=\left(z, z^{\prime}\right)$ (cf. n.1 and n.5, § 1.2).)

$$
\lambda_{X^{*}}:=\mu_{X^{*}} \times \mathbb{R}_{1}^{+} .
$$

Next we set:

$$
\operatorname{Coh}^{*}\left(X^{*}\right)_{\mathrm{p} . \mathrm{g}}:=\operatorname{Coh}^{\prime}(\tilde{\mathbb{X}})_{\mathrm{p} . \mathrm{g}} \text { or } \operatorname{Coh}^{\prime \prime}\left(\mathbb{X}^{\prime}\right)_{\mathrm{p} . \mathrm{g}}
$$

(cf. $(1.4)_{9}$ and $\left.(1.18)_{1}\right)$.

(Recall that such collections consist of the p.g. coherent sheaves over $\mathbb{X}^{*}$ satisfying certain algebraic conditions (as in $(1.4)_{9}$ and $\left.(1.18)_{1}\right)$ ) ) For an element $\mathfrak{S}$ $\in \operatorname{Coh}^{*}\left(X^{*}\right)_{\text {p.g }}$, the q-structures $\theta_{\mathfrak{r}}, \theta_{\mathfrak{g}}^{\prime}$ will be as in $(4.1)_{2}$. Also for an element $(P ; r ; a) \in \lambda_{X^{*}}\left(\subset \mathbb{X}_{1}^{*} \times \mathbb{R}^{+} \times \mathbb{R}_{1}^{+}\right)$, the set of the cochains $\Gamma\left(\tilde{U}_{r}(P), \mathfrak{H} ; \tilde{\theta}_{\mathfrak{g}^{2}}\right)_{a}$, where $\hat{\theta}_{\mathfrak{n}}=\theta_{\mathfrak{j}}$ or $\theta_{\mathfrak{n}}^{\prime}$, will have the similar meaning to $(4.1)_{2}$. (Here, as in $(4.1)_{2}$, we set $\tilde{U}_{r}(P):=\left\{Q \in \mathbb{X}^{*} ; d(P, Q)<r\right\}$, with the natural metric $d$ in $\mathbb{C}^{n}(z)$ $\times \mathbb{C}^{n}\left(z^{\prime}\right)$ or $\mathbb{C}^{n}(z)$.)

*) As in Chapter I, we use the symbol $\tilde{X}$ also for its underlying variety $\tilde{X}$. Also recall that the affine variety $\boldsymbol{X}^{\prime}$ in $(1.11)_{0}$ consists of the data $\left(\mathbb{C}^{n}(z), X^{\prime}, H_{X^{\prime}}\right)$, where $X^{\prime}$ is the underlying variety of $X^{\prime}$ and $H_{X^{\prime}}$ is the resolution of the structure sheaf of $X^{\prime}$ (cf. (1.11) $)$. We also use the symbol $\boldsymbol{X}^{\prime}$ for its underlying variety $X^{\prime}$.

**) Similar global results to Lemma 4.2, Lemma 4.3, also hold for $\mathbb{X}^{*}=\tilde{\mathbf{X}}$ or $\mathbb{X}^{\prime}$. Also, for $\boldsymbol{X}^{*}=\tilde{\boldsymbol{X}}$, similar global facts for Lemma 4.4-Lemma 4.6 hold. But we do not use such facts (cf. §2). 
Lemma 4.8 $\mathbf{1}_{\mathbf{1}}$ (Uniform estimation with bound for $\boldsymbol{X}^{*}=\tilde{\mathbf{X}}$ or $\left.\boldsymbol{X}^{\prime}\right)$. There are maps*) $\varepsilon_{\mathbf{X}^{*}}: \operatorname{Coh}^{*}\left(\boldsymbol{X}^{*}\right)_{\mathrm{p} . \mathrm{g}}$ $\ni \mathfrak{S} \rightarrow \tilde{\boldsymbol{M}} \ni \tilde{M}_{\mathfrak{S}}$ and $\varepsilon_{\mathbf{X}^{*}}^{\prime}: \mathbb{Z}^{+} \rightarrow \mathbf{M}$, which satisfy the factorization in Figure I and with which we have the following**) for each $\mathfrak{S} \in \operatorname{Coh}^{*}\left(\boldsymbol{X}^{*}\right)_{\text {p.g }}$ :

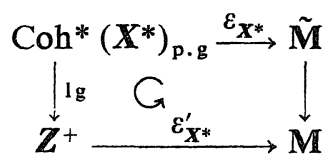

Figure $I^{*}$.

$$
i^{*} \Gamma\left(\widetilde{U}_{r}(P), \mathfrak{T} ; \theta_{\mathfrak{g}}^{\prime}\right)_{a} \subset \Gamma\left(\widetilde{U}_{r^{\prime}}(P), \mathfrak{H} ; \theta_{\mathfrak{g}}\right)_{a^{\prime}},
$$

with $\left(r^{\prime} ; a^{\prime}\right)=\tilde{M}_{\mathfrak{g}}(r ; a)$. Here $(P ; r ; a)$ is in $\lambda_{\mathbf{X}^{*}}\left(\subset X_{1}^{*} \times \boldsymbol{R}^{+} \times \boldsymbol{R}_{1}^{+}\right)$.

We prove Lemma $4.8_{1}$ in $n .3$, and we derive Lemma 1.2 from Lemma $4.8_{1}$ in Section 4.4.

2. Globalization of Lemma 4.4-Lemma 4.6 to $\boldsymbol{X}^{\prime}$. In $n .2$ we let the set $f^{\prime}=\left(f_{j}^{\prime}\right)_{j=1}^{s} \subset \Gamma\left(\boldsymbol{X}^{\prime}, \mathfrak{D}_{\boldsymbol{X}^{\prime}}\right)_{\mathrm{p} . \mathrm{g}}$, the $m$-th Koszul complex $\mathscr{F}^{\prime m}$ for $f^{\prime}: 0 \rightarrow \mathfrak{D}_{\mathbf{X}^{\prime}}$

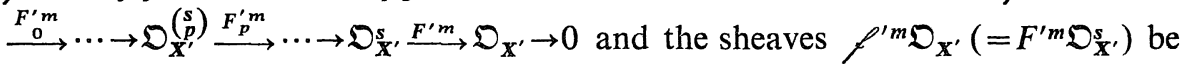
as in Lemma 2.5, Section 2.3. In Lemma $4.8_{2,3}$ we globalize Lemma 4.4Lemma 4.6 to $\boldsymbol{X}^{\prime}$, by using the sheaves $\rho^{\prime m} \mathfrak{D}_{\mathbf{X}^{\prime}}, \ldots$. The estimation maps in Lemma $4.8_{2-4}$ will be in $\mathbf{E}_{\mathrm{a} \text {.d }}^{\prime}$ (cf. (4.2) $)_{7}^{\prime \prime}$ ). We use the symbols $\pi_{1}, \pi_{2}$ for the assignments: $\mathbf{E}_{\mathrm{a} . \mathrm{d}}^{\prime} \ni E^{\prime} \rightarrow M, L$, where $M, L$ are the first and a.d. parts of $E^{\prime}$. Then, taking a suitable linear function $L_{0}=L_{0, X}(t)=c_{0} t ; c_{0}>0$, we globalize Lemma 4.4 to $\boldsymbol{X}^{\prime}$ in the following manner:

Lemma 4.8 $\left(\right.$ Open map property for the Koszul complexes $\left\{\mathscr{F}^{\prime m}\right\}_{m=1}^{\infty}$ ). There is a map $\varepsilon_{\mathbf{X}^{\prime}}: \boldsymbol{Z}^{+} \ni \tilde{m} \rightarrow \mathbb{E}_{\mathrm{a} . \mathrm{d}}^{\prime} \ni E_{\mathbf{X}^{\prime}, \tilde{m}}$, which satisfies the similar factorization to Figure III, Lemma 4.6, and with which we have the following for each $(\tilde{m}, m) \in \mathbb{Z}^{+} \times Z^{+}$satisfying $\tilde{m}>L_{0}(m)(1 \leqq p<s)$ :

$$
\begin{aligned}
i^{*}\left(\Gamma\left(\tilde{U}_{r}(P), f^{\prime \tilde{m}} \mathfrak{V}_{X^{\prime}}^{(s)}\right)_{a} \cap\left(F_{p}^{\prime m}\right)^{-1}(0)\right) & \\
& \subset F_{p-1}^{\prime m} \Gamma\left(\tilde{U}_{r^{\prime}}(P), f^{\tilde{m}^{\prime}} \mathfrak{D}_{X^{\prime}}^{(p-1}\right)_{a^{\prime}},
\end{aligned}
$$

with $\left(r^{\prime} ; \tilde{m}^{\prime} ; a^{\prime}\right)=E_{X^{\prime}, \tilde{m}}(r ; \tilde{m} ; a)$. Here $(P ; r ; a) \in\left(\boldsymbol{X} \times \boldsymbol{R}^{+} \times \boldsymbol{R}_{1}^{+}\right)$is as in Lemma $4.8_{1}$.

Next letting the sheaf $\mathfrak{H}^{\prime}\left(\subset \mathfrak{D}_{\mathbf{X}^{\prime}}^{k}\right)$ and the homomorphism $K_{0}^{\prime}: \mathfrak{D}_{\mathbf{X}^{\prime}}^{k_{1}} \rightarrow \mathfrak{H}^{\prime}$ be as in***) Lemma 2.7, we define a sheaf $\mathfrak{S}_{m}^{\prime}$ and a homomorphism

*) For the sets $\tilde{\mathbf{M}}, \mathbf{M}$ of the estimation maps and the map ' $/ g^{\prime}, \ldots$, see Lemma 4.1, Section 4.1.

**) $i=$ inclusion: $\tilde{U}_{r^{\prime}}(P) \hookrightarrow \tilde{U}_{r}(P)$. When there is no fear of confusions, we use the symbol ' $i$ ' for the inclusion in question, without mentioning it (cf. also §4.1, §4.2).

***) The map $K_{0}^{\prime}$ is the first resolution, denoted by $\omega_{H^{\prime}}$ in the sheaf $H^{\prime}$ in Lemma 2.7. The symbol ' $K_{0}^{\prime \prime}$ is concordant to the one in Lemma 4.5 , Lemma 4.6, and is convenient for the arguments on Lemma $4.8_{3}$. 
$K_{0, m}^{\prime}: \mathfrak{O}_{\mathbf{X}^{\prime}}^{k_{1}+s k} \rightarrow \mathfrak{S}_{m}^{\prime}\left(m \in \mathbb{Z}^{+}\right)$in the similar manner to $(4.2)_{7}^{\prime}:$

$$
\begin{aligned}
K_{0, m}^{\prime}: \mathfrak{D}_{\mathbf{X}^{\prime}}^{k_{1}}+\mathfrak{D}_{\mathbf{X}^{\prime}}^{s k} \ni \varphi_{1}+\varphi_{2} \longrightarrow & \mathfrak{D}_{\mathbf{X}^{\prime}}^{k} \ni K_{0}^{\prime} \varphi_{1}+F^{\prime m} \varphi_{2}, \\
& \text { and } \mathfrak{S}_{m}^{\prime}:=K_{0, m}^{\prime} \mathfrak{D}_{\mathbf{X}^{\prime}}^{k_{1}+s k}\left(\subset \mathfrak{D}_{\mathbb{X}^{\prime}}^{k}\right) .
\end{aligned}
$$

Moreover, taking suitable $\sigma=\sigma_{\mathfrak{g}^{\prime}} \in \mathbb{R}_{1}^{+2}$ and $\bar{m}=\bar{m}_{\mathfrak{g}^{\prime}} \in \mathbb{Z}^{+}$, we form parameter spaces:

$(4.6)_{3}^{\prime \prime} \quad \mu_{\mathfrak{g}^{\prime}}:=\left\{(P ; r) \in \mathbb{X}^{\prime} \times \mathbb{R}^{+} ; r<\left\{\sigma g_{\mathbf{X}^{\prime}}(P)\right\}^{-1}\right\}, \tau_{\mathfrak{p}^{\prime}}:=\boldsymbol{\mu}_{\mathfrak{F}^{\prime}} \times \mathbb{Z}_{\mathfrak{m}}^{+} \times \mathbb{R}_{1}^{+}$.

Lemma 4.8. $\mathbf{8}_{\mathbf{3}}$. There is a map $\varepsilon_{\mathfrak{g}^{\prime}}: \mathbb{Z}^{+} \ni \tilde{n} \rightarrow \mathbb{E}_{\mathrm{a}, \mathrm{d}}^{\prime} \ni E_{\mathfrak{g}^{\prime}, \tilde{m}}$, which satisfies the familiar factorization to Figure III, Lemma 4.6, with which we have the following:

(1) (Open map property for the sheaf $\left.\mathfrak{S}^{\prime}\right)$ :

$$
i^{*}\left(\Gamma\left(\tilde{U}_{r}(P), f^{\prime \tilde{m}} \mathfrak{D}_{X^{\prime}}^{k}\right)_{a} \cap \Gamma\left(\tilde{U}_{r}(P), \mathfrak{S}^{\prime}\right)\right) \subset K_{0}^{\prime} \Gamma\left(\tilde{U}_{r^{\prime}}(P), f^{\prime} \tilde{m}^{\prime} \mathfrak{D}_{X^{\prime}}^{k_{1}}\right)_{a^{\prime}}
$$

(2) (Comparison of filtrations):

$$
i^{*}\left(\Gamma\left(\widetilde{U}_{r}(P), \mathfrak{D}_{X^{\prime}}^{k}\right)_{a} \cap \Gamma\left(U_{r}(P), \mathfrak{S}_{\tilde{m}}^{\prime}\right)\right) \subset K_{0, \tilde{m}^{\prime}}^{\prime} \Gamma\left(\widetilde{U}_{r^{\prime}}(P), \mathfrak{D}_{X^{\prime}}^{k_{1}+s k}\right)_{a^{\prime}}
$$

In the above $\left(r^{\prime} ; \tilde{m}^{\prime} ; a^{\prime}\right)=E_{\mathfrak{E}, \tilde{m}}(r ; \tilde{m} ; a)$, and $(P ; r ; \tilde{m} ; a)$ is in $\tau_{\mathfrak{g}^{\prime}}\left(\subset \mathbb{X}^{\prime} \times \mathbb{R}^{+}\right.$ $\times \mathbb{Z}^{+} \times \mathbb{R}^{+}$.

We use Lemmas $4.8_{2,3}$ in the proof of Lemmas 2.3, 2.5 and Lemma 2.7 (cf. §4.2).

3. Proof of Lemmas $4.8_{1-3}$. (i) For the proof of Lemmas $4.8_{1-3}$, we will give a natural compactification (=completion) of $\mathbb{X}^{*}=\tilde{\mathbb{X}}$ or $=\mathbb{X}^{\prime}$. For this we first set:

(4.7) ${ }_{1} \quad P^{n}(\mathbb{C}):=\cup_{j=0}^{n} \mathbb{C}_{j}^{n}$, with $\mathbb{C}_{0}^{n}:=\mathbb{C}^{n} . \quad$ (Namely, $P^{n}(\mathbb{C})=$ projective space, which is the natural completion of $\mathbb{C}^{n}$. Moreover, $\mathbb{C}_{j}^{n}$ are euclidean spaces, which cover $P^{n}(\mathbb{C})$.)

$(4.7)_{2} \quad \bar{X}^{*}:=P^{n}(\boldsymbol{C}) \times U_{1}^{\prime}$ (cf. $(4.6)_{0}$ ) or the completion $\bar{X}^{\prime}$ of $\mathbb{X}^{\prime}$ in $P^{n}(\mathbb{C})$, and $(4.7)_{3} \quad D^{*}:=D \times U_{1}^{\prime}$ or $D \cap \overline{\mathbf{X}}^{\prime}$, with $D:=P^{n}(\mathbb{C})-\mathbb{C}^{n}$.

We then take a point $P \in D^{*}$ and a small neighborhood $\tilde{U}$ of $P$ in $\bar{X}^{*}$. Also taking an element $\sigma=\sigma_{P} \in \boldsymbol{R}_{1}^{+2}$, we form parameter spaces $\boldsymbol{\ell}_{P}, \boldsymbol{\lambda}_{P}$ similarly to $(4.6)_{0}$ :

$$
\mu_{P}:=\left\{(Q ; r) \in\left(\tilde{U}-D^{*}\right) \times \mathbb{R}^{+} ; r<\left\{\sigma g_{X^{*}}(Q)\right\}^{-1}\right\}, \lambda_{P}:=\mu_{P} \times \mathbb{R}_{1}^{+} .
$$

Then the following analogue of Lemma 4.1, Lemma 4.4 at the 'point at infinity' 
$P \in D^{*}$ will suffice to insure Lemmas $4.8_{1,2}$ :

Lemma 4.1". Take a suitable map $\varepsilon_{P}: \operatorname{Coh}\left(\mathbb{X}^{*}\right)_{\mathrm{p} . \mathrm{g}} \ni \mathfrak{S} \rightarrow \tilde{\mathbb{M}} \ni \tilde{M}_{5}$. Then $\varepsilon_{P}$ satisfies the similar factorization to Figure I, Lemma $4.8_{1}$, and we have the following for each $\mathfrak{S} \in \operatorname{Coh}^{*}\left(\mathbb{X}^{*}\right)_{\mathrm{p} . \mathrm{g}}$ :

$$
i^{*} \Gamma\left(\tilde{U}_{r}(Q), \mathfrak{H} ; \theta_{\mathfrak{g}}^{\prime}\right)_{a} \subset \Gamma\left(\widetilde{U}_{r^{\prime}}(Q), \mathfrak{H} ; \theta_{\mathfrak{g}}\right)_{a^{\prime}},
$$

with $\left(r^{\prime} ; a^{\prime}\right)=\tilde{M}_{\mathfrak{E}}(r ; a)$. Here $(Q ; r ; a)$ is in $\lambda_{P}\left(\subset\left(\tilde{U}-D^{*}\right) \times \mathbb{R}^{+} \times \mathbb{R}_{1}^{+}\right)$.

Lemma 4.4'。 Take a suitable map $\varepsilon_{P}: \mathbb{Z}^{+} \ni \tilde{m} \rightarrow \mathbb{E}_{\text {a.d }}^{\prime} \ni E_{p, \tilde{n}}$ and a linear function $L_{0}=L_{0, P}(t)=c_{0} t ; c_{0}>0$. Then we have the similar factorization to Figure III, Lemma 4.6 and we also have the following for each $(\tilde{m}, m) \in \mathbb{Z}^{+} \times$ $\mathbb{Z}^{+}$satisfying $\tilde{m}>L_{0}(m)(1 \leqq s<p)$ :

$$
\begin{aligned}
i^{*}\left(\Gamma\left(\tilde{U}_{r}(Q), f^{\prime \tilde{m}} \mathfrak{D}_{X^{\prime}}^{(s)}\right)_{a} \cap\right. & \left.\left(F_{p}^{\prime m}\right)^{-1}(0)\right) \\
& \subset F_{p-1}^{\prime m} \Gamma\left(\tilde{U}_{r^{\prime}}(Q), f^{\prime \tilde{m}^{\prime}} \mathfrak{D}_{X^{\prime}-1}^{(p)}\right)_{a^{\prime}},
\end{aligned}
$$

with $\left(r^{\prime} ; \tilde{m}^{\prime} ; a^{\prime}\right)=E_{P, \tilde{m}}(r ; \tilde{m} ; a)$, where $(Q ; r ; a)$ is in $\lambda_{P}$.

Actually, take finite points $\left\{P_{u}\right\}_{u=1}^{u 0}$ in $D^{*}$ and neighborhoods $\tilde{U}_{u}$ of $P_{u}$ in $\bar{X}^{*}$, so that Lemma $4.1^{\prime \prime}$ holds*) for $\left(\tilde{U}_{u}, \tilde{U}_{u} \cap D^{*}, \mathfrak{S}\right)$. Also take finite points $\left\{P_{v}\right\}_{v=1}^{v_{0}^{0}}$ in $X^{*}$ so that Lemma 4.1 holds for*) $\left(\widetilde{U}_{v}, \phi ; \mathfrak{H}\right)$. By the compactness of $\overline{\mathbb{X}}^{*}$, we may assume that $\overline{\mathbb{X}}^{*} \subset\left(U_{u} \tilde{U}_{u}\right) \cup\left(U_{v} \tilde{U}_{v}\right)$. Thus, to each $Q \in \mathbb{X}^{*}$, one can apply Lemma 4.1 or Lemma $4.1^{\prime \prime}$ (resp. Lemma 4.4 or Lemma $4.4^{\prime}$ ); comparing the explicit formulation of Lemma $4.8_{1}$ to that of Lemma 4.1, Lemma $4.1^{\prime \prime}$ we have the implication:

$$
\text { Lemma } 4.1+\text { Lemma } 4.1^{\prime \prime} \longrightarrow \text { Lemma } 4.8_{1} \text {. }
$$

Similarly to the above, we have**):

$$
\text { Lemma } 4.4+\text { Lemma } 4.4^{\prime} \longrightarrow \text { Lemma } 4.8_{2} \text {. }
$$

The proof of Lemma $4.8_{3}$ is given similarly to $(4.8)_{4}$, by taking Lemma 4.5 and Lemma 4.6 as the starting point and by giving the corresponding facts to these lemmas at the point at infinity***). We omit the proof of Lemma $4.8_{3}$.

*) $\tilde{U}_{v}$ is a neighborhood of $P_{v}$ in $\overline{\boldsymbol{X}}^{*}$. Also, for the terminology 'Lemma 4.1 for ( $\tilde{U}_{v}$, $\phi ; \mathfrak{5})$ ', see (4.3) $)_{1}$. We use 'Lemma $4.1^{\prime \prime}$ for $\left(P_{u}, \ldots\right)$ ' in the similar manner.

**) The estimation maps in Lemma $4.4^{\prime}$, Lemma $4.8_{1}$ and in Lemma 4.4 are respectively a series of maps in $\mathbb{E}_{\mathrm{a}, d}^{\prime}$ and in $\mathbb{E}_{\mathrm{a}, d}$. This gap is filled by the comparison of these maps in Remark 4.2 (cf n.6, part B, §4.1).

***) We do not give here the explicit form of these facts. However, it is similar to Lemma 4.4 , and one can give it easily from the proof of Lemma 4.4' soon below in (iii). 
(ii) Check of Lemma $4.1^{\prime \prime}$ and Lemma $4.4^{\prime}$. For the proof of the lemmas in the title, we let $z^{j}=\left(z_{h}^{j}\right)_{h=1}^{n}$ the standard coordinates of $\mathbb{C}_{j}^{n}$ (cf. (4.7) $)_{1}$, and we denote by $T_{j}$ the transformation: $\mathbb{C}^{n} \rightarrow \mathbb{C}_{j}^{n}$ :

$$
z^{j}=T^{j}(z), \quad \text { where } \quad z_{h}^{j}=z_{h} / z_{j}(k=j), z_{j}^{j}=1 / z_{j} .
$$

We then set:

$$
(4.8)_{4}^{\prime \prime}\left\{\begin{array}{l}
T_{i}^{*}:=\left\{\begin{array}{l}
\left.T_{j} \times \text { identity }: \mathbb{C}^{n} \times \mathbb{C}^{n^{\prime}} \longrightarrow \mathbb{C}_{i}^{n} \times \mathbb{C}^{n^{\prime}}\right\}, \\
\left(\mathbb{C}_{j}^{* n}, \mathbb{C}_{j}^{*}\right):=\left\{\begin{array}{l}
\left(\mathbb{C}^{n} \times \mathbb{C}_{j}^{n}\right. \\
\left(\mathbb{C}^{n}, \mathbb{C}_{j}^{n}\right)
\end{array}, \mathbb{C}_{j}^{n} \times \mathbb{C}^{n^{\prime}}\right)
\end{array}\right\}, \\
\left(z^{* j}, z^{*}\right):=\left\{\begin{array}{l}
\left(\tilde{z},\left(z^{j}, z^{\prime}\right)\right) \\
\left(z, z^{i}\right)
\end{array}\right\},\left(d^{*}, d_{j}^{*}\right)=\text { natural distance in }\left(\mathbb{C}^{* n}, \mathbb{C}_{j}^{* n}\right) .
\end{array}\right.
$$

Next we fix an element $j \in[1, \ldots, n]$ satisfying $\mathbb{C}_{j}^{* n} \supset \tilde{U}$. For an element $(Q ; r)$ $\in\left(\tilde{U}-D^{*}\right) \times \mathbb{R}^{+}$we set:

$$
\widetilde{U}_{r}^{j}(Q):=\left\{R \in \mathbb{X}^{*} \cap \mathbb{C}_{j}^{* n} ; d_{j}^{*}(R, Q)<r\right\} .
$$

Then the following distance comparison between $d^{*}$ and $d_{j}^{*}$ is checked easily, by using $(4.8)_{4}^{\prime}$ :

Proposition 4.3. For a suitable positive monomial $M_{P} \in \mathbb{R}$, we have the following for each $(Q ; r) \in\left(\tilde{U}-D^{*}\right) \times \mathbb{R}^{+}$satisfying $r<\left\{\tilde{\sigma} g_{X^{+}}(Q)\right\}^{-1}$, with a suitable $\tilde{\sigma} \in \mathbb{R}_{1}^{+2}$.

$(4.8)_{5} \quad T^{* j}\left(\tilde{U}_{r^{\prime}}(Q)\right) \subset \tilde{U}_{r}^{j}(Q)$, and $T^{* j}\left(\tilde{U}_{r}(Q)\right) \supset \tilde{U}_{r^{\prime}}^{j}(Q)$, with $r^{\prime}=M_{P}(r)$, where the open set $\tilde{U}_{r}(Q)$ is as in Lemma $4.8_{1}$.

Now, Lemma $4.1^{\prime \prime}$ is checked as follows: taking an open set $\hat{U}(\ni P)$ in the ambient space $\mathbb{C}_{j}^{* n}$ of $\tilde{U}\left(\subset \overline{\mathbb{X}}^{*}\right)$ satisfying $\hat{U} \cap \overline{\mathbb{X}^{*}}=\tilde{U}$, we define a local analytic variety $\boldsymbol{X}_{P}^{*} \in \mathbf{A n}_{1 a}\left(\mathrm{cf} .(1.8)_{0}\right)$ by

$$
\mathbb{X}_{P}^{*}:=\left(\mathbb{C}_{j}^{* n}\left(z^{* j}\right), \hat{U}, \tilde{U}, z_{j}^{j}, P_{0}\right),
$$

and we attach the collection $\operatorname{Coh}^{\prime \prime}\left(\mathbb{X}_{P}^{*}\right)_{\text {p.g }}$ to $\mathbb{X}_{P}^{*}$ by $(1.18)_{1}$. Then, remarking that $D^{*}$ is the divisor of $z_{j}^{i}$, we have:

$$
\operatorname{Coh}^{*}\left(\mathbb{X}^{*}\right)_{p \cdot g} \subset \operatorname{Coh}^{\prime \prime}\left(\mathbb{X}_{P}^{*}\right)_{\mathrm{p} \cdot \mathrm{g}}\left(\mathrm{cf} .(4.6)_{0}^{\prime \prime}\right) \text {. }
$$

By this we apply Lemma 4.1 to*) $\mathfrak{H} \in \operatorname{Coh}\left(\mathbb{X}^{*}\right)_{\text {p.g. }}$. Then, we have the similar inclusion to $(4.8)_{1}$, Lemma $4.1^{\prime \prime}$, by changing the open set $\tilde{U}_{r}(P)$, which is

*) Recall that Lemma 4.1 is also applied to such a sheaf $\mathfrak{5}$ by (1), Remark 4.1 at the end of Part A, Section 4.1. 
required in Lemma $4.1^{\prime \prime}$, to $\tilde{U}_{r}^{j}(Q)$ : using Proposition 4.3 , we can replace $\tilde{U}_{r^{\prime}}^{j}(Q)$ by $\tilde{U}_{r^{\prime}}(Q)$, and we have Lemma $4.1^{\prime \prime}$ from Lemma 4.1 .

Next, the check of Lemma $4.4^{\prime}$ is similar to the above, and is as follows: first we remark that

(c) 1

$$
\tilde{f}^{\prime}:=\left(z_{j}^{j}\right)^{d} f^{\prime} \subset \Gamma\left(\tilde{U}, \mathfrak{D}_{\tilde{U}}\right) \text {, with a suitable } d \in \mathbb{Z}^{+},
$$

and that $\tilde{f}^{\prime m} \mathfrak{D}_{\boldsymbol{X}^{\prime}}=f^{\prime m} \mathfrak{D}_{\mathbf{X}^{\prime}}$ in $\tilde{U}-D^{*}$. Then we easily have:

$(\mathrm{c})_{2}$

$$
\begin{aligned}
& \Gamma\left(\tilde{U}_{r}, \tilde{f}^{\prime m} \mathfrak{D}_{\mathbf{X}^{\prime}}\right)_{a} \subset \Gamma\left(\tilde{U}_{r}, f^{\prime m} \mathfrak{D}_{\mathbf{X}^{\prime}}\right)_{a}, \\
& \Gamma\left(\tilde{U}_{r}, f^{\prime m} \mathfrak{D}_{\mathbf{X}^{\prime}}\right)_{a} \subset \Gamma\left(\tilde{U}_{r}, \tilde{f}^{\prime m} \mathfrak{O}_{\mathbf{X}^{\prime}}\right)_{a^{\prime}},
\end{aligned}
$$

where $a^{\prime}=M_{P, m}(a / r)$, with a positive monomial $M_{P, m}$, which is independent from $Q \in \tilde{U}-D^{*}$. (Here we write $\tilde{U}_{r}(Q)$ as $\tilde{U}_{r}$.)

Using a similar argument to the proof of Lemma $4.1^{\prime \prime}$, we see easily that the comparisons of the distance and cochains as in Proposition 4.3 and (c) ${ }_{2}$ lead to the implication:

$$
\text { Lemma } 4.4 \longrightarrow \text { Lemma } 4.4^{\prime} \text {. }
$$

(Here we apply Lemma 4.4 to the set $\tilde{f}^{\prime}$ at the point at infinity $P \in D$.) Thus we checked Lemma 4.1" and Lemma 4.4', and we also have Lemma $4.8_{1}$ and Lemma $4.8_{2}$ (cf. (1)).

4. Finally we add here an elementary uniform estimation on local parametrization of the variety $\boldsymbol{X}=\left(\boldsymbol{C}^{n}(z), U_{0}, X_{0}, X_{0}^{\prime}, P_{0}\right)$ (cf. $\left.(4.1)_{0}\right)$. We set $X=X_{0}-X_{0}^{\prime}$, and we assume that $X_{0}^{\prime}=X_{0, \text { sing. Also taking a suitable open }}$ subset $U_{1, X}\left(\exists P_{0}\right)$ of $U_{0}$ and an element $\sigma_{X} \in \boldsymbol{R}_{1}^{+2}$, we form a parameter space $\boldsymbol{\mu}_{\boldsymbol{X}}:=\left\{(Q ; r) \in\left(U_{1, X} \cap X\right) \times \boldsymbol{R}^{+} ; r<\left\{\sigma_{\boldsymbol{X}} \cdot g_{\boldsymbol{X}}(P)\right\}^{-1}\right\}$, with $g_{\boldsymbol{X}}(P):=d\left(P, X_{0}^{\prime}\right)^{-1}$.

Proposition 4.4. For a suitable positive monomial $M_{\mathbf{X}} \in \mathbf{M}$ we have the following for each $(P ; r) \in \boldsymbol{\mu}_{\mathbf{X}}\left(\subset \boldsymbol{X} \times \boldsymbol{R}^{+}\right)$:

(4.9) there is an analytic map $\omega: U_{r^{\prime}}(P) \rightarrow \widetilde{U}_{r}(P)$, which is the identity on $\tilde{U}_{r^{\prime}}(P)$, with $r^{\prime}:=M_{\mathbf{x}}(r)$. Here $U_{r}(P):=\left\{Q \in C^{n}: d(Q, P)<r\right\}$, and $\tilde{U}_{r}(P)$ : $=U_{r}(P) \cap \boldsymbol{X}$.

We use Proposition 4.4 for the proof of Lemma 1.4 (for the local variety $\boldsymbol{X} \in \mathbf{A} \mathbf{n}_{1 a}$ as in Lemma 1.4). The check of Proposition 4.4 is given in Part A, Apendix I.

Next letting the affine variety $\boldsymbol{X}^{\prime} \subset \boldsymbol{C}^{n}(z)$ and the divisor $S \subset \boldsymbol{C}^{n} \times U_{0}$ be as in Lemma 1.4 and Lemma 1.3, we take elements $\sigma=\sigma_{\mathbf{X}^{\prime}}, \tilde{\sigma}=\tilde{\sigma}_{S} \in \boldsymbol{R}_{1}^{+2}$ and an 
open subset $U^{\prime}$ of $U_{0}$, and we form parameter spaces:

$$
\begin{aligned}
& \boldsymbol{\mu}_{\mathbf{X}^{\prime}}:=\left\{(P ; r) \in \boldsymbol{X}^{\prime} \times \boldsymbol{R}^{+} ; r<\left\{\sigma g_{\mathbf{X}^{\prime}}(P)\right\}^{-1}\right\}, \text { and } \\
& \boldsymbol{\mu}_{S}:=\left\{(P ; r) \in\left(S \cap\left(\mathbb{C} \times U_{0}\right)\right) \times \mathbb{R}^{+} ; r<\left\{\tilde{\sigma} g_{S}(P)\right\}^{-1}\right\},
\end{aligned}
$$

where the p.g. function $g_{\boldsymbol{X}^{\prime}}$ of $\boldsymbol{X}^{\prime}$ is as in Lemma 1.4, and we set $g_{S}:=|w|$, with the coordinate of $\boldsymbol{C}$ (cf. Lemma 1.3).

We then have the following analogue of Proposition 1.4 for $\mathbb{X}^{\prime}$ and $S$.

Proposition 4.4'. For suitable positive monomials $M_{\mathbf{X}^{\prime}}, M_{S}$, we have the following for each $(P ; r) \in \mu_{X^{\prime}}$ and $\left(P^{\prime} ; r\right) \in \mu_{S}$ :

$(4.9)^{\prime}$ there are analytic maps $\omega: U_{r^{\prime}}(P) \rightarrow\left(U_{r}(P) \cap X^{\prime}\right)$ and $\omega^{\prime}: U_{r^{\prime \prime}}\left(P^{\prime}\right)$ $\rightarrow\left(U_{r}\left(P^{\prime}\right) \cap S\right)$, which are the identities on $\left(U_{r^{\prime}}(P) \cap X^{\prime}\right),\left(U_{r^{\prime \prime}}\left(P^{\prime}\right) \cap S\right)$, where $r^{\prime}=M_{X^{\prime}}(r)$ and $r^{\prime \prime}=M_{S}(r)$. Also the discs $U_{r}(P), U_{r}\left(P^{\prime}\right)$ in $\mathbb{C}^{n}(z), \mathbb{C}(w) \times \mathbb{C}^{n}(z)$ have the similar meaning to the one $U_{r}(P)$ in Proposition 4.4.

We use Proposition $4.4^{\prime}$ for the proof of Lemma 1.4 for $\mathbb{X}^{\prime}$ and of Lemma 1.3.

Proof. Let $\overline{\boldsymbol{X}}^{\prime}$ and $\bar{S}$ denote the completions of $\mathbb{X}^{\prime}, S$ in $\mathbb{P}^{n}(\mathbb{C}), \mathbb{P}(\mathbb{C})$ $\times U_{0}$. Then, taking points $P_{0} \in \bar{X}^{\prime}-X^{\prime}$ and $P_{0}^{\prime} \in(P(\mathbb{C})-\mathbb{C}) \times U_{0}$, we have the similar fact to Proposition 4.4 for $\left(\overline{\boldsymbol{X}}^{\prime}, P_{0}\right)$ and $\left(\bar{S}, P_{0}^{\prime}\right)$ (using similar arguments to Lemma 4.1"). Then, using the distance comparison, Proposition 4.3, and the similar arguments to the ones in n.3, we have Proposition $4.4^{\prime}$ from the above analogues of Proposition 4.4 at the points at infinity $P_{0}, P_{0}^{\prime}$ and from Proposition 4.4 (applied to finite points $P \in X^{\prime}$ and $P^{\prime} \in S$ ).

q.e.d.

\section{\$4.2. Proof of the Lemmas in Chapter $\mathbb{I}$}

In n.1-n.3 we give a cohomological version of Lemmas 4.1-4.8, and, using such a result, we prove the lemmas in Chapter I, Lemma 1.2 and Lemma 2.3, Lemma 2.5 as well as Lemma 2.7, which concern the uniform estimations on the sheaf homomorphisms. Also, using the results in n.1-n.3, we prove Proposition 4.2 in n.4. Moreover, we prove Lemma 1.3, 1.4 in n.5, n.6, by using Proposition 4.4.

1. Comparison of cohomological and non cohomological estimations. Here we give propositions, which play a key role in the proof of the first set of the lemmas just above (cf. Proposition $4.5_{1,2}$ ). Such propositions will be given in an abstract manner in terms of q-sheaves and is more general than the one 
used in the proof of the lemmas. In n.1 we fix q-sheaves*) $\left(\mathfrak{R}, 0_{\Re}\right),\left(\mathfrak{H}, \theta_{\mathfrak{5}}\right)$ and a homomorphism $\omega: \Omega \rightarrow \mathfrak{S}$ (of abelian sheaves). Also we fix a p.g. function $g: X \rightarrow \boldsymbol{R}_{1}^{+}$and a distance function $d: X \times X \rightarrow[0, \infty]$ satisfying $d=0$ on the diagonal $\Delta_{X}(\subset X \times X)$ (cf. Definition 1.4 4 and Definition 1.6 1 ). Moreover, we fix an element $\tilde{\sigma} \in \mathbb{R}_{1}^{+2}$, and we assume the following for each $P \in X$ :

$$
\begin{aligned}
& g(Q) / 2<g(P)<2 g(Q) \\
& \quad \text { for each } Q \in U_{\tilde{\sigma}}(P ; g):=\left\{Q \in X ; d(P, Q)<\{\tilde{\sigma} g(P)\}^{-1}\right\},
\end{aligned}
$$

$(4.10)_{0}^{\prime}$ the triangle inequality: $d\left(Q_{1}, Q_{3}\right) \leqq d\left(Q_{1}, Q_{2}\right)+d\left(Q_{2}, Q_{3}\right)$ : holds for any $Q_{i} \in U_{\tilde{\sigma}}(P ; g)(1 \leqq i \leqq 3)$.

For a point $P \in X$ and an element $(r ; a) \in \mathbb{R}^{+} \times \boldsymbol{R}_{1}^{+}$we set:

$(4.10)_{0}^{\prime \prime} \quad \Gamma\left(\tilde{U}_{r}(P), \mathfrak{H} ; \theta_{\mathfrak{n}}\right)_{a}:=\left\{\varphi \in \Gamma\left(\tilde{U}_{r}(P), \mathfrak{H}\right) ;|\varphi(Q)|_{\mathfrak{g}}<a \quad\right.$ in $\left.\quad \tilde{U}_{r}(P)\right\}$, where ||$_{\mathfrak{E}}=\theta_{\mathfrak{n}}$-absolute value*) and $\tilde{U}_{r}(P):=\{Q \in X ; d(P, Q)<r\}$.

(We use the similar notation for $\left(\Omega, \theta_{\Omega}\right)$.) Also, for the formulation of Propositions $4.5_{1,2}$, we fix a subset $Y$ of $X$, and we form the following parameter spaces (cf. also $\left.(4.1)_{4}\right)$ :

$$
\mu_{Y}:=\left\{(P ; r) \in Y \times \mathbb{R}^{+} ; r<\{\tilde{\sigma} g(P)\}^{-1}\right\}, \lambda_{Y}:=\mu_{Y} \times \mathbb{R}_{1}^{+} .
$$

(i) Bdd estimations $\rightarrow$ p.g. estimations**). First taking an estimation map $\tilde{M}=\left(M_{1}, M_{2}\right) \in \tilde{\mathbb{M}}=\mathbf{M} \times \mathbb{M}$ (cf. 11.1, §4.1), we assume the following uniform estimation for $\omega: \mathfrak{R \rightarrow \mathfrak { S }}$ on $Y$ :

$$
i^{*} \Gamma\left(\tilde{U}_{r}(P), \mathfrak{S} ; \theta_{\mathfrak{g}}\right)_{a} \subset \omega \Gamma\left(\tilde{U}_{r^{\prime}}(P), \mathfrak{A} ; \theta_{\Re}\right)_{a^{\prime}},
$$

with $\left(r^{\prime} ; a^{\prime}\right)=\tilde{M}(r ; a)$, where $(P ; r ; a)$ is in $\lambda_{Y}\left(\subset Y \times \boldsymbol{R}^{+} \times \boldsymbol{R}_{1}^{+}\right)$and $i=$ inclusion: $\tilde{U}_{r \cdot}(P) \hookrightarrow \tilde{U}_{r}(P)$.

Note that $(4.10)_{1}$ is of similar form to the estimation in Lemma $4.8_{1}$. In Proposition $4.5_{1}$ soon below we give a cohomological version of $(4.10)_{1}$, which is of similar form to the estimations in Lemma 1.2. In Proposition 4.5 1 we fix an estimation map***) $\tilde{\mathscr{L}} \in \tilde{\mathbb{L}}: \boldsymbol{R}^{+2} \times \mathbb{R}^{+2} \rightarrow \mathbb{R}^{+2} \times \mathbb{R}^{+2}$, which is determined by $\tilde{M} \in \tilde{\text { M. }}$. (For the explicit dependence of $\tilde{\mathscr{L}}$ on $\tilde{M}$, see $(4.10)_{7}$, (iii) in the proof of Proposition $4.5_{1}$.)

Proposition $4.5_{1}$ (Bdd estimations $\rightarrow$ p.g. estimations). For each element

*) Cf. Definition $1.4_{1}$.

**) The estimations of the left and right sides in the title of (i), (ii), concern respectively non cohomological and cohomological uniform estimations (cf. Lemmas $4.8_{1-3}$ and Lemma 1.2, Lemmas $2.5,2.7 \ldots$ ).

*:**) $\tilde{\mathbf{L}}=\mathbb{L}: \mathbf{L}$, with the collection $\mathbf{L}$ of all el-maps (cf. n.5, §1.2). 
$\left(Y^{\prime} ; \sigma ; \alpha\right) \in 2^{Y} \times \mathbb{R}_{\tilde{\sigma}}^{+2} \times \mathbb{R}_{1}^{+2}$ we have:

$(4.10)_{2} s^{*} C^{q}\left(\mathscr{A}_{\sigma}\left(Y^{\prime}\right), \mathfrak{H} ; \theta_{\tilde{n}}\right)_{\alpha} \subset \omega C^{q}\left(\mathscr{A}_{\sigma^{\prime}}\left(Y^{\prime}\right), \Re: \theta_{\Re}\right)_{\alpha^{\prime}}$, with $\left(\sigma^{\prime} ; \alpha^{\prime}\right)=\tilde{\mathscr{L}}(\sigma ; \alpha)$, where

$(4.10)_{2}^{\prime}\left\{\begin{array}{c}\mathscr{A}_{\sigma}\left(Y^{\prime}\right) \\ C^{q}\left(\mathscr{A}_{\sigma}\left(Y^{\prime}\right), \mathfrak{S} ; \theta_{\mathfrak{s}}\right)_{\alpha}\end{array}\right\}:=\left\{\begin{array}{l}g-p . g . \text { covering of } Y^{\prime} \text { in } X \text { of size } \sigma \text { (Defini- } \\ \left.\text { tion } 1.6_{1}\right), \text { set of }\left(g, \theta_{\tilde{n}}\right)-\alpha-\text { growth cochains } \\ \text { with value in } \mathfrak{H}\left(c f^{\prime} .(1.3)_{6}\right) .\end{array}\right.$

Moreover, $s$ is the p.g. refining map: $\mathscr{A}_{\sigma^{\prime}}\left(Y^{\prime}\right) \hookrightarrow \mathscr{A}_{\sigma}\left(Y^{\prime}\right)\left(c f\right.$. Definition 1.6 $\left.6_{2}\right)$.

We prove Proposition $4.5_{1}$ in (iii). In n.2 we use Proposition $4.5_{1}$ for the proof of Lemma 1.2.

(ii) A.d. estimations $\rightarrow$ d.p. estimations*).

We give here a key proposition, Proposition $4.5_{2}$, for the implication:

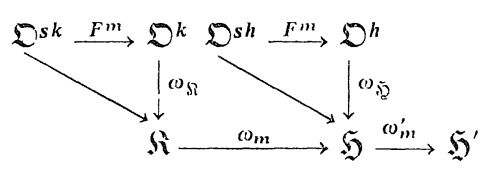

Figure I.

$(4.10)_{3}^{\prime \prime \prime} \quad$ Lemmas $4.8_{2,3} \longrightarrow$ Lemmas 2.5, 2.7 and Lemma 2.3.

For this we fix a sheaf $\mathfrak{D}$ of ring over $X$, and we assume that (1) $\mathfrak{R}, \mathfrak{S}$ are $\mathfrak{D}$ modules, and (2) $\mathfrak{R}, \mathfrak{5}$ ) are homomorphic images of $\mathfrak{D}^{k}, \mathfrak{D}^{h}(h, k>0): \mathfrak{D}^{k} \stackrel{\omega_{\Omega}}{\longrightarrow} \mathfrak{\Omega}$ $\rightarrow 0, \mathfrak{D}^{h} \stackrel{\omega_{\mathfrak{B}}}{\longrightarrow} \mathfrak{S} \rightarrow 0$. Moreover, we fix an abelian sheaf $\mathfrak{S}^{\prime}$ and series of homomorphisms $\omega_{m}: \mathfrak{S} \rightarrow \mathfrak{H}, \omega_{m}^{\prime}: \mathfrak{H} \rightarrow \mathfrak{S}^{\prime}(m=1,2, \ldots)$ satisfying $\omega_{m}^{\prime} \cdot \omega_{m}=0$. Furthermore, we fix a subset $f=\left(f_{j}\right)_{j=1}^{s} \subset \Gamma\left(X, \mathfrak{D}_{X} ; g\right)_{\mathrm{p} . \mathrm{g}}$ (cf. $\left.(1.3)_{1}^{\prime \prime \prime}\right)$, and we use the symbol $F^{m}(=m$-th homomorphism for $f): \mathfrak{D}^{s} \rightarrow \mathfrak{D}$ also for its $k$-and $h$-direct

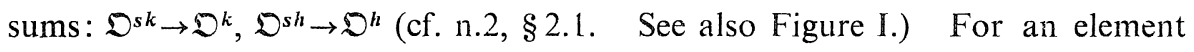
$(P ; r ; \tilde{m} ; a) \in X \times \mathbb{R}^{+} \times \mathbb{Z}^{+} \times \mathbb{R}^{+}$we set:

$(4.10)_{3}^{\prime \prime} \quad \Gamma\left(\tilde{U}_{r}(P), f^{\tilde{m}} \Re\right)_{a}:=\omega_{\Omega} F^{\tilde{m}} \Gamma\left(\tilde{U}_{r}(P), D^{s h}\right)_{a}$ (cf. $(4.10)_{0}^{\prime \prime}$ and Figure I).

(We use the similar notation for $\mathfrak{g}$.) Now taking a linear function $L_{0}(t)=c_{0} t$; $c_{0}>0$ and an a.d. map $E^{\prime} \in \mathbb{E}_{\text {a.d }}$ (cf. n.1, §4.2), we assume the following uniform estimations for the series $\left\{\omega_{m}\right\},\left\{\omega_{m}^{\prime}\right\}\left(m \in \mathbb{Z}^{+}\right)$:

$(4.10)_{3}$ For each $(\tilde{m}, m) \in \mathbb{Z}^{+} \times \mathbb{Z}^{+}$satisfying $\tilde{n}>L_{0}(m)$ we have:

$(4.10)_{3}^{\prime} \quad i^{*}\left(\Gamma\left(\widetilde{U}_{r}(P), f^{\tilde{m}} \mathfrak{S}\right)_{a} \cap \omega_{m}^{\prime-1}(0)\right) \subset \omega_{m} \Gamma\left(\tilde{U}_{r^{\prime}}(P), f^{\tilde{m}^{\prime}} \Re\right)_{a^{\prime}}$,

with $\left(r^{\prime} ; \tilde{m}^{\prime} ; a^{\prime}\right)=E^{\prime}(r ; \tilde{m} ; a)$, where $(P ; r ; a)$ is in $\lambda_{Y}\left(\subset Y \times \mathbb{R}^{+} \times \mathbb{R}_{1}^{+}\right)(c f$. $\left.(4.10)_{0}^{\prime \prime \prime}\right)$.

Note that $(4.10)_{3}$ is a similar inclusion to Lemma 4.4. In Proposition $4.5_{2}$ soon below, taking a d.p. estimation map $E \in \mathbb{E}_{\text {d.p }}($ cf. n.4, §2.1)*), we give a

*) See the footnote $* *)$, p. 454 . 
cohomological version of $(4.10)_{3}$, which is similar to Lemma $4.8_{2}$ :

Proposition $4.5_{2}$ (A.d. estimation $\rightarrow$ d.p. estimation). For each $(\tilde{m}, m) \in \mathbb{Z}^{+}$ $\times Z^{+}$satisfying $\tilde{m}>L_{0}(m)$ we have:

$(4.10)_{4} \quad s^{*}\left(C_{l}^{q}\left(\mathscr{A}_{\sigma}\left(Y^{\prime}\right), f^{\tilde{m} \mathfrak{G}}\right)_{\alpha} \cap \omega_{m}^{\prime-1}(0)\right) \subset \omega_{m} C_{l}^{q}\left(\mathscr{A}_{\sigma^{\prime}}\left(Y^{\prime}\right), f^{\tilde{m}^{\prime} \mathfrak{R}}\right)_{\alpha^{\prime}}$, with $\left(\sigma^{\prime} ; \tilde{m}^{\prime}\right.$; $\left.\alpha^{\prime}\right)=E(\sigma ; m ; \alpha)$, where $\left(Y^{\prime} ; \sigma ; \alpha\right)$ is in $2^{Y} \times \boldsymbol{R}_{\tilde{\sigma}}^{+2} \times \boldsymbol{R}_{1}^{+2}$, and

$(4.10)_{4}^{\prime} \quad C_{l}^{q}\left(\mathscr{A}_{\sigma}\left(Y^{\prime}\right), f^{m} \mathfrak{H}\right)_{\alpha}:=$ left $(\not \rho, g)$-d.p. filtered set of cochains with value in $\mathfrak{H}\left(c f .(2.3)_{3,5}\right)$.

(iii) Proof of Propositions 4.5 , $^{\circ}$ (1) Letting the positive monomials $M_{1}$, $M_{1}^{\prime}$ be the first parts of the estimation maps $\bar{M}, E^{\prime}$ in $(4.10)_{1,3}$, we first define el-maps $\overline{\mathscr{L}}_{1}, \overline{\mathscr{L}}_{1}^{\prime}: \boldsymbol{R}^{+2} \ni \sigma \rightarrow \boldsymbol{R}^{+2} \ni \sigma^{\prime}, \sigma^{\prime \prime}$ by the equation:

(4.10) $)_{5}^{\prime}\left(\sigma^{\prime} t\right)^{-1}=M_{1}\left((\sigma t)^{-1}\right),\left(\sigma^{\prime \prime} t\right)^{-1}=M_{1}^{\prime}\left((\sigma t)^{-1}\right)$, where $t$ is a variable. Then taking an el-map $\mathscr{L}_{0}: \boldsymbol{R}^{+2} \ni\left(\alpha_{1}, \alpha_{2}\right) \rightarrow \boldsymbol{R}^{+2} \ni\left(4 \alpha_{1} 2^{\alpha_{2}}, \alpha_{2}\right)$, we define:

$$
\mathscr{L}_{1}=\mathscr{L}_{0} \cdot \overline{\mathscr{L}}_{1} \cdot \mathscr{L}_{0}, \quad \mathscr{L}_{1}^{\prime}=\mathscr{L}_{0} \cdot \overline{\mathscr{L}}_{1}^{\prime} \cdot \mathscr{L}_{0}
$$

Such el-maps will be the first components of the desired estimation maps $\tilde{\mathscr{L}}, E$ in Propositions $4.5_{1,2}$ (cf. (4.10) $)_{7}$ ). Then, letting $\sigma \in \boldsymbol{R}_{1}^{+2}$ be as in Propositions 4.5 1,2 , we set:

$(4.10)_{5}^{\prime \prime} \quad \sigma^{\prime \prime}=\mathscr{L}_{0}(\sigma), \tilde{\sigma}^{\prime \prime}=\tilde{\mathscr{L}}_{1}\left(\sigma^{\prime \prime}\right)$ and $\tilde{\sigma}^{\prime}=\mathscr{L}_{0}\left(\tilde{\sigma}^{\prime \prime}\right)\left(=\tilde{\mathscr{L}}_{1}(\sigma)\right)$, where $\left(\tilde{\mathscr{L}}_{1}, \tilde{\mathscr{L}}_{1}\right)=$ $\left(\overline{\mathscr{L}}_{1}, \mathscr{L}_{1}\right)$ or $\left(\overline{\mathscr{L}}_{1}^{\prime}, \mathscr{L}_{1}^{\prime}\right)$.

Next taking an element $\mathscr{A}^{\prime} \in \mathscr{N}^{q+1} \mathscr{A}_{\tilde{\sigma}^{\prime}}\left(Y^{\prime}\right)$ (cf. Propositions 4.5 1,2 ) and a point $Q \in\left|\mathscr{A}^{\prime}\right|$, we set: $\mathscr{A}=s\left(\mathscr{A}^{\prime}\right) \in \mathscr{N}^{q+1} \mathscr{A}_{\sigma}\left(Y^{\prime}\right)$, where $s=$ p.g. refining map: $\mathscr{A}_{\tilde{\sigma}^{\prime}}\left(Y^{\prime}\right)$ $\hookrightarrow \mathscr{A}_{\sigma}\left(Y^{\prime}\right)$ (Definition $1.6_{2}$ ). Then we have the following from (4.10)" and $(4.10)_{0},(4.10)_{0}^{\prime}$ :

(4.10) ${ }_{5}^{\prime \prime \prime}|\mathscr{A}| \supset \tilde{U}_{r}(Q) \supset \tilde{U}_{r^{\prime}}(Q) \supset\left|\mathscr{A}^{\prime}\right|$, where $r=\left\{\sigma^{\prime \prime} g(Q)\right\}^{-1}, r^{\prime}=\left\{\tilde{\sigma}^{\prime \prime} g(Q)\right\}^{-1}$.

(Note that, by $(4.10)_{5}^{\prime \prime}$, we have: $r^{\prime}=\tilde{M}_{1}(r)$, with $\tilde{M}_{1}=M_{1}$ or $M_{1}^{\prime}$ (cf. $(4.10)_{1,3}$ ). The relation (4.10) $)_{5}^{\prime \prime \prime}$ will be a key fact for the proof of Propositions $4.5_{1,2}$.

(2) Now taking elements $\varphi \in C^{q}\left(\mathscr{A}_{\sigma}\left(Y^{\prime}\right), \mathfrak{H} ; \theta_{\mathfrak{g}}\right)_{\alpha}$ and $\tilde{\Psi} \in C_{l}^{q}\left(\mathscr{A}_{\sigma}\left(Y^{\prime}\right)\right.$, $\left.f^{\tilde{m}} \mathfrak{H}\right)_{\alpha}$ satisfying $\omega_{m}^{\prime} \tilde{\Psi}=0$, we write $\tilde{\Psi}$ explicitly as $\tilde{\Psi}=\omega_{\mathfrak{Y}} F^{m} \Psi$, with $\Psi \in C^{q}\left(\mathscr{A}_{\sigma}\left(Y^{\prime}\right), \mathfrak{D}^{s k}\right)_{\alpha}$ (cf. $\left.(4.10)_{2,4}\right)$. Then from $(4.10)_{0}$ we have :

$(4.10)_{6}^{\prime \prime}\left|\varphi_{\mathscr{A}}(R)\right|,\left|\Psi_{\mathscr{A}}(R)\right|<a:=\alpha^{\prime} \cdot g(Q)$ in $\tilde{U}_{r}(Q)$, where $\alpha^{\prime}=\left(\alpha_{1} 2^{\alpha_{2}}, \alpha_{2}\right)$ with $\alpha=\left(\alpha_{1}, \alpha_{2}\right)$.

*) As in Proposition $4.5_{1}$, the map $E \in \mathbf{E}_{\mathrm{d} . \mathrm{p}}$ is determined by $E^{\prime} \in \mathbf{E}_{\mathrm{a}, \mathrm{d}}$ by in a simple fashion (cf. $\left.(4.10)_{7}\right)$. 
We will apply $(4.10)_{1,3}$ to $\varphi_{\mathscr{A}}, \tilde{\Psi}_{\mathscr{A}}$ in $\tilde{U}_{r}(Q)$. Then there are elements $\varphi_{1}$ $\in \Gamma\left(\tilde{U}_{r^{\prime}}(Q), \Re\right)_{a^{\prime}}$ and $\tilde{\Psi}_{1} \in \Gamma\left(\tilde{U}_{r^{\prime \prime}}(Q), \ell^{\tilde{m}^{\prime}} \mathfrak{R}\right)_{a^{\prime \prime}}$ satisfying $(4.10)_{6}^{\prime} \quad \varphi_{\mathscr{A}}=\omega \varphi_{1}, \tilde{\Psi}_{\mathscr{A}}=\omega_{m} \tilde{\Psi}_{1}$, with $\left(r^{\prime} ; a^{\prime}\right)=\tilde{M}(r ; a)$ and $\left(r^{\prime \prime} ; \tilde{m}^{\prime} ; a^{\prime \prime}\right)=E^{\prime}(r ;$ $\tilde{m} ; a)$, where the estimation maps $\tilde{M} \in \tilde{\mathbf{M}}$ and $E^{\prime} \in \mathbb{E}_{\text {a.d }}$ are as in $(4.10)_{1-3}$.

We write $\tilde{\Psi}_{1}$ as $\tilde{\Psi}_{1}=\omega_{K} F^{m} \Psi_{1}$, with $\Psi_{1} \in \Gamma\left(\tilde{U}_{r^{\prime \prime}}(Q), D^{s k}\right)_{a^{\prime \prime}}$ (cf. Figure I). We note that $(4.10)_{6}^{\prime}$ implies :

$$
\left|\varphi_{1}\right|<a^{\prime} \text { and }\left|\Psi_{1}\right|<a^{\prime \prime} .
$$

Then from that $r=\left\{\tilde{\sigma}^{\prime \prime} g(Q)\right\}^{-1}$ (cf. $\left.(4.10)_{5}\right)$ and (4.10) $)_{0}$, together with the explicit forms of the estimation maps $\tilde{M}, E^{\prime}$, we easily see that $(4.10)_{6}^{\prime \prime}$ is rewritten in the form:

$(4.10)_{6} \quad\left\{\begin{array}{l}\left|\varphi_{1}(R)\right|<\alpha^{\prime} \cdot g(R) \\ \left|\Psi_{1}(R)\right|<\alpha^{\prime \prime} \cdot g(R)\end{array}\right\}$ with $\left\{\begin{array}{l}\alpha^{\prime}=\mathscr{L}_{2}(\alpha+\sigma) \\ \alpha^{\prime \prime}=\exp M(\tilde{m}) \cdot \mathscr{L}_{2}^{\prime}(\alpha+\sigma)\end{array}\right\}$,

where the el-maps $\mathscr{L}_{2}, \mathscr{L}_{2}^{\prime}$ and the positive monomial $M$ are determined by the maps $\tilde{M}, E^{\prime}$ in $(4.10)_{3}$.

Finally, letting $L: \mathbb{Z}^{+} \rightarrow \mathbb{Z}^{+}$be the a.d. part of $E^{\prime}$ (cf. $(4.2)_{1}$ ), we define a map $\tilde{\mathscr{L}} \in \tilde{\mathbf{L}}=\mathbf{L} \times \mathbf{L}$ and a d.p.c. map $E \in \mathbb{E}_{\text {d.p }}$ by the following.

$$
\left\{\begin{aligned}
\tilde{\mathscr{L}}: \mathbb{R}^{+2} \times \mathbb{R}^{+2} \ni(\sigma ; \alpha) \longrightarrow \mathbb{R}^{+2} \times \mathbb{R}^{+2} \ni\left(\mathscr{L}_{1}(\sigma), \mathscr{L}_{2}(\alpha+\sigma)\right) \\
E: \boldsymbol{R}^{+2} \times \mathbb{R}^{+2} \times \mathbb{Z}^{+} \ni(\sigma ; \alpha ; \tilde{m}) \longrightarrow \mathbb{R}^{+2} \times \mathbb{R}^{+2} \times \mathbb{Z}^{+} \ni\left(\mathscr{L}_{1}^{\prime}(\sigma),\right. \\
\left.\exp M(m) \cdot \mathscr{L}_{2}^{\prime}(\alpha+\sigma), L(\tilde{m})\right) .
\end{aligned}\right.
$$

We take the estimation maps $\tilde{L}$ and $E$ to be the desired ones in Propositions $4.5_{1,2}$. Then remarking that the restrictions $\varphi^{\prime}, \widetilde{\Psi}^{\prime}$ of $\varphi_{1}, \widetilde{\Psi}_{1}$ to $\mathscr{A}^{\prime}$ satisfy:

$$
s^{*} \varphi=\omega \varphi^{\prime} \quad \text { and } \quad s^{*} \tilde{\Psi}=\omega_{m} \widetilde{\Psi}^{\prime},
$$

we have $(4.10)_{2,4}$.

q.e.d.

Proposition $4.5_{2}$ will be used in the proof of Lemma 2.5, when the variety is the local one $\boldsymbol{X} \in \mathrm{An}_{1 a}$ (cf. n.3, §4.2). Here we give a slice modification of Proposition $4.5_{2}$, which is used in the proof of the other lemmas in Section 2.

Remark 4.3. (i) First take a series $\left\{F_{\tilde{m}}\right\}_{\tilde{m}=1}^{\infty}$ of estimation maps $F_{\tilde{m}} \in \mathbb{E}_{\text {a.d }}^{\prime}$ (cf. $(4.2)_{7}^{\prime \prime}$ ), which satisfies the similar factorization to Figure III, Lemma 4.6, and we make the following change of the estimation in $(4.10)_{3}^{\prime}$ :

$$
(r ; \tilde{m} ; a) \longrightarrow\left(r^{\prime} ; \tilde{m}^{\prime} ; a^{\prime}\right)=F_{\tilde{m}}(r ; \tilde{m} ; a) .
$$


Then, letting the el-map $\mathscr{L}_{1}^{\prime}: \boldsymbol{R}^{+2} \rightarrow \boldsymbol{R}^{+2}$ and the linear map $L$ be as in $(4.10)_{5,7}$, we have the following inclusion, which is similar to $(4.10)_{4}$, from the arguments in the proof of Proposition $4.5_{2}$ (cf., in particular, $(4.10)_{5-8}$ ):

$(4.10)_{9} \quad s^{*}\left(C_{1}^{q}\left(\mathscr{A}_{\sigma}\left(Y^{\prime}\right), \ell^{\tilde{m}} \mathfrak{H}\right)_{\mathrm{p} . \mathrm{g}} \cap \omega_{m}^{\prime-1}(0)\right) \subset \omega_{m} C_{1}^{q}\left(A_{\alpha^{\prime}}\left(Y^{\prime}\right), f^{\tilde{m}^{\prime}} \mathfrak{R}\right)_{\mathrm{p} . \mathrm{g}}$, with $\left(\sigma^{\prime} ; \tilde{m}^{\prime}\right)$ $=\left(\mathscr{L}_{1}^{\prime}(\sigma),[L(i \tilde{n})]\right)$. (For the p.g. subgroup as above, see $(2.3)_{6}$.)

We use the above remark in the proof of Lemma 2.5, when the variety is $\boldsymbol{X}^{\prime} \in \mathbf{A f f}$.

(2) Next we assume that the homomorphisms $\omega_{m}, \omega_{m}^{\prime}$ in Proposition $4.5_{2}$ are independent of $m \in \mathbb{Z}^{+}: \omega=\omega_{1}=\omega_{2}=, \ldots$ and $\omega^{\prime}=\omega_{1}^{\prime}=\omega_{2}^{\prime}=, \ldots$ Also take an element $\tilde{m} \in \mathbb{Z}^{+}$. Then, assuming the similar inclusion to $(4.10)_{3}^{\prime}$ for each*) $\tilde{m} \geqq \bar{m}$, we obviously have the similar inclusion to $(4.10)_{4}$ for such $\tilde{m} \in \mathbb{Z}^{+}$. We use this fact for the proof of Lemma 2.7 (given to $\mathbb{X} \in \mathbf{A n}_{1 a}$ ). Finally, we assume that the similar inclusion to $(4.10)_{3}$ holds for each $\tilde{m} \geqq \bar{m}$, by changing the estimation in $(4.10)_{3}$ to $(4.10)_{9}$. Then we have the similar inclusion to $(4.10)_{9}$ for each $m \geqq \bar{m}$. We use this for the proof of Lemma 2.7 (given to $\mathbb{X}^{\prime} \in \mathbf{A f f}$ ). (We also use a slice modification of Proposition $4.5_{2}$ in the proof of Lemma 2.3. Such a modification is given in the proof of Lemma 2.3 in $n .3, \S 4.2$.)

2. Proof of Lemma 1.2. Here we derive Lemma 1.2 from Lemma 4.1, Lemma $4.8_{1}$, by using Proposition $4.5_{1}$ :

$(4.11)_{1}$ non cohomological estimation in Lemma 4.1, Lemma 4.8 $\stackrel{\text { Prop.4.51 }}{\longrightarrow}$ cohomological estimation in Lemma 1.2.

For this we set:

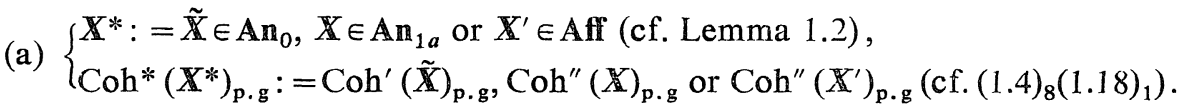

Also we denote by $\varepsilon_{\mathbf{X}^{*}}$ the map: $\operatorname{Coh}^{*}\left(\boldsymbol{X}^{*}\right)_{\mathrm{p} . \mathbf{g}} \ni \mathfrak{H} \rightarrow \mathbb{M} \ni M_{\text {占 }}$ as in Lemma $4.1^{* *)}$ or Lemma $4.8_{1}$ (according as $\boldsymbol{X}^{*}=\boldsymbol{X}$ or $\tilde{\boldsymbol{X}}, \boldsymbol{X}^{\prime}$ ). Moreover, we set:

(b) $\tilde{\boldsymbol{Y}}^{*}:=\boldsymbol{C}^{n} \times U_{1}^{\prime}(\subset \tilde{\boldsymbol{X}}), U_{1} \cap \boldsymbol{X}(\subset \boldsymbol{X})$ or $\boldsymbol{X}^{\prime}$. (For the open set $U_{1}^{\prime} \subset \mathbb{C}^{n^{\prime}}$, $U_{1} \subset C^{n}$, see $(4.1)_{4}$ and $(4.7)_{2}$.)

Then one can apply, to each $\mathfrak{H} \in \operatorname{Coh}^{*}\left(\boldsymbol{X}^{*}\right)_{\text {p.g }}$ and $Q \in \tilde{Y}^{*}$, the estimation in the left side of $(4.11)_{1}$ (cf. also the explicit formulations in Lemma 4.1, Lemma

*) Precisely, we replace the inequality: $\tilde{m}>L_{0}(m)$ in $(4.10)_{3}$ by $\tilde{m} \geqq \bar{m}$.

**) When we apply Lemma 4.1 to $\boldsymbol{X}^{*}=\boldsymbol{X} \in \mathbf{A n}_{1 a}$, we understand that $\boldsymbol{X}=X_{0}-X_{0}^{\prime}$ in Lemma 4.1 is of the form: $\boldsymbol{X}=X_{0}-D$, where the divisor $D$ of $X_{0}$ is as in Section 1. 
4.8 $)$. Now let the manifold*) $\mathbb{Y}^{*}:=\tilde{\mathbb{X}}_{r}\left(P^{\prime}\right), \mathbb{X}_{r}(P)$ or $\mathbb{X}^{\prime}$, the p.g. covering $A_{\sigma}\left(Y^{*}\right)$ and the sets of the cochains $C^{q}\left(\mathscr{A}_{\sigma}\left(\mathbb{Y}^{*}\right), \mathfrak{H} ; \Psi\right)_{\alpha}, C^{q}\left(\mathscr{A}_{\sigma}\left(\mathbb{Y}^{*}\right), \mathfrak{H} ; \Psi^{\prime}\right)_{\alpha}$ be as in Lemma 1.2. Then, by applying Proposition $4.5_{1}$ to the non cohomological estimation mentioned soon above, we get the desired inclusion of the cohomological form in Lemma 1.2 (cf. §1.3):

(c) $s^{*} C^{q}\left(\mathscr{A}_{\sigma}\left(Y^{*}\right), \mathfrak{5} ; \Psi^{\prime}\right)_{\alpha} \subset C^{q}\left(\mathscr{A}_{\sigma^{\prime}}\left(Y^{*}\right), \mathfrak{S} ; \Psi\right)_{\alpha^{\prime}}$, where $\left(\sigma^{\prime} ; \alpha^{\prime}\right)=\mathscr{L}_{\mathfrak{L}}(\sigma ; \alpha)$, with the element $\mathscr{L}_{\mathfrak{5}} \in \tilde{\mathbb{L}}(=\mathbb{L} \times \mathbb{L})$ determined by $M_{\mathfrak{E}}$ (cf. (a)) in the manner in (4.10) 7 . Next recall that, in Lemma 1.2, we imposed the factorization on the map: $\operatorname{Coh}^{*}\left(\mathbb{X}^{*}\right)_{\text {p.g }} \ni \mathfrak{S} \rightarrow \tilde{\mathbb{L}} \ni \tilde{\mathscr{L}}_{\mathfrak{5}}$ (cf. Figure III, Lemma 1.2)**). This follows from the corresponding facts in Lemma 4.1, Lemma 4.8, and we finish the proof of Lemma 1.2.

q.e.d.

3. Proof of Lemma 2.5, Lemma 2.7 and Lemma 2.3. The proof of the first two lemmas is similar to Lemma 1.2. We summarize the key point of it. First letting the Koszul homomorphism $\left.F_{p}^{m}: \mathfrak{D}_{X}^{\left(\begin{array}{l}s \\ p\end{array}\right)} \rightarrow \mathfrak{D}_{X}^{\left(p^{s}+1\right.}\right)$ for the local variety $\boldsymbol{X} \in \mathbb{A n}_{1 a}$ be as in Lemma 2.5 , we set $\left(\omega_{m}, \omega_{m}^{\prime}\right)=\left(F_{p-1}^{m}, F_{p}^{m}\right)$. Applying Proposition $4.5_{2}$ to $\left(\omega_{m}, \omega_{m}^{\prime}\right)$, we have:

(4.11) 2 Lemma $4.4 \rightarrow$ Lemma 2.5, when the variety is $\mathbb{X} \in \mathbb{A} \mathbb{B}_{1 a}$.

Next, from (1), Remark 4.3, we have the following in the similar manner to $(4.11)_{2}$ :

$(4.11)_{2}^{\prime} \quad$ Lemma $4.8_{2} \rightarrow$ Lemma 2.5 , when the variety is $\mathbb{X}^{\prime} \in \mathbb{A}$ ff.

Moreover, from (2), Remark 4.3, we have:

$(4.11)_{3} \quad$ Lemma 4.5, Lemma $4.8_{3} \rightarrow$ Lemma 2.7 for $\mathbb{X} \in \mathbb{A m}_{1 a}, \mathbb{X}^{\prime} \in \mathbb{A f f f}$.

Finally, the proof of Lemma 2.3 is a little involved than Lemma 2.5, Lemma 2.7, and is as follows. (The proof of Lemma 2.3 for $\mathbb{X}^{*}=\mathbb{X} \in \mathbb{A m}_{1 a}$ or $=\mathbb{X}^{\prime}$ $\in \mathbb{A f f}$ is similar. Here we prove Lemma 2.3 for $\mathbb{X} \in \mathbb{A m}_{1 a}$.) Letting the sheaf $\mathfrak{S}$ and the homomorphism $K_{0, m}: \mathfrak{D}_{X}^{k_{1}+s k} \rightarrow \mathfrak{S}_{m}$ be as in Lemma 4.6, the key fact for the proof of Lemma 2.3 is the following inclusion:

*) By Remark 1.4 (cf. the end of $\$ 1.3$ ), the proof of Lemma 1.2 for such a sheaf $\mathfrak{F}$ suffices for that of Lemma 1.2 in its original form as in n.2, Section 1.3. $\tilde{X}_{r}\left(P^{\prime}\right)=\mathbb{C}^{n}$ $\times U_{r}\left(P^{\prime}\right)$ and $\mathbb{X}_{r}(P)=\mathbb{X} \cap U_{r}(P)$, with the discs $U_{r}\left(P^{\prime}\right), U_{r}(P)$ in $\mathbb{C}^{n^{\prime}}, \mathbb{C}^{n}$ of center $P, P$ and radius $r$ (cf. Lemma 1.2). Also note that $Y^{*} \subset \tilde{Y}^{*}$ (cf. Lemma 1.2), and one can apply Lemma 1.2 to $\mathbf{Y}^{*}$.

**) Letting $M_{1}: \mathbb{R}^{+} \rightarrow \mathbb{R}^{+}$be the first part of the estimation $\tilde{M}_{.5} \in \tilde{M}$ in Lemma $4.1, \ldots$, the first part $\mathscr{L}_{1}: \boldsymbol{R}^{+2} \rightarrow \boldsymbol{R}^{+2}$ of $\mathscr{L}$ is determined uniquely by $M_{1}\left(\operatorname{cf}(4.10)_{5}\right)$. We use this fact in getting the factorization in Lemma 1.2. 
(4.11) $)_{4} s^{*}\left(C^{q}\left(\mathscr{A}_{\mu}, \mathfrak{S}_{m}\right) \cap C^{q}\left(\mathscr{A}_{\mu}, \mathfrak{D}_{X}^{k}\right)_{\mathrm{p.g}}\right) \subset K_{0, m^{\prime}} C^{q}\left(\mathscr{A}_{\mu^{\prime}}, \mathfrak{D}_{\mathbf{X}}^{k_{1}+s k}\right)_{\mathrm{p} . \mathrm{g}}$, with $m^{\prime}=$ $\left[L_{\mathfrak{S}}(m)\right]$. Here the p.g. covering $\mathscr{A}_{\mu}$ is as in Lemma 2.3, and the suffix $\mu^{\prime}$ of the p.g. covering $\mathscr{A}_{\mu^{\prime}}$ satisfies: $\mu^{\prime}>\mu$ (cf. $(2.8)_{0}$ ). Moreover, $L_{\S}$ is the a.d. part of the estimation map $E_{\mathfrak{g}, m} \in \mathbf{E}_{\mathbf{a}, \mathrm{d}}^{\prime}$ (as in Lemma 4.6).

This follows from Lemma 4.6 as follows: letting the open set $\widetilde{U}_{r}(P), \ldots$, be as in Lemma 4.6, we write $(4.2)_{7}$, Lemma 4.6 explicitly as follows:

$(4.11)_{4}^{\prime} \quad i^{*}\left(\Gamma\left(\tilde{U}_{r}(P), \mathfrak{D}_{\mathbb{X}}^{k}\right)_{a} \cap \Gamma\left(\tilde{U}_{r}(P), \mathfrak{H}_{m}\right)\right) \subset K_{0, m^{\prime}} \Gamma\left(\tilde{U}_{r^{\prime}}(P), \mathfrak{D}_{\mathbf{X}}^{k_{1}+s k}\right)_{a^{\prime}}$, with $\left(r^{\prime} ;\right.$ $\left.a^{\prime}\right)=\left(M_{1}(r), M_{2 m}(a / r)\right)$, where $M_{1}$ is the first part*) of $E_{5,}$, and the positive monomial $M_{2, m}$ is also determined by $E_{\mathfrak{5}, m}$ (cf. also (4.2) $)_{7}$ ).

We see easily that the application of Proposition $4.5_{1}$, together with*) the independence of $M_{1}$ from $m \in Z^{+}$, leads to (4.11) $)_{4}$.

Now we derive Lemma $2.3^{\prime}$ from $(4.11)_{4}$ as follows. (Also recall that Lemma 2.3 follows from Lemma $2.3^{\prime}$.) First we write $\mathfrak{H}$ explicitly as: $\mathfrak{D}_{\mathbf{X}}^{k}$ $\stackrel{K_{1}}{\longrightarrow} \mathfrak{D}_{X}^{k_{1}} \stackrel{K_{0}}{\longrightarrow} \mathfrak{H}\left(\subset \mathfrak{D}_{X}^{k}\right) \rightarrow 0$, and we define the homomorphism $K_{1, m}: \mathfrak{D}_{\mathbf{X}}^{k_{2}+s_{1}}$ $\rightarrow \mathfrak{D}_{\mathbf{X}}^{k_{1}}$ similarly to $K_{0, m}$ (cf. also (4.2) $)$. We set $\mathfrak{H}_{1, m}:=K_{1, m}\left(\mathfrak{D}_{\mathbf{X}}^{k_{2}+s k_{1}}\right)\left(\subset \mathfrak{D}_{\mathbf{X}}^{k_{1}}\right)$.

Then we have the exact sequence:

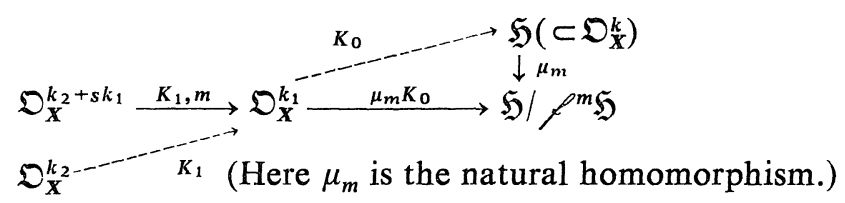

Next, by the definition of the right d.p. filtration (cf. $(2.3)_{6}$ ), we have:

$$
C_{r}^{q}\left(\mathscr{A}_{\mu}, f^{m} \mathfrak{H}\right)_{\mathrm{p} . \mathrm{g}}=K_{0}\left(C^{q}\left(\mathscr{A}_{\mu}, \mathfrak{D}_{\mathbf{x}}^{k_{1}}\right)_{\mathrm{p} . \mathrm{g}} \cap C^{q}\left(\mathscr{A}_{\mu}, \mathfrak{H}_{1, m}\right)\right) .
$$

Applying $(4.11)_{4}$ to the sheaf $\mathfrak{S}_{1, m}$, we have:

(4.11) $)_{4}^{\prime \prime \prime} \quad s^{*} C_{r}^{q}\left(\mathscr{A}_{\mu}, f^{m} \mathfrak{H}\right)_{\mathrm{p} . \mathrm{g}} \subset K_{0} K_{1, m^{\prime}} C^{q}\left(\mathscr{A}_{\mu^{\prime}}, \mathfrak{D}_{\mathbf{X}}^{k_{2}+s k_{1}}\right)_{\mathrm{p} . \mathrm{g}}$, with $m^{\prime}=[L(m)]$. (Here the linear map $L$ is determined by $\left\{K_{1, m}\right\}_{m=1}^{\infty}$.)

Recalling that $K_{1, m^{\prime}}:=K_{1}+F^{m^{\prime}}: \mathfrak{D}_{\mathbf{X}}^{k_{2}}+\mathfrak{D}_{\mathbf{X}}^{s k_{1}} \rightarrow \mathfrak{D}_{\mathbf{X}}^{k_{1}}$, we have the desired inclusion**) in Lemma $2.3^{\prime}$ :

$(4.11)_{4}^{\mathrm{iv}} \quad s^{*} C_{r}^{q}\left(\mathscr{A}_{\mu}, f^{m} \mathfrak{H}\right)_{\mathrm{p} . \mathrm{g}} \subset K_{0} F^{m^{\prime}} C^{q}\left(\mathscr{A}_{\mu^{\prime}}, \mathfrak{O}_{\mathbf{X}}^{\left.s_{1}\right)_{\mathrm{p} . \mathrm{g}}}\left(=C_{1}^{q}\left(\mathscr{A}_{\mu^{\prime}}, f^{m^{\prime}} \mathfrak{S}\right)_{\mathrm{p} . \mathrm{g}}\right)\right.$,

*) By the factorization in Lemma 4.6, the map $M_{1}$ is independent from $m \in Z^{+}$.

**) When the length of $\mathfrak{S}=1$ (i.e., $0 \longrightarrow D_{X}^{k_{1}} \stackrel{K_{0}}{\longrightarrow} \mathfrak{S}\left(\subset D_{X}^{k}\right) \longrightarrow 0$, we understand that $K_{1, m}$ : $=F^{m}: \bigvee_{X}^{s k_{1}} \longrightarrow D_{X}^{k}$ and that $\mathfrak{L}_{1, m}:=f^{m D_{X}^{k}}$. For such $\left(K_{1, m}, \mathfrak{S}_{1, m}\right)$, the similar inclusion to $(4.11)_{4}$ holds (cf. n.6, §5.2), and we also have its cohomological version, which is of the form in $(4.11)_{4}$. 
and we finish the proof of Lemma 2.3.

4. Proof of Proposition 4.2. Here, using the implications (4.11) $2-4$, we prove Proposition 4.2. First we prove $(4.5)_{3}$ (cf. Proposition 4.2), which is a key fact for the proof of Lemma 4.4 (cf. §5.2). For this letting the element $h \in \Gamma\left(X_{0}, D_{X_{0}}\right)$ and the divisor $D$ of $h$ be as in (4.5) $)_{3}$, we have the following from $(4.11)_{2}$ :

(a) 'Lemma 4.4 for $\left(X_{0}, D ; f\right)^{\prime} \longrightarrow$ 'Lemma 2.5 for $\left(X_{0}, D ; f\right)$ '.

Now, letting the parameters $(P ; r) \in \boldsymbol{\mu}_{h}\left(\subset\left(D-X_{0, \text { sing }}\right) \times \mathbb{R}^{+}\right), a \in \mathbb{R}_{1}^{+}$and the elements $(\tilde{m}, m) \in \mathbb{Z}^{+} \times \mathbb{Z}^{+}$be as in (4.5) $)_{3}$, we define:

$$
\alpha:=(a, 1) \in \mathbb{R}_{1}^{+2}, \tilde{r}:=r / 2 \text { and } \sigma:=(2 / r, 1) \in \mathbb{R}_{1}^{+2} .
$$

Then, for the set of the holomorphic functions as in $(4.5)_{3}$, we easily have:

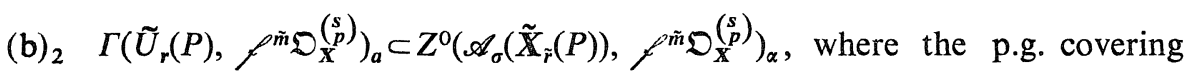
$\mathscr{A}_{\sigma}\left(\tilde{\mathbf{X}}_{\tilde{r}}(P)\right)$ and the set $Z^{0}(\cdots)$ in the right side are as in Lemma 2.5 (cf. $\S 2.3$ ).

Applying Lemma 2.5 to the right side (cf. (a)), we have:

$$
\begin{aligned}
& s^{*}\left(Z^{0}\left(\mathscr{A}_{\sigma}\left(\tilde{\mathbf{X}}_{\tilde{r}}(P)\right), f^{\left.\left.\tilde{m} \mathfrak{D}_{\tilde{X}}^{(s}\right)^{(}\right)}\right)_{\alpha} \cap\left(F_{p}^{m}\right)^{-1}(0)\right) \\
& \subset F_{p-1}^{m} Z^{0}\left(\mathscr{A}_{\sigma^{\prime}}\left(\tilde{\mathbb{X}}_{r^{\prime}}(P)\right), f^{\tilde{m}^{\prime}} \mathfrak{O}_{\mathbf{X}}^{(p-1)}\right)_{\alpha^{\prime}},
\end{aligned}
$$

with $\left(r^{\prime} ; \sigma^{\prime} ; \tilde{m}^{\prime} ; \alpha^{\prime}\right)=E(r ; \sigma ; \tilde{m} ; \alpha)$, where the d.p. map $E$ is as in Lemma 2.5 .

On the other hand, applying*) Theorem $2.2_{2}$ to the right side, we have:*),**)

(b) ${ }_{4} s^{*} Z^{0}\left(\mathscr{A}_{\sigma^{\prime}}\left(\tilde{X}_{r^{\prime}}(P)\right), f^{\tilde{m}^{\prime}} \mathfrak{D}_{\mathbf{X}}\right)_{\alpha^{\prime}} \subset F^{\tilde{m}^{\prime \prime}} Z^{0}\left(\mathscr{A}_{\sigma^{\prime \prime}}\left(\tilde{\mathbf{X}}_{r^{\prime \prime}}(P)\right), \mathfrak{D}_{\mathbf{X}}^{s}\right)_{\alpha^{\prime \prime}}$, with $\left(r^{\prime \prime} ; \sigma^{\prime \prime} ; \tilde{m}^{\prime \prime} ;\right.$ $\left.\alpha^{\prime \prime}\right)=E^{\prime}\left(r^{\prime} ; \sigma^{\prime} ; \tilde{m^{\prime}} ; \alpha^{\prime}\right)$, where the d.p. map $E^{\prime} \in \mathbb{E}_{\text {d.p }}$ is as in Theorem $2.2_{2}$.

From $(b)_{3,4}$, we have:

(b) $5 \quad$ (left side of $\left.(b)_{3}\right) \subset F_{p-1}^{m}\left(F^{\tilde{m}^{\prime \prime}} Z^{0}\left(\mathscr{A}_{\tilde{\sigma}^{\prime \prime}}\left(\boldsymbol{X}_{\tilde{r}^{\prime \prime}}(P)\right), \mathfrak{D}_{X}^{\left.(p-1)^{s}\right)}\right)_{\tilde{\alpha}^{\prime \prime}}\right.$, with $\left(\tilde{r}^{\prime \prime} ; \tilde{\sigma}^{\prime \prime} ; \tilde{m}^{\prime \prime}\right.$; $\left.\tilde{\alpha}^{\prime \prime}\right)=\widetilde{E}(r ; \sigma ; \tilde{m} ; \alpha)$, where the d.p. map $\widetilde{E}$ is determined by $E, E^{\prime}$.

Finally we write $\tilde{\alpha}^{\prime \prime} \in \boldsymbol{R}_{1}^{+2}$ as $\left(\tilde{a}^{\prime \prime}, \tilde{d}^{\prime \prime}\right)$ and $\left[d^{\prime \prime}+1\right]$ as $d$. Then we easily have the following inclusion, which is converse to $(b)_{2}$ :

$$
h^{d} Z^{0}\left(\mathscr{A}_{\tilde{\sigma}^{\prime \prime}}\left(\boldsymbol{X}_{\tilde{r}^{\prime \prime}}(P)\right), \mathfrak{D}_{\mathbf{X}}\right)_{\tilde{\alpha}^{\prime \prime}} \subset \Gamma\left(\tilde{U}_{\tilde{r}^{\prime \prime}}(P), \mathfrak{D}_{\mathbf{X}}\right)_{\tilde{a}^{\prime \prime}} .
$$

Also recalling the explicit form of the d.p. map $E \in \mathbb{E}_{\text {d.p }}$ (cf. n.4, § 2.1), one can

*) Recall that Lemma 2.5 implies Theorem $2.2_{2}$ and the inclusion of the form $(b)_{3}$ (cf. Remark 2.2 at the end of $\S 2$ ).

**) For the homomorphism $F^{m}=F_{s-1}^{m}$, see n.2, Section 2.1 and Section 2.3. 
easily write the elements $d \in \mathbb{Z}^{+}$and $\left(\tilde{r}^{\prime \prime} ; \tilde{m}^{\prime \prime} ; \tilde{a}^{\prime \prime}\right) \in \boldsymbol{R}^{+} \times \boldsymbol{Z}^{+} \times \boldsymbol{R}_{1}^{+}$as follows:

(b) ${ }_{7} d=[L(1)]$ and $\left(\tilde{r}^{\prime \prime} ; \tilde{m}^{\prime \prime} ; \tilde{a}^{\prime \prime}\right)=\tilde{E}^{\prime}(r ; m ; a)$, with a linear map $L$ and an a.d. map $\tilde{E}^{\prime}$ (Definition 4.2), which are defined by $\tilde{E} \in \mathbb{E}_{\text {d.p }}$.

(In (b) $)_{7}$, the element ' $1 \in \mathbb{Z}^{+}$in $d=[L(1)]$ ' is second component of $\alpha \in \mathbb{R}_{1}^{+2}$.) Finally from (b) ${ }_{2,5}$ and (b) 6 , we have

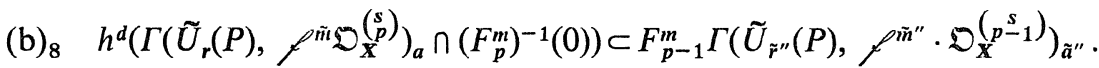

This inclusion, together with (b) 7 , insures the desired inclusion (4.2) $)_{3}$, which is given to the Koszul complexes $\mathscr{F}^{m}$ (cf. Proposition 4.2).

Next, the proof of $(4.5)_{4}$ is given similarly to the above, by taking 'Lemma 2.7 for $\mathbb{X} \in \mathrm{An}_{1 a}$ ' as the starting point (cf. (4.11) $)_{3}$ )*) Moreover, for the proof of $(4.5)_{2}$, we note that the application of Proposition $4.5_{2}$ to 'Lemma 4.3 for $\left(X_{0}, D ; f, \mathscr{G}\right)^{\prime}$ insures its cohomological version, which is similar to Lemma 2.5, Lemma 2.7. Then using the similar arguments to the above, we have $(4.5)_{2}$. Finally, the proof of $(4.5)_{1}$ is given similarly to the above, by taking 'Lemma 4.2 for $\left(X_{0}, D ; \not\right)^{\prime}$ ' as the starting point, and we finish the proof of Proposition 4.2.

q.e.d.

5. Proof of Lemma 1.4. (i) Take an open set $U$ of a euclidean space $\boldsymbol{C}^{n}(z)$ and a subset $X$ of $U$. Also taking a p.g. function $g: U \rightarrow \boldsymbol{R}_{1}^{+}$and an element $\tilde{\sigma} \in \boldsymbol{R}_{1}^{+2}$ we assume (cf. also $(4.10)_{0}$ ):

(4.12) $0 \quad g(Q) / 2<g(P)<2 g(Q)$ for each $P \in U$ and $Q \in U_{\tilde{\sigma}}(P ; g) \cap U$, where $U_{\tilde{\sigma}}(P ; g):=\left\{Q \in \mathbb{C}^{n} ; d(P, Q)<\{\tilde{\sigma} \cdot g(P)\}^{-1}\right\}$.

For an open subset $U^{\prime}$ of $U$ we say that $U$ is a $(g, \tilde{\sigma})$-d-envelope of $U^{\prime}$, if

$$
U_{\tilde{\sigma}}(Q ; g) \subset U \text { for each } Q \in U^{\prime} .
$$

We fix such an open set $U^{\prime}$ in the remainder of n.4. Now take an el-map $\mathscr{L}_{0}$ of the form $\mathscr{L}_{0}: \mathbb{R}^{+2} \ni\left(\sigma_{1}, \sigma_{2}\right) \rightarrow \boldsymbol{R}^{+2} \ni\left(4 \sigma_{1} \cdot \alpha^{\sigma_{2}}, \sigma_{2}\right)$ (cf. n.1).

Then setting

$$
U_{\sigma}(X ; g):=\cup_{P \in X} U_{\sigma}(P ; g),
$$

we easily have $(\sigma>\tilde{\sigma})$ :

$(4.12)_{2} U_{\sigma^{\prime}}(Q ; g) \cap X=\phi$ for each $Q \in U^{\prime}-U_{\sigma}(X ; g)$, where $\sigma^{\prime}=\mathscr{L}_{0}(\sigma)$.

$\Rightarrow$ Note that this cohomological version in terms of ' $C^{q}$ ' instead of ' $Z^{q}$ '. Using the similar remark to Remark 2.2, one can replace $C^{q}$ by $Z^{q}$, and we get a similar inclusion to $(b)_{2,3}$ (for the homomorphism $K_{0}$ as in (4.5) $)_{4}$ ). 
(This follows also if $U$ is a $\left(g, \sigma^{\prime \prime}\right)$-d-envelope of $U^{\prime}$, where $\sigma^{\prime \prime} \in \mathbb{R}_{1}^{+2}$ satisfies: $\sigma^{\prime \prime}>\sigma^{\prime}$.)

(ii) Extension of cochains. Here we give a key proposition for Lemma 1.4. For this we assume that $X$ is an analytic variety in $U$ and that there are open sets $\tilde{U}, \tilde{U}_{0}$ in $\mathbb{C}^{n}$ and varieties $\tilde{X}, \tilde{X}_{0}$ in $\tilde{U}, \tilde{U}_{0}$ satisfying

(a) $\tilde{U}, \tilde{U}_{0}$ are $(g, \tilde{\sigma})$-d-envelope of $U, \tilde{U}$, and $\tilde{X}=\tilde{X}_{0} \cap \tilde{U}, X=\tilde{X} \cap U$.

Also taking a positive monomial $M$ (cf. n.5, §1.1), we assume the following uniform estimation (cf. Proposition 4.4): for each $Q \in \tilde{X}$ and $r \in \mathbb{R}^{+}$satisfying $r<\{\tilde{\sigma} g(Q)\}^{-1}$, there is an analytic map

$(4.12)_{3} \omega: U_{r^{\prime}}(P) \hookrightarrow \widetilde{U}_{r}(P)$, which is the identity on $\widetilde{U}_{r^{\prime}}(P)$. Here $r^{\prime}=M(r)$, and we set $U_{r}(P):=\left\{Q \in \mathbb{C}^{n} ; d(P, Q)<r\right\}, \tilde{U}_{r}(P)=U_{r}(P) \cap \tilde{X}_{0}$.

For an element $\sigma \in \mathbb{R}_{1}^{+2}$ we set:

$$
\left\{\begin{array}{c}
\mathscr{A}_{\sigma}(X) \\
\mathscr{B}_{\sigma}\left(U^{\prime}\right)
\end{array}\right\}:=g \text {-p.g.-covering of }\left\{\begin{array}{l}
X \\
U^{\prime}
\end{array}\right\} \text { in }\left\{\begin{array}{l}
\tilde{X} \\
\mathbb{C}^{n}
\end{array}\right\} \text { of size } \sigma
$$

(Definition 1.6.6).

Next we define an el-map $\mathscr{L}^{\prime}: \mathbb{R}^{+2} \rightarrow \mathbb{R}^{+2}$ from $M$ in the manner in $(4.10)_{5}$, and we set $\tilde{\mathscr{L}}^{\prime}:=\mathscr{L}_{0} \circ \mathscr{L}^{\prime} \circ \mathscr{L}_{0}$, where $\mathscr{L}_{0}$ is as in (4.12) $)_{2}$. Also denoting by $\mathscr{L}_{0}^{\prime}$ the el-map: $\boldsymbol{R}^{+2} \ni \sigma \rightarrow \boldsymbol{R}^{+2} \ni \mathscr{L}_{0}(2 \cdot \sigma)$, we set:

$$
\mathscr{L}:=\mathscr{L}_{0}^{\prime} \circ \tilde{\mathscr{L}}^{\prime}
$$

Then, denoting by $\mathfrak{D}_{X}, \mathfrak{D}$ and $\omega_{X}^{*}$ respectively the structure sheaf of $X, \mathbb{C}^{n}$ and the natural homomorphism: $\mathfrak{D} \rightarrow \mathfrak{D}_{X}$, we have:

Proposition 4.6 (Extension of cochains). For any $\sigma \in \mathbb{R}_{\tilde{\sigma}}^{+2}$ and $\alpha=\left(\alpha_{1}, \alpha_{2}\right)$ $\in \mathbb{R}_{1}^{+2}$ we have a map:

$$
\begin{aligned}
& e^{*}: Z^{q}\left(\mathscr{A}_{\sigma}(X), \mathfrak{D}_{X}\right)_{\alpha} \hookrightarrow C^{q}\left(\mathscr{B}_{\sigma^{\prime}}\left(U^{\prime}\right), D_{\alpha^{\prime}},\right. \\
& \text { where } \alpha^{\prime}=\left(4 \alpha_{1} 2^{\alpha_{2}}, \alpha_{2}\right), \sigma^{\prime}=\mathscr{L}(\sigma),
\end{aligned}
$$

which satisfies $\omega_{X}^{*} \delta e^{*}=0$ and

$$
\varsigma^{*}=\omega_{X}^{*} e^{*} \text {, with the p.g. r efining map s: } \mathscr{A}_{\sigma^{\prime}}(X) \hookrightarrow \mathscr{A}_{\sigma}(X) \text {. }
$$

(The similar facts to the above holds by changing $\left(U^{\prime}, \sigma^{\prime}\right)$ to $\left(\tilde{U}^{\prime}, \tilde{\sigma}^{\prime}\right)$, with an open subset $\tilde{U}^{\prime}$ of $U^{\prime}$ and an element $\tilde{\sigma}^{\prime} \in \mathbb{R}_{1}^{+2}$ satisfying ${ }^{*} \tilde{\sigma}^{\prime}>\sigma^{\prime}$.)

Proof. (1) First we extend cochains on $X$ to its small p.g. neighborhood.

*) Note that this implies that $U$ is a $\left(g, \tilde{\sigma}^{\prime}\right)$-d-envelope of $\tilde{U}^{\prime}$. 
For this setting $\mathscr{B}_{\sigma}(X):=g$-p.g.-covering of $X$ in $\mathbb{C}^{n}$ of size $\sigma$ (Definition $1.6_{1}$ ), we show the existence of a map:

$(4.12)_{7} \quad e^{* *}: Z^{q}\left(\mathscr{A}_{\sigma}(X), \mathfrak{D}_{X}\right)_{\alpha} \hookrightarrow C^{q}\left(\mathscr{B}_{\sigma^{\prime \prime}}(X), \mathfrak{D}\right)_{\alpha^{\prime}}$, satisfying $\omega_{X^{*}} \delta e^{*}=0$ and $s^{*}=$ $\omega_{X}^{*} e^{\prime *}$, with the p.g. refining map $s: \mathscr{A}_{\sigma^{\prime \prime}}(X) \hookrightarrow \mathscr{A}_{\sigma}(X)$,

where the element $\sigma^{\prime \prime} \in \boldsymbol{R}_{1}^{+2}$ is defined as follows:

$$
\tilde{\sigma}^{\prime \prime}=\mathscr{L}_{0}(\sigma), \bar{\sigma}^{\prime \prime}=\mathscr{L}^{\prime}\left(\tilde{\sigma}^{\prime \prime}\right) \text {, and } \sigma^{\prime \prime}=\mathscr{L}_{0}\left(\bar{\sigma}^{\prime \prime}\right)\left(=\tilde{\mathscr{L}}^{\prime}(\sigma)\right) \text {. }
$$

Take an element $\mathscr{B}^{\prime}=\left\{B_{j}^{\prime}\right\}_{j=1}^{q+1} \in \mathscr{N}^{q+1} \mathscr{B}_{\sigma^{\prime \prime}}(X)$ satisfying $\left|\mathscr{B}^{\prime}\right| \cap X \neq \phi$, and we set $\mathscr{A}^{\prime}=\left\{A_{j}^{\prime}\right\}_{j=1}^{q+1}$, with $A_{j}^{\prime}=B_{j}^{\prime} \cap \tilde{X}$, and $\mathscr{A}=s\left(\mathscr{A}^{\prime}\right) \in \mathscr{N}^{q+1} \mathscr{A}_{\sigma}(X)$. Then, taking a point $Q \in\left|\mathscr{A}^{\prime}\right|$, we have:

(a) ${ }_{1}|\mathscr{A}| \supset \tilde{U}_{r}(Q), U_{r^{\prime}}(Q) \supset\left|\mathscr{B}^{\prime}\right|$, with $r=\left\{\tilde{\sigma}^{\prime \prime} g(Q)\right\}^{-1}, r^{\prime}=\left\{\bar{\sigma}^{\prime \prime} g(Q)\right\}^{-1}(=M(r))$ (cf. $(4.12)_{5}$ and $(4.10)_{5}$ ).

By $(4.12)_{3}$ take an analytic map $\omega: U_{r}(Q) \hookrightarrow \widetilde{U}_{r}(Q)$, which is the identity on $\tilde{U}_{r^{\prime}}(Q)$. Now, for an element $\varphi \in Z^{q}\left(\mathscr{A}_{\sigma}(X), \mathfrak{D}_{X}\right)_{\alpha}$, we set $\varphi_{\mathscr{G}^{\prime}}^{\prime}:=\omega^{*} \varphi_{\mathscr{A}} \in$ $\Gamma\left(\left|\mathscr{B}^{\prime}\right|, \mathfrak{D}\right)$. Then we have:

(a) 2

$$
\omega_{X}^{*} \varphi_{\mathscr{B}}^{\prime}=\varphi_{\mathscr{A ^ { \prime }}} \text {, and }\left|\varphi_{\mathscr{B}}^{\prime}(R)\right|<\alpha^{\prime} g(R) \text { in }\left|\mathscr{B}^{\prime}\right| \text {. }
$$

We then define an element $\varphi^{\prime}:=e^{*} \varphi$ by

(a) $\varphi_{3} \varphi_{\mathscr{B}^{\prime}}^{\prime}:=\omega_{X}^{*} \varphi_{\mathscr{B}^{\prime}}^{\prime}$ or $=0$, according as $\left|\mathscr{B}^{\prime}\right| \cap \tilde{X} \neq \phi$ or $=\phi$.

Then it is easy to see that (a) ${ }_{2}$ insures $(4.12)_{7}$.

(2) Next setting $\bar{\sigma}^{\prime}=2 \cdot \sigma^{\prime \prime}$ and $\sigma^{\prime}=\mathscr{L}_{0}\left(\bar{\sigma}^{\prime}\right)\left(=\mathscr{L}_{0}^{\prime}\left(\sigma^{\prime \prime}\right)\right)$, we set $\mathscr{B}^{\prime}:=$ $\left\{U_{\sigma^{\prime}}(Q ; g) ; Q \in U^{\prime} \cap \operatorname{supp} \mathscr{B}_{\bar{\sigma}^{\prime}}(X)\right\}$ (cf. (4.12) $)$. Then we have a refining map

(b) $1 \quad t: \mathscr{B}^{\prime} \hookrightarrow \mathscr{B}_{\sigma^{\prime \prime}}(X)$ so that $t\left(U_{\sigma^{\prime}}(Q ; g)\right)=U_{\sigma^{\prime \prime}}(Q ; g)$, if $Q \in X \cap U^{\prime}$.

Also we note that $(4.12)_{2}$ insures:*)

(b) 2

$$
U_{\sigma^{\prime}}(Q ; g) \cap X=\phi \text {, if } Q \in U^{\prime} \text {-supp } \mathscr{B}_{\tilde{\sigma}^{\prime}}(X),
$$

Then we set:

(b) ${ }_{3} \mathscr{N}^{q+1} \mathscr{B}_{\sigma^{\prime}}\left(U^{\prime}\right)=\mathscr{N}^{q+1} \mathscr{B}^{\prime} \cup \mathscr{B}^{\prime \prime}$, where $\mathscr{B}^{\prime \prime}:=\left\{\mathscr{B}_{\mu}^{\prime \prime} \in \mathscr{N}^{q+1} \mathscr{B}_{\sigma^{\prime}}\left(U^{\prime}\right)\right.$; where one of elements $U_{\sigma^{\prime}}(Q ; g) \in \mathscr{B}_{\mu}^{\prime \prime}$ satisfies: $\left.Q \notin \operatorname{supp} \mathscr{B}_{\bar{\sigma}^{\prime}}\right\}$.

Note that $(b)_{2}$ implies:

(b) 4

$$
\left|\mathscr{B}_{\mu}^{\prime \prime}\right| \cap X=\phi \quad \text { if } \quad \mathscr{B}_{\mu}^{\prime \prime} \in \mathscr{B}^{\prime \prime}
$$

*) $(b)_{2}$ holds for the pair $\left(\tilde{U}^{\prime \prime}, \bar{\sigma}^{\prime}\right)$ as in the remark soon below Proposition 4.6, and also the remark itself holds. 
Now letting $\varphi^{\prime}=e^{\prime *} \varphi$ be as in $(\mathrm{a})_{3}$, we define an element $\Psi=e^{*} \varphi \in C^{q}\left(\mathscr{B}_{\sigma^{\prime}}\left(U^{\prime}\right)\right.$, D) ${ }_{\alpha^{\prime}}$ by the following:

$$
\Psi=t^{*} \varphi^{\prime} \quad \text { on } \mathscr{V}^{q+1} \mathscr{B}^{\prime} \text {, and }=0 \text { on } \mathscr{B}^{\prime \prime} .
$$

By $(b)_{1-4}$ and $(a)_{3}$ we easily have $(4.12)_{6}$.

q.e.d.

(iii) Proof of Lemma 1.4. Now Lemma 1.4 follows from Proposition 4.6 almost directly as follows. First let the local variety $\mathbb{X} \in \mathbf{A n}_{1 a}$ be as in Lemma 1.4. Then letting the parameter space $\boldsymbol{\mu}_{\mathbf{X}}\left(\subset \mathbb{X} \times \mathbb{R}^{+}\right)$and the positive monomial $M_{\mathrm{X}}$ be as in Proposition 4.4, we assume the estimation (4.9) in Proposition 4.4 for $\left(\mu_{\mathbf{X}}, M_{\mathbf{X}}\right) .^{*)}$ The symbol $\mathscr{L}_{\mathbf{X}}$ denotes the el-map: $\mathbb{R}^{+2} \rightarrow \mathbb{R}^{+2}$, which is formed from $M_{\mathbf{X}}$ in the manner in Proposition 4.6. Next let the manifolds $\mathbb{Y}_{r}(P)=U_{r}(P)-D_{0}, \mathbb{X}_{r}(P)=\mathbb{Y}_{r}(P) \cap X$ and their p.g. coverings $\mathscr{A}_{\sigma}\left(\mathbb{Y}_{r}(P)\right)$, $\mathscr{A}_{\sigma}\left(\boldsymbol{X}_{r}(P)\right)$ in $\mathbb{C}^{n}, \boldsymbol{X}$ be as in Lemma 1.4.**) Then choosing suitable neighborhoods $U^{\prime}, U^{\prime \prime}$ of the origin $P_{0}$ of $\mathbb{X}$ (cf. $\left.(1.8)_{0}\right)$ in $\mathbb{C}^{n}$, and an element $\tilde{\sigma} \in \mathbb{R}_{1}^{+2}$, we have:

$$
\begin{array}{r}
U^{\prime}-D_{0}\left(\text { resp. } U^{\prime \prime}-D_{0}\right) \text { is }{ }^{* * *)} \text { a }\left(g_{\mathbf{X}}, \sigma^{\prime \prime}\right) \text {-d-envelope of } \\
U^{\prime \prime}-D_{0}\left(\text { resp. } Y_{r}(P)\right) .{ }^{* *}
\end{array}
$$

Thirdly, let $E_{\mathbf{X}}^{\prime}$ be the first part of the p.g.c. map $E_{\mathbf{X}}$ as in Lemma 1.4. Then setting $\left(r^{\prime} ; \sigma^{\prime}\right)=E_{X}^{\prime}(r ; \sigma)$ we have the following from the explicit form of the map $E_{\mathbf{X}}^{\prime}$ (cf. n.5, §1.1).

$$
\mathbb{Y}_{r}(P) \text { is a }\left(g_{\mathbf{X}}, \sigma^{\prime}\right) \text {-d-envelope of } \boldsymbol{Y}_{\boldsymbol{r}^{\prime}}(P) \text {, and } \sigma^{\prime}>\mathscr{L}_{\mathbf{X}}(\sigma) .
$$

By (a), (b), one can apply Proposition 4.6 to $\left(\mathscr{A}_{\sigma^{\prime}}\left(\boldsymbol{Y}_{r^{\prime}}(P)\right), \mathscr{A}_{\sigma}\left(\mathbb{X}_{r}(P)\right)\right)$ and the structure sheaves $\mathfrak{D}_{X}, \mathfrak{D}$ of $\mathbb{X}, \mathbb{C}^{n}$ : writing the p.g. resolution $\mathfrak{S}_{\mathbf{X}}$ of $\mathfrak{D}_{\mathbf{X}}$ over $U_{0}-D_{0}$ in the form: $\rightarrow \mathfrak{D} \stackrel{\omega_{X}^{*}}{\longrightarrow} \mathfrak{H}_{\mathbf{X}} \rightarrow 0$, where $\mathfrak{H}_{\mathbf{X}}$ coincides with $\mathfrak{D}_{\mathbf{X}}$ as the coherent sheaf over $U-D_{0}$, we have a map $e^{*}$ from (4.12) 6 in Proposition 4.6:

(c) $e^{*}: Z^{q}\left(\mathscr{A}_{\sigma}\left(\mathbb{X}_{r}(P)\right), \mathfrak{D}_{X}\right)_{\alpha} \rightarrow Z^{q}\left(\mathscr{A}_{\sigma^{\prime}}\left(\mathbb{Y}_{r^{\prime}}(P)\right), \mathfrak{H}_{\mathbf{X}}\right)_{\alpha^{\prime}}$, which satisfies: $s^{*}=\omega_{\mathbb{X}}^{*} e^{*}$.

(Here $s=$ p.g. refining map: $\mathscr{A}_{\sigma^{\prime}}\left(\mathbb{X}_{r^{\prime}}(P)\right) \hookrightarrow \mathscr{A}_{\sigma}\left(\mathbb{X}_{r}(P)\right)$, and we use the symbol $\omega_{X}^{*}: \mathfrak{D} \rightarrow \mathfrak{H}_{X}$ also for the obvious homomorphism: $\mathfrak{D}_{\mathbf{X}} \rightarrow \mathfrak{D}_{\mathbf{X}}$. We note that the

*) When we apply Proposition 4.4 to $X$, we assume that the pair $\left(X_{0}, X_{0}^{\prime}\right)$ in Proposition 4.4 is of the form: $\left(X_{0}, X_{0}^{\prime}\right)=\left(X_{0}, D\right)$, with the divisor $D$ of $X_{0}$ as in Lemma 1.4. $\left(=D_{0} \cap X_{0}\right.$, with the divisor $D_{0}$ of $\left.U_{0}\right)$.

**) $U_{r}(P):=\left\{Q \in C^{n} ; d(P, Q)<r\right\}$ (cf. (1.17)

***) From the explicit form of the parameter space $\mu_{X}$, one can take $U^{\prime}, U^{\prime \prime}$ independently from the manifold $\boldsymbol{Y}_{r}(P)$ in Lemma 1.4.

****) $g_{X}\left(=\left|h_{\boldsymbol{X}}\right|^{-1}\right.$, where $h_{\mathrm{r}} \in \Gamma\left(U_{0}, \wp_{U_{0}}\right)$ ) is the p.g. function for $\boldsymbol{X}$ (cf. $\S 1$ ). 
map $\omega_{\boldsymbol{X}}^{*}$ in Lemma 1.4 was used in the latter sense.)

It is clear that (c) insures the desired inclusion in Lemma 1.4:

$$
s^{*} Z^{q}\left(\mathscr{A}_{\sigma}\left(\mathbb{X}_{r}(P)\right), \mathfrak{D}_{\mathbf{X}}\right)_{\alpha} \subset \omega_{\mathbb{X}}^{*} Z^{q}\left(\mathscr{A}_{\sigma^{\prime}}\left(\boldsymbol{Y}_{r^{\prime}}(P)\right), \mathfrak{S}_{\mathbf{X}}\right)_{\alpha^{\prime}},
$$

and we have Lemma 1.4 for the local variety $\mathbb{X} \in \mathbf{A n}_{1 a}$. For the affine variety $\boldsymbol{X}^{\prime} \in \mathrm{Aff}$, we note that the ambient space $\mathbb{C}^{n}$ is $\left(g_{\mathbf{X}}, \sigma\right)$-d-envelope of $\mathbb{C}^{n}$ itself for any $\sigma \in \boldsymbol{R}_{1}^{+2}$. Using this remark and Proposition 4.4 for $\mathbf{X}^{\prime}$, the proof of Lemma 1.4 for $\boldsymbol{X}^{\prime} \in \mathbf{A f f}$ is given similarly (and more easily) to the case of $\mathbb{X} \in \mathbf{A n}_{1 a}$, and we finish the proof of Lemma 1.4.

6. Proof of Lemma 1.3. Let the divisor $S \subset \tilde{\mathbb{X}}:=\mathbb{C}(w) \times U_{0}(\subset \mathbb{C}(w) \times$ $\left.\mathbb{C}^{n}(z)\right)$ be as in Lemma 1.3 (cf. also (1.16) $)$. Then for points $\widetilde{Q} \in S, Q \in \mathbb{X}=$ $U_{0}-D$ and $\sigma \in \mathbb{R}_{1}^{+2}$, we set (cf. Lemma 1.3):

$(4.13)_{0}\left\{\begin{array}{l}\tilde{U}_{\sigma}\left(\widetilde{Q} ; g_{S}\right) \\ \tilde{U}_{\sigma}\left(Q ; g_{X}\right)\end{array}\right\}:=\left\{\begin{array}{l}R \in S: d(\widetilde{Q}, R)<\left(\sigma g_{S}(\widetilde{Q})\right)^{-1} \\ R \in \mathbb{X} ; d(Q, R)<\left(\sigma g_{X}(Q)\right)^{-1}\end{array}\right\}$, with $\left\{\begin{array}{l}g_{s} \\ g_{X}\end{array}\right\}:=\left\{\begin{array}{l}|w| \\ \left|h_{\mathbf{X}}^{-1}\right|\end{array}\right\}$.

(Here $h_{\mathbf{X}} \in \Gamma\left(U_{0}, \mathfrak{D}_{U_{0}}\right)$ and its divisor $D$ in $U_{0}$ are as in Lemma 1.3 (cf. also $\left.(1.15)_{4}\right)$. Next take a suitable open subset $U_{\boldsymbol{X}}$ of $U_{0}$, an element $\sigma=\sigma_{\mathbf{X}} \in \mathbb{R}_{1}^{+2}$ and an el-map $\mathscr{L}_{\mathbf{X}}: \mathbb{R}^{+2} \rightarrow \mathbb{R}^{+2}$ (cf. n.5, §1.1). Then, from a simple observation, we have the following comparison of the p.g. properties of $S$ and $X$ :

Proposition 4.7. $\cdot$ (1) $g_{\mathbf{X}}(Q) / 2<g_{S}(\widetilde{Q})<2 g_{\mathbf{X}}(Q)$.

(2) $\pi_{\boldsymbol{X}}\left(\tilde{U}_{\sigma^{\prime}}(\widetilde{Q})\right) \subset \tilde{U}_{\sigma}(Q)$ and $\pi_{\mathbf{X}}\left(\tilde{U}_{\tilde{\sigma}}(\widetilde{Q})\right) \supset \tilde{U}_{\sigma^{\prime}}(Q)$, with $\sigma^{\prime}=\mathscr{L}_{X}(\sigma)$.

Here $(Q ; \sigma)$ is in $\left(U_{\mathbf{X}}-D\right) \times \mathbb{R}^{+2}$, and $\tilde{Q}=\pi_{\mathbf{X}}^{-1}(Q)$. Moreover, $\pi_{\mathbf{X}}$ is the natural projections: $S \rightarrow \mathbb{X}=U_{0}-D$ (cf. $\left.n .4, \S 1.3\right)$. Also we write $U_{\sigma}\left(\widetilde{Q} ; g_{S}\right)$ as $U_{\sigma}(\widetilde{Q}), \ldots$

Letting the point $P \in D$ and the element $r \in \mathbb{R}^{+}, \sigma \in \mathbb{R}_{1}^{+2}$ be as in Lemma 1.3, we set:

(a) $)_{1} \quad S_{r}(P):=S \cap \tilde{\mathbf{X}}_{r}(P)$, with $\tilde{\mathbb{X}}_{r}(P):=\boldsymbol{C} \times U_{r}(P)$, and $\boldsymbol{X}_{r}(P):=U_{r}(P)-D_{0}($ cf. $\left.\left.(1.16)_{2}\right), *\right)$

(a) $)_{2}\left\{\begin{array}{l}\mathscr{A}_{\sigma}\left(S_{r}(P)\right) \\ \mathscr{A}_{\sigma}\left(\tilde{\mathbf{X}}_{r}(P)\right) \\ \mathscr{A}_{\sigma}\left(\boldsymbol{X}_{r}(P)\right)\end{array}\right\}:=\left\{\begin{array}{l}g_{S} \\ g_{S} \\ g_{\mathbf{X}}\end{array}\right\}$-p.g. covering of $\left\{\begin{array}{l}S_{r}(P) \\ \tilde{\mathbf{X}}_{r}(P) \\ \boldsymbol{X}_{r}(P)\end{array}\right\}$ of size $\sigma$ in $\left\{\begin{array}{l}S \\ \tilde{\mathbf{X}} \\ \tilde{C}^{n}\end{array}\right\}$.

Then from Proposition 4.7 we easily have:

*) $U_{r}(P):=\left\{R \in C^{n} ; d(R, P)<r\right\}$ (cf. n.4, §1.3). 
Proposition $4.7_{2}$. There are (natural) refining maps $s_{S}$ and $s_{X}$ :

$(4.13)_{2}\left\{\begin{array}{l}s_{S}: \mathscr{A}_{\sigma^{\prime}}\left(S_{r}(P)\right) \ni \tilde{U}_{\sigma^{\prime}}(\widetilde{Q}) \longrightarrow \pi_{X}^{-1} \mathscr{Q}_{\sigma}\left(\mathbb{X}_{r}(P)\right) \ni \pi_{\mathbf{X}}^{-1}\left(\tilde{U}_{\sigma}\left(\pi_{\mathbf{X}}(\widetilde{Q})\right)\right) \\ s_{X}: \mathscr{A}_{\sigma^{\prime}}\left(\mathbb{X}_{r}(P)\right) \ni \tilde{U}_{\sigma^{\prime}}(Q) \longrightarrow \pi_{X} \mathscr{A}_{\sigma}\left(S_{r}(P)\right) \ni \pi_{\boldsymbol{X}}\left(\tilde{U}_{\sigma}\left(\pi_{X}^{-1}(\widetilde{Q})\right)\right)\end{array}\right\}$, with $\sigma^{\prime}=\mathscr{L}_{\mathbf{X}}(\sigma)$.

Thirdly let the estimation: $(r ; \sigma ; \alpha) \rightarrow\left(r^{\prime}: \sigma^{\prime} ; \alpha^{\prime}\right)=E_{\mathbb{X}}(r ; \sigma ; \alpha)$, where $(r ; \sigma$; $\alpha) \in \mathbb{R}^{+} \times \mathbb{R}_{1}^{+2} \times \mathbb{R}_{1}^{+2}$ and $E_{\mathbf{X}} \in \mathbb{E}_{\mathrm{p} . \mathrm{g}}$, be as in Lemma 1.3 . Then from the explicit form of $E_{\boldsymbol{X}}$ (cr. Definition 1.5), we easily have:

$$
\mathbb{X}_{r}(P) \text { is a }\left(g_{\mathbf{X}}, \sigma\right) \text {-d-envelope of } \mathbb{X}_{r^{\prime}}(P) \text { (cf. (i), n.5, §4.2). }
$$

By this we apply Proposition 4.6 to $\left(\mathscr{A}_{\sigma}\left(S_{r}(P)\right), \mathscr{A}_{\sigma}\left(\tilde{\mathbb{X}}_{r}(P)\right)\right.$ ) (cf. also Proposition 4.4), and we have:

Proposition $\mathbf{4 . 7}_{\mathbf{3}}$ (Extension of cochains). There is a map:

$(4.13)_{3} \quad e^{*}: Z^{q}\left(\mathscr{A}_{\sigma}\left(S_{r}(P)\right), D_{S}\right)_{\alpha} \longrightarrow Z^{q}\left(\mathscr{A}_{\sigma^{\prime}}\left(\mathbb{X}_{r^{\prime}}(P)\right), \mathfrak{S}_{S}\right)_{\gamma}$, with $\quad\left(r^{\prime} ; \sigma^{\prime} ; \alpha^{\prime}\right)=$ $E_{X}(r ; \sigma ; \alpha)$, which satisfies: $s^{*}=\omega_{S}^{*} e^{*}$. Here the homomorphism $\omega_{S}: \mathfrak{5}_{S} \rightarrow \mathbb{D}_{S}$ is as in Lemma 1.3 and $s:=$ p.g. refining map: $\mathscr{A}_{\sigma^{\prime}}\left(S_{r}(P)\right) \hookrightarrow \mathscr{A}_{\sigma}\left(S_{r}(P)\right.$ ). (Note that Proposition $4.7_{3}$ insures, in the similar manner to n.5, the following inclusion:

(4.13) $)_{4} \quad \omega_{S}^{*} Z^{q}\left(\mathscr{A}_{\sigma^{\prime}}\left(\tilde{\mathbf{X}}_{r^{\prime}}(P)\right), \mathfrak{H}_{S}\right)_{\alpha^{\prime}} \supset s^{*} Z^{q}\left(\mathscr{A}_{\sigma}\left(\mathfrak{S}_{r}(P)\right), \mathfrak{D}_{S}\right)_{\alpha}$, where the correspondence $(r ; \sigma ; \alpha) \rightarrow\left(r^{\prime} ; \sigma^{\prime} ; \alpha^{\prime}\right)$ is as in $(4.13)_{3}$.)

Now, it is easy to get the comparison of the sets of the cochains $Z^{q}\left(\mathscr{A}_{\sigma}\left(\tilde{\mathbb{X}}_{r}(P)\right), \mathfrak{S}_{S}\right)_{\alpha}$ and $Z^{q}\left(\mathscr{A}_{\sigma}\left(\mathbb{X}_{r}(P)\right), \mathfrak{D}_{X}\right)_{\alpha}$ in Lemma 1.3 , which are defined respectively for $\mathbb{C} \times \mathbb{C}^{n}$ and $U_{0}-D$, from Propositions $4.7_{2,3}$ and (4.13) . Thus we have Lemma 1.3, and we also finish the proof of all the lemmas in Chapter I, which is postponed in Section 4.

\section{§5. Proof of Lemma 4.1-Lemma 4.6}

In Section 5.1 we prove Lemma 4.1, and, in Section 5.2, we prove Lemma 4.2-Lemma 4.6. The geometric datum $\mathbb{X}=\left(\mathbb{C}^{n}(z), U_{0}, X_{0}, X_{0}^{\prime}, P_{0}\right)$ consisting of varieties $X_{0} \supset X_{0}^{\prime}\left(\ni P_{0}\right)$ in $U_{0} \subset \operatorname{Ouv}\left(\mathbb{C}^{n}(z)\right)$ will be the one in Section 4. Also letting the subvarieties $X_{1}, X_{2}$ of $X_{0}$ be as in Section 4 , we assume that $\left(X_{1}, X_{2}\right)$

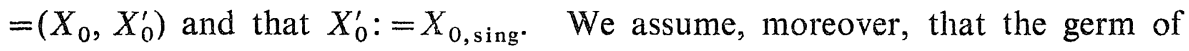
$X_{0}$ at $P_{0}$ is irreducible. From (4.3) $)_{3}^{\prime \prime}$ and a simple observation, the above assumptions are harmless for the proof of Lemma 4.1-Lemma 4.6. 


\section{§5.1. Proof of Lemma 4.1}

In n.1-n.4 we prove Lemma $4.1^{\prime}$, and in n.5, we prove Lemma 4.1.

1. Reduction of Lemma 4.1'. Letting the matrix $K: \mathfrak{D}_{X}^{v} \rightarrow \mathfrak{D}_{X}^{u}\left(X=X_{0}\right.$ $\left.-X_{0}^{\prime}\right)$ and its image $\mathfrak{R}:=K \mathfrak{D}_{X}^{v}\left(\subset \mathfrak{D}_{X}^{u}\right)$ be as in Lemma $4.1^{\prime}$, we make some reductions of Lemma 4.1'. First we see that the proof of the following special case of Lemma $4.1^{\prime}$ suffices for the proof of Lemma $4.1^{\prime}$ in its original form in n.4, Section 4.1:

Lemma 5.1'. Lemma 4.1' holds for $\left(X_{0}, X_{0}^{\prime} ; K\right)$, when $\left(X_{0}, X_{0}^{\prime}\right)=\left(U_{0}, \phi\right)$.

(For the terminology as above, see $(4.3)_{1}$.) Here we check the implication:

Proposition 5.1. Lemma 5.1' $\rightarrow$ Lemma 4.1'.

Proof. We let $\left(X_{0}, X_{0}^{\prime}\right)$ be of the general form as in Lemma 4.1', and we take an open subset $U_{1}\left(\ni P_{0}\right)$ of $U_{0}$. We then take matrices $\tilde{K}, \widetilde{G}$, whose entries are*) in $\Gamma\left(U_{1}, \mathfrak{D}_{U_{0}}\right)$, which satisfy the following:**)

(a) ${ }_{1} \pi(\tilde{K})=K$, and $\pi(\widetilde{G})=0$, where $\pi:=$ natural homomorphism: $\mathfrak{D}_{U_{0}} \rightarrow \mathfrak{D}_{X}$.

(a) ${ }_{2} \quad \pi\left(\varphi_{P}\right) \in \mathfrak{R}_{P} \Longleftrightarrow \varphi_{P} \in \tilde{\mathfrak{F}}_{P}$ for each $P \in U_{1}$ and $\varphi_{P} \in \mathfrak{D}_{U_{0}, P}^{\prime \prime}$, where***) $\tilde{\mathfrak{F}}$ : $=\tilde{F} \mathfrak{D}_{U_{0}}^{v+v^{\prime}}$, with the matrix $\tilde{F}:=[\tilde{K}, \tilde{G}]$.

Now, take an element $\varphi \in \Gamma\left(\tilde{U}_{r}(P), \Re, \theta_{\hat{\Omega}}^{\prime}\right)_{a}$ as in Lemma $4.1^{\prime}$. Then, by Proposition 4.4, we have an analytic map:

$$
\omega: U_{r^{\prime}}(P) \rightarrow \tilde{U}_{r}(P) \text {, which is the identity on } \tilde{U}_{r^{\prime}}(P) \text {. }
$$

Here $r^{\prime}:=M_{X}(r)$, with the positive monomial $M_{X}$ as in Proposition 4.4, and

(b) ${ }^{\prime} \quad U_{r}(P):=\left\{Q \in \mathbb{C}^{n} ; d(Q, P)<r\right\}$. (Also, as in Section 4.1, $\tilde{U}_{r}(P):=U_{r}(P) \cap$ $X$.

We apply 'Lemma 4.1 ' for $\left(U_{0}, \phi ; \tilde{\mathbb{F}}\right)$ ' to $\tilde{\varphi}=\omega^{*} \varphi$. Then we have an element ${ }^{* * * *)} \tilde{\Psi} \in \Gamma\left(U_{r^{\prime \prime}}(P), \mathfrak{O}_{U_{0}}^{++v^{\prime}}\right)_{a^{\prime}}$, satisfying $\tilde{F} \widetilde{\Psi}=\tilde{\varphi}$, where $\left(r^{\prime \prime} ; a^{\prime}\right)=M_{F}\left(r^{\prime} ; a\right)$

*) $\mathfrak{D}_{U_{0}}:=$ structure sheaf of $U_{0}$.

**) Take a base $f_{1}, \ldots, f_{w}$ of the ideal of $X_{0}$ (over $U_{1}$ ), and we set $\mathfrak{f}:=\left[f_{1}, \ldots, f_{w}\right]$. Then we form the $u>v^{\prime}$-matrix $G$, where $v^{\prime}:=u \times w$, to be: $\left.\tilde{G}:=\left[\begin{array}{c}f, 0, \ldots, \\ \vdots \\ \vdots \\ 0, \ldots, \ldots \\ \vdots\end{array}\right]\right\} u$. Then we easily see that $\tilde{F}:=[\tilde{K}, \tilde{G}]$ satisfies $(\text { a })_{1,2}$.

***) $v^{\prime}:=$ length of colums of $\tilde{G}$ (cf. the remark in **) soon above).

****) $\Gamma\left(U_{r}(P), \mathfrak{D}_{U_{0}}\right)_{a}:=\Gamma\left(U_{r}(P), \mathfrak{D}_{U_{0}} ; \theta\right)_{a}$, with the standard q-structure $\theta$ of $\mathfrak{D}_{U_{0}}$ (cf. Definition $1.4_{1}$ and $(4.1)_{2}$ ). We use the similar abbreviation in Section 5 . 
with an element $M_{F} \in \mathbf{M}$ (cf. $(4.1)_{3}$ ); by restricting $\widetilde{\Psi}$ to $X$, we have 'Lemma 4.1' for $\left(X_{0}, X_{0}^{\prime} ; K\right)^{\prime}$ (cf. also $\left.(\mathrm{a})_{1,2}\right)$, and we have Proposition 5.1. q.e.d.

2. We will prove Lemma $5.1^{\prime}$ inductively on $n=\operatorname{dim} U_{0}$. For this we add a lemma, which is slicely stronger than Lemma $5.1^{\prime}$ and is convenient for the inductive proof: take a variety $V\left(\ni P_{0}\right)$ in $U_{0}$ arbitrarily. Then taking a suitable subvariety $V^{\prime}$ of $V$ and an element $\sigma=\sigma_{V . V^{\prime}} \in \boldsymbol{R}_{1}^{+2}$, we form a parameter space $\boldsymbol{\mu}=\boldsymbol{\mu}_{V, V^{\prime}}$ in the similar manner to $(4.1)_{4}$ :

$$
\boldsymbol{\mu \ell}:=\left\{(P ; r) \in\left(U_{1} \cap\left(V-V^{\prime}\right)\right) \times \boldsymbol{R}^{+} ; r<\{\sigma g(P)\}^{-1}\right\},
$$

where $U_{1}=U_{1, V, V^{\prime}}$ is a suitable open subset $\left(\ni P_{0}\right)$ of $U_{0}$ and $g(P):=d\left(P, V^{\prime}\right)^{-1}$. We set $\lambda:=\boldsymbol{\mu} \times \boldsymbol{R}_{1}^{+}$. Then taking an estimation map $\tilde{M}=\tilde{M}_{V, V^{\prime}} \in \tilde{M}: \mathbb{R}^{--} \times \mathbb{R}^{+}$ $\rightarrow \boldsymbol{R}^{+} \times \boldsymbol{R}^{+}$, we have:

Lemma 5.1. We have the following inclusion:

$$
i^{*}\left(\Gamma\left(\widetilde{U}_{r}(P), \mathfrak{D}_{U_{0}}^{u}\right)_{a} \cap \mathfrak{S}_{P}\right) \subset K \Gamma\left(U_{r^{\prime}}(P), \mathfrak{D}_{U_{0}}^{v}\right)_{a^{\prime}},
$$

where $\left(r^{\prime} ; a^{\prime}\right)=\tilde{M}(r ; a)$ and $(P ; r ; a)$ is in $\lambda\left(\subset\left(V-V^{\prime}\right) \times \mathbb{R}^{+} \times \mathbb{R}_{1}^{+}\right)$. Moreover, we set:

(left side of $\left.(5.1)_{2}\right):=\left\{\varphi \in \Gamma\left(U_{r}(P), \mathfrak{D}_{U_{0}}^{u}\right)_{a} ; \varphi_{P} \in \mathfrak{R}_{P}\right\}$

(The matrix $K$ is as in the beginning of $n .1$, by understanding that $\left(X_{0}, X_{0}^{\prime}\right)$ $=\left(U_{0}, \phi\right)$.)

Actually, we clearly have: (left side of $\left.(5.1)_{2}\right) \supset \Gamma\left(\tilde{U}_{r}(P), \Omega ; \theta_{\Re}^{\prime}\right)_{a}$, and we have the implication: Lemma $5.1 \rightarrow$ 'Lemma $4.1^{\prime}$ for $\left(V, V^{\prime} ; K\right)^{\prime}$ (cf. (4.3) $)$. From this and from the chain property for sheaf homomorphisms (cf. Proposition 4.1), we easily see that Lemma 5.1 leads to Lemma 5.1'. Next we make some remarks on Lemma 5.1: first we check that the proof of Lemma 5.1 for the case of $u(=$ number of columns of $K)=1$ suffices for the case of $u \geqq 2$ :

Proposition 5.21. Lemma 5.1 for $u=1 \rightarrow$ that for $u \geqq 1$

Proof. Assuming that $u \geqq 2$, we write $K$ as ${ }^{t} K=\left[{ }^{t} K_{1},{ }^{t} K_{2}\right]$, where $K_{1}$ is of type $(1, v)$. Denote by $K_{1}^{\prime}\left(\subset \mathfrak{D}_{u_{0}}^{v}\right)$ the kernel of the homomorphism $K_{1}$ : $\mathfrak{D}_{u_{0}}^{v} \rightarrow \mathfrak{D}_{U_{0}}$. Choosing elements $\left\{g_{i}\right\}_{i=1}^{t} \subset \Gamma\left(U_{1}, \mathfrak{D}_{U_{0}}^{v}\right)$, which generate $K_{1}^{\prime}$, we set $K^{\prime \prime}:=\left[g_{1}, \ldots, g_{t}\right]$ and $K_{2}^{\prime}:=K_{2} \cdot K^{\prime \prime}$. Then the equation $K x=y$, where ${ }^{t} y=\left(y_{1}, \ldots, y_{u}\right)$ is solved as follows: first find a solution $x^{0}$ of $K_{1} x^{0}=y$, and we solve: $K_{2}^{\prime} x^{1}=y^{\prime}-K_{2} x^{0}$, where ${ }^{t} y^{\prime}:=\left[y_{2}, \ldots, y_{v}\right]$. Then we have: $K\left(x^{0}+K^{\prime \prime} x^{1}\right)$ $=y$. Using this remark, we check easily Proposition 5.2 . 
By Proposition 5.2 we henceforth assume that $u=1$ and that $K:=\left[f_{1}, \ldots\right.$, $f_{v}$ ] satisfies: $f_{1} \not \equiv 0$. Then setting $Y:=$ divisor of $f_{1}$, we have:

Proposition 5.2. Lemma 5.1 holds for $(V, V \cap Y ; K)$.

Proof. From the explicit form of Lemma 5.1 and from Lojasiewicz inequality, we easily see that the proof of Proposition $5.2_{2}$ in the case: $V=U_{0}$ leads to the general case: $V \subset U_{0}$. Now take a point $P \in U_{1}-Y$ and an element $r \in \mathbb{R}^{+}$satisfying $r<\left\{\sigma^{0} g(P)\right\}^{-1}$, where $g(P):=d(P, Y)^{-1}$ and $\sigma^{0}$ is a suitable element of $\mathbb{R}_{1}^{+2}$. Then Lojasiewicz inequality insures:

$$
\left|f_{1}(P)\right|>c d(P, Y) \text {, with a suitable } c=c_{f_{1}} \in \mathbb{R}^{+2} .
$$

From this we easily have:

$$
2\left|f_{1}(Q)\right|>\left|f_{1}(P)\right|>\left|f_{1}(Q) / 2\right| \text { in } U_{r}(P) .
$$

Take an element $\varphi \in \Gamma\left(U_{r}(P), \mathfrak{D}_{U_{0}}\right)_{a}$. Then we clearly have $\varphi=K y$, with ${ }^{t} y$ : $=\left(\varphi / f_{1}, 0, \ldots, 0\right)$. Using (a), (b) and that $r<\left(\sigma^{0} g(P)\right)^{-1}$, we easily have:

$$
\left|\varphi / f_{1}\right|<a \cdot M_{c, \sigma^{0}}\left(r^{-1}\right) \text {, with a positive monomial } M_{c, \sigma^{0}},
$$

and we have 'Lemma 5.1 for $\left(U_{0}, Y ; K\right)$ '.

q.e.d.

3. Now we prove Lemma 5.1 inductively on $n=\operatorname{dim} U_{0}$. First, if $n=1$, then Proposition 5.2 insures that the check of Lemma 5.1 for the case of $(V$, $\left.V^{\prime} ; K\right)=\left(P_{0}, \phi ; K\right)$ suffices for the proof of Lemma 5.1 itself (cf. also Proposition 4.1). To see the former, take an element $\varphi \in \Gamma\left(U_{r}(P), \mathfrak{D}_{U_{0}}\right)_{a}$ (cf. $\left.(5.1)_{2}\right)$, and we assume that $m:=\operatorname{deg} f_{1}>\operatorname{deg} f_{j}(j \geqq 2)$. Then we have: $\left|f_{1}(Q)\right|>c_{f_{1}}$. $\left|z^{m}\right|$, with an element $c_{f_{1}} \in \mathbb{R}^{+}$, in a neighborhood of $P_{0}$ (in $\mathbb{C}$ ). (Here $z$ is the coordinate of $C)$. Remarking $\varphi_{P_{0}} \equiv 0\left(f_{1}\right)$, we estimate the holomorphic function $\varphi / f_{1}$ at the boundary of $U_{r}(P)$ (by means of the maximal principle). Then we have: $\left|\varphi / f_{1}\right| \leqq c_{f_{1}} a / r^{m}$ in $U_{r / 2}(P)$. This insures Lemma 5.1 for $n=1$. We henceforth assume that $n \geqq 2$ and that Lemma 5.1 holds for $\tilde{n}<n$. By Proposition $5.2_{2}$ we assume that $V \subset Y$ (=locus of $f_{1}$ ). Moreover, without loss of generality, we assume that

$(5.1)_{4}$ the germ of $V$ at $P_{0}$ is irreducible, $f_{j}(1 \leqq j \leqq t)$ are Weierstrass polynomials*) at $P_{0}$, and $m>\operatorname{deg} f_{j}$ at $P_{0}(j \geqq 2)$, where $m=\operatorname{deg} f_{1}$ at $P_{0}$.

We then define an element $m \in \mathbb{Z}^{+}$and a subvariety $V^{\prime \prime} \subset V$ by:

(a) $\operatorname{deg} f_{1}=m$ at each $P \in V-V^{\prime \prime}$, and $\operatorname{deg} f_{1}>m$ at each point $P \in V^{\prime \prime}$.

*) Weierstrass Polynomial in the last coordinate $z_{n}$ of $z$. 
Now we prove the implication:

$$
\text { Lemma } 5.1 \text { for } \tilde{n}=n-1 \longrightarrow \text { that for } \tilde{n}=n,
$$

using the standard methods of the proof of the coherency theorem of K. Oka (cf. [14], [4]).

4. Proof of $(\mathbf{5 . 1})_{\mathbf{5}}$. We begin n.4 by summarizing some (elementary) estimations, which are used in the proof of $(5.1)_{5}$. For this we take suitable elements $\sigma=\sigma_{V, V^{\prime}}, \alpha=\alpha_{V, V^{\prime}} \in \mathbb{R}^{+2}$ and an estimation map $\tilde{M}=\tilde{M}_{V, V^{\prime}} \in \tilde{\mathbb{M}}$. Then letting the open set $U_{1}\left(\ni P_{0}\right)$ of $U_{0}$ be as in Lemma 5.1, we have the following for each $P \in U_{1} \cap\left(V-V^{\prime}\right)$ and each $r \in \mathbb{R}^{+}$satisfying $r<\{\sigma g(P)\}^{-1}$, with $g(P)=d\left(P, V^{\prime}\right)^{-1}$.

Proposition 5.3. (1) One can write $f_{1}$ as $f_{1}=\varepsilon \cdot f_{1}^{\prime}$, with $f_{1}^{\prime}, \varepsilon \in \Gamma\left(U_{r}(P)\right.$, $\left.\mathfrak{D}_{U_{0}}\right)$. Here $f_{1}^{\prime}$ is a Weierstrass polynomial at $P$ and $\varepsilon$ is a unit in $\Gamma\left(U_{r}(P)\right.$, $\left.\mathfrak{D}_{U_{0}}\right)$. Moreover, $\varepsilon$ satisfies:

$$
|\varepsilon(Q)|,\left|\varepsilon^{-1}(Q)\right|<\alpha \cdot\left\{d\left(Q, V^{\prime}\right)^{-1}\right\} \text { for each } Q \in U_{r}(P) .
$$

(2) For an element $\varphi \in \Gamma\left(U_{r}(P), \mathfrak{D}_{U_{0}}\right)_{a}\left(a \in \mathbb{R}_{1}^{+}\right)$we have:

$(5.1)_{7} \varphi=\varphi_{1} f_{1}^{\prime}+\varphi_{2}$, where $\varphi_{1}, \varphi_{2}$ are in $\Gamma\left(U_{r^{\prime}}(P), \mathfrak{D}_{U_{0}}\right)_{a^{\prime}}$, and $\varphi_{2}$ is a polynomial in $z_{n}$ of degree $<m$. Moreover, $\left(r^{\prime} ; a^{\prime}\right)=\tilde{M}(r ; a)$.

We prove Proposition 5.3 in Appendix I.

Proof of $(5.1)_{5}$. Letting $\varphi$ and $\varphi_{2}$ be as in $(5.1)_{7}$, we assume that $\varphi \in \mathfrak{S}_{P}$. Then $\tilde{\varphi}_{2}:=\varepsilon \cdot \varphi_{2}$ is also in $\boldsymbol{\Lambda}_{P}$. Remarking that $\tilde{\varphi}_{2}$ is a polynomial of degree $\tilde{m}-1$ in $z_{n}$, we write $\tilde{\varphi}_{2}=\sum_{k=0}^{\tilde{n}-1} g_{k} z_{n}^{k}$, and we form a vector $x:=\left(g_{k}\right)_{k=0}^{m-1}$. Moreover, we denote by $\tau$ the projection: $\mathbb{C}^{n}(z) \rightarrow \mathbb{C}^{n-1}\left(z_{1}, \ldots, z_{n-1}\right)$. Without loss of generality for the proof of Lemma 5.1, we assume that $U_{0}$ is of the form $U_{0}$ $=U_{0}^{\prime} \times U_{0}^{\prime \prime}$, where $U_{0}^{\prime \prime}$ is a polydisc in $\mathbb{C}^{n-1}$ and $U_{0}^{\prime \prime}$ is a disc in $\mathbb{C}$. We set $P^{\prime}=\tau(P)$.

(i) First we have the following estimation:

$$
x \in \Gamma\left(U_{r^{\prime}}^{\prime}\left(P^{\prime}\right), \mathfrak{D}_{U_{0}^{\prime}}^{\tilde{n}}\right)_{a^{\prime}}, \text { with }\left(r^{\prime} ; a^{\prime}\right)=\tilde{M}_{f_{1}}(r ; a),
$$

where the estimation map $\tilde{M}_{f_{1}}$ is in $\tilde{\mathbb{M}}$, and we set $U_{r}^{\prime}\left(P^{\prime}\right):=\left\{Q \in \mathbb{C}^{n-1} ; d\left(Q, P^{\prime}\right)\right.$ $\left.<r^{\prime}\right\}$.

To check this we first note that the inequality $|\varepsilon(Q)|^{-1}>\alpha \cdot d\left(Q, V^{\prime}\right)$ leads*) easily

*) Also note that the element $r \in \mathbb{R}^{+}$in Proposition 5.3 satisfies: $r<\left(\sigma \cdot d\left(P, V^{\prime}\right)^{-1}\right)^{-1}$. Using this, we get easily (a) ${ }_{1}$ (cf. also (c) in the proof of Proposition 5.2 ${ }_{2}$. 
to the inequality:

$$
|\varepsilon(Q)|<M_{f_{1}}\left(1 / r^{\prime}\right) \text {, with a positive monomial } M_{f_{1}} .
$$

From this and from that $\left|\varphi_{2}\right|<a^{\prime}$ (cf. $\left.(5.1)_{7}\right)$, we have:

$(\mathrm{a})_{2}$

$$
\left|\tilde{\varphi}_{2}\right|<\bar{a}^{\prime}:=a^{\prime} \cdot M_{K}\left(1 / r^{\prime}\right) .
$$

Writing $\tilde{\varphi}_{2}$ as $\tilde{\varphi}_{2}=\sum_{k=0}^{\tilde{m}-1} g_{k}^{\prime}\left(z_{n}-z_{n}(P)\right)^{k}$, we estimate $g_{k}^{\prime}$ in the following form (by means of Cauchy integral formula):

$(\mathrm{a})_{3}$

$$
g_{k}^{\prime} \in \Gamma\left(U_{r^{\prime \prime}}^{\prime}\left(P^{\prime}\right), \mathfrak{D}_{U_{0}^{\prime}}^{\prime}\right)_{a^{\prime \prime}} \text {, with }\left(r^{\prime \prime} ; a^{\prime \prime}\right)=\left(r^{\prime} / 4 ; \bar{a}^{\prime} \cdot\left(2 / r^{\prime}\right)^{\tilde{m}}\right) .
$$

Finally, rewriting $\tilde{\varphi}_{2}$ as $\tilde{\varphi}_{2}=\sum_{k=0}^{m-1} g_{k} z_{n}^{k}$, we have (a).

(ii) Next, applying the standard method of the proof of the coherency theorem (cf. [4]) to the pair $\left(K, \tilde{\varphi}_{2}\right)$, we have elements $\Phi_{j} \in \mathfrak{D}_{U_{0}, P}$ satisfying

(b) $\quad \tilde{\varphi}_{2}=\sum_{j=1}^{v} \Phi_{J} f_{j}$, and $\Phi_{j}$ are polynomials of $z_{n}$ of degree $\leqq \tilde{m}-1$.

Here the key fact is, as in the proof of the coherency theorem, that the coefficients $f_{j}$ of the above equation are independent from $P \in V-V^{\prime}$. We write $\Phi_{j}=$ $\sum_{k=0}^{\tilde{m}-1} g_{j k} z_{n}^{k}$, and we form vectors $\tilde{x}, y$ by ${ }^{*}{ }^{t} \tilde{x}:=\left(0,{ }^{t} x\right)$ and ${ }^{t} y:=\left(y_{1}, \ldots, y_{v}\right)$, with $y_{j}:=\left(g_{j k}\right)_{k=0}^{\tilde{m}-1}$. Then (b) is equivalent to

(c) $x=K^{\prime} y$, with a matrix $K^{\prime}$, whose entries are the polynomials of the coefficients of $f_{j}(1 \leqq j \leqq v)$, and are holomorphic in $U_{0}^{\prime}$.

(iii) Now we derive Lemma 5.1 for $\tilde{n}=n$ from that for $\tilde{n}=n-1$ (applied to the matrix $K^{\prime}$ ). For this recalling that $V \subset Y$ (=locus of the Weierstrass polynomial $f_{1}$ ), the map $\tau: V \rightarrow \widetilde{V}:=\tau(V)$ is integral ${ }^{* *}$ ) and is surjective. Then, taking a suitable subvariety $\widetilde{V}^{\prime}$ of $\widetilde{V}$, the induction hypothesis insures 'Lemma 5.1 for $\left(\tilde{V}, \tilde{V}^{\prime} ; K^{\prime}\right)^{\prime}$. Setting $V^{\prime \prime \prime}:=\tau^{-1}\left(\tilde{V}^{\prime}\right) \cap V$ and $V^{\prime \prime}=V^{\prime \prime \prime} \cup V^{\prime}$, we assume that the point $P$ is in $V-V^{\prime \prime}$. Also we assume that the element $r \in \boldsymbol{R}^{+}$in (5.1) satisfies***): $r<\left\{\sigma \cdot d\left(P, V^{\prime \prime}\right)^{-1}\right\}^{-1}$, with an element $\sigma=\sigma_{V, V^{\prime \prime}} \in \boldsymbol{R}_{1}^{+2}$. Setting $\mathfrak{\Omega}^{\prime}:=K^{\prime} \mathfrak{D}_{U_{0}^{\prime}}^{\tilde{v}}\left(\subset \mathfrak{D}_{U_{0}^{\prime}}^{\tilde{u}_{0}}\right)$, where $\tilde{v}:=m \cdot v$, we note that $x \in \mathfrak{\Re}_{P^{\prime}}^{\prime}$. Then, applying 'Lemma 5.1 for $\left(V, V^{\prime} ; K^{\prime}\right)$ ' to the element $x$, we have:

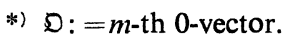

**) Precisely, 'integral' at the origin $P_{0}$ of $V$.

***) Note that, to apply 'Lemma 5.1 for $\left(\tilde{V}, \tilde{V}^{\prime} ; K^{\prime}\right)$ ', the element $r \in \boldsymbol{R}^{+}$should satisfy: $r<\left(\tilde{\sigma} \cdot d\left(P, \tilde{V}^{\prime}\right)^{-1}\right)^{-1}$, with an element $\bar{\sigma}=\bar{\sigma}_{\tilde{V}}, \tilde{\nu}^{\prime} \in \boldsymbol{R}_{1}^{+2}$. But from the Lojasiewicz inequality and a simple observation, we have: $d\left(P, V^{\prime \prime}\right) \leqq c d\left(P^{\prime}, V^{\prime}\right)$, with an element $c \in \boldsymbol{R}^{+2}$. From this one can assume that the inequality soon above is satisfied (because $r^{\prime \prime}<\left(\left(\sigma \cdot d\left(P, V^{\prime \prime}\right)^{-1}\right)^{-1}\right)$. 
(d) $x \in K^{\prime} \Gamma\left(\tilde{U}_{r^{\prime \prime}}^{\prime}\left(P^{\prime}\right), \mathfrak{D}_{U_{0}^{\prime}}^{v}\right)_{a^{\prime \prime}}$, where $\left(r^{\prime \prime} ; a^{\prime \prime}\right)=\tilde{M}_{K^{\prime}}(r ; a)$ with an estimation map $\tilde{M}_{K^{\prime}} \in \tilde{\mathbf{M}}$.

By the equivalence of (b) and (c), we have*):

(e) $\varphi_{2}=\sum_{j} \tilde{\Phi}_{j} \cdot f_{j}$, with $\tilde{\Phi}_{j} \in \Gamma\left(\tilde{U}_{\tilde{r}^{\prime \prime}}(P), \mathfrak{D}_{U_{0}}\right)_{\tilde{a}^{\prime \prime}}$, with $\left(\tilde{r}^{\prime \prime} ; \tilde{a}^{\prime \prime}\right)=\tilde{M}_{K}(r ; a)$.

Finally, setting $\Phi_{j}^{\prime}:=\Phi_{j} / \varepsilon$, we have: $\varphi_{2}=\sum_{j} \Phi_{j}^{\prime} \cdot f_{j}$. Also, applying the similar argument to $(a)_{1}$ to the estimation of $\left|\varepsilon^{-1}\right|$ in $(5.1)_{6}$, we have the similar estimation to (e) for $\Phi_{j}^{\prime}$. This implies 'Lemma 5.1 for $\left(V, V^{\prime \prime} ; K\right)$ ', and we also finish the proof of Lemma 5.1 (and so that of Lemma 4.1').

5. Proof of Lemma 4.1. Here we derive Lemma 4.1 from Lemma 4.1', by using a cohomological method as follows: (i) first, for a subset $Y$ of $X=X_{0}$ $-X_{0}^{\prime}$ and elements $t, a \in \mathbb{R}^{+}$, we define:

$$
\left\{\begin{array}{c}
\mathscr{A}_{t}(Y):=\left\{\tilde{U}_{t}(Q) ; Q \in Y\right\}, \text { where } \tilde{U}_{t}(Q):=\{R \in X ; d(R, Q)<t \\
\left.\left(\text { cf. }(4.1)_{1}\right)\right\}, \\
C^{q}\left(\mathscr{A}_{t}(Y), \mathfrak{D}_{X}\right)_{a}:=\left\{\varphi \in C^{q}\left(\mathscr{A}_{t}(Y), \mathfrak{D}_{X}\right) ;\left|\varphi_{\mathscr{A}}(Q)\right|<a\right. \\
\left.\quad \text { for each } \mathscr{A} \in \mathscr{N}^{q+1} \mathscr{A}_{t}(Y)\right\} \\
Z^{q}\left(\mathscr{A}_{t}(Y), \mathfrak{D}_{X}\right)_{a}:=C^{q}\left(\mathscr{A}_{t}(Y), \mathfrak{D}_{\mathbf{X}}\right)_{a} \cap \delta^{-1}(0)
\end{array}\right.
$$

Also taking an open set $U_{X}^{\prime}$ of $U_{0}$ and an element $\sigma_{X} \in \mathbb{R}_{1}^{+2}$, we form the following parameter space:

$(5.2)_{0}^{\prime} \quad \lambda_{X}:=\left\{(P ; r ; t ; a) \in\left(U_{X}^{\prime} \cap X\right) \times \mathbb{R}^{+} \times \mathbb{R}^{+} \times \mathbb{R}_{1}^{+} ; r, t<\left\{\sigma_{X} g_{X}(P)\right\}^{-1}\right\}$, with $g_{X}(P):=d\left(P, X_{0}^{\prime}\right)^{-1}$.

Moreover, setting $\overline{\mathbf{M}}:=\mathbf{M} \times \mathbf{M} \times \mathbf{M}$, where $\mathbf{M}:=$ collection of all positive monomials (cf. n.5, §1.1), we define the following map for each $\bar{M}=\left(M_{i}\right)_{i=1}^{3}$ $\in \overline{\mathbf{M}}$ :

$(5.2)_{0}^{\prime \prime} \quad \bar{M}: \boldsymbol{R}^{+} \times \boldsymbol{R}^{+} \times \boldsymbol{R}^{+} \ni(r ; t ; a) \rightarrow \boldsymbol{R}^{+} \times \boldsymbol{R}^{+} \times \boldsymbol{R}^{+} \ni\left(M_{1}(r), M_{2}(r t), M_{3}(a / r t)\right)$.

Then taking an estimation map $\bar{M}_{X} \in \bar{M}$, the following lemma will play a key fact for the proof of Lemma $4.1^{* *)}$ :

Lemma 5.2. $s^{*} Z^{q}\left(\mathscr{A}_{t}\left(\tilde{U}_{r}(P)\right), \mathfrak{D}_{\mathbf{X}}\right)_{a} \subset \delta C^{q-1}\left(\mathscr{A}_{t^{\prime}}\left(\tilde{U}_{r^{\prime}}(P)\right), \mathfrak{D}_{\mathbf{X}}\right)_{a^{\prime}}(q \geqq 1)$, with $\left(r^{\prime} ; t^{\prime} ; a^{\prime}\right)=\bar{M}_{\mathbf{X}}(r ; t ; a)$, where the parameter $(P ; r ; t ; a)$ is in $\lambda_{X}\left(\subset X \times \mathbb{R}^{+}\right.$

*) In (e) we understand that the estimation map $\tilde{M}_{K}$ is of the desired form as in Lemma 5.1 .

**) The refining map $s$ in Lemma 5.2 is defined similarly to Definition $1.6_{2}: s: \mathscr{A}_{t^{\prime}}\left(\tilde{U}_{r^{\prime}}(P)\right)$ $\ni \tilde{U}_{t}^{\prime}(Q) \longrightarrow \mathscr{A}_{t}\left(\tilde{U}_{r}(P)\right) \ni \tilde{U}_{t}(Q)$. 
$\left.\times \boldsymbol{R}^{+} \times \boldsymbol{R}_{1}^{+}\right)$

For convenience of the proof of Lemma 5.1, we add here the following weaker version of Lemma 5.2:

Lemma 5.2'. Lemma 5.2 is true, if $\left(X_{0}, X_{0}^{\prime}\right)=\left(U_{0}, \phi\right)$.

We prove Lemma 5.2' at the end of Section 6. Also we derive Lemma 5.2 from Lemma $5.2^{\prime}$ in (iii). In (ii) we derive Lemma 4.1 from Lemma 4.1' and Lemma 5.2.

(ii) 'Double complex'. Next letting the sheaf $\mathfrak{H} \in \operatorname{Coh}\left(X_{0} ; \not\right)$ be as in $(4.1)_{6}$, we assume that $\# \mathscr{L}=1$ (cf. (iv), n.7, §4.1). For notational convenience, we write $\mathfrak{H}$ as follows:

$$
0 \longrightarrow \mathfrak{D}_{X}^{k_{0}} \stackrel{\delta_{\mathfrak{g}}}{\longrightarrow} \cdots \mathfrak{D}_{X}^{k_{p}-1} \stackrel{\delta_{\mathfrak{g}}}{\longrightarrow} \mathfrak{D}_{X}^{k_{p}} \longrightarrow \mathfrak{D}_{X}^{k_{p}} / \mathfrak{H} \longrightarrow 0
$$

where we use the symbol $\delta_{5}$ for the matrices $K_{0}, \ldots$ in $(4.1)_{6}$, and the homomorphism in the final stage is the natural one: $\mathfrak{D}_{X}^{k_{p}} \rightarrow \mathfrak{D}_{X}^{k_{p}} / \mathfrak{S}$ (with $\mathfrak{S}=\delta_{\mathfrak{g}} \mathfrak{D}_{X}^{k_{p-1}}$ ). Then, for the parameter $\lambda_{2}=(P ; r ; t ; a) \in \lambda_{X}\left(\subset\left(U_{X}^{\prime} \cap X\right) \times \mathbb{R}^{+} \times \mathbb{R}^{+} \times \mathbb{R}_{1}^{+}\right)$, we set:

$$
\left\{\begin{array}{l}
C^{\tilde{p} q}(\lambda) \\
Z^{\prime \tilde{p} q}(\lambda) \\
Z^{\tilde{p} q}(\lambda)
\end{array}\right\}:=\left\{\begin{array}{l}
C^{q}\left(\mathscr{A}_{t}\left(\tilde{U}_{r}(P)\right), \mathfrak{D}_{X}^{k \tilde{p}}\right)_{a}(0 \leqq \tilde{p} \leqq p, q \geqq 0) \\
C^{\tilde{p} q}(\lambda) \cap \delta_{\mathfrak{g}}^{-1}(0), Z^{\prime \prime} \tilde{p} q(\lambda):=C^{\tilde{p} q}(\lambda) \cap \delta^{-1}(0) \\
Z^{\prime \tilde{p} q}(\lambda) \cap Z^{\prime \prime} \tilde{p} q(\hat{\lambda})
\end{array}\right\} .
$$

Next taking an element $\sigma_{\mathfrak{5}} \in \mathbb{R}_{1}^{+2}$, we form a parameter space $\lambda_{\mathfrak{g}}\left(\subset\left(U_{X}^{\prime} \cap X\right)\right.$ $\left.\times \boldsymbol{R}^{+} \times \boldsymbol{R}^{+} \times \boldsymbol{R}_{1}^{+}\right)$similarly to $(5.2)_{0}^{\prime}$, by changing the condition on the third term: ' $t<\left\{\sigma_{X} g_{X}(P)\right\}^{-1}$ ' by ' $t<\left\{\sigma_{\tilde{n}} g_{D}(P)\right\}^{-1}$ ': with $g_{D}(P):=d(P, D)^{-1}$ where $D:=$ divisor of $h$ on $X_{0}$.

Proposition 5.4. . We have the following inclusions:

$$
\begin{array}{r}
\left\{\begin{array}{l}
Z^{\prime \tilde{p} q}(P ; r ; t ; a) \subset \delta_{\mathfrak{5}} C^{\tilde{p}-1} q\left(P ; r ; t^{\prime} ; a^{\prime}\right)(\tilde{p} \geqq 1) \\
Z^{\prime \prime \tilde{p} q}(P ; r ; t ; a) \subset \delta C^{\tilde{p} q-1}\left(P ; r^{\prime} ; t^{\prime} ; a^{\prime}\right)(q \geqq 1)
\end{array}\right\}, \text { with } \\
\left\{\begin{array}{l}
\left(t^{\prime} ; a^{\prime}\right)=\tilde{M}_{\mathfrak{\mathfrak { d }}}(t ; a) \\
\left(r^{\prime} ; t ; a^{\prime}\right)=\bar{M}_{X}(r ; t ; a)
\end{array}\right\},
\end{array}
$$

where the parameter $(P ; r ; t ; a)$ is in $\lambda_{\mathfrak{g}}$ or in $\lambda_{X}\left(\subset X \times \mathbb{R}^{+} \times \boldsymbol{R}^{+} \times \boldsymbol{R}_{1}^{+}\right)$, and the estimation maps $\tilde{M}_{\mathfrak{5}}, \bar{M}_{X}$ are in $\tilde{\mathbf{M}}$ and $\overline{\mathbf{M}}$ *) $^{*}$

Proof. The second inclusion is a rewriting of Lemma 5.2. The first one is

*) In Proposition 5.4 , the first factor $\left(U_{X}^{\prime} \cap X\right)$ of $\lambda_{\mathfrak{5}}$ is independent from the individual $\mathfrak{H} \in \operatorname{Coh}\left(X_{0} ; \not h\right)$. This is insured by $(2)$, Remark $4.1_{1}$. 
derived from Lemma 4.1', using a similar procedure to Proposition $4.5_{1}$ : letting the estimation: $(r ; t) \rightarrow\left(M_{1}(r), M_{2}(a / t)\right)$ be as in Lemma $4.1^{\prime}$, we define a map $\tilde{M}_{\text {. }} \in: \tilde{\mathbb{N}}(t ; a) \rightarrow\left(M_{1}^{\prime}(t), M_{2}(a / 4 t)\right)$, where $M_{1}^{\prime}:=M_{0} \cdot M_{1} \cdot M_{0}$, with a map $M_{0}: t \rightarrow t / 4$. Then using the similar inclusion to $(4.10)_{5}$ in Proposition $4.5_{1}$, we have the first inclusion, which is a cohomological version of Lemma 4.1'.

q.e.d.

Next letting the estimation map $\bar{M} \in \overline{\mathbb{M}}$ be as in $(5.2)_{0}^{\prime \prime}$, we call $M_{1}: \mathbb{R}^{+} \ni r$ $\rightarrow \mathbb{R}^{+} \ni M_{1}(r)$ the first part of $\bar{M}$, and we use the symbol $\pi$ for the projection: $\overline{\mathbb{M}} \ni \bar{M} \rightarrow \mathbb{M} \ni M_{1}$.

Proposition 5.4. $4_{2}$. There is a map $\varepsilon_{\varsigma}$ : $\operatorname{Coh}\left(X_{0} ; \not\right) \ni \mathfrak{H} \rightarrow \overline{\mathbb{M}} \ni \bar{M}_{\mathfrak{n}}$, which is factored as in Figure I, and with which we have the following $\operatorname{map}(p \geqq \tilde{p} \geqq 1)$ :

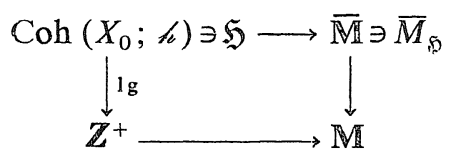

Figure I. $(5.2)_{4}\left\{\begin{array}{l}\theta: Z^{\tilde{p} 0}(P: r ; t ; a) \hookrightarrow Z^{0} \tilde{p}\left(P ; r^{\prime} ; t^{\prime} ; a^{\prime}\right) \\ \theta^{\prime}: Z^{0 \tilde{p}}(P ; r ; t ; a) \hookrightarrow Z^{\tilde{p} 0}\left(P ; r^{\prime} ; t^{\prime} ; a^{\prime}\right)\end{array}\right.$, with $\left(r^{\prime} ; t^{\prime} ; a^{\prime}\right)=\bar{M}_{\tilde{\mathfrak{g}}}(r ; t ; a)$, which satisfy:

$$
\left\{\begin{array}{c}
\left(1-0^{\prime} \cdot \theta\right) Z^{\tilde{p} 0}(P ; r ; t ; a) \hookrightarrow \delta_{5} Z^{\prime \prime \tilde{p}-10}\left(P ; r^{\prime} ; t^{\prime} ; a^{\prime}\right) \\
\theta \delta_{\tilde{5}} Z^{\prime \prime \tilde{p}-10}(P ; r ; t ; a) \hookrightarrow \delta Z^{\prime 0 \tilde{p}-1}\left(P ; r^{\prime} ; t^{\prime} ; a^{\prime}\right) \\
\text { with }\left(r^{\prime} ; t^{\prime} ; a^{\prime}\right)=\bar{M}_{\mathfrak{g}}(r ; t ; a)
\end{array}\right.
$$

and the similar facts for $\left(1-\theta \cdot \theta^{\prime}\right) Z^{0 \tilde{p}}$ and $\delta Z^{0} \tilde{p}^{-1}$ (by changing the role of $\delta_{j}$ and $\left.\delta\right)$. (In the above the parameter $(P ; r ; t ; a)$ is in $\lambda_{5}\left(\subset X \times \mathbb{R}^{+} \times \mathbb{R}^{+}\right.$ $\left.\times \mathbb{R}_{1}^{+}\right)$.)

Proof. It suffices to check the similar fact for $Z^{\tilde{p} q}:=Z^{\tilde{p} q}(P ; r ; t ; a)$ and $Z^{\tilde{p}-1 q+1}:=Z^{\tilde{p}-1 q+1}(P ; r ; t ; a)$, where $1 \leqq \tilde{p} \leqq p, q \geqq 0$. For this take an element $\varphi \in Z^{\tilde{p} q}$ (resp. $\left.\varphi^{\prime} \in Z^{\tilde{p}-1} q^{+1}\right)$. Then, by Proposition 5.4 , we take elements $\varphi_{1}, \varphi_{1}^{\prime}$ from the right sides in (5.2) $)_{3}$ satisfying $\delta_{\mathfrak{5}} \varphi_{1}=\varphi, \delta \varphi_{1}^{\prime}=\varphi^{\prime}$. We set $\tilde{\varphi}=\delta \varphi_{1}$ and $\tilde{\varphi}^{\prime}=\delta_{\tilde{n}} \varphi_{1}^{\prime}$. Then we easily*) see that the maps $\theta_{\tilde{p} q}: \varphi \rightarrow \tilde{\varphi}, \theta_{\tilde{p} q}^{\prime}$ : $\varphi^{\prime} \rightarrow \tilde{\varphi}^{\prime}$ satisfy the similar fact to $(5.2)_{4}$.

q.e.d.

(Proposition 5.4, correspond to the degeneracy theorem in the spectral sequence arguments. Our arguments as above are suitable for the explicit

*) Note that the estimation map $\tilde{M}_{X} \in \tilde{\mathbb{M}}$ in Proposition $5.4_{1}$ is independent from the individual $\mathfrak{F}_{\mathrm{C}} \in \operatorname{Coh}\left(X_{0} \measuredangle\right)$, while the map $\tilde{M}_{\tilde{n}} \in \tilde{\mathbb{M}}$ does not operate out the first term $r \in \mathbb{R}^{+}$(or, operates on $r$ as the identity). The factorization in Figure $I$ follows from these remarks. 
estimations, and are patterned on the algebraic arguments in [20].) Now from Proposition $5.4_{1,2}$ we easily have:

Proposition 5.5. $Z^{p 0}(P ; r ; t ; a) \subset \delta_{5} Z^{\prime p-10}\left(P ; r^{\prime} ; t^{\prime} ; a^{\prime}\right)(p \geqq \tilde{p} \geqq 1)$, where the estimation: $(r ; t ; a) \rightarrow\left(r^{\prime} ; t^{\prime} ; a^{\prime}\right)$ is as in Proposition $5.4_{1,2}$.

Finally we define an obvious map:

$$
\mu: Z^{\prime \prime \tilde{p}} 0(P ; r ; t ; a) \hookrightarrow \Gamma\left(\tilde{U}_{r}(P), \bigvee_{X}^{k \tilde{p}}\right) .
$$

We easily see:

$$
\mu^{-1}: \Gamma\left(\tilde{U}_{r}(P), \mathfrak{D}_{X}^{k_{p}}\right)_{a} \hookrightarrow Z^{\prime \prime \tilde{p} 0}(P ; r / 2 ; r / 2 ; a) .
$$

From (5.2) 5.6 and Proposition 5.5 we have:

$$
\left(\Gamma\left(\tilde{U}_{r}(P), \mathfrak{D}_{\mathbf{X}}^{k \tilde{p}}\right)_{a} \cap \delta_{\mathfrak{\xi}^{-1}}^{-1}(0)\right) \subset \delta_{\tilde{\xi}} \Gamma\left(\tilde{U}_{r^{\prime}}(P), \mathfrak{D}_{\mathbf{X}}^{k \tilde{p}^{-1}}\right)_{a^{\prime}}(1 \leqq \tilde{p} \leqq p),
$$

where the correspondence: $(r ; a) \rightarrow\left(r^{\prime} ; a^{\prime}\right)$ is as in Proposition $5.4_{2}$.

This inclusion and the factorization in Figure I, Proposition $5.4_{2}$ insure Lemma 4.1, and we finish the proof of Lemma 4.1.

(iii) Here we check the implication: Lemma 5.2' $\rightarrow$ Lemma 5.2. In (iii) we assume that $X_{0}$ is irreducible at the origin $P_{0}$ of $X_{0}$. This assumption is harmless for the proof of Lemma 5.2. Now letting the parameter $(P ; r ; t)$ $\in X \times \boldsymbol{R}^{+} \times \boldsymbol{R}^{+}$be as in Lemma 5.2, we take a suitable projection*) $\tau: \boldsymbol{C}^{n}(z)$ $\rightarrow \boldsymbol{C}^{k}\left(z^{\prime}\right)$, where $z^{\prime}=\left(z_{i_{1}}, \ldots, z_{i_{k}}\right)$ with $k=\operatorname{dim} Z_{0}$, and we set $P^{\prime}:=\tau(P)$. Then we have the following refining maps:

$(5.2)_{8}\left\{\begin{array}{l}s_{P}: \mathscr{A}_{t^{\prime}}\left(\tilde{U}_{r^{\prime}}(P)\right) \ni \tilde{U}_{t^{\prime}}(Q) \longrightarrow \tau^{-1}\left(\mathscr{A}_{t}\left(\tilde{U}_{r}\left(P^{\prime}\right)\right) \ni \tau^{-1}\left(\tilde{U}_{t}(\tau(Q))\right)\right. \\ s_{P^{\prime}}: \mathscr{A}_{t^{\prime}}\left(\tilde{U}_{r^{\prime}}\left(P^{\prime}\right)\right) \ni \tilde{U}_{t^{\prime}}(Q) \longrightarrow \tau\left(\mathscr{A}_{t}\left(\tilde{U}_{r}(P)\right) \ni \tau\left(\tilde{U}_{t}\left(\tau^{-1}(Q)\right)\right)\right.\end{array}\right\}$,

with $\left(r^{\prime} ; t^{\prime}\right)=\left(M_{X}(r), M_{X}(t)\right)$, where $M_{X}$ is a positive monomial. (This follows from Proposition 2, Appendix II.) It is then easy to see that, by using the above cofinal relation between $\mathscr{A}_{t}\left(\tilde{U}_{r}(P)\right)$ and $\mathscr{A}_{t}\left(\tilde{U}_{r}\left(P^{\prime}\right)\right)$, Lemma 5.2 (given to $X$ ) is reduced to Lemma 5.2' (given to $C^{k}$ ). As was mentioned, the check of Lemma $5.2^{\prime}$ is given in the end of Section 6.3.

\section{§5.2. Proof of Lemma 4.2-Lemma 4.6}

Here we prove the lemmas in the title, by using Proposition 4.2 and some elementary computations on the a.d. properties of coherent sheaves (cf. Lemma

*) 'suitable' $=$ suitable choice of coordinates $z^{\prime} \in z$. After fixing the coordinates $z^{\prime}, \tau$ is the natural one: $\boldsymbol{C}^{n}(z) \ni z \rightarrow \boldsymbol{C}^{k}\left(z^{\prime}\right) \ni z^{\prime}$. 
5.3 and Lemma 5.5). In n.1-n.4 we prove Lemma 4.2-Lemma 4.4. We prove Lemma 4.5 and Lemma 4.6 in n.5 and n.6. As in Section 5.1, our underlying variety is $X:=X_{0}-X_{0}^{\prime}$, with $X_{0}^{\prime}:=X_{0, \text { sing }}$.

1. Key lemmas for Lemma 4.2-Lemma 4.4. (i) First letting the sets $f$, $g \subset \Gamma\left(X_{0}, \mathfrak{D}_{X_{0}}\right)$ and the Koszul complexes $\mathscr{F}^{m}, \mathscr{G}$ (which are determined by $f, g)$ be as in Lemma 4.2-Lemma 4.4, we prove the following in n.1-n.4:

$(5.3)_{0} \quad$ Lemmas 4.2, 4.4 for $\left(X_{0}, X_{0}^{\prime} ; f\right)$ and Lemma 4.3 for $\left(X_{0}, X_{0}^{\prime} ; \rho ; \mathscr{G}\right)$. (By $(4.3)_{3}$, the proof of $(5.3)_{0}$ suffices for the proof of Lemma 4.2-Lemma 4.4 in its original form as in $n .2-n .4, \S 4.2)$. We give here a lemma, which is our starting point of the proof of $(5.3)_{0}$ :

Lemma 5.3. . There is a subvariety $Y$ of $X_{0}$ satisfying*)

$$
Y \supset X_{0}^{\prime}\left(=X_{0, \text { sing }}\right) \text {, and } \operatorname{dim}\left(Y-X_{0}^{\prime}\right) \leqq \operatorname{dim} X_{0}-2 \text {, }
$$

and with which the following three uniform estimations hold:

$$
\begin{aligned}
& \text { 'Lemma 4.2, Lemma } 4.4 \text { for }\left(X_{0}, Y ; \not \mathcal{f}\right) \text { ' } \\
& \text { and 'Lemma } 4.3 \text { for }\left(X_{0}, Y ; \mathcal{f}, \mathscr{G}\right) \text { '. }
\end{aligned}
$$

We check Lemma 5.3 in Part B, Appendix I. Note that Lemma 5.3 implies:

Proposition 5.6. If $\operatorname{dim} X_{0}=1$, then Lemma 4.2-Lemma 4.4 hold.

Setting $k:=\operatorname{dim} X_{0}$, we assume the following for the remainder of n.1n.4:

$$
k \geqq 2 \text {, and Lemma 4.2-Lemma } 4.4 \text { hold for } \tilde{k}<k \text {. }
$$

Remark 5.2. We may phrase Lemma $5.3_{1}$ by saying that 'the estimations in Lemma 4.2-4.4 hold for general points of $X:=X_{0}-X_{0}^{\prime}$ ' (cf. also the proof of Lemma 4.2-4.4 in Appendix I). Also as we will see in the course of n.1-n.4, our proof of Lemma 4.2-4.4 may be regarded as insuring the implication:

$(5.3)_{2}^{\prime}$ 'estimations in Lemma 4.2-4.4 for general points of $X$ ' $\rightarrow$ 'those for all points of $X$ '.

*) Precisely, we require (5.3) $)_{1}^{\prime}$ for the germs of $Y, \ldots$ at the origin $P_{0}$ of $X_{0}$ (cf. the begining of $\S 5$ ). For the local variety $X_{0}$, we use similar terminology to (5.3) ${ }_{1}$ in later arguments (cf. Lemma 5.3 and Proposition 5.7). 
We prove this by using an inductive argument on $k$ and Proposition 4.2, which is essentially a cohomological result (cf. the proof of Proposition 4.2 in n.4, $\S 4.2)$. The implication (5.3)' 2 and its proof may be interesting in connection with treatments of the a.d. properties of analytic varieties.

Now, the essential part of the proof of Lemma 4.2-4.4 is given in 11.2 n.4. Here we make some reductions of the above lemmas: For this we fix a variety $Y$ as in Lemma $5.3_{1}$, and we assume that $\left(Y-X_{0}^{\prime}\right) \neq \phi$. (Otherwise, Lemma 4.2-4.4 are true.) Then the following lemma suffices to insure Lemma 4.2-4.6:

Lemma 5.3. $\mathbf{3}_{2}$. There is a subvariety $Y^{\prime}$ of $Y$ satisfying (1) $Y^{\prime} \supset X_{0}^{\prime}$, (2) $Y^{\prime}-X_{0}^{\prime} \varsubsetneqq Y-X_{0}^{\prime}$, and with which we have (cf. n.7, Part $\left.A, \S 4.1\right)$ :

'Lemma 4.2, 4.4 for $\left(Y, Y^{\prime} ; \rho\right)$ ' and 'Lemma 4.3 for $\left(Y, Y^{\prime} ; f, \mathscr{G}\right)$ '.

Actually, by the chain property in Proposition 4.1, (5.3) 1,3 insures the following:

$(5.3)_{4} \quad$ 'Lemmas 4.2, 4.4 for $\left(X_{0}, Y^{\prime} ; f\right)^{\prime}$ ' and 'Lemma 4.3 for $\left(X_{0}, Y^{\prime} ; \not, \mathscr{G}\right)$ '. Remarking that (2), Lemma 5.32 insures: $X_{0}-Y^{\prime} \varsubsetneqq X_{0}-Y$, we easily see that a finite repetition of the above procedures leads to $(5.3)_{0}$. In (ii) soon below, we will give explicitly a variety $Y^{\prime}$ as in Lemma $5.3_{2}$.

(ii) Take a suitable element $h \in \Gamma\left(X_{0}, D_{X_{0}}\right)$, and we denote by $D$ the divisor of $h$. Then from a simple observation, we have:

Proposition 5.7. Taking a suitable subvariety $D^{\prime}$ of $D$, we have:

$(5.3)_{5} \quad D \supset Y \cup X_{0}^{\prime}, D^{\prime} \supset X_{0}^{\prime}, D^{\prime} \not \supset Y$, and $d h \neq 0$ at any $P \in D-D^{\prime}$. (Here $d=e x-$ terior differential operator on $X_{0}$.)

$(5.3)_{6} \quad$ The elements $f_{i} \in \mathcal{f}, g_{j} \in \mathscr{G}(1 \leqq i \leqq s, 1 \leqq j \leqq t)$ satisfy: $f_{i}, g_{j} \neq 0\left(D_{u}\right)$ for each irreducible component $D_{u}$ of the germ $D$ at $P_{0}$ satisfying $D_{u} \not \subset D^{\prime}$.

We set $Y^{\prime}:=Y \cap D^{\prime}$. Then we easily see that (1), (2) in Lemma $5.3_{2}$ hold. To show $(5.3)_{3}$ in Lemma $5.3_{2}$, it suffices to prove the following:

Lemma 5.4. The following four facts hold (cf. also n.7, part $A, \S 4.1$ ):

(5.3) 7 'Lemmas 4.2, 4.4 for $\left(D, D^{\prime} ; f\right)$ ' and 'Lemma 4.2 for $\left(D, D^{\prime} ; f, \mathscr{G}\right)$ '.

Actually, from that $D \supset Y \cup X_{0}^{\prime}$, one check easily that one can replace $\left(D, D^{\prime}\right)$ in Lemma 5.4 by $\left(Y, Y^{\prime}\right)$. Thus, by $(5.3)_{4}$, the remaining task for the proof of Lemma $4.2-4.4$ (cf. also $(5.3)_{1}$ ) is to prove Lemma 5.4. This will be done in 
n.2-n.4 soon below.

2. Proof of Lemma 5.4. First we give a proposition, which reduces Lemma 5.4 to Proposition 4.2. For this we fix a suitable*) $d=d_{D} \in \mathbb{Z}^{+}$, an element $(\sigma ; \bar{m})=\left(\sigma_{D}, \bar{m}_{D}\right) \in \mathbb{R}_{1}^{+2} \times \mathbb{Z}^{+}$and a linear function $L_{0}\left(=c_{0, D} t ; c_{0, D}>0\right)$. Moreover, letting the open set $U_{1, D}\left(\ni P_{0}\right)$ in $\mathbb{C}^{n}$, we form a parameter space $\mu_{D}\left(\subset\left(D-D^{\prime}\right) \times \mathbb{R}^{+}\right)$by $\mu_{D}:=\left\{(P ; r) \in\left(\left(D-D^{\prime}\right) \cap U_{1, D}\right) \times \mathbb{R}^{+} ; r<\{\sigma g(P)\}^{-1}\right\}$, with $g(P):=d\left(P, D^{\prime}\right)^{-1}$. Then, letting the Koszul homomorphisms $F_{p}^{m}, G_{p}$, be as in Lemma 4.2-Lemma 4.4, we have**):

Proposition 5.8. (1) For a suitable $E_{D} \in \mathbb{E}_{\mathrm{a} . \mathrm{d}}$ we have:

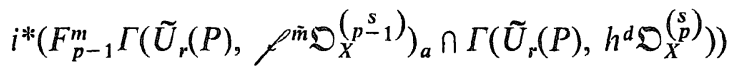

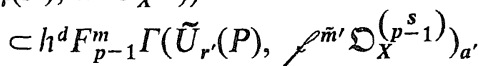

$(0<p<s)$, with $\left(r^{\prime} ; \tilde{m}^{\prime} ; a^{\prime}\right)=E_{D}(r ; \tilde{m} ; a)$. Here $(\tilde{m} ; m) \in \mathbb{Z}^{+} \times \mathbb{Z}^{+}$satisfies: $\tilde{m}>L_{0}(m)$.

$(5.4)_{2} \quad i^{*}\left(\Gamma\left(\tilde{U}_{r}(P), f^{m} \mathfrak{D}_{X}\right)_{a} \cap \Gamma\left(\tilde{U}_{r}(P), h^{d} \mathfrak{D}_{X}\right)\right) \subset h^{d} \Gamma\left(\widetilde{U}_{r^{\prime}}(P), f^{m^{\prime}} \mathfrak{D}_{X}\right)_{a^{\prime}}$, with $\left(r^{\prime} ; m^{\prime} ; a^{\prime}\right)=E_{D}(r ; m ; a)$. Here $m \in \mathbb{Z}^{+}$satisfies: $m \geqq \bar{m}$.

(2) For a suitable a.d. map $E_{D, \mathscr{s}} \in \mathbb{E}_{\mathrm{a} . \mathrm{d}}$, we have $(0<p<t)$ :

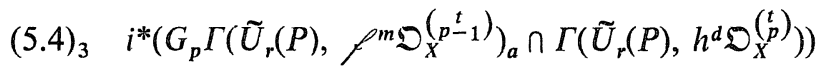

$$
\subset h^{d} G_{p-1} \Gamma\left(\tilde{U}_{r^{\prime}}(P), f^{m^{\prime}} \mathbb{D}_{X}^{\left(p^{t}-1\right.}\right)_{a^{\prime}},
$$

with $\left(r^{\prime} ; m^{\prime} ; a^{\prime}\right)=E_{D . \mathscr{g}}(r ; m ; a)$. Here $m \in \mathbb{Z}^{+}$satisfies: $m>\bar{m}$.

In the above $(P ; r ; a)$ is in $\boldsymbol{\ell}_{D} \times \mathbb{R}_{1}^{+}\left(\subset\left(D-D^{\prime}\right) \times \mathbb{R}^{+} \times \mathbb{R}_{1}^{+}\right)$.

We prove Proposition 5.8 in n.3, n.4. Here we check:

$(5.5)_{1} \quad$ Prop. 5.8 + Prop. 4.2 $\rightarrow$ 'Lemmas $4.2,4.4$ for $\left(D, D^{\prime} ;, f\right)$ ' and 'Lemma 4.3 for $\left(D, D^{\prime} ; \not \subset, \mathscr{G}\right)^{\prime}$.

Actually, by $(4.5)_{3}$, Proposition 4.2, we have:

$\left.(5.5)_{1}^{\prime} \quad i^{*} h^{d}\left(\Gamma\left(\tilde{U}_{r}(P), f^{m} \mathfrak{D}_{X}^{\left(\begin{array}{l}s \\ p\end{array}\right)}\right)_{a} \cap\left(F_{p}^{m}\right)^{-1}(0)\right) \subset F_{p-1}^{m} \Gamma\left(\tilde{U}_{r^{\prime}}(P), f^{\check{m}^{\prime}} \mathfrak{D}_{X}^{(p-1}\right)_{a^{\prime}}^{s}\right)$

with $\left(r^{\prime} ; i \tilde{n}^{\prime} ; a^{\prime}\right)=E_{X}(r ; \tilde{n} ; a)$. Here the a.d. map $E_{X} \in \mathbb{E}_{\mathrm{a.d}}$ is as in Proposition 4.2. From this and $(5.4)_{1}$ we have the desired inclusion to insure 'Lemma 4.2 for $\left(D, D^{\prime} ; f\right)^{\prime}$ :

*) When we are concerned with $(5.4)_{3}$, the data $d_{D}, \ldots$ depend also on $\mathscr{G}: d_{D}=d_{D}, \mathscr{G}, \ldots$.

**) For the sets of the cochains in Proposition 5.8, see (4.2) $)_{2}$ and Proposition 4.2. 


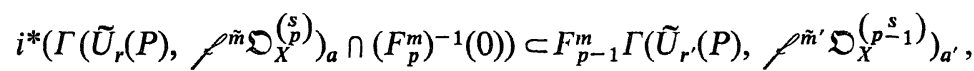

where $\left(\boldsymbol{r}^{\prime} ; \tilde{m}^{\prime} ; a^{\prime}\right)=\widetilde{E}_{\boldsymbol{D}}(r ; m ; a)$, with an a.d. map $E_{\boldsymbol{D}} \in \mathbf{E}_{\mathrm{a} . \mathrm{d}}$, which is determined by $E_{D}, E_{X}$ as in $(5.4)_{1},(5.5)_{1}^{\prime}$.

Thus we have the first fact in the right side of $(5.5)_{1}$. The remaining two facts in the right side of $(5.5)_{1}$ follow from $(5.4)_{2,3}$ and from (4.5) $)_{1,3}$ in Proposition 4.2. Thus, the remaining task for the proof of Lemma 5.4 (and so Lemma 4.2-4.4) is to prove Proposition 5.8. This is done in n.3, n.4, soon below.

Remark 5.3. By a simple induction on $d \in Z^{+}$we easily see that the proof of Proposition 5.8 for $d=1$ leads to that in the general case: $d \geqq 2$. The proof of Proposition 5.8 will be given for the case of $d=1$.

3. Proof of Proposition 5.8-(1). Note that the uniform estimations in Proposition 5.8 were given to each $P \in U_{1} \cap\left(D-D^{\prime}\right)$ and to Koszul complexes $F^{m}, \ldots$, which are defined on $X_{0}$. We will derive Proposition 5.8 from the uniform estimations on Koszul complexes,... on the divisor $D_{0}$ of $X_{0}$, which are insured by the induction hypothesis (5.3) ${ }_{2}$ (cf. (5.6) ${ }_{4}^{\prime}$ and Proposition 5.9 soon below). For applications of such estimations, we begin $n .3$ by arranging some geometric data, which are attached to $D$ : first we set:

$(5.6)_{1}^{\prime} \quad \mathfrak{D}_{D}=$ structure sheaf of $D$, and $\pi=$ natural homomorphism: $\mathfrak{D}_{X_{0}} \rightarrow \mathfrak{D}_{D}$,

$$
\tilde{f}=\left(\tilde{f}_{i}\right)_{i=1}^{s}, \tilde{g}=\left(\tilde{g}_{j}\right)_{j=1}^{t} \text {, where } \tilde{f}_{i}=\pi\left(\tilde{f}_{i}\right), \tilde{g}_{j}=\pi\left(\tilde{g}_{j}\right) \text {. }
$$

We use the symbol $\tilde{\mathscr{F}}^{m}$ (resp. $\tilde{f}$ ) for the $m$-th Koszul (resp. Koszul)-complex for $\tilde{\rho}$ (resp. for $\tilde{g})$ :

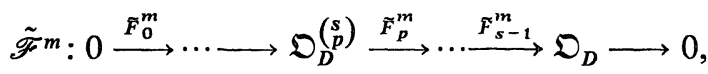

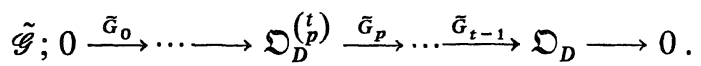

We then use the induction hypothesis $(5.3)_{2}^{\prime}$ in the following specified form:

(5.6)' 'Lemma 4.2, Lemma 4.4 for $\left(D, D^{\prime} ; \tilde{\rho}\right)$ ' as well as 'Lemma 4.3 for $\left(D, D^{\prime} ; \tilde{f}, \tilde{G}\right)^{\prime}$ hold.

In the remainder of $n .3$ we summarize some (direct) consequences of (5.6)' First we check that the a.d. property of the holomorphic functions is preserved by the extension from $D$ to $X_{0}$ :

Proposition 5.9. (Extension of a.d. property). For a suitable a.d. map $E_{D} \in \mathbf{E}_{\mathrm{a} . \mathrm{d}}$, we have: 
$(5.6)_{1}$

$$
\begin{aligned}
i^{\prime *} \Gamma\left(\tilde{U}_{r}^{\prime}(P), \tilde{f^{m}} \mathfrak{D}_{D}\right)_{a} \subset \pi \Gamma\left(\tilde{U}_{r^{\prime}}(P), \tilde{f}^{m^{\prime}} \mathfrak{D}_{X}\right)_{a^{\prime}}, \\
\text { with }\left(r^{\prime} ; m^{\prime} ; a^{\prime}\right)=E_{D}(r ; m ; a) .
\end{aligned}
$$

Here the parameter $(P ; r ; m ; a)$ is, as in Proposition 5.8 , in $\mu_{D} \times \mathbb{Z}_{\tilde{m}}^{+} \times \mathbb{R}_{1}^{+}$ $\left(\subset\left(D-D^{\prime}\right) \times \mathbb{R}^{+} \times \mathbb{Z}^{+} \times \mathbb{R}_{1}^{+}\right)$. Also we set:

$$
\tilde{U}_{r}^{\prime}(P)=\tilde{U}_{r}(P) \cap D(=\{Q \in D ; d(Q, P)<r\}),
$$

and $i^{\prime}$ : =inclusion: $\tilde{U}_{r^{\prime}}^{\prime}(P) \hookrightarrow \widetilde{U}_{r}^{\prime}(P)$.

Proof. First, by its definition, the left side of $(5.6)_{1}$ is written as follows:

$$
\left.\Gamma\left(\tilde{U}_{r}^{\prime}(P), \tilde{f}^{\tilde{m}} \mathfrak{D}_{D}\right)_{a}=\tilde{F}^{\tilde{m}} \Gamma\left(\tilde{U}_{r}^{\prime}(P), \mathfrak{D}_{D}^{s}\right)_{a} \quad \text { (cf. }(4.2)_{2}\right) .
$$

Next by Proposition 4.4 there is an analytic map $\omega: \tilde{U}_{r^{\prime}}(P) \rightarrow \tilde{U}_{r}^{\prime}(P)$, which is the identity on $\tilde{U}_{r^{\prime}}^{\prime}(P)$. (Here $r^{\prime}=M_{D}(r)$, with a positive monomial $M_{D}$ as in Proposition 4.4.) This implies: $\Gamma\left(\tilde{U}_{r^{\prime}}^{\prime}(P), \mathfrak{D}_{D}\right)_{a} \subset \pi \Gamma\left(\tilde{U}_{r^{\prime}}(P), \mathfrak{D}_{X}\right)_{a}$, and we have:

$$
i^{\prime *} \Gamma\left(\tilde{U}_{r}^{\prime}(P), \tilde{\rho^{\tilde{m}}} \mathfrak{D}_{D}\right)_{a} \subset \pi F^{\tilde{m}} \Gamma\left(\tilde{U}_{r^{\prime}}^{\prime}(P), \mathfrak{D}_{X}^{s}\right)_{a} .
$$

Letting the a.d. maps $E_{D}, E_{D, g}$ be as in Proposition 5.8, we give the following slicely weaker version of (5.4) $)_{1,3}$ (cf. Proposition 5.8):

Proposition 5.9. We have the following inclusions:

$$
\begin{aligned}
& i^{*}\left(F_{p}^{m} \Gamma\left(\tilde{U}_{r}(P), \rho^{\tilde{m}} \mathfrak{D}_{X}^{\left(\begin{array}{c}
s \\
p
\end{array}\right)}\right)_{a} \cap \Gamma\left(\tilde{U}_{r}(P), h \mathfrak{D}_{\mathbf{X}}^{\left(p^{s}+1\right.}\right)\right) \subset \\
& F_{p}^{m}\left(\Gamma\left(\tilde{U}_{r^{\prime}}(P), f^{\left.\tilde{m}^{\prime} \mathfrak{D}_{X}^{(s} \begin{array}{l}
(s \\
p
\end{array}\right)}\right)_{a^{\prime}} \cap \Gamma\left(\tilde{U}_{r^{\prime}}(P), h \mathfrak{D}_{\mathbf{X}}^{\left(\begin{array}{l}
s \\
p
\end{array}\right)}\right)\right) \\
& \text { with }\left(r^{\prime}, \tilde{m}^{\prime}, a^{\prime}\right)=E_{D}(r ; m ; a)
\end{aligned}
$$

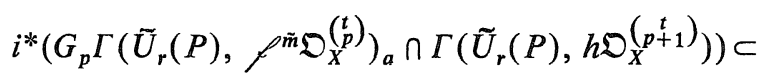

$$
\begin{aligned}
& \left.G_{p}\left(\Gamma\left(\tilde{U}_{r}(P), \rho^{\tilde{m}^{\prime}} \mathfrak{D}_{X}^{\left(\begin{array}{c}
t \\
p
\end{array}\right)}\right)_{a^{\prime}} \cap \Gamma\left(\tilde{U}_{r^{\prime}}(P), h \mathfrak{D}_{X^{(}}^{(t)}\right)\right)\right) \\
& \text { with }\left(\tilde{r}^{\prime} ; \tilde{m}^{\prime} ; a^{\prime}\right)=E_{D, \mathscr{\ell}}(r ; m ; a)
\end{aligned}
$$

Proof. Take elements $\varphi=F_{p}^{m} \varphi^{\prime}, \Psi=G_{p} \Psi^{\prime}$ from the left sides, where

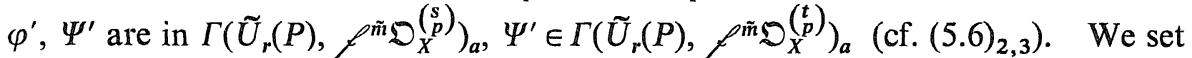
$\tilde{\varphi}^{\prime}=\pi \varphi^{\prime}$ and $\tilde{\Psi}^{\prime}=\pi \Psi^{\prime}$. Then, by 'Lemma 4.4 for $\left(D, D^{\prime} ; \tilde{f}\right)$ ' and 'Lemma 4.3 for $\left(D, D^{\prime} ; \tilde{f} ; \tilde{\mathscr{G}}\right)$, we have: $\tilde{\varphi}^{\prime}=F_{p-1}^{m} \tilde{\varphi}_{1}^{\prime}, \tilde{\Psi}^{\prime}=G_{p-1}^{\prime} \tilde{\Psi}_{1}^{\prime}$, with $\tilde{\varphi}_{1}^{\prime} \in \Gamma\left(\tilde{U}_{r^{\prime}}^{\prime}(P)\right.$, $\left.\tilde{\rho}^{\tilde{m}^{\prime}} \mathfrak{D}_{D}^{(p-1}\right)_{a^{\prime}}$ and $\tilde{\Psi}_{1}^{\prime} \in \Gamma\left(\tilde{U}_{r^{\prime}}^{\prime}(P), \tilde{\rho}_{\tilde{\boldsymbol{m}} \tilde{m}^{\prime}} \mathfrak{O}_{D}^{(p-1}\right)_{a^{\prime}}$, where $\left(r^{\prime} ; \tilde{m}^{\prime} ; a^{\prime}\right)=E_{D}(r ; \tilde{m} ; a)$ and $\left(r^{\prime} ; \tilde{m}^{\prime} ; a^{\prime}\right)=E_{D, \mathscr{g}}(r ; \tilde{m} ; a)$. (In the above we assume that $p \geqq 1$. If $p=0$, then (5.6) $)_{2.3}$ is obvious, because $F_{0}^{m}, G_{0}$ are injective.) By Proposition 5.9 1 we extend $\tilde{\varphi}_{1}^{\prime}, \tilde{\Psi}_{1}^{\prime}$ to $X_{0}$. Writing the extensions as $\varphi_{1}^{\prime}, \Psi_{1}^{\prime}$, we set $\varphi_{2}^{\prime}=\varphi^{\prime}-F_{p-1}^{m} \varphi_{1}^{\prime}$, $\Psi_{2}^{\prime}=\Psi^{\prime}-G_{p-1} \Psi_{1}^{\prime}$. We then have: $\pi \varphi_{2}^{\prime}=\pi \Psi_{2}^{\prime}=0$ and $F_{p}^{m} \varphi_{2}^{\prime}=\varphi, G_{p-1} \Psi_{2}^{\prime}=\Psi$. From this and the estimations: $(r ; \tilde{m} ; a) \rightarrow\left(r^{\prime} ; \tilde{m}^{\prime} ; a^{\prime}\right), \ldots$ soon above as well as 
the one in Proposition 5.9 , we easily have $(5.6)_{2,3}$.

q.e.d.

4. Proof of Proposition 5.8-(2). We first check the implication:

$$
(5.6)_{2} \longrightarrow(5.4)_{2} \text {. }
$$

To see this, take an element $\varphi \in \Gamma\left(\tilde{U}_{r}(P), f^{m} \mathfrak{D}_{X}\right)_{a} \cap \Gamma\left(\tilde{U}_{r}(P), h \mathfrak{D}_{X}\right)$ (cf. $\left.(5.4)_{2}\right)$. We take a suitable linear map*) $L: \mathbb{R}^{+} \ni m \rightarrow \boldsymbol{R}^{+} \ni \mathrm{cm} ; c>0$. Letting $m^{\prime}=$ $[L(m)], \tilde{m}=m-m^{\prime}$, we have:

$$
\left.\left.\varphi \in F^{m} \Gamma\left(\tilde{U}_{r}(P), \rho^{\tilde{m}} \bigvee_{X}^{s}\right)_{a} \cap \Gamma\left(\tilde{U}_{r}(P), h D_{X}\right) \quad \text { (cf. (5.6) }\right)_{2}\right) .
$$

By the choice of the map $L$, one can assume*): $L_{0}\left(m^{\prime}\right)<i \tilde{l}$, with the linear function $L_{0}$ in $(5.6)_{2}$, Proposition $5.9_{2}$; applying $(5.6)_{2}$ to the right side of $(a)_{1}$, we have:

(a) ${ }_{2} \quad i^{\prime *} \varphi \in F^{m^{\prime}}\left(\Gamma\left(\tilde{U}_{r^{\prime}}(P), f^{\tilde{m}^{\prime}} \mathfrak{D}_{X}^{s}\right)_{a^{\prime}} \cap \Gamma\left(\tilde{U}_{r^{\prime}}(P), h \mathfrak{D}_{X}^{s}\right)\right)$, where $\quad\left(r^{\prime} ; \tilde{n}^{\prime} ; a^{\prime}\right)=$ $E_{X}(r ; \tilde{m} ; a)$ is as in $(5.6)_{2}$.

Remarking that**) $\Gamma\left(U_{r^{\prime}}(P), f^{\tilde{m}^{\prime}} \mathfrak{D}_{X}^{s}\right)_{a} \subset \Gamma\left(U_{r^{\prime}}(P), \mathfrak{D}_{X}^{s}\right)_{a^{\prime}}$, we apply the bdd. uniform estimation, Lemma 5.1, (applied to the homomorphism $h: \mathfrak{D}_{X}^{s} \ni \varphi \rightarrow D_{X}^{s}$ $\ni h \varphi)$ to the right side of $(\mathrm{a})_{2}$. Then we have:

$$
i^{\prime \prime *} \varphi \in F^{m^{\prime}} \cdot h \cdot \Gamma\left(\tilde{U}_{r^{\prime \prime}}(P), D_{X}^{s}\right)_{a^{\prime \prime}} \text {, with }\left(r^{\prime \prime} ; a^{\prime \prime}\right)=M_{h}\left(r^{\prime} ; a^{\prime}\right) .
$$

Here the map $M_{h}: \boldsymbol{R}^{+} \times \mathbb{R}^{+} \rightarrow \mathbb{R}^{+} \times \mathbb{R}^{+}(\in \mathbf{M})$ is determined by the homomorphism $h$ as in Lemma 5.1. This inclusion is of the desired form in $(5.4)_{2}$. Moreover, we easily check that the map: $(r ; m ; a) \rightarrow\left(r^{\prime} ; m^{\prime} ; a^{\prime}\right)$, which is determined by the maps $L, E_{X}$ and $M_{h}$ as in (a) $)_{1-3}$, is also of the desired form in (5.4) $)_{2}$ (Namely, that correspondence is written as $\left(r^{\prime} ; m^{\prime} ; a^{\prime}\right)=E(r ; m ; a)$, with an a.d. map $E$ determined by $L, E_{X}$ and $M_{h}$.) Thus we have the implication (a). Next, it is easy to see that the application of $(5.4)_{2}$ to the right side of $(5.6)_{2,3}$ leads to the following implication:

$$
(5.6)_{2}+(5.4)_{2} \rightarrow(5.4)_{1} \text {, and }(5.6)_{3}+(5.4)_{2} \rightarrow(5.4)_{3} .
$$

Thus we have Proposition 5.8, and we have Lemma 5.4 and so Lemma $5.3_{2}$ as well as our original lemmas, Lemma 4.2-Lemma 4.4 (cf. $(5.5)_{1},(5.3)_{7,4}$ and $\left.(5.3)_{0}\right)$.

5. Proof of Lemma 4.5. Letting the sheaf $\mathfrak{H}$ be as in Lemma 4.5, we

*) For the treatment of the 'a.d. exponents' $m, \ldots$ here, see also (iii), n.3, Section 2.3 (in the proof of Lemma 2.6), where the similar arguments are given.

**) Recalling that $f^{\prime}=0$ at $P_{0}$, one can assume that $|f|<1$. 
assume that $\mathfrak{S}$ is of the form in $(5.2)_{1}$. We write $\delta_{\tilde{D}} \mathfrak{D}_{X}^{h \tilde{p}}\left(\subset \mathfrak{D}_{X}^{h \tilde{p}}\right)$ as $\mathfrak{S}_{\tilde{p}}(1 \leqq \tilde{p} \leqq p)$. (Thus $\mathfrak{S}_{2}=\mathfrak{S}_{p}$.) Also we denote by $D$ the divisor of*) $h$ on $X_{0}$. Then, letting the variety $Y \subset X_{0}$ be as**) in Lemma $5.3_{1}$, the following lemma will be our starting point of Lemma 4.5 :

Lemma 5.5. Lemina 4.5 holds for $\left(X_{0}, Y: \mathfrak{5}_{\tilde{p}}\right)(1 \leqq \tilde{p} \leqq p)$, and $Y \supset D$, $Y-D \subset V\left(=\right.$ locus of,$\left.^{\prime}\right)$

(For the proof of Lemma 5.5. see Part B. Appendix I.) Next taking an open subset $U_{s}$ of $U_{0}$ and an element $\sigma_{n} \in \mathbb{R}_{1}^{+2}$, we form parameter spaces:

$(5.7)_{0} \quad \lambda_{5,}:=\left\{(P ; r ; a) \in\left(U_{5,} \cap(Y-D)\right) \times \mathbb{R}^{+} \times \mathbb{R}_{1}^{+} ; r<\left\{\sigma_{\text {, }} g_{D}(P)\right\}^{-1}\right\}$, with $g_{D}(P):$ $=d(P, D)^{-1}$, and $\tau_{5,}:=\lambda_{\xi} \times \mathbb{Z}^{+}$.

(If $Y \subset D$, then Lemma 4.5 holds. We henceforth assume that $Y \not \subset D$.) For convenience of the proof of Lemma 4.5, we fix a set $g=\left(g_{\dot{q}}\right)_{\vec{q}=1}^{q} \subset \Gamma\left(X_{0}, \mathfrak{D}_{X_{0}}\right)$ satisfying $Y=\cap_{\tilde{q}=1}^{q} D_{\tilde{q}}$, where $D_{\tilde{q}}$ :=divisor of $g_{\tilde{q}}$ on $X_{0}$. Also taking an element $d \in \mathbb{Z}^{+}$, we set $g^{d}:=\left(g_{\tilde{q}}^{d}\right)_{\tilde{q}=1}^{q}$. Thirdly taking a parameter $(P ; r ; a ; m)$ $\in \tau_{\text {s }}\left(\subset(Y-D) \times \mathbb{R}^{+} \times \mathbb{R}_{1}^{+} \times \mathbb{Z}^{+}\right)$, we set:

$(5.7)_{1} \quad C_{d}^{\tilde{p} \tilde{q}}(P ; r ; a ; m):=\sum_{I}\left(g_{I}^{d}\right)^{-1} \cdot \Gamma\left(\tilde{U}_{r}(P), f^{m} \mathfrak{D}_{X}^{h}\right)_{a}(\tilde{q} \geqq 0,0 \leqq \tilde{p} \leqq p)$, where $I$ exhaust all indices of the form $I=\left(i_{1}<\cdots<i_{\tilde{q}+1}\right)$ and $g_{I}^{d}:=g_{i_{1}}^{d} \cdots g_{i_{\tilde{p}+1}}^{d}$.

The homomorphism $\delta_{\tilde{g}}$ operates on $C_{i}^{p q}(\tau)$, with $\tau=(P: r ; a ; m): \delta_{\tilde{n}}: C_{\tilde{d}}^{\tilde{\tilde{q}} \tilde{q}}(\tau)$ $\rightarrow C_{d}^{\tilde{p}+1 \tilde{q}}(\tau)$. We denote by $\delta_{g^{d}}$ the Koszul homomorphism for $C_{d}^{\tilde{\tilde{q}} \tilde{q}}(\tau)$ :

$(5.7)_{2} \quad \delta_{g^{d}}: C_{d}^{\tilde{p} \tilde{q}}(\tau) \ni \sum_{I} \varphi_{I} / g_{I}^{d}$, with $\quad \varphi_{I} \in \Gamma\left(\tilde{U}_{r}(P), f^{m} \mathfrak{D}_{X}^{k \tilde{p}}\right)_{a}$

$$
\longrightarrow C_{d}^{\tilde{p} \tilde{q}+1}(\tau) \ni \sum_{J} \varphi_{J} / g_{J}^{d},
$$

where $\left(\varphi_{I}\right)_{I} \rightarrow\left(\varphi_{J}\right)_{J}$ is defined by the Koszul homomorphism defined for $g^{d}$ (cf. n.3, part B, §4.1).

We then set:

$$
\begin{aligned}
& Z_{d}^{\prime p q}(\tau):=C_{d}^{p q}(\tau) \cap \delta_{s^{-1}}^{-1}(0), Z_{d}^{\prime p q}(\tau):=C_{d}^{p q}(\tau) \cap \delta_{g d}^{-1}(0), \text { and } \\
& Z_{d}^{p q}(\tau):=Z_{d}^{\prime p q}(\tau) \cap Z_{d}^{\prime p q}(\tau) .
\end{aligned}
$$

Take a suitable element***) $e=e_{\hat{n}} \in \mathbb{E}^{+}$and an a.d. map $E_{\text {fุ }} \in \mathbb{E}_{\text {a.d }}$ (cf. n.1, B,

*) The element $h \in \Gamma\left(X_{0}, D_{X_{0}}\right)$ is also as in $(5.2)_{1}$.

*) Precisely, we understand that the divisor $D$ plays the role of the subvariety $X_{0}^{\prime}$ in Lemma 5.3. In Lemma 5.3, we assumed: $\operatorname{dim}\left(Y-X_{0}^{\prime}\right) \leqq \operatorname{dim} X_{0}-2$. Here we do not assume the corresponding fact: $\operatorname{dim}(Y-D) \leqq \operatorname{dim} X_{0}-2$.

***) When we are concerned with the second inclusion in $(5.7)_{4}$, the data $\left(e_{5,}, E_{5}\right)$ depend also on $g: c_{5, j}=e_{i g}, g$ and $E_{i j}=E_{i, g, g}$. 
§4.1). Then we have:

Proposition 5.10. We have the following inclusions:

$(5.7)_{4}\left\{\begin{array}{l}Z_{d}^{\prime \tilde{p} \tilde{q}}(P ; r: a ; m) \subset \delta_{\tilde{g}} C_{e}^{\tilde{p}-1} \tilde{q}\left(P ; r^{\prime} ; a^{\prime} ; m^{\prime}\right)(\tilde{p} \geqq 1) \\ Z_{d}^{\prime \prime} \tilde{p} \tilde{q}(P ; r ; m ; a) \subset \delta_{g^{d}} C^{\tilde{p} \tilde{q}-1}\left(P ; r^{\prime} ; a^{\prime} ; m^{\prime}\right)(\tilde{q} \geqq 1)\end{array}\right\}$,

$$
\text { with }\left(r^{\prime} ; a^{\prime} ; m^{\prime}\right)=E_{\text {占 }}(r ; m ; a) \text {. }
$$

Proof. The first inclusion follows from Lemma 5.5 and Proposition 4.2. Moreover, the second follows from Lemma 4.3 or Lemma 4.2, according as $\tilde{q}<$ or $=q$ (cf. also the proof of Lemma $\left.4.3^{\prime}\right)$.

q.e.d.

Applying the similar algebraic arguments to n.5, Section 5.1, we get the following from Proposition 5.10 .

Proposition $\mathbf{5 . 1 0}_{\mathbf{2}}$. We have the following inclusion $(\tilde{p} \geqq 1)$ :

$$
Z_{d}^{p 0}(P ; r ; a ; m) \subset \delta_{\mathfrak{5}} Z_{e}^{\prime \prime p-10}\left(P ; r^{\prime} ; a^{\prime} ; m^{\prime}\right),
$$

where the correspondence: $(r ; a ; m) \rightarrow\left(r^{\prime} ; a^{\prime} ; m^{\prime}\right)$ is similar to Proposition $5.10_{1}$.

Finally, by comparing the set of the cocycles, $Z^{p 0}(P ; r ; a ; m)$ and the set of the holomorphic functions $\Gamma\left(\tilde{U}_{r}(P), f^{m} \mathfrak{D}_{X}^{k_{p}}\right)_{a}$ in a similar manner to n.5, Section 5.1, we get easily the desired inclusion in Lemma 4.5:

$(5.7)_{6} \quad i^{*}\left(\Gamma\left(\widetilde{U}_{r}(P), f^{m} \mathfrak{D}_{X}^{k \tilde{y}}\right)_{a} \cap \delta_{\mathfrak{g}}^{-1}(0)\right) \subset \delta_{\tilde{g}} \Gamma\left(\tilde{U}_{r^{\prime}}(P), f^{m^{\prime}} \cdot \mathfrak{D}_{X^{k}-1}^{k \tilde{p}}\right)_{a^{\prime}} \quad(1 \leqq \tilde{p} \leqq p)$,

where the correspondence: $(r ; a ; m) \rightarrow\left(r^{\prime} ; a^{\prime} ; m^{\prime}\right)$ is similar to Proposition $5.10_{2}$. (Precisely, from the explicit form of the parameter space $\tau_{5 \mathfrak{g}},(5.7)_{6}$ insures 'Lemma 4.5 for $\left(Y, D ; \mathfrak{S}_{\tilde{p}}\right)$ ' $(1 \leqq \tilde{p} \leqq p)$. But, using the chain property in Proposition 4.1 , we have 'Lemma 4.5 for $\left(X_{0}, D ; \mathfrak{H}\right)$ ' from the above (cf. also n.1, § 5.2), and we also have Lemma 4.5 (cf. also (iii), n.8, B, §4.1).)

6. Proof of Lemma 4.6. Finally we derive Lemma 4.6 from Lemma 4.5 and Lemma 4.4. In n.6, the sheaves $\mathfrak{S}_{\tilde{p}}=\delta_{\mathfrak{g}} \mathfrak{D}_{X}^{k \tilde{p}-1}\left(\subset \mathfrak{D}_{X^{2}}^{k \tilde{p}}\right)(1 \leqq \tilde{p} \leqq p)$ are as in n.5. Next take a positive monomial $M$ and an a.d. map $E \in \mathbf{E}_{\text {a.d. }}$. We then define a map

$(5.8)_{0} \quad E: \boldsymbol{R}^{+} \times \boldsymbol{R}^{+} \times \boldsymbol{R}^{+} \times \boldsymbol{Z}^{+} \ni(r ; t ; a ; m) \rightarrow \boldsymbol{R}^{+} \times \boldsymbol{R}^{+} \times \boldsymbol{R}^{+} \times \boldsymbol{Z}^{+} \ni\left(r^{\prime} ; t^{\prime} ; a^{\prime} ; m^{\prime}\right)$, where $r^{\prime}=M(r)$ and $\left(t^{\prime} ; a^{\prime} ; m^{\prime}\right)=E(r t ; a ; m)$.

We write the collection of all such maps as $\mathbf{E}$. Moreover, the parameter space $\lambda_{X}\left(\subset X \times \boldsymbol{R}^{+} \times \boldsymbol{R}^{+} \times \boldsymbol{R}_{1}^{+}\right)$is as in (5.2) $)_{0}$, and we set $\tau_{\mathbf{X}}:=\lambda_{X} \times \boldsymbol{Z}^{+}$. 
Now, we give two propositions, which are the key fact for the proof of Lemma 4.6. (In Propositions 5.11 $1_{1,2}$, we fix an element $m_{X} \in \mathbb{Z}^{+}$.)

Proposition 5.11 $1_{1}$ For a suitable estimation map $E_{X} \in \tilde{E}$ we have:

$(5.8)_{1} \quad s^{*} Z^{q}\left(\mathscr{A}_{t}\left(\tilde{U}_{r}(P)\right), \mathscr{f}^{m} \mathfrak{D}_{X}\right)_{a} \subset F^{m^{\prime}} Z^{q}\left(\mathscr{A}_{t^{\prime}}\left(\tilde{U}_{r^{\prime}}(P)\right), \mathfrak{D}_{X}^{s}\right)_{a^{\prime}}$, with $\left(r^{\prime} ; t^{\prime} ; a^{\prime} ; m^{\prime}\right)$ $=E_{X}(r ; t ; a ; m)$, where we set:

(5.8) ${ }_{1}^{\prime} \quad C^{q}\left(\mathscr{A}_{t}\left(\tilde{U}_{r}(P)\right), f^{m} \mathfrak{D}_{X}\right)_{a}:=F^{m} C^{q}\left(\mathscr{A}_{t^{\prime}}\left(\tilde{U}_{r^{\prime}}(P)\right), \mathfrak{D}_{X}^{s}\right)_{a^{\prime}} \quad$ (cf. (5.2) $\left.)_{0}\right)$, and $Z^{q}\left(\mathscr{A}_{t}\left(\tilde{U}_{r}(P)\right), \mathscr{f}^{m} \mathfrak{D}_{X}\right)_{a}:=($ left side $) \cap \delta^{-1}(0)$.

Proposition $5.11_{2}$. For a suitable $E_{\tilde{1}} \in \mathbf{E}$, we have $(1 \leqq \tilde{p} \leqq p)$ :

$(5.8)_{2} \quad s^{*} Z^{q}\left(\mathscr{A}_{t}\left(\tilde{U}_{r}(P)\right), f^{m} \mathfrak{D}_{X}^{k p}\right)_{a} \cap \delta_{\tilde{n}}^{-1}(0) \subset \delta_{\mathfrak{g}^{2}} Z^{q}\left(\mathscr{A}_{t^{\prime}}\left(\tilde{U}_{r^{\prime}}(P)\right), f^{m^{\prime}} \cdot \mathfrak{D}_{X}\right)_{a^{\prime}}$, with $\left(r^{\prime} ; t^{\prime} ; a^{\prime} ; m^{\prime}\right)=E_{\mathfrak{n}}(r ; t ; a ; m)$.

In the above*), the parameter $(P ; r ; t ; a)$ is in $\lambda_{X}\left(\subset X \times \mathbb{R}^{+} \times \mathbb{R}^{+} \times \mathbb{R}_{1}^{+}\right)$, and the element $m \in \mathbb{Z}^{+}$satisfy: $m \geqq m_{X}$.

Note that, by applying Lemma 5.2 to the right side of $(5.8)_{1,2}$, we have

$(5.8)_{3}$ left side of $\left\{\begin{array}{l}(5.8)_{1} \\ (5.8)_{2}\end{array}\right\} \subset\left\{\begin{array}{l}\delta F^{m} C^{q-1}\left(\mathscr{A}_{t^{\prime}}\left(\tilde{U}_{r^{\prime}}(P)\right), \mathcal{f}^{m} \mathfrak{D}_{X}^{s}\right)_{a^{\prime}} \\ \delta_{\mathfrak{g}} C^{q-1}\left(\mathscr{A}_{t^{\prime}}\left(\widetilde{U}_{r^{\prime}}(P)\right), \mathcal{f}^{m} \mathfrak{D}_{X}^{s}\right)_{a^{\prime}}\end{array}(q \geqq 1)\right\}$.

Also note we gave cohomological versions of Lemma 4.4 and Lemma 4.5 in Lemma 2.6' and (2.14) in the proof of Lemma 2.6 (cf. (iii), n.3, §2.3). Propositions $5.11_{1,2}$ are similar to those results, but are more simple than them, because Propositions 5.11 1,2 do not contain no p.g. conditions on neither the sets of the cochains nor the coverings $\mathscr{A}_{t}\left(\tilde{U}_{r}(P)\right)$ in $(5.8)_{1}$. The proof of Propositions $5.11_{1,2}$ is given in a similar algebraic pattern to that of Lemma $2.6^{\prime}$ and (2.14). The estimations in the former are much simpler than the latter. We omit the check of Propositions 5.11 1,2 .

Proof of Lemma 4.6. We derive here Lemma 4.6 from Propositions $5.11_{1,2}$. For this taking a positive monomial $M$ and an estimation map $E^{\prime} \in \mathbb{E}^{\prime}$ (cr. $\left.(4.2)_{7}^{\prime}\right)$, we define an estimation map:

$(5.8)_{2}^{\prime} \quad \tilde{E}^{\prime}: \boldsymbol{R}^{+} \times \boldsymbol{R}^{+} \times \boldsymbol{R}_{1}^{+} \times \boldsymbol{Z}^{+} \ni(r ; t ; a ; m) \rightarrow \mathbb{R}^{+} \times \mathbb{R}^{+} \times \boldsymbol{R}_{1}^{+} \times \mathbb{E}^{+} \ni\left(r^{\prime} ; t^{\prime} ; a^{\prime} ; m^{\prime}\right)$, with $r^{\prime}=M(r)$ and $\left(t^{\prime} ; a^{\prime} ; m^{\prime}\right)=E(r t ; a ; m)$.

Writing the estimations: $\boldsymbol{R}^{+} \ni r \rightarrow \mathbb{R}^{+} \ni r^{\prime}$ and $\mathbb{Z}^{+} \ni m \rightarrow \mathbb{Z}^{+} \ni m^{\prime}$ as $M, L$ we call the maps $M, L$ the first and a.d. parts of $\tilde{E}^{\prime}$. We use the symbols $\tilde{\mathbb{E}}^{\prime}$ and $\mathbf{M}, \mathbb{L} \mathbf{n}$

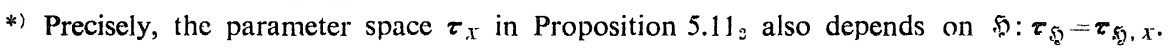


for the collections of all maps as in (5.8) ${ }_{2}^{\prime}$ and of their first and a.d. parts. Moreover, we use the symbol $\pi$ for the map $\tilde{\mathbb{E}}^{\prime} \ni \widetilde{E}^{\prime} \rightarrow \mathbf{M} \times \mathbb{L} \mathbf{n} \ni(M, L)$.

Now, let the homomorphism $K_{0, m}=K_{0}+F^{m}: \mathfrak{D}_{X}^{k_{1}}+\mathfrak{D}_{X}^{s_{k}^{k}} \rightarrow \mathfrak{S}_{m}\left(\subset \mathfrak{D}_{X}^{k}\right)$ be as in Lemma 4.6. Then we have:

Proposition 5.11 $\mathbf{1}^{\circ}$. There is a map $\varepsilon_{\mathfrak{r}}: \mathbb{Z}^{+} \ni m \rightarrow \widetilde{E}^{\prime}$ $\ni \widetilde{E}_{\mathfrak{f}, m}$ satisfying the diagram in Figure $I$, and with which we have:

$$
\begin{aligned}
s^{*}\left(Z ^ { q } \left(\mathscr{A}_{t}\left(\tilde{U}_{r}(P)\right),\right.\right. & \left.\left.\left.\mathfrak{D}_{X}^{k}\right)_{a} \cap Z^{q}\left(\mathscr{A}_{t}\left(\tilde{U}_{r}(P)\right), \mathbb{S}_{m}\right)\right)\right) \\
& \subset K_{0, m^{\prime}} Z^{q}\left(\mathscr{A}_{t^{\prime}}\left(\tilde{U}_{r^{\prime}}(P)\right), \mathfrak{D}_{X}^{k_{1}+s h}\right)_{a^{\prime}},
\end{aligned}
$$

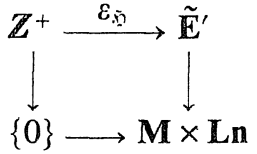

Figure I.

with $\left(r^{\prime} ; t^{\prime} ; a^{\prime} ; m^{\prime}\right)=E_{\mathfrak{g}}(r ; t ; a ; m)$, where the parameter $(P ; r ; t ; a ; m) \in$ $X \times \mathbb{R}^{+} \times \mathbb{R}^{+} \times \mathbb{R}_{1}^{+} \times \mathbb{Z}^{+}$has the similar meaning to Proposition $5.11_{2}$.

(For the diagram in Figure I, compare the similar one in Figure III, Lemma 4.6.)

Note that, by comparing the sets of the cochains in $(5.8)_{3}$ (in the case of $q=0)$ and the sets of the holomorphic functions in Lemma 4.6, we see easily that Proposition $5.11_{3}$ implies Lemma 4.6. We prove Proposition $5.11_{3}$ soon below. We add here an inclusion, which we use in the proof of Proposition $5.11_{3}$ :

$(5.8)_{4} \quad S^{*}\left(Z^{q}\left(\mathscr{A}_{t}\left(\tilde{U}_{r}(P)\right), \mathfrak{D}_{X^{p}}^{p}\right)_{a} \cap \delta_{\mathfrak{g}^{-1}}^{-1}(0)\right) \subset \delta_{\mathfrak{g}^{\prime}} C^{q-1}\left(\mathscr{A}_{t^{\prime}}\left(\tilde{U}_{\boldsymbol{r}^{\prime}}(P)\right), \mathfrak{D}_{X^{p-1}}^{k}\right)_{a^{\prime}}$, with $\left(r^{\prime} ; t^{\prime} ; a^{\prime}\right)=M_{5}(r ; t ; a)$, where the parameter $(P ; r ; t ; a) \in \lambda_{\mathfrak{g}}\left(\subset X \times \mathbb{R}^{+} \times \mathbb{R}^{+}\right.$ $\times \mathbb{R}_{1}^{+}$) is as in Proposition 5.11 and the estimation map $M_{,}$is in $\mathbf{M}$ (cf. $\left.(5.2)_{0}\right)$.

(This follows easily from Proposition 5.5 and Lemma 5.2.)

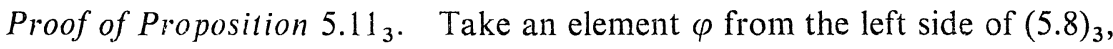
and we apply $(5.2)_{3}$ to the homomorphism $K_{0, m}$. Then one can write $\varphi$ as follows:

(a) $\varphi_{1}=K_{0} \varphi_{1}+F^{m} \varphi_{2}$, with $\varphi_{1} \in C^{q}\left(\mathscr{A}_{r t^{\prime}}, \mathfrak{D}_{X}^{k_{1}}\right)_{a^{\prime}}$ and $\varphi_{2} \in C^{q}\left(\mathscr{A}_{r t^{\prime}}, \mathfrak{D}_{X}^{s_{2} k_{2}}\right)_{\boldsymbol{a}^{\prime}}$, where $\left(t^{\prime} ; a^{\prime}\right)=M_{\mathfrak{n}, m}(t ; a)$, with an estimation map $\tilde{M}_{\mathfrak{s}, m} \in \mathbf{M}$ (cf. (4.1) $)_{3}$ ). (Here we write $\mathscr{A}_{t}\left(\tilde{U}_{r}(P)\right)$ as $\left.\mathscr{A}_{r t}.\right) \quad$ Next we apply $(5.8)_{2}^{\prime}$ to $\varphi:=K_{0} \delta \varphi_{1}$, and we have:

(a) ${ }_{2} \delta K_{0}\left(\varphi_{1}-\varphi_{1}^{\prime}\right)=0$, with an element $\varphi_{1}^{\prime} \in C^{q}\left(\mathscr{A}_{r^{\prime} t^{\prime \prime}}, \rho^{m^{\prime \prime}} \mathfrak{D}_{X}^{k_{1}}\right)_{a^{\prime \prime}}$, with $\left(r^{\prime} ; t^{\prime \prime}\right.$; $\left.a^{\prime \prime} ; m^{\prime \prime}\right)=E_{\tilde{5}}\left(r ; t^{\prime} ; a^{\prime} ; m^{\prime}\right)$. (The estimation map $E_{\tilde{g}} \in \mathbb{E}$ is as in $(5.8)_{2}$.)

Thirdly, writing the sheaf $\mathfrak{S}$ explicitly as: $\rightarrow \mathfrak{D}_{X}^{K^{2}} \stackrel{K_{1}}{\longrightarrow} \mathfrak{O}_{X}^{K} \stackrel{K_{0}}{\longrightarrow} \mathfrak{H} \rightarrow 0$, we apply 
$(5.8)_{3}^{\prime}$ to $\left(K_{1}, \delta\left(\varphi_{1}-\varphi_{1}^{\prime}\right)\right)$. Then we have:

(a) $)_{3} \quad\left(\varphi_{1}-\varphi_{1}^{\prime}+K_{1} \varphi_{1}^{\prime \prime}\right)=0$, with an element $\varphi_{1}^{\prime \prime} \in C^{q}\left(\mathscr{A}_{r i \tilde{t}^{\prime \prime}}, \mathfrak{D}_{X}^{k 2}\right)_{\tilde{a}^{\prime \prime}}$. Here $\left(\tilde{t}^{\prime \prime} ; \tilde{a}^{\prime \prime}\right)$ $=\tilde{M}_{\mathfrak{S}}\left(t^{\prime \prime} ; a^{\prime \prime}\right)$, with an estimation map $\tilde{M}_{\mathfrak{5}} \in \tilde{\mathbb{M}}$ (cf. $\left.(4.1)_{3}\right)$.

Now, we set $\tilde{\varphi}_{1}:=\varphi_{1}-\varphi_{1}^{\prime}+K_{1} \varphi_{1}^{\prime \prime}$. Then one can write:

(a) ${ }_{4} \quad \varphi=\left(K_{0} \varphi_{1}+F^{m} \varphi_{2}\right)=K_{0} \tilde{\varphi}_{1}+F^{m} \varphi_{2}-K_{0} \varphi_{1}^{\prime}$ (and we have $\left.\delta \tilde{\varphi}_{1}=0\right)$.

Setting $\tilde{\varphi}_{2}$ : $=F^{m} \varphi_{2}-K_{0} \varphi_{1}^{\prime}$, we have the following from $(\mathrm{a})_{1,2}$ and $(\mathrm{a})_{4}$ :

$$
\tilde{\varphi}_{2} \in Z^{q}\left(\mathscr{A}_{r^{\prime} \tilde{t}^{\prime \prime}}, f^{m^{\prime \prime}} \mathfrak{D}_{X}\right)_{\tilde{a}^{\prime \prime}}
$$

Finally, we apply $(5.8)_{1}^{\prime}$ to $\tilde{\varphi}_{2}$. Then we have the desired inclusion in $(5.8)_{4}$ :

(a) $s_{6} \quad s^{*} \varphi \in K_{0} Z^{q}\left(\mathscr{A}_{\bar{r}^{\prime \prime}}, \mathfrak{D}_{X}^{h^{1}}\right)_{\bar{a}^{\prime \prime}}+F^{\bar{m}^{\prime \prime}} Z^{q}\left(\mathscr{A}_{\bar{r}^{\prime \prime} \bar{t}^{\prime \prime}}, \mathfrak{D}_{X}^{s h}\right)_{\bar{a}^{\prime \prime}}$,

$$
\text { with }\left(\bar{r}^{\prime \prime} ; \bar{t}^{\prime \prime} ; \bar{a}^{\prime \prime} ; \bar{m}^{\prime \prime}\right)=E_{X}\left(r^{\prime} ; \tilde{t}^{\prime \prime} ; \tilde{a}^{\prime \prime} ; \tilde{m}\right),
$$

where the estimation map $E_{X} \in \mathbb{E}$ is as in $(5.8)_{1}^{\prime}$.

Thus we finish the proof of Lemma 4.6. (We should check that the estimations in (a) $)_{1-6}$ yields the one of the form in $(5.8)_{3}$. But this is checked by a tedious observation).

By the proof of Lemma 4.6, we finish all the non cohomological uniform estimations in Section 4.1, which are the basis of the cohomological estimations in Section 1, Section 2. Finally, we make remarks oil the p.g. Poincaré lemmas (Lemma 3.3 and Proposition 3.1), which we used in the application to de Rham theory in Section 3.

Remark 3.4. We summarize here the key facts for the proof of the p.g. Poincaré lemma, Proposition 3.1 and Lemma 3.3. The details of the content here will be given elsewhere*). First we remark that the p.g. Poincaré lemma in Proposition 3.1 is of similar form to Lemma 1.2 (except that the sheaf homomorphism in Proposition 3.1 is the exterior cifferential operator $d$, while that in Lemma 1.2 is an $\mathfrak{D}_{X}$-homomorphism.) Thus, by Proposition 4.5 , we see that similar non cohomological uniform estimation (for the operator $d$ ) to the one in Lemma 4.1 suffices for the check of Proposition 3.1. But such a non cohomological estimation is checked very easily, and Proposition 3.1 is essentially of very elementary nature***).

*) Cf. [17].

**) When the pair $\left(X_{0}, X_{0}^{\prime}\right)$ (as in Lemma 4.1') satisfies: $X_{0}=U_{0}\left(\in \operatorname{Ouv}\left(\mathbb{C}^{n}\right)\right.$, such an estimation is easily checked. In the general case, we get the estimation from those for $X_{0}=U_{0}$, by using the simillar arguments to n.5, Section 5.1 . 
Next, we recall that the p.g. Poincaré lemma, Lemma 3.3, is a formal consequence of the p.g. open map property, Lemma 3.2. On the other hand, by Proposition 4.6, we have the implication: Lemma 4.7 $\rightarrow$ Lemma 3.2. Thus, for the proof of Lemma 3.3, the proof of Lemma 4.7 is sufficient. The proof of Lemma 4.7 has, in its algebraic structure, similarities to the one of Lemma 4.5. We summarize here the key facts for the proof of Lemma 4.6: first, by an elementary computation, we have:

(5.9) $)_{1}$ Lemma 4.7 holds for $\left(X_{0}, Y\right.$; $\left.d\right)$, where $Y$ is a subvariety of $X_{0}$ satisfying (1) $\operatorname{dim} Y \leqq \operatorname{dim} X_{0}-2$ and (2) $Y \subset V$ (=locus of $f$ ).

(This terminology should be understood similarly to $(4.3)_{1}$. In the proof of Lemma 4.5, we gave a similar fact to $(5.9)_{1}$ (cf. Lemma 5.5). The proof of $(5.9)_{1}$ is given differently from the one of Lemma 5.5. But the proof of $(5.9)_{1}$ is very elementary, and we omit it.) Assuming (5.9) ${ }_{1}$, the key point of the proof of Lemma 4.7 is as follows: first we let the finite set $g^{d}=\left(g_{\tilde{q}}^{d}\right)_{\tilde{q}=1}^{d} \subset \Gamma\left(X_{0}, D_{X_{0}}\right)$ and the parameter $(P ; r ; a ; m) \in(Y-D) \times \boldsymbol{R}^{+} \times \boldsymbol{R}_{1}^{+} \times \boldsymbol{Z}^{+}$be as in $(5.7)_{1}$. (Here we assume, as in $(5.7)_{1}$, that $\cap_{\tilde{q}} D_{\tilde{q}}=Y$.)

$(5.9)_{2} \quad C^{p q}(P ; r ; a ; m):=\sum_{|I|=\tilde{q}}\left(g_{I}^{d}\right)^{-1} \circ \Gamma\left(\tilde{U}_{r}(P), f^{m} \Omega_{X}^{\tilde{p}}\right)(\tilde{p}, \tilde{q} \geqq 0)$, where $g_{I}^{d}$ is as in $(5.7)_{1}$, and the set of the holomorphic differential forms in the right side is as in $(4.2)_{9}$.

(5.9) ${ }_{2}^{\prime} \quad Z^{\prime p q}(\tau):=C^{p q}(\tau) \cap d^{-1}(0), Z^{\prime p q}(\tau):=C^{p q}(\tau) \cap \delta_{g^{d}}^{-1}(0)$, and $Z^{p q}(\tau):=$ $Z^{\prime p q}(\tau) \cap Z^{\prime \prime p q}(\tau)$, where we set $\tau=(P ; r ; a ; m)$.

In the above $\delta_{g^{d}}$ is the Koszul homomorphism as in n.5, Section 5.2 and $d$ denotes the exterior differential operator.

Then the key fact for the proof of Lemma 4.7 is as follows:

Proposition 5.12. . We have the inclusions:

$(5.9)_{3} \quad Z^{\prime p q}(P ; r ; a ; m) \subset d C^{p-1} q\left(P ; r^{\prime} ; a^{\prime} ; m^{\prime}\right)$

$(5.9)_{4} \quad Z^{\prime \prime p q}(P ; r ; a ; m) \subset \delta_{g d d} C^{p q^{-1}}\left(P ; r^{\prime} ; a^{\prime} ; m^{\prime}\right)$, with $\left(r^{\prime} ; a^{\prime} ; m^{\prime}\right)=E_{X}(r ; a ; m)$, where the estimation $E_{X}$ is in $\mathbf{E}_{\mathrm{a} . \mathrm{d}}$ (cf. Definition 4.1) and the element $e \in \mathbf{Z}^{+}$is similar to Proposition $5.10_{1}$.

The first inclusion is same as the second one in (5.7) $)_{4}$. Also we see easily that the similar argument to Proposition $5.10_{2}$ insures the implication:

Prop. 5.12 $\longrightarrow$ Lemma 4.7 .

In the remainder we summarize the key points of the proof of $(5.9)_{3}$, which is 
based on Theorem $2.2_{2}$ and an a.d. estimations on $C^{\infty}$-differential forms (cf. Proposition 5.124): first we note that, from a simple observation, the proof of $(5.9)_{3}$ for the case of $\# g=1$ leads to that of the case: $\# g \geqq 2$. We henceforth assume that $\# g=1$. In order to make the notation concordant to the one in Section 1, Section 2, we write $g$ as $g=\{h\}$. Also letting the parameter space $\boldsymbol{\mu}_{X}\left(\subset D \times \mathbb{R}^{+} \times \boldsymbol{R}_{1}^{+2}\right)$ be as in $(1.9)_{4}$, we form a product $\tau_{X}:=\boldsymbol{\mu}_{X} \times \mathbb{R}_{1}^{+} \times \mathbb{Z}^{+}$ $\left(\subset D \times \boldsymbol{R}^{+} \times \boldsymbol{R}_{1}^{+2} \times \boldsymbol{R}_{1}^{+2} \times \boldsymbol{Z}^{+}\right)$. Taking an parameter $\tau=(P ; r ; \sigma ; a ; m)$ in $\boldsymbol{\tau}_{X}$ $\left(\subset D \times \boldsymbol{R}^{+} \times \boldsymbol{R}_{1}^{+2} \times \boldsymbol{R}_{1}^{+2} \times \boldsymbol{Z}^{+}\right)$, we form a double complex as follows:

$(5.10)_{1} C_{d}^{p q}(\tau):=\left\{\varphi \in C^{q}\left(\mathscr{A}_{\sigma}\left(\tilde{X}_{r}(P)\right), \Omega_{X}^{p}\right) ;|\varphi| \cdot\left|h^{d}\right|<a|f|^{m}\right\}$, where the p.g. covering $\mathscr{A}_{\sigma}\left(X_{r}(P)\right)$ is as in Theorem 1.1.

$(5.10)_{2} \quad Z_{d}^{\prime p q}(\tau):=C_{d}^{p q}(\tau) \cap d^{-1}(0), Z_{d}^{\prime \prime p q}(\tau):=C_{d}^{p q}(\tau) \cap \delta^{-1}(0)$,

$$
\text { and } Z_{d}^{p q}(\tau):=Z_{d}^{\prime p q}(\tau) \cap Z_{d}^{\prime p q}(\tau) \text {. }
$$

Now, let the a.d. map $E_{X}$ and the element $e \in \mathbb{Z}^{+}$be as in Proposition 5.12.

Proposition 5.12. We have the following inclusion:

$(5.10)_{3} \quad Z_{d}^{\prime p q}(P ; r ; \sigma ; a ; m) \subset d C_{e}^{p-1 q}\left(P ; r^{\prime} ; \sigma^{\prime} ; a^{\prime} ; m^{\prime}\right)$

$(5.10)_{4} \quad Z_{d}^{\prime \prime p q}(P ; r ; \sigma ; a ; m) \subset \delta C_{e}^{p q-1}\left(P ; r^{\prime} ; \sigma^{\prime} ; a^{\prime} ; m^{\prime}\right)$, where $\left(r^{\prime} ; \sigma^{\prime} ; m\right)=E_{X}(r ; a ; m)$ and $\sigma^{\prime}$ is an element ${ }^{*)}$ of $\mathbb{R}_{1}^{+2}$.

Proof. First, using the entirely parallel arguments to the proof of Proposition $4.9_{2}$ (cf. n. $4, \S 4.2$ ), we have $(5.10)_{4}$ from Theorem 2.2. . (As in n.4, $\S 4.2,(5.10)_{4}$ is a non cohomological version of Theorem $2.2_{2}$ (which is the cohomological inclusion as in Section 2.) Next we get $(5.10)_{3}$ from $(5.9)_{1}$ easily as follows: first, using Proposition $4.5_{2}$, we have the cohomological version of $(5.9)_{1}$ (in the manner as in Proposition $4.5_{2}$ ). Then, using the similar arguments to the ones in $n .4$, Section 4.2 , we have:

$(5.9)_{1} \stackrel{\text { Prop. 5.41 }}{\longrightarrow}$ cohomological version of $(5.9)_{1} \rightarrow(5.10)_{4}$.

This finishes the proof of Proposition 5.12.

q.e.d.

Next, to the double complexes $C_{d}^{p q}(\tau)$, we will apply the similar arguments to Proposition 5.4. Then we have**):

Proposition 5.12. There are maps $\theta: Z_{d}^{p}{ }^{o}(P ; r ; \sigma ; a ; m) \rightarrow Z_{e}^{0 p}\left(P ; r^{\prime}\right.$; $\left.\sigma^{\prime} ; a^{\prime} ; m^{\prime}\right)$ and $\theta^{\prime}: Z_{d}^{0}(P ; r ; \sigma ; a ; m) \rightarrow Z_{e}^{p}{ }^{0}\left(P ; r^{\prime} ; \sigma^{\prime} ; a^{\prime} ; m^{\prime}\right)$, which satisfy:

*) The dependence of the element $\sigma^{\prime} \in \boldsymbol{R}_{1}^{+2}$ is clear from the proof soon below. We do not use the explicit form of $\sigma^{\prime} \in \boldsymbol{R}_{1}^{+2}$.

**) The estimation: $(r ; \sigma ; a ; m) \rightarrow\left(r^{\prime} ; \sigma^{\prime} ; a^{\prime} ; m^{\prime}\right), \ldots$ are as in Proposition $5.12_{2}$. 


$$
\begin{aligned}
\left(1-\theta^{\prime} \cdot \theta\right) Z_{d}^{p}{ }^{0}(P ; r ; \sigma ; a ; m) \subset d \rightarrow Z_{e}^{\prime p-10}\left(P ; r^{\prime} ; \sigma^{\prime} ; a^{\prime} ; m^{\prime}\right) & \\
& d Z^{\prime \prime p-10}(P ; r ; \sigma ; a ; m) \subset \delta Z_{e}^{\prime 0}{ }^{p-1}\left(P ; r^{\prime} ; \sigma^{\prime} ; a^{\prime} ; m^{\prime}\right)
\end{aligned}
$$

(and the similar relation for $\theta \cdot 0^{\prime}, \ldots$ (cf. also Proposition $5.4_{2}$ ).

Now, using Proposition $5.12_{3}$, the proof of the following suffices for the proof of $(5.9)_{3}$.

Proposition 5.124. $\quad Z_{d}^{p 0}(P ; r ; \sigma ; a ; m) \subset \delta Z_{d}^{\prime 0}{ }^{p-1}\left(P ; r^{\prime} ; \sigma^{\prime} ; a^{\prime} ; m^{\prime}\right)$.

Actually, by Proposition $5.12_{3}$, the inclusion just above insures:

$$
Z_{d}^{p 0}(P ; r ; \sigma ; a ; m) \subset d Z_{e}^{\prime 0-1}\left(P ; r^{\prime} ; \sigma^{\prime} ; a^{\prime} ; m^{\prime}\right) .
$$

Then, comparing the sets $Z_{d}^{p 0}$ in Proposition $5.12_{3}$ and the sets $Z_{d}^{\prime p 0}$ in (5.9) (using the similar arguments to $(5.2)_{5-7}$ and $(5.7)_{6}$ ), we easily see that $(5.10)_{6}$ insures the desired inclusion $(5.9)_{3}$ ).

Finally we remark that the both sides in Proposition $5.12_{3}$ are contained in $Z^{q}\left(\mathscr{A}_{\sigma}\left(\tilde{X}_{r}(P)\right), \mathbb{C}\right)$ and $C^{q-1}\left(\mathscr{A}_{\sigma}\left(\tilde{X}_{r}(P)\right), \boldsymbol{C}\right)$ and are endowed with the a.d. properties. Thus Proposition $5.12_{3}$ is of topological nature, and the key fact for the proof of Proposition 5.12 ${ }_{4}$ differs from our arguments on the coherent sheaves in the present paper. The details of the proof of $(5.10)_{6}$ is in [17]. Here we only remark that (1) Proposition $5.12_{4}$ follows from our estimations on the a.d. properties of the (topological) contractibility of (local real analytic varieties) (cf. $[15]_{2}$ ). Applying the standard chain homotopy arguments in the proof of $C^{\infty}$-Poincaré lemma to the explicit contraction in Lemma $2,[15]_{2}$, one get a similar $C$-version of (5.9) $)_{3}$. From such an inclusion, we get rather easily Proposition 5.124 . (2) The a.d. property of the (topological) contraction mentioned just above is elementary once we assume the existerice of what we call normalized series of stratified spaces and some quantitative properties of such stratified spaces (cf. §3. See also [15] ${ }_{2-4}$ and [17]). The details of Proposition 5.12 and of what are mentioned in (1), (2) just above will be given in [17].

\section{Appendix I. Elementary Computations - 1}

Here we prove Proposition 5.3, Proposition 4.4 and Lemma 5.3, Lemma 5.4. The geometric datum $X=\left(\boldsymbol{C}^{n}(z), U_{0}, X_{0}, X_{0}^{\prime}, P_{0}\right)$ will be as in (4.1), and the structure sheaf $\mathfrak{D}_{U_{0}}$ of $U_{0}, \ldots$ will have the similar meaning to Section 4 , Section 5. We prove Proposition 5.3, Proposition 4.4 in Part A and Lemma $5.3_{1}$, Lemma 5.5 in $\mathrm{B}$. 
A

1. An estimation of Weierstrass polynomials. In A we assume that $U_{0}$ $\left(\subset \mathbb{C}^{n}\right)$ is of the form $U_{0}=U_{0}^{\prime} \times U_{0}^{\prime \prime}$, where $U_{0}^{\prime}$ and $U_{0}^{\prime \prime}$ are polydiscs in $\mathbb{C}^{n-1}\left(z^{\prime}\right)$, $C\left(z_{n}\right)$, where $z=\left(z^{\prime}, z_{n}\right)$. We assume that the radius of $U_{0}^{\prime}, U_{0}^{\prime \prime}$ coincides and that $d$ (=this radius) $<1$. We use the symbols $\pi_{1}, \pi_{2}$ for the projections: $\mathbb{C}^{n} \rightarrow \mathbb{C}^{n-1}, \boldsymbol{C}$.

(i) Take an element $f \in \Gamma\left(U_{0}, D_{U_{0}}\right)$. We assume that $f$ is already defined in $\tilde{U}_{0}=\tilde{U}_{0}^{\prime} \times \tilde{U}_{0}^{\prime \prime}$, where the polydiscs $\tilde{U}_{0}^{\prime}, \tilde{U}_{0}^{\prime \prime}$ have the same center as $U_{0}^{\prime}, U_{0}^{\prime \prime}$ and the radius of them $=2 d$. We set:

(1) $\quad K:=\max \left(1, K^{\prime}\right)$, with $K^{\prime}:=\sup _{Q \in U_{0}}|f(Q)|+\sum_{j=1}^{n}\left|\partial f(Q) / \partial z_{j}\right|$.

Next take a point $P \in \hat{U}_{0}=\hat{U}_{0}^{\prime} \times \hat{U}_{0}^{\prime \prime}$, where the polydiscs $\hat{U}_{0}^{\prime}, \hat{U}_{0}^{\prime \prime}$ have the same center as $U_{0}^{\prime}, U_{0}^{\prime \prime}$ and the radius of them $=2^{-1} \cdot d$. We assume that $m:=\operatorname{deg} f$ in $z_{n}$ at $P>0$. Setting $\tilde{z}^{\prime}:=z^{\prime}-z^{\prime}(P), \tilde{z}_{n}:=z_{n}-z_{n}(P)$, we expand $f$ at $P$ as $f\left(\tilde{z}^{\prime} ; \tilde{z}_{n}\right)=\sum_{j=0}^{\infty} f_{j}\left(\tilde{z}^{\prime}\right) \tilde{z}_{n}^{j}$. Then, for $\tilde{z}^{\prime}=0$, we have:

(1) $)_{1}^{\prime} f\left(0, \tilde{z}_{n}\right)=\tilde{z}_{n}^{m}\left(f_{m}(0)+\tilde{z}_{n} f_{m}^{\prime}\left(0, \tilde{z}_{n}\right)\right)$, with a holomorphic function $f_{m}^{\prime}$, which is estimated for $\left|\tilde{z}_{n}\right|<d / 8$ as follows*):

(1) $)_{1}^{\prime \prime}\left|f_{m}^{\prime}\left(0, \tilde{z}_{n}\right)\right|<\tilde{M}_{m}(K / d)$, where the positive monomial $\tilde{M}_{m}$ depends only on $m \in \mathbb{Z}^{+}$. (We note that, for $\tilde{M}_{m}^{\prime}=\left(8^{m+1} K / d^{m+1}\right)$, the above inequality holds.)

From this we easily have:

$(1)_{1}^{\prime \prime \prime} \quad\left|f\left(0, \tilde{z}_{n}\right)\right| \geqq 2^{-1}\left|\tilde{z}_{n}^{m}\right| \cdot\left|f_{m}(0)\right|$ for $\left|\tilde{z}_{n}\right|<\min \left(d / 8,2^{-1} \cdot\left|f_{m}(0)\right| \cdot \tilde{M}_{m}(K / d)\right)$.

For convenience of the estimation soon below, we set:

$$
e_{m}:=\min \left(1,\left|f_{m}(0)\right|\right) \text {. }
$$

Now take positive monomials $M_{m}, M_{m}^{\prime}$, which depend**) only on $m$, and we take an element $r=\left(r_{1}, r_{2}\right) \in \mathbb{R}^{+2}$ satisfying

$$
r_{2} \leqq M_{m}\left(d e_{m} / K\right), \quad r_{1} \leqq M_{m}^{\prime}\left(r_{2}\right) .
$$

We set $U_{r}:=U_{r_{1}}^{\prime} \times U_{r_{2}}^{\prime \prime}$, where $U_{r_{1}}^{\prime}, U_{r_{2}}^{\prime \prime}$ are the polydiscs in $\mathbb{C}^{n-1}, \mathbb{C}$ of radius $r_{1}, r_{2}$ and center $\pi_{1}(P), \pi_{2}(P)$. Moreover, we take an element $c=\left(c_{1}, c_{2}\right)$ $\in \mathbb{R}^{+2}$, which depends**) only on $m$. And we set:

*) This is gotten by applying Cauchy integral formula to $f_{m}^{\prime}$.

**) One can take $M_{m}$ to be $(t / 2)$. Starting with this positive monomial, the choice of $M^{\prime}$ $c \in \mathbb{R}^{+2}$ is clear from the proof of Proposition 1 . 
Proposition 1. We have the following inclusion.

$$
U_{r} \supset W_{r c} \supset U_{r} \cap X \text {, where } X:=\text { divisor of } f .
$$

Proof. Take an element $\tilde{z}_{n} \in \boldsymbol{C}$ satisfying $\left|\tilde{z}_{n}\right|=r_{2}$, and we take an element $\tilde{z}^{\prime}$ satisfying

$$
\left|\tilde{z}^{\prime}\right| \leqq \min \left(d / 2,(2 K)^{-1} \cdot\left|f_{m}(0)\right| \cdot\left|\tilde{z}_{n}^{m}\right|\right) .
$$

Then we have the following from (1) $)_{1}^{\prime \prime \prime}$.

$$
\left|f\left(\tilde{z}^{\prime} ; \tilde{z}_{n}\right)\right| \neq 0 \text {. }
$$

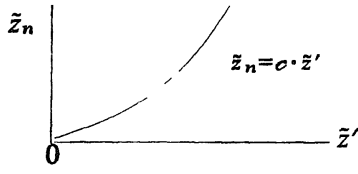

Figure I.

Now, by a choice of the positive monomial $M_{m}^{\prime}$, we have: $M_{m}^{\prime}\left(r_{2}\right)<$ right side of (a) (cf. also (1) $)_{1}^{y}$ ), and we have (b) for $\left|\tilde{z}^{\prime}\right| \leqq M_{m}^{\prime}\left(r_{2}\right)$. Finally, setting $u=M_{m}^{\prime}(t)$, we define an element $c \in \mathbb{R}^{+2}$ by $t=c \cdot u$. Then, clearly, we have the first inclusion in (1) . Also, by (b), the inequality $\left|\tilde{z}_{n}\right|<c \cdot\left|\tilde{z}^{\prime}\right|$ insures: $f\left(\tilde{z}, \tilde{z}^{\prime}\right) \neq 0$, and we have the second inclusion in $(1)_{1}$.

Proof of Proposition 5.3. We set $r_{2}^{0}:=M_{m}\left(d e_{m} / K\right)$, and $r_{1}^{0}:=M_{m}^{\prime}\left(r_{2}^{0}\right) / 2$. For each $\left|\tilde{z}^{\prime}\right|<r_{1}^{0}$, let $\zeta_{j}\left(z^{\prime}\right)(1 \leqq j \leqq m)$ denote the roots of $f\left(\tilde{z}_{n} ; \tilde{z}^{\prime}\right)=0$, and we form Weierstrass polynomial $f^{\prime}\left(\tilde{z}_{n} ; \tilde{z}^{\prime}\right)$ by

$$
f^{\prime}\left(\tilde{z}_{n} ; \tilde{z}^{\prime}\right):=\prod_{j=1}^{m}\left(\tilde{z}_{n}-\zeta_{j}\left(z^{\prime}\right)\right) \text {. }
$$

Note that $(1)_{1}$ implies that $\left|\tilde{z}_{n}-\zeta_{j}\left(\tilde{z}^{\prime}\right)\right|>r_{2}^{0} / 2$, for $\left|\tilde{z}_{n}\right|=r_{2}^{0}$, and we have:

$$
\left|f^{\prime}\left(\tilde{z}_{n} ; \tilde{z}^{\prime}\right)\right| \geqq\left(r_{2}^{0} / 2\right)^{m} \text { for }\left|\tilde{z}_{n}\right|=r_{2}^{0} .
$$

Also, from the explicit form of $r_{2}^{0}$ and $M_{m}^{\prime}$, we have (cf. also (1) ${ }_{1}^{\prime \prime}$ and (a), (b) in the proof of Proposition 1).

$$
\left|f\left(\tilde{z}^{\prime}, \tilde{z}_{n}\right)\right| \geqq \bar{M}_{m}\left(r_{2}^{0}\right) \text { for }|z|=r_{2}^{0} \text { and }\left|z^{\prime}\right|=r_{1}^{0},
$$

with a positive monomial $\bar{M}_{m}$, which depends only on $m$.

Remarking that $|f| \leqq K$ in $U_{0}$ and $\left|f^{\prime}\right| \leqq\left(r_{2}^{0}\right)^{m}$, we easily have

(1) $)_{3} \quad \varepsilon:=f \mid f^{\prime}$ and $\varepsilon^{\prime}:=f^{\prime} \mid f$ satisfies: $|\varepsilon|,\left|\varepsilon^{\prime}\right|<M_{m}^{*-1}\left(d e_{m} / K\right)$ in $U_{r_{1}}^{0} \times U_{r_{2}}^{0}$, with a positive monomial $M_{m}^{*}$ depending only on $m \in Z^{+}$.

Comparing this with the explicit formulation in $(5.1)_{6}$, Proposition 5.3, we see easily that $(1)_{3}$ insures $(5.1)_{6}$, Proposition 5.3.

Next take a holomorphic function $\varphi \in \Gamma\left(U_{r}, \mathfrak{D}_{U_{0}}\right)$ satisfying $|\varphi|<a$ in $U_{r}$. (See $(5.1)_{7}$, Proposition 5.3. Here we understand that $U_{r}$ : = polydisc in $\boldsymbol{C}^{n}$ 
of radius $r$ and center $P$, and we assume that $r<r_{2}^{0}$.) Then, setting $r_{2}^{\prime}:=r_{2}^{0} / 4$ and $r_{1}^{\prime}:=M_{m}^{\prime}\left(r_{2}^{\prime}\right)$, we write $\varphi=\varphi_{1} f^{\prime}+\varphi_{2}$ (cf. $\left.(5.1)_{7}\right)$ in $U_{r_{1}}^{\prime} \times U_{r_{2}}^{\prime \prime}$. Here $U_{r_{1}^{\prime}}^{\prime}$ and $U_{r_{2}^{\prime}}^{\prime \prime}$ are the polydiscs in $\mathbb{C}^{n-1}$ and $\mathbb{C}$ of radius $r_{1}^{\prime}, r_{2}^{\prime}$ and of center $\pi_{1}(P)$, $\pi_{2}(P)$. Then $\varphi_{1}$ is given by the following integral formula (cf. p. 70, [4]):

$(1)_{4} \quad(2 \pi \sqrt{-1}) \varphi_{1}=\int_{\gamma_{1}} \theta_{1}\left(\tilde{z}^{\prime} ; \zeta\right) /\left(\zeta-\tilde{z}_{n}\right) d \zeta$, where $\gamma_{1}:=\left\{\zeta ;|\zeta|=r_{2}^{\prime} / 2\right\}$ and $\theta_{1}:=$ $\varphi\left(\tilde{z}^{\prime} ; \zeta\right) / f^{\prime}\left(\tilde{z}^{\prime} ; \zeta\right)$.

From this and from $(1)_{3}$, we easily have:

$(1)_{5}\left|\varphi_{1}\right|<M_{f, m}(a / r)$ in $U_{r_{1}}^{\prime} \times U_{r_{2}^{\prime}}^{\prime}$, where the positive monomial $M_{f, m}$ is independent from the point $P$ satisfying $\operatorname{deg}_{z_{n}} f(P)=m$.

Moreover, $\varphi_{2}=\varphi-\varphi_{1} f$ is estimated in the similar manner to $(1)_{5}$, and we have $(5.1)_{7}$, Proposition 5.3. Thus we finish the proof of Proposition 5.3.

2. Proof of Proposition 4.4. In n.2 we assume that $X_{0}$ is irreducible at the origin $P_{0}$ of $X_{0}$. By a simple observation, this assumption is harmless for the proof of Proposition 4.4. We fix a set $f=\left(f_{j}\right)_{j=1}^{n-k} \subset \Gamma\left(U_{0}, \mathfrak{D}_{U_{0}}\right), k=\operatorname{dim} X_{0}$, satisfying (1) $f \equiv 0\left(X_{0}\right)$ and (2) $\tilde{g}_{1}:=|\operatorname{det} \partial f| \partial\left(z_{k+1}, \ldots, z_{n}\right) \mid \not \equiv 0\left(X_{0}\right)$. We assume that $f$ is defined in $\hat{U}_{0}$ (cf. the beginning of (i), n.1), and we define:

$$
K:=\sup _{z \in U_{0}} 1+\sum_{i=1}^{n-k}\left(\left|f_{i}\right|+\sum_{j=1}^{n}\left|\partial f_{i} / \partial z_{j}\right|\right) .
$$

Moreover, we fix an open subset $U_{1}\left(\ni P_{0}\right)$ of $U_{0}$, and, for a point $P \in U_{1} \cap X_{0}$ satisfying $\tilde{g}_{k}(P) \neq 0$, we define:

$$
g_{t}(P):=\min \left(1, \tilde{g}_{\ell}(P)\right) .
$$

Next we choose positive monomials $M_{i}=M_{i ; n, k}(n \geqq i \geqq 1)$, which depend only on $(n, k)$, and we take an element $r=\left(r_{j}\right)_{j=1}^{n} \in \mathbb{R}^{+n}$ satisfying

$$
r_{n} \leqq M_{n}\left(d g_{f}(P) / K\right), \quad r_{j} \leqq M_{j}\left(r_{j+1}\right) \quad(n-1 \geqq j \geqq 1) .
$$

We then define:

$(2)_{2} \quad U_{r}(P):=\prod_{j=1}^{n} U_{r_{j}}(P) \subset \mathbb{C}^{n}(\subset \mathbb{C} \times \cdots \times \mathbb{C}), U_{r}^{k}(P):=\prod_{j=1}^{k} U_{r_{j}}\left(P_{j}\right)$,

where $U_{r_{j}}(P)$ denotes the disc in the $j$-th component $\mathbb{C}$ of $\mathbb{C}^{n}$, whose radius is $r_{j}$ and center is $P_{0}:=\pi_{j n}(P)$. (Here $\pi_{j n}$ denotes the projection: $\mathbb{C}^{n}(z) \rightarrow \mathbb{C}^{n}\left(z^{j}\right)$, with $z^{j}:=\left(z_{1}, \ldots, z_{j}\right)$.) Moreover, taking an element $c=c_{n, k} \in \mathbb{R}^{+2}$, which depends only on $(n, k)$, we set:

(2) ${ }_{3} \quad W_{r c}(P):=\left\{z=\left(z^{k} ; z_{k+1}, \ldots, z_{n}\right) \in U_{r}^{k}(P) \times \mathbb{C}^{n-k}\left(\subset \mathbb{C}^{n}\right)\right.$, where $\left|z_{t}-z_{t}(P)\right|$ $\left.<c \cdot\left|\left(\pi_{k n}(z)-\pi_{k n}(P)\right)\right|(n \geqq t \geqq k+1)\right\}$. 
Then, setting $X:=X_{0}-\left(\right.$ locus of $\left.\tilde{g}_{p}\right)$, we have:

Proposition 2. We have the inclusion:

(3) ${ }_{1}^{n, k} \quad U_{r}(P) \supset W_{r o}(P) \supset U_{r}(P) \cap X$, and

$(3)_{2}^{n, k} \quad \pi_{k n}:\left(U_{r}^{r}(P) \cap X\right) \rightarrow U_{r}^{k}(P)$ is biregular (and is surjective).

Proof. First we note that (3) $)_{1,2}^{n-1}$ follows easily from Proposition 1 . We also note that this implies Proposition 2 for the case of $n=2$. We assume that $n \geqq 3$ and that Proposition 2 holds for $\tilde{n}<n$. We will derive $(3)_{1,2}^{n, k}$ for $k \leqq n-2$ from $(3)_{1,2}^{n, n-1}$ and $(3)_{1,2}^{n-1, k}$. For this we set:

(a) $\tilde{g}_{f_{1}}:=\left|\partial f_{1} / \partial z_{k+1}\right|, f^{\prime}:=\left(f_{2}, \ldots, f_{n}\right)$, and $\tilde{g}_{\ell^{\prime}}:=\left|\operatorname{det} \partial f^{\prime}\right| \partial\left(z_{k+2}, \ldots, z_{n}\right) \mid$.

Then, assuming that the indices $(1, \ldots, n)$ are suitably chosen, we have:

$$
\tilde{g}_{f_{1}}(P), \tilde{g}_{l}(P)>\tilde{g}_{l}(P) / M_{n, k}(P) \text {, with a suitable } M_{n, k} \in \mathbb{M} \text {. }
$$

(To check this, we first note that $\tilde{g}_{f_{1}}(P) \cdot \tilde{g}_{t}(P) \geqq \tilde{g}_{k}(P) / n$. From this and the relation $\tilde{g}_{f_{1}}(P), \tilde{g}_{t^{\prime}}(P) \leqq M_{n, k}^{\prime}(K)$, which is easily checked, we have (a) $)_{1}$.)

(i) Denote by $Y_{1}$ the locus of $f_{1}$ in $U_{0}$. We summarize here the application of $(3)_{1,2}^{n, n-1}$ to $Y_{1}$. For this we choose positive monomials $M_{i}^{\prime}=M_{i ; n, k}^{\prime}$ $(n \geqq i \geqq 1)$ suitably, and take an element $r^{\prime}=\left(r_{j}^{\prime}\right)_{j=1}^{n} \in \mathbb{R}^{+n}$ satisfying

$$
r_{n}^{\prime}<M_{n}^{\prime}\left(d \cdot g_{(}(P) / K\right), \quad r_{j}^{\prime}<M_{j}^{\prime}\left(r_{j+1}^{\prime}\right) \quad(n-1 \geqq j \geqq 1) .
$$

Also we choose an element $c^{\prime}=c_{n, k}^{\prime} \in \boldsymbol{R}^{+2}$, which depends only on $(n, k)$, and we form manifolds $U_{r^{\prime}}(P), W_{r^{\prime} c^{\prime}}(P)$ and $U_{r^{\prime}}^{n-1}(P)$ in the similar manner to $(2)_{2.3}$. To make clear that we are concerned with the indices $(n, n-1)$, we write the first two manifolds as $U_{r^{\prime}}^{n, n-1}(P)$ and $W_{r^{\prime}, c^{\prime}}^{n, n-1}(P)$. Then we have:

$(3)_{3}^{\prime n, n-1} \quad U_{r^{\prime}}^{n, n-1}(P) \supset W_{r^{\prime}, c^{\prime}}^{n, n-1}(P) \supset\left(U_{r^{\prime}}^{n, n-1}(P) \cap Y_{1}\right)$, and $\pi_{n-1 n}:\left(U_{r^{\prime}}^{n, n-1}(P) \cap\right.$ $\left.Y_{1}\right) \rightarrow U_{r^{\prime}}^{n-1}(P)$ is biregular.

(To check this, we first define the invariants $K_{1}$ of $f_{1}$ in the manner in $(2)_{0}$, and we set $g_{f_{1}}(P):=\min \left(1, \tilde{g}_{f_{1}}(P)\right)$. Also, we let $M_{i}=M_{i ; n, n-1}$ be as in (3) ${ }_{1}$. Then, for an element $r=\left(r_{j}\right)_{j=1}^{n}$ satisfying: $r_{n}<M_{n}\left(d g_{f_{1}}(P) / K_{1}\right)$ and $r_{j}<M_{j}\left(r_{j+1}\right)$ $(n-1 \geqq j \geqq 1)$, we have the similar fact to $(3)_{3}^{\prime n, n-1}$ from $(3)_{1}^{n, n-1}$. But, assuming that $M_{n}^{\prime}$ is suitably chosen, $(\mathrm{a})_{1}$ insures:

(b) ${ }_{2}$

$$
M_{n}^{\prime}\left(d g_{\ell}(P) / K\right)<M_{n}\left(d g_{f_{1}}(P) / K_{1}\right) .
$$

Thus, by understanding that $M_{j}^{\prime}=M_{j}(n \geqq j \geqq 1)$, we have $(3)_{3}^{\prime n, n-1}$.

For purpose of the arguments henceforth, we arrange some data: we define 
an element $\tilde{r}:=\left(\tilde{r}_{j}\right)_{j=1}^{n} \in \mathbb{R}^{+n}$ by

$$
\tilde{r}_{n}:=M_{n}^{\prime}\left(d g_{f_{1}}(P) / K\right), \quad \tilde{r}_{j}:=M_{j}^{\prime}\left(\tilde{r}_{j+1}\right) \quad(n-1 \geqq j \geqq 1) .
$$

Also, setting $P^{\prime}:=\pi_{n-1 n}(P)$, we define: $U_{0}^{\prime}\left(P^{\prime}\right):=\prod_{j=1}^{n-1} U_{\tilde{r}_{j}}\left(P^{\prime}\right) \subset \mathbb{C}^{n-1}$ (cf. $\left.(2)_{2}\right)$. Moreover, we set $\tilde{f}^{\prime}:=\left(\tilde{f}_{j}^{\prime}\right)_{j=1}^{n}$, with $\tilde{f}_{j}^{\prime}:=\left(\pi_{n-1}^{-1}\right)^{*}\left(f_{j}\right)$. We regard that $\tilde{f}^{\prime}$ is defined in $U_{0}^{\prime}\left(P^{\prime}\right)$.

(2) Denote by $Y_{2}$ the locus of $\tilde{f^{\prime}}$ ii $U_{0}^{\prime}\left(P^{\prime}\right)$, and we summarize the application of $(3)_{1,2}^{n-1, k}$ to $Y_{2}$. For this take positive monomials $M_{j}^{\prime \prime}=M_{j: n-1, k}^{\prime \prime}$ $(n-1 \geqq j \geqq k)$. We then define an element $r^{\prime \prime}=\left(r_{j}^{\prime \prime}\right)_{j=1}^{n-1} \in \mathbb{R}^{+n-1}$ by

$$
r_{n-1}^{\prime \prime}: \leqq M_{n-1}^{\prime \prime}\left(d g_{f}(P) / K\right), \quad r_{j}^{\prime \prime}<M_{i}\left(r_{j+1}^{\prime \prime}\right) \quad(n-2 \geqq j \geqq 1) .
$$

Now, letting the manifolds $U_{r^{\prime \prime}}^{n-1, k}\left(P^{\prime}\right) \subset \mathbb{C}^{n-1}, \ldots$ have the similar meaning to $(3)_{3}^{\prime n, n-1}$ (cf. also (2) 3 ), we have:

$(3)_{4}^{\prime n-1, k} \quad U_{r^{\prime \prime}}^{n-1, k}\left(P^{\prime}\right) \supset W_{r^{\prime \prime} c^{\prime \prime}}^{n-1, k}\left(P^{\prime}\right) \supset U_{r^{\prime \prime}}^{n-1, k}(P) \cap Y_{2}$, and $\pi_{k n-1}:\left(U_{r^{\prime \prime}}^{n-1, k}(P) \cap Y_{2}\right)$ $\rightarrow U_{r^{\prime \prime}}^{k}(P)$ is biregular.

(The element $c^{\prime \prime}=c^{\prime \prime n-1, k} \in \mathbb{R}^{+-2}$ depends only on $(n-1, k)$.) We check $(3)_{4}^{\prime n-1, k}$ soon below. Here we remark:

$$
(3)_{1}^{\prime n, n-1}+(3)_{4}^{\prime n-1, k} \longrightarrow(3)_{1,2}^{n, k} \text {. }
$$

(This is easily checked, once we remark that, letting $U_{r}(P)$ be as in $(3)_{1 ; 2}^{n ; k}$, we have: $U_{r}(P) \cap X=\left(U_{r}(P) \cap Y_{1}\right)=\left(\right.$ locus of $\left.f^{\prime}\right)$.)

Now the check of $(3)_{4}^{\prime n-1, k}$ is as follows: first, we define the radius $d^{\prime}$ of $U_{0}^{\prime}\left(P^{\prime}\right)$ to be: $d^{\prime}:=\tilde{r}_{1}$ (cf. (b) $)_{3}$ ). Also we define the invariant $K^{\prime \prime}$ of $\tilde{f}^{\prime}$ (which is defined in $\left.U_{0}^{\prime}\left(P^{\prime}\right)\right)$ in the manner in $(2)_{0}$. Also we define: $g_{\tilde{r}^{\prime}}:=$ $\left|\operatorname{det} \partial \tilde{f}^{\prime}\right| \partial\left(z_{k+2}, \ldots, z_{n}\right) \mid$ and $g_{\ell^{\prime}}(P):=\min \left(1, g_{t^{\prime}}(P)\right)$. Moreover, letting the positive monomials $M_{j}=M_{j ; n-1, k}(n-1 \geqq j \geqq 1)$ have the similar meaning to the one used in $(3)_{1,2}^{n-1, h}$ (cf. also $\left.(2)_{1}\right)$, we define: an element $r=\left(r_{j}\right)_{j=1}^{n-1}$ by

$$
r_{n}: \leqq M_{n-1}\left(d^{\prime} g_{\tilde{i}^{\prime}} / K^{\prime \prime}\right) \text {, and } r_{j} \leqq M_{j}\left(r_{j+1}\right)(n-2 \geqq j \geqq 1) \text {. }
$$

Also, letting the element $c \in \mathbb{R}^{+2}$ be the one used in $(3)_{1,2}^{n-1, k}$, we have the similar fact to $(3)_{1.2}^{\prime n-1, k}$ (given in terms of $(r ; c)$ instead of $\left(r^{\prime \prime} ; c^{\prime \prime}\right)$ ) from $(3)_{1,2}^{n-1, k}$. But, choosing a suitable positive monomial $M_{n, k}$, we have:

(c) ${ }_{3} \quad d^{\prime}>\hat{M}_{n, k}\left(d \tilde{g}_{l} / K\right), K^{\prime}<\hat{M}_{n, k}^{-1}\left(d \tilde{g}_{l}(P) / K\right)$ and $\tilde{g}_{\ell^{\prime}}(P)>\hat{M}_{n, k}\left(d \tilde{g}_{l}(P) / K\right)$.

(We check this soon below.) Thus, assuming that $M_{n}$ is suitably chosen, we have: 
(c) 4

$$
M_{n-1}^{\prime \prime}\left(d g_{f} / K\right)>M_{n-1}\left(d^{\prime} g_{\tilde{i}} / K^{\prime \prime}\right),
$$

and, by understanding that $M_{j}=M_{j}^{\prime \prime}(n-2 \geqq j \geqq 1)$, we have $(3)_{4}^{\prime n-1, k}$ from $(3)_{4}^{n-1, k}$ (in the form given soon above $\left.(c)_{3}\right)$. Finally, the first inequality in (c) $)_{3}$ is checked easily from the explicit form of $d=r_{1}$ (cf. (b) $)_{3}$ ). To check the second and third, we remark: $\partial \tilde{f}_{i}^{\prime} / \partial z_{j}=f_{i j}+\left(f_{1, j} / f_{1, k+1}\right) \cdot f_{i, k+1}$ with $f_{i j}:=\partial f_{i} / \partial z_{j}$ $(2 \leqq i \leqq n-k, n \geqq j \geqq k+2)$. The second inequality follows from this relation and $(\mathrm{a})_{1}$. Moreover, by a simple computation, we have: $\tilde{g}_{\tilde{l}^{\prime}}=\left(g_{f_{1}}\right)^{-n-1} \cdot \tilde{g}_{f}$, and from $(a)_{1}$ we easily derive the third inequality in (c) $)_{3}$. Thus we have (c) and $(3)_{4}^{\prime n-1, k}$. By (d) we also finish the proof of $(3)_{1,2}^{n, k}$.

q.e.d.

\section{B. Proof of Lemma 5.3 and Lemma 5.5}

1. First we prove the part of Lemma $5.3_{1}$, which concerns Lemma 4.2 and Lemma 4.4 (cf. n.1, §5.2). For this we let the set $f=\left(f_{j}\right)_{j=1}^{s} \subset \Gamma\left(X_{0}, D_{X_{0}}\right)$ and the Koszul homomorphism $F_{p}^{m}: \mathfrak{D}_{X}^{\left(\begin{array}{c}s \\ p\end{array}\right)} \rightarrow \mathfrak{D}_{X}^{\left(p^{s}\right)}$ be as in Lemma $5.3_{1}$ (cf. also Lemma 4.2).

(i) Take an element $\varphi=\left(\varphi_{I}\right)_{I} \in \mathfrak{D}_{X}^{\left(\begin{array}{c}s \\ p\end{array}\right)}(1 \leqq s \leqq p-1)$, where $I$ exhausts all indices of the form: $I=\left(i_{1}<\cdots<i_{p}\right)$, and we assume that $F_{p}^{m} \varphi=0$. Then we easily have:

$$
\varphi=0 \text {, if } \varphi_{I}=0 \text { for each } I \text { of the form: } I=\left(1<i_{2}<\cdots<i_{p}\right) .
$$

(ii) Next letting the subvariety $X_{1}, X_{2}$ of $X_{0}$ be as in Section 4.1, we take an open subset $U_{1}\left(\ni P_{0}\right)$ of $U_{0}$ and an element $\sigma \in \boldsymbol{R}_{1}^{+2}$. For each $P \in U_{1} \cap \tilde{X}_{1}$, where $\tilde{X}_{1}=X_{1}-X_{2}$ is as in Section 4.1 , we set: $\tilde{U}_{\sigma}(P ; g):=\{Q \in X ; d(Q, P)$ $\left.<(\sigma g(P))^{-1}\right\}$, with $g(P):=d\left(P, X_{2}\right)^{-1}$. Moreover, taking an element $c=$ $\left(c_{1}, c_{2}\right) \in \boldsymbol{R}^{+2}$ satisfying $c_{1} \leqq 1 \leqq c_{2}$, we have:

Proposition $1_{1}$. If the following holds for each $P \in U_{1} \cap \tilde{X}_{1}$ :

$$
\left|f_{j}(Q)\right| \geqq c \cdot|f(Q)| \text { in } \tilde{U}_{\sigma}(P ; g),
$$

where $j$ is an element of $[1, \ldots, s]$, then Lemma 4.2 and Lemma 4.4 hold for $\left(X_{1}, X_{2} ; f\right)$ (cf. (4.3) $)_{1}$.

Proof. Take an element $(r ; \tilde{n} ; a) \in \boldsymbol{R}^{+} \times \mathbb{Z}^{+} \times \boldsymbol{R}_{1}^{+}$satisfying $r<\{\sigma g(P)\}^{-1}$ and an element $\varphi \in \Gamma\left(\tilde{U}_{r}(P), \mathfrak{D}_{X}\right)_{a}^{\tilde{m}}\left(\mathrm{cf} .(4.2)_{2}\right)$. Then, taking an element $m \in \mathbb{Z}^{+}$ satisfying $m c_{2}<\tilde{m}$, we have:

$$
\left|\varphi(Q) / f_{j}^{m}(Q)\right| \leqq a^{\prime}|f(Q)|^{m^{\prime}} \text {, with }\left(a^{\prime} ; m^{\prime}\right)=\left(a / c_{1}^{m} ; \tilde{m}-c_{2} m\right) .
$$

This insures that $\varphi^{\prime}:=\varphi / f_{j}^{m}$ is in $\Gamma\left(\tilde{U}_{r}(P), \mathfrak{D}_{X}\right)_{a^{\prime \prime}}\left(\mathrm{cr} .(4.2)_{2}\right)$, where 
(b) $a^{\prime \prime}=a^{\prime}$ or $a^{\prime \prime}=a^{\prime} \cdot M^{\tilde{m}}$, with an element $M=M_{t} \in \mathbb{R}_{1}^{+}$, according as*) $f\left(P_{0}\right)=$ or $\neq 0$.

The condition: $\tilde{m}>c_{2} m$ is satisfied, by setting $m:=\left[\tilde{m} / 2 c_{2}\right]$. From the explicit form of the estimation: $(a ; \tilde{m}) \rightarrow\left(a^{\prime \prime} ; m^{\prime}\right)$ as above, we have 'Lemma 4.2 for $\left(X_{1}, X_{2} ; f\right)^{\prime}$ (cf. (4.3) $)_{1}$ and the explicit formulation of Lemma 4.2). Next assuming that $j=1$, we take an element $\varphi=\left(\varphi_{I}\right)_{I} \in \Gamma\left(\tilde{U}_{r}(P), \rho^{\tilde{m}} \mathfrak{D}_{X}^{\left(\begin{array}{l}s \\ p\end{array}\right)}\right)_{a}(1 \leqq p$ $\leqq s-1)$ satisfying $F_{p}^{m} \varphi=0\left(m \in \mathbb{Z}^{+}\right)$. We then define an element $\varphi^{\prime}=\left(\varphi_{J}^{\prime}\right)_{J}$ $\in \Gamma\left(\tilde{U}_{r}(P), \mathfrak{D}_{X}^{(p-1)}\right)$ by

(b) $\varphi_{J}^{\prime}:=\left\{\begin{array}{l}\varphi_{J^{\prime}}^{\prime} / f_{1}^{m}, \text { wherc } J^{\prime}=(1, J), \text { if } J \text { is of the form }\left(j_{1}<\cdots<j_{p-1}\right), \\ \text { with } j_{1}>1, \\ 0, \text { otherwise. }\end{array}\right.$

By (4) $)_{0}$ we have: $\varphi-F_{p-1}^{m} \varphi^{\prime}=0$. Moreover, if $\tilde{m}>2 c_{2} m$, then (a) implies:

(c) $\left|\varphi^{\prime}\right| \leqq a^{\prime} \cdot|f|^{\tilde{m} / 2}$, where the element $a^{\prime} \in \mathbb{R}_{1}^{+}$is given in the manner in (a).

We then apply 'Lemma 4.2 for $\left(X_{1}, X_{2} ; f\right)$ ', whose explicit form is given soon above. Then we have $\varphi^{\prime} \in \Gamma\left(\tilde{U}_{r}(P), \mathcal{f}^{\tilde{m}^{\prime}} \mathfrak{D}_{X}^{(p-1)}\right)_{\tilde{a}^{\prime}}$, with $\left(\tilde{a}^{\prime} ; \tilde{m}^{\prime}\right)=$ $\left(M_{1}(a) \exp M_{2}(\tilde{m}),[L(\tilde{m})]\right)$, where the positive monomials $M_{i}$ and the linear map $L$ are determined by the element $c \in \mathbb{R}^{+2}$. Thus we have 'Lemma 4.4 for $\left(X_{1}, X_{2} ; f\right)^{\prime}$ (cf. (4.3) $)_{1}$ and the explicit form of Lemma 4.4).

q.e.d.

(iii) Thirdly let $X_{0}^{\prime}$ and $V$ denote $X_{0 . \text { sing }}$ and the locus of $f$.

Proposition $\mathbb{1}_{2}$. The pair $\left(X_{1}, X_{2}\right)=\left(X_{0}, X_{0}^{\prime} \cup V\right)$ satisfies $(4)_{1}$.

Proof. First, by Lojasiewicz inequality, we have**):

(a) $|f(P)|>\alpha g(P)$ for each $P \in \tilde{U}_{1} \cap\left(X_{1}-X_{2}\right)$, with an element $\alpha \in \mathbb{R}^{+2}$.

Take an index $j$ satisfying $\left|f_{j}(P)\right| \geqq|f(P) / s|$. Then, assuming that the element $\sigma \in \mathbb{R}_{1}^{+2}$ is sufficiently large, we have:

$$
\begin{aligned}
2\left|f_{j}(Q)\right| \geqq\left|f_{j}(P)\right| \geqq\left|f_{j}(Q) / 2\right|, & \\
& \text { and }|2 f(Q)| \geqq|f(P)| \geqq|f(Q) / 2| \text { in } U_{\sigma}(P ; g) .
\end{aligned}
$$

The condition (4) is easily derived from (a), (b).

q.e.d.

Next we assume that $\operatorname{dim}\left(V-X_{0}^{\prime}\right)=k-1$, where $k=\operatorname{dim} X_{0}$. We choose a

*) $P_{0}$ is the origin of the variety $X_{0}$ (cf. the beginning of this appendix).

**) Recall that $g(P)=d\left(P, X_{2}\right)$ (cf. the beginnig of (ii)). We use the symbol ' $g$ ' in this sense in the remainder of Appendix I. Note that the variety $X_{\mathrm{a}}$ depends on the geometric situation in question. 
suitable subvariety $V^{\prime}$ of $V$ satisfying $\operatorname{dim}\left(V^{\prime}-X_{0}^{\prime}\right) \leqq k-2$.

Proposition $\mathbb{1}_{3}$. The pair $\left(X_{1}, X_{2}\right)=\left(V \cup X_{0}^{\prime}, V^{\prime} \cup X_{0}^{\prime}\right)$ satisfies (4) $)_{1}$.

Proof. Denote by $V_{1}$ the locus of $f_{1}$, and we write $V_{1}$ as $V_{1}=V \cup V_{1}^{\prime}$, where $V_{1}^{\prime}$ has no common irreducible components with $V$ at $P_{0}$ (=origin of $X_{0}$ ). We set $V^{\prime}:=V \cap V_{1}^{\prime}$. Then, letting the neighborhood $U_{\sigma}(P ; g)$ be as in Proposition $1_{2}$, we have: $d(Q, V)=d\left(Q, V_{1}^{\prime}\right)$ in $\tilde{U}_{\sigma}(P ; g)$. By Lojasiewicz inequality, we have:

(a) $\left|f_{1}(Q)\right| \geqq \beta \cdot|f(Q)|$ in $U_{\sigma}(P ; g)$, where $\beta \in \mathbb{R}^{+2}$ is independent from $P$, and we have Proposition $1_{3}$.

It is easy to see that Propositions $1_{2,3}$ insure the part of Lemma 5.3 concerns Lemma 4.2 and Lemma 4.4.

2. Next we check the part of Lemma 5.3, which concerns Lemma 4.3. The set $g \in \Gamma\left(X_{0}, \mathfrak{D}_{X_{0}}\right)$ and its locus $W\left(\right.$ on $\left.X_{0}\right)$ are as in Lemma 4.3.

Proposition 21. Lemma 4.3 holds for $\left(X_{0}, W \cup X_{0}^{\prime} ; \not f, \mathscr{G}\right)$.

Proof. Setting $\left(X_{1}, X_{2}\right)=\left(X_{0}, X_{0}^{\prime} \cup W\right)$, we apply Proposition $1_{2}$ to $g$ and $U_{\sigma}(P ; g)$. Then we have:

$$
\left|g_{j}(Q)\right|>\alpha|g(Q)| \text { in } U_{\sigma}(P ; g) \text {, with a suitable } \alpha \in \mathbb{R}_{1}^{+2} .
$$

Thus, assuming that $\sigma \in \mathbb{R}_{1}^{+2}$ is sufficiently large, we have: $\left|1 / g_{j}(Q)\right|<1 / r$ for each $r \in \mathbb{R}^{+}$satisfying $r<(\sigma g(P))^{-1}$. Now take an element $\varphi \in \Gamma\left(\tilde{U}_{r}(P), f^{\tilde{m}} \mathfrak{D}_{X}\right)_{a}$, where the element $(r ; a ; \tilde{m}) \in \mathbb{R}^{+} \times \mathbb{R}_{1}^{+} \times \mathbb{Z}^{+}$is as in Proposition $1_{1}$. Then, from the inequality just above, we directly have:

$$
\left(\varphi / g_{j}\right) \in \Gamma\left(\tilde{U}_{r}(P), f^{\tilde{m}} \mathfrak{D}_{X}\right)_{a^{\prime}} \text {, with } a^{\prime}:=a / r .
$$

Finally, taking an element $\left.\varphi \in \Gamma\left(\widetilde{U}_{r}(P), f^{\tilde{m} \mathfrak{D}_{X}^{(t)}}\right)_{a}\right)_{a}$ satisfying $G_{p} \varphi=0$, we define an element $\varphi^{\prime} \in \Gamma\left(\tilde{U}_{r}(P), f^{\tilde{m}} \mathfrak{D}_{X}^{\left(p^{t}\right)}\right)$ satisfying $\varphi=G_{p-1} \varphi^{\prime}$ in the manner in Proposition $1_{1}$ (by changing $\left(F_{p}^{m}, f_{1}\right)$ to $\left(G_{p}, g_{j}\right)$ ). Then, from (b), we have: $\varphi^{\prime} \in \Gamma\left(\widetilde{U}_{r}(P), f^{\tilde{m}} \mathfrak{D}_{X}\left({ }^{t}-1\right)\right)_{a^{\prime}}$, and we have Proposition $2_{1}$ (cf. also the explicit formulation of Lemma 4.3).

q.e.d.

Assume that $\operatorname{dim}\left(W-X_{0}^{\prime}\right)=k-1$, and we choose a suitable subvariety $Y$ of $W$ satisfying $\operatorname{dim}\left(W-X_{0}^{\prime}-Y\right) \leqq k-2$. Then we have:

Proposition $2_{2}$. Lemma 4.3 holds for $\left(X_{1}, X_{2}\right)=\left(W \cup X_{0}^{\prime}, Y \cup X_{0}^{\prime}\right)$.

Proof. Letting $W_{1}$ be the locus of $g_{1}$, we write $W_{1}$ as $W_{1}=W \cup W_{1}^{\prime}$, where 
$W_{1}$ has no common irreducible components with $W$. Recall that $V \supset W$ (cf. $(4.2)_{4}$ ), and we write $V$ as $V=W \cup V_{1}$ in the similar manner to the above. Then, setting $Y:=W \cap\left(W_{1} \cup V_{1}\right)$, we have the following similarly to Proposition $1_{3}$ : $d(Q, W)=d\left(Q, W_{1}\right)=d(Q, V)$ in $U_{\sigma}(P ; g)$. Applying Lojasiewicz inequality to $\left(g_{1}, f\right)$, we have:

$$
\left|g_{1}(Q)\right| \geqq \alpha|f(Q)| \text { in } \tilde{U}_{\sigma}(P ; g) \text {, with an clement } \alpha \in \mathbb{R}^{+2} .
$$

Now let the element $\varphi \in \Gamma\left(\widetilde{U}_{r}(P), f^{\tilde{m} \mathfrak{D}_{X}}\right)_{a}$ be as in Proposition $2_{1}$. Then taking an element $d \in \mathbb{Z}^{+}$, we write $\varphi$ as $\varphi=F^{\hat{m}-d} \varphi^{\prime}$, with $\varphi^{\prime} \in \Gamma\left(\tilde{U}_{r}(P), f^{d} \mathscr{D}_{x}\right)_{a}$. (Here we take $d \in Z^{+}$to be: $d>\alpha_{2}$, with the second component $\alpha_{2}$ to $\alpha$, and we may regard that $d \in \mathbb{Z}^{+}$depends only on $\alpha \in \mathbb{R}_{1}^{+2}$.) By (a), we have:

(b) $\varphi / g_{1} \in \Gamma\left(\tilde{U}_{r}(P), f^{\tilde{m}-d} \mathfrak{O}_{X}\right)_{a^{\prime}}$, with $a^{\prime}:=a \cdot K$, with an element $K=K_{f, g} \in \mathbb{R}_{1}^{+}$. Finally, for an element $\Psi \in \Gamma\left(\widetilde{U}_{r}(P), f^{\tilde{m}} \mathfrak{O}_{X}^{(t)}\right)_{a}$ satisfying $G_{p} \Psi=0$, we define an element $\Psi^{\prime} \in \Gamma\left(\tilde{U}_{r}(P), \mathfrak{D}_{X}^{\left(p_{-1}^{t}\right)}\right)$ satisfying $G_{p-1} \Psi^{\prime}=\Psi$ in the similar manner to Proposition $22_{1}$. But, by (b), we have:

$$
\Psi^{\prime} \in \Gamma\left(\tilde{U}_{r}(P), f^{\left.\tilde{m}-d \mathfrak{D}_{X}^{(p-1}\right)}\right)_{a^{\prime}},
$$

and we finish the proof of Proposition $2_{2}$.

q.e.d.

It is clear that Propositions $2_{1,2}$ insure the part of Lemma 5.3, which concerns Lemma 4.3, and we also finish the proof of Lemma 5.3.

3. Finally the proof of Lemma 5.5 is quite simple, and is as follows: letting the variety $X_{0}$ and its divisor $D$ be as in Lemma 5.5, we take the subvariety $Y$ (required in Lemma 5.5) to be the locus of $f$. Also we take a matrix $K: \mathfrak{D}_{X}^{t} \rightarrow \mathfrak{D}_{X}^{s}(s, t>0)$, whose entries are meromorphic functions over $X_{0}$ (with the pole $D)$. Now take a point $P \in U_{1} \cap\left(X_{0}-(Y \cup D)\right)$ and an element $(r ; \alpha ; m)$ $\in \boldsymbol{R}^{+} \times \boldsymbol{R}_{1}^{+} \times \mathbb{Z}^{+}$satisfying $r<\{\sigma d(P, Y \cup D)\}^{-1}$. (For the open set $U_{1}$ and the element $\sigma \in \boldsymbol{R}_{1}^{+2}$, see the beginning of part B.) Also we take an element $\varphi$ $\in \Gamma\left(U_{r}(P), f^{m D_{X}}\right)_{a} \cap \Gamma\left(\widetilde{U}_{r}(P), \mathfrak{S}\right)$, with $\mathfrak{S}:=K D_{X}^{s}\left(\subset D_{X}^{t}\right)$. By (b) in the proof of Proposition $1_{2}$, we have: $\left(2^{-1}\right) \cdot|f(Q)|<|f(P)|<2 \cdot|f(Q)|$ in $\tilde{U}_{r}(P)$, and we also have: $|\varphi(Q)|<a^{\prime}:=\left(2^{m} \cdot\left|f(P)^{m}\right|\right)$. Wc apply Lemma $4.1^{\prime}$ to $(K, \varphi)$, and we have an element $\varphi^{\prime} \in \Gamma\left(\tilde{U}_{r^{\prime}}(P), \mathfrak{D}_{X}^{t}\right)_{a^{\prime \prime}}$, satisfying $\varphi=K \varphi^{\prime}$, where the estimation: $\left(r ; a^{\prime}\right) \rightarrow\left(r^{\prime} ; a^{\prime \prime}\right)=\left(M_{1 K}(r) ; M_{2 K}\left(a^{\prime} / r\right)\right)$ (with the positive monomials $M_{i K}$ ) are given as in Lemma 4.1'. From this explicit estimation, one can rewritc the estimation as above in the form:

(a) $\left|\varphi^{\prime}(Q)\right|<\tilde{a}^{\prime} \cdot\left|f(Q)^{m^{\prime}}\right|$, with $\left(r^{\prime} ; \tilde{a}^{\prime} ; m^{\prime}\right)=E_{K}(r ; a ; m)$, where the a.d. map $E_{K}$ 
(Definition 4.1) is determined by $M_{1, K}$.

We apply Lemma 4.2 for $\left(X_{0}, Y \cup D ; f\right)$ to $\varphi^{\prime}$, and we have: $\varphi^{\prime} \in \Gamma\left(\tilde{U}_{\vec{r}^{\prime}}(P)\right.$, $\left.f^{\bar{m}^{\prime}} \mathfrak{D}_{X}^{s}\right)_{\bar{a}^{\prime}}$, where the estimation: $\left(r^{\prime} ; \tilde{a}^{\prime} ; m^{\prime}\right) \rightarrow\left(\bar{r}^{\prime} ; \bar{a}^{\prime} ; \bar{m}^{\prime}\right)$ is given similarly to (a). It is clear that this, estimation, together with the equation: $K \varphi^{\prime}=\varphi$, insures Lemma 5.5, and we also finish the proof of all the facts, which are used in Section 4 and Section 5 and whose proof are remained in Appendix II.

\section{Chapter III. Polynomial Growth Uniform Estimations for the Structure Sheaves of Complex Euclidean Spaces}

The main purpose of this chapter is to prove Theorem 1.7, which concerns the uniform estimations in the title. The proof is given by the reduction:

Theorem $1.7 \rightarrow$ p.g. estimations for Cousin integrals on $\boldsymbol{C}$.

The main part of (1) will be certain algebraic arguments on types of filtrations, which are suitably applied to the sets of the cochains in Theorem 1.7 and its variants (\$6.1). The contents of Chapter III are as follows. In Section 6.1 we give the variants of Theorem 1.7 which are convenient for the use of the filtrations. The algebraic arguments on the filtrations are given in Section 6.2 and in Part A, Section 7. Using these arguments, the proof of Theorem 1.7 is given in Section 6.3 and Part B, Section 7. Very roughly our algebraic arguments enable us to give the following implication:

$$
E_{1, \mu}^{0, q} \cong 0(q \geqq 1, \mu \in \mu) \Rightarrow H^{q}\left(K^{*}\right) \cong E_{2}^{q, 0} \quad(q \geqq 0),
$$

where $K^{*}$ is a filtered complex in question and $E_{r}^{p, q}$ is its spectral sequence. Also $E_{r, \mu}^{p, q}$ is the spectral sequence of a complex $K_{\mu}^{*}$, where the complex $\left\{K_{\mu}^{*}\right\}_{\mu}$ is defined from the geometric situation in question. Note that the standard degeneracy theorem, applied to our situation, is read as follows:

$$
E_{1}^{p, q-p} \cong 0(q>p \geqq 0) \Longrightarrow H^{q}\left(K^{*}\right) \cong E_{2}^{q, 0}(q \geqq 0) .
$$

Thus, our sharpening (2), where the assumption is given at the lowest filtration degree $p=0$, will give a strong sharpening of $(2)_{1}$ as well as the main part of the reduction (1). The key facts for (2) are the construction of the families of the complexes $K_{\mu}^{*}$ and some reduction arguments using such families. These will be given in Section 6.2 and in Part A, Section 7. (For the first, see n.3, Section 6.2. The main part of $A$, Section 7 will be devoted to the latter.)

Cohomology theories with growth conditions have been developed 
systematically since the works of L. Hörmander (cf. [8], [9]), chiefly by means of the $\bar{\partial}$-estimations. On the other hand, the very basic method of Cousin integrals in function theory seems to have been regarded as not so adequate for the cohomology theories with growth conditions (cf. for example, p. 90, [8]). Our algebraic arguments on the filtrations enable us to take the Cousin integrals as the analytic base for our p.g. cohomology theory. Also, our sharpening of the degeneracy theorem indicated in (2) (cf. also Lemmas 7.2-7.5) may be worthwhile pointing out; the content of Chapter III may owe its own interests aparting from the applications to Chapters I, II.

Remark. The necessary p.g. estimations on Cousin integrals and the algebraic arguments on the filtrations will be found in Part A, Appendix II and in Section 6.2, Part A, Section 7. Interested readers in the above analytic and algebraic arguments may first read those parts. The estimations and the algebraic arguments mentioned soon above may deserve to be tried their applicabilities to more wider situations. The author hopes to try possible generalizations in a future.

\section{§6. Cousin Integrals and Spectral Sequences}

\section{§6.1. Key Theorems for Theorem 1.7}

1. Elementary coverings. We begin Section 6.1 by introducing certain coverings of domains of a complex euclidean space $\mathbb{C}^{n}(z)(n>0)(\mathrm{cf} . \mathrm{n} .1, \S 1.2)$, which are of quite simple nature and are suitable for explicit estimations on Cousin integrals (cf. Appendix II).

First let $U=(a, a+b) ; b>0$ be an open interval in a real euclid line $\boldsymbol{R}$. We mean by elementary covering of $U$ of size $\eta=(k ; \varepsilon) \in\left(\mathbb{Z}^{+} \cup 0\right)$

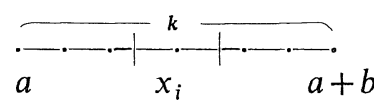

Figure I. $\times(0,1 / 2)$ the following element of*) $\operatorname{Cov}_{0}(\mathbb{R})$ :

$(6.1)_{1}\left\{\begin{array}{l}\left\{U_{i}\right\}_{i=0}^{k}, \text { where } U_{i}:=\left\{x \in \mathbb{R} ;\left|x-x_{i}\right|<\tilde{\varepsilon} b \mid k\right\} \quad(k>0) \\ \left\{U^{0}\right\}, \text { where } U^{0}:=\left\{x \in \mathbb{R} ;\left|x-x^{0}\right|<\tilde{\varepsilon} b\right\} \quad(k=0) .\end{array}\right.$

Here $x_{i}:=a+(i b / k), x^{0}:=a+(b / 2)$ and $\tilde{\varepsilon}:=\varepsilon+2^{-1}$.

Next by an elementary figure in $\mathbb{C}^{n}(z)$ we mean a product $U=\prod_{j=1}^{2 n} U_{j}$

*) For a topological space $X$, we set: $\operatorname{Cov}_{0}(X):=2^{\text {our }}(\mathrm{r})$ (cf. the end of the introduction of Chapter l). 
$\subset \mathbb{R}^{2 n}\left(\cong \boldsymbol{C}^{n}\right)$, where $U_{j}$ is an open interval in the $j$-th component of $\boldsymbol{R}^{2 n}$. For an element $\tilde{\eta}=\left(k_{c} ; \varepsilon\right) \in\left(\mathbb{Z}^{+} \cup 0\right)^{2 n} \times(0,1 / 2)$, where $\hbar_{a}=\left(k_{j}\right)_{j=1}^{2 n}$, we make:

Definition 6.1. $1_{1}$. By elementary covering of $U$ of size $\tilde{\eta}$, we mean an element $\mathscr{A}_{\tilde{\eta}}(U) \in \operatorname{Cov}_{0}\left(\boldsymbol{C}^{n}\right)$ as follows:

$$
\mathscr{A}_{\tilde{i}}(U):=\left\{\prod_{j=1}^{2 n} \mathscr{A}_{j \eta_{j}}\left(\subset \mathbb{R}^{2 n} \cong \mathbb{C}^{n}\right)\right\},
$$

where $\mathscr{A}_{j \eta_{j}}$ runs through the elementary covering of $U_{j}(\subset \mathbb{R})$ of size $\eta_{j}:=\left(k_{j} ; \varepsilon\right)$.

When $k_{1}=k_{2}=\cdots(=k)$, we write $\mathscr{A}_{\tilde{\eta}}(U)$ also as $\mathscr{A}_{\eta}(U)$, with $\eta=(k ; \varepsilon)$.

Thirdly, we define a covering of $\mathbb{C}^{n}$, which may be a most simple one among elements of $\operatorname{Cov}_{0}\left(\mathbb{C}^{n}\right)$. For this we decompose $\mathbb{C}^{n}$ in the form:

$(6.1)_{3} \quad \mathbb{C}^{n}=\cup_{I \in Z^{2 n}} T_{I}$, where $T_{I}=\prod_{j=1}^{2 n}\left(i_{j}, i_{j}+1\right) \subset \mathbb{C}^{n}\left(\cong \mathbb{R}^{2 n}\right)$, with $I=\left(i_{1}, \ldots\right.$, $\left.i_{2 n}\right)$.

Then, taking an element $\eta=(k, \varepsilon) \in \mathbb{Z}^{+} \times(0,1 / 2)$, we make:

Definition $6 . \mathbb{1}_{2}$. By elementary covering of $\mathbb{C}^{n}$ of size $\eta$, we mean an element of $\operatorname{Cov}_{0}\left(\mathbb{C}^{n}\right)$ as follows:

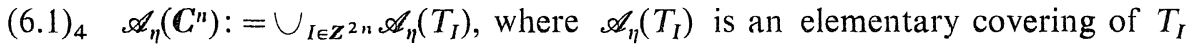
of size $\eta$.

It is classically well known that such a covering is suitable for computation of Cousin integrals. Our key theorem, Theorem 6.1, will be formulated in terms of such a covering (cf. n.2 soon below).

Remark $6.1_{1}$. Let the elements $\tilde{\eta}=(\hbar, \varepsilon) \in\left(\mathbb{Z}^{+} \cup 0\right)^{2 n} \times(0,1 / 2), \eta=(k, \varepsilon)$ $\in \mathbb{Z}^{+} \times(0,1 / 2)$ and the elementary coverings $\mathscr{A}_{\hat{\eta}}(U), \mathscr{A}_{\eta}\left(\mathbb{C}^{n}\right)$ be as in $(6.1)_{2,4}$. In our arguments henceforth, we use such coverings in the case: $\varepsilon=1 / 4$. For notational simplification, we use the symbols $\mathscr{A}_{k}(U), \mathscr{A}_{k}\left(\mathbb{C}^{n}\right)$ for the elementary coverings $\mathscr{A}_{\tilde{\eta}}(U), \mathscr{A}_{\eta}\left(\mathbb{C}^{n}\right)$.

Finally, for purpose of the formulation of Theorem 6.1, we fix a euclidean space $\boldsymbol{C}^{n^{\prime}}\left(z^{\prime}\right)(n>0)$. Wc write $\left(\bar{z}, z^{\prime}\right)$ as $\tilde{z}$. The symbol $\mathfrak{D}$ will denote the structure shear of $\mathbb{C}^{n} \times \mathbb{C}^{n^{\prime}}$.

2. A key theorem. Now, letting $k$ be an element of $\mathbb{Z}^{+}$, we take a suitable $k^{\prime} \in \mathbb{Z}^{+}$and an el-map $\mathscr{L}_{n, k}: \boldsymbol{R}^{+2} \rightarrow \boldsymbol{R}^{+2}$, which depends only on $(n, k)$. Then we have $(q \geqq 1)$ :

Theorem 6.1 $q_{q^{*}}^{n}$ For each $\alpha \in \mathbb{R}_{1}^{+2}$ and an open set $D^{\prime} \subset C^{n^{\prime}}$, we have: 
(6.2) $s^{*} Z^{q}\left(\mathscr{A}_{k}\left(\mathbb{C}^{n}\right) \times D^{\prime}, \mathfrak{D}\right)_{\alpha} \subset \delta C^{q-1}\left(\mathscr{A}_{k^{\prime}}\left(\mathbb{C}^{n}\right) \times D^{\prime}, \mathfrak{D}\right)_{\alpha^{\prime}}$, where $\alpha^{\prime}=\mathscr{L}_{n, k}(\alpha)$ and $s$ is a suitable refining map: $\mathscr{A}_{k^{\prime}}\left(\mathbb{C}^{n}\right) \times D^{\prime} \hookrightarrow \mathscr{A}_{k}\left(\mathbb{C}^{n}\right) \times D^{\prime}$.

Here we set:

(6.2) $C^{a}\left(\mathscr{A}_{k}\left(\mathbb{C}^{n}\right) \times D^{\prime}, \mathfrak{D}\right)_{\alpha}:=$ set of $(|\tilde{z}|+1)-\alpha$-growth cochains with values in $\mathfrak{D}\left(c f .(1.3)_{6}\right.$ and $\left.(1.4)_{8}\right)$,

and we write the element $\left\{D^{\prime}\right\} \in \operatorname{Cov}_{0}\left(\mathbb{C}^{n^{\prime}}\right)$ as $D^{\prime}$.

(The element $D^{\prime} \in \operatorname{Cov}_{0}\left(\mathbb{C}^{n^{\prime}}\right)$ is just additional to $\mathscr{A}_{h}\left(\mathbb{C}^{n}\right)$. But it is convenient to include this element for our application of Theorem 6.1 to the proof of Theorem 1.7 and for the proof of Theorem 6.1 itself (cf. $\$ 6.2$ and $\S 7$ ).)

Remark 6.1.$_{2}$. In Theorem 6.1 we do not give an explicit form*) of the correspondence: $\mathbb{Z}^{+} \ni k \rightarrow \mathbb{Z}^{+} \ni k^{\prime}$. Also we do not give an explicit dependence of the el-map $\mathscr{L}_{n, k}$ on $k \in \mathbb{Z}^{+}$; the estimation in Theorem 6.1 is of relaxed form (relative to the ones in Chapter I). But, the formulation of Theorem 6.1 suffices for the proof of Theorem 1.7. (In the proof of Theorem 1.7, we use Theorem 6.1 only in the case of $k=1$; see Section 6.2.)

Remark $6.1_{3}$. Except our concrete formulation, Theorem 6.1 is of familiar type in $\bar{\partial}$-estimations (cf. [9]). However, as we will see in the course of Chapter III, our proof of Theorem 6.1 differs entirely from the ones depending on $\bar{\partial}$-estimations (cf. also the introduction of Chapter III).

3. An another key theorem. Recall that the elementary covering $\mathscr{A}_{k}\left(\mathbb{C}^{n}\right)$ in Theorem 6.1 is much simpler than the p.g. coverings in Theorem 1.7. We give here a theorem, which will fill the gap between Theorem 6.1 and Theorem 1.7 stemming from the above difference of the types of the coverings.

(i) P.g. elementary coverings. First we give a type of coverings of domains in $\mathbb{C}^{n} \times \mathbb{C}^{n^{\prime}}$, which is convenient to fill the gap just above: taking an element $\zeta=(\zeta ; \varepsilon) \in \mathbb{Z}^{+2} \times(0,1 / 2)$ and an elementary figure $U^{\prime} \subset \mathbb{C}^{n^{\prime}}$, we set:

$(6.3)_{1}^{\prime} \quad S_{I, \varepsilon}:=\prod_{j=1}^{2 n}\left(-\tilde{\varepsilon}+i_{j}, i_{j}+\tilde{\varepsilon}\right) \subset \mathbb{C}^{n}\left(\cong \mathbb{R}^{2 n}\right)$, with $\tilde{\varepsilon}:=\varepsilon+2^{-1}$, and $l=$ $\left(i_{j}\right)_{j=1}^{2 n} \in \mathbb{Z}^{2 n}\left(\cong \mathbb{C}^{n}\right)$.

$(6.3)_{1} \mathscr{A}_{\xi}^{\prime}\left(S_{I, \varepsilon} \times U^{\prime}\right):=$ elementary covering of $S_{I, \varepsilon} \times U^{\prime}$ of size $(\zeta(|I|+1), \varepsilon)$ (Def. 6.1 $1_{1}$ ), with $|I|:=\left|i_{1}\right|+\cdots+\left|i_{2 n}\right|$.

*) We can take $k^{\prime}:=\left[L_{n}(k)\right]$, with a linear map $L_{n}$ depending only on $\mathrm{n}$. (Here [ ] is the Gauss symbol, cf. Appendix II and §6.3.) However, we do not use this fact in this paper. 
We then make:

Definition 6.2. By p.g. elementary covering of $\boldsymbol{C}^{n} \times U^{\prime}$ of size $\xi=(\zeta, \varepsilon)$, we mean the following element of $\operatorname{Cov}_{0}\left(\boldsymbol{C}^{n} \times \boldsymbol{C}^{n^{\prime}}\right)$ :

$$
\mathscr{A}_{\xi}^{\prime}\left(\boldsymbol{C}^{n} \times U^{\prime}\right):=\cup_{I \in Z^{2 n}} \mathscr{A}_{\xi}^{\prime}\left(S_{I \varepsilon} \times U^{\prime}\right) .
$$

Concerning the p.g. elementary covering $\mathscr{A}_{\xi}^{\prime}=\mathscr{A}_{\xi}^{\prime}\left(\boldsymbol{C}^{n} \times U^{\prime}\right)$ we remark that $(6.3)_{3}$ each element of $\mathscr{A}_{\xi}^{\prime}$ is an elementary figure in $\boldsymbol{C}^{n} \times \boldsymbol{C}^{n^{\prime}}$, and that

$(6.3)_{4}$ the size of the elements of $\mathscr{A}_{\xi}^{\prime}$ becomes smaller in the p.g. order with respect to $g=|\tilde{z}|+1$.

By $(6.3)_{4,3}$, the p.g. elementary coverings have similarities to the p.g. and elementary coverings as in Theorem 1.7 and Theorem 6.1; the p.g. elementary coverings, which are essentially of technical nature, are suitable to fill the gap between Theorem 1.7 and Theorem 6.1 (cf. §6.2).

Remark 6.2. If $\varepsilon \notin Q$ (=field of rational numbers), then, by a simple observation, we see that the expression $(6.3)_{2}$ is the disjoint union:

$$
\mathscr{A}_{\xi}^{\prime}\left(\boldsymbol{C}^{n} \times U^{\prime}\right)=\coprod_{I \in Z^{2 n}} \mathscr{A}_{\xi}^{\prime}\left(S_{I \varepsilon} \times U^{\prime}\right),
$$

and one can define a map

$$
\mathscr{A}_{\xi}^{\prime}\left(\mathbb{C}^{n} \times U^{\prime}\right) \ni A \stackrel{\pi_{\xi}}{\longrightarrow} \mathbb{Z}^{2 n} \ni I, \text { where } \mathscr{A}_{\xi}^{\prime}\left(S_{I \varepsilon} \times U^{\prime}\right) \ni A
$$

We use this map in later arguments. Henceforth, by a p.g. elementary covering $\mathscr{A}_{\xi \varepsilon}^{\prime}\left(\boldsymbol{C}^{n} \times U^{\prime}\right)$, we mean such a one with $\zeta \notin \boldsymbol{Q}$.

(ii) Now we give an another key theorem, Theorem 6.2, for Theorem 1.7, by using the p.g. elementary covering. For this we first set:

(6.3) $)_{6} \operatorname{Ouv}_{\mathrm{el}}\left(\boldsymbol{C}^{n^{\prime}}\right):=$ collection of all elementary figures in $\boldsymbol{C}^{n^{\prime}}$,

(6.3) $\operatorname{Ouv}_{\mathrm{el}}^{\prime}\left(\boldsymbol{C}^{n^{\prime}}\right):=\left\{U^{\prime} \in \operatorname{Ouv}_{\mathrm{el}}\left(\boldsymbol{C}^{n}\right) ; d_{U^{\prime}}^{+}<1\right\}$, where, writing $U^{\prime}$ as $U^{\prime}=$ $\prod_{j=1}^{2 n}\left(a_{j}, a_{j}+b_{j}\right)\left(\subset C^{n^{\prime}}\right)\left(b_{j}>0\right)$, we set: $d_{U^{\prime}}^{+}:=\max _{j}\left(\max \left(b_{j}, 1\right)\right)$.

Also, for later convenience, it is convenient to set:

$$
d_{U}^{-}:=\min _{j}\left(\min \left(b_{j}, 1\right)\right) .
$$

Moreover, for an element $m \in \boldsymbol{R}^{+}$, we set:

(6.4) ${ }_{1}^{\prime} U_{m}^{\prime}$ :=elementary figure in $\boldsymbol{C}^{n^{\prime}}$ with center=that of $U^{\prime}$ and size= $m \times\left(\right.$ size of $\left.U^{\prime}\right)$, where, writing $U^{\prime}$ as $\prod_{j=1}^{2 n^{\prime}}\left(a_{j}, a_{j}+b_{j}\right)\left(b_{j}>0\right) \subset \boldsymbol{C}^{n^{\prime}}\left(\cong \boldsymbol{R}^{2 n^{\prime}}\right)$, 
the 'size of $U^{\prime}$ : $:=\left(b_{j}\right)_{j=1}^{2 n^{\prime}} \subset \mathbb{R}^{+2 n^{\prime}}$.

In the remainder of (ii) we fix an element*) $\varepsilon=\varepsilon_{\tilde{n}} \in((0,1 / 2)-Q)$, where $\tilde{n}$ : $=\left(n, n^{\prime}\right)$, and we form the following collection of elementary coverings in $\mathbb{C}^{n} \times \mathbb{C}^{n^{\prime}}$ :

$$
\mathscr{A}^{\prime \tilde{n}}:=\left\{\mathscr{A}_{\zeta \varepsilon}^{\prime}\left(\mathbb{C}^{n} \times U^{\prime}\right) ;\left(U^{\prime} ; \zeta\right) \in \mathrm{Ouv}_{\mathrm{el}}^{\prime}\left(\mathbb{C}^{n^{\prime}}\right) \times \mathbb{Z}^{+2}\right\} .
$$

For an element $\mathscr{A}_{\zeta \varepsilon}^{\prime} \in \boldsymbol{A}^{\prime \tilde{n}}$ and an element $\alpha \in \mathbb{R}_{1}^{+2}$ we set:

$(6.4)_{2} C^{q}\left(\mathscr{A}_{\zeta \varepsilon}^{\prime}, \mathfrak{D}\right)_{\alpha}:=$ set of $(|\tilde{z}|+1)-\alpha$-growth cochains with value in $\mathfrak{D}$ (cf. $\left.(1.4)_{7}\right)$.

Next we form a collection of estimation maps as follows:

(6.4) $\boldsymbol{E}_{3}:=\mathbb{L} \times(\boldsymbol{M} \times \mathbb{L})$, where $\mathbb{L}$ and $\boldsymbol{M}$ are the collections of all el-maps and positive monomials (cf. n.5, §1.1).

To an element $E^{\prime}=\left(\mathscr{L}_{1},\left(M, \mathscr{L}_{2}\right)\right) \in \mathbb{E}^{\prime}=\mathbb{L} \times(\mathbb{R} \times \mathbb{L})$ we attach the following map:

$(6.4)_{3}^{\prime} \quad E^{\prime}: \mathbb{Z}^{+2} \times\left(\mathbb{R}^{+} \times \mathbb{R}^{+2}\right) \ni(\zeta ; r ; \alpha) \rightarrow \mathbb{Z}^{+2} \times \mathbb{R}^{+2} \ni\left(\zeta^{\prime}, \alpha^{\prime}\right)$ by $\zeta^{\prime}=\left[\mathscr{L}_{1}(\zeta)\right]$ and $\alpha^{\prime}=M^{-1}(r) \cdot \mathscr{L}_{2}(\alpha+\zeta)$. (Here, [ ] is the Gauss symbol. More precisely, writing $\bar{\zeta}^{\prime}:=\mathscr{L}_{1}(\zeta)$ as $\left(\bar{\zeta}_{1}^{\prime}, \bar{\zeta}_{2}^{\prime}\right)$, we understand that $\zeta^{\prime}:=\left(\left[\bar{\zeta}_{1}^{\prime}\right],\left[\bar{\zeta}_{2}^{\prime}\right]\right)$.)

Now, using the sets of the cochains and the estimation maps as above, our key theorem for Theorem 1.7 is as follows:

Theorem 6.2 $2_{q^{*}}^{\tilde{n}}$. There are an element $\varepsilon^{\prime}=\varepsilon_{\tilde{n}}^{\prime} \in((0,1 / 2)-Q)$ and an estimation map $E_{\tilde{n}}^{\prime} \in \mathbb{E}^{\prime}$, with which we have:

$(6.5)_{1} \quad s^{*} Z^{q}\left(\mathscr{A}_{\zeta \varepsilon}^{\prime}\left(\mathbb{C}^{n} \times U^{\prime}\right), \mathfrak{D}\right)_{\alpha} \subset \delta C^{q-1}\left(\mathscr{A}_{\zeta^{\prime} \varepsilon^{\prime}}^{\prime}\left(\mathbb{C}^{n} \times U_{1 / 2}^{\prime}\right), \mathfrak{D}\right)_{\alpha^{\prime}}$, with $\left(\zeta^{\prime} ; \alpha^{\prime}\right)=$ $E_{\tilde{n}}^{\prime}\left(\zeta ; 1 / d_{U^{\prime}} ; \alpha\right)$ (cf. $\left.(6.3)_{8}\right)$, where the parameter $\left(U^{\prime} ; \zeta ; \alpha\right)$ is in Ouv $\operatorname{Ou}_{\mathrm{e} 1}^{\prime}\left(\mathbb{C}^{n^{\prime}}\right)$ $\times \mathbb{Z}^{+2} \times \boldsymbol{R}_{1}^{+2}$, and $s$ is a refining map: $\mathscr{A}_{\zeta^{\prime} \varepsilon^{\prime}}^{\prime} \hookrightarrow \mathscr{A}_{\zeta \varepsilon}^{\prime}$.

For the proof of Theorem 6.2, see Section 6.3. Here we check the following implication.

Proposition 6.1. Theorem $6.2 \rightarrow$ Theorem 1.7.

Proof. Let the elementary figure $U^{\prime}$ be as in Theorem 6.2. Then we set:

(a) $\mathscr{A}_{\sigma}\left(\mathbb{C}^{n} \times U^{\prime}\right):=\{|\tilde{z}|+1\}$-p.g. covering of $\mathbb{C}^{n} \times U^{\prime}$ in $\mathbb{C}^{n} \times \mathbb{C}^{n^{\prime}}$ of size $\sigma \in \mathbb{R}_{1}^{+2}$ (cf. Def. 1.6 1 ).

*) The element $\varepsilon_{\tilde{n}}$ is fixed (in an arbitrary manner) in the remainder of Chapter III. It is convenient to regard that $\varepsilon_{\tilde{n}}$ depends only on $\tilde{n}$. 
Then, taking a suitable el-map $\mathscr{L}_{\tilde{n}}: \boldsymbol{R}^{+2} \rightarrow \mathbb{R}^{+2}$ and a positive monomial $M_{n}$, we see that if there is a relation between $\sigma$ and $\zeta=\left(\zeta_{1}, \zeta_{2}\right) \in \boldsymbol{Z}^{+2}$ of the form:

$(6.6)_{1} \quad \sigma>K^{\zeta_{2}} \cdot \mathscr{L}_{\tilde{n}}(\zeta)\left(\right.$ resp. $\left.\left.\zeta>M_{n}\left(1 / d_{U}\right)^{\prime}\right) \mathscr{L}_{\tilde{n}}(\sigma)\right)$, with $K=1+\sup _{z^{\prime} \in U^{\prime}}\left|z^{\prime}\right|$, then we have a refining map*):

$(6.6)_{2} \quad s: \mathscr{A}_{\sigma}\left(\boldsymbol{C}^{n} \times U^{\prime}\right) \hookrightarrow \mathscr{A}_{\zeta \varepsilon}^{\prime}\left(\boldsymbol{C}^{n} \times U^{\prime}\right)\left(\right.$ resp. $\left.s^{\prime}: \mathscr{A}_{\zeta \varepsilon}^{\prime}\left(\boldsymbol{C}^{n} \times U^{\prime}\right) \hookrightarrow \mathscr{A}_{\sigma}\left(\boldsymbol{C}^{n} \times U^{\prime}\right)\right)$.

From this relation and from a comparison of the estimation map $E_{\tilde{n}}^{\prime} \in \boldsymbol{E}^{\prime}$ in Theorem $6.2^{n}$ and the p.g. estimation map in Theorem 1.7, we easily have Proposition 6.1.

q.e.d.

By Proposition 6.1, the proof of Theorem 6.2 suffices for the proof of Theorem 1.7. The proof of the former is given by the following implications:

$(6.6)_{3}$ Theorem $6.1^{n-1} \rightarrow$ Theorem $6.1^{n}(n \geqq 2)$, Theorem $6.1^{n} \rightarrow$ Theorem $6.2^{n}$. (For details, see §6.3.) The key facts for the above implications are certain filtrations and algebraic arguments concerning them. These will be discussed**) in Section 6.2 and in Section 7.

\section{§6.2. A Type of Filtration}

Here we introduce a type of filtration (Definition 6.3), and we arrange some data for such a filtration, which are used in later arguments.

1. A type of filitration. Let $X$ be a topological space, $S i$ an element of $\mathrm{Ab}(X)$ and $\mathscr{A}$ an element of $\operatorname{Cov}_{0}(X)$. We also take an (abstract) set $\mathscr{B}$ and a map $\pi: \mathscr{A} \rightarrow \mathscr{B}$. For a subset $C^{q}$ of $C^{q}(\mathscr{A}, \mathfrak{R})$, we make

Definition 6.3. By a filtration in $C^{q}$ induced from $\pi: \mathscr{A} \rightarrow \mathscr{B}$, we mean the following decreasing sequence of subsets of $C^{q}$ :

$$
F_{0} C^{q}:=C^{q} \supset F_{1} C^{q} \supset \cdots \supset F_{q+1} C^{q} \cong 0,
$$

where

$(6.7)_{1}^{\prime} \quad F_{p} C^{q}:=\left\{\varphi \in C^{q} ; \varphi_{\mathscr{A}}=0\right.$ for each $\mathscr{A}^{\prime} \in \mathscr{N}^{q+1} \mathscr{A}$ satisfying $\left.\# \pi\left(\mathscr{A}^{\prime}\right) \leqq p\right\}$.

Assuming that $C^{q}=C^{q}(\mathscr{A}, \mathfrak{R})$, we write the spectral sequence of $\left(C^{*}, F_{p}\right)$ as $E_{r}^{p, *-p}$. Note that $E_{1}^{q, 0} \cong Z_{1}^{q, 0}:=\left\{\varphi \in F_{q} C^{q} ; \delta \varphi \in F_{q+1} C^{q+1}\right\}$, and that

*) Cf. Part B, Appendix II. We summarise necessary estimations on refiningmaps (used in $\S \S 6,7)$ in Part B, Appendix II. The estimations will be found to be essentially of very elementary nature.

**) Cf. the introduction of Chapter III. 
$\left(E_{1}^{q, 0}, d_{1}\right) \cong\left(Z_{1}^{q, 0}, \delta\right)$ is a subcomplex of $C^{*}$ in a natural manner:

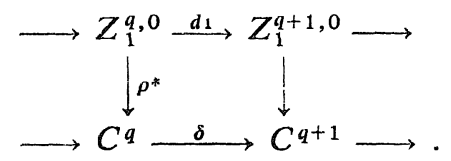

Next, for a subset $\mathscr{A}^{\prime}$ of $\mathscr{A}$, we mean by*) 'u-quotient' of $\mathscr{A}^{\prime}$ by $\pi$ the following element of $\operatorname{Cov}_{0}(X)$ :

$$
\left(\mathscr{A}^{\prime} \mid \pi\right)_{u}:=\left\{\operatorname{supp} \pi^{\prime-1}(B) ; B \in \pi\left(\mathscr{A}^{\prime}\right)\right\},
$$

where $\pi^{\prime}:=$ restriction of $\pi$ to $\mathscr{A}^{\prime}$. (Herc 'supp $\pi^{\prime-1}(B)$ ' is, as usual, defined to be: $\cup_{\mu} A_{\mu}$, where $A_{\mu} \in \pi^{\prime-1}(B)$.) Denoting by $r$ the refining map: $\mathscr{A} \ni A_{\mu} \rightarrow$ $(\mathscr{A} / \pi)_{U} \ni \operatorname{supp} \pi^{-1}(\pi(B))$, we easily have

Proposition 6.2. We have the following diagram:

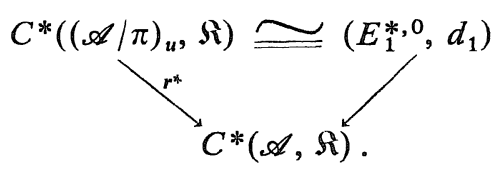

Proposition 6.3. We have the following implication (degeneracy theorem): $(6.7)_{3} \quad E_{1}^{p, q-p} \cong 0(q>p) \Rightarrow H^{*}(\mathscr{A}, \mathfrak{K}) \cong E_{2}^{q, 0}\left(\cong H^{q}\left(C^{*}\left((\mathscr{A} / \pi)_{u}, \mathfrak{R}\right)\right)\right)$.

2. Concrete filtrations. (i) First let $X_{1}, X_{2}$ be topological spaces and $\mathscr{A}_{1}, \mathscr{A}_{2}$ elements of $\operatorname{Cov}_{0}\left(X_{1}\right), \operatorname{Cov}_{0}\left(X_{2}\right)$. We denote by $\mathscr{A}$ the product $\mathscr{A}:=\mathscr{A}_{1} \times \mathscr{A}_{2}:=\left\{A_{1} \times A_{2} ; A_{i} \in \mathscr{A}_{i}(i=1,2)\right\} \in \operatorname{Cov}_{0}(X)$, with $X:=X_{1} \times X_{2}$, and we denote by $\pi$ the projection $\pi: \mathscr{A}=\mathscr{A}_{1} \times \mathscr{A}_{2} \ni\left(A_{1} \times A_{2}\right) \rightarrow \mathscr{A}_{2} \ni A_{2}$. Then we easily have

$$
(\mathscr{A} / \pi)_{u}=\left(\operatorname{supp} \mathscr{A}_{1}\right) \times \mathscr{A}_{2}\left(\in \operatorname{Cov}_{0}(X)\right) .
$$

Let $\mathscr{A}_{k}:=\mathscr{A}_{k}\left(\boldsymbol{C}^{n}\right)$ and $\mathscr{A}_{k}:=\mathscr{A}_{k}(U)$ be as in Definition $6.1_{2,1}$, and write $\mathbb{C}^{n}$ as $\mathbb{C}^{n}=\mathbb{C} \times \mathbb{C}^{n-1}, t_{0}$ as $\hbar_{c}=\left(k_{j}\right)_{j=1}^{2 n}$ and $U$ as $U=U_{1} \times U_{2}$, with elementary figures $U_{1}, U_{2}$ in $\mathbb{C}, \mathbb{C}^{n-1}$. We set $\mathscr{B}_{k}:=\mathscr{A}_{k}\left(\mathbb{C}^{n-1}\right)$ and $\mathscr{B}_{k}:=\mathscr{A}_{k^{\prime}}\left(U_{2}\right)$, with $\mathscr{t}^{\prime}:=\left(k_{j}\right)_{j=1}^{2 n-2}$, and we define maps

$$
\pi_{k}: \mathscr{A}_{k} \longrightarrow \mathscr{B}_{k} \text { and } \pi_{k}: \mathscr{A}_{k} \longrightarrow \mathscr{B}_{k}
$$

in the manner in the beginning of n.2, by using the product structures of $\mathscr{A}_{k}, \mathscr{A}_{k}$ as above. Then, by $(6.7)_{4}$, we have:

$(6.7)_{6}\left(\mathscr{A}_{k} / \pi_{k}\right)_{u}=C \times \mathscr{B}_{k}$ and $\left(\mathscr{A}_{k} / \pi_{k}\right)_{u}=\left(\operatorname{supp} \mathscr{A}_{l}^{\prime}\right) \times \mathscr{B}_{k}$, with $\mathscr{A}_{l}^{\prime}:=\mathscr{A}_{k_{1} k_{2}}\left(U_{1}\right)$.

*) ' $u$ ' is taken from the initial of 'union'. 
Next, take an open set $D^{\prime}$ of $\boldsymbol{C}^{n}$, and use the symbols $\pi_{k}, \pi_{k}$ also for the maps $\pi_{k}: \mathscr{A}_{k} \times D^{\prime} \ni\left(A \times D^{\prime}\right) \rightarrow \mathscr{B}_{k} \ni \pi_{k}(A)$ and $\pi_{k}: \mathscr{A}_{k} \times D^{\prime} \ni\left(A \times D^{\prime}\right) \rightarrow \mathscr{B}_{k} \ni \pi_{k}(A)$. We write the filtration induced from $\pi_{k}, \pi_{k}$ as $F_{p}$ (Definition 6.3), and write the spectral sequences of $\left(C^{*}\left(\mathscr{A}_{k} \times D^{\prime}, D\right), F_{p}\right)$ and $\left(C^{*}\left(\mathscr{A}_{k} \times D^{\prime}, D\right), F_{p}\right)$ as $E_{r, k}^{p, *-p}$ and $E_{r, k}^{p, *-p}$. Then Proposition 6.2 and (6.7) $)_{6}$ insure that

$(6.7)_{7} \quad E_{2, k}^{q, 0} \cong H^{q}\left(\mathscr{B}_{k} \times\left(\mathbb{C} \times D^{\prime}\right), \mathfrak{D}\right), E_{2, k}^{q, 0} \cong H^{q}\left(\mathscr{B}_{k} \times\left(D^{\prime \prime} \times D^{\prime}\right), \mathfrak{D}\right)$, with $D^{\prime \prime}:$ $=\operatorname{supp} \mathscr{A}_{k}^{\prime}(\subset C)$

Thus the $E_{2}^{q, 0}$-terms for $\mathscr{A}_{k}\left(\boldsymbol{C}^{n}\right), \mathscr{A}_{k}(U)$ (defined for $\boldsymbol{C}^{n}$ ) are isomorphic to the cohomology groups in the right sides, which are defined for $C^{n-1}$. We use a p.g. version of $(6.7)_{7}$ for our inductive proof of Theorem $6.1^{n}$ on $n=\operatorname{dim} C^{n}$ (cf. §6.3).

(ii) Next let the p.g. elementary covering $\mathscr{A}_{\xi}^{\prime}=\mathscr{A}_{\xi}^{\prime}\left(\boldsymbol{C}^{n} \times U^{\prime}\right)$ be as in Theorem 6.2, and let the map $\pi_{\xi}$ be as in $(6.3)_{5}$ :

$$
\pi_{\xi}: \mathscr{A}_{\xi}^{\prime} \longrightarrow \mathbb{Z}^{2 n}
$$

Then we easily see that the $u$-quotient of $\pi_{\xi}$ is written as follows:

$$
\left(\mathscr{A}_{\xi}^{\prime} / \pi_{\xi}\right)_{u}=\left\{\operatorname{supp} \mathscr{A}_{\xi}^{\prime}\left(S_{I \varepsilon} \times U^{\prime}\right) ; I \in \mathbb{Z}^{2 n}\right\},
$$

where $S_{I \varepsilon} \in \operatorname{Ouv}_{\mathrm{el}}\left(\mathbb{C}^{n}\right)$ and the elementary covering $\mathscr{A}_{\xi}^{\prime}\left(S_{I \varepsilon} \times U^{\prime}\right)$ are as in $(6.3)_{1}$. Thus, the $E_{2}$-term of the spectral sequence of $\left(C^{*}\left(\mathscr{A}_{\xi}^{\prime}, \mathfrak{D}\right), F_{p}\right)$, where the filtration $F_{p}$ is defined by $\pi_{\xi}$, is as follows (cf. $(6.7)_{3}$ ).

$$
E_{2}^{q, 0} \cong H^{q}\left(\left(\mathscr{A}_{\xi}^{\prime} / \pi_{\xi}\right)_{u}, \mathfrak{D}\right) \text {. }
$$

Concerning the right side, let $\mathscr{A}_{1 \varepsilon}=\mathscr{A}_{1 \varepsilon}\left(\mathbb{C}^{n}\right)$ be the elementary covering of $\boldsymbol{C}^{n}$ of size $(1 ; \varepsilon)$ (cf. Def. 6.2). We then easily check the existence of a unique refining map $i: \mathscr{A}_{1 \varepsilon} \times U^{\prime} \hookrightarrow\left(\mathscr{A}_{\xi}^{\prime} / \pi_{\xi}\right)_{u}$. Also taking an elementary covering $\mathscr{A}_{k}:=\mathscr{A}_{k}\left(\boldsymbol{C}^{n}\right), k>0$, of $\boldsymbol{C}^{n}$ and a refining map $u: \mathscr{A}_{k} \times U^{\prime} \hookrightarrow \mathscr{A}_{1 \varepsilon} \times U^{\prime}$ in an arbitrary manner, we have:

Proposition 6.4'. We have refining maps $s, v$ satisfying Figure I. (In Figure I, the map $r$ is defined as in Proposition 6.2 and the p.g. elementary covering $\mathscr{A}_{\xi^{\prime}}^{\prime}\left(=\mathscr{A}_{\xi^{\prime}}^{\prime}\left(\boldsymbol{C}^{n} \times U_{1 / 2}^{\prime}\right)\right)$ is as in Theorem 6.2.)

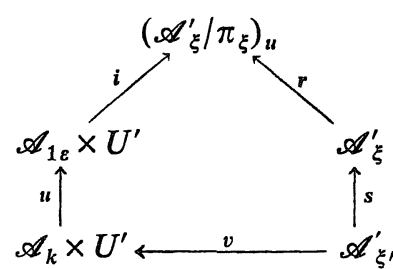

Figure I.

We check Proposition 6.4' in Part B, Appendix II. We note here that, letting the natural homomorphism $\rho^{*}: E_{2}^{q, 0} \rightarrow$ 
$H^{q}\left(\mathscr{A}_{\xi}^{\prime}, D\right)$ be as in Proposition 6.2, we have the following diagram from Porposition 6.4.

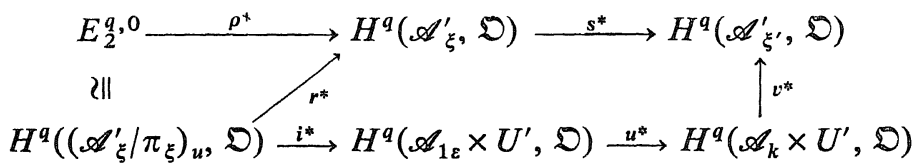

Roughly we may 'identify' $E_{2}^{q, 0} \cong H^{q}\left(\left(\mathscr{A}_{\xi}^{\prime} / \pi_{\xi}\right)_{u}, \mathfrak{D}\right)$ with $H^{q}\left(\mathscr{A}_{1 \varepsilon} \times U^{\prime}, \mathfrak{D}\right)$.

Proposition 6.4. We have the following implication:

$$
u^{*} H^{q}\left(\mathscr{A}_{1 \varepsilon} \times U^{\prime}, \mathfrak{D}\right) \cong 0 \Longrightarrow s^{*} \rho^{*} E_{2}^{q, 0} \cong 0 .
$$

A p.g. version of Proposition 6.4 will be a key fact for the reduction of Theorem 6.2 to Theorem 6.1 (cf. $\S 6.3$ and the end of $\S 6.1$ ).

3. Admissible family. Let the element $\mathscr{A} \in \operatorname{Cov}_{0}(X)$ be as in n.1. We attach to $\mathscr{A}$ a family of elements of $2^{\mathscr{A}}$ (i.e., a family of subsets of $\mathscr{A}$ ), which is important for our treatments of the filtrations in Definition 6.3. For this we first set $(p, q \geqq 1)$ :

$$
\left\{\begin{array}{l}
\mathscr{A}^{(q)}:=\left\{\mathscr{A}_{v} \subset \mathscr{A} ;|\mathscr{A}| \neq \phi, \# \mathscr{A}_{v}=q\right\}, \mathscr{A}_{p}^{(q)}:=\left\{\mathscr{A}_{v} \in \mathscr{A}^{(q)} ; \# \pi \mathscr{A}_{v}=p\right\}, \\
\mathscr{A}_{p}^{\infty}:=\coprod_{q=1} \mathscr{A}_{p}^{(q)} .
\end{array}\right.
$$

Recalling the explicit form of the filtration in Definition 6.3, we may regard $\mathscr{A}_{p}^{\infty}$ as un underlying set theoretical datum for the filtration. Analysys of $\mathscr{A}_{p}^{\infty}$ will be useful for studies of the filtrations. (See Part A, §7.) Here we make an observation for $\mathscr{A}_{p}^{\infty}$ : take a subset $\mathscr{F}_{p} \mathscr{A}$ of $2^{\mathscr{A}}$ satisfying

$$
\# \pi\left(\mathscr{A}_{\mu}\right)=p \text { for each } \mathscr{A}_{\mu} \in \mathscr{F}_{p} \mathscr{A} \text {. }
$$

Definition 6.4. We say that $\mathscr{F}_{p} \mathscr{A}$ is admissible, if there is a map (admissible map) $\tau: \mathscr{A}_{p}^{\infty} \rightarrow \mathscr{F}_{p} \mathscr{A}$ satisfying

$(6.8)_{1}^{\prime \prime} \quad \tau\left(\mathscr{A}_{v}\right) \supset \mathscr{A}_{v}$ for each $\mathscr{A}_{v} \in \mathscr{A}_{p}^{\infty}$, and $\tau\left(\mathscr{A}_{\mu}\right)=\tau\left(\mathscr{A}_{v}\right)$, if $\pi\left(\mathscr{A}_{\mu}\right)=\pi\left(\mathscr{A}_{v}\right)$.

Take an admissible family $\mathscr{F}_{p+1} \mathscr{A}$. We write the restriction of $\pi$ to $\mathscr{A}_{\mu} \in \mathscr{F}_{p+1} \mathscr{A}$ as $\pi_{\mu}$. Using the symbol $F_{p}$ also for the filtration induced from $\pi_{\mu}: \mathscr{A}_{\mu} \rightarrow \pi\left(\mathscr{A}_{\mu}\right)$, we write the spectal sequence of $\left(C^{*}\left(\mathscr{A}_{\mu}, \mathfrak{R}\right), F_{p}\right)$ as $E_{r \mu}^{p, *-p}$. The following proposition insures that the vanishing property of each $E_{1 \mu}^{p,-p}$; $\mathscr{A}_{\mu} \in \mathscr{F}_{p+1} \mathscr{A}$ suffices for that of $E_{1}^{p, *-p}$ of the original complex $\left(C^{*}(\mathscr{A}, \mathfrak{R}), F_{p}\right)$.

Proposition 6.5. $E_{1, \mu}^{p, q-p} \cong 0$ for each $\mathscr{A}_{\mu} \in \mathscr{F}_{p+1} \mathscr{A} \Rightarrow E_{1}^{p, q-p} \cong 0$.

Proof. Take an element $\varphi \in Z_{1}^{p, q-p}:=\left\{\varphi \in F_{p} C^{q}(\mathscr{A}, \mathfrak{\Re}) ; \delta \varphi \in F_{p+1} C^{q+1}(\mathscr{A}, \mathfrak{R})\right\}$. 
Then $i_{\mu}^{*} \varphi \in Z^{q}\left(\mathscr{A}_{\mu}, \mathfrak{\Omega}\right)$ for each $\mathscr{A}_{\mu} \in \mathscr{F}_{p+1} \mathscr{A}$, where $i_{\mu}:=$ inclusion: $\mathscr{A}_{\mu} \hookrightarrow \mathscr{A}$. Remarking that $F_{\tilde{p}+1} C^{q}\left(\mathscr{A}_{\mu}, \Re\right) \cong 0(\tilde{p} \geqq p)$ and $E_{1 \mu}^{p, q-p} \cong H^{q}\left(F_{p} C^{*}\left(\mathscr{A}_{\mu}, \Re\right)\right)$, we have an element $\varphi_{\mu}^{\prime} \in F_{p} C^{q-1}\left(\mathscr{A}_{\mu}, \Re\right)$ satisfying $\delta \varphi_{\mu}^{\prime}=i_{\mu}^{*} \varphi$. We define an element $\varphi^{\prime} \in F_{p+1} C^{q-1}(\mathscr{A}, \Omega)$ by

$$
\varphi_{\mathscr{Q}^{\prime}}^{\prime}:=\left\{\begin{array}{l}
0, \quad \text { if } \mathscr{A}^{\prime} \in \mathscr{N}^{q} \mathscr{A} \text { satisfies: } \# \pi\left(\mathscr{A}^{\prime}\right) \neq p+1 \\
\text { value of } \varphi_{\mu}^{\prime} \text { at } \mathscr{A}^{\prime}, \quad \text { if } \# \pi\left(\mathscr{A}^{\prime}\right)=p+1, \text { where the index } \mu \\
\quad \text { is defined by } \mathscr{A}_{\mu}=\tau\left(\mathscr{A}^{\prime}\right), \text { with the admissible map } \tau .
\end{array}\right.
$$

By $(6.8)_{1}^{\prime}$ we easily have $\varphi=\delta \varphi^{\prime} \in F_{p+1} C^{q}$.

q.e.d.

Remark 6.3. Detailed uses of the admissible family $\mathscr{F}_{p} \mathscr{A}$ will be given in Part A, Section 7. Here, for convenience of the arguments in the rest of Section 6, we illustrate uses of such a family in our studies of the filtration of the form in Definition 6.3: first note that Proposition 6.5 is given for a fixed $p \in \mathbb{Z}^{+}$. In later arguments we consider a series $\left\{\mathscr{F}_{p} \mathscr{A}_{p \in Z^{+}}\right.$of admissible families (cf. A, §7). We then define a set theoretical operation: $\mathscr{F}_{p+1} \mathscr{A} \rightarrow \mathscr{F}_{p} \mathscr{A}$ and a homomorphism from complexes defined for elements of $\mathscr{F}_{p+1} \mathscr{A}$ to those defined for elements of $\mathscr{F}_{p} \mathscr{A}$. (The above two operations will be our main parts of treatments of the filtrations in Definition 6.3; see Parts A and B, §7). Using such inductive treatmients of the families $\mathscr{F}_{p} \mathscr{A}$, we will have the implication of the following form:

$(6.8)_{2} \quad E_{1, \mu}^{0, q} \cong 0$ for each $\mathscr{A}_{\mu} \in \mathscr{F}_{1} \mathscr{A} \Longrightarrow E_{1, \mu}^{p, q-p} \cong 0(q>p)$ for each $\mathscr{A}_{\mu} \in$ $\mathscr{F}_{p+1} \mathscr{A}(p \geqq 1)$.

This, together with Proposition 6.5, insures:

$$
E_{1, \mu}^{0, q} \cong 0 ; \mathscr{A}_{\mu} \in \mathscr{F}_{1} \mathscr{A} \Longrightarrow E_{1}^{p, q-p} \cong 0(q>p) .
$$

(For the precise formulation, see Lemma 7.3 and Lemma 7.5. For technical reasons and for purposes of applications to our p.g. estimations, Lemma 7.3 and Lemma 7.5 are given slicely different form from (6.8) $)_{3}$.) Lemma 7.3 and Lemma 7.5 will be the sharpening of the degeneracy theorem mentioned in the introduction of Chapter III and are main tools for the proof of Theorem 6.1 and Theorem 6.2. In the remainder of Section 6, we first attach to the coverings $\mathscr{A}_{k}, \mathscr{A}_{\xi}^{\prime}$ (in Theorem 6.1, Theorem 6.2) admissible families in a concrete manner. We then formulate key lemmas for Theorem 6.1, Theorem 6.2 in terms of such admissible families.

Example 6.1. Let $\mathscr{A}$ be a collection of elementary figures in $\boldsymbol{C}^{n}(z)$ (cf. $\S 6.1$ ) 
and $\pi$ a map from $\mathscr{A}$ to $\mathscr{B}$. We assume:

$$
\# \pi^{-1}(B)<\infty \text { for each } B \in \mathscr{B} .
$$

Setting $\mathscr{B}^{[p]}:=\left\{\mathscr{B}^{\prime} \subset \mathscr{B} ; \# \mathscr{B}^{\prime}=p\right\}$, take an element $\mathscr{B}^{\prime} \in \mathscr{B}^{[p]}$ and an elementary figure $U^{\prime} \subset \mathbb{C}^{n}$. We form a subset $\mathscr{A}\left(\mathscr{B}^{\prime} ; U^{\prime}\right)$ of $\mathscr{A}$ as follows:

$$
\mathscr{A}\left(\mathscr{B}^{\prime} ; U^{\prime}\right):=\left\{A_{\mu} \in \mathscr{A} ; \pi\left(A_{\mu}\right) \in \mathscr{B}^{\prime}, A_{\mu} \cap U \neq \phi\right\} .
$$

We then set:

$(6.8)_{4} \mathscr{F}_{p}^{0} \mathscr{\mathscr { L }}:=\left\{\mathscr{A}_{\mu}^{\prime} \subset \mathscr{A} ; \mathscr{L}_{\mu}^{\prime}\right.$ is of the form $\left.\mathscr{A}_{\mu}^{\prime}=\mathscr{A}\left(\mathscr{B}^{\prime} ; U\right)\right\}$, where the pair $\left(\mathscr{B}^{\prime}, U\right)$ satisfies: $\left|\left(\pi^{-1}\left(\mathscr{B}^{\prime}\right) / \pi\right)_{u}\right| \supset U$ (cf. $\left.(6.7)_{2}\right)$.

Noting that

$(6.8)_{5}\left|\left(\pi^{-1}\left(\mathscr{B}^{\prime}\right) / \pi\right)_{u}\right|=U \mathscr{A}^{\prime \prime}\left|\mathscr{A}^{\prime \prime}\right|$, where $\mathscr{A}^{\prime \prime}$ is an element of $\mathscr{A}_{p}^{\infty}$ satisfying $\pi\left(\mathscr{A}^{\prime \prime}\right)=\mathscr{B}^{\prime}$.

we see easily that $\mathscr{F}_{p}^{0} \mathscr{A}$ satisfies $(6.8)_{1}^{\prime}$. Moreover, we have:

Proposition 6.5'. Assume that

$(6.8)_{6} \operatorname{supp} \pi^{-1}(B)$ is an elementary figure for each $B \in \mathscr{B}$. Then $\mathscr{F}_{p}^{0} \mathscr{A}$ is admissible.

Proof. We define an admissible map $\tau$ by

$(6.8)_{7} \quad \tau: \mathscr{A}_{p}^{\infty} \ni \mathscr{A}_{\mu} \longrightarrow \mathscr{F}_{p}^{0} \mathscr{A} \ni \mathscr{A}_{v}:=\mathscr{A}\left(\mathscr{B}^{\prime} ; U^{\prime}\right)$, with $\mathscr{B}^{\prime}:=\pi\left(\mathscr{A}_{\mu}\right)$ and $U^{\prime}:$ $\left.=\mid \pi^{-1}\left(\mathscr{B}^{\prime}\right) / \pi\right)_{u} \mid$.

(By $(6.8)_{6}, U^{\prime}$ is an elementary figure, and we have: $\mathscr{A}_{v} \in \mathscr{F}_{p}^{0} \mathscr{A}$.) The right side is determined by $\mathscr{B}^{\prime}=\pi\left(\mathscr{A}_{\mu}\right)$, and we have $(6.8)_{1}^{\prime \prime}$.

The family $\mathscr{F}_{p}^{0} \mathscr{A}$ is completely of experimental nature and is concordant to the inductive treatments on $p \in \mathbb{Z}^{+}$sketched soon above. (For detailed uses of $\mathscr{F}_{p}^{0} \mathscr{A}$, see n.6, A, §7.) We examine the structure of $\mathscr{F}_{1}^{0} \mathscr{A}$, which is most important for later applications. For this we set:

$(6.8)_{8} \operatorname{Cov}_{0}\left(\mathbb{C}^{n}\right)_{\mathrm{cl}}:=\left\{\mathscr{A}_{k}(U) ;(U ; \mathscr{L}) \in \operatorname{Ouv}_{\mathrm{el}}\left(\mathbb{C}^{n}\right) \times\left(\mathbb{Z}^{+} \cup 0\right)^{2 n}\right.$, where $\mathscr{A}_{k}(U)$ is the elementary covering of $U$ of size $k$ (Def. 6.1) $\}$.

We assume that, for each $B \in \mathscr{B}$, we have:

$$
\pi^{-1}(B) \in \operatorname{Cov}_{0}\left(\mathbb{C}^{n}\right)_{\mathrm{el}} .
$$

Then setting 


$$
\mathscr{F}_{1}^{0} \mathscr{A}_{\mid B}:=\left\{\mathscr{A}_{\mu} \in \mathscr{F}_{1}^{0} \mathscr{A} ; \pi\left(\mathscr{A}_{\mu}\right)=B\right\},
$$

we easily have the following.

Proposition 6.5". $\mathscr{F}_{1}^{0} \mathscr{A}_{\mid B}=\left\{\mathscr{A}_{\mu} \in \operatorname{Cov}_{0}\left(\mathbb{C}^{n}\right)_{\mathrm{e} 1} ;\right.$ where each element (=elementary figure) of $\mathscr{A}_{\mu}$ is contained in $\left.\pi^{-1}(B)\right\}$. Roughly, $\mathscr{F}_{1}^{0} \mathscr{A}_{B}$ is the collection of all elementary coverings of elementary figures, which are constructed from $\pi^{-1}(B) \in \operatorname{Cov}_{0}\left(C^{n}\right)_{\mathrm{e} 1}$.

(An interested reader may picture $\mathscr{F}_{1}^{0} \mathscr{A}_{\mid B}$ and will find that $\mathscr{F}_{1}^{0} \mathscr{A}_{\mid B}$ has a simple geometric meaning for $\pi^{-1}(B)$.) Let the p.g. elementary covering $\mathscr{A}_{\xi}^{\prime}$ $=\mathscr{A}_{\xi}^{\prime}\left(\boldsymbol{C}^{n} \times U^{\prime}\right)$ be as in Theorem 6.2 and the map $\pi_{\xi}: \mathscr{A}_{\xi}^{\prime} \rightarrow \mathscr{B}\left(=\boldsymbol{Z}^{2 n}\right)$ be as in $(6.3)_{5}$. We see easily that the map $\pi_{\xi}: \mathscr{A}_{\xi}^{\prime} \rightarrow \mathscr{B}$ satisfies (6.8) ${ }_{9}^{\prime}$. One can apply Proposition 6.5 to $\mathscr{A}_{\xi}^{\prime}$. We use the simple structure of $\mathscr{F}_{1}^{0} \mathscr{A}_{B B}$ in the proof of Theorem 6.2 (cf. §6.3).

Example 6.2. Let the elementary covering $\mathscr{A}_{k}:=\mathscr{A}_{k}\left(\boldsymbol{C}^{n}\right)$ be as in Theorem 6.1. Note that*) $\mathscr{A}_{k}$ does not satisfy the finiteness condition $(6.8)_{4}^{\prime}$. Here we attach to $\mathscr{A}_{k}$ an admissible series, which is useful for studies of $\mathscr{A}_{k}$. For this letting $\mathscr{B}_{k}^{(p)}:=\left\{\mathscr{B} \subset \mathscr{B}_{k} ; \# \mathscr{B}=p,|\mathscr{B}| \neq \phi\right\}$, we set:

$$
\mathscr{F}_{p}^{\prime 0} \mathscr{A}_{k}:=\left\{\pi_{k}^{-1}\left(\mathscr{B}^{\prime}\right) ; \mathscr{B}^{\prime} \in \mathscr{B}_{k}^{(p)}\right\} .
$$

We easily see that the map

$$
\tau:\left(\mathscr{A}_{k}\right)_{p}^{\infty} \ni \mathscr{A}^{\prime} \longrightarrow \mathscr{F}_{p}^{\prime 0} \mathscr{A}_{k} \ni \pi_{k}^{-1}\left(\pi_{k}\left(\mathscr{A}^{\prime}\right)\right) \text { is admissible. }
$$

We use the above admissible family in the proof of Theorem 6.1 (cf. §6.3). Note that $\pi_{k}^{-1}\left(\mathscr{B}^{\prime}\right)$ is written also as $\mathscr{A}_{k}(\boldsymbol{C}) \times\left|\mathscr{B}^{\prime}\right|(\mathrm{cf} . \mathrm{n.2}, \S 6.2)$ and that $\left.\mid \pi_{k}^{-1}\left(\mathscr{B}^{\prime}\right) / \pi_{k}\right)_{u}|=C \times| \mathscr{B}^{\prime} \mid$. Thus we have:

$$
\pi_{k}^{-1}\left(\mathscr{B}^{\prime}\right)=\mathscr{A}\left(\mathscr{B}^{\prime} ; U\right) \text {, with } U=C \times\left|\mathscr{B}^{\prime}\right| .
$$

This shows that our family $F_{p}^{\prime 0} \mathscr{A}_{k}$ has a similarity to the one in Example 6.1.

\section{§6.3. Main Lemmas}

Here we give our main lemmas for Theorems 6.1, 6.2 (cf. Lemmas 6.1, 6.2). These lemmas will be an application to Theorems $6.1,6.2$ of our sharpening of the degeneracy theorem (cf. also the introduction of Chapter III). The sharpening in general forms will be given in Section 7 (cf. Lemmas 7.3-7.5). We also

*) We understand that the map $\pi_{k}: \mathscr{A}_{k} \rightarrow \mathscr{B}_{k}$ is defined in the manner $(6.7)_{5}$. 
give some facts, which fill the gaps between Lemmas 6.1, 6.2 and Theorems $6.1,6.2$.

1. Sets of cochains. We should begin Section 6.3 by arranging certain sets of cochains, which we use in Lemmas 6.1, 6.2: first let $\mathscr{A}$ be a collection consisting of (1) elementary figures $A_{\mu}$ in $\mathbb{C}^{n} \times \mathbb{C}^{n^{\prime}}$ or (2) geometric figures of the form $A_{\mu}^{\prime} \times D^{\prime}$ with an elementary figure $A_{\mu}^{\prime} \subset \mathbb{C}^{n}$ and a (fixed) open set $D^{\prime} \subset \mathbb{C}^{n^{\prime}}$. Also take a map $\pi: \mathscr{A} \rightarrow \mathscr{B}$, where $\mathscr{B}$ is an (abstract) set. We use the symbol $F_{p}$ for the filtration induced from $\pi$ (Def. 6.3). First, concordantly the notation in spectral sequence theory, we set*):

$(6.10)_{1}\left\{\begin{array}{l}Z_{1}^{p, q-p}(\mathscr{A}, \mathfrak{D}) \\ B_{0}^{p, q-p}(\mathscr{A}, \mathfrak{D}) \\ Z_{\infty}^{p, q-p}(\mathscr{A}, \mathfrak{D})\end{array}\right\}:=\left\{\begin{array}{l}\left\{\varphi \in F_{p} C^{q}(\mathscr{A}, \mathfrak{D}) ; \delta \varphi \in F_{p+1} C^{q+1}(\mathscr{A}, \mathscr{D})\right\} \\ \delta F_{p} C^{q-1}(\mathscr{A}, \mathfrak{D}) \\ \left\{\varphi \in Z_{1}^{p, q-p}(\mathscr{A}, \mathfrak{D}) ; \delta \varphi=0\right\}\end{array}\right\}$.

We use the symbol $i$ for the injection: $Z_{\infty}^{p, q-p}(\mathscr{A}, \mathfrak{D}) \hookrightarrow Z^{q}(\mathscr{A}, \mathfrak{D})$. Next, for an element $\alpha \in \boldsymbol{R}_{1}^{+2}$ we define:

$(6.10)_{1}^{\prime}\left\{\begin{array}{l}Z_{c}^{p, q-p}(\mathscr{A}, \mathfrak{D})_{\alpha} \\ B_{0}^{p, q-p}(\mathscr{A}, \mathfrak{D})_{\alpha}\end{array}\right\}=\left\{\begin{array}{l}Z_{c}^{p, q-p}(\mathscr{A}, \mathfrak{D}) \cap C^{q}(\mathscr{A}, \mathfrak{D})_{\alpha}, \text { where } c=1 \text { or } \infty \\ \delta F_{p} C^{q-1}(\mathscr{A}, \mathfrak{D})_{\alpha},\end{array}\right.$

where

$(6.10)_{1}^{\prime \prime} C^{q}(\mathscr{A}, \mathfrak{D})_{\alpha}:=$ set of all $(|\tilde{z}|+1)$ - $\alpha$-growth cochains in $C^{q}(\mathscr{A}, \mathfrak{D})$.

Thirdly, assuming that $\mathscr{A}$ is of the form in (2), we write $\mathscr{A}$ as $\mathscr{A}^{\prime} \times D^{\prime}$, with a collection $\mathscr{A}^{\prime}$ of elementary figures in $\mathbb{C}^{n}$. Denoting by $\tilde{\mathscr{A}}$ one of $\mathscr{A}$ (as in (1)) or $\mathscr{A}^{\prime}$, we set:

$$
\tilde{\mathscr{A}_{m}}:=\left\{A_{\mu m} ; A_{\mu} \in \tilde{\mathscr{A}}\right\}\left(m \in \mathbb{R}^{+}\right) \quad\left(\mathrm{cf} .(6.4)_{1}^{\prime}\right) .
$$

We define:

$(6.10)_{2}^{\prime}\left\{\begin{array}{l}C^{q}(\mathscr{A}, \mathfrak{D})_{\alpha}^{m} \\ C^{q}\left(\mathscr{A}^{\prime} \times D^{\prime}, \mathfrak{D}\right)_{\alpha}^{m}\end{array}\right\}:=i_{m}\left\{\begin{array}{l}C^{q}\left(\mathscr{A}_{m}, \mathfrak{D}\right)_{\alpha} \\ C^{q}\left(\mathscr{A}_{m}^{\prime} \times D^{\prime}, \mathfrak{D}\right)_{\alpha}\end{array}\right\}$,

$$
\left\{\begin{array}{l}
\text { where } i_{m}:=\text { refining map: } \\
\mathscr{A} \ni A_{\mu} \longrightarrow \mathscr{A}_{m} \ni A_{\mu m} \text { or } \mathscr{A}^{\prime} \times D^{\prime} \ni A^{\prime} \times D^{\prime} \longrightarrow \mathscr{A}_{m}^{\prime} \times D^{\prime} \ni A_{\mu m}^{\prime} \times D^{\prime} .
\end{array}\right.
$$

(We define $C^{q}(\mathscr{A}, \mathfrak{D})^{m}, C^{q}\left(\mathscr{A}^{\prime} \times D^{\prime}, \mathfrak{D}\right)^{m}$ by dropping the term $\alpha$ from $(6.10)_{2}$.)

Then letting $\mathscr{A}$ be one of the form in (1) or (2), we define:

$(6.10)_{2} \quad\left\{\begin{array}{l}Z_{c}^{p, q}\left(\mathscr{A}, \mathfrak{D}_{\alpha}^{m}\right):=Z_{c}^{p, q-p}(A, \mathfrak{D}) \cap C^{q}(\mathscr{A}, \mathfrak{D})_{\alpha}^{m}(c=1 \text { or } \infty) \\ B_{0}^{p, q-p}(\mathscr{A}, \mathfrak{D})_{\alpha}^{m}:=\delta F_{p} C^{q-1}(\mathscr{A}, \mathfrak{D})_{\alpha}^{m} .\end{array}\right.$

We define $Z_{c}^{p, q-p}(\mathscr{A}, \mathfrak{D})^{m}$ and $B_{0}^{p, q-p}(\mathscr{A}, \mathfrak{D})^{m}$ by dropping $\alpha$ from $(6.10)_{2}$.

*) As hitherto, the symbol $\mathbb{D}$ denotes the structure sheaf of $\mathbb{C}^{n} \times \mathbb{C}^{n \prime}$. 
(Such sets will be useful, when we are concerned with refining maps of the form in Theorems $6.1,6.2, \ldots$ ) Finally, for a collection $\mathscr{A}$ of elementary figures in $\boldsymbol{C}^{n}$, take elements $A_{\mu}, A_{v} \in \mathscr{A}$. We write them as $A_{\mu}=\prod_{j=1}^{2 n}\left(a_{j \mu}, a_{j \mu}+b_{j \mu}\right)\left(b_{j \mu}>0\right)$ $\subset \boldsymbol{C}^{n}\left(\cong \boldsymbol{R}^{2 n}\right), \ldots$ We set $a_{\mu \nu}:=\max _{j} b_{j \mu} / b_{j v}$, and we define ratio number of $\mathscr{A}$ by

$$
a_{\mathscr{A}}:=\sup a_{\mu v}, \text { where } A_{\mu}, A_{v} \in \mathscr{A} \text { satisfy: } A_{\mu} \cap A_{v} \neq \phi .
$$

(This number will play a role in our treatments of p.g. properties of elementary figures.) Letting $\mathscr{A}=\mathscr{A}_{k}(U)$ or $=\mathscr{A}_{k}\left(\boldsymbol{C}^{n}\right)$ be as in Definition $6.1_{1,2}$, we easily have:

$$
a_{\mathscr{A}}=1 \text {. }
$$

For the p.g. elementary covering $\mathscr{A}_{\xi}^{\prime}:=\mathscr{A}_{\zeta \varepsilon}^{\prime}\left(\boldsymbol{C}^{n} \times U^{\prime}\right), \zeta=\left(\zeta_{1}, \zeta_{2}\right) \in \mathbb{Z}^{+2}$, as in Theorem 6.2, we easily have:

$(6.10)_{5} \quad a_{\mathscr{x}_{\xi}^{\prime}} \leqq c_{\tilde{n}}^{\zeta_{2}^{2}}$, with an element $c_{\tilde{n}} \in \boldsymbol{R}_{1}^{+}$which depends only on $\tilde{n}=\left(n, n^{\prime}\right)$. We use $(6.10)_{4,5}$ in later arguments.

2. Main lemmas. Here we give main lemmas for Theorem 6.1 and Theorem 6.2. The data for such lemmas are as follows:

$(6.11)_{0} \quad$ refining map $s:\left\{\begin{array}{r}\mathscr{A}_{k^{\prime}} \times D^{\prime} \hookrightarrow \mathscr{A}_{k} \times D^{\prime}, \text { where } \mathscr{A}_{k^{\prime}}:=\mathscr{A}_{k^{\prime}}\left(\boldsymbol{C}^{n}\right), \ldots \\ \mathscr{A}_{\xi^{\prime}}^{\prime}:=\mathscr{A}_{\xi^{\prime}}^{\prime}\left(\boldsymbol{C}^{n} \times U^{\prime}\right) \hookrightarrow \mathscr{A}_{\xi}^{\prime}\left(\boldsymbol{C}^{n} \times U^{\prime}\right), \text { where } \xi= \\ (\zeta ; \varepsilon), \xi^{\prime}=\left(\zeta^{\prime} ; \varepsilon\right) \text { are in } \boldsymbol{Z}^{+2} \times(0,1 / 2) .\end{array}\right.$

(The data as above have similar meanings to the data in Theorem 6.1 and Theorem 6.2).

According as we are concerned with Theorem 6.1 or Theorem 6.2 , the admissible families:

$$
\mathscr{F}_{1}^{\prime 0} \mathscr{A}_{k^{\prime}}\left(=\mathscr{F}_{1}^{\prime 0} \mathscr{A}_{k^{\prime}}\left(\boldsymbol{C}^{n}\right)\right) \text { and } \mathscr{F}_{1}^{0} \mathscr{A}_{\xi}^{\prime}\left(=\mathscr{F}_{1}^{0} \mathscr{A}_{\xi}^{\prime}\left(\mathbb{C}^{n} \times U^{\prime}\right)\right)
$$

will play the basic roles (cf. Examples 6.1 and 6.2, §6.2). For each $\mathscr{A}_{\mu} \in \mathscr{F}_{1}^{\prime 0} \mathscr{A}_{k^{\prime}}$ and $\mathscr{A}_{\mu}^{\prime} \in \mathscr{F}_{1}^{0} \mathscr{A}_{\xi}^{\prime}$ we set:

$(6.11)_{0}^{\prime \prime} \quad s_{\mu}:=$ restriction of $s$ to $\mathscr{A}_{\mu}, \mathscr{A}_{\mu}^{\prime}$, and $\pi_{k \mu}, \pi_{\xi_{\mu}}$ are the restrictions of the maps $\pi_{k}, \pi_{\xi}$ to $\mathscr{A}_{\mu}, \mathscr{A}_{\mu}^{\prime}$. (For the maps $\pi_{k}, \pi_{\xi}$ see $(6.7)_{5}$ and $\left.(6.3)_{5}.\right)$ )

When we are concerned with the original $\mathscr{A}_{k^{\prime}}, \mathscr{A}_{\xi}^{\prime}$, we use the filtrations $F_{p}$ induced from $\pi_{k^{\prime}}, \pi_{\zeta}$ (cf. Def. 6.3 and n.2, §6.2). When we are concerned with $\mathscr{A}_{\mu}, \mathscr{A}_{\mu}^{\prime}$, the filtrations $F_{p}$ will be the ones induced from $\pi_{k \mu}, \pi_{\xi \mu}$. Using such filtrations, the sets of the cochains in Lemmas $6.1,6.2$ soon below will 
be of the form in $(6.10)_{1-3}$, which are specified to $\mathscr{A}_{h}, \mathscr{A}_{\xi}^{\prime}$ and $\mathscr{A}_{\mu}, \mathscr{A}_{\xi}^{\prime}$.

Lemma 6.1" (Sharpening of degeneracy theorem for Theorem 6.1). Assume the following for each $\mathscr{A}_{\mu} \in \mathscr{F}_{1}^{\prime 0} \mathscr{A}_{l^{\prime}}$ :

$(6.11)_{1}^{0, q} s_{\mu}^{*} Z_{1}^{0, q}\left(s_{\mu}\left(\mathscr{A}_{\mu}\right), \mathfrak{D}\right)_{\alpha} \subset B_{0}^{0, q}\left(\mathscr{A}_{\mu}, \mathfrak{D}\right)_{\alpha^{\prime}}$, where the estimation $\alpha^{\prime}=L(\alpha)$ is as in Theorem 6.1 .

Then we have a map $\tau^{*}: s^{*} Z^{q}\left(\mathscr{A}_{k}, \mathfrak{D}\right) \rightarrow Z_{\infty}^{q, \cap}\left(\mathscr{A}_{k^{\prime}}, \mathfrak{D}\right)$ satisfying

(6.11) $)_{1}^{q}\left(1-i^{*} \tau^{*}\right) s^{*} Z^{q}\left(\mathscr{O}_{k}, \mathfrak{D}\right)_{\alpha} \subset \delta C^{q-1}\left(\mathscr{C}_{k}, \mathfrak{D}\right)_{\alpha^{\prime}}$, with the inclusion $i^{*} Z_{\infty}^{q, 0} \hookrightarrow Z^{q}$ (cf. $\left.(6.10)_{1}\right)$.

Lemma 6.2 ${ }^{n}$ (Sharpening of degeneracy thenrem for Theorem 6.2). Assume the following for each $\mathscr{A}_{\mu}^{\prime} \in \mathscr{F}_{1}^{0} \mathscr{A}_{\xi}^{\prime}$ :

$(6.11)_{2}^{0, q} Z_{1}^{0, q}\left(\mathscr{A}_{\mu}^{\prime}, \mathfrak{D}\right)_{\alpha}^{m} \subset B_{0}^{0, q}\left(\mathscr{A}_{\mu}^{\prime}, \mathfrak{D}\right)_{\alpha^{\prime}}^{m^{\prime}}(q \geqq 1)$, where the estimation: $\alpha \rightarrow \alpha^{\prime}$ is as in Theorem 6.2. Moreover, the elements $m, m^{\prime} \in \mathbb{R}_{1}^{+}$are of the form: $(6.11)_{2}^{q} m>c_{n} a_{\xi}, m^{\prime}=m /\left(d_{n} a_{\xi}\right)$, with the ratio number $a_{\xi}$ of $\mathscr{A}_{\xi}^{\prime}$ (cf. (6.10) $\left.)_{3}\right)$, and the elements $c_{\tilde{n}}, d_{\tilde{n}} \in \boldsymbol{R}^{+2}$ which depends only on $\tilde{n}:=\left(n, n^{\prime}\right)$.

Then we have a map $\tau^{*}: s^{*} Z^{q}\left(\mathscr{A}_{\xi}^{\prime}, \mathfrak{D}\right)_{\alpha} \rightarrow Z_{\infty}^{q, 0}\left(\mathscr{A}_{\xi^{\prime}}^{\prime}, \mathfrak{D}\right)_{\alpha^{\prime}}$ satisfying

$$
\left(1-i^{*} \tau^{*}\right) s^{*} Z^{q}\left(\mathscr{A}_{\xi}^{\prime}, \mathfrak{D}\right)_{\alpha} \subset \delta C^{q-1}\left(\mathscr{A}_{\xi^{\prime}}^{\prime}, \mathfrak{D}\right)_{\alpha^{\prime}} .
$$

(The integer $n$ in Lemma 6.1 (or 6.2) satisfies: $n \geqq 2$ (or $\geqq 1$ ). Also the integer $q$ satisfies: $q \geqq 1$.)

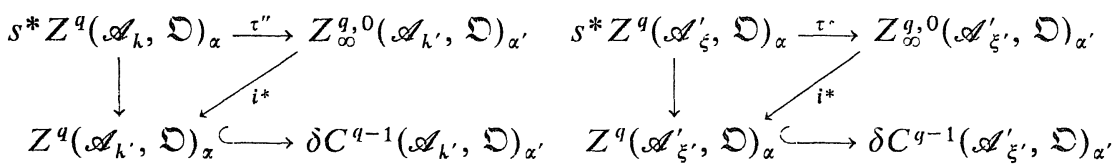

Figure I.

Lemmas 6.1, 6.2 will play the most basic roles in the proof of Theorems 6.1, 6.2, and will be a special case of Lemma 7.5. The latter lemma will be a sharpening of the degeneracy theorem (cf. Introduction) in a more or less general set up (cf. B, §7). Note that the conclusions $(6.11)_{1,2}^{q}$ in Lemmas $6.1,6.2$ are regarded as a p.g. version of the standard consequence of the degeneracy theorem (cf. Proposition 6.3). By this reason, we used the term sharpening of the degeneracy theorem' in Lemmas 6.1, 6.2. The proof of Lemmas 6.1, 6.2 will be given in $\mathrm{B}$, Section 7, after giving some algebraic arguments on the filtration of the type in Definition 6.3. Here we give a consequence of Lemmas 6.1, 6.2.

Proposition 6.6. We have the following implications: 


$$
\left\{\begin{array}{l}
\text { Theorem } 6.1^{n-1} \longrightarrow \text { Theorem } 6.1^{n} \\
\text { Theorem } 6.1^{n} \longrightarrow \text { Theorem } 6.2^{n}
\end{array}\right.
$$

Proof. This is proved by giving a p.g. version of (6.7) $)_{7}$ and Proposition 6.4. First, corresponding to $(6.7)_{7}$ we easily have:

$$
Z_{\infty}^{q, 0}\left(\mathscr{A}_{k}\left(\boldsymbol{C}^{n}\right) \times D^{\prime}, \mathfrak{D}\right)_{\alpha} \cong Z^{q}\left(\mathscr{A}_{k}\left(\boldsymbol{C}^{n-1}\right) \times\left(\boldsymbol{C} \times D^{\prime}\right)\right)_{\alpha^{\prime}} .
$$

Applying Theorem $6.1^{n-1}$ to the right side, we have the first implication. To check the second, we have the diagram corresponding to $(6.7)_{9}$ :

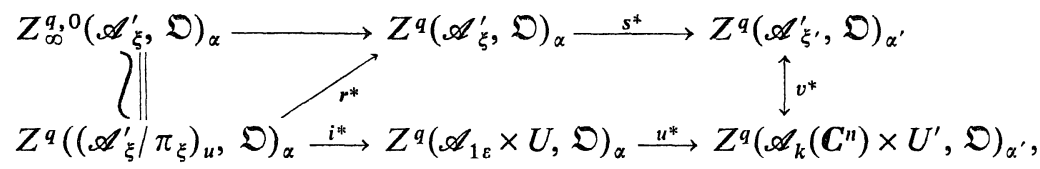

where the p.g. elementary covering $\mathscr{A}_{\xi}^{\prime}:=\mathscr{A}_{\xi}^{\prime}\left(\boldsymbol{C}^{n} \times U^{\prime}\right)$ is as in Theorem 6.2. Also the refining maps in (b) have the similar meanings to (6.7) ${ }_{9}$. Apply Theorem 6.2 to $z^{*} Z^{q}\left(\mathscr{A}_{1 \varepsilon} \times U^{\prime}, \mathfrak{D}\right)_{\alpha}$. Then, from (b) and from the comparison of the explicit forms of the estimations in Theorem 6.1 and Theorem 6.2, we have the second implication.

q.e.d.

By Proposition 6.6 1 , the remaining task for the proof of Theorem 6.1 and Theorem 6.2 is to prove Theorem $6.1^{1}$ and the assumptions $(6.11)_{1 ; 2}^{0, q}$ in Lemmas 6.1, 6.2. The former is proven in Appendix II by giving explicit estimations of Cousin integrals. In $n .3$ soon below, we check $(6.11)_{1}^{0, q}, 2$.

3. Note that the assumption $(6.11)_{2}^{0, q}$ in Lemma 6.2 concerns finite collections $\mathscr{A}_{\mu} \in \mathscr{F}_{1}^{0} \mathscr{A}_{\xi}^{\prime}$ of elementary figures. Here we give a p.g. estimation for finite elementary coverings. For this, letting the collection $\operatorname{Cov}_{0}\left(\boldsymbol{C}^{n}\right)_{\mathrm{e} 1}$ of finite elementary coverings be as in $(6.8)_{8}$, we form a parameter space:

$$
\Lambda^{n}:=\operatorname{Cov}_{0}\left(\boldsymbol{C}^{n}\right)_{\mathrm{e} 1} \times \operatorname{Ouv}\left(\boldsymbol{C}^{n^{\prime}}\right) \times \boldsymbol{R}_{1}^{+2} .
$$

For an element $\left(\mathscr{A}_{\lambda}, D^{\prime}, \alpha\right) \in \Lambda^{n}=\operatorname{Cov}_{0}\left(\boldsymbol{C}^{n}\right)_{\mathrm{e} 1} \times \operatorname{Ouv}\left(\boldsymbol{C}^{n^{\prime}}\right) \times \boldsymbol{R}_{1}^{+2}$, we set:

$(6.13)_{0}^{\prime} \quad C^{q}\left(\mathscr{A}_{\lambda} \times D^{\prime}, \mathfrak{D}\right)_{\alpha}:=$ set of all $(|\tilde{z}|+1)$ - $\alpha$-growth cochains with valuc in $\mathfrak{D}$ (cf. $(1.3)_{6}$ and $\left.(1.4)_{8}\right)$.

Next, for a finite collection $\mathscr{A}$ of elementary figures in $\boldsymbol{C}^{n}$, we set:

$$
l_{\mathscr{A}}:=\# \mathscr{A}, r_{\mathscr{A}}^{+}:=\max (1, \text { diameter*) of } \operatorname{supp} \mathscr{A}) \text {, }
$$

*) Letting $Y$ be a subset of $C^{n}$, we understand that $\operatorname{diam}(Y):=\sup _{P, Q} d(P, Q)$, where $P$, $Q$, are points in $Y$. 
and $r_{\mathscr{A}}^{-}:=\min _{\mu}\left(\min \left(1, d_{A_{\mu}}^{-}\right)\right)$, where $A_{\mu}$ runs through $\mathscr{A}$ (cf. also $\left.(6.3)_{8}\right)$. For each $\mathscr{A}_{\mu} \in \operatorname{Cov}_{0}\left(\mathbb{C}^{n}\right)_{\mathrm{el}}$, we write $l_{\mathscr{A}_{\mu}}, r_{\mathcal{A}_{\mu}}^{ \pm}$as $l_{\mu}, r_{\mu}^{ \pm}$. Such numerical invariants are used in the p.g. estimation just below. Thirdly, for the explicit estimation below, we make:

Definition 6.5 By an elementary p.g. estimation map, we mean a map of the form:

$(6.13)_{0}^{\prime \prime \prime} \quad E: \mathbb{R}^{+2} \times \boldsymbol{R}^{+2} \ni\left(\left(u_{1}, u_{2}\right), \alpha=\left(\alpha_{1}, \alpha_{2}\right)\right), \mathbb{R}^{+2} \ni \alpha^{\prime}:=M_{1}\left(u_{1}\right) \cdot M_{2}\left(u_{2}\right)^{u_{3}}$. $\mathscr{L}(\alpha)$, where $u_{3}=M_{3}\left(\alpha_{2}\right)$ and $M_{i}, \mathscr{L}$ are positive monomials and an el-map $(\mathrm{n} .5, \S 1.1)$.

Now taking elements $c_{n}, d_{n} \in \mathbb{R}_{1}^{+}$and an elementary p.g. map $E$ we have:

Lemma $6.3^{n}$ ( $\left.n \geqq 1\right)$. We have the following inclusion.

(6.13) ${ }_{1} \quad Z^{q}\left(\mathscr{A}_{\lambda} \times D^{\prime}, \mathfrak{D}\right)_{\alpha}^{m} \subset \delta C^{q-1}\left(\mathscr{A}_{\lambda} \times D^{\prime}, \mathfrak{D}\right)_{\alpha^{\prime}}^{m^{\prime}}(q>0)$, with $\alpha^{\prime}=E_{n}\left(l_{\lambda} / r_{\lambda}^{-}, m r_{\lambda}^{+}\right.$, $\alpha)$, where the parameter $\left(\mathscr{A}_{\lambda}, D^{\prime \prime}, \alpha\right)$ is in $\Lambda^{\prime \prime}$ and $m, m^{\prime} \in \mathbb{R}_{1}^{+}$satisfy: $m>c_{n}$ and $m^{\prime}=m / d_{n}$.

As may be clear from the formulation, Lemma 6.3 is a finite version of Theorem $6.1^{n}$. The proof of Lemma 6.3 is accordingly easier than that of Theorem $6.1^{n}$. Here we give a lemma for Lemma $6.3^{n}$, which corresponds to Lemma 6.1 in the proof of Theorem 6.1. For this we assume that $n \geqq 2$. For notational simplification, we set $\boldsymbol{A}^{n}:=\operatorname{Cov}_{0}\left(\boldsymbol{C}^{n}\right)_{\mathrm{el}}$. We write an element $\mathscr{A}_{\lambda} \in \boldsymbol{A}^{n}$ as $\mathscr{A}_{\lambda}=\mathscr{A}_{\lambda}^{\prime} \times \mathscr{B}_{\lambda}$, with $\mathscr{A}_{\lambda}^{\prime} \in \mathscr{A}^{1}, \mathscr{B}_{\lambda} \in \mathscr{A}^{n-1}$, and we define the map $\pi_{\lambda}: \mathscr{A}_{\lambda} \rightarrow \mathscr{B}_{\lambda}$ in the manner in n.2, Section 6.2. We then set:

$$
\mathbb{B}^{n}:=\left\{\mathscr{B}_{\lambda}\right\}_{\lambda}, \pi^{n}:=\left\{\pi_{\lambda}\right\}_{\lambda}, \text { where } \mathscr{A}_{\lambda} \in \mathscr{A}^{n}, \text { and } \tilde{A}^{n}:=\left(\mathscr{A}^{n}, \mathbb{B}^{n}, \pi^{n}\right) .
$$

(Such notation is also used in Part B, §7.) For each $\mathscr{A}_{\lambda} \in \boldsymbol{A}^{n}$, we define a family $\mathscr{F}_{p}^{0} \mathscr{A}_{\lambda} \in 2^{\mathscr{A} \lambda}$ by $(6.8)_{4}$, and we set:

$(6.13)_{2}^{\prime} \mathscr{F}_{p}^{0} \tilde{A}^{n}:=\left\{\mathscr{A}_{\mu} ;\right.$ where $\mathscr{A}_{\mu}$ is in $\mathscr{F}_{p}^{0} \mathscr{A}_{\lambda}$, with an element $\left.\mathscr{A}_{\lambda} \in \mathscr{A}^{n}\right\}$.

For each $\mathscr{A}_{\mu} \in \mathscr{F}_{p}^{0} \tilde{\mathscr{A}}^{n}$ we denote by $\mathscr{A}_{\lambda_{\mu}}$ the element of $\mathscr{A}^{n}$ satisfying $\mathscr{A}_{\mu}$ $\in \mathscr{F}_{p}^{0} \mathscr{A}_{\lambda_{\mu}}$. Then letting $(D, \alpha) \in \operatorname{Ouv}\left(\boldsymbol{C}^{n}\right) \times \mathbb{R}_{1}^{+2}$ be as in Lemma 6.3, we have:

Lemma $6 . \mathscr{A}^{n}(n \geqq 2) \quad$ Assume the following for each $\mathscr{A}_{\mu} \in \mathscr{F}_{1}^{0} \tilde{A}^{n}$.

$$
Z_{1}^{0, q}\left(\mathscr{A}_{\mu} \times D^{\prime}, \mathfrak{D}\right)_{\alpha}^{m} \subset B_{0}^{0, q}\left(\mathscr{A}_{\mu} \times D^{\prime}, \mathfrak{D}\right)_{\alpha^{\prime}}^{m^{\prime}}(q>0),
$$

where $\alpha^{\prime}=E_{n}\left(l_{\lambda_{\mu}} / r_{\lambda_{\mu}}^{-}, m r_{\lambda_{\mu}}^{+}, \alpha\right)$ and the correspondence: $m \rightarrow m^{\prime}$ are as in Lemma 6.3. Then, for each $\mathscr{A}_{\lambda} \in A^{n}$, we have the following diagram (similar 
to the one in Lemma 6.1):

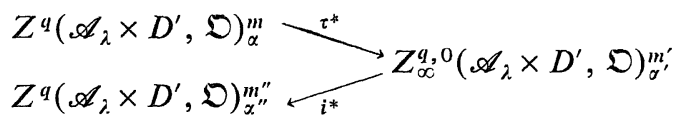

such that

$$
\left(1-i^{*} \tau^{*}\right) Z^{q}\left(\mathscr{A}_{\lambda} \times D^{\prime}, \mathfrak{D}\right)_{\alpha}^{m} \subset \delta C^{q-1}\left(\mathscr{A}_{\lambda} \times D^{\prime}, \mathfrak{D}\right)_{\alpha^{\prime \prime}}^{m \prime \prime}
$$

where the estimation: $(m ; \alpha) \rightarrow\left(m^{\prime} ; \alpha^{\prime}\right)$ and $\left(m^{\prime}, \alpha^{\prime}\right) \rightarrow\left(m^{\prime \prime}, \alpha^{\prime \prime}\right)$ are as in Lemma 6.3 .

Similarly to Proposition $6.6_{1}$, we have the following implication:

Proposition 6.62. Lemma $6.3^{n-1} \rightarrow$ Lemma $6.3^{n}(n \geqq 2)$.

Proof. Letting $\mathscr{A}_{\lambda} \in \operatorname{Cov}_{0}\left(\mathbb{C}^{n}\right)_{\mathrm{e} 1}$ be as in Lemma 6.3, we write $\mathscr{A}_{\lambda}$ explicitly as follows: $\mathscr{A}_{\lambda}=\mathscr{A}_{k}(U)=\mathscr{A}_{h^{\prime}}\left(U^{\prime}\right) \times \mathscr{A}_{h^{\prime \prime}}\left(U^{\prime \prime}\right)$, with $U=U^{\prime} \times U^{\prime \prime}$ $\in \operatorname{Ouv}_{\mathrm{e} 1}\left(\boldsymbol{C}^{n}\right)\left(=\operatorname{Ouv}_{\mathrm{e} 1}\left(\boldsymbol{C}^{n-1}\right) \times \operatorname{Ouv}_{\mathrm{e} 1}(\boldsymbol{C})\right)$ and $k=\left(k^{\prime \prime}, k^{\prime \prime}\right) \in \boldsymbol{Z}^{2 n}\left(=\mathbb{Z}^{2 n-2} \times \mathbb{Z}^{2}\right)$. Then, the p.g. version of $(6.7)_{7}$ implies:

(a) $\quad Z_{\infty}^{q, 0}\left(\mathscr{A}_{k}(U) \times D^{\prime}, \mathfrak{D}\right)_{\alpha}^{m} \cong Z^{q}\left(\mathscr{A}_{k^{\prime}}\left(U^{\prime}\right) \times\left(D^{\prime \prime} \times D^{\prime}\right), \mathfrak{D}\right)_{\alpha}^{m}$, with $D^{\prime \prime}:=$ Supp $\mathscr{A}_{k^{\prime \prime}}\left(U^{\prime \prime}\right)_{m}$.

Applying Lemma $6.3^{n-1}$ to the right side, we have Lemma $6.3^{n}$.

Check of the assumptions in Lemmas 6.1, 6.2. Here we check the assumptions $(6.11)_{1,2}^{0, \frac{q}{2}}$ in Lemmas 6.1, 6.2, which are given for the lowest degree $p=0$ :

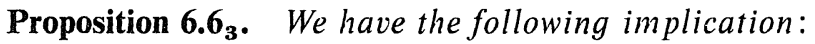

$$
\text { Theorem } 6.1^{1} \longrightarrow(6.11)_{1}^{0, q}, \quad \text { Lemma } 6.3 \longrightarrow(6.11)_{2}^{0, q} \text {. }
$$

We also have the following implication for the assumption in Lemma 6.4.

Proposition 6.6.4 Lemma $6.3^{1} \longrightarrow(6.13)^{0, q}$.

The proof of Proposition $6.6_{4}$ is easier than that of Proposition $6.6_{3}$. Here we prove only Proposition $6.6_{3}$.

Proof. Let the coverings $\mathscr{A}_{k^{\prime}}:=\mathscr{A}_{k^{\prime}}\left(\boldsymbol{C}^{n}\right)$ and $\mathscr{A}_{\xi}^{\prime}:=\mathscr{A}_{\xi}^{\prime}\left(\boldsymbol{C}^{n} \times U^{\prime}\right)$ be as in Lemmas 6.1, 6.2, and the maps $\pi_{k}, \pi_{\xi}$ the ones attached to $\mathscr{A}_{k}, \mathscr{A}_{\xi}^{\prime}$ (cf. (6.7) $)_{5,8}$ ). We set $\mathscr{B}_{k}:=\pi_{k}\left(\mathscr{A}_{k}\right)$ and $\mathscr{B}_{\xi}:=\pi_{\xi}\left(\mathscr{A}_{\xi}^{\prime}\right)$. By the definition, these sets are of the following forms (cf. $\left.(6.7)_{5,8}\right)$ : 
(a)

$$
\mathscr{B}_{k}=\mathscr{A}_{h}\left(\mathbb{C}^{n-1}\right) \text { and } \mathscr{B}_{\xi}=\mathbb{Z}^{2 n} \text {. }
$$

For an element $B \in \mathscr{B}_{k}$ or $\mathscr{B}_{\xi}$, we easily have:

(b) $\pi_{k}^{-1}(B)=\mathscr{A}_{h}(C) \times B$ and $\pi^{-1}(B)=\mathscr{A}_{\xi}\left(S_{B_{\varepsilon}} \times U^{\prime}\right.$ ). (For the latter set, see $(6.3)_{1}^{\prime}$.)

Next recall that the assumptions $(6.11)_{1,2}^{0,4}$ are given to the admissible families $\mathscr{F}_{1}^{\prime 0} \mathscr{A}_{h}$ and $\mathscr{F}_{1}^{0} \mathscr{N}_{\xi}^{\prime}$. Setting $\left(\mathscr{A}, \tilde{\mathscr{F}}_{1}\right):=\left(\mathscr{A}_{h}, \mathscr{F}_{1}^{\prime 0}\right)$ or $\left(\mathscr{A}_{\xi}^{\prime}, \mathscr{F}_{1}^{0}\right)$, we thes the filtration of $\widetilde{F}_{1} \mathscr{A}_{\mid B}$ as in Proposition 6.5". Remarking that $\# \pi^{-1}(B)=\infty$ or $<\infty$, according as $\mathscr{A}:=\mathscr{A}_{k}$ or $\mathscr{A}_{\xi}^{\prime}$, we divide the arguments henceforth in two parts :

(i) The case $\mathscr{A}=\mathscr{A}_{k}\left(C^{n}\right)$. We easily see that $\mathscr{F}_{1}^{\prime 0} \mathscr{A}_{k \mid B}$ consists of the single element $\mathscr{A}_{\mu}:=\mathscr{A}_{k}(\mathbb{C}) \times B$. Apply Theorem $6.1^{1}$ to $\mathscr{A}_{\mu}$, and we have: (c) $s_{1} s^{*} Z^{q}\left(\mathscr{A}_{k}(\mathbb{C}) \times\left(B \times D^{\prime}\right), \mathfrak{D}\right)_{\alpha} \subset \delta C^{q-1}\left(\mathscr{A}_{k^{\prime}}(\mathbb{C}) \times\left(B \times D^{\prime}\right), \mathfrak{D}\right)_{\alpha^{\prime}}$, where the estimation: $\alpha \rightarrow \alpha^{\prime}$ and the refining map $s$ is defined in the manner as in Theorem $6.1^{1}$.

Take a suitable refining map $s_{2}: \mathscr{A}_{k^{\prime}}\left(\mathbb{C}^{n-1}\right) \hookrightarrow \mathscr{A}_{k}\left(\mathbb{C}^{n-1}\right)$, and we set: $s:=s_{1} \times s_{2}$ : $\mathscr{A}_{k^{\prime}}\left(\mathbb{C}^{n}\right) \times D^{\prime}\left(=\left(\mathscr{A}_{h}(\mathbb{C}) \times D^{\prime}\right) \times \mathscr{A}_{k^{\prime}}\left(\mathbb{C}^{n-1}\right)\right) \hookrightarrow \mathscr{A}_{k}\left(\mathbb{C}^{n}\right)\left(=\left(\mathscr{A}_{k}(\mathbb{C}) \times D^{\prime}\right) \times \mathscr{A}_{k}\left(\mathbb{C}^{n-1}\right)\right)$. From (c) ${ }_{1}$ have:

(c) $)_{2} s_{\mu}^{*} Z^{q}\left(s_{\mu}\left(\mathscr{A}_{\mu} \times D^{\prime}\right), \mathscr{D}\right)_{\alpha} \subset \delta C^{q-1}\left(\mathscr{A}_{\mu} \times D^{\prime}, \mathfrak{D}\right)_{\alpha^{\prime}}$, with the refining map $s_{\mu}$ : $\mathscr{A}_{\mu} \times D^{\prime} \hookrightarrow s_{\mu}\left(\mathscr{A}_{\mu}\right) \times D^{\prime}$ as in Lemma 6.1.

Finally, noting that $\# B=1$, we change the symbol $Z^{q}, \delta C^{q-1}$ in (c) $)_{2}$ to $Z_{1}^{q, 0}$ and $B_{0}^{0, q}$. Then we have $(6.11)_{1}^{0, q}$.

(ii) The case $\mathscr{A}=\mathscr{A}_{\xi}^{\prime}$. In this case the check of $(6.11)_{2}^{0, q}$ is essentially an easy consequence of Lemma 6.3. The unique key point is to check the explicit forms of the estimations in (6.11) 2 and Lemma 6.3. First the collection $\mathscr{F}_{1}^{0} \mathscr{A}_{\mid B}$ is of the form: $\left\{\mathscr{A}_{\mu}\right\}_{\mu}$, where $\mathscr{A}_{\mu}$ is an element of $\operatorname{Cov}_{0}\left(\mathbb{C}^{n} \times \mathbb{C}^{n^{\prime}}\right)_{\mathrm{e} 1}$. (This follows from Proposition 6.5" and the explicit form of $B$ in (a).) Also note that $\mathscr{A}_{\mu}$ consists of elementary figures in $\mathscr{A}_{\xi}^{\prime}\left(S_{B \varepsilon} \times U^{\prime}\right)$ (cf. $\left.(6.3)_{1}^{\prime}\right)$. Applying Lemma 6.3 to $\mathscr{A}_{\mu}$, we have:

(c) $)_{3} \quad Z^{q}\left(\mathscr{A}_{\mu}, \mathfrak{D}\right)_{\alpha}^{m} \subset \delta C^{q-1}\left(\mathscr{A}_{\mu}, \mathfrak{D}\right)_{\sigma^{\prime}}^{m^{\prime}}$, with $\alpha^{\prime}=E_{n+n^{\prime}}\left(l_{\mu} / r_{\mu}^{-} ; m r_{\mu}^{+} ; \alpha\right)$,

where the estimation map $E_{n+n^{\prime}}$ and the elements $\left(m, m^{\prime}\right)$ are as in Lemma 6.3. Also the numerical invariants $l_{\mu}, r_{\mu}^{ \pm}$of $\mathscr{A}_{\mu}$ are defined in the manner in (6.13)”. The desired estimation $(6.11)_{2}^{0, q}$ follows from (c) $)_{3}$ as follows: first from a simple observation, the invariants $l_{\mu}, r_{\mu}^{ \pm}$are estimated in the following fashion: 
(d) $)_{1} \quad l_{\mu}=[\zeta(|B|+1)]^{2\left(n+n^{\prime}\right)}, r_{\mu}^{-} \geqq\left(d_{U^{\prime}}^{-} / l_{\mu}\right)$, and $r_{\mu}^{+} \leqq c_{n \cdot n^{\prime}}$, with an element $c_{n, n^{\prime}} \in$ $\boldsymbol{R}_{1}^{+}$depending only on $\left(n, n^{\prime}\right)$.

For each $z \in\left|\mathscr{A}_{\mu}\right|$, we easily have*) $:|z-B|<c_{n, n^{\prime}}^{\prime}$, with an element $c_{n, n^{\prime}}^{\prime} \in R_{1}^{+}$. From this we easily have:

(d) $)_{2} \quad \zeta \cdot(|B|+1)<\zeta^{\prime}(|z|+1)$, with $\zeta^{\prime}=\mathscr{L}_{n, n^{\prime}}(\zeta)$, where the el-map $\mathscr{L}_{n, n^{\prime}}$ (cf. n.5, $\S 1.1)$ depends only on $\left(n, n^{\prime}\right)$.

Now it is easy to rewrite the estimation in $(c)_{3}$ in the form in $(6.11)_{2}^{0, q}$ (by using $(d)_{1,2}$ ), and we finish the proof of Proposition 6.6.

Conclusion: Now we conclude Section 6.3 by the following.

Proposition 6.7. For the proof of Theorem 6.1, Theorem 6.2 and Lemma 6.3, the proof of the following suffices:

(6.14) Theorem 6.4' Lemma $6.3^{1}$ as well as Lemmas 6.1, 6.2 and 6.4.

Proof. This follows easily from Proposition 6.6.

The proof of the first two facts are given in Appendix II. The second three facts are proven in B, Section 7 after we develop certain algebraic arguments for the filtrations in Definition 6.3.

Remark 6.2. Here we check that Lemma 5.2' follows easily from Lemma 6.3. The implication: Lemma $6.3 \rightarrow$ Lemma $5.2^{\prime}$ is very elementary. We summarize only the key points of the proof of the implication: Lemma $6.3 \rightarrow$ Lemma 5.2'. Take an elementary covering $\mathscr{A}_{\lambda}=\mathscr{A}_{k}(U) \in \boldsymbol{A}^{n}\left(=\operatorname{Cov}_{0}\left(\boldsymbol{C}^{n}\right)_{\mathrm{e} 1}\right)$, where $(U ; k)$ is in $\operatorname{Ouv}\left(\boldsymbol{C}^{n}\right)_{\mathrm{cl}} \times \boldsymbol{Z}^{+2 n}$. We assume that $t_{0}$ is of the form: $\mathfrak{k}_{0}=\left(k_{j}\right)_{j=1}^{2 n}$, with $k_{1}=k_{2}=\cdots$. (We write $k_{1}, k_{2}, \ldots$ as $k$.) Letting the invariants $r_{\lambda}^{t}$ of $\mathscr{A}_{\lambda}$ be as in (6.12) $)_{0}^{\prime \prime \prime}$, we easily see that the inequality:

(6.15) ${ }_{1}^{\prime} \quad c_{n} r_{\lambda}^{+} / k<t$ (resp. $\left.t<c_{n} r_{\lambda}^{-} / k\right)$, with an element $c_{n} \in \boldsymbol{R}_{1}^{+}$, which depend only on $n=\operatorname{dim} C^{n}$, insures the existence of a refining map:

$(6.15)_{1} \quad s: \mathscr{A}_{k}(U) \hookrightarrow \mathscr{A}_{t}(U)$ (resp. $s^{\prime}: \mathscr{A}_{t}(U) \hookrightarrow \mathscr{A}_{k}(U)$ ), where the covering $\mathscr{A}_{t}(U)$ is defined in the manner as in (5.2) 0 .

The above fact suffices to get the implication: Lemma $6.3 \rightarrow$ Lemma $5.2^{\prime}$. Actually, we first recall that the underlying geometric figure in Lemma $5.2^{\prime}$ is the disc $U_{r}$ in $\boldsymbol{C}^{n}$, while that in Lemma 6.3 is the geometric figures $U$ as above.

*) Note that $B$ is in $Z^{n}$ and is in $C^{n}$. Using this $|z-B|$ is well defined. 
The difference of these two geometric figures is quite small, and one can easily get the similar relation among the coverings $\mathscr{A}_{k}(U)$ and $\mathscr{A}_{t}(U)$. Also, by checking the explicit estimations in Lemma 5.2' and Lemma 6.3, we easily get Lemma 5.2' from Lemma 6.3 (by using $(6.15)_{1}$ ).

\section{§7. A Sharpening of Degeneracy Theorem and Polynomial Growth Uniform Estimations}

The main purpose of Section 7 is to give the sharpening of the degerency theorem (cf. Lemma 7.3 and Lemma 7.5) and to complete the proof of Theorem 6.1 and Theorem 6.2 by using such a sharpening. In Part A we give some detailed computations on spectral sequences, which are used in the proof of the sharpening mentioned just above. The applications of the arguments in $\mathrm{A}$ will be given in $\mathrm{B}$.

\section{A. A Localization Method in Filtrations}

In Part A the topological space $X$, the element $\mathscr{A} \in \operatorname{Cov}_{0}(X)$ and the map $\pi: \mathscr{A} \rightarrow \mathscr{B}$ will be the ones in $\mathrm{n.1}$, Section 6.2. We make the following additional condition on $\pi: \mathscr{A} \rightarrow \mathscr{B}$.

$(7.0)_{1} \quad \pi: \mathscr{A} \rightarrow \mathscr{B}$ is surjective and $p:=\# \mathscr{B}-1 \geqq 1$.

The main results in A will be Lemma 7.1-Lemma 7.1"'. The applications of such lemmas are found in the proof of Lemma 7.3 and Lemma 7.5 (cf. n.1 and n.2, B §7).

1. An additional filtration. Letting $p \in \mathbb{Z}^{+}$be the one fixed in $(7.0)_{1}$, we set $^{*)}$ :

$$
K^{q}:=F_{p} C^{q}(\mathscr{A}, \Re)(q \geqq 0) .
$$

The purpose of $A$ will be then to reduce investigations of $K^{*}:=\sum_{q \geqq 0} K^{q}$ to those of certain complexes at the filtration degree $\tilde{p}=p-1$ and $p=0$ (Lemma 7.1). For this we fix a total order $\prec$ of $\mathscr{B}$, and we set:

$$
B:=\text { maximal element of } \mathscr{B} \text {, and } \tilde{\mathscr{A}}:=\pi^{-1}(B) \text {. }
$$

Using the order $\prec$, we introduce a filtration in $K^{q}$ :

Definition 7.1. By filtration of $K^{q}$ induced from $\prec$, we mean the following

\footnotetext{
*) The sheaf $\Re$ is also as in n.1, $\S 6.2$.
} 
decreasing sequence of subsets of $K^{q}$ :

$$
G_{0} K^{q}:=K^{q} \supset \cdots \supset G_{t} K^{q} \supset \cdots \supset G_{q-p+1} K^{q} \cong 0,
$$

where

$(7.1)_{1}^{\prime} \quad G_{t} K^{q}:=\left\{\varphi \in K^{q} ; \varphi_{. \alpha^{\prime}}=0\right.$ for each $\mathscr{A}^{\prime} \in \mathcal{A}^{\wedge+1} \mathscr{A}$ satisfying $\#\left(\tilde{\mathscr{A}} \cap \mathscr{A}^{\prime}\right)$ $\leqq i\}$.

We write the spectral sequence for $\left(K^{*}, G_{t}\right)$ as $E_{r}^{\prime t, *-t}$. In $n .2$ and n.3 we give a detailed structure of $E_{1}^{\prime t}$-terms.

2. Decomposition of $\mathbf{E}_{1}^{\prime}$-terms. First, taking a subset $\mathscr{A}^{\prime}$ of $\mathscr{A}$ and integers $q, \tilde{p}, t \geqq 1$, we arrange the following notation, which is convenient for later arguments (cf. also n.3, §6.2):

$$
\left\{\begin{array}{l}
\mathscr{A}^{\prime(q)}:=\left\{\mathscr{A}^{\prime \prime} \subset \mathscr{A}^{\prime} ;\left|\mathscr{A}^{\prime \prime}\right| \neq \phi, \# \mathscr{A}^{\prime \prime}=q\right\}, \mathscr{A}_{\tilde{p}}^{\prime(q)}:=\left\{\mathscr{A}^{\prime \prime} \in \mathscr{A}^{\prime(q)} ; \# \pi\left(\mathscr{A}^{\prime \prime}\right)=\tilde{p}\right\}, \\
\mathscr{A}_{\tilde{p} \mid t}^{\prime(q)}:=\left\{\mathscr{A}^{\prime \prime} \in \mathscr{A}_{\tilde{p}}^{\prime(q)} ; \#\left(\mathscr{A}^{\prime \prime} \cap \tilde{\mathscr{A}}\right)=t\right\} .
\end{array}\right.
$$

Moreover, for an element $\mathscr{D} \in \tilde{\mathscr{A}}^{(t)}$, we set:

$$
\mathscr{A}_{\tilde{p} \mid \mathscr{D}}^{\prime(q)}:=\left\{\mathscr{A}^{\prime \prime} \in \mathscr{A}_{\tilde{p}}^{(q)} ;\left(\mathscr{A}^{\prime \prime} \cap \tilde{\mathscr{A}}\right)=\mathscr{D}\right\} .
$$

Then we obviously have:

$$
\mathscr{A}_{\tilde{p} \mid t}^{\prime(q)}:=\Perp_{\mathscr{Q}^{\prime}} \mathscr{A}_{\tilde{p} \mid \mathscr{O}}^{\prime(q)}, \text { where } \mathscr{D} \text { runs through } \tilde{\mathscr{A}}^{(t)} .
$$

Next, we use the symbol ' $v$ ' for the map:

(7.1) ${ }_{3}^{\prime \prime \prime} \quad v: \mathscr{N}^{q} \mathscr{A}^{\prime} \ni \mathscr{A}_{\mu}^{\prime} \rightarrow \mathscr{A}^{\prime(q)} \ni \mathscr{A}_{\mu}^{\prime}$, where we forget the order of $\mathscr{A}_{\mu}^{\prime}$ in the right side,

and we define:

$$
\begin{aligned}
& \mathscr{N}_{\tilde{p}}^{q} \mathscr{A}^{\prime}:=v^{-1}\left(\mathscr{A}_{\tilde{p}}^{\prime(q)}\right), \mathscr{N}_{\tilde{p}}^{q}(\mathscr{A})_{\mid t}:=v^{-1}\left(\mathscr{A}_{\tilde{p} \mid t}^{(q)}\right), \quad \text { and } \\
& \mathscr{N}_{\tilde{p}}^{q}(\mathscr{A})_{\mid \mathscr{D}}:=v^{-1}\left(\mathscr{A}_{\tilde{p} \mid \mathscr{D}}^{\prime(q)}\right)
\end{aligned}
$$

Now, taking an element $\mathscr{D} \in \tilde{\mathscr{A}}^{(t+1)}$, we define:

$$
K_{t \mid \mathscr{D}}^{q}:=\left\{\varphi \in K^{q} ; \varphi_{\mathscr{\mathscr { A } ^ { \prime }}}=0 \text {, unless } \mathscr{A}^{\prime} \in \mathscr{N}_{p+1}^{q+1}(\mathscr{A})_{\mid \mathscr{O}}\right\},
$$

and we set:

$$
K_{t}^{q}:=\oplus_{\mathscr{D}} K_{t \mid \mathscr{D}}^{q} \text {, where } \mathscr{D} \text { runs through } \tilde{\mathscr{A}}^{(t+1)} .
$$

It is then easy to see:

$$
\left(G_{t} K^{q} / G_{t+1} K^{q}\right) \cong K_{t}^{q} \text { (as abelian groups) }
$$


We want to make this isomorphism as the one of complexes. For this, denote by $i_{\mathscr{D}}$ the inclusion: $K_{t \mid \mathscr{Q}}^{q} \hookrightarrow K^{q}$ and by $r_{\mathscr{Q}}$ the restriction map:

$$
\begin{aligned}
(7.1)_{4}^{\prime} \quad r_{\mathscr{D}}: K^{q} \ni \varphi \longrightarrow K_{t \mid \mathscr{D}}^{q} \ni \varphi^{\prime}, \text { where } \varphi_{\mathscr{\mathscr { A }}}^{\prime}=\varphi_{\mathscr{\mathscr { A }}}^{\prime} \\
\text { for each } \mathscr{\mathscr { A }}^{\prime} \in \mathscr{N}_{p+1}^{q+1}(\mathscr{A})_{\mid \mathscr{D}},
\end{aligned}
$$

and we define a degree one map $\delta_{\mathscr{D}}: K_{t \mid \mathscr{D}}^{q} \ni \varphi \rightarrow K_{t \mid \oslash}^{q+1} \ni \varphi^{\prime}$

by Figure I. The explicit description of $\varphi^{\prime}=\delta_{\mathscr{D}}(\varphi)$ is as follows: taking an element $\mathscr{A}^{\prime} \in \mathscr{N}_{p+1}^{q+2}(\mathscr{A})_{\mid \mathscr{O}}$, we assume that the order of $\mathscr{A}^{\prime}$ is given in the form:

$(7.1)_{4}^{\prime \prime} \quad \mathscr{A}^{\prime}=\left(\mathscr{A}^{\prime \prime}, \mathscr{D}\right)$, where $\mathscr{D}$ is ordered in an arbitray

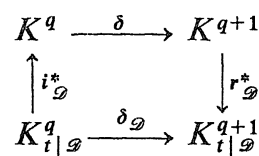

Figure I. manner and $\mathscr{A}^{\prime \prime}$ is an element of $\mathscr{N}_{p-1}^{q+1-t}(\mathscr{A})$ satisfying $\pi\left(\mathscr{A}^{\prime \prime}\right)=\mathscr{B}-B$. Moreover, $A_{v}<A_{\mu}$ for each $\left(A_{v}, A_{\mu}\right) \in \mathscr{A}^{\prime \prime} \times \mathscr{D}$.

Then $\varphi_{. \alpha^{\prime}}^{\prime}$ is given as follows:

(7.1) $\varphi_{4}^{\prime \prime \prime} \quad \varphi_{\mathscr{A}}^{\prime \prime, \mathscr{A}}=\sum_{j=1}^{q+1-t}(-1)^{j} \cdot \varphi_{\mathscr{s}^{\prime \prime}, \mathscr{D}}$, with $\mathscr{A}_{j}^{\prime \prime}:=\mathscr{A}^{\prime \prime}-(j$-th component of $\left.\mathscr{A}^{\prime \prime}\right)$.

(From the explicit form of $K_{t \mid \mathscr{Q}}^{*}$ (cf. $(7.1)_{3}^{\prime}$ ), the attachment of $\varphi_{\mathscr{A ^ { \prime }}}^{\prime}$ for the element $\mathscr{A}^{\prime} \in \mathcal{V}_{p+1}^{q+2}(\mathscr{A})_{\mid \mathscr{Q}}$ suffices for the definition of $\varphi^{\prime}=\delta_{\mathscr{\Omega}}(\varphi)$.) Then setting $\delta_{t}:=\oplus_{\oplus}^{\delta}: K_{t}^{q} \rightarrow K_{t}^{q+1}$, we easily have the following.

Proposition 7.1. $\left(K_{t}^{*}, \delta_{t}\right) \cong\left(\oplus_{t} G_{t} K^{*} / G_{t+1} K^{*}, \delta\right)$, where $\delta$ is induced from $K^{q} \rightarrow K^{q+1}$, and the isomorphism is induced from $\oplus_{g} i_{g}$.

By the definition of $E_{1}^{\prime}$-term (cf. n.1), we have:

Corollary 7.1. $E_{1}^{\prime t, q-t} \cong H^{q}\left(K_{t}^{*}\right) \cong \oplus_{\mathscr{D}} H^{q}\left(K_{t \mid \mathscr{Q}}^{*}\right)$.

In $\mathrm{n} .3$ we give an another description of $E_{1}^{\prime}$-term in a convenient form for our inductive treatments of the filtration $F_{p}$ on $p=0,1, \ldots$

3. Localization procedure. First taking an element $\mathscr{D} \in \tilde{\mathscr{A}}^{(t)}$, we make:

Definition 7.2. By localization of $\mathscr{A}$ with respect to $\mathscr{D}$ (resp. $|\mathscr{D}|$ ), we mean the following collections:

$(7.2)_{1}\left\{\begin{array}{l}\mathscr{A}_{\mathscr{D}} \\ \mathscr{A}_{|\mathscr{D}|}\end{array}\right\}:=\left\{\begin{array}{l}\left.\mathscr{A}_{\mu} \in \mathscr{A} ; \pi\left(\mathscr{A}_{\mu}\right) \subset \mathscr{B}-B,\left|\mathscr{A}_{\mu}\right| \cap|\mathscr{D}| \neq \phi\right\}\left(\subset \pi^{-1}(\mathscr{B}-B)\right. \\ \left.\left|\mathscr{A}_{\mu}\right| \cap|\mathscr{D}|: \mathscr{A}_{\mu} \in \mathscr{A}_{\mathscr{D}}\right\}\left(\subset \operatorname{Cov}_{0}(|\mathscr{D}|)\right)\end{array}\right\}$.

We use the localization $\mathscr{A}_{|\mathscr{D}|}$ in n.3. The localization $\mathscr{A}_{\mathscr{D}}$ will be convenient for our explicit p.g. estimations (cf. n.4, Part A). We arrange here some data which we use in later arguments: first we define an obvious bijective map: 
$(7.2)_{1} \quad \operatorname{res}_{\mathscr{D}}: \mathscr{A}_{\mathscr{D}} \ni A_{\mu} \longrightarrow \mathscr{A}_{|\mathscr{D}|} \ni A_{\mu}^{\prime}:=A_{\mu} \cap|\mathscr{D}|$,

and we use the symbols $\pi_{\mathscr{D}}, \pi_{|\mathscr{O}|}$ for the restrictions of $\pi$ to $\mathscr{A}_{\mathscr{D}}$ and $\pi_{\mathscr{D}} \cdot \operatorname{res}_{\mathscr{D}}^{-1}$ (cf. Figure II). Also, concordantly to n.1. we set:

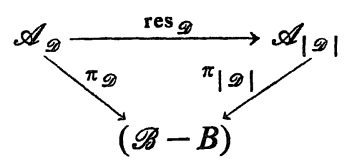

Figure II.

$$
\left(\mathscr{A}_{|\mathscr{P}|}\right)_{p}^{(q)}:=\left\{\mathscr{A}_{\mu} \in\left(\mathscr{A}_{|\mathscr{D}|}\right)^{(q)} ; \# \pi_{|\mathscr{D}|}\left(\mathscr{A}_{\mu}\right)=p\right\}, \mathscr{N}_{p}^{q}\left(\mathscr{A}_{|\mathscr{P}|}\right):=v^{-1}\left(\left(\mathscr{A}_{|\mathscr{P}|}\right)_{p}^{(q)}\right) .
$$

The map $\operatorname{res}_{\mathscr{D}}^{-1}$ induces an injective map: $\mathscr{N}_{p}^{q}\left(\mathscr{A}_{|\mathscr{D}|}\right) \rightarrow \mathscr{N}_{p}^{q}\left(\mathscr{A}_{\mathscr{D}}\right)$ in a natural manner. (This map is, in general, not surjective.) We write this map also as $\operatorname{res}_{\mathscr{D}}^{-1}$. Now, using the symbol $F_{\tilde{p}}$ also for the filtration induced from the map $\pi_{|\mathscr{D}|}$, we set:

$$
K_{|\mathscr{D}|}^{*}:=F_{p-1} C^{*}\left(\mathscr{A}_{|\mathscr{D}|}, \mathfrak{\Omega}\right), \text { where } \mathscr{D} \in \tilde{\mathscr{A}}^{(t+1)} .
$$

We show that $K_{|\mathscr{}|}^{*}$ and $K_{t \mid \mathscr{D}}^{*}$ are isomorphic*). For this we first define (set theoretical) maps $\theta_{|\mathscr{D}|}, \theta_{\mathscr{D}}$ by Figure III. (In Figure III we fix an order of

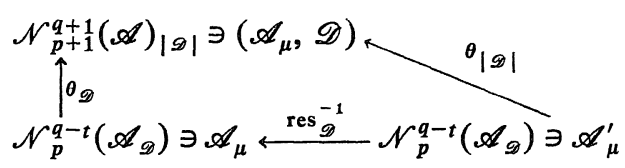

Figure III.

$\mathscr{D}$ arbitrarily**), and the order of $\left(\mathscr{A}_{\mu}, \mathscr{D}\right)$ is given similarly to (7.1) $)_{4}^{\prime \prime}$. Also note that $\theta_{\mathscr{D}}$ is defined for the image of $\operatorname{res}_{\mathscr{\mathscr { C }}}^{-1}$.) Now, using the maps $\theta_{\mathscr{D}}, \theta_{|\mathscr{Q}|}$, we define:

Definition 7.3. By reduction and reverse maps $\theta_{|\mathscr{Q}|}^{*}, \omega_{|\mathscr{Q}|}^{*}$, we mean the following isomorphisms of complexes:

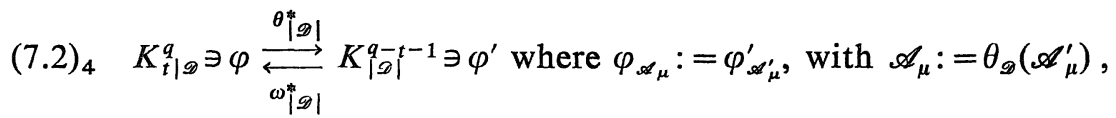
for each $\mathscr{A} \in \mathscr{N}_{p}^{q-t}\left(\mathscr{A}_{|\mathscr{D}|}\right)$.

(By Figure III we easily have $\left|\theta_{|\mathscr{D}|}\left(\mathscr{A}_{\mu}^{\prime}\right)\right|=\left|\mathscr{A}_{\mu}^{\prime}\right|$, and one can easily check the well definedness and the isomorphisms of the maps $\left.\theta_{\mathscr{D}}^{*}, \omega_{\mathscr{D}}^{*}\right)$. From Definition 7.3 and Proposition 7.1, we have:

Proposition 7.2 $\cdot E_{1}^{\prime t, q-t} \cong \oplus_{\mathscr{D}} H^{q-t-1}\left(K_{|\mathscr{D}|}^{*}\right)$.

*) Note that $K_{t \mid \mathscr{D}}^{*}$ is the subcomplex of $K^{*}=F_{p} C^{*}(\mathscr{A}, \Re)(\mathrm{cf} . \mathrm{n} .2)$, while $K_{|\mathscr{Q}|}^{*}=F_{p-1}$. $C^{*}\left(\mathscr{A}_{\mathscr{D}}, \Re\right)\left(\mathrm{cf} .(7.2)_{3}\right)$. The author believes that there is no notational confusions between the above two complexes.

**) Thus the maps $\theta_{\mathscr{D}}, \theta_{\mathscr{D}}$ and the resulting homomorphisms $\theta_{|\mathscr{D}|}^{*}, \omega_{|\mathscr{D}|}^{*}$ in Definition 7.3 depend on the order of $\mathscr{D}$. As we will see in the course of the arguments, the latter arguments on $\theta_{|\mathscr{D}|}^{*}, \omega_{|\mathscr{D}|}^{*}$ will work well for any choice of the order of $\mathscr{D}$. 
Using the injection $i_{\mathscr{D}}^{*}: K_{t \mid \mathscr{D}}^{*} \hookrightarrow K^{*}$ and the restriction $r_{\mathscr{D}}^{*}: K^{*} \rightarrow K_{t \mid \mathscr{D}}^{*}$ (cf. n.2), we have the homomorphisms $\theta_{\mathscr{D}}^{*} r_{\mathscr{D}}^{*}: K^{*} \rightarrow K_{\mathscr{D}}^{*-t-1}$ and $i_{\mathscr{D}}^{*} \omega_{\mathscr{D}}^{*}: K_{\mathscr{D}}^{*-t-1}$ $\rightarrow K^{*}$. When there is no fear of confusions we call such maps also reduction and reverse maps and use the symbols $\theta_{\mathscr{Q}}^{*}, \omega_{\mathscr{D}}^{*}$ also for such maps.

Remark 7.1. Let $\varphi \in K_{t \mid \mathscr{D}}^{q}$ and the elements $\mathscr{A}_{\mu}^{\prime}, \mathscr{A}_{\mu}=\theta_{\mathscr{D}}\left(\mathscr{A}_{\mu}^{\prime}\right)\left(=\left(\mathscr{A}_{\mu}^{\prime}, \mathscr{D}\right)\right)$ be as in $(7.2)_{4}$. We then regard $\mathscr{A}_{\mu}=\left(\mathscr{A}_{\mu}^{\prime}, \mathscr{D}\right)$ as the variable for the cochain map: $\varphi: N_{p+1}^{q+1}(\mathscr{A})_{\mid \mathscr{D}} \ni \mathscr{A}_{\mu} \rightarrow \Gamma\left(\mathscr{A}_{\mu}, \mathfrak{K}\right)$. Then the idea of Definition 7.3 is to regard the first and second variables $\mathscr{A}_{\mu}^{\prime}$ and $\mathscr{D}$ respectively as the moving and fixed variables. Thus our reduction method is concordant to standard reduction methods obtained by regarding some parts of variables in questions as the fixed ones. We also note that we use a similar reduction method for our cohomology theories for certain stratified spaces (cf. [18]). The reduction maps as in Definition 7.3 will be a key tool in the later arguments of Section 7.

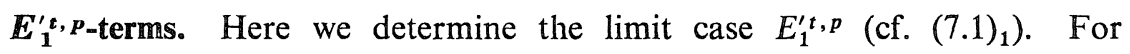
this take an element $\mathscr{D} \in \tilde{\mathscr{A}}^{(t+1)}$, and we set:

$$
J_{\mathscr{D}}:=\left\{\begin{array}{l}
\Gamma\left(\left|\left(\mathscr{A}_{\mathscr{D}} / \pi_{\mathscr{D}}\right)_{u}\right|, \mathfrak{R}\right), \text { if*) } \mathscr{A}_{p+1 \mid \mathscr{D}}^{(p+q+1)} \neq \phi\left(\mathrm{cf} .(7.1)_{2}\right) \\
0, \text { otherwise. }
\end{array}\right.
$$

We set $J_{\mathscr{d}}^{t}:=\oplus_{\mathscr{A}} J_{\mathscr{A}}$, with $\mathscr{D} \in \tilde{\mathscr{A}}^{(t+1)}$, and we define a degree one map:

$(7.2)_{5}^{\prime} \quad \delta_{\tilde{\mathscr{A}}}: J_{\mathscr{\mathscr { A }}}^{t} \ni \varphi \rightarrow J_{\tilde{\mathscr{A}}}^{t+1} \ni \varphi^{\prime}$, where $\varphi_{\mathscr{D}}^{\prime}:=\sum_{j=1}^{t+2}(-1)^{j} \varphi_{\mathscr{D}_{j}}$, for each $\mathscr{D} \in$ $\mathscr{r}^{t+2} \tilde{\mathscr{A}}$. (Here $\mathscr{D}_{j}:=\mathscr{D}-\left(j\right.$-th component of $\mathscr{D}$.) Precisely, $\varphi_{\mathscr{D}_{j}}:=\rho_{j} \varphi_{\mathscr{D}_{j}}$, with the restriction**) $\rho_{j}: \Gamma\left(\left|\left(\mathscr{A}_{\left|\mathscr{D}_{j}\right|} / \pi_{\mid \mathscr{D} j}\right)_{u}\right|, \mathfrak{R}\right) \rightarrow \Gamma\left(\left|\left(\mathscr{A}_{|\mathscr{D}|} \mid \pi_{|\mathscr{Q}|}\right)_{u}\right|, \mathfrak{R}\right)$.)

Next remarking that $E_{1}^{\prime t, p}:=Z_{1}^{\prime t, p}:=\left\{\varphi \in G_{t} K^{t+p} ; \delta \varphi \in G_{t+1} K^{t+p+1}\right\}\left(\subset K^{t+p}\right)$, we define an element $\varphi^{\prime} \in J_{\mathscr{A}}^{t}$ by attaching the following value to each $\mathscr{D} \in$ $\mathscr{N}^{t+1} \mathscr{A}$. (We write the correspondence: $E_{1}^{\prime t, p} \ni \varphi \rightarrow J_{\mathscr{A}}^{t} \ni \varphi^{\prime}$ as $\chi_{\text {.) }}$

$(7.2)_{6} \quad \varphi_{\mathscr{D}}^{\prime}:=\varphi_{\mathscr{D}, \mathscr{A}^{\prime}}$ in $\left|\mathscr{A}^{\prime}\right| \cap|\mathscr{D}|$ for each $\mathscr{A}^{\prime} \in \mathscr{N}^{p} \mathscr{A}$ satisfying $\pi\left(\mathscr{A}^{\prime}\right)=\mathscr{B}-B$, $\left|\mathscr{A}^{\prime}\right| \cap|\mathscr{D}| \neq \phi$. (Here, writing $\mathscr{A}^{\prime}$ as $\mathscr{A}^{\prime}=\left(A_{1}^{\prime} \prec \cdots \prec A_{p}^{\prime}\right)$, we understand that $\pi\left(A_{1}^{\prime}\right) \prec \cdots \prec \pi\left(A_{p}^{\prime}\right)$.)

(Remark that $\left|\left(\mathscr{A}_{\mathscr{D}} \mid \pi_{\mathscr{O}}\right)_{u}\right|=\cup_{\mathscr{A}}\left(\left|\mathscr{A}^{\prime}\right| \cap|\mathscr{D}|\right)$, where $\mathscr{A}^{\prime} \in \mathscr{N}^{p} \mathscr{A}$ is as in $(7.2)_{6}$ (cf. the footnote to $(7.2)_{5}$ ). Also we see easily that $\varphi \in E_{1}^{\prime t, p}$ implies: $\varphi_{\mathscr{O}, \mathscr{A}^{\prime}}$ $=\varphi_{\mathscr{O}, \mathscr{Q}}^{\prime \prime}$ in $|\mathscr{D}| \cap\left|\mathscr{A}^{\prime}\right| \cap\left|\mathscr{A}^{\prime \prime}\right|$ for such $\mathscr{A}^{\prime}, \mathscr{A}^{\prime \prime}$. From the above we see that

*) By $(6.8)_{5}$ this is same to say that $\pi_{\mathscr{X}}\left(\mathscr{A}_{\mathscr{Q}}\right)=\mathscr{B}-B$ and $\left|\left(\mathscr{A}_{\mathscr{O}} / \pi_{\mathscr{g}}\right)_{u}\right| \neq \phi$. We use this fact in later arguments.

**) When $\mathscr{A}_{p+11 \mathscr{Q}}^{(p+t+1)}=\phi$, we understand that $\rho_{j}$ is the zero map. 
$\varphi_{\mathscr{D}}^{\prime}$ is an element of $J_{\mathscr{D}}=\Gamma\left(\left|\left(\mathscr{A}_{\mathscr{D}} / \pi_{\mathscr{D}}\right)_{u}\right|, \mathfrak{R}\right)$.) From the explicit relation in $(7.2)_{6}$ we easily have:

Proposition 7.22. $\left(E_{1}^{\prime *, p}, d_{1}\right) \cong\left(J_{\mathscr{\mathscr { A }}}^{*}, \delta_{. \tilde{q}}\right)$.

Finally we summarize the arguments in n.1-n.3. For this we use the symbol $F_{p}$ for the filtration in $C^{*}(\mathscr{A}, \Re)$ and $C^{*}\left(\mathscr{A}_{|\mathscr{D}|}, \mathfrak{\Omega}\right)$, which are induced from the maps $\pi: \mathscr{A} \rightarrow \mathscr{B}$ and $\pi_{|\mathscr{D}|}: \mathscr{A}_{|\mathscr{D}|} \rightarrow \mathscr{B}-B$. Also we use ' $F_{p}$ ' for the filtration in $J_{\mathscr{A}}$, which is induced from the map $\pi: \mathscr{A}\left(=\pi^{-1}(\mathscr{B})\right) \rightarrow \mathscr{B}$ (cf. (7.0) $)_{2}$ ). Note that $\# B=1$ implies that $F_{0} J_{\tilde{\mathscr{q}}} \cong J_{\tilde{\alpha}}$ and $F_{p} J_{\tilde{\alpha}} \cong 0(p \geqq 1)$. We then set:

$$
\left\{\begin{array}{l}
\left(E_{r, \mathscr{A}}^{p, q-p}, E_{r,|\mathscr{D}|}^{p, q-p}, E_{r, \mathscr{A}}^{p, q-q}\right):=\text { spectral sequneces of }\left(\left(C^{*}(\mathscr{A}, \mathfrak{S}), F_{p}\right),\right. \\
\left.\left(C^{*}\left(\mathscr{A}_{|\mathscr{D}|}, \mathfrak{S}\right), F_{p}\right),\left(J_{\mathscr{\mathscr { f }}}^{*}, F_{p}\right)\right) .
\end{array}\right.
$$

Lemma 7.1. Assume that

$$
E_{1,|\mathscr{P}|}^{p-1} \cong 0 \text { for each } 0 \leqq t<q-p \text { and } \mathscr{D} \in \tilde{\mathscr{A}}^{(t+1)} .
$$

Then we have the isomorphism:

$$
E_{1, \mathscr{\infty}}^{p, q-p} \cong E_{1, \tilde{q}}^{0, q-p}
$$

Proof. First, from that $\# \pi \mathscr{A}=p+1$ and $\# \pi \mathscr{A}_{|\mathscr{D}|}=p$, we have: $F_{\tilde{p}} C^{*}\left(\mathscr{A}_{\mathscr{D}}, \mathfrak{\Omega}\right)$ $\cong 0(\tilde{p}>p)$ and $F_{\tilde{p}} C^{*}\left(\mathscr{A}_{\mathscr{Q}}, \mathfrak{R}\right) \cong 0(\tilde{p}>p-1)$. This implies:

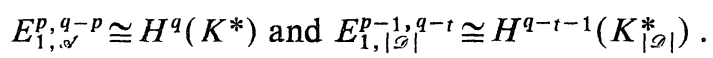

(For this also recall that $K^{*}=F_{p} C^{*}(\mathscr{A}, \mathfrak{\Omega})$ and $K_{|\mathscr{D}|}^{*}=F_{p} C^{*}\left(\mathscr{A}_{|\mathscr{D}|}, \mathfrak{R}\right)$ (cf. $(7.0)_{3}$ and $(7.2)_{3}$.) Moreover, by Proposition $7.2_{1}$, we see that (a) implies the following:

$$
(7.2)_{7}^{\prime} \longrightarrow E_{1}^{\prime t, q-t} \cong 0(0 \leqq t<p)
$$

and, by the degeneracy theorem (applied to $E_{1}^{\prime t, *}$-terms) and by Proposition $7.2_{2}$, we have:

$$
H^{q}\left(K^{*}\right)\left(\cong E_{1, \propto}^{p, q-p}\right) \cong E_{1, \alpha}^{\prime q-p, p}\left(\cong H^{q-p}\left(J_{\mathscr{\alpha}}^{*}\right)\right) .
$$

Finally, remarking that $F_{p} J_{\mathscr{q}}^{*} \cong 0(p \geqq 1)$ implies: $H^{q-p}\left(J_{\mathscr{A}}^{*}\right) \cong E_{1, \mathscr{d}}^{0, q-p}$, we have $(7.2)_{7}$.

q.e.d.

Corollary 7.2. If $E_{1, \mathscr{q}}^{0, q-p} \cong 0$, then $E_{1, \propto}^{p, q-p} \cong 0$.

Lemma 7.1 and Corollary 7.2 insure that the $E_{1}^{p}$-terms for the original complex $C^{*}(\mathscr{A}, \mathfrak{R})$ is 'determined' by the $E_{1}^{p-1}$-and $E_{1}^{0}$-terms for $C^{*}\left(\mathscr{A}_{|\mathscr{D}|}, \mathfrak{R}\right)$ and for $J_{\mathscr{A}}^{*}$, and will play a basic role in getting our sharpening of the degerency 
theorem in Part B*). In n.4, n.5, we give p.g. versions of Lemma 7.1. Such facts will be basic in the proof of Lemma 6.1-Lemma 6.3 (cf. n.3, Part B).

4. Reduction maps for localization $\mathscr{A}_{\mathscr{D}}$. Here we develop similar arguments to $\mathrm{n} .3$ for the localization $\mathscr{A}_{\mathscr{D}}$ (cf. $\left.(7.2)_{1}\right)$. In $\mathrm{n} .4$ we assume that $\mathscr{A}$ consists of elementary figures in $\mathbb{C}^{n}(z)$ (cf. $\S 6.1$ ). We make this assumption for some technical reasons*) and, more importantly, for purpose of the applications to the proof of Lemma 6.1-Lemma 6.3 (cf. Part B, §7). We also assume that $(7.3)_{0}$ the ratio number a of $\mathscr{O}<\infty$ (cf. $\left.(6.10)_{3}\right)$.

We begin $n .4$ by giving a proposition on elementary properties of elementary figures, which we use in the later arguments:

Proposition 7.3. $\cdot$ (1) If $A_{i} \in \mathscr{A}(1 \leqq i \leqq 3)$ satisfy: $A_{i} \cap A_{j} \neq \phi(1 \leqq i, j \leqq 3)$, then $\cap_{i=1}^{3} A_{i}=\phi$. Moreover, if $A_{1} \cap A_{2} \neq \phi$, then we have:

$(7.3)_{1} \quad A_{1, m} \supset A_{2, m^{\prime}}$ for an! $m, m^{\prime} \in \mathbb{R}_{1}^{+}$satisfying $m>3 m^{\prime} a$.

(2) For the maps res ${ }_{\mathscr{D}}^{-1}$ and $\theta_{\mathscr{D}}\left(c f .(7.2)_{2,3}\right)$, we have:

(7.3) $)_{1}^{\prime \prime} \quad\left\{\begin{array}{l}\operatorname{res}_{\mathscr{D}}^{-1}: \mathcal{V}_{p}^{q q}\left(\mathscr{A}_{|\mathscr{Q}|}\right) \longrightarrow \mathscr{A}_{p}^{q}\left(\mathscr{A}_{\mathscr{D}}\right) \text { is bijective } \\ \theta_{\mathscr{D}}: \mathscr{N}_{p}^{q-i}\left(\mathscr{A}_{\mathscr{D}}\right) \ni \mathscr{A}_{\mu}^{\prime} \longrightarrow \mathscr{N}_{p+1}^{q+1}(\mathscr{A}) \ni \mathscr{A}_{\mu} \text { satisfies }:\left|\mathscr{A}_{\mu m}\right| \supset\left|\mathscr{A}_{\mu m^{\prime}}^{\prime}\right| .\end{array}\right.$

(In (7.3) $)_{1, m}$ : = elementary figure with the same center as $A_{1}$ and with the size $=m \times\left(\right.$ size of $\left.A_{1}\right)\left(c f .(6.4)_{1}^{\prime}\right)$. Also, writing $\mathscr{A}=\left\{A_{\mu}\right\}_{\mu}$, we recall that $\mathscr{A}_{m}=\left\{A_{\mu m}\right\}_{\mu}\left(c f .(6.10)_{2}^{\prime \prime}\right)$.)

Proof. (1) is very elementary, while (2) follows easily from (1).

In the arguments soon below, we define reduction and reverse maps for $\mathscr{A}_{\mathscr{D}}$ from those maps for $\mathscr{A}_{\mid \mathscr{}}$ (Definition 7.3). The bijectivity in (7.3)" is used in the definition. The second condition is also used, as a technical convenience, in such a definition (cf. Proposition 7.3 soon below).

Next we assume that the aheaf $\Omega$ is the structure sheaf $\mathfrak{D}$ of $\mathbb{C}^{n}$. We use the 'principle of analytic continuation' for $\mathfrak{D}$ in the definition mentioned just above. Taking an element $m \in \mathbb{R}_{1}^{+}$, our complex will be of the following form: $(7.3)_{0}^{\prime \prime} \quad C^{*}(\mathscr{A}, \mathfrak{D})^{m}\left(=i_{m}^{*} C^{*}\left(\mathscr{A}_{m}, \mathfrak{D}\right)\right)\left(\subset C^{*}(\mathscr{A}, \mathfrak{D})\right)\left(\mathrm{cf} .(6.10)_{2}\right)$, with the refining map $i_{m}: \mathscr{A} \ni A_{\mu} \rightarrow \mathscr{A}_{m} \in A_{\mu m}$.

We use the symbol $F_{p}$ also for the filtration in $C^{*}(\mathscr{A}, D)^{m}$, which is induced from the map $\pi: \mathscr{A} \rightarrow \mathscr{B}$. In a concordant manner to $(7.0)_{3},(7.1)_{4}$, we define:

*) cf. Proposition 7.3 - Proposition 7.3. 
$(7.3)_{0}^{\prime \prime \prime}$

$$
\begin{aligned}
K^{*}(m):=F_{p} C^{*}(\mathscr{A}, \mathfrak{D})^{m}, K_{t \mid \mathscr{D}}^{*}(m):=K_{t \mid \mathscr{D}}^{*} \cap K^{*}(m), \\
\text { and } K_{\mathscr{D}}^{*}(m):=F_{p-1} C^{*}\left(\mathscr{A}_{\mathscr{D}}, \mathfrak{D}\right)^{m} .
\end{aligned}
$$

(We use the symbol $F_{p-1}$ also for the filtration in $C^{*}\left(\mathscr{A}_{\mathscr{D}}, \mathfrak{D}\right)^{m}$, induced from $\pi_{\mathscr{D}}: \mathscr{A}_{\mathscr{D}} \rightarrow \mathscr{B}-B$ (cf. n.2).)

Now take elements $m, m^{\prime}$ and $m^{\prime \prime} \in \boldsymbol{R}_{1}^{+}$satisfying:

$$
m>3 m^{\prime} \cdot a \text {, and } m^{\prime \prime}>3 m^{\prime} a \text {. }
$$

We then have the following analogues of Proposition 7.2 and Definition 7.3:

Proposition 7.3. . For each $\mathscr{D} \in \tilde{\mathscr{A}}^{(t+1)}(t \geqq 0)$, there are homomorphisms

$$
\left\{\begin{array}{l}
\theta_{\mathscr{D}}^{*}: K_{t \mid \mathscr{D}}^{*}(m) \longrightarrow K_{\mathscr{P}}^{*-t-1}\left(m^{\prime}\right) \\
\omega_{\mathscr{D}}^{*}: K_{\mathscr{D}}^{*-t-1}(m) \longrightarrow K_{t \mid \mathscr{D}}^{*-t}\left(m^{\prime}\right)
\end{array}\right.
$$

which are characterized by

Figure IV-1, and the homomorphism $\omega_{\mathscr{D}}^{*} \cdot \theta_{\mathscr{D}}^{*}$ satisfies Figure IV-2 (and $\theta_{\mathscr{D}}^{*} \cdot \omega_{\mathscr{D}}^{*}$ satisfies the similar diagram to Figure IV $-2)$.

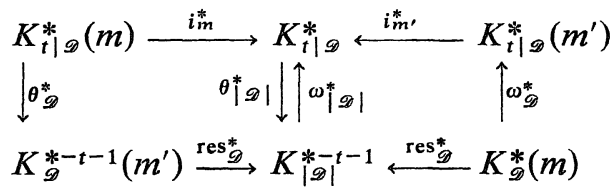

Figure IV-1.

(In Figures IV-1, IV-2, $i_{m^{\prime \prime} m}:=$ refining map: $\mathscr{A}_{m^{\prime \prime}} \ni A_{m^{\prime \prime}} \rightarrow \mathscr{A}_{m} \ni A_{m}, \ldots$, and we set $i_{m}:=i_{1 m}$.)

We call $\theta_{\mathscr{D}}^{*}$ and $\omega_{\mathscr{D}}^{*}$ also reduction and reverse maps. Moreover, as in n.3,

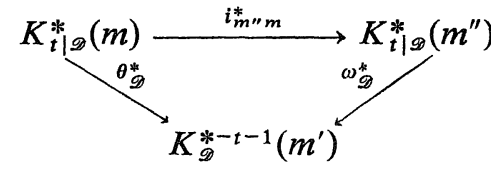

Figure IV-2. we call the homomorphisms:

$(7.3)_{3} \quad \theta_{\mathscr{D}}^{*} \cdot r_{\mathscr{D}}^{*}: K^{*}(m) \longrightarrow K_{\mathscr{P}}^{*-t-1}\left(m^{\prime}\right), i_{\mathscr{D}}^{*} \cdot \omega_{\mathscr{D}}^{*}: K_{\mathscr{D}}^{*-t-1}(m) \longrightarrow K^{*}\left(m^{\prime}\right)$, with the injection $i_{\mathscr{D}}^{*}: K_{t \mid \mathscr{D}}^{*} \hookrightarrow K^{*}$ and the restriction $r_{\mathscr{D}}^{*}: K^{*} \rightarrow K_{t \mid \mathscr{D}}^{*}$, also reduction and reverse maps (and we use the symbols $\theta_{\mathscr{Q}}^{*}, \omega_{\mathscr{Q}}^{*}$ also for such maps). (Proposition 7.3 follows easily from Propositions 7.2, 7.3. Here we summarize the technical key points of the proof. First setting $\varphi^{\prime}=\theta_{\mathscr{D}}^{*}(\varphi), \tilde{\varphi}^{\prime}=\omega_{\mathscr{D}}^{*}(\tilde{\varphi})$, where $\varphi, \tilde{\varphi} \in K^{*}(m), K_{\mathscr{D}}^{*-t-1}(m)$, we remark that $\varphi^{\prime}, \tilde{\varphi}^{\prime}$ are explicitly as follows: (7.3) $)_{2} \varphi_{\mathscr{Q}_{\mu}^{\prime}}^{\prime}=\varphi_{\mathscr{A}_{\mu}}, \tilde{\varphi}_{\mathscr{A}_{\mu}}^{\prime}=\tilde{\varphi}_{\mathscr{A}_{\mu}^{\prime}}$ for each $\mathscr{A}_{\mu}^{\prime} \in \mathscr{N}_{p}^{q-t}\left(\mathscr{A}_{\mathscr{D}}\right)$, with $\mathscr{A}_{\mu}=\theta_{\mathscr{D}}\left(\mathscr{A}_{\mu}^{\prime}\right)$. (We understand that $\varphi_{\mathscr{S}_{\mu}^{\prime}}^{\prime}=$ restriction of $\varphi_{\mathscr{A}_{\mu}} \in \Gamma\left(\left|\mathscr{A}_{\mu m}\right|, \mathfrak{D}\right)$ to $\Gamma\left(\left|\mathscr{A}_{\mu m^{\prime}}^{\prime}\right|, \mathfrak{D}\right)$ and $\tilde{\varphi}_{\mathscr{A}_{\mu}}$ should be understood similarly. Note that $\left|\mathscr{A}_{\mu}\right| \varsubsetneqq\left|\mathscr{A}_{\mu}^{\prime}\right|$ in general. But (7.3) $)_{1}$ implies: $\left.\left|\mathscr{A}_{\mu m}\right| \supset\left|\mathscr{A}_{\mu m^{\prime}}^{\prime}\right|.\right) \quad$ The existence of the maps $\theta_{\mathscr{D}}^{*}, \omega_{\mathscr{Q}}^{*}$ follows from 
$(7.3)_{2}$, while the uniqueness follows from the principle of the analytic continuation for $D$, applied to $\left|\mathscr{A}_{\mu m^{\prime}}^{\prime}\right| \subset\left|\mathscr{A}_{\mu m}\right|$.

$\mathbb{E}_{\mathbf{1}}^{\prime *}, \boldsymbol{p}$-terms. Here we give an analogue of Proposition $7.2_{2}$. We assume the following for each $\mathscr{D} \in \tilde{\mathscr{A}}^{(t+1)}$ :

$$
\mathscr{A}_{p+1 \mid \mathscr{D}}^{(q+++1)} \neq \phi\left(\text { cf. }(7.2)_{5}\right) \text {, and }\left|\left(\mathscr{A}_{\mathscr{Q}} / \pi_{\mathscr{D}}\right)_{u}\right| \text { is connected } .
$$

Corresponding to Proposition $7.2_{2}$ we write the spectral sequence of $\left(K^{*}(m), G_{t}\right)$ as $E_{r}^{\prime t, *-t}(m)$.

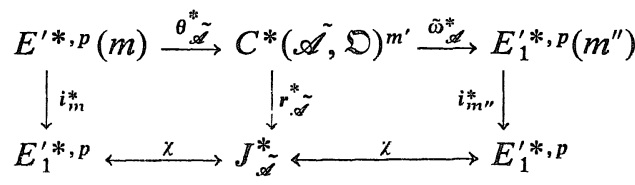

Proposition $7.3_{2}$. There

Figure IV-3.

are homomorphisms:

$$
\left\{\begin{array}{l}
\theta_{\tilde{A}}^{*}: E_{1}^{\prime *, p}(m) \longrightarrow C^{*}(\tilde{\mathscr{A}}, 0)^{m^{\prime}} \\
\omega_{\tilde{A}}^{*}: C^{*}(\tilde{\mathscr{A}}, \mathfrak{D})^{m} \longrightarrow E_{1}^{\prime *, p}\left(m^{\prime \prime}\right)
\end{array}\right.
$$

characterized by Figure IV-3. Moreover, $\omega_{\mathscr{\mathscr { A }}}^{*} \cdot \theta_{\mathscr{\mathscr { q }}}^{*}$ satisfies Figure IV-4 (and $\theta_{\mathscr{\mathscr { A }}}^{*} \cdot \omega_{\mathscr{\mathscr { A }}}^{*}$

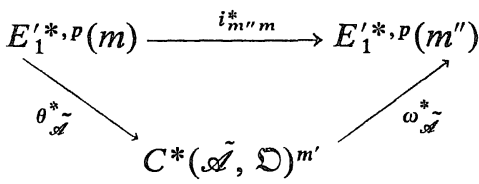

Figure IV-4. satisfies the similar diagram.)

We call $\theta_{\mathscr{A}}^{*}, \omega_{\mathscr{\mathscr { A }}}^{*}$ also reduction and reverse maps. (The map $\chi$ just above is as in Proposition 7.22. The map $r_{\tilde{\mathscr{A}}}^{*}$ is a natural map given soon below.) Proposition 7.3 follows easily from Proposition 7.2. Here we also summarize technical key points in the check of Proposition $7.3_{2}$ : first recall that $J_{\mathscr{A}}^{t}=$ $\left.\oplus_{\mathscr{D}} J_{\mathscr{D}}\left(=\Gamma\left(\left|\mathscr{A}_{\mathscr{D}}\right| \pi_{\mathscr{O}}\right)_{u} \mid, \mathfrak{D}\right)\right)\left(\mathrm{cf} .(7.2)_{5}\right)$, and we take $r_{\mathscr{\mathscr { A }}}^{*}$ to be the induced from the restriction: $\Gamma(|\mathscr{D}|, \mathfrak{D}) \rightarrow J_{\mathscr{D}}$. Thus, for an element $\varphi \in E_{1}^{\prime *, p}(m)$, the value of $\varphi^{\prime}=\theta_{\mathscr{A}}(\varphi)$ on $\mathscr{D}$ is the restriction of $\varphi_{\mathscr{D}, \mathscr{A}^{\prime}}$ to $\left|\mathscr{D}_{m^{\prime}}\right|$, with an arbitrary $\mathscr{A}^{\prime}$ $\in \mathscr{N}_{p}^{p}\left(\mathscr{A}_{\mathscr{Q}}\right)$. (For convenience of the arguments, we understood that the order of $\mathscr{A}^{\prime}$ is concordant to $\pi\left(\mathscr{A}^{\prime}\right)=\mathscr{B}^{\prime}-B^{\prime}$.) By the first condition*) in (7.3) 3 such an element $\mathscr{A}^{\prime}$ exists*). Also by the second ${ }^{*)}, \theta_{\mathscr{Q}}^{*}$ is independent of the choice of such an element $\mathscr{A}^{\prime}$. The definition of $\omega_{\mathscr{A}^{\prime}}^{*}$ and Figure IV-4 follow easily from Proposition 7.2.

Finally, we give an analogue of Lemma 7.1. For this we set:

$(7.3)_{5}^{\prime} \quad\left(E_{r, \&}^{p, *-p}(m), E_{r, 2}^{p, *-p}(m), E_{r, \mathscr{A}}^{p, *-p}(m)\right):=$ spectral sequence of $\left(\left(K^{*}(m)\right.\right.$, $\left.\left.F_{p}\right),\left(K_{\mathscr{D}}^{*}(m), F_{p}\right),\left(C^{*}(\mathscr{A}, \mathfrak{D})^{m}, F_{p}\right)\right)$,

*) For the existence of such an element $\mathscr{A}^{\prime}$, see also the footnote to (7.2) . Let $\mathscr{A}^{\prime}, \mathscr{A}^{\prime \prime}$ be such elements. Then the connectivity condition in (7.3) ${ }_{3}$ implies the existence of such elements $\left\{\mathscr{A}_{u}^{\prime}\right\}_{u=1}^{v}$, with $\mathscr{A}_{1}^{\prime}=\mathscr{A}^{\prime}, \mathscr{A}_{v}^{\prime}=\mathscr{A}^{\prime \prime}$ and $\left|\mathscr{A}_{u}^{\prime}\right| \cap\left|\mathscr{A}_{u+1}^{\prime}\right| \neq \phi$. The welldefinedness of $\theta_{\mathscr{D}}^{*}$ follows from this. 
where the filtration $F_{p}$ in $K^{*}(m), K_{\mathscr{D}}^{*}(m)$ and $C^{*}(\tilde{\mathscr{A}}, \mathfrak{D})^{m}$ is the one induced from $\pi: \mathscr{A} \rightarrow \mathscr{B}, \pi_{\mathscr{D}}: \mathscr{A}_{\mathscr{D}} \rightarrow \mathscr{B}-B$ and $\pi: \tilde{\mathscr{A}}=\pi^{-1}(B) \rightarrow B$ (as in n.3).

Now taking elements $c, d \in \boldsymbol{R}_{1}^{+2}$, we assume the following for each $0 \leqq t$ $<q-p$ and $\mathscr{D} \in \tilde{A}^{(t+1)}$ :

(7.3) $)_{6}^{\prime} \quad i_{m^{\prime}, m}^{*} E_{1, \mathscr{D}}^{p-1, q-p-t}(m) \cong 0$, where $m, m^{\prime} \in \boldsymbol{R}_{1}^{+}$satisfy: $m>c a, m^{\prime}=m / d a$.

Lemma 7.1'. There are homomorphisms:

$(7.3)_{6}\left\{\begin{array}{l}\theta_{\tilde{\mathscr{q}}}^{*}: E_{1, \mathscr{q}}^{p, q-p}(\tilde{m}) \longrightarrow E_{1, \mathscr{q}}^{0, q-p}\left(\tilde{m}^{\prime}\right) \\ \omega_{\tilde{\mathscr{q}}}^{*}: E_{1, \tilde{\mathscr{q}}}^{0, q-p}(\tilde{m}) \longrightarrow E_{1, \mathscr{q}}^{p, q-p}\left(\tilde{m}^{\prime}\right),\end{array}\right.$

satisfying Figure IV-5 (and the similar d'a Jram to $\left.\theta_{\mathscr{\mathscr { A }}}^{*} \cdot \omega_{\mathscr{\mathscr { A }}}^{*}\right)$. Here the elements $\tilde{m}, \tilde{m}^{\prime}$ and $\tilde{m}^{\prime \prime} \in \boldsymbol{R}_{1}^{+}$satisfy:

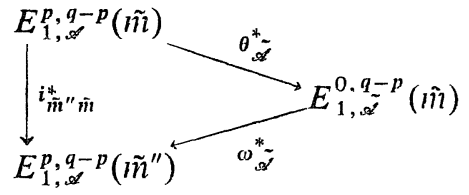

Figure IV-5.

(7.3)" $\quad \tilde{m}>c_{q, p} a, \tilde{m}^{\prime}=\tilde{m} / d_{q, p} \cdot a$ and $\tilde{m}^{\prime \prime}=\tilde{m}^{\prime} / d_{q, p} \cdot a$, where

$(7.3)_{6}^{\prime \prime \prime} \quad c_{q, p}=\mathscr{L}_{q, p}(c), d_{q, p}=\mathscr{L}_{q, p}(d)$, with an el-map $\mathscr{L}_{q, p}$, which depends only on $(q, p)$ (cf. n.5, § 1.1).

Proof. The algebraic structure of the implication: Proposition $7.3_{1,2} \rightarrow$ Lemma $7.1^{\prime}$ is parallel to the proof of Lemma 7.1. The condition on the elements $\tilde{m}, \ldots$ is checked, by recalling that we imposed the condition $(7.3)_{2}^{\prime}$ in Proposition $7.3_{1,2}$.

q.e.d.

Corollary 7.2. Assume that

$$
i_{m^{\prime} m}^{*} E_{1, \mathscr{A}}^{0, q-p}(m) \cong 0, \text { where } m, m^{\prime} \in \boldsymbol{R}_{1}^{+} \text {are as in }(7.3)_{6} \text {. }
$$

Then we have:

$$
i_{\tilde{m}^{\prime}, \tilde{m}}^{*} E_{1, q}^{p, q-p}(\tilde{m}) \cong 0
$$

5. A p.g. version of Lemma 7.1'. Here we give a p.g. version of Lemma 7.1 in a convenient form for the later arguments: for a subset $\mathscr{A}^{\prime}$ of $\mathscr{A}$, an open set $D^{\prime} \subset \boldsymbol{C}^{n^{\prime}}\left(z^{\prime}\right)$ (cf. §6) and an element $\alpha \in \boldsymbol{R}_{1}^{+2}$, we use the sets of the cochains*) $Z_{1}^{p, q-p}(\mathscr{A} \times D, D)_{\alpha}^{m}$. (For such sets of cochains, see $(6.10)_{1,2}$.) Next, for convenience of our explicit estimations, we take an $s$-times product $\boldsymbol{R}_{1}^{+s}$ of $\boldsymbol{R}_{1}^{+}$, and we fix a collection $E$ consisting of maps: $\boldsymbol{R}_{1}^{+s} \times \boldsymbol{R}_{1}^{+2} \rightarrow \mathbb{R}_{1}^{+2}$. (We permit the case $s=0$, or equivalently, the case $\boldsymbol{R}_{1}^{+s} \times \boldsymbol{R}_{1}^{+2}=\boldsymbol{R}_{1}^{+2}$.) For maps $E_{\lambda}, E_{\mu} \in \boldsymbol{E}$, we write $E_{\lambda} \succ E_{\mu}$, if $E_{\lambda}(\beta ; \alpha)>E_{\mu}(\beta ; \alpha)$ for any $(\beta ; \alpha) \in \boldsymbol{R}_{1}^{+s} \times \boldsymbol{R}_{1}^{+2}$. Moreover, we

*) The filtration here is induced from the map: $\mathscr{A} \times D^{\prime} \ni\left(A \times D^{\prime}\right) \rightarrow \mathscr{B} \ni \pi(A)$, 
define a composition $E_{v}:=E_{\lambda} \cdot E_{\mu}$ by

$$
E_{v}: \mathbb{R}_{1}^{+s} \times \mathbb{R}_{1}^{+2} \ni(\beta ; \alpha) \longrightarrow \mathbb{R}_{1}^{+2} \ni \alpha^{\prime}:=E_{\lambda}\left(\beta ; E_{\mu}(\beta ; \alpha)\right) .
$$

Next we fix a map $\eta_{1}: \mathbb{E} \times \mathbb{E} \ni\left(E_{\mu}, E_{v}\right) \rightarrow \mathbb{E} \ni E_{\lambda}$ satisfying $E_{\lambda} \succ E_{\mu} \cdot E_{\nu}$. We then define maps $\eta_{p}(p \geqq 2)$ inductively as follows ${ }^{*}$ :

$$
\eta_{p}: \mathbb{E}_{p+1}=\mathbb{E}^{p} \times \mathbb{E} \ni\left(E_{\mu}, E_{v}\right) \longrightarrow \mathbb{E} \ni E_{\lambda}:=\eta_{1}\left(\eta_{p-1}\left(E_{\mu}\right), E_{v}\right) .
$$

For each $p \geqq 1$ we define a map:

$$
\tilde{\eta}_{p}: \mathbb{E} \ni E \longrightarrow \mathbb{E} \ni E^{\prime}:=\eta_{p}(\underbrace{E, \ldots, E}_{p+i}) .
$$

Also, for convenicnce of the estimation here, we fix an element $\beta=\beta_{\gamma^{*}} \in \mathbb{R}_{1}^{\prime-2}$. Now we assume the following for each $0 \leqq t<q-p$ and $\mathscr{D} \in \tilde{\mathscr{A}}^{(t+1)}$ :

$$
Z_{1}^{p-1, q-p-t}\left(\mathscr{A}_{\mathscr{G}} \times D^{\prime}, \mathfrak{D}\right)_{\alpha}^{m} \subset B_{0}^{p-1, q-p-t}\left(\mathscr{A}_{\mathscr{A}} \times D^{\prime}, \mathfrak{D}\right)_{\alpha^{\prime}}^{m \prime}, \text { with } \alpha^{\prime}=E(\beta ; \alpha) \text {. }
$$

where $\alpha$ is in $\mathbb{R}_{1}^{+2}$, and $m, m^{\prime} \in \mathbb{R}_{1}^{+}$satisfy: $m>c a, m^{\prime}=m / d a$ (cf. Lemma 7.1').

Lemma 7.1". The reduction and reverse maps*) $\theta_{\mathscr{\alpha}}^{*}$ and $\omega_{\tilde{\alpha}}^{*}$ preserve the p.g. conditions:

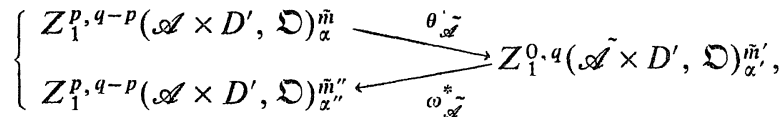

$$
\begin{aligned}
& \text { with }\left\{\begin{array}{l}
\tilde{n}^{\prime}=\tilde{m} / d_{q, p} a, \tilde{n}^{\prime \prime}=\tilde{n}^{\prime} / d_{q, p} a \\
\alpha^{\prime}=E_{q, p}(\beta ; \alpha), \alpha^{\prime \prime}=E_{q, p}\left(\beta ; \alpha^{\prime}\right),
\end{array}\right.
\end{aligned}
$$

and also satisfy:

$$
\left(1-\omega_{\mathscr{q}}^{*} \theta_{\mathscr{\mathscr { A }}}^{*}\right) Z_{1}^{p, q-p}\left(\mathscr{A} \times D^{\prime}, \mathfrak{D}\right)_{\alpha}^{\tilde{m}} \subset B_{0}^{p, q-p}\left(\mathscr{A} \times D^{\prime}, \mathcal{O}\right)_{\sigma^{\prime \prime \prime}}^{\tilde{m}^{\prime \prime}}
$$

(and a similar inclusion to $\theta_{\tilde{\alpha}}^{*} \cdot \omega_{\tilde{\alpha}}^{*}$ ). In the above, the element $\alpha_{p, q} \in \mathbb{R}_{1}^{+2}$ is as in Lemma 7.1, and the map $E_{q, p}$ is as follows:

(7.4) $)_{6}^{\prime \prime} \quad E_{q, p}=\tilde{\eta}_{e}(E)$, with an element $e=e_{q, p} \in Z^{+}$, which depends only on $(q, p)$.

The proof of Lemma 7.1" is given similarly to Lemma 7.1', and is omitted.

Our main application of Lemma 7.1" will be given in Part B (cf. Lemma

7.5). Here we derive a kcy proposition for the proof of Lemma 6.1:

A key proposition for Lemma 6.1. Let the collection $\mathscr{A}_{k^{\prime}}\left(\mathbb{C}^{n}\right)$ of elementary figures be as in Theorem 6.1, and the map $\pi_{k^{\prime}}: \mathscr{A}_{k^{\prime}}\left(\mathbb{C}^{n}\right) \rightarrow \mathscr{B}_{k^{\prime}}=\mathscr{A}_{k^{\prime}}\left(\mathbb{C}^{n-1}\right)$ as well as the family $\mathscr{F}_{p+1}^{\prime 0} \mathscr{A}_{k}(p \geqq 1)$ be as in $(6.7)_{5}$ and (6.9) . Taking an element $\mathscr{A}_{\mu}^{\prime}$

*) Here we assume that $E$ is closed under the composition in the sense that if $E_{\mu} E_{\nu} \in \mathbb{E}$, then there is a map $E_{\lambda} \in \mathbb{E}$ satisfying $E_{\lambda}>E_{\mu} E_{\nu}$. 
$\in \mathscr{F}_{p+1}^{\prime 0} \mathscr{A}_{k^{\prime}}$, we set:

$$
\mathscr{B}_{\mu}^{\prime}:=\pi_{\mu}\left(\mathscr{A}_{\mu}^{\prime}\right) \text {, with the restriction } \pi_{\mu} \text { of } \pi_{k} \text { to } \mathscr{A}_{\mu}^{\prime} \text {, }
$$

and we fix an order $\prec$ of $\mathscr{B}_{\mu}^{\prime}$, and we set $\tilde{\mathscr{A}}_{\mu}^{\prime}:=\pi^{-1}\left(B_{\mu}^{\prime}\right)$, with the maximal element $B_{\mu}^{\prime}$ of $\mathscr{B}_{\mu}^{\prime}$.

Proposition 7.4. . Take elements*) $m_{n}, d_{n} \in \boldsymbol{R}_{1}^{+}$and an el-map $\mathscr{L}_{n, h}$. Then there are maps $\theta^{*}, \omega^{*}$ :

$$
\left\{\begin{array}{l}
Z_{1}^{p, q-p}\left(\mathscr{A}_{\mu}^{\prime} \times D^{\prime}, \mathfrak{D}\right)_{\alpha}^{m_{n}}-\theta^{*} \\
Z_{1}^{p, q-p}\left(\mathscr{A}_{\mu}^{\prime} \times D^{\prime}, \mathfrak{D}\right)_{\alpha \prime \prime}^{m \prime \prime} \\
\text { with }\left\{\begin{array}{l}
m^{\prime}=m_{n} / d_{n}, m^{\prime \prime}=m^{\prime} / d_{n} \\
\alpha^{\prime}=L_{n, k}(\alpha), \alpha^{\prime \prime}=L_{n, k}\left(\alpha^{\prime}\right),
\end{array}\right.
\end{array}\right.
$$

which satisfy:

$(7.5)_{2} \quad\left(1-\omega^{*} \cdot \theta^{*}\right) Z_{1}^{p, q-p}\left(\mathscr{A}_{\mu}^{\prime} \times D^{\prime}, \mathfrak{D}\right)_{\alpha}^{m_{n}} \subset B_{0}^{p, q-p}\left(\mathscr{A}_{\mu}^{\prime} \times D^{\prime}, \mathfrak{D}\right)_{\alpha^{\prime \prime}}^{m^{\prime \prime}}($ and the similar relation for $\left.\theta^{*} \cdot \omega^{*}\right)$.

Proof. Here we take the set of parameters $\left(\boldsymbol{R}_{1}^{+s} \times \boldsymbol{R}_{1}^{+2}\right)$ and the collection $E$ of estimation maps in Lemma $7.1^{\prime \prime}$ to be: $\left(\boldsymbol{R}_{1}^{+s} \times \boldsymbol{R}_{1}^{+2}\right)=\boldsymbol{R}_{1}^{+2}$ and $E:=$ set of all el-maps**) $\boldsymbol{R}^{+2} \rightarrow \boldsymbol{R}^{+2}$. Then, by Lemma 7.1", it suffices to check the following***) for each $0 \leqq t<q-p$ and $\mathscr{D} \in \tilde{\mathscr{A}}_{\mu}^{\prime(t+1)}$ for the proof of $(7.5)_{1,2}$ :

$$
Z_{1}^{p-1, q-p-t}\left(\mathscr{A}_{\mu \mathscr{D}}^{\prime} \times D^{\prime}, \mathfrak{D}\right)_{\alpha}^{m_{n}} \subset B_{0}^{p-1, q-p-t}\left(\mathscr{A}_{\mu \mathscr{D}}^{\prime} \times D^{\prime}, \mathfrak{D}\right)_{\alpha^{\prime}}^{m^{\prime}},
$$

where the estimation: $\left(m_{n}, \alpha\right) \rightarrow\left(m^{\prime} ; \alpha^{\prime}\right)$ is as in $(7.5)_{1}$.

To check this, we remark that the localization $\mathscr{A}_{\mu \supset}^{\prime}$ is a finite set, and is, moreover, $\mathscr{A}_{\mu \mathscr{D}}^{\prime}$ is written as:

(a) $\mathscr{A}_{\mu \mathscr{D}}^{\prime}$ is in $\mathscr{F}_{p}^{0} \mathscr{A}_{\lambda_{\mu}}$ (cf. (6.8) $)_{4}$ ), with a finite elementary covering $\mathscr{A}_{\lambda_{\mu}}^{\prime}$ $\in \operatorname{Cov}_{0}\left(\boldsymbol{C}^{n}\right)_{\mathrm{el}}\left(\mathrm{cf} .(6.12)_{2}\right)$, which consists of elements of $\mathscr{A}_{k^{\prime}}$.

We give here a proposition, which is applied to the finite collection $\mathscr{A}_{\mu \mathscr{D}}^{\prime}$ : let the collection $\boldsymbol{A}^{n}\left(=\operatorname{Cov}_{0}\left(\boldsymbol{C}^{n}\right)_{\mathrm{el}}\right), \boldsymbol{B}^{n}=\left\{\boldsymbol{B}_{\lambda}\right\}_{\lambda}$ and $\pi^{n}=\left\{\pi_{\lambda}\right\}_{\lambda}$ be as in (6.12)' Then we have the following for each $\mathscr{A}_{\mu} \in \mathscr{F}^{0} \mathscr{A}_{k}\left(p \geqq 1\right.$ ) (cf. $\left.(6.8)_{4}\right)$ :

Proposition 7.4 $\cdot Z_{1}^{p, q-p}\left(\mathscr{A}_{\mu} \times D^{\prime}, \mathfrak{D}\right)_{\alpha}^{m} \subset B_{0}^{p, q-p}\left(\mathscr{A}_{\mu} \times D^{\prime}, \mathfrak{D}\right)_{\alpha^{\prime}}^{m^{\prime}}$, where $\alpha^{\prime}=E_{n}\left(l_{\mu} / r_{\mu}^{-} ; m r_{\mu}^{+} ; \alpha\right)$. (Here the open set $D^{\prime} \subset C^{n^{\prime}}$, the element $(m ; \alpha)$

*) $m_{n}, d_{n}$ and $\mathscr{L}_{n, k}$ depend, respectively, only on $n=\operatorname{dim} \boldsymbol{C}^{n}$ and $(n, k)$.

**) Cf. n.5, Section 1.1.

***) $\mathscr{A}_{\mu \mathscr{Q}}^{\prime}=$ localization of $\mathscr{A}_{\mu}^{\prime}$ (Definition 7.2). 
$\in \boldsymbol{R}_{1}^{+} \times \boldsymbol{R}_{1}^{+2}$ and the estimation map $E_{n}$ have the similar meaning to Lemma 6.4.)

Proposition $7.4_{2}$ follows directly from our algebraic result*), Lemma 7.4 in Part B, Section 7, which is proven independently from the content here concerning Lemma 6.1. Our use of Lemma 7.4 for Proposition $7.4_{2}$ is legitimate. Now letting the localization $\mathscr{A}_{\mu \mathscr{Q}}^{\prime}$ be as in $(\mathrm{a})_{1}$, the numerical invariants $l_{\mu \mathscr{O}}^{\prime}$, $r_{\mu \supset}^{\prime \pm}$ of $\mathscr{A}_{\mu \curvearrowright}^{\prime}$ (cf. (6.12) $)_{0}^{\prime \prime \prime}$ ) satisfy:

(a) ${ }_{2} l_{\mu \mathscr{D}}^{\prime}, r_{\mu \mathscr{D}}^{\prime+}$ and $1 / r_{\mu \mathscr{I}}^{\prime}<c_{n, k}$, with an element $c_{n, k} \in \mathbb{R}_{1}^{+}$, which depends only on $(n, k)$. (This is checked easily by remarking that the radius of $|\mathscr{D}|$ is estimated similarly to $(a)_{2}$ and by recalling the explicit form of the localization $\mathscr{A}_{\mu \rightarrow}^{\prime}$ (cf. Definition 7.2).)

We then apply Proposition $7.4_{1}$ to $A_{\mu \mathscr{D}}^{\prime}$. Then we have the similar inclusion to $(7.5)_{3}$, by the following change of the estimation:

(a) $\tilde{\alpha}_{3}=E_{n}\left(l_{\mu \Omega}^{\prime} / r_{\mu \mathscr{\Omega}}^{\prime} ; m_{n} r_{\mu \Omega}^{\prime+} ; \alpha\right)$, with the estimation map $E_{n}$ as in Proposition $7.4_{2}$.

But, by $(\mathrm{a})_{2}$ and the explicit form of the map $E_{n}$ (cf. Definition 6.5), we easily see that the element $\alpha^{\prime}$ in $(7.5)_{3}$ satisfies: $\alpha^{\prime} \succ \tilde{\alpha}^{\prime}$, and we have $(7.5)_{3}$. Finally, by Lemma $7.1^{\prime \prime}$, we know that $(7.5)_{3}$ insures $(7.5)_{1,2}$, and we have Proposition $7.4_{1}$.

q.e.d.

Next we derive a key proposition for Lemma 6.1 from Proposition $7.4_{1}$. For this letting the refining map $s: \mathscr{A}_{k^{\prime}} \times D^{\prime} \hookrightarrow \mathscr{A}_{k} \times D^{\prime}$ be as in Lemma $6.1^{n}$, we also take a suitable $k^{\prime \prime} \in Z^{+}$and a refining map $s^{\prime}: \mathscr{A}_{k^{\prime \prime}} \times D^{\prime} \hookrightarrow \mathscr{A}_{k^{\prime}} \times D^{\prime}$, where we set $\mathscr{A}_{k^{\prime \prime}}:=A_{k^{\prime \prime}}\left(\boldsymbol{C}^{n}\right)$. We then take an element $\mathscr{A}_{\mu}^{\prime \prime} \in F_{p^{+1}}^{\prime 0} \mathscr{A}_{k^{\prime \prime}}$ (cf. (6.9) $)_{1}$ ), and we set $\mathscr{A}_{\mu}^{\prime} \times D^{\prime}=s_{\mu}^{\prime}\left(\mathscr{A}_{\mu}^{\prime \prime} \times D^{\prime}\right), \mathscr{A}_{\mu} \times D^{\prime}=s_{\mu}\left(\mathscr{A}_{\mu}^{\prime} \times D^{\prime}\right)$, with the restrictions $s_{\mu} s_{\mu}^{\prime}$ of $s^{\prime}, s$ to $\mathscr{A}_{\mu}^{\prime \prime} \times D^{\prime}, \mathscr{A}_{\mu}^{\prime} \times D^{\prime}$. Also, letting the map $\pi_{\tilde{k}}: \mathscr{A}_{\tilde{k}} \rightarrow \mathscr{B}_{\tilde{k}}:=\mathscr{A}_{\tilde{k}}$ $\left(\boldsymbol{C}^{n-1}\right)$, where $k=k, k^{\prime}$ or $k^{\prime \prime}$, by the one defined by $(6.7)_{5}$, we set:

(b) 1

$\left\{\begin{array}{l}\pi_{\mu}, \pi_{\mu}^{\prime} \text { and } \pi_{\mu}^{\prime \prime}=\text { restriction of } \pi_{\tilde{k}} \text { to } A_{\mu}, A_{\mu}^{\prime} \text { and } A_{\mu}^{\prime \prime} \\ \mathscr{B}_{\mu}, \mathscr{B}_{\mu}^{\prime} \text { and } \mathscr{B}_{\mu}^{\prime \prime}:=\pi\left(\mathscr{A}_{\mu}\right), \pi\left(\mathscr{A}_{\mu}^{\prime}\right) \text { and } \pi\left(\mathscr{A}_{\mu}^{\prime \prime}\right) .\end{array}\right.$

We fix an order of $\mathscr{B}_{\mu}^{\prime \prime}$, and we use the orders of $\mathscr{B}_{\mu}^{\prime}$,

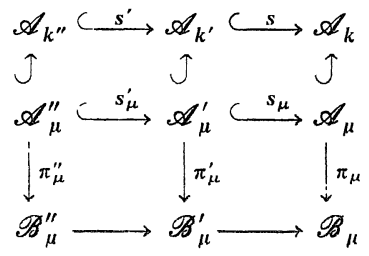

Figure $\mathrm{J}$. $\mathscr{B}_{\mu}$, which are induced from $\mathscr{B}^{\prime \prime}$ (cf. Figure I). We write the maximal elements of $\mathscr{B}_{\mu}^{\prime \prime}, \mathscr{B}_{\mu}^{\prime}$ and $\mathscr{B}_{\mu}$ as $B_{\mu}^{\prime \prime}, B_{\mu}^{\prime}$ and $B_{\mu}$, and we set

*) Precisely, 'Lemma 7.4 applied to $\left(\mathbb{A}^{n}, \mathbb{B}^{n}, \pi^{n}\right)$ '. 
$\tilde{\mathscr{A}_{\mu}^{\prime \prime}}:=\pi_{\mu}^{\prime \prime-1}\left(B_{\mu}^{\prime \prime}\right), \tilde{\mathscr{A}}_{\mu}^{\prime}=\pi_{\mu}^{\prime-1}\left(B_{\mu}^{\prime}\right)$ and $\tilde{\mathscr{A}}_{\mu}=\pi_{\mu}^{-1}\left(B_{\mu}\right)$. We assume that $(6.11)_{1}^{0, q}$ holds.

Proposition $7.4_{3}$. We have the following diagram:

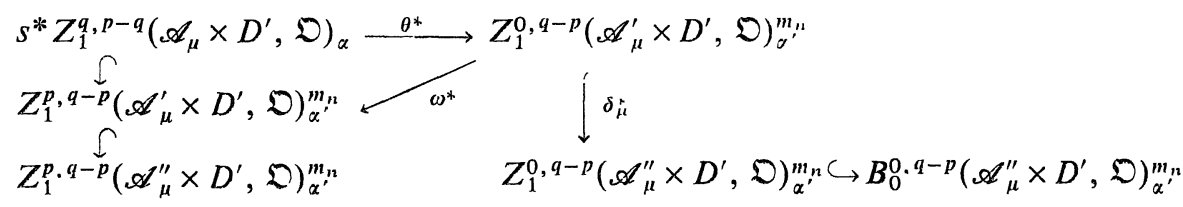

Proof. First wc easily have:

$$
s\left(A^{\prime} \times D^{\prime}\right) \supset A_{m_{n}}^{\prime} \times D^{\prime} \text { for each } A^{\prime} \in \mathscr{A}_{k^{\prime}},
$$

and we have the first diagram in (7.5) $)_{4}$ from Proposition $7.4_{1}$. By applying $(6.11)_{1}^{0, q}$ to $Z_{1}^{0, q-p}\left(\mathscr{A}_{\mu}^{\prime} \times D^{\prime}, \mathfrak{D}\right)^{m_{n}}$ (cf. (7.5) $)$, we have the diagram in the right side of $(7.5)_{+}$. The commutativity of $\omega^{*}, \omega^{\prime *}$ and $s^{\prime *}$ is easily checked, we have $(7.5)_{4}$.

q.e.d.

Now setting $\tilde{s}:=s^{\prime} \cdot s$ we have:

Corollary to Proposition $7.4_{3}$. For each $\mathscr{A}_{\mu}^{\prime \prime} \in \mathscr{F}_{p+1}^{\prime 0} \mathscr{A}_{k^{\prime \prime}}$ we have:

$$
s_{\mu}^{*} Z_{1}^{p, q-\nu}\left(\mathscr{A}_{\mu}^{\prime \prime} \times D^{\prime}, \mathfrak{D}\right)_{\alpha} \subset B_{0}^{p, q-p}\left(\mathscr{A}_{u}^{\prime \prime} \times D^{\prime}, \mathfrak{D}\right)_{\alpha^{\prime}},
$$

where we set $\tilde{s}_{\mu}:=$ restriction of $s$ to $\mathscr{A}_{\mu}^{\prime \prime}$ and $\mathscr{A}_{\mu}:=s\left(\mathscr{A}_{\mu}^{\prime \prime}\right)$. Moreover, the estimation: $\alpha \rightarrow \alpha^{\prime}$ is as in Lemma 6.1.

This covers the essential part of the proof of Lemma 6.1 (cf. B, §7).

6. Reduction condition. Let the collection $\mathscr{A}$ of elementary figures in $\mathbb{C}^{n}(z)$, the map $\pi: \mathscr{A} \rightarrow \mathscr{B}$ and the families $\mathscr{F}_{p}^{0} \mathscr{A} \subset 2^{\mathscr{A}}(p \geqq 1)$ be as in Example 6.1, n.2, Section 6.2. Take an element $\mathscr{A}_{\mu}=\mathscr{A}\left(\mathscr{B}^{\prime} ; U^{\prime}\right) \in \mathscr{F}_{p+1}^{0} \mathscr{A}$, where $\mathscr{B}^{\prime}$ is a subset of $\mathscr{B}$ satisfying $\# \mathscr{B}^{\prime}=p$ and $U^{\prime}$ is an elementary figure in $C^{n}(z)$. We then fix an order in $\mathscr{B}_{\mu}\left(=\pi_{\mu}\left(\mathscr{A}_{\mu}\right)\right)$, where $\pi_{\mu}:=$ restriction of $\pi$ to $\mathscr{A}_{\mu}$, in an arbitrary manner. Setting $B_{\mu}:=$ maximal element of $\mathscr{B}_{\mu}, \tilde{\mathscr{A}}_{\mu}:=\pi_{\mu}^{-1}\left(B_{\mu}\right)$, we easily have:

$$
\left.\tilde{\mathscr{A}}_{\mu}=\mathscr{A}\left(B_{\mu} ; U^{\prime}\right) \text {, and is in } \mathscr{F}_{1}^{0} \mathscr{A} \quad \text { (cf. }(6.8)_{4}^{\prime}\right) \text {. }
$$

Also taking an element $\mathscr{D} \in \tilde{\mathscr{A}}^{(t+1)}(t \geqq 0)$, we also easily check:

(7.6) $)_{2}$ the localization $\mathscr{A}_{\mu \mathscr{D}}$ is of the form $\mathscr{A}_{\mu \mathscr{D}}=\mathscr{A}\left(\mathscr{B}_{\mu}-B_{\mu} ; U^{\prime} \cap|\mathscr{D}|\right)$, and $\left|\left(\mathscr{A}_{\mathscr{O}} / \pi_{\mu}\right)_{u}\right|$ is connected (cf. $\left.(7.3)_{3}\right)$. 
By $(7.6)_{1,2}$, the series $\mathbb{F}^{0} \mathscr{A}:=\left\{\mathscr{F}_{p}^{0} \mathscr{A}_{p=1}^{\infty}\right.$ is closed under the localization procedure: $\mathscr{A}_{\mu} \rightarrow \mathscr{A}_{\mu \mathscr{D}}$ and the procedure: $\mathscr{A}_{\mu} \rightarrow \tilde{\mathscr{A}}_{\mu}:=\pi_{\mu}^{-1}\left(B_{\mu}\right)$. This fact, together with the second condition in $(7.5)_{2}$, makes it possible to apply the localization methods in n.1-n.5 to the series $\mathbb{F}^{0} \mathscr{A}$. Now taking a series $\mathbb{F}_{\mathscr{L}} \mathscr{A}=\left\{\mathscr{F}_{p}, \mathscr{A}\right\}_{p=1}^{\infty}$ of families $\mathscr{F}_{p} \mathscr{A} \subset 2^{\mathscr{N}}$ satisfying $(6.8)_{1}^{\prime}$, we make:

Definition 7.4. We say that $\mathbb{F} \mathscr{A}$ satisfies reduction condition, if the following holds for each $\mathscr{A}_{\mu} \in \mathscr{F}_{p+1} \mathscr{A}(p \geqq 1)$ :

$(7.6)_{3}$ fix a suitable order $\prec$ of $\mathscr{B}^{\prime}:=\pi_{\mu}\left(\mathscr{A}_{\mu}\right)$, where $\pi_{\mu}:=$ restriction of $\pi$ to $\mathscr{A}_{\mu}$. Then setting $B_{\mu}:=$ maximal element of $\mathscr{B}_{\mu}$ and $\tilde{\mathscr{A}}_{\mu}:=\pi_{\mu}^{-1}\left(B_{\mu}\right)$, we have: $(7.6)_{3}^{\prime} \quad \tilde{\mathscr{A}}_{\mu} \in \mathscr{F}_{1}^{0} \mathscr{A}$. Moreover, for each $\mathscr{D} \in \tilde{\mathscr{A}}^{(t+1)}(t>0)$, the localization $\mathscr{A}_{\mu \mathscr{D}}$ is in $\mathscr{F}_{p} \mathscr{A}$, and $\left|\left(\mathscr{A}_{\mu \mathscr{D}} / \pi_{\mu}\right)_{u}\right|$ is connected.

We rewrite $(7.6)_{1,2}$ in the following form:

Proposition 7.5. The series $\boldsymbol{F}^{0} \mathscr{A}$ satisfies the reduction condition.

In Part B we give a sharpening of the degeneracy theorem in the form of Proposition 6.3 for a series $\mathbb{A}$ as above, which satisfies the reduction condition.

\section{B. A Sharpening of Degeneracy Theorem}

In $n .1$ and $n .2$ we give our main applications of the localization arguments in Part A, and in n.3, we prove the main lemmas, Lemma 6.1-Lemma 6.3, in Section 6.3 , by using the results of $n .1, n .2$.

1. First let the collection $\mathscr{A}$ of elementary figures in $\mathbb{C}^{n}(z)$, the map $\pi$ : $\mathscr{A} \rightarrow \mathscr{B}$ and the series $\boldsymbol{F} \mathscr{A}=\left\{F_{p} \mathscr{A}\right\}_{p=1}^{\infty}$ of families $\mathscr{F}_{p} \mathscr{A} \subset 2^{\mathscr{A}}$ be as in Definition 7.4. We assume:

$(7.7)_{0} \quad\left\{\begin{array}{l}\text { ratio number } a \text { of } \mathscr{A}<\infty\left(\mathrm{cf} .(6.10)_{3}\right) \\ F \mathscr{A} \text { satisfies the reduction condition (Definition 7.4). }\end{array}\right.$

For each $\mathscr{A}_{\mu} \in \mathscr{F}_{p} \mathscr{A}$, we set $\pi_{\mu}:=$ restriction of $\pi$ to $\mathscr{A}_{\mu}$, and let $E_{r, \mu}^{p, q-p}(m)$, $m \geqq 1$, denote the spectral sequence ${ }^{*)}$ of $\left(C^{*}\left(\mathscr{A}_{\mu}, \mathfrak{D}\right)^{m}, F_{p}\right)$, where the filtration $F_{p}$ is induced from $\pi_{\mu}$ (Definition 6.3). We fix elements $c_{1}, d_{1} \in \mathbb{R}_{1}^{+2}$.

Lemma 7.2. Assume the following for each $\mathscr{A}_{\mu} \in \mathscr{F}_{1} \mathscr{A}$ : $(7.7)_{1}^{0, q} \quad i_{m^{\prime} m}^{*} E_{1, \mu}^{0, q}(m) \cong 0(q \geqq 1)$, where $m, m^{\prime} \in \boldsymbol{R}_{1}^{+}$satisfy: $m>c_{1} a, m^{\prime}=m / d_{1} a$.

*) For the complex $C^{*}\left(\mathscr{A}_{\mu}, D\right)^{m}$, see $(6.10)_{2}^{\prime}$. 
Then we have the following for each $\mathscr{A}_{\mu} \in \mathscr{F}_{p+1} \mathscr{A}(p \geqq 1)$ :

$(7.7)_{1}^{p, q} \quad i_{\tilde{m}^{\prime} \tilde{m}} E_{1, \mu}^{p, q-p}(\tilde{m}) \cong 0(q>p)$, where $\tilde{m}, \tilde{m}^{\prime} \in \boldsymbol{R}_{1}^{+}$satisfy: $\tilde{m}>c_{q, p} a, \tilde{m}^{\prime}=$ $\tilde{m} / d_{q, p} a$, with elements $c_{q, p}, d_{q, p} \in \boldsymbol{R}_{1}^{+2}$, which are determined $\left.{ }^{*}\right)$ by $\left(c_{1}, d_{1}\right)$ and $(q, p)$.

Lemma 7.3. Assume, in addition to (7.7) ${ }_{1}^{0, q}$, the following:

$(7.7)_{0}^{\prime}$ each $\mathscr{F}_{p} \mathscr{A}(p \geqq 1)$ is admissible (Definition 6.4).

Then we have a homomorphism

$(7.7)_{2} \quad \tau^{*}: H^{q}\left(C^{*}(\mathscr{A}, \mathfrak{D})^{\tilde{m}}\right) \rightarrow E_{2, \mathscr{A}}^{q, 0}\left(\tilde{m}^{\prime}\right)$

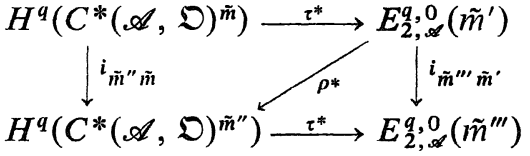

Figure I.

satisfying Figure I.

In the above, $E_{2, \infty}^{q, 0}(m)$ denotes the spectral sequence of the 'original complex' $\left(C^{*}(\mathscr{A}, \mathfrak{D})^{m}, F_{p}\right)$, and the homomorphism $\rho^{*}$ is the natural one: $E_{2, \infty}^{q, 0} \rightarrow$ $H^{q}\left(C^{*}(\mathscr{A}, \mathfrak{D})^{m}\right)$. Moreover, the correspondence: $\tilde{m} \rightarrow \tilde{m}^{\prime}$ is as in Lemma 7.2 and: $\tilde{m}^{\prime} \rightarrow \tilde{m}^{\prime \prime}$ is given similarly to $\tilde{m} \rightarrow \tilde{m}^{\prime}$.

Proof. The proof of Lemma 7.2 is given inductively on $p$ as follows: taking an integer $p \geqq 1$, we assume $(7.7)_{1}^{\tilde{p}, q}$ for each $\tilde{p}<p$. For an element $\mathscr{A}_{\mu} \in \mathscr{F}_{p+1} \mathscr{A}$, let the localization $\mathscr{A}_{\mu \mathscr{\mathscr { S }}} \in \mathscr{F}_{p} \mathscr{A}$ and the inverse image $\tilde{\mathscr{A}}_{\mu}$ : $=\pi_{\mu}^{-1}\left(B_{\mu}\right) \in \mathscr{F}_{1} \mathscr{A}$ have the similar meaning to (7.6)' ${ }_{3}^{\prime}$. By the induction condition we see that $(7.7)_{1}^{p-1, q-p-t}$ and $(7.7)_{1}^{0, q-p}$ hold for $\tilde{A}_{\mu}, \mathscr{A}_{\mu \mathscr{D}}$. On the otherhand, the key lemma, Lemma 7.1', insures that the above facts for $\mathscr{A}_{\mu \mathscr{D}}$, $\tilde{\mathscr{A}}_{\mu}$ imply (7.7) ${ }_{1}^{p, q}$ for $\mathscr{A}_{\mu}$, and we have Lemma 7.2.

Next, to check Lemma 7.3, we recall that the admissibility of $\mathscr{F}_{p+1} \mathscr{A}$, together with $(7.7)_{1}^{p, q}$, for each $\mathscr{A}_{\mu} \in \mathscr{F}_{p+1} \mathscr{A}$, insures:

$(7.7)_{2}^{p, q} \quad i_{\tilde{m}^{\prime} \tilde{m}} E_{1, q}^{p, q-p}(\tilde{m}) \cong 0(q>p \geqq 0)$ (cf. Proposition 6.5).

Thus, applying the standard degeneracy theorem**) to this inclusion, we have Lemma 7.3.

q.e.d.

Lemma 7.2 and Lemma 7.3 are our sharpening of the degeneracy theorem

*) The map: $\left(c_{1}, d_{1}\right) \rightarrow\left(c_{q, p}, d_{q, p}\right)$ is given in the manner as in (7.3) $)_{6}^{\prime \prime \prime}$.

**) In Lemma 7.3, we are concerned with the subcomplex $C^{*}(\mathscr{A}, D)^{m}$ of $C^{*}(\mathscr{A}, \mathbb{D})$ (instead of $\mathscr{C}^{*}(\mathscr{A}, \mathscr{D})$ ), and we use the homomorphism $i \tilde{m}^{\prime}, \tilde{m}$. However, this gap is quite small, and one get Lemma 7.3 from a slice modification of the standard proof of the degeneracy theorem (cf. [22]). 
which is mentioned previously*), and the key point is that the assumption for the 'isomorphism' in Figure I is reduced to the vanishing property for $(7.7)_{1}^{0, q}$ at the lowest filtration degree $p=0$. In n.2 we give a p.g.version of Lemma 7.2, Lemma 7.3, which may be our substantial main lemmas in our treatments of the p.g. uniform estimations in Chapter III.

2. A p.g. version of Lemma 7.3. In $n .2$ we start with a family $\tilde{A}=\left\{\tilde{\mathcal{A}_{\lambda}}\right.$; $\lambda \in \Lambda\}$ consisting of data $\tilde{\boldsymbol{A}}_{\lambda}$ as follows:

$(7.8)_{0} \tilde{\boldsymbol{A}}_{\lambda}=\left(\mathscr{A}_{\lambda}, \mathscr{B}_{\lambda}, \pi_{\lambda}\right)$, where $\mathscr{A}_{\lambda}$ is a collection of elementary figures in $\mathbb{C}^{n}(z), \mathscr{B}_{\lambda}$ is an abstract set, and $\pi_{\lambda}$ is a map: $\mathscr{A}_{\lambda} \rightarrow \mathscr{B}_{\lambda}$. For notational convenience, we write:

$(7.8)_{0}^{\prime} \mathscr{A}=\left\{\mathscr{A}_{\lambda}\right\}_{\lambda}, \mathscr{B}=\left\{\mathscr{B}_{\lambda}\right\}$, and $\pi=\left\{\pi_{\lambda}\right\}_{\lambda}$, where $\lambda$ runs through $\Lambda$.

We assume the following for each $\mathscr{A}_{\lambda} \in \mathscr{A}$ :

$(7.8)_{0}^{\prime \prime}$ the ratio number $a_{\lambda}$ of $\mathscr{A}_{\lambda}<\infty$ (cf. $\left.(6.10)_{3}\right)$.

Moreover, for each $A_{\lambda} \in \mathscr{A}$, we fix a series $\mathscr{F}_{\lambda}=\left\{\mathscr{F}_{p} \mathscr{A}_{\lambda}\right\}_{p=1}^{\alpha}$ of families $\mathscr{F}_{p} \mathscr{A}_{\lambda} \subset 2^{\mathscr{A} \lambda}$. We assume that each $\mathscr{F}_{p} \mathscr{A}_{\lambda}$ satisfies $(6.8)_{1}^{\prime}$ and that

(7.8) $)_{0}^{\prime \prime \prime} \quad F_{\lambda}$ satisfies the reduction condition (Definition 7.4).

We then define:

(7.8) iv $^{\text {iv }} \mathscr{F}_{p} \tilde{\boldsymbol{A}}:=\left\{\mathscr{A}_{\mu}\right.$; where $\mathscr{A}_{\mu}$ is an element of $\mathscr{F}_{p} \mathscr{A}_{\lambda}$, with an element $\left.\mathscr{A}_{\lambda} \in \mathscr{A}\right\}$.

For each $\mathscr{A}_{\mu} \in \mathscr{F}_{p} \tilde{\boldsymbol{A}}$, we denote by $\mathscr{A}_{\lambda_{\mu}}$ the element of $\mathscr{A}$ satisfying $\mathscr{F}_{p} \mathscr{A}_{\lambda_{\mu}}$ $\ni \mathscr{A}_{\mu}$.

Next, for the explicit estimation here, let the set of parameters $\mathbb{R}_{1}^{+s} \times \mathbb{R}_{1}^{+2}$ and the collection $\boldsymbol{E}$ of maps: $\left(\boldsymbol{R}_{1}^{+s} \times \boldsymbol{R}_{1}^{+2}\right) \rightarrow \boldsymbol{R}_{1}^{+2}$ be as in Lemma 7.1". Moreover, for each $\mathscr{A}_{\lambda} \in \mathscr{A}$, we fix an element $\beta_{\lambda} \in \mathbb{R}_{1}^{+s}$.

Now, take elements $c_{1}, d_{1} \in \boldsymbol{R}_{1}^{+2}$ and a map $E_{1} \in \mathbb{E}$, and we assume the following uniform estimation for $\mathscr{F}_{1} \tilde{\mathscr{A}}(q>0)$ :

$(7.8)_{1}^{0, q} \quad Z_{1}^{0, q}\left(\mathscr{A}_{\mu} \times D^{\prime}, \mathfrak{D}\right)_{\alpha}^{m} \subset B_{0}^{0, q}\left(\mathscr{A}_{\mu} \times D^{\prime}, \mathfrak{D}\right)_{\alpha^{\prime}}^{m^{\prime}}$ for any $\left(\mathscr{A}_{\mu} ; D^{\prime} ; \alpha\right) \in \mathscr{F}_{1} \tilde{\mathscr{A}} \times$ $\operatorname{Ouv}\left(\boldsymbol{C}^{n}\right) \times \boldsymbol{R}_{1}^{+2}$, where $\alpha^{\prime}=\left(E_{1}\left(\beta_{\lambda_{\mu}} ; \alpha\right)\right.$, and $m, m^{\prime} \in \boldsymbol{R}_{1}^{+}$satisfy: $m>c_{1} a_{\lambda_{\mu}} m^{\prime}=$ $m / d_{1} a_{\lambda_{\mu}}$

Then we have the following p.g.version of Lemma 7.2, which is easily derived

*) Cf. the introduction of Chapter III, and the end of Section 6.3. 
from Lemma 7.1", by using the similar inductive arguments on $p \in Z^{+}$as in Lemma 7.2:

Lemma 7.4. For each $\mathscr{A}_{\mu} \in \mathscr{F}_{p+1} \tilde{\mathscr{A}}(p \geqq 1)$, we have:

$(7.8)_{1}^{p, q} Z_{1}^{p, q-p}\left(\mathscr{A}_{\mu} \times D^{\prime}, \mathfrak{D}\right)_{\alpha}^{\tilde{m}} \subset B_{0}^{p, q-p}\left(\mathscr{A}_{\mu} \times D^{\prime}, \mathfrak{D}\right)_{\alpha^{\prime}}^{\tilde{m}^{\prime}}(q>p)$, where $\alpha^{\prime}=E_{q, p}\left(\beta_{\lambda_{\mu}}\right.$; $\alpha$ ), and $\tilde{m}, \tilde{n}^{\prime} \in \mathbb{R}_{1}^{+}$satisfy: $\tilde{m}>c_{q, p} a_{\lambda_{\mu}}, \tilde{n}^{\prime}=\tilde{m} / d_{p, q} a_{\lambda_{\mu}}$. Here the elements $c_{q, p}$ and $\left.\alpha_{p, p} b y^{*}\right)\left(c_{1}, d_{1}\right)$ and $(q, p)$. Also the map $E_{q, p} \in \boldsymbol{E}$ is given in the form:

$(7.8)_{1}^{\prime p, q} \quad E_{q, p}=\tilde{\eta}_{e}\left(E_{1}\right)$, with an element $e=e_{q, p} \in \mathbb{Z}^{+}$, which is determined by $(q, p)$. (For the map $\tilde{\eta}_{e}: E \rightarrow E$, see $(7.4)_{3}$.)

Next we assume the following for each $\mathscr{A}_{\lambda} \in \mathscr{A}$ :

(7.8) ${ }_{2}^{\prime \prime}$ each $\mathscr{F}_{p} \mathscr{A}_{\lambda}(p \geqq 1)$ is admissible (Definition 6.4).

Then, using the similar arguments to Lemma 7.3, we have the following p.g. version of Lemma 7.3 :

Lemma 7.5. For each $\mathscr{A}_{\lambda} \in \mathscr{A}$, we have***) $\operatorname{map} \tau_{\lambda}$ :

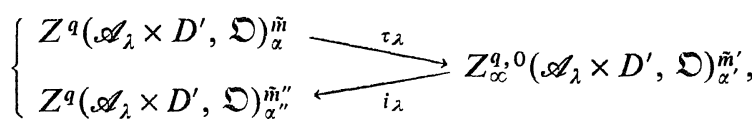

$$
\begin{aligned}
& \text { with }\left\{\begin{array}{l}
\tilde{n}^{\prime}=\tilde{m} / d_{q} a_{\lambda}, \tilde{m}^{\prime \prime}=\tilde{n}^{\prime} / d_{q} a_{\lambda}, \\
\alpha^{\prime}=E_{q}\left(\beta_{\lambda} ; \alpha\right), \alpha^{\prime \prime}=E_{q}\left(\beta_{\lambda} ; \alpha^{\prime}\right),
\end{array}\right.
\end{aligned}
$$

satisfying

$(7.8)_{3}^{\prime} \quad\left(1-i_{\lambda} \rho_{\lambda}\right) Z^{q}\left(\mathscr{A}_{\lambda} \times D^{\prime}, \mathfrak{D}\right)_{\sigma}^{\tilde{m}} \subset \delta C^{q-1}\left(\mathscr{A}_{\lambda} \times D^{\prime}, \mathfrak{D}\right)_{\alpha^{\prime}}^{\tilde{m}^{\prime \prime}}$, and the similar relation for $i_{\lambda} \cdot \tau_{\lambda}$.

In the above, the element $\tilde{m} \in \boldsymbol{R}_{1}^{+}$satisfies: $\tilde{m}>c_{q} a_{\lambda}$, and $c_{q}, d_{q} \in \boldsymbol{R}_{1}^{+2}$ are determined $b y^{* * *)}\left(c_{1}, d_{1}\right)$ and $q$. Moreover, the map $E_{q} \in \mathbb{E}$ is given as follows: $(7.8)_{3}^{\prime \prime} \quad E_{q}=\eta_{e_{q}}\left(E_{1}\right)$, with an element $e_{q} \in \mathbb{Z}^{+}$, which depends only on $q \in \mathbb{Z}^{+}$.

Applications of Lemma 7.5 will be given in $n .3$ soon below.

3. Proof of Lemma 6.1-Lemma 6.3. (i) First we derive Lemma 6.2 from Lemma 7.5. For this letting the collection $\boldsymbol{A}^{\prime \tilde{n}}, \tilde{n}=\left(n, n^{\prime}\right)$, of the p.g. elementary coverings be as in Lemma 6.2 (cf. also $(6.4)_{1}$ ), take an element $\mathscr{A}_{\xi}^{\prime} \in \boldsymbol{A}^{\prime} \tilde{\text { n}}$.

$*, * *)$ The correspondence: $\left(c_{1}, d_{1}\right) \rightarrow\left(c_{q, p}, d_{q, p}\right)$ is as in Lemma 7.2 (cf. also (7.3) $\left.)_{6}^{\prime \prime \prime}\right)$. Moreover, one can take $\left(o_{1}, d_{q}\right)$ in Lemma 7.5 to be: $\left(c_{q}, d_{q}\right)=\left(c_{q, q}, d_{q, q}\right)$.

***) For the set $Z_{\infty}^{q, 0}$ and the map $i_{2}$ in $(7.8)_{3}$, see $(6.10)_{1},(6.10)_{2}^{\prime}$. 
Then, letting the map $\pi_{\xi}: \mathscr{A}_{\xi}^{\prime} \rightarrow \mathbb{Z}^{2 n}$ be as in $(6.7)_{8}$, we set:

(7.9) $\mathbb{B}^{\prime n}:=\left\{B_{\xi}^{\prime}\right\}_{\xi}, \pi^{\prime n}:=\left\{\pi_{\xi}\right\}$, where we write $\mathbb{Z}^{2 n}\left(=\pi_{\xi}\left(\mathscr{A}_{\xi}^{\prime}\right)\right)$ as $\mathscr{B}_{\xi}^{\prime}$.

Next letting the element $c_{n} \in \mathbb{R}_{1}^{+2}$ be as in the assumption $(6.11)_{2}^{0, q}$ in Lemma 6.2, we define an element $c_{n, q} \in \mathbb{R}_{1}^{+2}$ by $c_{n, q}=\mathscr{L}_{n, q}\left(c_{n}\right)(q>0)$, where the el-map $\mathscr{L}_{n, q}$ is as in $(7.3)_{6}^{\prime \prime \prime}$. Now, let the refining map $s: \mathscr{A}_{\xi}^{\prime} \hookrightarrow \mathscr{A}_{\xi}^{\prime}$ be as in Lemma 6.2. We write the p.g. elementary coverings $\mathscr{A}_{\xi}^{\prime}, \mathscr{A}_{\xi}^{\prime}$, explicitly as $\mathscr{A}_{\xi}^{\prime}=\mathscr{A}_{\xi}^{\prime}\left(\mathbb{C}^{n}\right.$ $\left.\times U^{\prime}\right)$ and $\mathscr{A}_{\xi^{\prime} \varepsilon}^{\prime}\left(\boldsymbol{C}^{n} \times U^{\prime}\right)$, with $\bar{\zeta}=(\zeta ; \varepsilon), \xi^{\prime}=\left(\zeta^{\prime} ; \varepsilon\right) \in \mathbb{Z}^{+2} \times(0,1 / 2)$ and $U^{\prime}$ $\in \operatorname{Ouv}^{\prime}\left(\boldsymbol{C}^{n^{\prime}}\right)_{\mathrm{el}}$ (cf. $\left.(6.3)_{7}\right)$. We define ${ }^{*)}: \beta_{\xi^{\prime}}:=\left(d_{U^{\prime}}^{-} ; \zeta^{\prime}\right) \in \mathbb{R}_{1}^{+} \times \mathbb{R}_{1}^{+2}$. Now we apply**) Lemma 7.5 to $\mathscr{A}_{\xi^{\prime}}^{\prime}$. Then we have the diagram:

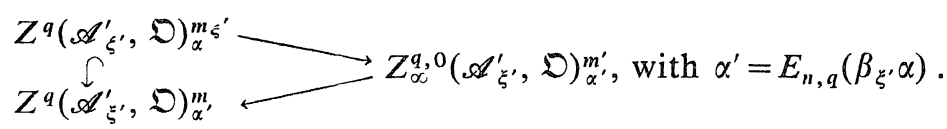

In the above we set $m_{\xi^{\prime}}:=c_{n, q} \cdot a_{\xi^{\prime}}$, with the ratio number $a_{\xi^{\prime}}$ of $\mathscr{A}_{\xi}^{\prime}$ (cf. $\left.(6.10)_{3}\right)$. Moreover, the maps: $m_{\xi^{\prime}} \rightarrow m^{\prime}, m^{\prime} \rightarrow m^{\prime \prime}$ are defined in the manner in Lemma 7.5. Furthermore, the estimation map $E_{n, q}$ is, as in Lemma $6.2^{* * *)}$, in $\mathbb{E}=\mathbb{E}$ $\times(\mathbf{M} \times \mathbf{L})\left(\mathrm{cf} .(6.4)_{3}\right)$.

On the otherhand, we have (cf. (5), Part B, Appendix II):

$$
s\left(\mathscr{A}_{\mu}^{\prime}\right) \mathscr{A}_{\mu m_{\xi^{\prime}}}^{\prime} \text { for each } \mathscr{A}_{\mu}^{\prime} \in \mathscr{A}_{\xi^{\prime}}^{\prime} \text {. }
$$

Thus one can apply $(7.9)_{2}$ to $s^{*} Z^{q}\left(\mathscr{A}_{\xi}^{\prime}, \mathfrak{D}\right)_{\alpha}$, and we have the conclusion****) $(6.11)_{2}^{q}$ in Lemma 6.2. This finishes the proof of Lemma 6.2.

(ii) Proof of Lemma 6.3. The proof of Lemma $6.3^{1}$ is given in Part A, Appendix II. Recall that the proof of Lemma $6.4^{n}(n \geqq 2)$ suffices for the proof of Lemma $6.3^{n}$ ( $n \geqq 2$ ) (cf. $\S 6.3$ ). But we easily see that, by applying Lemma 7.5 to the triple $\left(A^{n}, B^{n}, \pi^{n}\right)$ as in $(6.12)_{2}$, we have Lemma 6.4. (In the application, the estimation map $E$ should be the elementary p.g. map as in Definition 6.4. Moreover, for an element $\mathscr{A}_{\lambda} \in \mathcal{A}^{n}$, the invariant $\beta_{\lambda}$ is defined by*****) $\beta_{\lambda}=$ $\left(l_{\lambda} / r_{\lambda}^{-} ; m r_{\lambda}^{+}\right)$, with the invariants $l_{\lambda}, r_{\lambda}^{+}$of $\mathscr{A}_{\lambda}$ as in $(6.12)_{0}^{\prime \prime \prime}$. Using these data, the proof of Lemma 6.3 is given similarly to Lemma 6.5.)

*) For the invariant $d_{U^{\prime}}^{-}$of $U^{\prime}$, see $(6.3)_{8}$.

**) Precisely, 'Lemma 7.5 for $\left(\mathcal{A}^{\prime n}, \boldsymbol{B}^{\prime n}, \pi^{\prime n}\right)$.

***) The estimation: $\alpha \rightarrow \alpha^{\prime}=E_{n, q}\left(\beta_{\xi^{\prime}} ; \alpha\right)$ is given explicitly as in $(6.4)_{3}^{\prime}$.

****) Precisely, the estimation (6.11) $)_{2}^{q}$ is of the form $\alpha^{\prime}=E_{n, q}\left(1 / d_{U} ; \zeta ; \partial\right)$. But, from the explicit form of the map $E_{n, q}$ (cf. $\left.(6.4)_{3}^{\prime}\right)$, one can change the term $\zeta^{\prime}$ in $(7.9)_{3}$ to $\zeta$.

*****) The term $m \in \boldsymbol{R}_{1}^{+}$is independent from $\mathscr{A}_{\lambda} \in \mathcal{A}^{n}$ (cf. (6.12) $)_{0}$ ), and is not an invariant of $\mathscr{A}_{2}$. But we check easily that the proof of Lemma 7.4 and Lemma 7.5 is applied to the element $\beta_{\lambda}=\left(l_{\lambda} / r_{2}^{-} ; m r_{i}^{+}\right)$at the present situation. 
(iii) Proof of Lemma 6.1. Assume the assumption (6.11) ${ }_{1}^{0, q}$ in Lemma 6.1, and let the refining map $s: \mathscr{A}_{k^{\prime \prime}} \times D^{\prime} \hookrightarrow \mathscr{A}_{k} \times D^{\prime}$ be as in Proposition 7.4. . (Here we write $\mathscr{A}_{k}\left(\mathbb{C}^{n}\right), \ldots$, as $\left.\mathscr{A}_{k}, \ldots\right)$ Then, using the similar algebraic arguments as in Lemma 7.5, we see easily that the check of the following suffices for the proof of the conclusion $(6.11)_{2}^{q}$ in Lemma 6.1:

$$
s^{*} Z_{1}^{p, q-p}\left(\mathscr{A}_{k} \times D^{\prime}, \mathfrak{D}\right)_{\alpha} \subset B_{0}^{p, q-p}\left(\mathscr{A}_{k^{\prime \prime}} \times D^{\prime}, \mathfrak{D}\right)_{\alpha^{\prime}}(q>p \geqq 0),
$$

where the estimation: $\alpha \rightarrow \alpha^{\prime}$ is as in Proposition 7.4 (or, equivalently, as in Lemma 6.1). But Proposition 7.4 $4_{3}$ insures the similar inclusion for each $\mathscr{A}_{\mu}$ $\in \mathscr{F}_{p+1}^{\prime 0} \mathscr{A}_{k^{\prime \prime}}$. By the admissibility of $\mathscr{F}_{p+1}^{\prime 0} \mathscr{A}_{h^{\prime \prime}}$ (cf. Proposition 6.5), the latter insures $(7.9)_{4}$, and we finish the proof of Lemma 6.1.

By Proposition 6.7 and Proposition 6.1, the proof of Lemma 6.1-Lemma 6.3 insures Theorem 6.1, Theorem 6.2 and our original concern, Theorem 1.7, and we finish the proof of Theorem 1.7, which is our basis for the geometric arguments in Chapters I, II.

\section{Appendix II. Elementary Computations - 2}

In Part A we prove Lemma $6.3^{1}$ and Theorem $6.1^{1}$ (for the complex euclid line), by using Cousin integrals. In B we summarize some estimations on the refining maps, which are used in Section 6, Section 7.

\section{A}

In $\mathrm{A}$ we fix a complex euclid line $\boldsymbol{C}(z), z=x+\sqrt{-1} \cdot y$, and a complex euclid space $\mathbb{C}^{n^{\prime}}\left(z^{\prime}\right)\left(n^{\prime}>0\right)$ (cf. n.1, §1.2). We set $\tilde{z}=\left(z, z^{\prime}\right)$ and $\mathfrak{D}:=$ structure sheaf of $\boldsymbol{C} \times \boldsymbol{C}^{n^{\prime}}$. We use Ouv $\left(\boldsymbol{C}^{n^{\prime}}\right) \times \boldsymbol{R}_{1}^{+2}$ as the parameter space for sets of the cochains in A.

Notation. Take a set $\mathscr{A}=\left\{A_{\mu}\right\}_{\mu}$ of elementary figures in $C$ and a map $\pi: \mathscr{A} \rightarrow \mathscr{B}$, where $\mathscr{B}$ is an abstract set. For elements*) $\left(D^{\prime} ; \alpha\right) \in \operatorname{Ouv}\left(\boldsymbol{C}^{n^{\prime}}\right)$ $\times \boldsymbol{R}_{1}^{+2}$ and $m \in \boldsymbol{R}_{1}^{+}$, we define the following sets of the cochains in the manner in $(6.10)_{1,2}$ :

(0) $C_{1} C^{q}\left(\mathscr{A} \times D^{\prime}, \mathfrak{D}\right)_{\alpha}^{m}:=i_{m}^{*} C^{q}\left(\mathscr{A}_{m} \times D^{\prime}, \mathfrak{D}\right)_{\alpha}$, where**) $\mathscr{A}_{m}:=\left\{A_{\mu m}\right\}_{\mu}$, and $i_{m}$ : = refining map: $\mathscr{A} \times D^{\prime} \ni A_{\mu} \times D^{\prime} \rightarrow \mathscr{A}_{m} \times D^{\prime} \ni A_{\mu m} \times D^{\prime}$. Moreover, the right

*) Ouv $\left(\boldsymbol{C}^{n^{\prime}}\right)$ : = collection of all open sets in $\boldsymbol{C}^{n^{\prime}}$.

**) $A_{\mu m}:=$ elementary figure with same center with $A_{\mu}$ and size $=m \times\left(\right.$ size of $\left.A_{\mu}\right)$ (cf. $(6.10)_{1,2}$ ). 
side is the set of all $\alpha-(|\tilde{z}|+1)$-growth cochains (cf. $(1.3)_{6}$ and $\left.(1.4)_{8}\right)$.

$(0)_{2} \quad Z_{1}^{p, q-p}\left(\mathscr{A} \times D^{\prime}, \mathfrak{D}\right)_{\alpha}^{m}:=\left\{\varphi \in F_{p} C^{q}\left(\mathscr{A} \times D^{\prime}, \mathfrak{D}\right)_{\alpha}^{m} ; \delta \varphi \in F_{p+1} C^{q+1}\left(\mathscr{A} \times D^{\prime}, \mathfrak{D}\right)\right\}$, and $B_{0}^{p, q-p}\left(\mathscr{A} \times D^{\prime}, \mathfrak{D}\right)_{\alpha}^{m}:=\delta F_{p} C^{q-1}\left(\mathscr{A} \times D^{\prime}, \mathscr{D}\right)_{\alpha}^{m}$, where $F_{p}$ is the filtration induced from $\pi: \mathscr{A} \rightarrow \mathscr{B}$.

1. Cousin integrals. The argument here is divided into two parts, according as the collection $\mathscr{A}$ is a finite or infinite set.

(i) Finite case. Take a rectangle $U=(a, b) \times\left(a^{\prime}, b^{\prime}\right) \subset \mathbb{C}\left(\cong \mathbb{R}^{2}\right)$ and elements $\tilde{k}=\left(k, k^{\prime}\right) \in\left(\mathbb{Z}^{+} \cup 0\right)^{2}, \varepsilon \in(0,1 / 2)$, and we set:

(a) $\mathscr{A}_{\eta}(U):=$ elementary covering of $U$ of size $\eta:=(\tilde{k} ; \varepsilon)\left(\right.$ Definition $\left.6.1_{1}\right)$.

We write $\mathscr{A}_{\eta}:=\mathscr{A}_{\eta}(U)$ explicitly as $\mathscr{A}_{\eta}=\left\{U_{I} ; I \in[0, k] \times\left[0, k^{\prime}\right]\right\}$, where the center $P_{I}$ of the elementary figure $U_{I}$ is characterized by the following condition:

(a) ${ }_{1}^{\prime} \quad x\left(P_{I}\right)<x\left(P_{I^{\prime}}\right), y\left(P_{I}\right)<y\left(P_{I^{\prime \prime}}\right)$, with $I^{\prime}:=I+(1,0), I^{\prime \prime}:=I+(0,1)$.

Next setting $\mathscr{B}_{\eta}:=\left[0, k^{\prime}\right]$, we define a map:

$(\mathrm{a})_{2}$

$$
\pi_{\eta}: \mathscr{A}_{\eta} \ni U_{I} \longrightarrow \mathscr{B}_{\eta} \ni j \text {, with } I=(i ; j) .
$$

The symbol $\left(\mathscr{A}_{\eta m} / \pi_{\eta}\right)_{u}$ denotes the $u$-quotient of $\mathscr{A}_{\eta m}$ by $\pi_{\eta}$ (cf. $\left.(6.7)_{2}\right)$. Note that, if $k, k^{\prime}>0$, then $\left(\mathscr{A}_{\eta m} / \pi_{\eta}\right)_{u}$ is explicitly as follows:

(a) ${ }_{3} \quad\left(\mathscr{A}_{\eta m} / \pi_{\eta}\right)_{u}=\left\{U_{j}(m)\right\}_{j=0}^{k}$, where $U_{j}(m):=\{z \in \mathbb{C} ;-m r \tilde{\varepsilon}+a<x<b+m r \tilde{\varepsilon}$, $\left.\left|y-y_{j}\right|<m r^{\prime} \tilde{\varepsilon}\right\}$, with $r=(b-a) / k, r^{\prime}:=\left(b^{\prime}-a^{\prime}\right) / k^{\prime}, \tilde{\varepsilon}:=\varepsilon+2^{-1}$ and $y_{j}:=a^{\prime}$ $+j r^{\prime}$. Now, defining the numerical invariants $l_{\eta}, r_{\eta}^{ \pm}$of $\mathscr{A}_{\eta}$ by $(6.12)_{0}^{\prime \prime \prime}$, we have:

Proposition 1. Take suitable $c_{1}, d_{1} \in \mathbb{R}_{1}^{+}$and an elementary p.g.map $E$ (Definition 6.5), which depend only on $1=\operatorname{dim} \mathbb{C}$. Then we have:

(1) $Z_{1}^{0,1}\left(\mathscr{A}_{\eta} \times D^{\prime}, \mathfrak{D}\right)_{\alpha}^{m} \subset B_{0}^{0,1}\left(\mathscr{A}_{\eta} \times D^{\prime}, \mathfrak{D}\right)_{\alpha^{\prime}}^{m^{\prime}}+F_{1} C^{1}\left(\mathscr{A}_{\eta} \times D^{\prime}, \mathfrak{D}\right)_{\alpha^{\prime}}^{m^{\prime}}$

(1) $Z_{2} Z^{1}\left(\left(\mathscr{A}_{\eta m} / \pi_{\eta}\right)_{u} \times D^{\prime}, \mathfrak{D}\right)_{\alpha} \subset \delta C^{0}\left(\left(\mathscr{A}_{\eta m^{\prime}} / \pi_{\eta}\right)_{u} \times D^{\prime}, \mathfrak{D}\right)_{\alpha^{\prime}}$

$$
\text { with }\left\{\begin{array}{l}
m^{\prime}=m / d_{1} \\
\alpha^{\prime}=E\left(l_{\eta} / r_{\eta}^{-} ; \mathrm{mr}_{\eta}^{+} ; \alpha\right),
\end{array}\right.
$$

where the parameter $\left(D^{\prime} ; \alpha\right)$ is in Ouv $\left(\mathbb{C}^{n^{\prime}}\right) \times \boldsymbol{R}_{1}^{+2}$ and the element $m \in \mathbb{R}_{1}^{+}$ satisfies: $m>c_{1}$. Moreover, the filtration $F_{1}$ and the sets $Z_{1}^{0,1}, \ldots$ are defined in the manner in $(0)_{2}$, by the map $\pi_{\eta}$.

(ii) Infinite case. The arguments here are divided into further two parts: first taking an element $(a ; b ; \varepsilon) \in\left(\boldsymbol{R} \times \mathbb{R}^{+}\right) \times(0,1 / 2)$, we set:

(2) $\mathscr{A}_{\varepsilon}^{\prime}(a ; b):=\left\{U_{i \varepsilon}\right\}_{i=-\infty}^{\infty}$, where $U_{i \varepsilon}:=\left\{z \in \mathbb{C} ;|x-i|<\varepsilon+2^{-1},|y-a|<b+\varepsilon\right\}$, 
$(2)_{0}^{\prime} \quad c:=\max |a \pm b|$.

Now take an element $\varphi \in Z^{1}\left(\mathscr{A}_{\varepsilon}^{\prime}(a ; b) \times D^{\prime}, \mathcal{D}\right)_{\alpha}$, where $\left(D^{\prime} ; \alpha\right)$ is in Ouv $\left(\mathbb{C}^{n^{\prime}}\right)$ $\times \boldsymbol{R}_{1}^{+2}$.

Proposition 2. There is an element $\varphi^{\prime} \in C^{0}\left(\mathscr{A}_{\varepsilon^{\prime}}^{\prime}(a ; b) \times D^{\prime}, \mathfrak{D}\right)$ satisfying (2) $\varphi_{1} \varphi=\delta \varphi^{\prime}$ and $\left|\varphi_{A^{\prime}}^{\prime}(P)\right|<\alpha^{\prime} \cdot(|\tilde{z}|+c+1)$ in $\left|A^{\prime}\right|$ for each $A^{\prime} \in \mathscr{A}_{\varepsilon^{\prime}}^{\prime}(a ; b)$. Here $\alpha^{\prime}:=M_{1}(1 / \varepsilon) \mathscr{L}_{1}(\alpha)$ and $\varepsilon^{\prime}=\varepsilon / d_{1}$, with a positive monomial $M_{1}$, an elmap $\mathscr{L}_{1}$ and an element $d_{1} \in \mathbb{R}_{1}^{+}$, which depend only on $1=\operatorname{dim} \mathbb{C}$.

We prove Proposition 2 in (iv). Next, for an element $\varepsilon \in(0,1 / 2)$, we use the symbol $\mathscr{A}_{\varepsilon}(\boldsymbol{C})$ for the elementary covering of $\boldsymbol{C}$ of size $(1 ; \varepsilon)$ (Definition $6.1_{2}$ ). We define a map:

$(2)_{2}^{\prime} \quad \pi: \mathscr{A}_{\varepsilon}(\mathbb{C}) \ni A \longrightarrow \mathbb{Z} \ni j$, where $j=y$-coordinate of the center of $A$.

Then the $u$-quotient $\left(\mathscr{A}_{\varepsilon}(\mathbb{C}) / \pi\right)_{u}$ of $\mathscr{A}_{\varepsilon}(\boldsymbol{C})$ by $\pi$ (cf. $\left.(6.7)_{2}\right)$ is explicitly as follows: $(2)_{2}^{\prime \prime} \quad\left(\mathscr{A}_{\varepsilon}(\boldsymbol{C}) / \pi\right)_{u}=\left\{V_{j \varepsilon}\right\}_{j=-\infty}^{\infty}$, with $V_{j \varepsilon}:=\left\{z \in \mathbb{C} ;|y-j|<\varepsilon+2^{-1}\right\}$.

Then, letting the el-map $\mathscr{L}_{1}$, the positive monomial $M_{1}$ and the element $d_{1} \in \boldsymbol{R}_{1}^{+}$ have the similar meaning to Proposition 2, we have:

Proposition 3. We have the inclusions:

(2) $Z_{1} \quad Z_{1}^{0,1}\left(\mathscr{A}_{\varepsilon}(\mathbb{C}) \times D^{\prime}, \mathfrak{D}\right)_{\alpha} \in B_{0}^{0,1}\left(\mathscr{A}_{\varepsilon^{\prime}}(\boldsymbol{C}) \times D^{\prime}, \mathfrak{D}\right)_{\alpha^{\prime}}+F_{1} C^{1}\left(A_{\varepsilon^{\prime}}(C) \times D^{\prime}, \mathfrak{D}\right)_{\alpha^{\prime}}$, (2) $Z_{3} \quad Z^{1}\left(\left(\mathscr{A}_{\varepsilon}(\mathbb{C}) / \pi\right)_{u} \times D^{\prime}, \mathfrak{D}\right)_{\alpha} \subset \delta C^{0}\left(\left(\mathscr{A}_{\varepsilon^{\prime}}(\mathbb{C}) / \pi\right)_{u} \times D^{\prime}, \mathfrak{D}\right)_{\alpha^{\prime}}$, where $\alpha^{\prime}=M_{1}(1 / \varepsilon) \mathscr{L}_{1}(\alpha)$ and $\varepsilon^{\prime}=\varepsilon / d_{1}$. Here $\left(D^{\prime} ; \alpha\right)$ is in Ouv $\left(\boldsymbol{C}^{n^{\prime}}\right) \times \boldsymbol{R}_{1}^{+2}$.

The proof of Proposition 3 is also given in (iv).

(iii) Proof of Proposition 1.*) For an index $I=(i, j) \in[0, k] \times\left[0, k^{\prime}\right]$ (resp. $j \in\left[0, k^{\prime}\right]$ ) we define arcs $\gamma_{I}^{ \pm}$(resp. $\gamma_{j}^{ \pm}$) by the following con dition (cf. also Figure I):

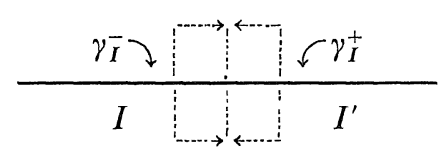

Figure I.

$$
\begin{aligned}
(\mathrm{a})_{1} \quad\left\{\begin{array}{l}
\gamma_{I}^{+} \cup \gamma_{I}^{-} \\
\gamma_{j}^{+} \cup \gamma_{j}^{-}
\end{array}\right\}:=\left\{\begin{array}{l}
\partial\left(U_{I, m^{\prime \prime}} \cap U_{I^{\prime}, m^{\prime \prime}}\right) \\
\partial\left(U_{j}\left(m^{\prime \prime}\right) \cap U_{j}\left(m^{\prime \prime}\right)\right)
\end{array}\right\}, \text { with }\left\{\begin{array}{l}
I^{\prime}:=I+(0,1) \\
m^{\prime \prime}:=m / 2
\end{array}\right\}, \\
(\mathrm{a})_{2} \quad\left\{\begin{array}{l}
x\left(\gamma_{I}^{+}\right) \geqq x_{i} \geqq x\left(\gamma_{I}^{-}\right) \\
x\left(\gamma_{j}^{+}\right) \geqq y_{j} \geqq y\left(\gamma_{j}^{-}\right)
\end{array}\right\}, \text {with }\left\{\begin{array}{l}
x_{i}:=a+\left(i+2^{-1}\right) r \\
y_{j}:=a^{\prime}+\left(j+2^{-1}\right) r
\end{array}\right\}, \\
\text { where }\left\{\begin{array}{l}
r:=(b-a) / k \\
r^{\prime}:=\left(b^{\prime}-a^{\prime}\right) / k^{\prime}
\end{array}\right\} .
\end{aligned}
$$

*) We prove $(1)_{1,2}$ for the case $k, k^{\prime}>0$. The proof of the case $k=0, k^{\prime}=0$ is given by a slice modification of the proof given here. 
(The inequality ' $x\left(\gamma_{I}^{+}\right) \geqq x_{i}$ ' indicates that $x \geqq x_{i}$ for each $x \in \gamma_{I}^{+}, \ldots$ ). Now taking elements $\varphi, \tilde{\varphi}$ from the left sides of $(1)_{1,2}$, we define:

$(b)_{1} \quad\left\{\begin{array}{c}\varphi^{\prime} \frac{ \pm}{I} \\ \tilde{\varphi}_{j}^{\prime} \frac{ \pm}{j}\end{array}\right\}:=\left\{\begin{array}{l}\int \varphi \cdot(\zeta-z)^{-1} d \zeta \\ \int \varphi \cdot(\zeta-z)^{-1} d \zeta\end{array}\right\}$, where the integral is taken over $\left\{\begin{array}{l}\gamma_{I}^{ \pm} \\ \gamma_{j}^{ \pm}\end{array}\right\}$.

We then define elements $\Phi \in C^{0}\left(\mathscr{A}_{\eta} \times D^{\prime}, D\right)_{\alpha}^{m^{\prime}}$ and $\widetilde{\Phi} \in C^{0}\left(\left|\left(\mathscr{A}_{\eta m^{\prime}} \mid \pi_{\eta}\right)_{u}\right|, \mathcal{D}\right)$, where $m^{\prime}:=m / 4$, by the following:

$$
\Phi_{i, j}:=\sum_{t=0}^{i-1} \varphi_{1 j}^{\prime-}-\sum_{t=i}^{h-i} \varphi_{t, j}^{\prime+}, \widetilde{\Phi}_{j}:=\sum_{t=0}^{i-1} \tilde{\varphi}_{t}^{\prime-}-\sum_{t=j}^{h-1} \tilde{\varphi}_{t}^{\prime+} .
$$

It is clear that $\varphi-\delta \Phi \in F_{1} C^{1}\left(\mathscr{A}_{\eta} \times D^{\prime}, \mathfrak{D}\right)$ and $\tilde{\varphi}-\delta \tilde{\Phi}=0$. Moreover, for all indices $I=(t, j)$ and $t$, which appear in $(b)_{2}$, we have:

(c) $\left|\varphi^{\prime} \frac{ \pm}{I}(z)\right|,\left|\tilde{\varphi}_{j}^{\prime}(z)\right|<\left(8 / r_{\eta}^{-}\right)\left(8 m r_{\eta}^{+}\right) \cdot \alpha^{\prime}\left(|\tilde{z}|+\left(8 m r_{\eta}^{+}\right)+1\right)$ in $U_{I, m^{\prime}}, U_{j}\left(m{ }^{\prime}\right)$, with $\alpha^{\prime}=\mathscr{L}_{1}(\alpha)$. Here the el-map $\mathscr{L}_{1}: \mathbb{R}^{+2} \rightarrow \mathbb{R}^{+2}$ depends only on $1=\operatorname{dim} \mathbb{C}$. (The first and second terms in (c) appear from the estimations of $|\zeta-z|^{-1}$ and the length of $\gamma_{I}^{ \pm}, \gamma_{j}^{ \pm}$. The third term appears from the estimation of $\varphi, \tilde{\varphi}$ on $\gamma_{I}^{ \pm}, \gamma_{j}^{ \pm}$. Precisely, we have: $|\zeta-z|<8 m r_{\eta}^{+}$in $U_{I \cdot m^{\prime}}$ and $U_{j}\left(m^{\prime}\right)$. From this and from that $|\varphi|,|\tilde{\varphi}|<\alpha\left(|z|+\left|z^{\prime}\right|+1\right)$ on $\gamma_{I}^{ \pm}, \gamma_{j}^{ \pm}$, we have the third term.) From $(b)_{2}$ and (c) and from the relation soon below (b) $)_{2}$, we have $(1)_{1,2}$ in Proposition 1.

q.e.d.

(iv) Proof of Proposition 2 and Proposition 3. We first prove Proposition 2. For this, for each $i \in \mathbb{Z}$, we define $\operatorname{arcs} \gamma_{i}^{ \pm}$by:

(a) $\gamma_{i}^{+} \cup \gamma_{i}^{-}=\partial\left(U_{i \varepsilon^{\prime}} \cap U_{i+1 \varepsilon^{\prime}}\right), \varepsilon^{\prime}=\varepsilon / 2$,

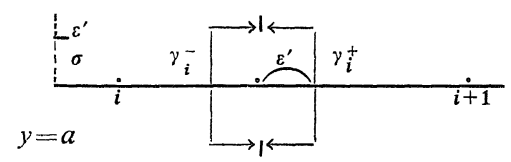
and ${ }^{*)} x\left(\gamma_{i}^{+}\right) \geqq i+2^{-1} \geqq x\left(\gamma_{i}^{-}\right)$(cf. Figure I).

Figure I.

Letting the element $\varphi \in Z^{1}\left(\mathscr{A}_{\varepsilon}^{\prime}(a ; b) \times D^{\prime}, \mathfrak{D}\right)_{\alpha}$ be as in Proposition 2, we define:

(b) $12 \pi \sqrt{-I}\left\{\begin{array}{l}\varphi_{i}^{ \pm}(\tilde{z}) \\ \varphi_{i}^{ \pm}\left(z^{\prime}\right)\end{array}\right\}:=\left\{\begin{array}{l}\int \varphi \cdot(\zeta-z)^{-1} d \zeta \\ \int \varphi \cdot(\zeta-a \sqrt{-1})^{-j-1} d \zeta\end{array}\right\}$ where the integral is taken over $\gamma_{i}^{ \pm}$.

We expand $\left.{ }^{* * *}\right) \quad \varphi_{i}^{+}(z)(i \geqq 0)$ at the 'origin' $(0, a \sqrt{-1}): \varphi_{i}^{+}(z)=\sum_{j} \varphi_{i j}^{+}$.

*) The inequality here has the similar meaning to $(a)_{2}$, (iii).

**) Precisely, $\varphi_{i}^{+}$is, as in $(b)_{1}$, the function of $\tilde{z}$. In the proof of Proposition 2, we regard $z^{\prime}$ as the parameter and $z$ as the variable. We write $\varphi_{i}^{\prime+}(\tilde{z})$ also as $\varphi_{l}^{+}(z)$. This notation will clarify the main genmetric situation in question. 
$(z-a \sqrt{-1})^{j}$, and we set:

(b) ${ }_{2} \varphi_{i}^{\prime+}:=\sum_{j=0}^{\beta} \varphi_{i j}^{+} \cdot(z-a \sqrt{-1})^{j}, \varphi_{i}^{\prime \prime+}:=\varphi_{i}^{+}-\varphi_{i}^{\prime+}$, where $\beta=\left[\alpha_{2}+3\right]$, with the second component $\alpha_{2}$ of $\alpha \in \boldsymbol{R}_{1}^{+2}$.

Next we define an element $\Psi_{i} \in \Gamma\left(U_{i \tilde{\varepsilon}} \times D^{\prime}, \mathfrak{D}\right), \tilde{\varepsilon}:=\varepsilon / 4$, by:

$$
\Psi_{i}:=\sum_{i=0}^{i-1}\left(\varphi_{\bar{i}}^{-}+\varphi_{i}^{\prime+}\right)-\sum_{i \geqq i} \varphi_{i}^{\prime \prime} \quad(i \geqq 0) .
$$

Then we easily see that

$$
\Psi_{i+1}-\Psi_{i}=\varphi_{i} \text { in }\left(U_{i \tilde{\varepsilon}} \cap U_{i+1 \tilde{\varepsilon}}\right) \times D^{\prime} \quad(i \geqq 0) .
$$

Next we will estimate $\Psi_{i}$ as follows: first, using the expression (b) ${ }_{1}$, we have:

(c) 1

$$
\left\{\begin{array}{c}
\left|\varphi_{i, j}^{+}\right|<(4 / \varepsilon)(2 b+2) \cdot J_{i} \cdot\left(i+2^{-1}\right)^{-j-1}, \text { with } J_{i}:=\alpha_{1}\left(d_{1}^{\alpha_{2}}\right) \cdot\left(i+c+\left|z^{\prime}\right|+1\right)^{\alpha_{2}} \\
\left|\varphi_{i}^{+}(z)\right|<(4 / \varepsilon)(2 b+2) J_{i} \text { for } z \in \boldsymbol{C} \text { satisfying: }|y-a|<b+(\varepsilon / 4), \\
x<i+2^{-1}+(\varepsilon / 4) .
\end{array}\right.
$$

(In the above one can take $d_{1}$ to be, for example, $d_{1}=4$.) The terms $J_{i}, \varepsilon^{-1}$ and $(2 b+2)$ appear from the estimation of $\varphi$ on $\gamma_{i}^{+},|\zeta-z|^{-1}$ and the length of $\gamma_{i}^{+}$.) From $(\mathrm{c})_{1},(\mathrm{~b})_{2}$ we easily get:*)

(c) ${ }_{2}\left|\varphi_{i}^{\prime \prime}(z)\right|<(4 / \varepsilon)(2 b+2) \cdot J_{i} \cdot\left(i^{\prime}-|z-a \sqrt{-1}|\right)^{-1}\left|(z-a \sqrt{-1}) / i^{\prime}\right|^{\beta+1}$

$$
\text { for }|z-a \sqrt{-1}|)<i^{\prime}:=i+2^{-1} \text {. }
$$

Also from (c) $)_{1}$ we have**):

(c) $)_{3}\left|\varphi_{i}^{\prime}(z)\right|<(4 / \varepsilon) J_{i} \cdot \alpha^{\prime} \cdot(|\tilde{z}|+c+1)$ for any $z \in C$, where $\alpha^{\prime}=\tilde{\mathscr{L}}_{1}(\alpha)$, with an el-map $\tilde{\mathscr{L}}_{1}: \boldsymbol{R}^{+2} \rightarrow \boldsymbol{R}^{+2}$, which depends only on $1=\operatorname{dim} \boldsymbol{C}$,

and, from $(c)_{1,3}$, and from $(b)_{2}$, we see that***)****)

(c) $\varphi_{4} \varphi_{i}^{\prime \prime}$ is estimated in the similar manner to (c) $)_{3}$ for $z \in C$ satisfying the same condition as in $(\mathrm{c})_{1}$.

Now, using $(\mathrm{c})_{1-4}$, we have:

(d) $)_{1}\left|\Psi_{i}(\tilde{z})\right|<(1 / \varepsilon) \cdot \alpha^{\prime} \cdot(|\tilde{z}|+c+1)$ in $U_{i \tilde{\varepsilon}}$, where $\alpha^{\prime}=\mathscr{L}_{1}(\alpha)$, with an el-map $\mathscr{L}_{1}$, which depends only on $1=\operatorname{dim} \boldsymbol{C}$.

*) $(c)_{2}$ holds by dropping the term $(4 / \varepsilon)$. The addition of the term $(4 / \varepsilon)$ is convenient for later arguments (cf. $\left.(d)_{1}\right)$.

**) Nothing that $|a|, b<c$, we replace $(b+1)$ by $(c+1)$ and $|z-a \sqrt{-1}|^{j}$ by $\left.(|z|+1)+(c+1)\right)^{j}$ $<\left(2(|z|+1)(c+1)^{\beta}(j \leqq \beta)\right.$. Using this we easily get $(c)_{3}$ from $(c)_{1}$.

***) The estimation in $(c)_{4}$ is less sharper than $(c)_{2}$. But $(c)_{4}$ holds for more general $z \in C$ than $(c)_{2}$. (We use $(c)_{4}$ in the estimation of $\left.(b)_{3}\right)$.

****) Herc we use the similar trick to the ones in $* *)$. 
Actually, we see easily that the first two terms in (b) $)_{3}$ are estimated*) in the similar manner to $(d)_{1}$, by using $(c)_{1,4}$. (Here we use the fact that $\varphi_{i}^{-}(z)$ is estimated in the form of (c) $)_{1}$ in $U_{\tilde{i} \tilde{\varepsilon}}(\tilde{l}<i)$.) Next we write the third term of $(b)_{3}$ in the form:

$$
\sum_{i=1}^{i-1} \varphi_{i}^{\prime \prime+}+\sum_{i \geqq i} \varphi_{i}^{\prime+}, \text { where } \bar{i}:=[i+c+3] .
$$

We estimate the first term of this in the form of $(d)_{1}$, by using $(c)_{4}$. Moreover, the second term, which may be the main term of $(b)_{3}$, is also estimated*) in the manner of $(d)_{1}$, by using $(c)_{2}$. Thus we have $(d)_{1}$.

The arguments hitherto are given for $i \geqq 0$. We repeat the similar argument to get $\Psi^{\prime i}(i \leqq 0)$, which satisfy the similar facts to $(b)_{4},(d)_{1}$. We then define $\widetilde{\Psi}_{i}(i \in \mathbb{Z})$ by

(d) $\widetilde{\Psi}_{i}:=\Psi_{i}-\int \Psi_{0}^{\prime \prime} d \zeta(i \geqq 0)$ and $:=\Psi_{i}^{\prime}+\int \Psi_{0}^{\prime \prime} d \zeta(i \leqq 0)$, where the integral is taken over $\gamma_{0}^{\mp}$ (cf. (a)), and we set: $\Psi_{0}^{\prime \prime}:=(2 \pi \sqrt{-1})^{-1} \cdot\left(\Psi_{0}-\Psi_{0}^{\prime}\right) /(\zeta-z)$. Then $\widetilde{\Psi}_{i}$ satisfies (b) for each $i \in \mathbb{Z}$, and is estimated in the manner in $(\mathrm{d})_{1}$, and we have Proposition 2.

q.e.d.

Proof of Proposition 3. First we check that $(2)_{2}$ follows almost directly from Proposition 2. To see this, letting the map $\pi: \mathscr{A}_{\varepsilon}(\mathbb{C}) \rightarrow \mathbb{Z}$ and the set $\mathscr{A}_{\varepsilon}^{\prime}\left(j ; 2^{-1}\right)(j \in \mathbb{Z})$ have the similar meaning to $(2)_{2}^{\prime},(2)_{0}$, we note:

$$
\pi^{-1}(j)=\mathscr{A}_{\varepsilon}^{\prime}\left(j ; 2^{-1}\right) \text {. }
$$

Now take an element $\varphi$ from the left side of $(2)_{2}$. Then, letting $i_{j}$ denote the injection: $\mathscr{A}_{\varepsilon}^{\prime}\left(j ; 2^{-1}\right) \hookrightarrow \mathscr{A}_{\varepsilon}(\boldsymbol{C})$, we fix an element $\varphi_{j} \in C^{0}\left(\mathscr{A}_{\varepsilon^{\prime}}^{\prime}\left(j ; 2^{-1}\right) \times D^{\prime}, \mathfrak{D}\right)_{\alpha^{\prime}}$ satisfying: $i_{j}^{*} \varphi=\delta \varphi_{j}$, where the estimation: $(\varepsilon ; \alpha) \rightarrow\left(\varepsilon^{\prime} ; \alpha^{\prime}\right)$ is as in $(2)_{1}$. Define an element $\varphi^{\prime} \in C^{0}\left(\mathscr{A}_{\varepsilon^{\prime}}(C) \times D^{\prime}, \mathfrak{D}\right)_{\alpha^{\prime}}$ by $: \varphi^{\prime}=\varphi_{j}$ on $\mathscr{A}_{\varepsilon^{\prime}}^{\prime}\left(j ; 2^{-1}\right) \times D^{\prime}$. Then it is clear that $\varphi-\delta \varphi^{\prime} \in F_{1} C^{1}\left(\mathscr{A}_{\varepsilon^{\prime}}(C) \times D^{\prime}, \mathfrak{D}\right)$, and $\varphi^{\prime}$ is estimated in the manner in $(2)_{2}$. Thus we have $(2)_{2}$.

Next, in order to prove $(2)_{3}$, we first summarize necessary facts from Proposition 2: first for each $p \in \mathbb{Z}^{+}$we set:

(b) ${ }_{1} \quad \mathscr{A}_{\varepsilon}^{\prime}\left(0 ; p+2^{-1}\right):=\left\{V_{j \varepsilon \mid p}\right\}_{j=-\infty}^{\infty}$, where $V_{j \varepsilon \mid p}:=V_{j \varepsilon}$

$$
\cap\left\{z \in \boldsymbol{C} ;|x|<p+2^{-1}+\varepsilon\right\} \text {, with } V_{j \varepsilon} \text { as in }(2)_{2}^{\prime \prime} \text {. }
$$

(Note that the collection $\mathscr{A}_{\varepsilon}^{\prime}$ is of the form in (2) , with the change of $x$ and $y$-coordinates.) Also we define a map**)

*) See the footnote $* * * *)$, p. 544 .

**) For the explicit form of the u-quotient $\left(\mathscr{A}_{\varepsilon}(\mathscr{C}) / \pi\right)_{u}$, see also $(2)_{\eta}^{*}$. 
$(b)_{1}^{\prime}$

$$
\theta_{p}: A_{\varepsilon}^{\prime}\left(0 ; p+2^{-1}\right) \ni V_{j \varepsilon \mid p} \longrightarrow\left(\mathscr{A}_{\varepsilon}(\boldsymbol{C}) / \pi\right)_{u} \ni V_{j \varepsilon} .
$$

Now take an element $\varphi$ from the left side of (2) $)_{3}$. Then, by Proposition 2, we fix an element $\varphi_{p} \in C^{0}\left(\mathscr{A}_{\varepsilon^{\prime}}^{\prime}\left(0 ; p+2^{-1}\right) \times D^{\prime}, \mathfrak{D}\right)_{\alpha^{\prime}}$ satisfying

(b) ${ }_{2} \quad \theta_{p}^{*} \varphi=\delta \varphi_{p}$, and $\left|\varphi_{p}(\tilde{z})\right|<\alpha^{\prime}(|\tilde{z}|+p+1)$, where $\alpha^{\prime}=\mathscr{L}_{1}(\alpha)$, with an el-map $\mathscr{L}_{1}$, which depends only on $l=\operatorname{dim} C$ (cf. (2) $)_{1}$.

Next setting $W_{\varepsilon^{\prime}}(p):=\operatorname{supp} \mathscr{A}_{\varepsilon^{\prime}}^{\prime}\left(0 ; p+2^{-1}\right)$, we define:

$(\mathrm{c})_{1}$

$$
\Psi_{p}:=\varphi_{p+1}-\varphi_{p} \in \Gamma\left(W_{\varepsilon^{\prime}}(p) \times D^{\prime}, \mathfrak{O}\right),
$$

and we expand $\Psi_{p}$ at the origin 0 of $C: \Psi_{p}=\sum_{k} \Psi_{p k} \cdot z^{k}$. Similarly to the proof of Proposition 2, we set:

(c) ${ }_{2}^{\prime} \Psi_{p}^{\prime}:=\sum_{k=0}^{\beta} \Psi_{p k} \cdot z^{k}, \Psi_{p}^{\prime \prime}:=\Psi_{p}-\Psi_{p}^{\prime}$, where $\beta=\left[\alpha_{2}^{\prime}+3\right]$, with the second component $\alpha_{2}^{\prime}$ of $\alpha^{\prime} \in \mathbb{R}_{1}^{+2}$ (cf. also (b) $)_{2}$ in the proof of Proposition 2).

Then setting

$(\mathrm{c})_{3}$

$$
\Phi:=\varphi_{p}-\sum_{s=1}^{p-1} \Psi_{s}^{\prime}+\sum_{s \geqq p} \Psi_{s}^{\prime \prime} \text { in } V_{j \varepsilon^{\prime} \mid p} \times D^{\prime}(\text { for each } j \in \mathbb{Z}),
$$

we easily see that

(c) 4

$$
\Phi \text { is independent of } p \in \boldsymbol{Z}^{+} \text {, and } \varphi=\delta \Phi \text {. }
$$

Now we estimate $\Phi$ as follows: first, using the integral representation: $2 \pi \sqrt{-1}$. $\Psi_{p j}=\int \varphi_{p}(\zeta) \cdot \zeta^{-j-1} d \zeta$, where the integral is taken over the circle of radius $p+2^{-1}$ and center 0 , we have:

$$
\left|\Psi_{p j}\right|<\alpha^{\prime}\left(\left|z^{\prime}\right|+2 p+2\right) \cdot\left(p+2^{-1}\right)^{-j},
$$

and we also have*) (cf. (c) $)_{2}^{\prime}$.

$(\mathrm{d})_{2}\left|\Psi_{p}^{\prime}(\tilde{z})\right|<\alpha^{\prime \prime}(|\tilde{z}|+p+1)$ for each $z \in \boldsymbol{C}$, with $\alpha^{\prime \prime}=\mathscr{L}_{1}^{\prime}(\alpha)$, where the el-map $\mathscr{L}_{1}^{\prime}$ depends only on $1=\operatorname{dim} \boldsymbol{C}$.

From $(\mathrm{d})_{2}$ and $(\mathrm{b})_{2}$ we easily have:*)

(d) $\Psi_{3} \Psi_{p}^{\prime \prime}(\tilde{z})$ is estimated in the similar manner to $(\mathrm{d})_{2}$ for $z \in V_{j \varepsilon^{\prime} \mid p}$.

Moreover, using $(\mathrm{d})_{1}, \Psi_{p}^{\prime \prime}$ is estimated in a sharper form than $(\mathrm{d})_{3}$ :

$$
\left|\Psi_{p}^{\prime \prime}(\tilde{z})\right|<\alpha^{\prime}\left(\left|z^{\prime}\right|+2 p+2\right)\left(p+2^{-1}\right)^{-\beta} \cdot|z|^{\beta+1} \text { for }|z|<p-2^{-1} \text {. }
$$

Finally taking a point $z \in \boldsymbol{C}$, we choose an element $(j, p) \in \mathbb{Z} \times \boldsymbol{Z}^{+}$satisfying $z \in V_{j \varepsilon^{\prime} \mid p}-\bar{V}_{j \varepsilon^{\prime} \mid p-1}$, and we set: $m:=|j|+p+3$. We write*) the third term of

*) Also compare similar arguments in the proof of Proposition 2. 
(c) $)_{3}$ as: (c) ${ }_{3}^{\prime} \sum_{s=p}^{m}+\sum_{s \geqq m+1}$. We then estimate*) the first two terms of (c) and the first term of $(\mathrm{c})_{3}^{\prime}$ by $(\mathrm{d})_{2}$ and $(\mathrm{b})_{2}$. Also we estimate the second term of $(c)_{3}^{\prime}$ by $(d)_{4}$. Then we have the desired estimation of $\Phi$ in $(3)_{3}$. q.e.d.

3. Proof of Lemma $6.3^{1}$. Let $\mathbb{A}^{1}$ be the collection $\left\{A_{\tilde{k}}(U) ;(U ; \tilde{k})\right.$ $\left.\in \operatorname{Ouv}(\mathbb{C}) \times\left(\mathbb{Z}^{+} \cup 0\right)^{2}\right\} \quad\left(\right.$ cf. $\left.(6.12)_{2}\right)$. For each $\mathscr{A}_{i}: \mathscr{A}_{\hat{h}}(U) \in \mathbb{A}^{1}, \tilde{k}=\left(k, k^{\prime}\right)$, we set $\mathscr{B}_{i}:=\left[0, k^{\prime}\right]$, and we denote by $\pi_{\lambda}$ the map: $\mathscr{A}_{\lambda} \rightarrow \mathscr{B}_{\lambda}$ as in $(\text { a })_{2}$, n.1. We then set:

(3) $\mathscr{A}^{1}:=\left(\mathscr{A}^{1}, \mathbb{B}^{1}, \pi^{1}\right)$, with $\mathbb{B}^{1}=\left\{\mathscr{B}_{\lambda}\right\}_{\lambda}, \pi^{1}:=\left\{\pi_{\lambda}\right\}_{\lambda}$, where $\mathscr{O}_{\lambda}$ runs through 1 '.

Then, from Lemma 7.5, we scc easily that the check of the following suffice for the proof*) of Lemma $6.3^{1}$ :

(3) ${ }_{1}^{0, q} \quad Z_{1}^{0, q}\left(\mathscr{A}_{\mu} \times D^{\prime}, \mathfrak{D}\right)_{\alpha}^{m} \subset B_{0}^{0, q}\left(\mathscr{A}_{\mu} \times D^{\prime}, \mathfrak{D}\right)_{\alpha^{\prime}}^{m^{\prime}} \quad$ with the estimation:

(3) $\left.{ }_{1}^{q} \quad Z_{\infty}^{q, 0}\left(\mathscr{A}_{\lambda} \times D^{\prime}, \mathfrak{D}\right)_{\alpha}^{m} \subset \delta C^{q-1}\left(\mathscr{A}_{\lambda} \times D^{\prime}, \mathfrak{D}\right)_{\sigma^{\prime}}^{m^{\prime}}\right\}(m ; \alpha) \longrightarrow\left(m^{\prime} ; \alpha^{\prime}\right)$

as in Proposition 1.

(Here $\mathscr{A}_{\lambda}$ is in $\mathscr{A}^{1}$ and $\mathscr{A}_{\mu}$ is in $\mathscr{F}_{1}^{0} \mathscr{A}_{\lambda_{\mu}}$, with an element $\mathscr{A}_{\lambda_{\mu}} \in \mathscr{A}^{1}$.) But (left side of $\left.(3)_{1}^{q}\right) \cong 0(q \geqq 2)$ and $\cong Z^{1}\left(\left|\left(\mathscr{A}_{\lambda m} / \pi_{\lambda}\right)_{u}\right| \times D^{\prime}, \mathfrak{D}\right)_{\alpha}(q=1)$ (cf. (1) $\left.)_{2}\right)$. Thus, applying $(1)_{2}$, Proposition 1 to $Z^{1}(\cdots)$, we have $(3)_{1}^{4}$. On the otherhand remarking that $\# \pi_{\lambda_{\mu}}\left(\mathscr{A}_{\mu}\right)=1$, one can write $\mathscr{A}_{\mu}$ as $\mathscr{A}_{\mu}=\mathscr{A}_{0, k^{\prime \prime}}(U)$, with an element $k^{\prime \prime} \in \mathbb{Z}^{+}$, and applying (1) $)_{1}$, Proposition 1 to $\mathscr{A}_{\mu}$, we have (3) $)_{1}^{0 . q}$.

q.e.d.

4. Proof of Theorem 6.1 $1^{1}$ Here we derive Theorem 6.1 $1^{1}$ from Proposition 2 and Proposition 3: letting the element $\varepsilon \in(0,1 / 2)$ and the parameter $\left(D^{\prime} ; \alpha\right) \in \operatorname{Ouv}\left(\mathbb{C}^{n}\right) \times \boldsymbol{R}_{1}^{+2}$ be as in Proposition 3, we have:

Proposition 4. We have the inclusion:

(4) ${ }_{1} Z^{q}\left(\mathscr{A}_{\varepsilon}(\boldsymbol{C}) \times D^{\prime}, \mathfrak{D}\right)_{\alpha} \subset \delta C^{q-1}\left(\mathscr{A}_{\varepsilon^{\prime}}(\mathbb{C}) \times D^{\prime}, \mathfrak{D}\right)_{\alpha^{\prime}}$, where the estimation: $(\varepsilon ; \alpha)$ $\rightarrow\left(\varepsilon^{\prime} ; \alpha^{\prime}\right)$ is as in Proposition 3. (Also $\mathscr{A}_{\varepsilon}(\mathbb{C})=\mathscr{A}_{1 \varepsilon}(\mathbb{C})$ as in Proposition 3.)

Proof. First, if $q \geqq 4$, the left side of $(4)_{1} \cong 0$. Also we see very easily that, for $q=3$, (4), holds by taking $(\varepsilon ; \alpha) \rightarrow\left(\varepsilon^{\prime} ; \alpha^{\prime}\right)$ to be the identity. Moreover, if $q=1$, we get easily (4) from (2) ${ }_{2,3}$ in Proposition 3. (This casc is, of course, the main part of Proposition 4.) Finally, assuming that $q=2$, we summarize the key fact for the proof of $(4)_{1}$ : first we let the map $\pi: \mathscr{A}_{c}(\mathbb{C}) \rightarrow Z$ be as in $(2)_{2}$. Then we easily have:

(a) $Z_{1}^{1}, 1\left(\tilde{\mathscr{A}}_{\varepsilon}, \mathfrak{D}\right)_{\alpha} \cong Z^{2}\left(\mathscr{A}_{\varepsilon}, \mathfrak{D}\right)_{\alpha}$, and $Z_{1}^{2,0}\left(\tilde{\mathscr{A}}_{\varepsilon}, \mathfrak{D}\right)_{\alpha} \cong 0$, where we set $\tilde{\mathscr{A}}_{\varepsilon}:=$

\footnotetext{
*) For the sets of the cochains here, see $(0)_{1}$, Proposition 1 and $(6.10)_{1}$. .
} 
$\mathscr{A}_{\varepsilon}(\boldsymbol{C}) \times D^{\prime}$, and the sets $Z_{1}^{1}, 1, \ldots$, are, as in Proposition 3 , defined by the filtration $F_{1}$, which is induced from the map $\pi$ (cf. (2) $)_{2}$ and Definition 6.3).

To analyze the set $Z_{1}^{1,1}$, we fix a subset $\mathscr{B}=\{j, j+1\} \subset \boldsymbol{Z}^{2}$, and we set:

(b) ${ }_{1} \quad \mathscr{A}_{\varepsilon \tilde{j}}:=\pi^{-1}(\tilde{j})(\tilde{j}=j$ or $j+1), \tilde{\mathscr{A}}_{\varepsilon \mathscr{B}}:=\pi^{-1}(\mathscr{B})\left(=\mathscr{A}_{\varepsilon j} \Perp \mathscr{A}_{\varepsilon j+1}\right)$, and $\tilde{\mathscr{A}}_{\varepsilon j}:=$ $\mathscr{A}_{\varepsilon j} \times D^{\prime}, \ldots$ We then define the following subset of $Z_{1}^{1,1}\left(\mathscr{A}_{\varepsilon \mathscr{G}}, \mathfrak{D}\right)$ :

(b) $Z_{1}^{\prime}{ }^{t, 2-t}\left(\tilde{\mathscr{A}}_{\varepsilon \mathscr{B}}, \mathfrak{D}\right)_{\alpha}:=\left\{\varphi \in G_{t} Z_{1}^{1}, 1\left(\tilde{\mathscr{A}_{\varepsilon \mathscr{B}}}, \mathfrak{D}\right)_{\alpha}: \delta \varphi \in G_{t+1} Z_{1}^{1}, 1\right\}$, where the filtration $G_{t}$ is defined by the order: $j<j+1$ in $\mathscr{B}$ (Definition 7.1).

Then, defining the collection $\mathscr{A}_{\tilde{\varepsilon}}\left(j+2^{-1} ; \tilde{\varepsilon}\right)$, where $\tilde{\varepsilon}=\varepsilon / 2$, by $(2)_{0}$, we easily have the following from Proposition $\left.7.2^{*}\right)$ :

$$
Z_{1}^{\prime 1,1}\left(\tilde{\mathscr{A}}_{\varepsilon \mathscr{B}}, \mathfrak{D}\right)_{\alpha} \cong Z^{1}\left(\tilde{\mathscr{A}}_{\varepsilon}\left(j+2^{-1} ; \tilde{\varepsilon}\right) \times D^{\prime}, \mathfrak{D}\right)_{\alpha}
$$

Apply Proposition 7.2 to the right side. Then, by Proposition 7.2, we have:

(c) $Z_{1}^{\prime 1,1}\left(\tilde{\mathscr{A}_{\varepsilon \mathscr{B}}}, \mathfrak{D}\right)_{\alpha} \subset \delta G_{1} C^{1}\left(\tilde{\mathscr{A}_{\varepsilon^{\prime} \mathscr{B}}}, \mathfrak{D}\right)_{\alpha^{\prime}}$, where the estimation: $(\varepsilon ; \alpha) \rightarrow\left(\varepsilon^{\prime} ; \alpha^{\prime}\right)$ is as in Proposition 4.

On the otherhand, for an element $D \in \mathscr{A}_{\varepsilon j+1}$, the localization $\mathscr{A}_{\varepsilon \mathscr{B}, D}$ (Definition 7.2) is easily seen to be of the form: $\mathscr{A}_{\varepsilon \mathscr{G}, D}=\left\{A_{j}\right\}_{j=1}^{3}$, where the elements $A_{j}$ $\in \pi^{-1}(j)$ are characterized by $A_{j} \cap|D| \neq \phi$. Applying Proposition 1 to $\mathscr{A}_{\varepsilon \mathscr{G}, D}$, we have: $Z^{1}\left(\mathscr{A}_{\varepsilon \mathscr{G}, D} \times D^{\prime}, \mathfrak{D}\right)_{\alpha} \subset \delta C^{0}\left(\mathscr{A}_{\varepsilon \mathscr{B}, D} \times D^{\prime}, \mathfrak{D}\right)_{\alpha^{\prime}}$, where the estimation: $(\varepsilon ; \alpha) \rightarrow\left(\varepsilon^{\prime} ; \alpha^{\prime}\right)$ is as in Proposition 4. (The estimation is first given in the form of Proposition 1, which is applied to $A_{\varepsilon \mathscr{G} . D}$. From the above explicit form of $\mathscr{A}_{E \mathscr{B}, D}$ (cf. also Definition 7.2), the estimation is rewritten in the form of Proposition 4.) Applying Corollary 7.1 to the inclusion just above (cf. also Definition 7.3), we have:

$(\mathrm{c})_{2}$

$$
Z_{1}^{\prime 0,2}\left(\mathscr{A}_{\varepsilon \mathscr{B}} \times D^{\prime}, \mathfrak{D}\right) \subset \delta C^{1}\left(\mathscr{A}_{\varepsilon^{\prime} \mathscr{B}} \times D^{\prime}, \mathfrak{D}\right)_{\alpha^{\prime}}+G_{1} C^{2}\left(\mathscr{A}_{\varepsilon^{\prime} \mathscr{B}} \times D^{\prime}, \mathfrak{D}\right)_{\alpha^{\prime}} .
$$

Using the similar argument to Lemma 7.1 and Corollary 7.2, we have the following from $(\mathrm{c})_{1,2}$ :

$$
Z_{1}^{1,1}\left(\mathscr{A}_{\varepsilon \mathscr{B}} \times D^{\prime}, \mathfrak{D}\right)_{\alpha} \subset \delta F_{1} C^{1}\left(\mathscr{A}_{\varepsilon^{\prime} \mathscr{B}} \times D^{\prime}, \mathfrak{D}\right)_{\alpha^{\prime}} .
$$

(To get (c) $)_{3}$ we also remark that $G_{t} Z_{1}^{1,1} \cong 0$ if $t \geqq 2$.) Finally, from the explicit form of $\pi^{-1}(B)$, we easily see that

(d) $\quad\left\{\pi^{-1}(\mathscr{B}) ; \mathscr{B}=\{j, j+1\}\right\}_{j=-\infty}^{\infty}$ is admissible (cf. Definition 6.4).

*) See also $(7.2)_{5}$ for the explicit form of $Z_{1}^{\prime 1,1}$. Writing the complex $J^{*}$ in $(7.2)_{5}$ explicitly (in the present situation), we easily get $(b)_{3}$. 


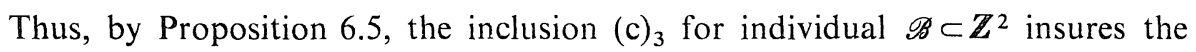
similar inclusion for the 'original collection' $\mathscr{A}_{\varepsilon}(\mathbb{C})$ :

(d) ${ }_{2}$

$$
Z^{2}\left(\mathscr{A}_{\varepsilon}(\boldsymbol{C}) \times D^{\prime}, \mathfrak{D}\right)_{\alpha} \subset \delta C^{1}\left(\mathscr{A}_{\varepsilon^{\prime}}(\mathbb{C}) \times D^{\prime}, \mathfrak{D}\right)_{\alpha^{\prime}}
$$

which is the desired inclusion in $(4)_{1}$.

q.e.d.

Finally, Theorem 6.1 is derived from Proposition 4 as follows: take an clement $k \in \mathbb{Z}^{+}$, and we set $\eta:=(k ; \varepsilon), \eta^{\prime}:=\left(k ; \varepsilon^{\prime}\right)$, where the elements $\varepsilon, \varepsilon^{\prime}$ $\in(0,1 / 2)-Q$ are as in Proposition 4. Letting $\pi_{k}$ denote the biregular map: $\mathbb{C} \ni z \rightarrow C \ni k z$, we have: $\pi_{h}: \mathscr{N}_{\eta} \simeq . \mathscr{A}_{1 s}(\mathbb{C}) \ldots$. Using this we have the following from a very simple observation:

(d) ${ }_{3} \quad Z^{q}\left(\mathscr{A}_{\eta}(\boldsymbol{C}) \times D^{\prime}, \mathfrak{D}\right)_{\alpha} \subset \delta C^{q-1}\left(\mathscr{A}_{\eta^{\prime}}(\mathbb{C}) \times D^{\prime}, \mathfrak{D}\right)_{\tilde{\alpha}^{\prime}}$, where $\tilde{x}^{\prime}=\left(\alpha_{1}^{\prime} k^{\alpha_{2}^{\prime}}, \alpha_{2}^{\prime}\right)$, with the element $\alpha^{\prime}=\left(\alpha_{1}^{\prime}, \alpha_{2}^{\prime}\right)$ as in Proposition 4.

Moreover, if an element $k$ satisfies: $k>4 / \varepsilon$, there is*) a refining map $s: \mathscr{A}_{k \tilde{\varepsilon}}(\mathbb{C})$ $\hookrightarrow \mathscr{A}_{1 \varepsilon}(\boldsymbol{C})$, with $\tilde{\varepsilon}=1 / 4$. Using this refining map, one can easily rewrite $(4)_{2}$ in the form of Theorem $6.1^{1}$, and we finish the proof of Theorem $6.1^{1}$.

\section{B}

1. Let $\mathbb{R}$ be a euclid line, $U=(a, a+b)(b>0)$ an interval in $\mathbb{R}$, and let $\mathscr{A}_{\eta}(U)$, where $\eta=(k ; \varepsilon) \in\left(\mathbb{Z}^{+} \cup 0\right) \times(0,1 / 2)$, be the elementary covering of $U$ of size $\eta$ (cf. $\left.(6.1)_{1}\right)$. For an element $\eta^{\prime}:=\left(k^{\prime}: \varepsilon^{\prime}\right) \in \mathbb{Z}^{+} \times(0,1 / 2)$ and for each $P \in U$, we set $\left(m \in \mathbb{R}_{1}^{+}\right)$:

$$
U_{\eta^{\prime}}(P)_{m}:=\left\{x \in \boldsymbol{R} ;|x-x(P)|<\tilde{\varepsilon}^{\prime} \cdot m b / k^{\prime}\right\}, \text { with } \tilde{\varepsilon}^{\prime}:=\varepsilon^{\prime}+2^{-1},
$$

and we define:

$$
\mathscr{B}_{\eta^{\prime}}(U)_{m}:=\left\{U_{\eta^{\prime}}(P)_{m} ; P \in \bar{U}\right\}
$$

Now we assume:

$$
\varepsilon^{\prime} \geqq \varepsilon \text { and } k^{\prime}>4 m \tilde{k} / \varepsilon, \text { with } \tilde{k}:=\max (1, k) .
$$

Then we check easily the following:

$$
\text { there is a refining map } s: \mathscr{B}_{\eta^{\prime}}(U)_{m} \hookrightarrow_{\mathscr{A}_{11}}(U) \text {. }
$$

The estimations on the refining maps in Sections 6 and 7 are derived from the above simple fact:

*) cf. (5) $)_{2}$ in Part B of this appendix. 
(i) Assume that $k>0$ and that (5) holds. Then, for the elementary coverings $\mathscr{A}_{\eta}\left(\mathbb{C}^{n}\right), \mathscr{A}_{\eta^{\prime}}\left(\boldsymbol{C}^{n}\right)(n \geqq 1)$ (cf. Definition $\left.6.1_{2}\right)$, we easily have:

$$
\text { there is a refining map } s: \mathscr{A}_{\eta^{\prime}}\left(\mathbb{C}^{n}\right)_{m} \hookrightarrow \mathscr{A}_{\eta}\left(\mathbb{C}^{n}\right) \text {. }
$$

(For the collections $\mathscr{A}_{\eta}\left(\mathbb{C}^{n}\right)_{m}, \ldots$, see $(6.10)_{2}$.)

(ii) Next take elements $k=\left(k_{j}\right), k^{\prime}=\left(k_{j}^{\prime}\right)(1 \leqq j \leqq 2 n) \in\left(\mathbb{Z}^{+} \cup 0\right)^{2 n}$, and we set $\eta=(k ; \varepsilon), \eta^{\prime}:=\left(k^{\prime} ; \varepsilon^{\prime}\right)$. Taking an elementary figure $U$ (cr. n.1, $\S 6.1$ ) in $C^{n}$ we denote $\mathscr{A}_{n}(U), \mathscr{A}_{\eta^{\prime}}(U)$ the elementary coverings of $U$ of size $\eta, \eta^{\prime}$. Then if

$(5)_{3}^{\prime} \quad \varepsilon^{\prime} \geqq \varepsilon, k_{j}^{\prime}>4 m \tilde{k}_{j} / \varepsilon$, with $\tilde{k}_{j}:=\max \left(1, k_{j}\right)(1 \leqq j \leqq 2 n)$, then we have:

$(5)_{3}$ there is a refining map $s: \mathscr{A}_{\eta^{\prime}}(U) \hookrightarrow \mathscr{A}_{\eta}(U)$.

(iii) Thirdly take elements $\xi=(\zeta ; \varepsilon), \xi^{\prime}=\left(\zeta^{\prime} ; \varepsilon^{\prime}\right) \in \mathbb{Z}^{+2} \times(0,1 / 2)$ and an elementary figure*' $U^{\prime}$ of $\mathbb{C}^{n^{\prime}}$, and let $\mathscr{A}_{\xi}^{\prime}:=\mathscr{A}_{\xi}^{\prime}\left(\mathbb{C}^{n} \times U^{\prime}\right), \mathscr{A}_{\xi^{\prime}}^{\prime}:=\mathscr{A}_{\xi}^{\prime}\left(\mathbb{C}^{n} \times U^{\prime}\right)$ be the p.g. elementary coverings of $\mathbb{C}^{n} \times U^{\prime}$ of size $\xi$, $\check{\zeta}^{\prime}$ (cf. Definition 6.2). We summarize some estimations on the coverings $\mathscr{A}_{\xi}^{\prime}, \mathscr{A}_{\xi^{\prime}}^{\prime}$. For this we first recall that $\mathscr{A}_{\xi}^{\prime}$ is the disjoint union $\mathscr{A}_{\xi}^{\prime}=\Perp_{I \in Z^{2 n}} \mathscr{A}_{\xi I}^{\prime}$, where $\mathscr{A}_{\zeta I}^{\prime}:=\mathscr{A}_{\zeta}\left(S_{I \varepsilon} \times U^{\prime}\right)$ denotes the elementary covering of the elementary figure $S_{I \varepsilon} \times U^{\prime}$ of size $\zeta$. $(|I|+1)$ (cf. $(6.3)_{5}$.) See also $(6.3)_{1}$ for the elementary figure $S_{I \varepsilon}$.) For each $A^{\prime} \in \mathscr{A}_{\xi}^{\prime}$, let $P$ be the center of $\operatorname{pr}\left(A^{\prime}\right)$, where $\operatorname{pr}:=$ projection: $\mathbb{C}^{n} \times \mathbb{C}^{n^{\prime}} \rightarrow \mathbb{C}^{n}$. We then attach to $A^{\prime}$ an element $I=I_{P} \in \mathbb{Z}^{2 n}$ satisfying $P \in S_{I \varepsilon}$. Also we set $I^{\prime}:=\pi_{\xi}\left(A^{\prime}\right)$, with the map $\pi_{\xi}: \mathscr{A}_{\xi}^{\prime} \rightarrow \mathbb{Z}^{2 n}$ (cf. (6.3) $)$. Then we easily have the inequality:

$(5)_{4}^{\prime \prime} \quad c^{-1} \cdot\left(\left|I^{\prime}\right|+1\right)<(|I|+1)<c(|I|+1)$, with an element $c=c_{n} \in \mathbb{R}_{1}^{+}$, which depends only on $n$.

Now writing $\zeta, \zeta^{\prime} \in \mathbb{Z}^{+2}$ as $\left(\zeta_{1}, \zeta_{2}\right)$ and $\left(\zeta_{1}^{\prime}, \zeta_{2}^{\prime \prime}\right)$, we easily see from $(5)_{4}$ that the inequalities:

$(5)_{4}^{\prime}$

$$
\varepsilon \geqq \varepsilon^{\prime}, \zeta_{2}^{\prime} \geqq \zeta_{2} \text { and }(4 m / \varepsilon) \zeta_{1} c^{\zeta_{2}} \leqq \zeta_{1}^{\prime}
$$

insure the existence of a refining map:

$$
s: \mathscr{A}_{\xi}^{\prime} \hookrightarrow \mathscr{A}_{\xi}^{\prime} \text { satisfying } s(A)=I \text { for any } A^{\prime} \in \mathscr{A}_{\xi}^{\prime} .
$$

We use $(5)_{4}$ in getting Proposition 6.4' and in (7.9) $)_{3}$. (When we get Proposition 6.4', we take $I$ to be: $\omega \cdot u \cdot v(A)$, where the refining maps $v, u$ are as in Proposition 6.4' and $\omega$ denotes the map: $\mathscr{A}_{1 \varepsilon}\left(\mathbb{C}^{n}\right) \ni A \rightarrow \mathbb{Z}^{2 n} \ni I=$ center of $A$. When

*) As in Theorem 6.2 we assume that $d_{U^{\prime}}^{-}-1$. 
we are concerned with $(7.9)_{3}$, we lake $m \in \mathbb{R}_{1}^{+}$to be $: m=2 c_{n, q} \cdot a_{\xi}$, with the ratio number $a_{\xi}$ of $A_{\xi}^{\prime}$ and the element $c_{n, q} \in \mathbb{R}_{1}^{+2}$ as in (7.9) $)_{3}$. Moreover, we define $I \in \mathbb{Z}^{2 n}$ to be $: I=\pi_{\xi}(A)$, with the map $\pi_{\xi}: \mathscr{A}_{\xi}^{\prime} \rightarrow \mathbb{Z}^{2 n}$ as in $(6.7)_{8}$.)

(iv) Finally we check (6.6) $)_{1,2}$, which is the key fact in the proof of Proposition 6.1 : letting the p.g. covering $\mathscr{A}_{\sigma}\left(\mathbb{C}^{n} \times U^{\prime}\right)$ and the p.g. elementary covering $\mathscr{A}_{\xi}^{\prime}\left(\mathbb{C}^{n} \times U^{\prime}\right)$ be as in Proposition 6.1, we let the elementary figure $S_{I, \varepsilon}\left(I \in \mathbb{Z}^{2 n}\right)$ be the one defined in the manner as in (6.3) $)_{1}^{\prime}$. Also we use the symbols $\mathscr{A}_{\sigma}\left(S_{I \varepsilon}\right.$ $\left.\times U^{\prime}\right)$ and $\mathscr{A}_{\xi}^{\prime}\left(S_{I \varepsilon} \times U^{\prime}\right)$ for the p.g. covering of $S_{I \varepsilon} \times U^{\prime}$ of size $\sigma$ and for the elementary covering, which is defined in the manner as in (6.3) $)_{1}$. For the proof of the cofinal relation between the p.g. and p.g. elementary coverings $\mathscr{A}_{\sigma}\left(\mathbb{C}^{n} \times U^{\prime}\right)$ and $\mathscr{A}_{\xi}^{\prime}\left(\mathbb{C}^{n} \times U^{\prime}\right)$ as in $(6.6)_{1,2}$, it suffices to show the similar cofinal relation between $\mathscr{A}_{\sigma}\left(S_{I \varepsilon} \times U^{\prime}\right)$ and $\mathscr{A}_{\xi}^{\prime}\left(S_{I \varepsilon} \times U^{\prime}\right)$. But, from (5) $)_{1}$, we easily see that the relation $(6.6)_{1}$ in the proof of Proposition 6.1:

(a) $\sigma>K^{\zeta_{2}} \cdot \mathscr{L}_{n}(\zeta)\left(\operatorname{resp} . \zeta>M_{n}\left(1 / d_{U^{\prime}}\right) \cdot \mathscr{L}_{n}(\sigma)\right)$, with $K:=1+\sup _{z^{\prime} \in U^{\prime}}\left|z^{\prime}\right|$, where the positive monomial $M_{\tilde{n}}$ and the el-map $\mathscr{L}_{\tilde{n}}: \mathbb{R}^{+2} \rightarrow \mathbb{R}^{+2}$ depend only on $\tilde{n}=\left(n, n^{\prime}\right)$,

insures the existence of refining maps:

(b) $s_{I}: \mathscr{A}_{\sigma}\left(S_{I, \varepsilon} \times U^{\prime}\right) \hookrightarrow \mathscr{A}_{\xi}\left(S_{I, \varepsilon} \times U^{\prime}\right)\left(\right.$ resp. $\left.s_{I}^{\prime}: \mathscr{A}_{\xi}\left(S_{I, \varepsilon} \times U^{\prime}\right) \hookrightarrow \mathscr{A}_{\sigma}\left(S_{I, \varepsilon} \times U^{\prime}\right)\right)$.

From this we have refining maps:

(c) $s: \mathscr{A}_{\sigma}\left(\mathbb{C}^{n} \times U^{\prime}\right) \hookrightarrow \mathscr{A}_{\zeta}\left(\mathbb{C}^{n} \times U^{\prime}\right)\left(\right.$ resp. $\left.s^{\prime}: \mathscr{A}_{\xi}\left(\mathbb{C}^{n} \times U^{\prime}\right) \hookrightarrow \mathscr{A}_{\sigma}\left(\mathbb{C}^{n} \times U^{\prime}\right)\right)$, and we have $(6.6)_{1,2}$.

\section{References}

[1] Cartan, H., Sur les fonctions de plusiers complexes; les espaces analytiques, Proc. Int. Cong. of Math., Edinburgh, 1958.

[2] Cornalba, M. and Griffiths, P. A., Analytic cycles and vector bundles on non compact algebraic varieties, Invent. Math., 28 (1975), 11-106.

[3] Fujiki, A., Poincaré and Dolbeaut lemmas on analytic spaces, Problems on analytic varieties and stratified spaces, RIMS Kokyuroku, 372, Kyoto Univ., 1979, 1-16 (in Japanese).

[4] Gunning, R. and Rossi, H., Analytic functions of complex varicbles, Van Norstand, Princeton, New Jersey, 1966.

[5] Grothendieck, A., On the de Rham cohomology of algebraic varieties, Publ. Math. I.H.E.S., 29 (1966), 95-103.

[6] Kamiya, S., Some topics in P.G.-geometry, Problems on analytic varieties and stratified spaces, RIMS Kokyuroku, 372, Kyoto Univ., 1979, 141-156. 
[7] Hartshorne, R., On the de Rham cohomology of algebraic varieties, Publ. Math. I.H.E.S., 45 (1975), 6-99.

[8] Hörmander, L., $L^{2}$-estimations and existence theorems for $\bar{\partial}$-operator, Acta. Math., 113 (1965), 89-152.

[9] — An introduction to complex analysis in several variables, Van tostrand, 1966.

[10] Lojasiewicz, S., Sur les problems de divisions, Studia Math., 18 (1956), 87-136.

[11] Maltsioniosts, G., Gaga affine (d'apres P. Deligne), Seminaire de geometrie algebrique, Soc. Math. de France, Asterisque, 17 (1974), 141-160.

[12] Nagata, M., Local rings, John Wiliey, 1962.

[13] Noumi, M., Openness condition for filtered complexes, Problems on analytic varieties and stratified spaces, RIMS Kokyuroku, 372, Kyoto Univ., 1979, 171-178.

[14] Oka, K., Sur les fonctions analytiques de plusieurs variables, Iwanami Shoten, Tokyo, 1961.

[15]*) Sasakura, N., Complex analytic de Rham cohomology, I-IV, Proc. Japan Acad., 49 (1973), 718-722, 50 (1974), 292-295, 51 (1975), 7-11, 51 (1975), 535-539.

[16] — Divisible and asymptotic behaviors of coherent sheaves, RIMS Kokyuroku, 192, Kyoto Univ., 1973, 37-327.

[17] —- Polynomial growth $C^{\infty}$-de Rham cohomology and normalized series of prestratified spaces, Complex analysis and algebraic geometry, Iwanami Shoten, 1975, 383-395.

[18] - De Rham cohomologies and stratifications (to appear).

[19] Serre, J.-P., Algebre locale et multiplicities, Lecture Notes in Math., 11, Springer, 1965.

[20] Weil, A., Sur les theoreme de de Rham, Comm. Math. Helv., 26 (1952), 119-145.

[21] Yamaguchi, H., Gaga affine by polynomial growth, Problems on analytic varieties and stratified spaces, RIMS Kokyuroku, 372, Kyoto Univ., 1979, 157-170.

[22] Godement, R., Topologie algebrique et theorie des faisceaux, Actualites Sci. Ind., Hermann, Paris, 1958.

*) 'Complex analytic de Rham cohomology, I-IV' will be abbreviated as $[15]_{1}-[15]_{4}$. 